ORNL-6970

(Edition 23 of ORNL-5198)

Center for Transportation Analysis

Engineering Science \& Technology Division

\title{
TRANSPORTATION ENERGY DATA BOOK: EDITION 23
}

\author{
Stacy C. Davis \\ Susan W. Diegel \\ Oak Ridge National Laboratory
}

October 2003

\author{
Prepared for the \\ Office of Planning, Budget Formulation and Analysis \\ Energy Efficiency and Renewable Energy \\ U.S. Department of Energy
}

Prepared by the

Oak Ridge National Laboratory

Oak Ridge, Tennessee 37831-6073

Managed by

UT-BATTELLE, LLC

for the

U.S. DEPARTMENT OF ENERGY

under Contract No. DE-AC05-00OR22725 


\section{DOCUMENT AVAILABILITY}

Reports produced after January 1, 1996, are generally available free via the U.S. Department of Energy (DOE) Information Bridge:

Web site: http://www.osti.gov/bridge

Reports produced before January 1, 1996, may be purchased by members of the public from the following source:

National Technical Information Service

5285 Port Royal Road

Springfield, VA 22161

Telephone: 703-605-6000 (1-800-553-6847)

TDD: 703-487-4639

Fax: 703-605-6900

E-mail: info@ntis.fedworld.gov

Web site: http://www.ntis.gov/support/ordernowabout.htm

Reports are available to DOE employees, DOE contractors, Energy Technology Data Exchange (ETDE) representatives, and International Nuclear Information System (INIS) representatives from the following source:

Office of Scientific and Technical Information

P.O. Box 62

Oak Ridge, TN 37831

Telephone: $865-576-8401$

Fax: 865-576-5728

E-mail: reports@adonis.osti.gov

Web site: http://www.osti.gov/contact.html

This report was prepared as an account of work sponsored by an agency of the United States Government. Neither the United States government nor any agency thereof, nor any of their employees, makes any warranty, express or implied, or assumes any legal liability or responsibility for the accuracy, completeness, or usefulness of any information, apparatus, product, or process disclosed, or represents that its use would not infringe privately owned rights. Reference herein to any specific commercial product, process, or service by trade name, trademark, manufacturer, or otherwise, does not necessarily constitute or imply its endorsement, recommendation, or favoring by the United States Government or any agency thereof. The views and opinions of authors expressed herein do not necessarily state or reflect those of the United States Government or any agency thereof. 
Users of the Transportation Energy Data Book are encouraged to comment on errors, omissions, emphases, and organization of this report to one of the persons listed below. Requests for additional complementary copies of this report, additional data, or information on an existing table should be referred to Ms. Stacy Davis, Oak Ridge National Laboratory.

Stacy C. Davis

Oak Ridge National Laboratory

National Transportation Research Center

2360 Cherahala Boulevard

Knoxville, Tennessee 37932

Telephone:

(865) 946-1256

FAX:

(865) 946-1314

E-mail: DAVISSC@ornl.gov

Web Site Location: www-cta.ornl.gov

Philip D. Patterson

Office of Planning, Budget Formulation and Analysis

Energy Efficiency and Renewable Energy

Department of Energy, EE-30

Forrestal Building, Room 5F-034

1000 Independence Avenue, S.W.

Washington, D.C. 20585

Telephone:

(202) 586-9121

FAX:

(202) 586-9811

E-mail:

PHILIP.PATTERSON@hq.doe.gov

Web Site Location: www.eere.energy.gov

This and previous editions of the

Transportation Energy Data Book

can be found on the web at:

www-cta.ornl.gov/data 



\section{TABLE OF CONTENTS}

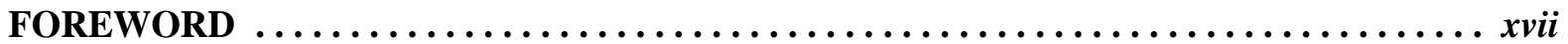

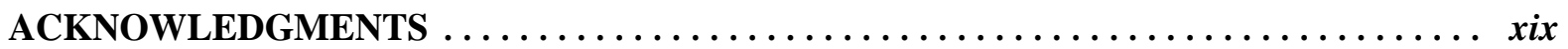

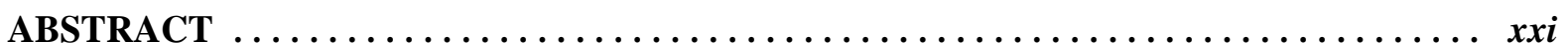

INTRODUCTION $\ldots \ldots \ldots \ldots \ldots \ldots \ldots \ldots \ldots \ldots \ldots \ldots \ldots \ldots \ldots \ldots \ldots \ldots \ldots \ldots \ldots \ldots \ldots \ldots \ldots$

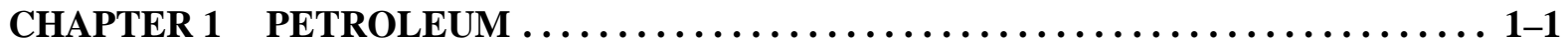

Table $1.1 \quad$ World Fossil Fuel Potential $\ldots \ldots \ldots \ldots \ldots \ldots \ldots \ldots \ldots \ldots \ldots \ldots \ldots \ldots$

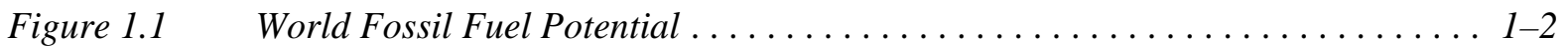

Table $1.2 \quad$ World Crude Oil Production, $1960-2002 \ldots \ldots \ldots \ldots \ldots \ldots \ldots \ldots \ldots$

Table $1.3 \quad$ World Petroleum Production, $1973-2002 \ldots \ldots \ldots \ldots \ldots \ldots \ldots \ldots \ldots \ldots$

Table $1.4 \quad$ World Petroleum Consumption, $1960-2002 \ldots \ldots \ldots \ldots \ldots \ldots \ldots \ldots \ldots$

Figure $1.2 \quad$ World Oil Reserves, Production and Consumption, $2002 \ldots \ldots \ldots \ldots \ldots$

Table $1.5 \quad$ World Oil Reserves, Production and Consumption, $2002 \ldots \ldots \ldots \ldots \ldots \ldots$

Figure $1.3 \quad$ World Natural Gas Reserves, Production, and Consumption, 2000 . . . . . . . 1-7

Table $1.6 \quad$ World Natural Gas Reserves, Production, and Consumption, $2000 \ldots \ldots$. . . 1-7

Table $1.7 \quad$ Petroleum Stocks of OECD Countries by Ownership, 1995-2002 . . . . . . . . 1-8

Figure $1.4 \quad$ Crude Oil Prices in Current and Constant Terms, 1870-2002 . . . . . . . . . . . 1-9

Table $1.8 \quad$ U.S. Petroleum Imports by World Region of Origin, $1960-2002 \ldots \ldots \ldots \ldots$. . 1-10

Figure $1.5 \quad$ Oil Price and Economic Growth, 1970-2002 . . . . . . . . . . . . . 1-11

Table 1.9 Summary of 1996 Military Expenditures for Defending Oil Supplies from the

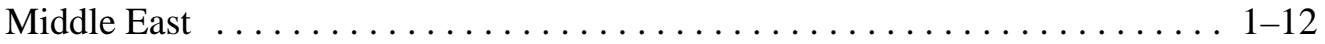

Figure $1.6 \quad$ Refinery Gross Output by World Region, 2002 . . . . . . . . . . . . . . . . 1-13

Table $1.10 \quad$ U.S. Refinery Input of Crude Oil and Petroleum Products, 1987-2002 . . . . 1-14

Table 1.11 Refinery Yield of Petroleum Products from a Barrel of Crude Oil, 1978-2002 . 1-15

Table 1.12 United States Petroleum Production, Imports and Exports, 1950-2002 . . . . 1-16 


\section{TABLE OF CONTENTS (Continued)}

Table 1.13 Petroleum Production and Consumption Ratios, 1950-2002 . . . . . . . . . 1-17

Figure $1.7 \quad$ United States Petroleum and Consumption, 1970-2025 . . . . . . . . . . . . 1-18

Table 1.14 Consumption of Petroleum by End-Use Sector, 1973-2002 . . . . . . . . . . . 1-19

Table 1.15 Ton-Miles of Petroleum and Petroleum Products in the U.S. by Mode,

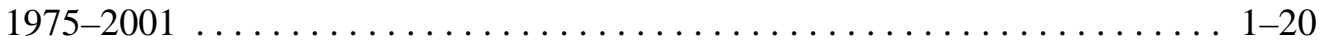

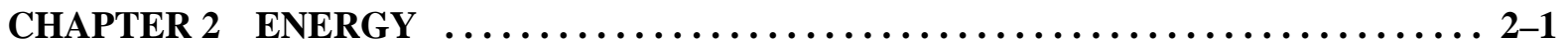

Figure $2.1 \quad$ World Consumption of Primary Energy, $2001 \ldots \ldots \ldots \ldots \ldots \ldots \ldots$

Table $2.1 \quad$ U. S. Consumption of Total Energy by End-Use Sector, 1973-2002 . . . . . . 2-3

Table 2.2 Distribution of Energy Consumption by Source, 1973 and $2002 \ldots \ldots$. . . 2-4

Table 2.3 Alternative Fuel and Oxygenate Consumption, 1992-2002 . . . . . . . . 2-5

Table 2.4 Domestic Consumption of Transportation Energy by Mode and

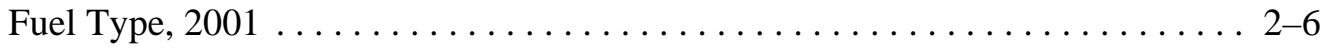

Table $2.5 \quad$ Transportation Energy Use by Mode, $2000-2001 \ldots \ldots \ldots \ldots \ldots \ldots \ldots$. . . . . .

Table 2.6 Highway Transportation Energy Consumption by Mode, 1970-2001 . . . . . 2-8

Table 2.7 Nonhighway Transportation Energy Consumption by Mode, 1970-2001 . . . . 2-9

Table 2.8 Off-Highway Use of Gasoline and Diesel, 1985-2001 . . . . . . . . . . 2 2-10

Table 2.9 Highway Usage of Gasoline and Special Fuels, 1973-2001 . . . . . . . . . . 2-11

Table $2.10 \quad$ U.S. Production and Imports of MTBE and Fuel Ethanol, 1985-2002 . . . . . 2 2-12

Table $2.11 \quad$ Passenger Travel and Energy Use, $2001 \ldots \ldots \ldots \ldots \ldots \ldots \ldots \ldots \ldots \ldots$

Table 2.12 Energy Intensities of Highway Passenger Modes, 1970-2001 . . . . . . . . . 2-14

Table 2.13 Energy Intensities of Nonhighway Passenger Modes, 1970-2001 . . . . . . . 2-15

Figure 2.2 Energy Intensities for Transit Rail, $2001 \ldots \ldots \ldots \ldots \ldots \ldots \ldots \ldots \ldots$

Figure 2.3 Energy Intensities for Selected Transit Bus Systems, 2001 . . . . . . . . . . 2-17

Table 2.14 Intercity Freight Movement and Energy Use in the United States, $2001 \ldots$. . 2-18

Table $2.15 \quad$ Energy Intensities of Freight Modes, $1970-2001 \ldots \ldots \ldots \ldots \ldots \ldots \ldots$. . . . . . . . .

CHAPTER 3 HIGHWAY VEHICLES AND CHARACTERISTICS $\ldots \ldots \ldots \ldots \ldots \ldots$. 


\section{TABLE OF CONTENTS (Continued)}

Table 3.1

Table 3.2

Table 3.3

Figure 3.1

Table 3.4

Table 3.5

Table 3.6

Table 3.7

Table 3.8

Figure 3.2

Table 3.9

Figure 3.3

Table 3.10

Figure 3.4

Table 3.11

Figure 3.5

CHAPTER 4 LIGHT VEHICLES AND CHARACTERISTICS

Table 4.1

Table 4.2

Table 4.3

Table 4.4

Table 4.5

Table 4.6

Table 4.7

Automobiles and Trucks in Use, 1970-2001
Automobile Registrations for Selected Countries, 1950-2001 $3-2$

Truck and Bus Registrations for Selected Countries, 1950-2001 . 3-3 . 3-5

Vehicles per Thousand People: U.S. Compared to Other Countries . . 3-6

Vehicle Stock and New Sales in the United States, 2001 Calendar Year . . . . . . 3-7

Shares of Highway Vehicle-Miles Traveled by Vehicle Type, 1970-2001 . . . 3-8

Automobiles in Operation and Vehicle Travel by Age, 1970 and 2001 . . . . . 3 3-9

Trucks in Operation and Vehicle Travel by Age, 1970 and 2001 . . . . . . . . 3-10

Average Age of Automobiles and Trucks in Use, 1970-2001 . . . . . . . . . 3-11

Average Age and Registrations of Automobiles and Trucks, 1970-2001 . . . . 3-12

Automobile Scrappage and Survival Rates 1970, 1980 and 1990 Model Years 3-13

Automobile Survival Rates . . . . . . . . . . . . . . . . . . . . . 3-14

Light Truck Scrappage and Survival Rates $\ldots \ldots \ldots \ldots \ldots \ldots \ldots \ldots$ 3-15

Light Truck Survival Rates . . . . . . . . . . . . . . . . . 3-16

Heavy Truck Scrappage and Survival Rates $\ldots \ldots \ldots \ldots \ldots \ldots \ldots$ 3-17

Heavy Truck Survival Rates . . . . . . . . . . . . . . . . . . . . 3-18

Summary Statistics for Passenger Cars, $1970-2001 \ldots \ldots \ldots \ldots \ldots$ 4 $\ldots$.2

Summary Statistics for Two-Axle, Four-Tire Trucks, 1970-2001 . . . . . . . . 4-3

Summary Statistics on Class 1, Class 2a, and Class 2b Light Trucks . . . . . . . 4-4

Sales Estimates of Class 1, Class 2a, and Class 2b Light Trucks, 1989-1999 . . . 4-4

New Retail Automobile Sales in the United States, 1970-2002 . . . . . . . . . . 4-5

New Retail Sales of Trucks 10,000 pounds GVW and Less in the

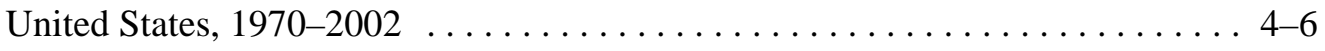

Period Sales, Market Shares, and Sales-Weighted Fuel Economies of New Domestic and Import Automobiles, Selected Sales Periods 1976-2002 $4-7$ 


\section{TABLE OF CONTENTS (Continued)}

Table 4.8 Period Sales, Market Shares, and Sales-Weighted Fuel Economies of New Domestic and Import Light Trucks, Selected Sales Periods 1976-2002 . . . . . . 4-8

Table 4.9 Light Vehicle Market Shares by Size Class, Sales Periods 1976-2002 _... . 4 4-9

Figure 4.1 Light Vehicle Market Shares, Sales Periods, 1976-2002 . . . . . . . . . . . 4-10

Table 4.10 Sales-Weighted Engine Size of New Domestic and Import Automobiles by Size Class, Sales Periods $1976-2002 \ldots \ldots \ldots \ldots \ldots \ldots \ldots \ldots$. . . . . . . . . .

Table 4.11 Sales-Weighted Engine Size of New Domestic and Import Light Trucks

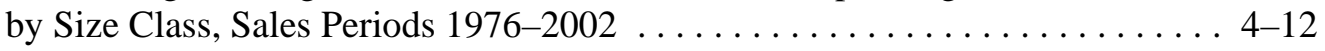

Table 4.12 Sales-Weighted Curb Weight of New Domestic and Import Automobiles by Size Class, Sales Periods $1976-2002 \ldots \ldots \ldots \ldots \ldots \ldots \ldots \ldots$. . . . . . . . . .

Table 4.13 Sales-Weighted Interior Space of New Domestic and Import Automobiles by Size Class, Sales Periods $1976-2002$. . . . . . . . . . . . . . . . . 4-14

Table 4.14 Sales-Weighted Wheelbase of New Automobiles and Light Trucks, Sales Periods $1976-2002 \ldots \ldots \ldots \ldots \ldots \ldots \ldots$. . . . . . . . . . . . . 4

Table 4.15 Average Material Consumption for a Domestic Automobile, 1978, 1985,

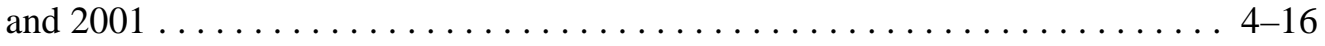

Table $4.16 \quad$ New Light Vehicle Dealerships and Sales, $1970-2000$. . . . . . . . . . . . 4 4-17

Table 4.17 Conventional and Alternative Fuel Refueling Stations . . . . . . . . . . 4-18

Table 4.18 Automobile Corporate Average Fuel Economy (CAFE) Standards versus Sales-Weighted Fuel Economy Estimates, 1978-2003 . . . . . . . . . . . . . 4 4-19

Table 4.19 Light Truck Corporate Average Fuel Economy (CAFE) Standards versus Sales-Weighted Fuel Economy Estimates, 1978-2003 . . . . . . . . . . . . 4-20

Table 4.20 Corporate Average Fuel Economy (CAFE) Fines Collected, 1983-2001 . . . . 4-21

Table 4.21 The Gas Guzzler Tax on New Cars $\ldots \ldots \ldots \ldots \ldots \ldots \ldots \ldots \ldots \ldots \ldots \ldots \ldots \ldots \ldots$

Table 4.22 Tax Receipts from the Sale of Gas Guzzlers, $1980-2001 \ldots \ldots \ldots \ldots$. . . . 4 4-23

Table $4.23 \quad$ Vehicle Specifications for Vehicles Tested in the 1997 Study . . . . . . . . . 4-25

Table 4.24 Fuel Economy by Speed, 1973, 1984 and 1997 Studies . . . . . . . . . . . 4-26

Figure 4.2 Fuel Economy by Speed, 1973, 1984 and 1997 Studies . . . . . . . . . . . 4-27

Table 4.25 Steady Speed Fuel Economy for Vehicles Tested in the 1997 Study . . . . . . . 4-28 


\section{TABLE OF CONTENTS (Continued)}

Figure 4.3

Figure 4.4

Figure 4.5

Figure 4.6

Figure 4.7

Table 4.26

Table 4.27

Table 4.28

Table 4.29

Table 4.30

Figure 4.8

Table 4.31

CHAPTER

Table 5.1

Table 5.2

Table 5.3

Table 5.4

Table 5.5

Table 5.6

Table 5.7

Table 5.8

Table 5.9

Table 5.10

Table 5.11
Urban Driving Cycle $4-29$

Highway Driving Cycle $4-29$

New York City Driving Cycle . $4-30$

Representative Number Five Driving Cycle $4-30$ US06 Driving Cycle $4-31$

Projected Fuel Economies from U.S., European, and Japanese Driving Cycles 4-32 Comparison of U.S., European, and Japanese Driving Cycles $\ldots . . \ldots \ldots$. 4-33 Occupant Fatalities by Vehicle Type and Nonoccupant Fatalities, 1975-2000 4-34 Light Vehicle Occupant Safety Data, $1975-2001 \ldots \ldots \ldots \ldots \ldots \ldots$. . . . . 4 35

Crashes by Crash Severity, Crash Type, and Vehicle Type, 2001 . . . . . . . 4-36 Percent Rollover Occurrence in Fatal Crashes by Vehicle Type, 2001 . . . . . 4-37 Summary Statistics on Light Transit Vehicles, $1994-2001 \ldots \ldots \ldots \ldots \ldots$. . . 4 48

\section{HEAVY VEHICLES AND CHARACTERISTICS $\ldots \ldots \ldots \ldots \ldots \ldots \ldots$ 5-1}

Summary Statistics for Other Single-Unit Trucks, 1970-2001 . . . . . . . . . . 5-2

Summary Statistics for Combination Trucks, $1970-2001 \ldots \ldots \ldots \ldots \ldots$. . . . .

New Retail Truck Sales by Gross Vehicle Weight, $1970-2002$. . . . . . . . . 5 5-4

Truck Statistics by Gross Vehicle Weight Class, 1997 . . . . . . . . . . . . . . 5-6

Truck Harmonic Mean Fuel Economy by Size Class, 1992 and 1997 . . . . . . . 5-6

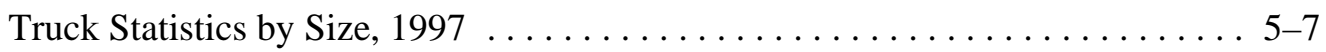

Percentage of Trucks by Size Ranked by Major Use, $1997 \ldots \ldots \ldots \ldots \ldots \ldots$. . . . 5-8

Percentage of Trucks by Fleet Size and Primary Fueling Facility, 1997 . . . . . . 5-9

Percentage of Trucks by Major Use and Primary Fueling Facility, 1997 . . . . . 5-10

Growth of Freight in the United States: Comparison of the 1997 and 1993 Commodity Flow Surveys . . . . . . . . . . . . . . 5-12

Growth of Freight Miles in the United States: Comparison of the 1997

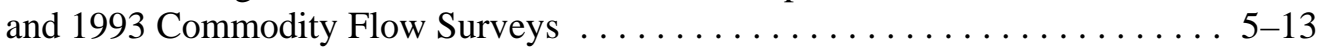




\section{TABLE OF CONTENTS (Continued)}

Table 5.12 Summary Statistics on Transit Buses, $1984-2001 \ldots \ldots \ldots \ldots \ldots \ldots \ldots \ldots$. . . . 5 .14

Table 5.13 Summary Statistics on Intercity and School Buses, 1970-2001 . . . . . . . . 5-15

CHAPTER 6 ALTERNATIVE FUEL AND ADVANCED TECHNOLOGY VEHICLES AND CHARACTERISTICS $\ldots \ldots \ldots \ldots \ldots \ldots \ldots \ldots \ldots .6 .6 .6$

Table 6.1 Estimates of Alternative Fuel Vehicles in Use, 1992-2002 . . . . . . . . . 6 6-3

Table 6.2 Estimates of Alternative Fuel Vehicles by Ownership, 1996 and $2002 \ldots$. . . 6 6-4

Table 6.3 Alternative Fuel Vehicles Available by Manufacturer, Model Year $2001 \ldots$. . . 6-5

Table $6.4 \quad$ Number of Alternative Refuel Sites by State and Fuel Type, $2002 \ldots \ldots$. . . . 6-6

Figure $6.1 \quad$ Clean Cities Coalitions $\ldots \ldots \ldots \ldots \ldots \ldots \ldots \ldots \ldots \ldots \ldots \ldots \ldots \ldots \ldots \ldots \ldots \ldots$

Table 6.5 Sales and Specifications of Available Advanced Technology Vehicles . . . . . 6-8

Table $6.6 \quad$ Hydrogen Production Methods $\ldots \ldots \ldots \ldots \ldots \ldots \ldots \ldots \ldots \ldots \ldots \ldots$

Table $6.7 \quad$ U.S. Hydrogen Production Plants and Storage Terminals . . . . . . . . . . . 6-11

Table $6.8 \quad$ U.S. and World Hydrogen Consumption by End-Use Category, 1999 . . . . . 6-12

Table 6.9 Hydrogen Storage Systems for On-Board Light Vehicles . . . . . . . . . 6-13

Table $6.10 \quad$ Properties of Conventional and Alternative Fuels . . . . . . . . . . . 6-14

Table $6.11 \quad$ Fuel Cell Type Comparison $\ldots \ldots \ldots \ldots \ldots \ldots \ldots \ldots \ldots \ldots \ldots \ldots \ldots \ldots \ldots \ldots$

CHAPTER 7 FLEET VEHICLES AND CHARACTERISTICS $\ldots \ldots \ldots \ldots \ldots \ldots \ldots$. 7 -1

Figure $7.1 \quad$ Fleet Vehicles in Service as of February 1, $2002 \ldots \ldots \ldots \ldots \ldots \ldots$. . . . . .

Table $7.1 \quad$ Light Vehicles in Fleets of 10 or More, $2000 \ldots \ldots \ldots \ldots \ldots \ldots \ldots$

Table $7.2 \quad$ New Light Fleet Vehicle Purchases by Vehicle Type, $2000 \ldots \ldots \ldots \ldots \ldots$. . $7-3$

Table 7.3 Average Length of Time Business Fleet Vehicles are in Service, 2001 . . . . . 7-4

Table $7.4 \quad$ Average Annual Vehicle-Miles of Travel for Fleet Vehicles, 2000 . . . . . . 7-4

Figure 7.2 Average Miles per Domestic Federal Vehicle by Vehicle Type, 2001 . . . . . . . 7-5

Table $7.5 \quad$ Federal Government Vehicles by Agency, Fiscal Year $2001 \ldots \ldots$. . . . . . 7-6

Table $7.6 \quad$ Federal Fleet Vehicle Acquisitions by Fuel Type, FY 1998-2001 . . . . . . 7 7-7

Table $7.7 \quad$ Fuel Consumed by Federal Government Fleets, FY 1998-2001 . . . . . . . . 7-7 


\section{TABLE OF CONTENTS (Continued)}

Table 7.8 Energy Policy Act Purchase Requirements of Light Alternative

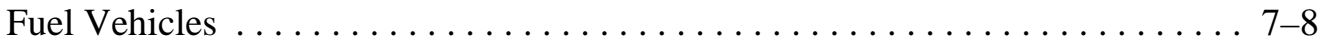

CHAPTER 8 HOUSEHOLD VEHICLES AND CHARACTERISTICS . . . . . . . 8 8-1

Table $8.1 \quad$ Population and Vehicle Profile, $1950-2001 \ldots \ldots \ldots \ldots \ldots \ldots \ldots$. . . . . . .

Table 8.2 Population and Vehicle Ratios, $1950-2001 \ldots \ldots \ldots \ldots \ldots \ldots \ldots$. . . . .

Table 8.3 Average Annual Expenditures of Households by Income, $2001 \ldots \ldots$. . . . . 8-4

Table $8.4 \quad$ Household Vehicle Ownership, $1960-2000$ Census $\ldots \ldots \ldots \ldots \ldots \ldots$. . . . 8-5

Table 8.5 Demographic Statistics, 1969, 1977, 1983, 1990, 1995 NPTS and 2001 NHTS . . 8-6

Table 8.6 Average Annual Vehicle-Miles, Vehicle Trips and Trip Length per Household 1969, 1977, 1983, 1990, 1995 NPTS and 2001 NHTS . . . . . . . . . . . 8-7

Table 8.7 Average Annual Person-Miles Traveled (PMT), Person Trips and Trip Length per Household by Selected Trip Purposes, 1983, 1990, 1995 NPTS

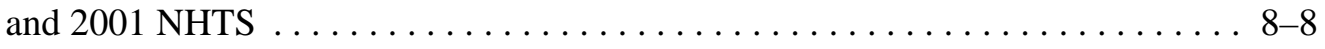

Table 8.8 Average Number of Vehicles and Vehicle Travel per Household, 1990 NPTS and 2001 NHTS . . . . . . . . . . . . . . . . . . . . . . . . 8-9

Figure 8.1 Average Vehicle Occupancy by Vehicle Type, 1995 NPTS and 2001 NHTS . . . 8-10

Figure 8.2 Average Vehicle Occupancy by Trip Purpose, 1977 NPTS and 2001 NHTS _. 8-11

Table 8.9 Average Annual Miles per Vehicle by Household Vehicle Ownership, 2001 NHTS . . . . . . . . . . . . . . . . . . . . . . . 8-12

Table 8.10 Average Age of Vehicles by Household Vehicle Ownership, 2001 NHTS . . . 8 8-12

Table 8.11 Average Annual Miles per Household Vehicle by Vehicle Age . . . . . . . . 8-13

Table 8.12 Self-Reported vs. Odometer Average Annual Miles, 1995 NPTS . . . . . . . . . 8 8-14

Table 8.13 Means of Transportation to Work, 1980, 1990 and 2000 Census ........ . 8-15

Table $8.14 \quad$ Workers by Commute Time, 1990 and 2000 Census $\ldots \ldots \ldots \ldots \ldots \ldots$. . . . . . .

Table $8.15 \quad$ Bicycle Sales, $1981-2002 \ldots \ldots \ldots \ldots \ldots \ldots \ldots \ldots \ldots \ldots \ldots \ldots$. $\ldots \ldots \ldots$

Table 8.16 Specialty Bicycle Sales by Year, $2000-2002 \ldots \ldots \ldots \ldots \ldots \ldots \ldots$. . . . . . . .

Figure $8.3 \quad$ Walk and Bike Trips by Trip Purpose, 2001 NHTS . . . . . . . . . . . . 8-19

Figure $8.4 \quad$ Long-Distance Trips by Destination, $1995 \ldots \ldots \ldots \ldots \ldots \ldots \ldots \ldots \ldots$. . . . . . . . . 


\section{TABLE OF CONTENTS (Continued)}

Table 8.17 Long-Distance Trips by Mode and Purpose, 1995 $8-21$

Figure 8.5 Shares of Long-Distance Person Trips by Mode and Household

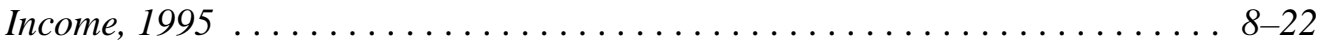

CHAPTER 9 NONHIGHWAY MODES $\ldots \ldots \ldots \ldots \ldots \ldots \ldots \ldots \ldots \ldots \ldots \ldots \ldots \ldots$ 9-1

Table $9.1 \quad$ Nonhighway Energy Use Shares, $1970-2001 \ldots \ldots \ldots \ldots \ldots \ldots \ldots$. . . . . . .

Table 9.2 Summary Statistics for U.S. Domestic and International Certificated Route Air Carriers (Combined Totals), 1970-2001 . . . . . . . . . . . . . . . . . . . 9 9-3

Table 9.3 Summary Statistics for General Aviation, $1970-2001 \ldots \ldots \ldots \ldots \ldots$. . . . . 9-4

Table 9.4 Tonnage Statistics for Domestic and International Waterborne Commerce, $1970-2001 \ldots \ldots \ldots \ldots \ldots$. . . . . . . . . . . . . . . 9-5

Table 9.5 Summary Statistics for Domestic Waterborne Commerce, 1970-2001 . . . . . . 9-6

Table 9.6 Breakdown of Domestic Marine Cargo by Commodity Class, $2001 \ldots$. . . . . . 9-7

Table 9.7 Recreational Boating Statistics, $1977-2001 \ldots \ldots \ldots \ldots \ldots \ldots \ldots$

Table 9.8 Class I Railroad Freight Systems in the United States Ranked by Revenue

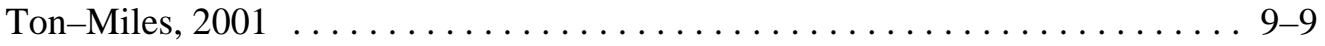

Table 9.9 Summary Statistics for Class I Freight Railroads, $1970-2001 \ldots \ldots \ldots$. . . 9-10

Table 9.10 Railroad Revenue Carloads by Commodity Group, 1974 and 2001 . . . . . . 9-11

Table $9.11 \quad$ Intermodal Rail Traffic, $1965-2001 \ldots \ldots \ldots \ldots \ldots \ldots \ldots \ldots \ldots . . \ldots \ldots$

Table 9.12 Summary Statistics for the National Railroad Passenger Corporation

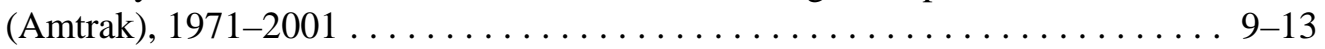

Table 9.13 Summary Statistics for Commuter Rail Operations, 1984-2001 . . . . . . . . . 9-14

Table $9.14 \quad$ Summary Statistics for Rail Transit Operations, 1970-2001 . . . . . . . . . 9-15

CHAPTER 10 TRANSPORTATION AND THE ECONOMY .............. 10-1

Table 10.1 Gasoline Prices for Selected Countries, $1978-2002 \ldots \ldots \ldots \ldots \ldots$. . . . . . 10-2

Figure 10.1 Gasoline Prices for Selected Countries, 1990 and 2002 . . . . . . . . . . 10--3

Table 10.2 Diesel Fuel Prices for Selected Countries, 1978-2002 . . . . . . . . . . . 10-4

Figure 10.2 Diesel Prices for Selected Countries, 1990 and 2002 . . . . . . . . . . . 10-5 


\section{TABLE OF CONTENTS (Continued)}

Table 10.3 Prices for a Barrel of Crude Oil and a Gallon of Gasoline, 1978-2002 _. . . 10-6

Table $10.4 \quad$ Retail Prices for Motor Fuel, $1978-2002 \ldots \ldots \ldots \ldots \ldots \ldots \ldots \ldots \ldots$. $\ldots \ldots$

Table 10.5 Refiner Sales Prices for Propane and No. 2 Diesel, 1978-2002 . . . . . . . 10 10

Table 10.6 Refiner Sales Prices for Aviation Gasoline and Jet Fuel, 1978-2002 . . . . . . 10-9

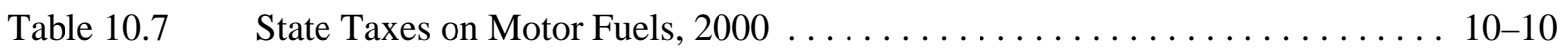

Table $10.8 \quad$ State Tax Exemptions for Gasohol, $2001 \ldots \ldots \ldots \ldots \ldots \ldots \ldots \ldots$. . . . . . . . . .

Table $10.9 \quad$ Federal Excise Taxes on Motor Fuels $\ldots \ldots \ldots \ldots \ldots \ldots \ldots \ldots \ldots \ldots$. . . . . . . . . .

Table 10.10 State Ethanol Incentives, $2003 \ldots \ldots \ldots \ldots \ldots \ldots \ldots \ldots \ldots \ldots \ldots \ldots \ldots \ldots$

Table 10.11 Average Price of a New Car, $1970-2001 \ldots \ldots \ldots \ldots \ldots \ldots \ldots \ldots$. . . . . . . . .

Table 10.12 Automobile Operating Cost per Mile, $1985-2002 \ldots \ldots \ldots \ldots \ldots \ldots \ldots$. . . . 10 .15

Table 10.13 Fixed Automobile Operating Costs per Year, 1975-2002 . . . . . . . . . 10 10 16

Table 10.14 Economic Indicators, $1970-2002 \ldots \ldots \ldots \ldots \ldots \ldots \ldots \ldots \ldots \ldots$. . . . . . . . . . . .

Table 10.15 Consumer Price Indices, $1970-2002 \ldots \ldots \ldots \ldots \ldots \ldots \ldots \ldots \ldots \ldots$. . . . 17

Table 10.16 Transportation-related Employment, 1993 and $2002 \ldots \ldots \ldots \ldots \ldots$. . . . . .

CHAPTER 11 GREENHOUSE GAS EMISSIONS $\ldots \ldots \ldots \ldots \ldots \ldots \ldots \ldots \ldots \ldots$ 11-1

Table 11.1 World Carbon Emissions from Energy Consumption, 1990 and $2001 \ldots$. . . 11-2

Table 11.2 Numerical Estimates of Global Warming Potentials Compared with

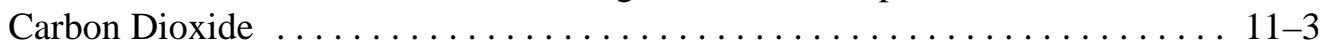

Table 11.3 Estimated U.S. Emissions of Greenhouse Gases, 1990-2001 . . . . . . . . . 11-4

Table $11.4 \quad$ U.S. Carbon Dioxide Emissions from Fossil Energy Consumption by End-Use Sector, $1990-2001$. . . . . . . . . . . . . . . . . . . . . . . . . . . . 11-5

Table $11.5 \quad$ U.S. Carbon Dioxide Emissions from Energy Use in Transportation

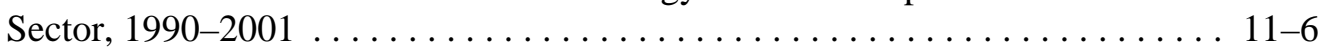

CHAPTER 12 CRITERIA AIR POLLUTANTS $\ldots \ldots \ldots \ldots \ldots \ldots \ldots \ldots \ldots \ldots \ldots$ 12-1

Table 12.1 Total National Emissions of the Criteria Air Pollutants by Sector, $2001 \ldots$. . 12-2

Table 12.2 Total National Emissions of Carbon Monoxide, $1980-2001$. . . . . . . . . 12-3 


\section{TABLE OF CONTENTS (Continued)}

Table 12.3 Emissions of Carbon Monoxide from Highway Vehicles, 1980-2001 . . . . . 12-4

Table 12.4 Total National Emissions of Nitrogen Oxides, $1980-2001 \ldots \ldots \ldots \ldots \ldots$. . . . 12-5

Table 12.5 Emissions of Nitrogen Oxides from Highway Vehicles, 1980-2001 . . . . . 12-6

Table 12.6 Total National Emissions of Volatile Organic Compounds, 1980-2001 . . . . 12-7

Table 12.7 Emissions of Volatile Organic Compounds from Highway Vehicles,

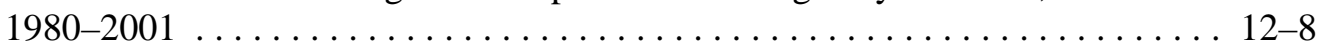

Table 12.8 Total National Emissions of Particulate Matter (PM-10), 1980-2001 . . . . . 12-9

Table 12.9 Emissions of Particulate Matter (PM-10) from Highway Vehicles, $1980-2001$. . . . . . . . . . . . . . . . . . . . . . . . . . . . . . . . . . 12-10

Table 12.10 Total National Emissions of Particulate Matter (PM-2.5), 1990-2001 . . . . 12-11

Table 12.11 Emissions of Particulate Matter (PM-2.5) from Highway Vehicles,

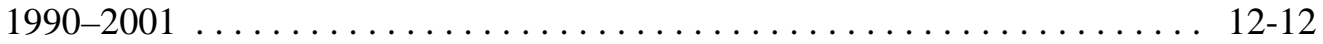

Table 12.12 Tier 2 Emission Standards for Cars and Light Trucks Effective for 2004-2009 Model Years ... . . . . . . . . . . . . . . . . . . . . . . . . . 12-14

Table 12.13 Light Vehicle Exhaust Emission Standards in Effect in 2009 when U.S.

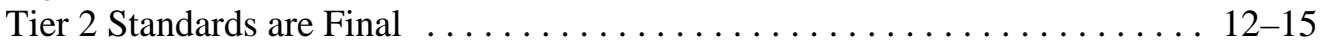

Table 12.14 Federal Exhaust Emission Certification Standards for Gasoline- and

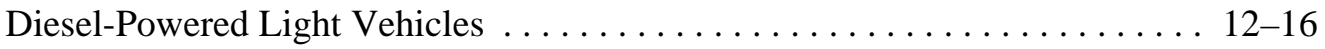

Table 12.15 Federal Exhaust Emission Certification Standards for Gasoline- and Diesel-Powered Light Trucks (Category LDT1) . . . . . . . . . . . . . . 12-17

Table 12.16 Federal Exhaust Emission Certification Standards for Gasoline- and Diesel-Powered Light Trucks (Category LDT2) . . . . . . . . . . . . . . . 12-18

Table 12.17 Federal Exhaust Emission Certification Standards for Gasoline- and Diesel-Powered Light Trucks (Category LDT3) . . . . . . . . . . . . . . . . 12-19

Table 12.18 Federal Exhaust Emission Certification Standards for Gasoline- and Diesel-Powered Light Trucks (Category LDT4) . . . . . . . . . . . . . . 12-20

Table 12.19 Federal Exhaust Emission Certification Standards for Gasoline- and Diesel-Powered Light Heavy Trucks . . . . . . . . . . . . . . . . . . . 12-21

Table 12.20 Federal Exhaust Emission Certification Standards for Gasoline- and Diesel-Powered Heavy Heavy Trucks . . . . . . . . . . . . . . . . . . . 12-22 


\section{TABLE OF CONTENTS (Continued)}

Table 12.21 California Passenger Cars and Light Trucks Emission Certification

Standards for Model Years 2001-2006 . . . . . . . . . . . . . . . . . . 12-23

Table 12.22 California Vehicle Emission Reduction for Passenger Cars and

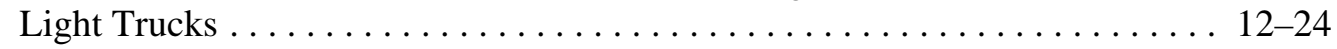

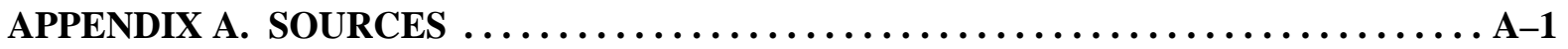

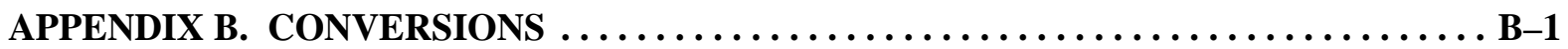

APPENDIX C. MAPS $\ldots \ldots \ldots \ldots \ldots \ldots \ldots \ldots \ldots \ldots \ldots \ldots \ldots \ldots \ldots \ldots \ldots \ldots \ldots \ldots \ldots$

GLOSSARY $\ldots \ldots \ldots \ldots \ldots \ldots \ldots \ldots \ldots \ldots \ldots \ldots \ldots \ldots \ldots \ldots \ldots \ldots \ldots \ldots \ldots \ldots \ldots \ldots \ldots$

INDEX $\ldots \ldots \ldots \ldots \ldots \ldots \ldots \ldots \ldots \ldots \ldots \ldots \ldots \ldots \ldots \ldots \ldots \ldots \ldots \ldots \ldots \ldots \ldots \ldots \ldots$ 


\section{FOREWORD}

Welcome to this 23rd edition of the Transportation Energy Data Book. I would like to bring to your attention some of the data that is new or of particular interest:

1. The Transportation Oil Gap shows that transportation oil use has exceeded U.S. oil production since 1987 and this gap is projected to continue to grow (Figure 1.7)

2. Between 1991 and 2001, heavy truck energy use grew at a faster rate than for any other mode (Tables 2.6 and 2.7)

3. Vehicles per thousand people varies greatly by region of the world (Figure 3.1)

4. The median lifetime of automobiles in the U.S. rose from 11.5 years for model year 1970 vehicles to 16.9 years for model year 1990 vehicles (Table 3.9)

5. The percent of automobiles that are imports or transplants reached $50 \%$ for the first time in 2001 (Table 4.5)

6. SUVs accounted for $6.8 \%$ of all light vehicle sales in 1990 and $24.6 \%$ in 2002 (Table 4.9)

7. The number of new light vehicle dealerships declined at an annual rate of $1.1 \%$ over the 1979-2000 period, but the vehicles sold per dealership grew at an annual rate of 3\% (Table 4.16)

8. CAFE fines collected were $\$ 34$ million in 2001, while tax receipts from the sale of gas guzzlers were $\$ 78$ million (Tables 4.20 and 4.22)

9. Data for hydrogen has been added that show production methods, production totals, consumption, storage systems, and fuel cell types (Tables 6.6 through 6.12)

I hope you find value in this data book. We welcome suggestions on how to improve it.

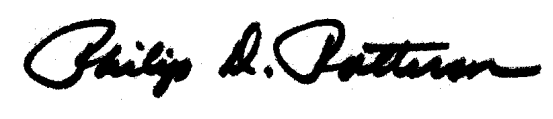




\section{xviii}

TRANSPORTATION ENERGY DATA BOOK: EDITION 23-2003 


\section{ACKNOWLEDGMENTS}

The authors would like to express their gratitude to the many individuals who assisted in the preparation of this document. First, we would like to thank Phil Patterson and the Energy Efficiency and Renewable Energy staff for their continued support of the Transportation Energy Data Book project. We would also like to thank Patricia Hu of Oak Ridge National Laboratory (ORNL) for her guidance and mentoring. This document benefits from the criticism and careful review of Phil Patterson, DOE; Elyse Steiner, National Renewable Energy Laboratory; James Moore, TAEngineering, Inc.; and Margaret Singh, Argonne National Laboratory. We would also like to thank Jamie Payne, ORNL, who designed the cover; Sherry Campbell Gambrell, ORNL, who prepared the title index; and Bob Boundy, Q Systems, who assisted with so many tasks we can't name them all. Finally, this book would not have been possible without the dedication of Debbie Bain, who masterfully prepared the manuscript. 


\begin{abstract}
The Transportation Energy Data Book: Edition 23 is a statistical compendium prepared and published by Oak Ridge National Laboratory (ORNL) under contract with the Office of Planning, Budget Formulation, and Analysis, under the Energy Efficiency and Renewable Energy (EERE) program in the Department of Energy (DOE). Designed for use as a desk-top reference, the data book represents an assembly and display of statistics and information that characterize transportation activity, and presents data on other factors that influence transportation energy use. The purpose of this document is to present relevant statistical data in the form of tables and graphs. The latest editions of the Data Book are available to a larger audience via the Internet (www-cta.ornl.gov/data).

This edition of the Data Book has 12 chapters which focus on various aspects of the transportation industry. Chapter 1 focuses on petroleum; Chapter 2 - energy; Chapter 3 - highway vehicles; Chapter 4 - light vehicles; Chapter 5 - heavy vehicles; Chapter 6 - alternative fuel vehicles; Chapter 7 - fleet vehicles; Chapter 8 - household vehicles; and Chapter 9- nonhighway modes; Chapter 10 - transportation and the economy; Chapter 11 - greenhouse gas emissions; and Chapter 12 - criteria pollutant emissions. The sources used represent the latest available data. There are also three appendices which include detailed source information for some tables, measures of conversion, and the definition of Census divisions and regions. A glossary of terms and a title index are also included for the readers convenience.
\end{abstract}




\section{INTRODUCTION}

In January 1976, the Transportation Energy Conservation (TEC) Division of the Energy Research and Development Administration contracted with Oak Ridge National Laboratory (ORNL) to prepare a Transportation Energy Conservation Data Book to be used by TEC staff in their evaluation of current and proposed conservation strategies. The major purposes of the data book were to draw together, under one cover, transportation data from diverse sources, to resolve data conflicts and inconsistencies, and to produce a comprehensive document. The first edition of the TEC Data Book was published in October 1976. With the passage of the Department of Energy (DOE) Organization Act, the work being conducted by the former Transportation Energy Conservation Division fell under the purview of the DOE's Office of Transportation Programs, then to the Office of Transportation Technologies. DOE, through the Office of Transportation Technologies, has supported the compilation of Editions 3 through 21. In the most recent DOE organization, Editions 22 and 23 fall under the purview of the Office of Planning, Budget Formulation, and Analysis in the Office of Energy Efficiency and Renewable Energy.

Policymakers and analysts need to be well-informed about activity in the transportation sector. The organization and scope of the data book reflect the need for different kinds of information. For this reason, Edition 23 updates much of the same type of data that is found in previous editions.

In any attempt to compile a comprehensive set of statistics on transportation activity, numerous instances of inadequacies and inaccuracies in the basic data are encountered. Where such problems occur, estimates are developed by ORNL. To minimize the misuse of these statistics, an appendix (Appendix A) is included to document the estimation procedures. The attempt is to provide sufficient information for the conscientious user to evaluate the estimates and to form their own opinions as to their utility. Clearly, the accuracy of the estimates cannot exceed the accuracy of the primary data, an accuracy which in most instances is unknown. In cases where data accuracy is known or substantial errors are strongly suspected in the data, the reader is alerted. In all cases it should be recognized that the estimates are not precise.

The majority of the statistics contained in the data book are taken directly from published sources, although these data may be reformatted for presentation by ORNL. Consequently, neither ORNL nor DOE endorses the validity of these data. 
xxiv

TRANSPORTATION ENERGY DATA BOOK: EDITION 23-2003 


\section{Chapter 1 Petroleum}

Summary Statistics from Tables/Figures in this Chapter

Source

Table 1.3 World Petroleum Production, 2002 (million barrels per day)

73.65

U.S. Production (million barrels per day)

7.63

U.S. Share

$10.4 \%$

Table 1.4 World Petroleum Consumption, 2002 (million barrels per day)

77.46

U.S. Consumption (million barrels per day)

19.76

U.S. Share

$25.5 \%$

Figure 1.5 Average refinery yield, 2002

OECD

North

Europe

America

Gasoline

$20.8 \%$

$41.5 \%$

Diesel fuel

$35.8 \%$

$22.7 \%$

Residual fuel

$16.3 \%$

$7.0 \%$

Kerosene

$6.0 \%$

$8.4 \%$

Other

$21.1 \%$

$20.4 \%$

Table 1.13 U.S. transportation petroleum use as a percent of U.S. petroleum production, $2002 \quad 161.9 \%$

Table 1.13 Net imports as a percentage of U.S. petroleum consumption, 2002

$52.8 \%$

Table 1.14 Transportation share of U.S. petroleum consumption, 2002

$67.1 \%$

In this document, petroleum is defined as crude oil (including lease condensate) and natural gas plant liquids.

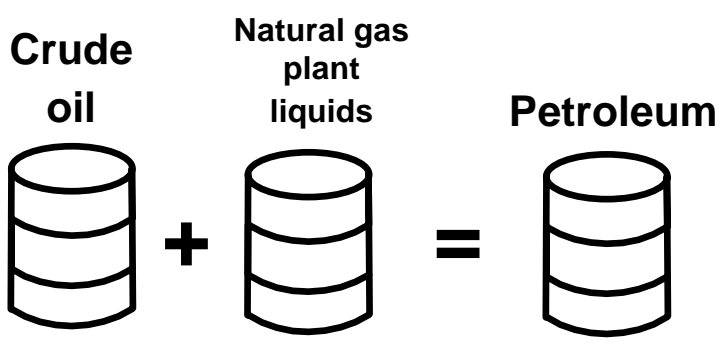


Although the world has consumed about $40 \%$ of estimated conventional oil resources, the total fossil fuel potential is huge. Methane hydrates-a potential source of natural gas-are included in the "additional occurrences" of unconventional natural gas, and constitute the largest resource.

Table 1.1

World Fossil Fuel Potential

(gigatonnes of carbon)

\begin{tabular}{|c|c|c|c|c|}
\hline & $\begin{array}{l}\text { Consumption } \\
\text { (1860-1998) }\end{array}$ & Reserves & Resources & $\begin{array}{l}\text { Additional } \\
\text { occurrences }\end{array}$ \\
\hline \multicolumn{5}{|l|}{ Oil } \\
\hline Conventional & 97 & 120 & 121 & 0 \\
\hline Unconventional & 6 & 102 & 305 & 914 \\
\hline \multicolumn{5}{|l|}{ Natural Gas } \\
\hline Conventional & 36 & 83 & 170 & 0 \\
\hline Unconventional & 1 & 144 & 364 & 14,176 \\
\hline Coal & 155 & 533 & 4,618 & $\mathrm{a}$ \\
\hline
\end{tabular}

Source:

Rogner, H.H., World Energy Assessment: Energy and the Challenge of Sustainability, Part II, Chapter 5, 2000, p. 149.

Figure 1.1. World Fossil Fuel Potential

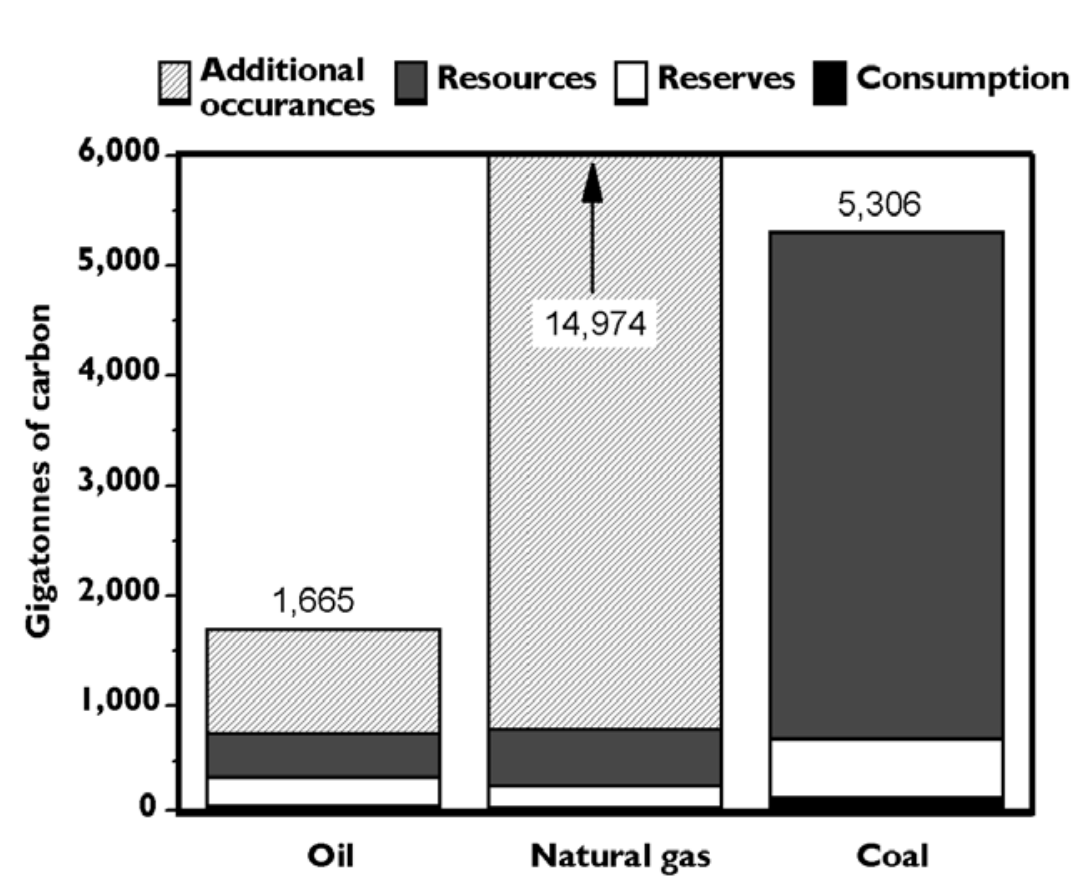

Source:

See Table 1.1.

${ }^{\text {a }}$ Data are not available 
In 2002, OPEC accounted for 40\% of world oil production. Responding to low oil prices in early 2000, Mexico, Norway, Russia, and Oman joined OPEC in cutting production. This group of oil countries, referred to here as OPEC+, account for almost $60 \%$ of world oil production.

Table 1.2

World Crude Oil Production, 1960-2002 (million barrels per day)

\begin{tabular}{|c|c|c|c|c|c|c|c|c|c|c|}
\hline Year & $\begin{array}{c}\text { United } \\
\text { States }\end{array}$ & $\begin{array}{l}\text { U.S. } \\
\text { share }\end{array}$ & $\begin{array}{l}\text { Total } \\
\text { OPEC }^{b}\end{array}$ & $\begin{array}{c}\text { OPEC } \\
\text { share }\end{array}$ & OPEC $+^{\mathrm{c}}$ & $\begin{array}{c}\text { OPEC + }{ }^{\mathrm{c}} \\
\text { share }\end{array}$ & $\begin{array}{l}\text { Total } \\
\text { non- } \\
\text { OPEC }\end{array}$ & $\begin{array}{l}\text { Persian } \\
\text { Gulf } \\
\text { nations }^{\mathrm{d}}\end{array}$ & $\begin{array}{l}\text { Persian } \\
\text { Gulf }^{\mathrm{d}} \\
\text { share }\end{array}$ & World \\
\hline 1960 & 7.04 & $33.5 \%$ & 8.70 & $41.4 \%$ & 12.25 & $58.3 \%$ & 12.29 & 5.27 & $25.1 \%$ & 20.99 \\
\hline 1965 & 7.80 & $25.7 \%$ & 14.35 & $47.3 \%$ & 19.83 & $65.4 \%$ & 15.98 & 8.37 & $27.6 \%$ & 30.33 \\
\hline 1970 & 9.64 & $21.0 \%$ & 23.30 & $50.8 \%$ & 31.16 & $67.9 \%$ & 22.59 & 13.39 & $29.2 \%$ & 45.89 \\
\hline 1975 & 8.37 & $15.8 \%$ & 26.77 & $50.7 \%$ & 37.56 & $71.1 \%$ & 26.06 & 18.93 & $35.8 \%$ & 52.83 \\
\hline 1980 & 8.60 & $14.4 \%$ & 26.61 & $44.6 \%$ & 41.07 & $68.9 \%$ & 32.99 & 17.96 & $30.1 \%$ & 59.60 \\
\hline 1985 & 8.97 & $16.6 \%$ & 16.18 & $30.0 \%$ & 31.81 & $58.9 \%$ & 37.80 & 9.63 & $17.8 \%$ & 53.98 \\
\hline 1986 & 8.68 & $15.4 \%$ & 18.28 & $32.5 \%$ & 34.05 & $60.6 \%$ & 37.95 & 11.70 & $20.8 \%$ & 56.23 \\
\hline 1987 & 8.35 & $14.7 \%$ & 18.52 & $32.7 \%$ & 34.72 & $61.3 \%$ & 38.15 & 12.10 & $21.4 \%$ & 56.67 \\
\hline 1988 & 8.14 & $13.9 \%$ & 20.32 & $34.6 \%$ & 36.66 & $62.4 \%$ & 38.42 & 13.46 & $22.9 \%$ & 58.74 \\
\hline 1989 & 7.61 & $12.7 \%$ & 22.07 & $36.9 \%$ & 38.50 & $64.3 \%$ & 37.79 & 14.84 & $24.8 \%$ & 59.86 \\
\hline 1990 & 7.36 & $12.2 \%$ & 23.20 & $38.3 \%$ & 39.12 & $64.6 \%$ & 37.37 & 15.28 & $25.2 \%$ & 60.57 \\
\hline 1991 & 7.42 & $12.3 \%$ & 23.27 & $38.6 \%$ & 38.53 & $64.0 \%$ & 36.94 & 14.74 & $24.5 \%$ & 60.21 \\
\hline 1992 & 7.17 & $11.9 \%$ & 24.40 & $40.5 \%$ & 37.67 & $62.6 \%$ & 35.81 & 15.97 & $26.5 \%$ & 60.21 \\
\hline 1993 & 6.85 & $11.4 \%$ & 25.12 & $41.7 \%$ & 37.65 & $62.5 \%$ & 35.12 & 16.71 & $27.7 \%$ & 60.24 \\
\hline 1994 & 6.66 & $10.9 \%$ & 25.51 & $41.8 \%$ & 37.67 & $61.8 \%$ & 35.48 & 16.96 & $27.8 \%$ & 60.99 \\
\hline 1995 & 6.56 & $10.5 \%$ & 26.00 & $41.7 \%$ & 38.24 & $61.4 \%$ & 36.33 & 17.21 & $27.6 \%$ & 62.33 \\
\hline 1996 & 6.46 & $10.1 \%$ & 26.46 & $41.5 \%$ & 39.15 & $61.5 \%$ & 37.25 & 17.37 & $27.3 \%$ & 63.71 \\
\hline 1997 & 6.45 & $9.8 \%$ & 27.71 & $42.2 \%$ & 40.69 & $61.9 \%$ & 37.98 & 18.10 & $27.6 \%$ & 65.69 \\
\hline 1998 & 6.25 & $9.3 \%$ & 28.77 & $43.0 \%$ & 41.61 & $62.2 \%$ & 38.19 & 19.34 & $28.9 \%$ & 66.92 \\
\hline 1999 & 5.88 & $8.9 \%$ & 27.58 & $41.9 \%$ & 40.50 & $61.5 \%$ & 38.27 & 18.67 & $28.4 \%$ & 65.85 \\
\hline 2000 & 5.82 & $8.5 \%$ & 29.26 & $42.8 \%$ & 42.92 & $62.8 \%$ & 39.08 & 19.89 & $29.1 \%$ & 68.34 \\
\hline 2001 & 5.80 & $8.5 \%$ & 28.32 & $41.6 \%$ & 42.61 & $62.6 \%$ & 39.74 & 19.21 & $28.2 \%$ & 68.06 \\
\hline \multirow[t]{2}{*}{2002} & 5.75 & $8.6 \%$ & 26.37 & $39.5 \%$ & 39.95 & $59.8 \%$ & 40.47 & 17.79 & $26.6 \%$ & 66.84 \\
\hline & \multicolumn{10}{|c|}{ Average annual percentage change } \\
\hline 1960-2002 & $-0.5 \%$ & & $2.7 \%$ & & $2.9 \%$ & & $2.9 \%$ & $2.9 \%$ & & $2.8 \%$ \\
\hline 1970-2002 & $-1.6 \%$ & & $0.4 \%$ & & $0.8 \%$ & & $1.8 \%$ & $0.9 \%$ & & $1.2 \%$ \\
\hline 1992-2002 & $-2.2 \%$ & & $0.8 \%$ & & $0.6 \%$ & & $1.2 \%$ & $1.1 \%$ & & $1.1 \%$ \\
\hline
\end{tabular}

\section{Source:}

U.S. Department of Energy, Energy Information Administration, Annual Energy Review 2002, Washington, DC, November 2003, Table 11.5. (Additional resources: www.eia.doe.gov)

${ }^{a}$ Includes lease condensate. Excludes natural gas plant liquids.

borganization of Petroleum Exporting Countries. See Glossary for membership.

'OPEC+ includes all OPEC nations plus Russia, Mexico, Norway and Oman.

${ }^{\mathrm{d}}$ See Glossary for Persian Gulf nations. 
This table shows petroleum production, which includes both crude oil and natural gas plant liquids. The U.S. was responsible for $10.4 \%$ of the world's petroleum production in 2002, but only $8.6 \%$ of the world's crude oil production (Table 1.2).

Table 1.3

World Petroleum Production, 1973-2002 (million barrels per day)

\begin{tabular}{|c|c|c|c|c|c|c|c|c|c|}
\hline Year & $\begin{array}{l}\text { United } \\
\text { States }\end{array}$ & $\begin{array}{l}\text { U.S. } \\
\text { share }\end{array}$ & $\begin{array}{l}\text { Total } \\
\text { OPEC }^{\mathrm{b}}\end{array}$ & $\begin{array}{l}\text { OPEC } \\
\text { share }\end{array}$ & $\begin{array}{c}\text { Total non- } \\
\text { OPEC }\end{array}$ & $\begin{array}{l}\text { Non- } \\
\text { OPEC } \\
\text { share }\end{array}$ & $\begin{array}{l}\text { Persian } \\
\text { Gulf } \\
\text { nations }^{\mathrm{c}}\end{array}$ & $\begin{array}{c}\text { Persian } \\
\text { Gulf }^{\mathrm{c}} \\
\text { share }\end{array}$ & World \\
\hline 1973 & 10.95 & $18.7 \%$ & 30.95 & $52.9 \%$ & 27.51 & $47.1 \%$ & 20.86 & $35.7 \%$ & 58.47 \\
\hline 1974 & 10.44 & $17.8 \%$ & 30.70 & $52.5 \%$ & 27.81 & $47.5 \%$ & 21.41 & $36.6 \%$ & 58.51 \\
\hline 1975 & 10.00 & $18.0 \%$ & 27.14 & $48.8 \%$ & 28.48 & $51.2 \%$ & 19.18 & $34.5 \%$ & 55.62 \\
\hline 1976 & 9.73 & $16.2 \%$ & 30.77 & $51.1 \%$ & 29.43 & $48.9 \%$ & 21.80 & $36.2 \%$ & 60.21 \\
\hline 1977 & 9.86 & $15.7 \%$ & 31.37 & $50.0 \%$ & 31.32 & $50.0 \%$ & 22.07 & $35.2 \%$ & 62.69 \\
\hline 1978 & 10.28 & $16.3 \%$ & 30.03 & $47.5 \%$ & 33.21 & $52.5 \%$ & 21.02 & $33.2 \%$ & 63.24 \\
\hline 1979 & 10.13 & $15.4 \%$ & 31.22 & $47.3 \%$ & 37.74 & $52.7 \%$ & 21.53 & $32.6 \%$ & 65.96 \\
\hline 1980 & 10.17 & $16.1 \%$ & 27.34 & $43.4 \%$ & 35.70 & $56.6 \%$ & 18.49 & $29.3 \%$ & 63.04 \\
\hline 1981 & 10.18 & $17.0 \%$ & 23.31 & $39.0 \%$ & 36.40 & $61.0 \%$ & 15.85 & $26.5 \%$ & 59.71 \\
\hline 1982 & 10.20 & $17.9 \%$ & 19.62 & $34.4 \%$ & 37.48 & $65.6 \%$ & 12.77 & $22.4 \%$ & 57.11 \\
\hline 1983 & 10.25 & $18.0 \%$ & 18.28 & $32.1 \%$ & 38.62 & $67.9 \%$ & 11.63 & $20.4 \%$ & 56.90 \\
\hline 1984 & 10.51 & $18.0 \%$ & 18.31 & $31.4 \%$ & 40.05 & $68.6 \%$ & 11.38 & $19.5 \%$ & 58.36 \\
\hline 1985 & 10.58 & $18.3 \%$ & 17.07 & $29.5 \%$ & 40.85 & $70.5 \%$ & 10.28 & $17.7 \%$ & 57.92 \\
\hline 1986 & 10.23 & $16.9 \%$ & 19.25 & $31.9 \%$ & 41.13 & $68.1 \%$ & 12.40 & $20.5 \%$ & 60.38 \\
\hline 1987 & 9.95 & $16.3 \%$ & 19.53 & $32.0 \%$ & 41.42 & $68.0 \%$ & 12.82 & $21.0 \%$ & 60.95 \\
\hline 1988 & 9.77 & $15.4 \%$ & 21.40 & $33.8 \%$ & 41.82 & $66.2 \%$ & 14.27 & $22.6 \%$ & 63.22 \\
\hline 1989 & 9.16 & $14.2 \%$ & 23.26 & $36.1 \%$ & 41.10 & $63.9 \%$ & 15.69 & $24.4 \%$ & 64.36 \\
\hline 1990 & 8.92 & $13.7 \%$ & 24.48 & $37.5 \%$ & 40.72 & $62.5 \%$ & 16.21 & $24.9 \%$ & 65.20 \\
\hline 1991 & 9.08 & $14.0 \%$ & 24.57 & $37.8 \%$ & 40.47 & $62.2 \%$ & 15.67 & $24.1 \%$ & 65.04 \\
\hline 1992 & 8.87 & $13.6 \%$ & 25.76 & $39.5 \%$ & 39.42 & $60.5 \%$ & 16.97 & $26.0 \%$ & 65.18 \\
\hline 1993 & 8.59 & $13.1 \%$ & 26.56 & $40.6 \%$ & 38.87 & $59.4 \%$ & 17.75 & $27.1 \%$ & 65.43 \\
\hline 1994 & 8.39 & $12.7 \%$ & 26.98 & $40.7 \%$ & 39.31 & $59.3 \%$ & 18.03 & $27.2 \%$ & 66.29 \\
\hline 1995 & 8.32 & $12.3 \%$ & 27.51 & $40.6 \%$ & 40.32 & $59.4 \%$ & 18.32 & $27.0 \%$ & 67.82 \\
\hline 1996 & 8.29 & $12.0 \%$ & 27.96 & $40.4 \%$ & 41.33 & $59.6 \%$ & 18.45 & $26.6 \%$ & 69.30 \\
\hline 1997 & 8.27 & $11.6 \%$ & 29.30 & $41.0 \%$ & 42.12 & $59.0 \%$ & 19.25 & $27.0 \%$ & 71.42 \\
\hline 1998 & 8.01 & $11.0 \%$ & 30.43 & $41.8 \%$ & 42.41 & $58.3 \%$ & 20.57 & $28.2 \%$ & 72.80 \\
\hline 1999 & 7.73 & $10.8 \%$ & 29.23 & $40.7 \%$ & 42.62 & $59.3 \%$ & 19.78 & $27.5 \%$ & 71.85 \\
\hline 2000 & 7.73 & $10.4 \%$ & 31.06 & $41.6 \%$ & 43.57 & $58.4 \%$ & 21.11 & $28.3 \%$ & 74.63 \\
\hline 2001 & 7.67 & $10.3 \%$ & 30.25 & $40.5 \%$ & 44.41 & $59.5 \%$ & 20.53 & $27.5 \%$ & 74.66 \\
\hline 2002 & 7.63 & $10.4 \%$ & 28.47 & $38.7 \%$ & 45.18 & $61.3 \%$ & 19.27 & $26.2 \%$ & 73.65 \\
\hline & \multicolumn{9}{|c|}{ Average annual percentage change } \\
\hline 1973-2002 & $-1.2 \%$ & & $-0.3 \%$ & & $1.7 \%$ & & $-0.3 \%$ & & $0.8 \%$ \\
\hline 1992-2002 & $-1.5 \%$ & & $1.0 \%$ & & $1.4 \%$ & & $1.3 \%$ & & $1.2 \%$ \\
\hline
\end{tabular}

\section{Source:}

U.S. Department of Energy, Energy Information Administration, International Petroleum Monthly, Tables 4.1 and 4.3. (Additional resources: www.eia.doe.gov)

${ }^{a}$ Includes natural gas plant liquids, crude oil and lease condensate. Does not account for all inputs or refinery processing gain.

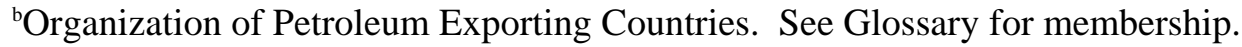

'See Glossary for Persian Gulf nations. 
The United States has accounted for approximately one-quarter of the world's petroleum consumption for the last two decades.

Table 1.4

World Petroleum Consumption, 1960-2002 (million barrels per day)

\begin{tabular}{|c|c|c|c|c|c|}
\hline Year & $\begin{array}{l}\text { United } \\
\text { States }\end{array}$ & $\begin{array}{l}\text { U.S. } \\
\text { share }\end{array}$ & Total OECD ${ }^{\mathrm{a}}$ & $\begin{array}{c}\text { Total } \\
\text { non-OECD }\end{array}$ & World \\
\hline 1960 & 9.80 & $45.9 \%$ & 15.78 & 5.56 & 21.34 \\
\hline 1965 & 11.51 & $37.0 \%$ & 22.81 & 8.33 & 31.14 \\
\hline 1970 & 14.70 & $31.4 \%$ & 34.49 & 12.32 & 46.81 \\
\hline 1975 & 16.32 & $29.0 \%$ & 38.82 & 17.38 & 56.20 \\
\hline 1976 & 17.46 & $29.3 \%$ & 41.39 & 18.28 & 59.67 \\
\hline 1977 & 18.43 & $29.8 \%$ & 42.43 & 19.40 & 61.83 \\
\hline 1978 & 18.85 & $29.4 \%$ & 43.62 & 20.54 & 64.16 \\
\hline 1979 & 18.51 & $28.4 \%$ & 44.01 & 21.21 & 65.22 \\
\hline 1980 & 17.06 & $27.0 \%$ & 41.41 & 21.66 & 63.07 \\
\hline 1981 & 16.06 & $26.4 \%$ & 39.14 & 21.76 & 60.90 \\
\hline 1982 & 15.30 & $25.7 \%$ & 37.45 & 22.05 & 59.50 \\
\hline 1983 & 15.23 & $25.9 \%$ & 36.59 & 22.15 & 58.74 \\
\hline 1984 & 15.73 & $26.3 \%$ & 37.43 & 22.40 & 59.83 \\
\hline 1985 & 15.73 & $26.2 \%$ & 37.23 & 22.86 & 60.09 \\
\hline 1986 & 16.28 & $26.4 \%$ & 38.28 & 23.48 & 61.76 \\
\hline 1987 & 16.67 & $26.5 \%$ & 38.96 & 24.04 & 63.00 \\
\hline 1988 & 17.28 & $26.7 \%$ & 40.24 & 24.58 & 64.82 \\
\hline 1989 & 17.33 & $26.3 \%$ & 40.88 & 25.04 & 65.92 \\
\hline 1990 & 16.99 & $25.7 \%$ & 40.92 & 25.16 & 66.08 \\
\hline 1991 & 16.71 & $25.0 \%$ & 41.40 & 25.32 & 66.72 \\
\hline 1992 & 17.03 & $25.4 \%$ & 42.42 & 24.51 & 66.93 \\
\hline 1993 & 17.24 & $25.7 \%$ & 42.98 & 24.14 & 67.12 \\
\hline 1994 & 17.72 & $25.9 \%$ & 44.17 & 24.25 & 68.42 \\
\hline 1995 & 17.73 & $25.3 \%$ & 44.92 & 25.07 & 69.99 \\
\hline 1996 & 18.31 & $25.6 \%$ & 46.04 & 25.54 & 71.58 \\
\hline 1997 & 18.62 & $25.5 \%$ & 46.61 & 26.49 & 73.10 \\
\hline 1998 & 18.92 & $25.6 \%$ & 46.84 & 27.02 & 73.86 \\
\hline 1999 & 19.52 & $25.8 \%$ & 47.65 & 27.96 & 75.61 \\
\hline 2000 & 19.70 & $25.6 \%$ & 47.88 & 29.02 & 76.90 \\
\hline 2001 & 19.65 & $25.5 \%$ & 47.63 & 29.50 & 77.13 \\
\hline 2002 & 19.76 & $25.5 \%$ & 47.59 & 29.87 & 77.46 \\
\hline & \multicolumn{5}{|c|}{ Average annual percentage change } \\
\hline 1960-2002 & $1.7 \%$ & & $2.7 \%$ & $4.1 \%$ & $3.1 \%$ \\
\hline 1970-2002 & $0.9 \%$ & & $1.0 \%$ & $2.8 \%$ & $1.6 \%$ \\
\hline 1992-2002 & $1.5 \%$ & & $1.2 \%$ & $2.0 \%$ & $1.5 \%$ \\
\hline
\end{tabular}

\section{Source:}

U.S. Department of Energy, Energy Information Administration, Annual Energy Review 2002, Washington, DC, July 2003, Table 11.9 and updates from the International Petroleum Monthly, July 2003. (Additional resources: www.eia.doe.gov)

\footnotetext{
${ }^{a}$ Organization for Economic Cooperation and Development. See Glossary for membership.
} 
Figure 1.2. World Oil Reserves, Production and Consumption, 2002

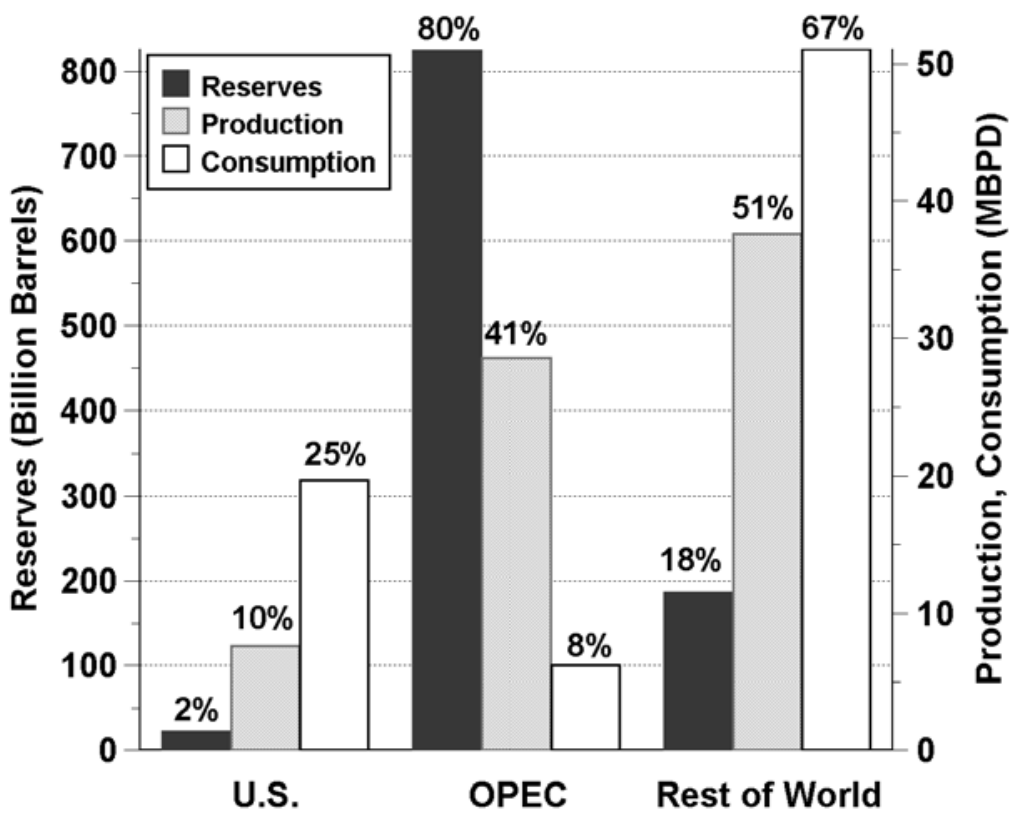

Table 1.5

World Oil Reserves, Production and Consumption, 2002

\begin{tabular}{|c|c|c|c|c|c|c|}
\hline & $\begin{array}{c}\text { Crude oil } \\
\text { reserves } \\
\text { (billion } \\
\text { barrels) }\end{array}$ & $\begin{array}{c}\text { Reserve } \\
\text { share }\end{array}$ & $\begin{array}{l}\text { Petroleum } \\
\text { production } \\
\text { (million } \\
\text { barrels } \\
\text { per day) }\end{array}$ & $\begin{array}{l}\text { Production } \\
\text { share }\end{array}$ & $\begin{array}{l}\text { Petroleum } \\
\text { consumption } \\
\text { (million } \\
\text { barrels } \\
\text { per day) }\end{array}$ & $\begin{array}{c}\text { Consumption } \\
\text { share }\end{array}$ \\
\hline U.S. & 22.4 & $2 \%$ & 7.6 & $10 \%$ & 19.7 & $25 \%$ \\
\hline OPEC & 823.5 & $80 \%$ & 28.5 & $41 \%$ & 6.2 & $8 \%$ \\
\hline Rest of world & 186.1 & $18 \%$ & 37.6 & $51 \%$ & 51.6 & $67 \%$ \\
\hline
\end{tabular}

\section{Source:}

Reserves - Energy Information Administration, International Energy Annual 2001, Table 8.1.

Production - Energy Information Administration, International Petroleum Monthly, July 2003, Tables 4.1a - 4.1c and 4.3

Consumption - Energy Information Administration, International Petroleum Monthly, July 2003, Table 4.6.

OPEC consumption (2001 data) - Energy Information Administration, International Energy Annual 2001, Table 1.2. (Additional resources: www.eia.doe.gov)

Note:

Total consumption is higher than total production due to refinery gains including alcohol and liquid products produced from coal and other sources.

OPEC countries include Venezuela, Iran, Iraq, Kuwait, Qatar, Saudi Arabia, United Arab Emirates, Algeria, Libya, Nigeria, Indonesia, Gabon, and Ecuador.

OPEC consumption data are for 2001. 
Figure 1.3. World Natural Gas Reserves, Production, and Consumption, 2000

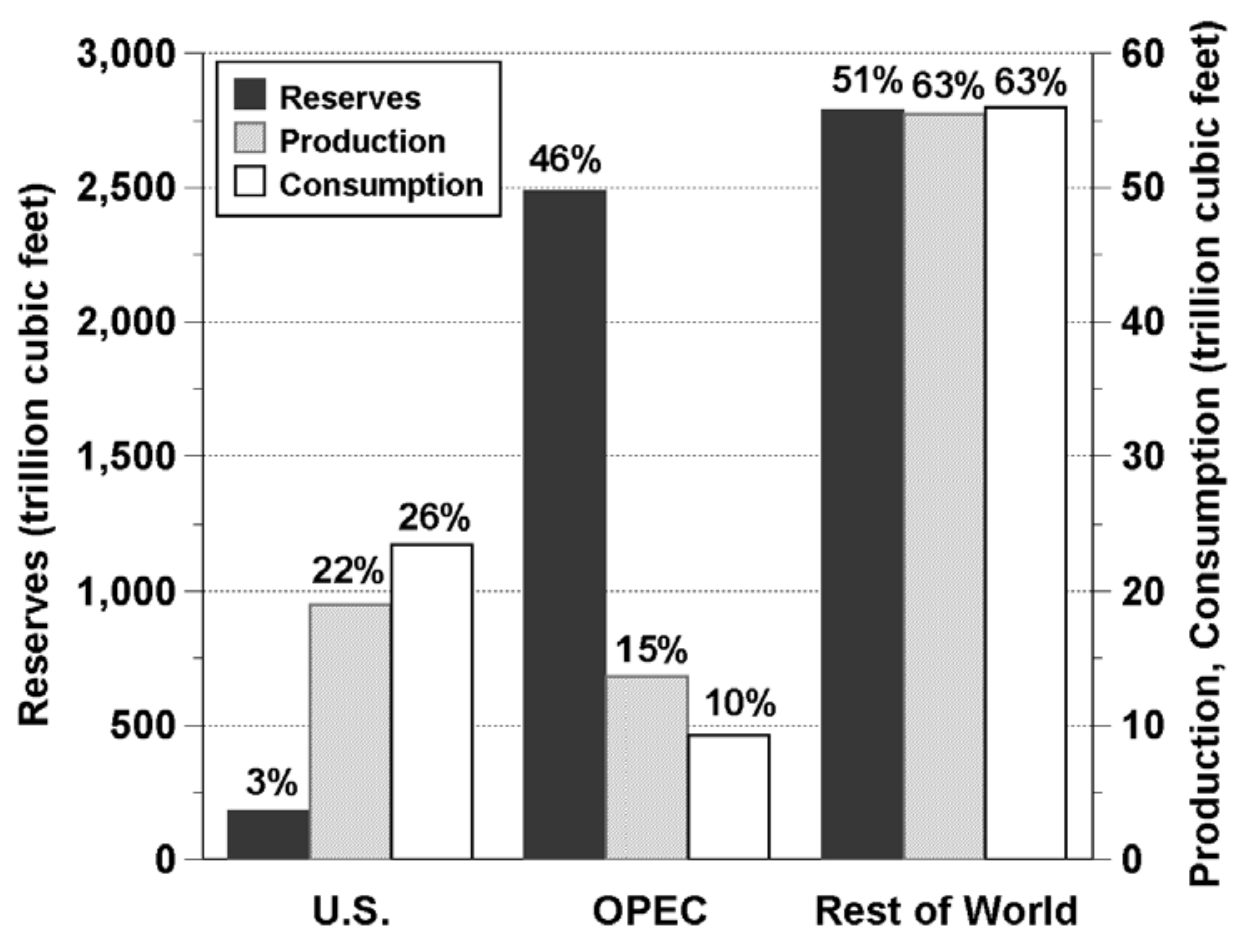

Table 1.6

World Natural Gas Reserves, Production and Consumption, 2000 (trillion cubic feet)

\begin{tabular}{lcccccc}
\hline & $\begin{array}{c}\text { Natural gas } \\
\text { reserves }\end{array}$ & $\begin{array}{c}\text { Reserve } \\
\text { share }\end{array}$ & $\begin{array}{c}\text { Natural gas } \\
\text { production }\end{array}$ & $\begin{array}{c}\text { Production } \\
\text { share }\end{array}$ & $\begin{array}{c}\text { Natural gas } \\
\text { consumption }\end{array}$ & $\begin{array}{c}\text { Consumption } \\
\text { share }\end{array}$ \\
\hline U.S. & 183.5 & $3 \%$ & 18.987 & $22 \%$ & 23.455 & $26 \%$ \\
OPEC & $2,485.1$ & $46 \%$ & 13.631 & $15 \%$ & 9.262 & $10 \%$ \\
Rest of world & $2,788.5$ & $51 \%$ & 55.5 & $63 \%$ & 56.0 & $63 \%$ \\
\hline
\end{tabular}

Source:

Energy Information Administration, International Energy Annual 2001, March 2003, Tables 4.2 and 8.1. (Additional resources: www.eia.doe.gov)

Note:

Reserves as of January 1, 2002. Production data are dry gas production. 
Total OECD government-owned petroleum stocks were slightly higher in 2002 than in 1995. The amount of petroleum held in government stocks is about one-third of what is held in commercial stocks.

Table 1.7

Petroleum Stocks of OECD Countries by Ownership, 1995-2002 (million barrels)

\begin{tabular}{|c|c|c|c|c|c|c|c|c|}
\hline \multirow[b]{2}{*}{ Year } & \multicolumn{2}{|c|}{ OECD Europe } & \multicolumn{2}{|c|}{ Japan } & \multicolumn{2}{|c|}{ United States $^{\mathrm{a}}$} & \multicolumn{2}{|c|}{ Total OECD ${ }^{\mathrm{b}}$} \\
\hline & Commercial & $\begin{array}{l}\text { Government- } \\
\text { owned }\end{array}$ & Commercial & $\begin{array}{l}\text { Government- } \\
\text { owned }\end{array}$ & Commercial & $\begin{array}{l}\text { Government- } \\
\text { owned }\end{array}$ & Commercial & $\begin{array}{l}\text { Government- } \\
\text { owned }\end{array}$ \\
\hline 1995 & 1,153 & 63 & 336 & 295 & 993 & 592 & 2,651 & 950 \\
\hline 1996 & 1,191 & 63 & 351 & 300 & 969 & 566 & 2,659 & 929 \\
\hline 1997 & 1,189 & 63 & 370 & 315 & 1,022 & 563 & 2,744 & 941 \\
\hline 1998 & 1,257 & 63 & 334 & 315 & 1,098 & 571 & 2,851 & 949 \\
\hline 1999 & 1,174 & 63 & 314 & 315 & 939 & 567 & 2,592 & 945 \\
\hline 2000 & 1,196 & 64 & 322 & 312 & 951 & 541 & 2,635 & 917 \\
\hline 2001 & 1,235 & 57 & 341 & 316 & 1,048 & 550 & 2,920 & 923 \\
\hline \multirow[t]{2}{*}{2002} & 1,208 & 57 & 298 & 321 & 888 & 599 & 2,715 & 977 \\
\hline & \multicolumn{8}{|c|}{ Average annual percentage change } \\
\hline $\begin{array}{l}1995- \\
2002\end{array}$ & $1.2 \%$ & $-1.7 \%$ & $0.2 \%$ & $1.2 \%$ & $0.9 \%$ & $-1.2 \%$ & $1.6 \%$ & $-0.5 \%$ \\
\hline
\end{tabular}

\section{Source:}

U.S. Department of Energy, Energy Information Administration, International Petroleum Monthly, June 2003, Table 1.6, and annual. (Additional resources: www.eia.doe.gov)

${ }^{a}$ Includes U.S. territories.

${ }^{b}$ Total OECD includes OECD Europe, Japan, United States, and other OECD countries. Look in the Glossary for a complete listing of OECD countries. 
This chart shows the volatility of crude oil prices since 1870. Given this volatility, it is difficult for anyone to predict future crude oil prices with any certainty.

Figure 1.4. Crude Oil Prices in Current and Constant Terms, 1870-2002
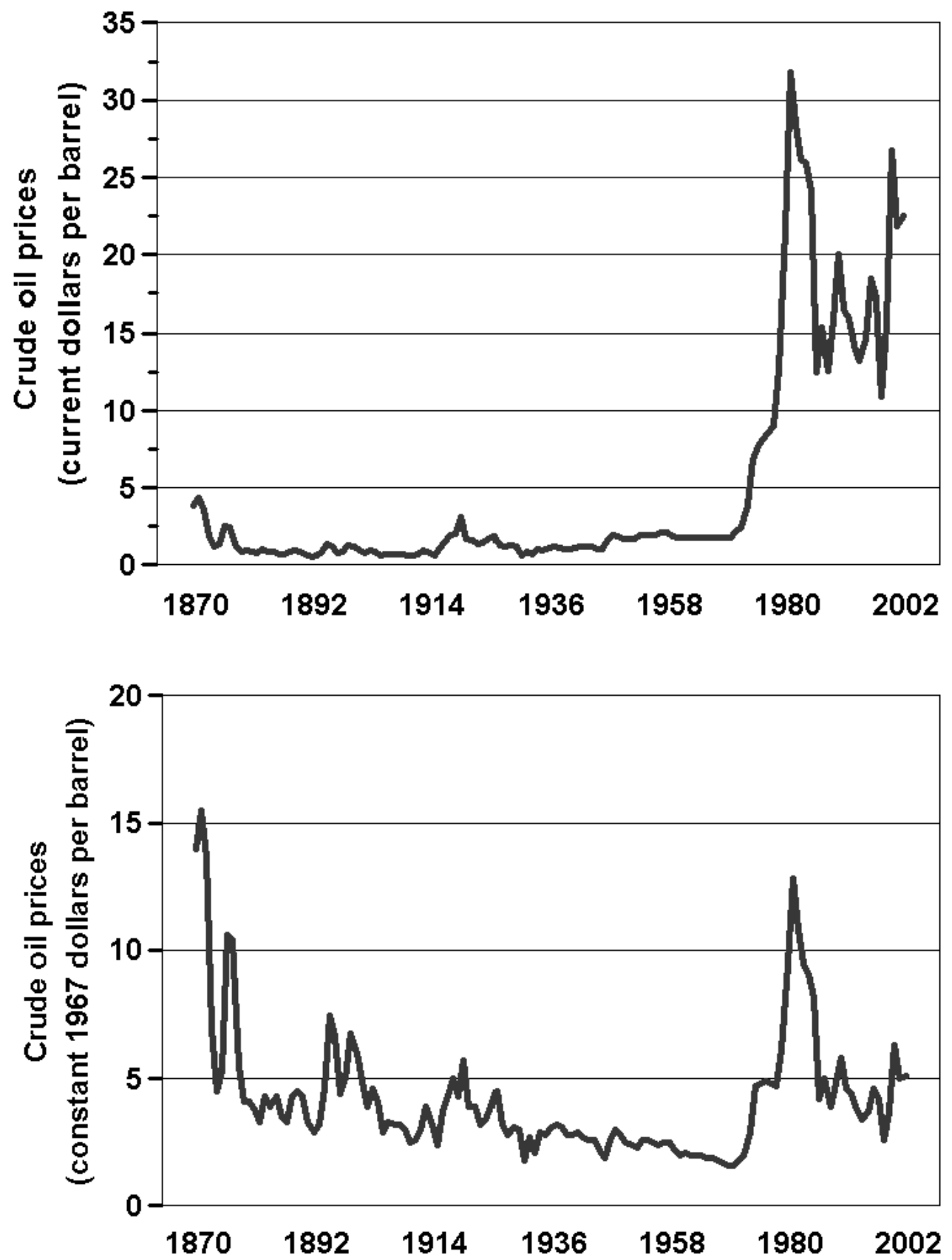

Source:

1870-1972 Crude oil prices - American Petroleum Institute, Basic Petroleum Data Book, Volume XXI, Number 2, August 2001.

1973-2001 Crude oil prices - U.S. Department of Energy, Energy Information Administration, Monthly Energy Review, April 2003, Table 9.1, domestic first purchase price. 
The share of petroleum imported to the U.S. can be calculated using total imports or net imports. Net imports, which is the preferred data, rose to $50 \%$ of U.S. petroleum consumption for the first time in 1998, while total imports reached $50 \%$ for the first time in 1993. OPEC share of net imports dropped from 51\% in 2001 to $43.7 \%$ in 2002.

Table 1.8

U.S. Petroleum Imports by World Region of Origin, 1960-2002 (million barrels per day)

\begin{tabular}{|c|c|c|c|c|c|c|c|}
\hline Year & $\begin{array}{c}\text { Net } \\
\text { OPEC } \\
\text { imports }\end{array}$ & $\begin{array}{c}\text { Net } \\
\text { OPEC } \\
\text { share }\end{array}$ & $\begin{array}{c}\text { Net } \\
\text { Persian } \\
\text { Gulf nation } \\
\text { imports } \\
\end{array}$ & $\begin{array}{c}\text { Net } \\
\text { Persian } \\
\text { Gulf } \\
\text { share } \\
\end{array}$ & $\begin{array}{c}\text { Net } \\
\text { imports }\end{array}$ & $\begin{array}{c}\text { Net imports } \\
\text { as a share of } \\
\text { U.S. } \\
\text { consumption }\end{array}$ & $\begin{array}{c}\text { Total } \\
\text { imports }\end{array}$ \\
\hline 1960 & 1.31 & $81.3 \%$ & $\mathrm{c}$ & $\mathrm{c}$ & 1.61 & $\mathrm{c}$ & 1.82 \\
\hline 1965 & 1.48 & $64.7 \%$ & c & c & 2.28 & c & 2.47 \\
\hline 1970 & 1.34 & $42.5 \%$ & с & с & 3.16 & с & 3.42 \\
\hline 1975 & 3.60 & $61.6 \%$ & c & c & 5.85 & $35.8 \%$ & 6.06 \\
\hline 1980 & 4.29 & $67.5 \%$ & $\because{ }^{\prime}$ & $\dddot{\mathrm{c}}$ & 6.37 & $37.3 \%$ & 6.91 \\
\hline 1981 & 3.32 & $61.4 \%$ & 1.22 & $22.5 \%$ & 5.40 & $33.6 \%$ & 6.00 \\
\hline 1982 & 2.14 & $49.7 \%$ & 0.69 & $16.1 \%$ & 4.30 & $28.1 \%$ & 5.11 \\
\hline 1983 & 1.84 & $42.7 \%$ & 0.44 & $10.2 \%$ & 4.31 & $28.3 \%$ & 5.05 \\
\hline 1984 & 2.04 & $43.2 \%$ & 0.50 & $10.6 \%$ & 4.72 & $30.0 \%$ & 5.44 \\
\hline 1985 & 1.82 & $42.5 \%$ & 0.31 & $7.2 \%$ & 4.29 & $27.3 \%$ & 5.07 \\
\hline 1986 & 2.83 & $52.0 \%$ & 0.91 & $16.7 \%$ & 5.44 & $33.4 \%$ & 6.22 \\
\hline 1987 & 3.06 & $51.7 \%$ & 1.07 & $18.2 \%$ & 5.91 & $35.5 \%$ & 6.68 \\
\hline 1988 & 3.51 & $53.3 \%$ & 1.53 & $23.2 \%$ & 6.59 & $38.1 \%$ & 7.40 \\
\hline 1989 & 4.12 & $57.3 \%$ & 1.86 & $25.8 \%$ & 7.20 & $41.6 \%$ & 8.06 \\
\hline 1990 & 4.29 & $59.8 \%$ & 1.96 & $27.4 \%$ & 7.16 & $42.2 \%$ & 8.02 \\
\hline 1991 & 4.07 & $61.3 \%$ & 1.83 & $27.7 \%$ & 6.63 & $39.6 \%$ & 7.63 \\
\hline 1992 & 4.07 & $58.7 \%$ & 1.77 & $25.6 \%$ & 6.94 & $40.8 \%$ & 7.89 \\
\hline 1993 & 4.25 & $55.8 \%$ & 1.77 & $23.3 \%$ & 7.62 & $44.2 \%$ & 8.62 \\
\hline 1994 & 4.23 & $52.6 \%$ & 1.72 & $21.4 \%$ & 8.05 & $45.4 \%$ & 9.00 \\
\hline 1995 & 3.98 & $50.5 \%$ & 1.56 & $19.8 \%$ & 7.89 & $44.5 \%$ & 8.84 \\
\hline 1996 & 4.19 & $49.3 \%$ & 1.60 & $18.8 \%$ & 8.50 & $46.4 \%$ & 9.48 \\
\hline 1997 & 4.54 & $49.6 \%$ & 1.75 & $19.1 \%$ & 9.16 & $49.2 \%$ & 10.16 \\
\hline 1998 & 4.88 & $50.0 \%$ & 2.13 & $21.8 \%$ & 9.76 & $51.6 \%$ & 10.71 \\
\hline 1999 & 4.93 & $49.8 \%$ & 2.46 & $24.8 \%$ & 9.91 & $50.8 \%$ & 10.85 \\
\hline 2000 & 5.18 & $49.7 \%$ & 2.48 & $23.8 \%$ & 10.42 & $52.9 \%$ & 11.46 \\
\hline 2001 & 5.43 & $51.0 \%$ & 2.73 & $25.7 \%$ & 10.64 & $55.5 \%$ & 11.62 \\
\hline 2002 & 4.61 & $43.7 \%$ & 2.27 & $21.5 \%$ & 10.55 & $52.8 \%$ & 11.53 \\
\hline & \multicolumn{7}{|c|}{ Average annual percentage change } \\
\hline 1960-2002 & $3.0 \%$ & & c & & $4.6 \%$ & & $4.5 \%$ \\
\hline 1970-2002 & $3.9 \%$ & & c & & $3.8 \%$ & & $3.9 \%$ \\
\hline 1992-2002 & $1.3 \%$ & & $4.2 \%$ & $2.5 \%$ & $4.3 \%$ & & $3.9 \%$ \\
\hline
\end{tabular}

\section{Source:}

U.S. Department of Energy, Energy Information Administration, Annual Energy Review 2002, Washington, DC, July 2002, Tables 5.4 and 5.7 and updates from the International Petroleum Monthly, July 2003, Table 4.10. Consumption: Transportation Energy Databook, Table 1.12.

\footnotetext{
a Organization of Petroleum Exporting Countries. See Glossary for membership.

b See Glossary for Persian Gulf nations.

${ }^{\mathrm{c}}$ Data are not available.
} 


\section{The Costs of Oil Dependence}

In the Costs of Oil Dependence: A 2000 Update, authors Greene and Tishchishyna indicate that the oil market upheavals caused by the OPEC cartel over the last 30 years have cost the U.S. in the vicinity of $\$ 7$ trillion (present value 1998 dollars) in total economic costs, which is about as large as the sum total of payment on the national debt over the same period.

Oil dependence is the product of (1) a noncompetitive world oil market strongly influenced by the OPEC cartel, (2) high levels of U.S. oil imports, (3) oil's critical role in the U.S. economy, and (4) the absence of economical and readily available substitutes for oil. Transportation is key to the problem because transportation vehicles account for $68 \%$ of U.S. oil consumption and nearly all of the high-value light products that drive the market.

Oil consuming economies incur three types of costs when monopoly power is used to raise prices above competitive market levels:

- Loss of potential gross domestic product (GDP) - the economy's ability to produce is reduced because a key factor of production is more expensive;

- Macroeconomic Adjustment Costs - sudden changes in oil prices increase unemployment, further reducing economic output; and

- Transfer of Wealth - some of the wealth of oil consuming states is appropriated by foreign oil producers.

Major oil price shocks have disrupted world energy markets four times in the past 30 years (1973-74, 1979-80, 199091, 1999-2000). Each of the first three oil price shocks was followed by an economic recession in the U.S.

Figure 1.5. Oil Price and Economic Growth, 1970-2002

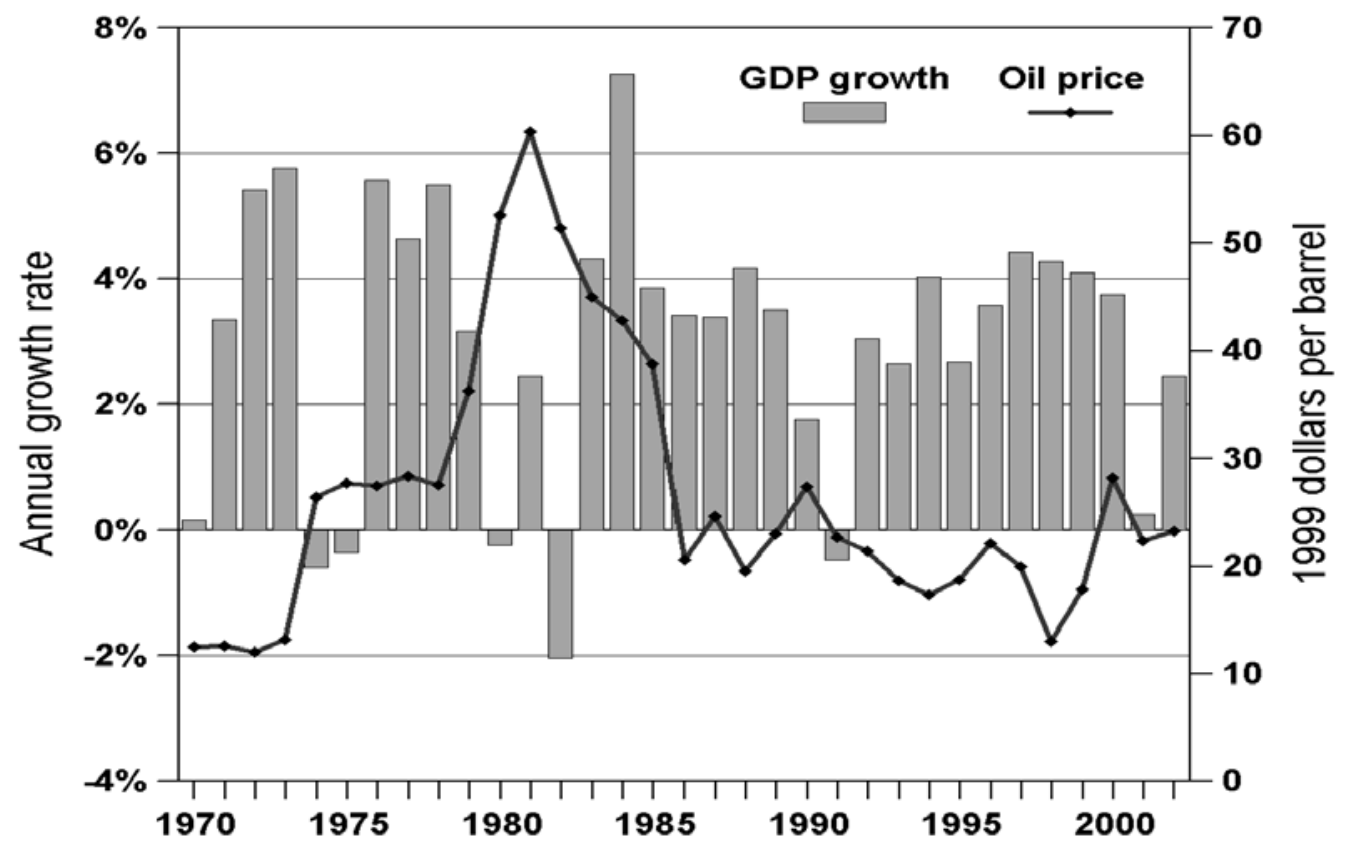

\section{Source:}

Greene, D.L. and N. I. Tishchishyna, Costs of Oil Dependence: A 2000 Update, Oak Ridge National Laboratory, ORNL/TM-2000/152, Oak Ridge, TN, 2000, and data updates, 2003.

(Additional resources: www-cta.ornl.gov/publications) 
Estimates of military expenditures for defending oil supplies in the Middle East range from $\$ 6$ to $\$ 60$ billion per year. This wide range in estimates reflects the difficulty in assigning a precise figure to the military cost of defending the U.S. interests in the Middle East. The two main reasons for the difficulty are 1) the Department of Defense does not divide the budget into regional defense sectors and 2) it is difficult to determine how much of the cost is attributable to defending Persian Gulf oil. The latest study, done by the National Defense Council Foundation, puts a price of $\$ 49$ billion dollars/year for the defense of oil.

Table 1.9

Summary of Military Expenditures f or Defending Oil Supplies from the Middle East

\begin{tabular}{lcc}
\hline \multicolumn{1}{c}{ Source } & $\begin{array}{c}\text { Original estimates } \\
\text { (billion dollars) }\end{array}$ & $\begin{array}{c}\text { Year of } \\
\text { original estimate }\end{array}$ \\
\hline General Accounting Office [1] & $\$ 33$ & 1990 \\
Congressional Research Service [2] & $\$ 6.4$ & 1990 \\
Greene and Leiby [3] & $\$ 14.3$ & 1990 \\
Kaufmann and Steinbruner [4] & $\$ 64.5$ & 1990 \\
Ravenal [5] & $\$ 50$ & 1992 \\
Delucchi and Murphy ${ }^{\mathrm{a}}$ [6] & $\$ 20-40$ & 1996 \\
National Defense Council Foundation [7] & $\$ 49.1$ & 2003 \\
\hline
\end{tabular}

[1] U.S. General Accounting Offices, Southwest Asia: Cost of Protecting U.S. Interests, GAO/NSIAD-91-250, Washington, DC, August 1991.

[2] Congressional Research Service, The External Costs of Oil Used in Transportation, prepared for the U.S. Alternative Fuels Council, Washington, DC, June 1992.

[3] Greene, D.L., and P. Leiby, The Social Costs to the U.S. of Monopolization of the World Oil Market, 1972-1991, ORNL-6744, Oak Ridge National Laboratory, Oak Ridge, TN, March 1993.

[5] Ravenal, E.C., Designing Defense for a New World Order: The Military Budget in 1992 and Beyond, Cato Institute, Washington, DC, 1991.

[4] Kaufmann, W.W., and J.D. Steinbruner, Decisions for Defense: Prospects for a New Order, The Brookings Institution, Washington, DC, 1991.

[6] Delucchi, M.A., and J. Murphy, U.S. Military Expenditures to Protect the Use of Persian-Gulf Oil for Motor Vehicles, UCD-ITS-RR-96-3 (15), University of California, Davis, California, April 1996.

[7] National Defense Council Foundation, Alexandria, VA, forthcoming publication, 2003.

Source:

Hu, P.S., "Estimates of 1996 U.S. Military Expenditures on Defending Oil Supplies from the Middle East: A Literature Review,” Oak Ridge National Laboratory, Oak Ridge, TN, March 1996.

${ }^{a}$ Annual cost to defend all U.S. interests in the Persian Gulf. 
Other parts of the world refine crude oil to produce more diesel fuel and less gasoline than does North

America. The OECD Pacific countries produce the lowest share of gasoline.

Figure 1.6. Refinery Gross Output by World Region, 2002

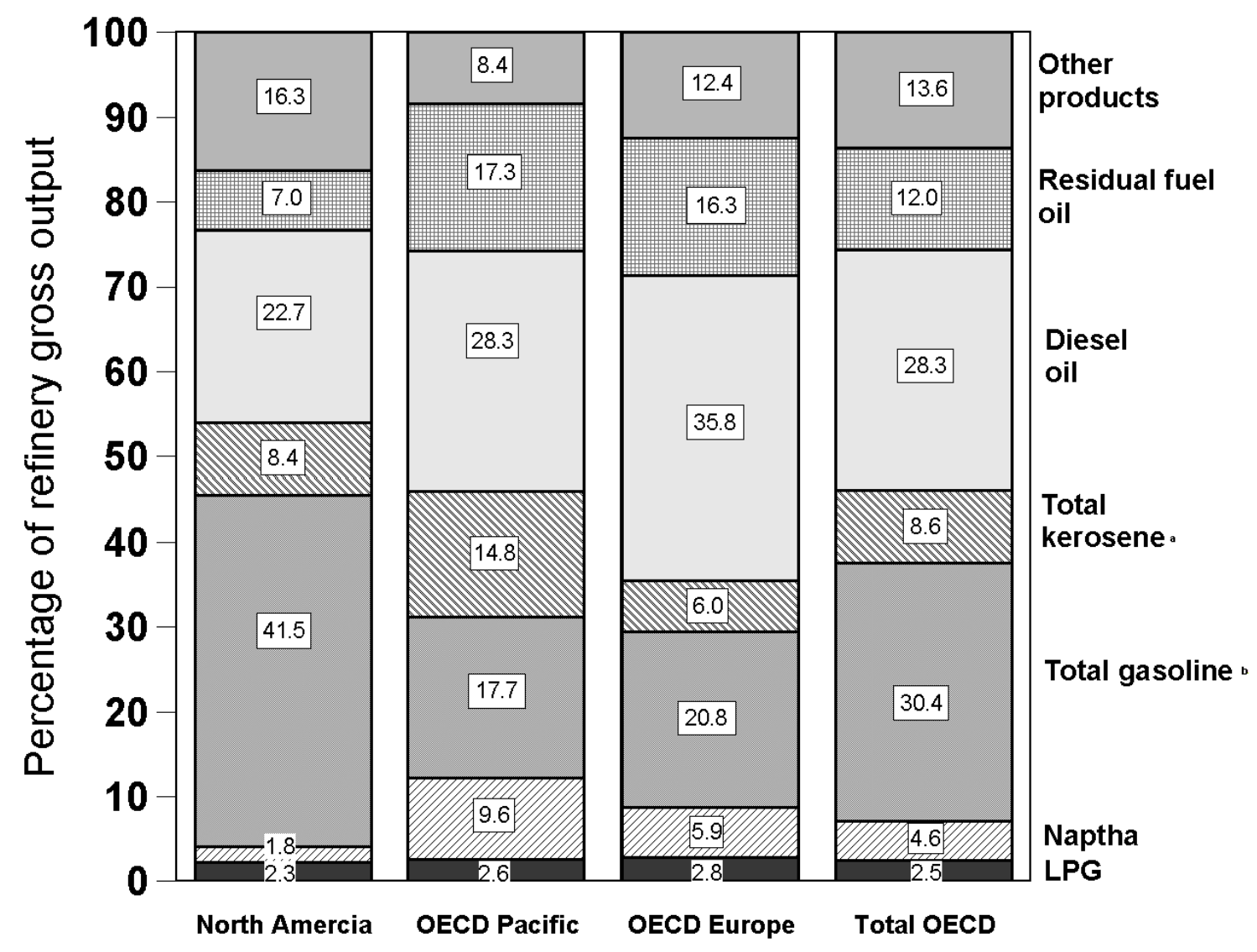

Source:

International Energy Agency, Monthly Oil Survey, January 2003, Paris, France, Table 7.

(Additional resources: www.iea.org)

${ }^{\mathrm{a}}$ Includes jet kerosene and other kerosene.

${ }^{\mathrm{b}}$ Includes motor gasoline, jet gasoline, and aviation gasoline.

${ }^{\mathrm{c}}$ Organization for Economic Cooperation and Development. See Glossary for membership. 
Oxygenate refinery input increased significantly in 1995, most certainly due to the Clean Air Act Amendments of 1990 which mandated the sale of reformulated gasoline in certain areas beginning in January 1995.

Table 1.10

U.S. Refinery Input of Crude Oil and Petroleum Products, 1987-2002

(thousand barrels)

\begin{tabular}{|c|c|c|c|c|c|c|c|c|c|}
\hline \multirow[b]{2}{*}{ Year } & \multirow[b]{2}{*}{ Crude oil } & \multirow[b]{2}{*}{$\begin{array}{c}\text { Natural gas } \\
\text { liquids }\end{array}$} & \multicolumn{4}{|c|}{ Oxygenates } & \multirow[b]{2}{*}{$\begin{array}{c}\text { Other } \\
\text { hydrocarbons }^{\mathrm{c}}\end{array}$} & \multirow[b]{2}{*}{$\begin{array}{c}\text { Other } \\
\text { liquids }\end{array}$} & \multirow[b]{2}{*}{$\begin{array}{c}\text { Total input to } \\
\text { refineries }\end{array}$} \\
\hline & & & $\begin{array}{c}\text { Fuel } \\
\text { ethanol }\end{array}$ & Methanol & $\mathrm{MTBE}^{\mathrm{a}}$ & $\begin{array}{c}\text { Other } \\
\text { oxygenates }^{\mathrm{b}}\end{array}$ & & & \\
\hline 1987 & $4,691,783$ & 280,889 & d & $\mathrm{d}$ & d & $\mathrm{d}$ & 23,304 & 220,296 & $5,105,392$ \\
\hline 1990 & $4,894,379$ & 170,589 & d & d & d & d & 28,642 & 231,466 & $5,325,076$ \\
\hline 1991 & $4,855,016$ & 172,306 & d & d & d & d & 31,574 & 248,691 & $5,307,587$ \\
\hline 1992 & $4,908,603$ & 171,701 & d & d & d & d & 47,918 & 224,758 & $5,352,980$ \\
\hline 1994 & $5,061,111$ & 169,868 & 3,620 & 242 & 52,937 & 1,676 & 14,130 & 179,678 & $5,483,262$ \\
\hline 1995 & $5,100,317$ & 172,026 & 9,055 & 246 & 79,396 & 3,876 & 14,668 & 175,743 & $5,555,327$ \\
\hline 1996 & $5,195,265$ & 164,552 & 11,156 & 126 & 79,407 & 3,444 & 20,587 & 193,695 & $5,668,232$ \\
\hline 1997 & $5,351,466$ & 151,769 & 11,803 & 496 & 86,240 & 3,750 & 22,976 & 178,292 & $5,806,792$ \\
\hline 1998 & $5,434,383$ & 146,921 & 11,722 & 675 & 89,362 & 3,363 & 22,759 & 183,376 & $5,892,561$ \\
\hline 1999 & $5,403,450$ & 135,756 & 13,735 & 813 & 94,784 & 3,334 & 21,447 & 204,332 & $5,877,651$ \\
\hline 2001 & $5,521,637$ & 156,479 & 16,929 & 1,431 & 87,116 & 3,113 & 24,903 & 167,729 & $5,979,337$ \\
\hline \multirow[t]{2}{*}{2002} & $5,455,530$ & 155,429 & 26,320 & 13 & 90,291 & 2,325 & 21,895 & 202,672 & $5,955,475$ \\
\hline & $1.1 \%$ & $-3.9 \%$ & e & Aver & $\underset{\mathrm{e}}{\text { annual per }}$ & $\underset{\mathrm{e}}{\text { ntage }}$ change & $-0.4 \%$ & $-0.6 \%$ & $1.0 \%$ \\
\hline 1993-2002 & $1.0 \%$ & $-1.6 \%$ & $25.7 \%$ & $-36.6 \%$ & $6.9 \%$ & $8.8 \%$ & $3.9 \%$ & $-2.9 \%$ & $0.9 \%$ \\
\hline
\end{tabular}

\section{Source:}

U.S. Department of Energy, Energy Information Administration, Petroleum Supply Annual, 2002, Vol. 1, June 2003, Table 16, and annual.

(Additional resources: www.eia.doe.gov)

${ }^{a}$ Methyl tertiary butyl ether (MTBE).

bIncludes ethyl tertiary butyl ether (ETBE), tertiary amyl methyl ether (TAME), tertiary butyl alcohol (TBA), and other aliphatic alcohols and ethers intended for motor gasoline blending.

'For 1987-92, includes other hydrocarbons/hydrogen/oxygenates. For 1993-on, includes other hydrocarbons/hydrogen.

${ }^{\mathrm{d}}$ Reported in "Other hydrocarbons” category in this year.

eData are not available. 
When crude oil and other hydrocarbons are processed into products that are, on average, less dense than the input, a processing volume gain occurs. Due to this gain, the product yield from a barrel of crude oil is more than 100\%. The processing volume gain has been growing over the years.

Table 1.11

Refinery Yield of Petroleum Products from a Barrel of Crude Oil, 1978-2002 (percentage)

\begin{tabular}{ccccccc}
\hline Year & $\begin{array}{c}\text { Motor } \\
\text { gasoline }\end{array}$ & $\begin{array}{c}\text { Distillate } \\
\text { fuel oil }\end{array}$ & Jet fuel & $\begin{array}{c}\text { Liquified } \\
\text { petroleum gas }\end{array}$ & Other $^{\mathrm{a}}$ & Total $^{\mathrm{b}}$ \\
\hline 1978 & 44.1 & 21.4 & 6.6 & 2.3 & 29.6 & 104.0 \\
1979 & 43.0 & 21.5 & 6.9 & 2.3 & 30.3 & 104.0 \\
1980 & 44.5 & 19.7 & 7.4 & 2.4 & 30.0 & 104.0 \\
1981 & 44.8 & 20.5 & 7.6 & 2.4 & 28.7 & 104.0 \\
1982 & 46.4 & 21.5 & 8.1 & 2.2 & 26.2 & 104.4 \\
1983 & 47.6 & 20.5 & 8.5 & 2.7 & 24.8 & 104.1 \\
1984 & 46.7 & 21.5 & 9.1 & 2.9 & 24.2 & 104.4 \\
1985 & 45.6 & 21.6 & 9.6 & 3.1 & 24.6 & 104.5 \\
1986 & 45.7 & 21.2 & 9.8 & 3.2 & 24.8 & 104.7 \\
1987 & 46.4 & 20.5 & 10.0 & 3.4 & 24.5 & 104.8 \\
1988 & 46.0 & 20.8 & 10.0 & 3.6 & 24.4 & 104.8 \\
1989 & 45.7 & 20.8 & 10.1 & 4.0 & 24.2 & 104.8 \\
1990 & 45.6 & 20.9 & 10.7 & 3.6 & 24.1 & 104.9 \\
1991 & 45.7 & 21.3 & 10.3 & 3.8 & 24.1 & 105.2 \\
1992 & 46.0 & 21.2 & 9.9 & 4.3 & 24.0 & 105.4 \\
1993 & 46.1 & 21.9 & 10.0 & 4.1 & 23.3 & 105.4 \\
1994 & 45.5 & 22.3 & 10.1 & 4.2 & 23.2 & 105.3 \\
1995 & 46.4 & 21.8 & 9.7 & 4.5 & 22.9 & 105.3 \\
1996 & 45.7 & 22.7 & 10.4 & 4.5 & 22.4 & 105.7 \\
1997 & 45.7 & 22.5 & 10.3 & 4.6 & 22.5 & 105.6 \\
1998 & 46.2 & 22.3 & 10.4 & 4.4 & 22.5 & 105.8 \\
1999 & 46.5 & 22.3 & 10.2 & 4.5 & 22.3 & 105.8 \\
2000 & 46.2 & 23.1 & 10.3 & 4.5 & 22.0 & 106.1 \\
2001 & 46.2 & 23.8 & 9.8 & 4.3 & 21.7 & 105.8 \\
2002 & 47.3 & 23.2 & 9.8 & 4.3 & 21.5 & 106.1 \\
\hline
\end{tabular}

\section{Source:}

Department of Energy, Energy Information Administration, Petroleum Supply Annual 2002, Vol. 1, June 2003, Table 19 and annual. (Additional resources: www.eia.doe.gov)

\footnotetext{
${ }^{a}$ Includes aviation gasoline(0.1\%), kerosene (0.4\%), residential fuel oil (3.9\%), naphtha and other oils for petrochemical feedstock use $(2.6 \%)$, special naphthas $(0.3 \%)$, lubricants $(1.1 \%)$, waxes $(0.1 \%)$, petroleum coke (5.1\%), asphalt and road oil (3.2\%), still gas (4.3\%), and miscellaneous products ( $0.4 \%)$.

${ }^{\mathrm{b}}$ Products sum greater than $100 \%$ due to processing gain. The processing gain for years 1978 to 1980 is assumed to be $4 \%$.
} 
Most of the petroleum imported by the United States is in the form of crude oil. The U.S. does export small amounts

of petroleum, mainly refined petroleum products which go to Canada and Mexico.

Table 1.12

United States Petroleum Production, Imports and Exports, 1950-2002 (million barrels per day)

\begin{tabular}{|c|c|c|c|c|c|c|c|c|c|}
\hline & \multicolumn{3}{|c|}{ Domestic Production } & \multicolumn{3}{|c|}{ Net Imports } & \multicolumn{3}{|c|}{ Exports } \\
\hline & $\begin{array}{c}\text { Crude } \\
\text { oil }\end{array}$ & $\begin{array}{l}\text { Natural } \\
\text { gas } \\
\text { plant } \\
\text { liquids }\end{array}$ & Total $^{\mathrm{a}}$ & $\begin{array}{c}\text { Crude } \\
\text { oil }\end{array}$ & $\begin{array}{l}\text { Petroleum } \\
\text { products }\end{array}$ & Total & $\begin{array}{c}\text { Crude } \\
\text { oil }\end{array}$ & $\begin{array}{l}\text { Petroleum } \\
\text { products }\end{array}$ & Total \\
\hline 1950 & 5.41 & 0.50 & 5.91 & 0.49 & 0.22 & 0.85 & 0.10 & 0.21 & 0.31 \\
\hline 1955 & 6.81 & 0.77 & 7.58 & 0.78 & 0.46 & 1.23 & 0.03 & 0.34 & 0.37 \\
\hline 1960 & 7.05 & 0.93 & 7.99 & 1.02 & 0.80 & 1.82 & 0.01 & 0.19 & 0.20 \\
\hline 1965 & 7.80 & 1.21 & 9.01 & 1.24 & 1.23 & 2.47 & 0.00 & 0.18 & 0.19 \\
\hline 1970 & 9.64 & 1.66 & 11.30 & 1.32 & 2.10 & 3.42 & 0.01 & 0.25 & 0.26 \\
\hline 1975 & 8.37 & 1.63 & 10.05 & 4.11 & 1.95 & 6.06 & 0.01 & 0.20 & 0.21 \\
\hline 1980 & 8.62 & 1.58 & 10.24 & 5.26 & 1.65 & 6.91 & 0.29 & 0.26 & 0.54 \\
\hline 1981 & 8.57 & 1.61 & 10.23 & 4.40 & 1.60 & 6.00 & 0.23 & 0.37 & 0.60 \\
\hline 1982 & 8.65 & 1.55 & 10.25 & 3.49 & 1.63 & 5.11 & 0.24 & 0.58 & 0.82 \\
\hline 1983 & 8.69 & 1.56 & 10.30 & 3.33 & 1.72 & 5.05 & 0.16 & 0.58 & 0.74 \\
\hline 1984 & 8.90 & 1.63 & 10.58 & 3.43 & 2.01 & 5.44 & 0.18 & 0.54 & 0.72 \\
\hline 1985 & 8.97 & 1.61 & 10.64 & 3.20 & 1.87 & 5.07 & 0.20 & 0.58 & 0.78 \\
\hline 1986 & 8.68 & 1.55 & 10.29 & 4.18 & 2.05 & 6.22 & 0.15 & 0.63 & 0.79 \\
\hline 1987 & 8.35 & 1.60 & 10.01 & 4.67 & 2.00 & 6.68 & 0.15 & 0.61 & 0.76 \\
\hline 1988 & 8.16 & 1.63 & 9.84 & 5.11 & 2.30 & 7.40 & 0.16 & 0.66 & 0.82 \\
\hline 1989 & 7.61 & 1.55 & 9.22 & 5.84 & 2.22 & 8.06 & 0.14 & 0.72 & 0.86 \\
\hline 1990 & 7.36 & 1.56 & 8.99 & 5.89 & 2.12 & 8.02 & 0.11 & 0.75 & 0.86 \\
\hline 1991 & 7.42 & 1.66 & 9.17 & 5.78 & 1.84 & 7.63 & 0.12 & 0.88 & 1.00 \\
\hline 1992 & 7.18 & 1.70 & 9.01 & 6.08 & 1.81 & 7.89 & 0.09 & 0.86 & 0.95 \\
\hline 1993 & 6.85 & 1.74 & 8.84 & 6.79 & 1.83 & 8.62 & 0.10 & 0.90 & 1.00 \\
\hline 1994 & 6.66 & 1.73 & 8.65 & 7.06 & 1.93 & 9.00 & 0.10 & 0.84 & 0.94 \\
\hline 1995 & 6.56 & 1.76 & 8.63 & 7.23 & 1.61 & 8.84 & 0.09 & 0.86 & 0.95 \\
\hline 1996 & 6.47 & 1.83 & 8.61 & 7.51 & 1.97 & 9.48 & 0.11 & 0.87 & 0.98 \\
\hline 1997 & 6.45 & 1.82 & 8.61 & 8.23 & 1.94 & 10.16 & 0.11 & 0.90 & 1.00 \\
\hline 1998 & 6.25 & 1.76 & 8.39 & 8.71 & 2.00 & 10.71 & 0.11 & 0.84 & 0.95 \\
\hline 1999 & 5.88 & 1.83 & 8.11 & 8.73 & 2.12 & 10.85 & 0.12 & 0.82 & 0.94 \\
\hline 2000 & 5.82 & 1.91 & 8.11 & 9.07 & 2.39 & 11.46 & 0.05 & 0.99 & 1.04 \\
\hline 2001 & 5.80 & 1.87 & 8.05 & 9.33 & 2.54 & 11.87 & 0.02 & 0.95 & 0.97 \\
\hline \multirow[t]{2}{*}{2002} & 5.82 & 1.88 & 8.12 & 9.05 & 2.31 & 11.36 & 0.01 & 0.97 & 0.98 \\
\hline & \multicolumn{9}{|c|}{ Average annual percentage change } \\
\hline 1950-2002 & $0.1 \%$ & $2.6 \%$ & $0.6 \%$ & $5.8 \%$ & $4.6 \%$ & $5.1 \%$ & $0.0 \%$ & $3.0 \%$ & $2.2 \%$ \\
\hline 1970-2002 & $-1.6 \%$ & $0.4 \%$ & $-1.0 \%$ & $6.2 \%$ & $0.3 \%$ & $3.8 \%$ & $0.0 \%$ & $4.3 \%$ & $4.2 \%$ \\
\hline 1992-2002 & $-2.1 \%$ & $1.0 \%$ & $-1.0 \%$ & $4.1 \%$ & $2.5 \%$ & $3.7 \%$ & $-19.7 \%$ & $1.2 \%$ & $0.3 \%$ \\
\hline
\end{tabular}

Source:

U.S. Department of Energy, Energy Information Administration, Annual Energy Review 2002, July 2003, Tables 5.3 and 5.5 and Monthly Energy Review, April 2003, Tables 3.1a and 3.1b.

${ }^{a}$ Total domestic production includes crude oil, natural gas plant liquids and small amounts of other liquids. 
The U.S. share of the world's petroleum consumption is approximately one-quarter. The U.S. relies heavily on imported petroleum. Imports accounted for 53\% of U.S. petroleum consumption in 2002.

Table 1.13

Petroleum Production and Consumption Ratios, 1950-2002

\begin{tabular}{|c|c|c|c|c|c|c|c|c|}
\hline & $\begin{array}{c}\text { Domestic } \\
\text { petroleum } \\
\text { production }^{\mathrm{a}}\end{array}$ & $\begin{array}{c}\text { Net } \\
\text { petroleum } \\
\text { imports } \\
\end{array}$ & $\begin{array}{c}\text { Transportation } \\
\text { petroleum } \\
\text { consumption } \\
\end{array}$ & $\begin{array}{c}\text { U.S. } \\
\text { petroleum } \\
\text { consumption } \\
\end{array}$ & $\begin{array}{c}\text { World } \\
\text { petroleum } \\
\text { consumption }\end{array}$ & $\begin{array}{c}\text { Net } \\
\text { imports } \\
\text { as a share of } \\
\text { U.S. }\end{array}$ & $\begin{array}{c}\text { U.S. } \\
\text { petroleum } \\
\text { consumption } \\
\text { as a share of } \\
\text { world }\end{array}$ & $\begin{array}{c}\text { Transportation } \\
\text { petroleum use } \\
\text { as a share of } \\
\text { domestic }\end{array}$ \\
\hline & \multicolumn{5}{|c|}{ (million barrels per day) } & consumption & consumption & production \\
\hline 1950 & 5.91 & 0.55 & 3.36 & 6.46 & \multirow{2}{*}{$\begin{array}{l}\mathrm{b} \\
\mathrm{b}\end{array}$} & $8.4 \%$ & \multirow{2}{*}{$\begin{array}{l}\mathrm{b} \\
\mathrm{b}\end{array}$} & $56.8 \%$ \\
\hline 1955 & 7.58 & 0.88 & 4.46 & 8.46 & & $10.4 \%$ & & $58.8 \%$ \\
\hline 1960 & 7.99 & 1.62 & 5.15 & 9.82 & 21.34 & $16.5 \%$ & $46.0 \%$ & $64.5 \%$ \\
\hline 1965 & 9.01 & 2.28 & 6.04 & 11.51 & 31.14 & $19.8 \%$ & $37.0 \%$ & $67.0 \%$ \\
\hline 1970 & 11.30 & 3.16 & 7.78 & 14.70 & 46.81 & $21.5 \%$ & $31.4 \%$ & $68.9 \%$ \\
\hline 1975 & 10.05 & 5.85 & 8.95 & 16.32 & 56.20 & $35.8 \%$ & $29.0 \%$ & $89.1 \%$ \\
\hline 1980 & 10.24 & 6.38 & 9.57 & 17.10 & 63.07 & $37.3 \%$ & $27.1 \%$ & $93.5 \%$ \\
\hline 1981 & 10.23 & 5.40 & 9.49 & 16.06 & 60.90 & $33.6 \%$ & $26.4 \%$ & $92.7 \%$ \\
\hline 1982 & 10.25 & 4.30 & 9.31 & 15.30 & 59.50 & $28.1 \%$ & $25.7 \%$ & $90.8 \%$ \\
\hline 1983 & 10.30 & 4.31 & 9.41 & 15.23 & 58.74 & $28.3 \%$ & $25.9 \%$ & $91.3 \%$ \\
\hline 1984 & 10.58 & 4.73 & 9.71 & 15.77 & 59.84 & $30.0 \%$ & $26.4 \%$ & $91.8 \%$ \\
\hline 1985 & 10.64 & 4.29 & 9.85 & 15.73 & 60.10 & $27.3 \%$ & $26.2 \%$ & $92.6 \%$ \\
\hline 1986 & 10.29 & 5.44 & 10.23 & 16.28 & 61.76 & $33.4 \%$ & $26.4 \%$ & $99.5 \%$ \\
\hline 1987 & 10.01 & 5.91 & 10.53 & 16.67 & 63.00 & $35.5 \%$ & $26.5 \%$ & $105.2 \%$ \\
\hline 1988 & 9.84 & 6.60 & 10.91 & 17.28 & 64.82 & $38.1 \%$ & $26.7 \%$ & $110.9 \%$ \\
\hline 1989 & 9.22 & 7.20 & 11.00 & 17.33 & 65.92 & $41.6 \%$ & $26.3 \%$ & 119.3\% \\
\hline 1990 & 8.99 & 7.16 & 10.97 & 16.99 & 65.98 & $42.2 \%$ & $25.7 \%$ & $122.0 \%$ \\
\hline 1991 & 9.17 & 6.63 & 10.80 & 16.71 & 66.73 & $39.6 \%$ & $25.0 \%$ & $117.8 \%$ \\
\hline 1992 & 9.01 & 6.94 & 10.97 & 17.03 & 66.92 & $40.8 \%$ & $25.4 \%$ & $121.8 \%$ \\
\hline 1993 & 8.84 & 7.62 & 11.18 & 17.24 & 67.12 & $44.2 \%$ & $25.7 \%$ & $126.5 \%$ \\
\hline 1994 & 8.65 & 8.05 & 11.48 & 17.72 & 68.42 & $45.4 \%$ & $25.9 \%$ & $132.7 \%$ \\
\hline 1995 & 8.63 & 7.89 & 11.72 & 17.73 & 69.99 & $44.5 \%$ & $25.3 \%$ & $135.8 \%$ \\
\hline 1996 & 8.61 & 8.50 & 11.99 & 18.31 & 71.58 & $46.4 \%$ & $25.6 \%$ & $139.3 \%$ \\
\hline 1997 & 8.61 & 9.16 & 12.12 & 18.62 & 73.10 & $49.2 \%$ & $25.5 \%$ & $140.8 \%$ \\
\hline 1998 & 8.39 & 9.76 & 12.46 & 18.92 & 73.86 & $51.6 \%$ & $25.6 \%$ & $148.5 \%$ \\
\hline 1999 & 8.11 & 9.91 & 12.83 & 19.52 & 75.61 & $50.8 \%$ & $25.8 \%$ & $158.2 \%$ \\
\hline 2000 & 8.11 & 10.42 & 13.12 & 19.70 & 76.90 & $52.9 \%$ & $25.6 \%$ & $161.8 \%$ \\
\hline 2001 & 8.05 & 10.90 & 13.51 & 19.65 & 77.13 & $55.5 \%$ & $25.5 \%$ & $167.8 \%$ \\
\hline \multirow[t]{2}{*}{2002} & 8.12 & 10.38 & 13.15 & 19.66 & b & $52.8 \%$ & b & $161.9 \%$ \\
\hline & \multicolumn{8}{|c|}{ Average annual percentage change } \\
\hline $1950-2002$ & $0.6 \%$ & $5.8 \%$ & $2.7 \%$ & $2.2 \%$ & b & & & \\
\hline $1970-2002$ & $-1.0 \%$ & $3.8 \%$ & $1.7 \%$ & $0.9 \%$ & $1.6 \%{ }^{c}$ & & & \\
\hline 1992-2002 & $-1.0 \%$ & $4.1 \%$ & $1.8 \%$ & $1.4 \%$ & $1.6 \%{ }^{c}$ & & & \\
\hline
\end{tabular}

Source:

U.S. Department of Energy, Energy Information Administration, Monthly Energy Review, April 2003, Tables 2.5, 3.1a, 3.1b, and A3. (Pre-1973 data from the Annual Energy Review).

World petroleum consumption - U.S. Department of Energy, Energy Information Administration, International Energy Annual 2001, March 2003, Table 1.1, and annual.

(Additional resources: www.eia.doe.gov)

${ }^{a}$ Total domestic production includes crude oil, natural gas plant liquids and small amounts of other liquids.

${ }^{\mathrm{b}}$ Data are not available.

${ }^{\mathrm{c}}$ Average annual percentage change is to the latest year possible. 
The transportation oil gap is the difference between the amount of petroleum the U.S. produces and the amount of petroleum used by the transportation sector. This gap has been getting wider not only due to increasing transportation demand, but also due to decreasing U.S. petroleum production.

Figure 1.7. United States Petroleum Production and Consumption, 1970-2025

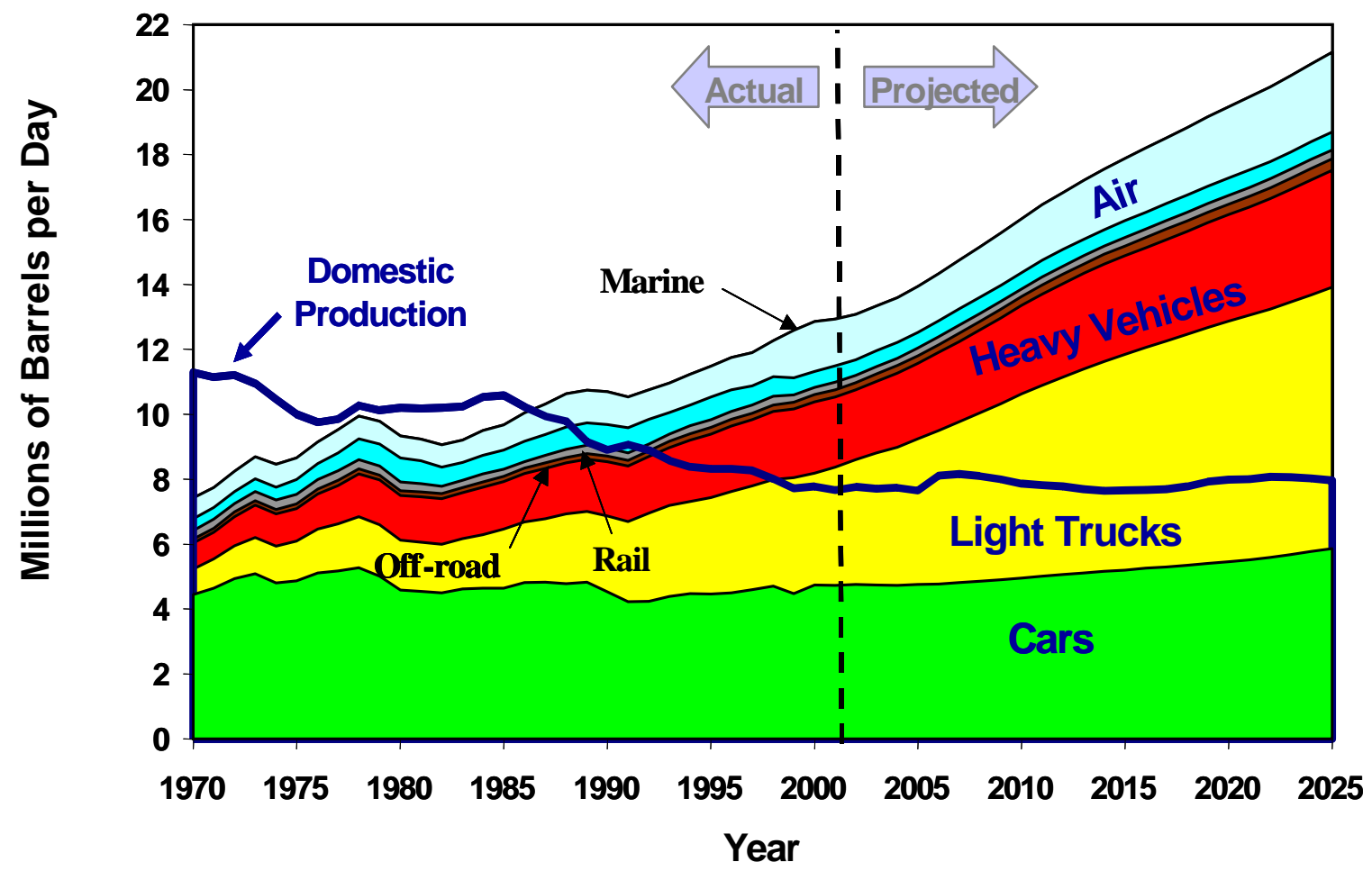

Source:

See Tables 1.12 and 2.5. Projections are from the Energy Information Administration, Annual Energy Outlook 2003, January 2003. 
Transportation accounts for more than two-thirds of the U.S. petroleum use. The residential sector and the commercial sector data which were previously combined are now available separately.

Table 1.14

Consumption of Petroleum by End-Use Sector, 1973-2002 (million barrels per day)

\begin{tabular}{rccccccc}
\hline Year & Transportation & Percentage & Residential & Commercial & Industrial & $\begin{array}{c}\text { Electric } \\
\text { utilities }\end{array}$ & Total \\
\hline 1973 & 9.06 & $52.3 \%$ & 1.49 & 0.75 & 4.48 & 1.54 & 17.31 \\
1974 & 8.84 & $53.1 \%$ & 1.36 & 0.68 & 4.30 & 1.48 & 16.65 \\
1975 & 8.95 & $54.8 \%$ & 1.32 & 0.63 & 4.04 & 1.39 & 16.32 \\
1976 & 9.40 & $53.8 \%$ & 1.43 & 0.70 & 4.46 & 1.52 & 17.51 \\
1977 & 9.76 & $53.0 \%$ & 1.42 & 0.72 & 4.82 & 1.71 & 18.43 \\
1978 & 10.16 & $53.9 \%$ & 1.38 & 0.69 & 4.87 & 1.75 & 18.85 \\
1979 & 10.01 & $54.1 \%$ & 1.09 & 0.63 & 5.34 & 1.44 & 18.52 \\
1980 & 9.57 & $56.1 \%$ & 0.91 & 0.61 & 4.85 & 1.15 & 17.10 \\
1981 & 9.49 & $59.1 \%$ & 0.81 & 0.52 & 4.27 & 0.96 & 16.06 \\
1982 & 9.31 & $60.8 \%$ & 0.76 & 0.48 & 4.06 & 0.69 & 15.30 \\
1983 & 9.41 & $61.8 \%$ & 0.74 & 0.55 & 3.86 & 0.68 & 15.23 \\
1984 & 9.71 & $61.7 \%$ & 0.71 & 0.58 & 4.20 & 0.56 & 15.77 \\
1985 & 9.85 & $62.6 \%$ & 0.79 & 0.50 & 4.10 & 0.48 & 15.72 \\
1986 & 10.23 & $62.9 \%$ & 0.78 & 0.53 & 4.10 & 0.64 & 16.28 \\
1987 & 10.53 & $63.2 \%$ & 0.81 & 0.52 & 4.25 & 0.55 & 16.67 \\
1988 & 10.90 & $63.1 \%$ & 0.84 & 0.51 & 4.40 & 0.69 & 17.33 \\
1989 & 11.00 & $63.5 \%$ & 0.85 & 0.47 & 4.35 & 0.75 & 17.42 \\
1990 & 10.97 & $64.5 \%$ & 0.70 & 0.45 & 4.18 & 0.57 & 16.86 \\
1991 & 10.80 & $64.6 \%$ & 0.72 & 0.42 & 4.55 & 0.53 & 17.02 \\
1992 & 10.97 & $64.4 \%$ & 0.73 & 0.40 & 4.44 & 0.44 & 16.96 \\
1993 & 11.18 & $64.8 \%$ & 0.77 & 0.37 & 4.64 & 0.50 & 17.44 \\
1994 & 11.48 & $64.8 \%$ & 0.74 & 0.37 & 4.54 & 0.47 & 17.59 \\
1995 & 11.72 & $66.1 \%$ & 0.76 & 0.35 & 4.80 & 0.33 & 17.96 \\
1996 & 11.99 & $65.5 \%$ & 0.84 & 0.37 & 4.92 & 0.36 & 18.48 \\
1997 & 12.12 & $65.1 \%$ & 0.81 & 0.35 & 4.81 & 0.41 & 18.51 \\
1998 & 12.46 & $65.9 \%$ & 0.75 & 0.33 & 4.80 & 0.58 & 18.92 \\
1999 & 12.83 & $65.7 \%$ & 0.84 & 0.34 & 4.98 & 0.53 & 19.52 \\
2000 & 13.12 & $66.4 \%$ & 0.87 & 0.37 & 4.89 & 0.51 & 19.76 \\
2001 & 13.01 & $66.2 \%$ & 0.86 & 0.37 & 4.85 & 0.56 & 19.66 \\
2002 & 13.15 & $67.1 \%$ & 0.84 & 0.37 & 4.85 & 0.40 & 19.60 \\
& & $A v 90$ ane & & & & & \\
$1973-2002$ & $1.3 \%$ & $-2.0 \%$ & $-2.4 \%$ & $0.3 \%$ & $-4.5 \%$ & $0.4 \%$ \\
$1992-2002$ & $1.8 \%$ & & $1.4 \%$ & $-0.8 \%$ & $0.9 \%$ & $-0.9 \%$ & $1.5 \%$ \\
\hline & & & & & & &
\end{tabular}

Source:

U.S. Department of Energy, Energy Information Administration, Monthly Energy Review, April 2003, Tables 2.2-2.6. Converted to million barrels per day using Table A3.

(Additional resources: www.eia.doe.gov) 
Pipelines accounted for two-thirds of the domestic movement of petroleum and petroleum products in 2001.

Table 1.15

Ton-Miles of Petroleum and Petroleum Products in the U.S. by Mode, 1975-2001

\begin{tabular}{|c|c|c|c|c|c|}
\hline \multirow[b]{2}{*}{ Year } & Pipelines $^{\mathrm{a}}$ & Water carriers & Motor carriers $^{\mathrm{b}}$ & Railroads & \multirow{2}{*}{$\frac{\text { Total }}{\text { (billion ton-miles) }}$} \\
\hline & \multicolumn{4}{|c|}{ (percent) } & \\
\hline 1975 & $59.9 \%$ & $35.2 \%$ & $3.3 \%$ & $1.7 \%$ & 846.7 \\
\hline 1976 & $59.4 \%$ & $35.4 \%$ & $3.8 \%$ & $1.5 \%$ & 867.7 \\
\hline 1977 & $59.1 \%$ & $36.1 \%$ & $3.2 \%$ & $1.6 \%$ & 923.4 \\
\hline 1978 & $50.5 \%$ & $45.7 \%$ & $2.7 \%$ & $1.1 \%$ & $1,160.2$ \\
\hline 1979 & $51.8 \%$ & $44.5 \%$ & $2.6 \%$ & $1.2 \%$ & $1,174.8$ \\
\hline 1980 & $47.2 \%$ & $49.6 \%$ & $2.2 \%$ & $1.0 \%$ & $1,245.3$ \\
\hline 1981 & $46.3 \%$ & $50.7 \%$ & $2.0 \%$ & $1.0 \%$ & $1,218.4$ \\
\hline 1982 & $46.4 \%$ & $50.6 \%$ & $1.9 \%$ & $1.1 \%$ & $1,218.2$ \\
\hline 1983 & $45.5 \%$ & $51.5 \%$ & $2.1 \%$ & $1.0 \%$ & $1,223.5$ \\
\hline 1984 & $48.1 \%$ & $48.4 \%$ & $2.5 \%$ & $1.0 \%$ & $1,180.2$ \\
\hline 1985 & $47.2 \%$ & $49.4 \%$ & $2.4 \%$ & $1.0 \%$ & $1,195.5$ \\
\hline 1986 & $48.7 \%$ & $47.8 \%$ & $2.5 \%$ & $1.0 \%$ & $1,187.8$ \\
\hline 1987 & $49.1 \%$ & $47.4 \%$ & $2.5 \%$ & $1.0 \%$ & $1,195.8$ \\
\hline 1988 & $50.6 \%$ & $45.8 \%$ & $2.6 \%$ & $1.1 \%$ & $1,188.1$ \\
\hline 1989 & $53.4 \%$ & $42.6 \%$ & $2.8 \%$ & $1.2 \%$ & $1,094.2$ \\
\hline 1990 & $54.2 \%$ & $41.7 \%$ & $2.8 \%$ & $1.3 \%$ & $1,076.8$ \\
\hline 1991 & $53.3 \%$ & $42.8 \%$ & $2.7 \%$ & $1.3 \%$ & $1,086.1$ \\
\hline 1992 & $53.9 \%$ & $42.1 \%$ & $2.6 \%$ & $1.4 \%$ & $1,091.7$ \\
\hline 1993 & $57.3 \%$ & $38.8 \%$ & $2.4 \%$ & $1.5 \%$ & $1,034.6$ \\
\hline 1994 & $56.5 \%$ & $39.3 \%$ & $2.7 \%$ & $1.5 \%$ & $1,046.7$ \\
\hline 1995 & $57.5 \%$ & $38.4 \%$ & $2.5 \%$ & $1.6 \%$ & $1,044.9$ \\
\hline 1996 & $60.6 \%$ & $34.9 \%$ & $2.9 \%$ & $1.6 \%$ & $1,022.2$ \\
\hline 1997 & $64.5 \%$ & $30.9 \%$ & $2.9 \%$ & $1.8 \%$ & 956.5 \\
\hline 1998 & $66.7 \%$ & $28.5 \%$ & $3.0 \%$ & $1.8 \%$ & 929.8 \\
\hline 1999 & $67.7 \%$ & $27.1 \%$ & $3.2 \%$ & $2.1 \%$ & 912.9 \\
\hline 2000 & $66.1 \%$ & $28.0 \%$ & $3.6 \%$ & $2.3 \%$ & 873.3 \\
\hline \multirow[t]{2}{*}{2001} & $66.2 \%$ & $28.1 \%$ & $3.5 \%$ & $2.2 \%$ & 869.8 \\
\hline & \multicolumn{5}{|c|}{ Average annual percentage change } \\
\hline 1975-2001 & & & & & $0.1 \%$ \\
\hline 1991-2001 & & & & & $-2.2 \%$ \\
\hline
\end{tabular}

Source:

Association of Oil Pipelines, Shifts in Petroleum Transportation, Washington, DC, May 2003, Table 1.

(Additional resources: www.aopl.org)

a The amounts carried by pipeline are based on ton-miles of crude and petroleum products for Federally regulated pipelines (84 percent) plus an estimated breakdown of crude and petroleum products of the ton-miles for pipelines not Federally regulated (16 percent).

${ }^{\mathrm{b}}$ The amounts carried by motor carriers are estimated. 


\section{Chapter 2 \\ Energy}

Summary Statistics from Tables in this Chapter

\section{Source}

Table 2.1 Transportation share of U.S. energy consumption, 2002

$27.3 \%$

Table 2.2 Petroleum share of transportation energy consumption, 2002

$96.8 \%$

Table 2.3 Alternative fuel and oxygenate consumption, 2002

(thousand gasoline equivalent gallons)

(share)

MTBE

$2,531,000$

$62.6 \%$

Ethanol in gasohol

$1,118,900 \quad 27.7 \%$

Liquified petroleum gas

$255,515 \quad 6.3 \%$

Compressed natural gas

$13,554 \quad 2.8 \%$

Liquified natural gas

$10,504 \quad 0.3 \%$

E85/E95

$10,075 \quad 0.0 \%$

Electricity

$4,460 \quad 0.0 \%$

M85/M100

$330 \quad 0.0 \%$

Table 2.5 Transportation energy use by mode, 2001

(trillion Btu) (share)

Automobiles

9,124 33.9\%

Light trucks

$6,654 \quad 24.7 \%$

Heavy trucks

$4,826 \quad 17.9 \%$

Air

$2,411 \quad 9.0 \%$

Water

$1,155 \quad 4.3 \%$

Off-highway

$1,036 \quad 3.8 \%$

Pipeline

889

$3.3 \%$

Rail

$615 \quad 2.3 \%$

Buses

$203 \quad 0.8 \%$


Petroleum accounted for 40\% of the world's energy use in 2000. Though petroleum is the dominant energy source for both OECD countries and non-OECD countries, the non-OECD countries rely on coal, natural gas, and hydroelectric power more than OECD countries do.

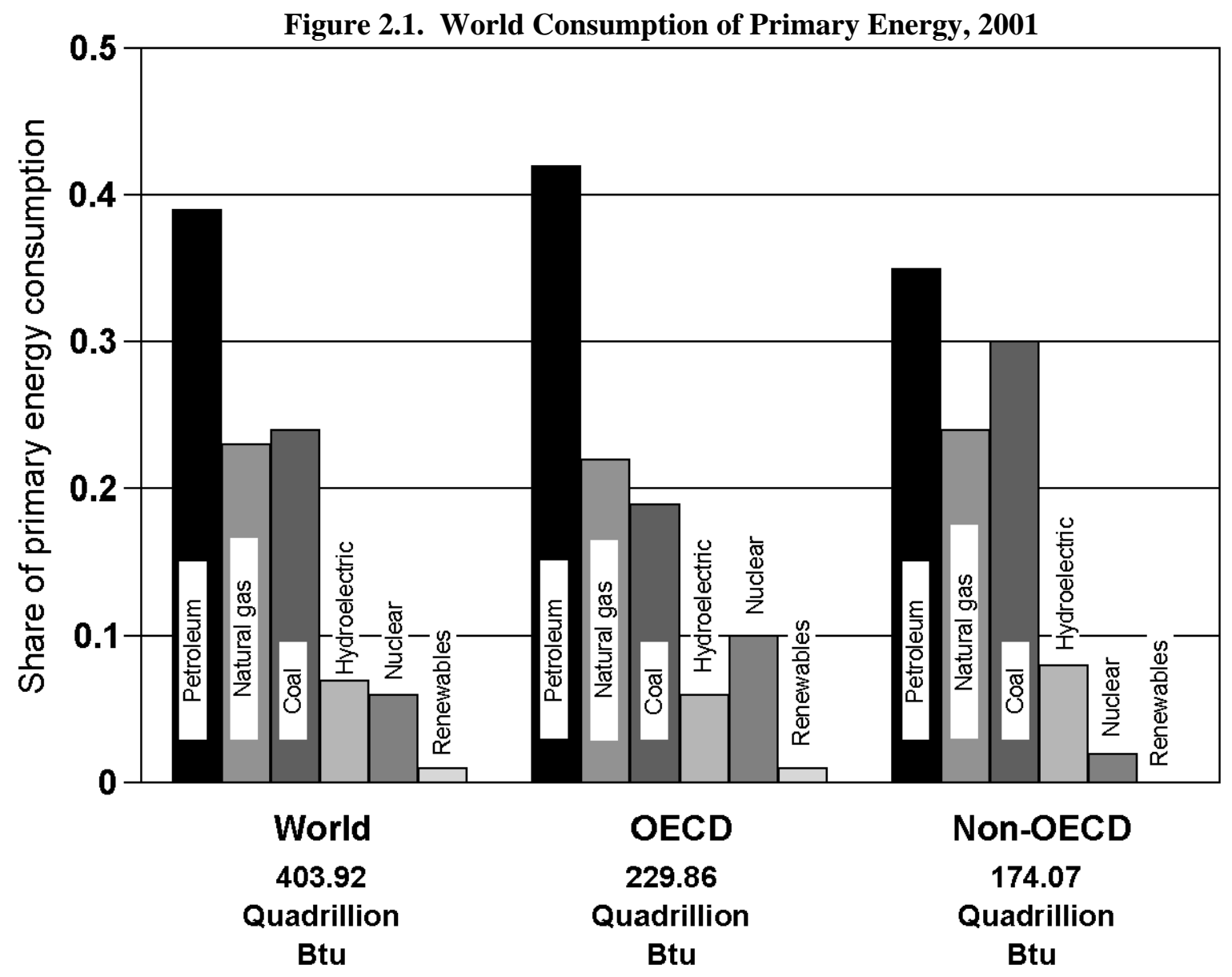

\section{Source:}

U.S. Department of Energy, Energy Information Administration, International Energy Annual 2001, Washington, DC, February 2003, Table 1.8. (Additional resources: www.eia.doe.gov) 
The Energy Information Administration revised the historical energy data series to include renewable energy in each sector. Also, the residential and commercial sector data are now separated. Total energy use was 97.9 quads in 2002 with transportation using $27.3 \%$.

Table 2.1

U. S. Consumption of Total Energy by End-Use Sector, 1973-2002 (quadrillion Btu)

\begin{tabular}{|c|c|c|c|c|c|c|}
\hline Year & Transportation & $\begin{array}{l}\text { Percentage } \\
\text { transportation } \\
\text { of total }\end{array}$ & Industrial & Commercial & Residential & Total \\
\hline 1973 & 18.6 & $24.6 \%$ & 32.7 & 9.5 & 14.9 & 75.7 \\
\hline 1974 & 18.1 & $24.5 \%$ & 31.8 & 9.4 & 14.7 & 74.0 \\
\hline 1975 & 18.2 & $25.3 \%$ & 29.4 & 9.5 & 14.8 & 72.0 \\
\hline 1976 & 19.1 & $25.1 \%$ & 31.4 & 10.0 & 15.4 & 76.0 \\
\hline 1977 & 19.8 & $25.4 \%$ & 32.3 & 10.2 & 15.7 & 78.0 \\
\hline 1978 & 20.6 & $25.8 \%$ & 32.7 & 10.5 & 16.2 & 80.0 \\
\hline 1979 & 20.5 & $25.3 \%$ & 34.0 & 10.6 & 15.8 & 80.9 \\
\hline 1980 & 19.7 & $25.2 \%$ & 32.2 & 10.6 & 15.9 & 78.3 \\
\hline 1981 & 19.5 & $25.6 \%$ & 30.8 & 10.6 & 15.4 & 76.3 \\
\hline 1982 & 19.1 & $26.0 \%$ & 27.7 & 10.9 & 15.6 & 73.2 \\
\hline 1983 & 19.1 & $26.2 \%$ & 27.5 & 11.0 & 15.5 & 73.1 \\
\hline 1984 & 19.8 & $25.8 \%$ & 29.6 & 11.5 & 15.8 & 76.7 \\
\hline 1985 & 20.1 & $26.3 \%$ & 29.0 & 11.5 & 15.9 & 76.4 \\
\hline 1986 & 20.8 & $27.1 \%$ & 28.4 & 11.6 & 15.9 & 76.7 \\
\hline 1987 & 21.5 & $27.1 \%$ & 29.5 & 12.0 & 16.2 & 79.2 \\
\hline 1988 & 22.3 & $27.0 \%$ & 30.8 & 12.6 & 17.1 & 82.8 \\
\hline 1989 & 22.6 & $26.6 \%$ & 31.4 & 13.2 & 17.8 & 84.9 \\
\hline 1990 & 22.5 & $26.6 \%$ & 31.9 & 13.3 & 16.9 & 84.6 \\
\hline 1991 & 22.1 & $26.2 \%$ & 31.5 & 13.5 & 17.4 & 84.5 \\
\hline 1992 & 22.5 & $26.2 \%$ & 32.7 & 13.4 & 17.3 & 85.9 \\
\hline 1993 & 22.9 & $26.1 \%$ & 32.7 & 13.8 & 18.2 & 87.6 \\
\hline 1994 & 23.5 & $26.3 \%$ & 33.6 & 14.1 & 18.1 & 89.2 \\
\hline 1995 & 24.0 & $26.3 \%$ & 33.9 & 14.7 & 18.7 & 91.2 \\
\hline 1996 & 24.5 & $26.0 \%$ & 34.9 & 15.2 & 19.6 & 94.2 \\
\hline 1997 & 24.8 & $26.2 \%$ & 35.2 & 15.7 & 19.1 & 94.7 \\
\hline 1998 & 25.4 & $26.8 \%$ & 34.8 & 16.0 & 19.1 & 95.1 \\
\hline 1999 & 26.1 & $27.0 \%$ & 34.7 & 16.4 & 19.6 & 97.8 \\
\hline 2000 & 26.7 & $27.0 \%$ & 34.7 & 17.2 & 20.5 & 99.0 \\
\hline 2001 & 26.4 & $27.5 \%$ & 32.4 & 17.3 & 20.3 & 96.2 \\
\hline 2002 & 26.7 & $27.3 \%$ & 33.1 & 17.5 & 20.9 & 97.9 \\
\hline \multicolumn{7}{|c|}{ Average annual percentage change } \\
\hline 1973-2002 & $1.3 \%$ & & $0.0 \%$ & $2.1 \%$ & $1.2 \%$ & $0.9 \%$ \\
\hline 1992-2002 & $1.7 \%$ & & $0.1 \%$ & $2.7 \%$ & $1.9 \%$ & $1.3 \%$ \\
\hline
\end{tabular}

Source:

U.S. Department of Energy, Energy Information Administration, Monthly Energy Review, April 2003, Washington, DC, Table 2.1. (Additional resources: www.eia.doe.gov)

${ }^{a}$ Electrical energy losses have been distributed among the sectors. 
The Energy Information Administration revised the historical energy data series to include renewable energy in each sector. In transportation, the alcohol fuels blended into gasoline to make gasohol (10\% ethanol or less) are now counted under "renewables" and have been taken out of petroleum. The petroleum category, however, still contains other blending agents, such as MTBE, that are not actually petroleum, but are not broken out into a separate category.

Table 2.2

Distribution of Energy Consumption by Source, 1973 and 2002 (percentage)

\begin{tabular}{|c|c|c|c|c|c|c|c|c|c|c|}
\hline \multirow{2}{*}{$\begin{array}{l}\text { Energy } \\
\text { source }\end{array}$} & \multicolumn{2}{|c|}{ Transportation } & \multicolumn{2}{|c|}{ Residential } & \multicolumn{2}{|c|}{ Commercial } & \multicolumn{2}{|c|}{ Industrial } & \multicolumn{2}{|c|}{ Electric utilities } \\
\hline & 1973 & 2002 & 1973 & 2002 & 1973 & 2002 & 1973 & 2002 & 1973 & 2002 \\
\hline Petroleum $^{\mathrm{a}}$ & 95.8 & 96.8 & 18.9 & 7.1 & 16.4 & 4.1 & 27.9 & 27.4 & 17.7 & 2.3 \\
\hline Natural gas ${ }^{\mathrm{b}}$ & 4.0 & 2.3 & 33.2 & 24.2 & 27.8 & 18.5 & 31.8 & 28.0 & 18.8 & 14.9 \\
\hline Coal & 0.0 & 0.0 & 0.7 & 0.1 & 1.6 & 0.6 & 12.4 & 6.3 & 43.5 & 52.4 \\
\hline Renewable & 0.0 & 0.7 & 2.4 & 2.0 & 0.1 & 0.6 & 3.6 & 5.2 & 15.4 & 9.1 \\
\hline Nuclear & 0.0 & 0.0 & 0.0 & 0.0 & 0.0 & 0.0 & 0.0 & 0.0 & 4.6 & 21.3 \\
\hline Electricity $^{\mathrm{c}}$ & 0.2 & 0.2 & 44.9 & 66.6 & 54.1 & 76.3 & 24.4 & 33.0 & 0.0 & 0.0 \\
\hline Other $^{\mathrm{d}}$ & 0.0 & 0.0 & 0.0 & 0.0 & 0.0 & 0.0 & 0.0 & 0.0 & 0.0 & 0.0 \\
\hline Total & 100.0 & 100.0 & 100.0 & 100.0 & 100.0 & 100.0 & 100.0 & 100.0 & 100.0 & 100.0 \\
\hline
\end{tabular}

\section{Source:}

U.S. Department of Energy, Energy Information Administration, Monthly Energy Review, April 2003, Washington, DC, pp. 27, 29, 31, 33. (Additional resources: www.eia.doe.gov)

\footnotetext{
${ }^{\mathrm{a}}$ In transportation, the petroleum category contains some blending agents which are not petroleum.

${ }^{\mathrm{b}}$ Includes supplemental gaseous fuels. Transportation sector includes pipeline fuel and natural gas vehicle use.

${ }^{\mathrm{c}}$ Includes electrical system energy losses.

${ }^{\mathrm{d}}$ Energy generated from geothermal, wood, waste, wind, photovoltaic, and solar thermal energy sources.
} 
Oxygenates are blended with gasoline to be used in conventional vehicles. The amount of oxygenate use dwarfs the alternative fuel use. Gasoline-equivalent gallons are used in this table to allow comparisons of different fuel types.

Table 2.3

Alternative Fuel and Oxygenate Consumption, 1992-2002 (thousand gasoline-equivalent gallons)

\begin{tabular}{|c|c|c|c|c|c|c|c|}
\hline Alternative fuel & 1993 & 1995 & 1998 & 2000 & 2001 & $2002^{\mathrm{a}}$ & $\begin{array}{c}2002 \\
\text { Percentage }\end{array}$ \\
\hline Liquified petroleum gas & 264,655 & 232,701 & 241,386 & 242,062 & 251,353 & 255,515 & $6.3 \%$ \\
\hline Compressed natural gas & 21,603 & 35,162 & 72,412 & 98,351 & 111,797 & 113,554 & $2.8 \%$ \\
\hline Liquified natural gas & 1,901 & 2,759 & 5,343 & 7,121 & 8,786 & 10,504 & $0.3 \%$ \\
\hline $\mathrm{M}^{\mathrm{b}} \mathrm{b}$ & 1,593 & 2,023 & 1,212 & 585 & 440 & 330 & $0.0 \%$ \\
\hline M100 & 3,166 & 2,150 & 449 & 437 & 406 & 0 & $0.0 \%$ \\
\hline $\mathrm{E} 85^{\mathrm{b}}$ & 48 & 190 & 1,727 & 7,074 & 8,736 & 10,075 & $0.2 \%$ \\
\hline E95 ${ }^{b}$ & 80 & 995 & 59 & 13 & 0 & 0 & $0.0 \%$ \\
\hline Electricity $^{\mathrm{c}}$ & 288 & 663 & 1,202 & 2,670 & 3,903 & 4,460 & $0.1 \%$ \\
\hline Subtotal & 229,334 & 276,643 & 323,790 & 363,313 & 385,421 & 394,438 & $9.8 \%$ \\
\hline \multicolumn{8}{|l|}{ Oxygenates } \\
\hline MTBE $^{\mathrm{d}}$ & $2,069,200$ & $2,691,200$ & $2,903,400$ & $3,087,900$ & $2,890,400$ & $2,531,000$ & $62.6 \%$ \\
\hline Ethanol in gasohol & 760,000 & 910,700 & 889,500 & $1,106,300$ & $1,117,500$ & $1,118,900$ & $27.7 \%$ \\
\hline Total & $3,122,534$ & $3,878,543$ & $4,116,690$ & $4,564,329$ & $4,418,752$ & $4,044,338$ & $100.0 \%$ \\
\hline
\end{tabular}

\section{Source:}

U.S. Department of Energy, Energy Information Administration, Alternatives to Traditional Transportation Fuels, 2000, Washington, DC, September 2002, web site www.eia.doe.gov/cneaf/alternate/page/datatables/atf113_00.html, Table 10. (Additional resources: www.eia.doe.gov)

Note:

These data were released in September 2002. Please check the source web site for updates which were not available when this document went to press.

${ }^{\text {a}}$ Based on plans or projections.

${ }^{b}$ Consumption includes gasoline portion of the mixture.

'Vehicle consumption only; does not include power plant inputs.

${ }^{d}$ Methyl Tertiary Butyl Ether. This category includes a very small amount of other ethers, primarily Tertiary Amyl Methyl Ether (TAME) and Ethyl Tertiary Butyl Ether (ETBE). 
As data about alternative fuel use become available, an attempt is made to incorporate them into this table. Sometimes assumptions must be made in order to use the data. Please see Appendix A for a description of the methodology used to develop these data.

Table 2.4

Domestic Consumption of Transportation Energy by Mode and Fuel Type, $2001^{\mathrm{a}}$ (trillion Btu)

\begin{tabular}{|c|c|c|c|c|c|c|c|c|c|}
\hline & Gasoline & Diesel fuel & $\begin{array}{l}\text { Liquified } \\
\text { petroleum } \\
\text { gas }\end{array}$ & Jet fuel & $\begin{array}{r}\text { Residual } \\
\text { fuel oil }\end{array}$ & $\begin{array}{c}\text { Natural } \\
\text { gas }\end{array}$ & Electricity & Methanol & Total \\
\hline HIGHWAY & $16,044.3$ & $4,750.8$ & 25.8 & & & 8.9 & 0.9 & 0.0 & $20,830.8$ \\
\hline Light vehicles & $15,489.9$ & 302.1 & 9.7 & & & 0.0 & & 0.0 & $15,801.7$ \\
\hline Automobiles & $9,072.7$ & 50.8 & & & & 0.0 & & 0.0 & $9,123.5$ \\
\hline Light trucks ${ }^{\mathrm{b}}$ & $6,393.4$ & 251.3 & 9.7 & & & 0.0 & & 0.0 & $6,654.4$ \\
\hline Motorcycles & 23.8 & & & & & & & & 23.8 \\
\hline Buses & 7.4 & 185.7 & 0.1 & & & 8.9 & 0.9 & 0.0 & 203.1 \\
\hline Transit & 0.2 & 81.4 & 0.1 & & & 8.9 & 0.9 & 0.0 & 91.6 \\
\hline Intercity $^{c}$ & & 32.3 & & & & & & & 32.3 \\
\hline School $^{\mathrm{d}}$ & 7.2 & 72.0 & & & & & & & 79.2 \\
\hline Medium/heavy & 547.0 & $4,263.0$ & 16.0 & & & & & & $4,826.0$ \\
\hline OFF-HIGHWAY & 163.5 & 872.7 & & & & & & & $1,036.2$ \\
\hline Construction & 63.3 & 375.6 & & & & & & & 438.9 \\
\hline Agriculture & 100.2 & 497.1 & & & & & & & 597.3 \\
\hline NONHIGHWAY & 351.9 & 831.0 & & $2,372.6$ & 550.9 & 643.3 & 319.5 & & $5,069.2$ \\
\hline Air & 38.4 & & & $2,372.6$ & & & & & $2,411.0$ \\
\hline General aviation & 38.4 & & & 126.7 & & & & & 165.1 \\
\hline Domestic air & & & & $1,892.4$ & & & & & $1,892.4$ \\
\hline International air & & & & 353.5 & & & & & 353.5 \\
\hline Water & 313.5 & 290.3 & & & 550.9 & & & & $1,154.7$ \\
\hline Freight & & 290.3 & & & 550.9 & & & & 841.2 \\
\hline Recreational & 313.5 & & & & & & & & 313.5 \\
\hline Pipeline & & & & & & 643.3 & 245.4 & & 888.7 \\
\hline Rail & & 540.7 & & & & & 74.1 & & 614.8 \\
\hline Freight (Class I) & & 517.3 & & & & & & & 517.3 \\
\hline Passenger & & 23.4 & & & & & 74.1 & & 97.5 \\
\hline Transit & & 0.0 & & & & & 48.6 & & 48.6 \\
\hline Commuter & & 10.0 & & & & & 15.9 & & 25.9 \\
\hline Intercity ${ }^{\mathrm{c}}$ & & 13.4 & & & & & 9.6 & & 23.0 \\
\hline TOTAL & $16,559.7$ & $6,454.5$ & 25.8 & $2,372.6$ & 550.9 & 652.2 & 320.4 & 0.0 & $26,936.2$ \\
\hline
\end{tabular}

Source:

See Appendix A for Energy Use Sources.

\footnotetext{
${ }^{a}$ Civilian consumption only. Totals may not include all possible uses of fuels for transportation (e.g., snowmobiles).

${ }^{\mathrm{b}}$ Two-axle, four-tire trucks.

c 2000 data. 2001 data are not yet available.
} 
The 2000 data have been revised to include the latest data available.

Table 2.5

Transportation Energy Use by Mode, 2000-2001

\begin{tabular}{|c|c|c|c|c|c|c|}
\hline & \multicolumn{2}{|c|}{ Trillion Btu } & \multicolumn{2}{|c|}{$\begin{array}{l}\text { Percentage of total } \\
\text { based on Btus }\end{array}$} & \multicolumn{2}{|c|}{$\begin{array}{l}\text { Thousand barrels per } \\
\text { day crude oil } \\
\text { equivalent }^{\mathrm{b}}\end{array}$} \\
\hline & 2000 & 2001 & 2000 & 2001 & 2000 & 2001 \\
\hline HIGHWAY & $20,762.7$ & $20,830.8$ & $76.1 \%$ & $77.3 \%$ & $10,629.6$ & $10,665.7$ \\
\hline Light vehicles & $15,737.4$ & $15,801.7$ & $57.7 \%$ & $58.7 \%$ & $8,233.2$ & $8,267.8$ \\
\hline Automobiles & $9,100.3$ & $9,123.5$ & $33.3 \%$ & $33.9 \%$ & $4,766.2$ & $4,779.0$ \\
\hline Light trucks ${ }^{c}$ & $6,610.9$ & $6,654.4$ & $24.2 \%$ & $24.7 \%$ & $3,453.3$ & $3,476.4$ \\
\hline Motorcycles & 26.2 & 23.8 & $0.1 \%$ & $0.1 \%$ & 13.7 & 12.4 \\
\hline Buses & 208.3 & 203.1 & $0.8 \%$ & $0.8 \%$ & 98.4 & 96.1 \\
\hline Transit & 96.8 & 91.6 & $0.4 \%$ & $0.3 \%$ & 45.6 & 43.3 \\
\hline Intercity & 32.3 & 32.3 & $0.1 \%$ & $0.1 \%$ & 15.2 & 15.2 \\
\hline School & 79.2 & 79.2 & $0.3 \%$ & $0.3 \%$ & 37.6 & 37.6 \\
\hline Medium/heavy trucks & $4,817.9$ & $4,826.0$ & $17.7 \%$ & $17.9 \%$ & $2,298.0$ & $2,301.8$ \\
\hline OFF-HIGHWAY & 943.7 & $1,036.2$ & $3.5 \%$ & $3.8 \%$ & 449.3 & 495.8 \\
\hline Construction & 383.0 & 438.9 & $1.4 \%$ & $1.6 \%$ & 181.4 & 209.7 \\
\hline Agriculture & 560.7 & 597.3 & $2.1 \%$ & $2.2 \%$ & 267.9 & 286.1 \\
\hline NONHIGHWAY & $5,586.1$ & $5,069.2$ & $20.5 \%$ & $18.8 \%$ & $2,201.4$ & $1,978.4$ \\
\hline Air & 2,548.6 & $2,411.0$ & $9.3 \%$ & $9.0 \%$ & $1,233.9$ & $1,167.3$ \\
\hline General aviation & 175.1 & 165.1 & $0.6 \%$ & $0.6 \%$ & 87.0 & 82.1 \\
\hline Domestic air carriers & $2,004.0$ & $1,892.4$ & $7.3 \%$ & $7.0 \%$ & 968.3 & 914.4 \\
\hline International air & 369.5 & 353.5 & $1.4 \%$ & $1.3 \%$ & 178.6 & 170.8 \\
\hline Water & $1,521.3$ & $1,154.7$ & $5.6 \%$ & $4.3 \%$ & 699.5 & 540.2 \\
\hline Freight & $1,210.1$ & 841.2 & $4.4 \%$ & $3.1 \%$ & 537.1 & 376.6 \\
\hline Recreational & 311.2 & 313.5 & $1.1 \%$ & $1.2 \%$ & 162.4 & 163.6 \\
\hline Pipeline & 908.4 & 888.7 & $3.3 \%$ & $3.3 \%$ & 11.3 & 12.8 \\
\hline Rail & 607.8 & 614.8 & $2.2 \%$ & $2.3 \%$ & 256.7 & 258.1 \\
\hline Freight & 516.0 & 517.3 & $1.9 \%$ & $1.9 \%$ & 242.7 & 243.3 \\
\hline Passenger & 91.8 & 97.5 & $0.3 \%$ & $0.4 \%$ & 14.0 & 14.8 \\
\hline Transit & 47.2 & 48.6 & $0.2 \%$ & $0.2 \%$ & 2.2 & 2.5 \\
\hline Commuter & 25.9 & 25.9 & $0.1 \%$ & $0.1 \%$ & 5.4 & 5.5 \\
\hline Intercity & 18.7 & 23.0 & $0.1 \%$ & $0.1 \%$ & 6.4 & 6.8 \\
\hline TOTAL & $27,292.5$ & $26,936.2$ & $100.0 \%$ & $100.0 \%$ & $12,831.0$ & $12,644.1$ \\
\hline
\end{tabular}

Source: See Appendix A for Energy Use Sources.

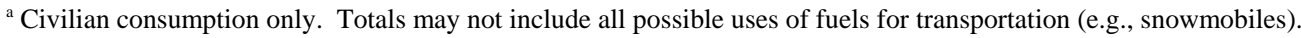

${ }^{b}$ This year, crude oil equivalent is not a simple conversion from Btu based on the average Btu in a barrel of oil. Each gallon of petroleum product was assumed to equal one gallon of crude oil. The oil used to produce electricity is also estimated. See Appendix A, p. 18 for details.

${ }^{\mathrm{c}}$ Two-axle, four-tire trucks. 
The highway sector is by far the largest part of transportation energy use. Light truck energy use has increased at the greatest rate, due to the increased use of light trucks as personal passenger vehicles. Light trucks include pick-ups, minivans, sport-utility vehicles, and vans.

Table 2.6

Highway Transportation Energy Consumption by Mode, 1970-2001 ${ }^{\mathrm{a}}$ (trillion Btu)

\begin{tabular}{|c|c|c|c|c|c|c|c|c|}
\hline Year & Autos & $\begin{array}{l}\text { Light } \\
\text { trucks }\end{array}$ & $\begin{array}{c}\text { Light } \\
\text { vehicles } \\
\text { subtotal }\end{array}$ & $\begin{array}{l}\text { Motor- } \\
\text { cycles }\end{array}$ & Buses & $\begin{array}{l}\text { Heavy } \\
\text { trucks }\end{array}$ & $\begin{array}{l}\text { Highway } \\
\text { subtotal }\end{array}$ & $\begin{array}{c}\text { Total } \\
\text { transportation }\end{array}$ \\
\hline 1970 & 8,479 & 1,539 & 10,018 & 7 & 129 & 1,553 & 11,707 & 15,321 \\
\hline 1975 & 9,298 & 2,384 & 11,682 & 14 & 124 & 2,003 & 13,823 & 17,356 \\
\hline 1976 & 9,826 & 2,602 & 12,428 & 15 & 134 & 2,114 & 14,691 & 18,426 \\
\hline 1977 & 9,928 & 2,797 & 12,724 & 16 & 137 & 2,344 & 15,222 & 19,157 \\
\hline 1978 & 10,134 & 3,020 & 13,153 & 18 & 141 & 2,607 & 15,919 & 20,126 \\
\hline 1979 & 9,629 & 3,055 & 12,685 & 22 & 144 & 2,697 & 15,547 & 20,135 \\
\hline 1980 & 8,800 & 2,975 & 11,774 & 26 & 143 & 2,686 & 14,629 & 18,979 \\
\hline 1981 & 8,693 & 2,963 & 11,655 & 27 & 145 & 2,724 & 14,551 & 19,120 \\
\hline 1982 & 8,673 & 2,837 & 11,510 & 25 & 151 & 2,707 & 14,393 & 18,560 \\
\hline 1983 & 8,802 & 2,989 & 11,791 & 22 & 152 & 2,770 & 14,735 & 18,677 \\
\hline 1984 & 8,837 & 3,197 & 12,034 & 22 & 146 & 2,873 & 15,075 & 19,323 \\
\hline 1985 & 8,932 & 3,413 & 12,345 & 23 & 154 & 2,883 & 15,404 & 19,659 \\
\hline 1986 & 9,138 & 3,629 & 12,767 & 23 & 160 & 2,958 & 15,908 & 20,277 \\
\hline 1987 & 9,157 & 3,819 & 12,976 & 24 & 164 & 3,061 & 16,225 & 20,742 \\
\hline 1988 & 9,158 & 4,077 & 13,235 & 25 & 169 & 3,118 & 16,548 & 21,280 \\
\hline 1989 & 9,232 & 4,156 & 13,388 & 26 & 169 & 3,199 & 16,782 & 21,580 \\
\hline 1990 & 8,688 & 4,451 & 13,139 & 24 & 167 & 3,334 & 16,663 & 21,689 \\
\hline 1991 & 8,029 & 4,774 & 12,803 & 23 & 177 & 3,402 & 16,405 & 21,279 \\
\hline 1992 & 8,169 & 5,117 & 13,286 & 24 & 184 & 3,468 & 16,963 & 21,939 \\
\hline 1993 & 8,368 & 5,356 & 13,723 & 25 & 183 & 3,577 & 17,509 & 22,393 \\
\hline 1994 & 8,470 & 5,515 & 13,984 & 26 & 183 & 3,778 & 17,976 & 22,997 \\
\hline 1995 & 8,489 & 5,695 & 14,183 & 25 & 184 & 3,937 & 18,334 & 23,536 \\
\hline 1996 & 8,634 & 5,917 & 14,551 & 24 & 186 & 4,045 & 18,813 & 24,042 \\
\hline 1997 & 8,710 & 6,168 & 14,879 & 25 & 192 & 4,086 & 19,187 & 24,404 \\
\hline 1998 & 8,936 & 6,305 & 15,241 & 26 & 196 & 4,218 & 19,686 & 24,839 \\
\hline 1999 & 9,134 & 6,605 & 15,738 & 26 & 202 & 4,638 & 20,610 & 26,034 \\
\hline 2000 & 9,100 & 6,611 & 15,711 & 26 & 208 & 4,819 & 20,764 & 26,350 \\
\hline 2001 & 9,123 & 6,654 & 15,778 & 24 & 203 & 4,826 & 20,830 & 25,899 \\
\hline & \multicolumn{8}{|c|}{ Average annual percentage change } \\
\hline 1970-2001 & $0.2 \%$ & $4.8 \%$ & $1.5 \%$ & $4.1 \%$ & $1.5 \%$ & $3.7 \%$ & $1.9 \%$ & $1.7 \%$ \\
\hline 1991-2001 & $1.3 \%$ & $3.4 \%$ & $2.1 \%$ & $0.4 \%$ & $1.4 \%$ & $3.6 \%$ & $2.4 \%$ & $2.0 \%$ \\
\hline
\end{tabular}

Source:

See Appendix A for Highway Energy Use.

\footnotetext{
${ }^{a}$ These data have been revised slightly. See Appendix A for detailed methodologies.

${ }^{\mathrm{b}}$ Total transportation figures do not include military and off-highway energy use and may not include all possible uses of fuel for transportation (e.g. snowmobiles).
} 
About $20 \%$ of transportation energy use is for nonhighway modes. Air travel accounts for nearly half of nonhighway energy use.

Table 2.7

Nonhighway Transportation Energy Consumption by Mode, 1970-2001 (trillion Btu)

\begin{tabular}{|c|c|c|c|c|c|c|}
\hline Year & Air & Water & Pipeline & Rail & $\begin{array}{c}\text { Nonhighway } \\
\text { subtotal }\end{array}$ & $\begin{array}{c}\text { Total } \\
\text { transportation }\end{array}$ \\
\hline 1970 & . & 753 & 995 & 558 & 3,614 & $15,321$. \\
\hline 1975 & 1,274 & 851 & 844 & 563 & 3,533 & 17,356 \\
\hline 1976 & 1,333 & 1,010 & 807 & 585 & 3,735 & 18,426 \\
\hline 1977 & 1,350 & 1,200 & 790 & 595 & 3,957 & 19,157 \\
\hline 1978 & 1,423 & 1,405 & 787 & 592 & 4,201 & 20,126 \\
\hline 1979 & 1,488 & 1,626 & 864 & 611 & 4,588 & 20,135 \\
\hline 1980 & 1,434 & 1,424 & 900 & 592 & 4,353 & 18,979 \\
\hline 1981 & 1,453 & 1,642 & 909 & 565 & 4,570 & 19,120 \\
\hline 1982 & 1,445 & 1,378 & 859 & 485 & 4,164 & 18,560 \\
\hline 1983 & 1,440 & 1,277 & 743 & 482 & 3,952 & 18,677 \\
\hline 1984 & 1,609 & 1,315 & 785 & 538 & 4,242 & 19,323 \\
\hline 1985 & 1,677 & 1,316 & 758 & 504 & 4,255 & 19,659 \\
\hline 1986 & 1,823 & 1,314 & 738 & 494 & 4,369 & 20,277 \\
\hline 1987 & 1,899 & 1,338 & 775 & 505 & 4,517 & 20,742 \\
\hline 1988 & 1,978 & 1,358 & 878 & 518 & 4,732 & 21,280 \\
\hline 1989 & 1,981 & 1,399 & 894 & 523 & 4,797 & 21,580 \\
\hline 1990 & 2,077 & 1,508 & 928 & 514 & 5,026 & 21,689 \\
\hline 1991 & 1,939 & 1,586 & 864 & 485 & 4,875 & 21,279 \\
\hline 1992 & 1,970 & 1,659 & 849 & 497 & 4,977 & 21,939 \\
\hline 1993 & 1,986 & 1,497 & 889 & 512 & 4,888 & 22,393 \\
\hline 1994 & 2,070 & 1,449 & 955 & 546 & 5,021 & 22,997 \\
\hline 1995 & 2,141 & 1,523 & 971 & 567 & 5,202 & 23,536 \\
\hline 1996 & 2,206 & 1,460 & 984 & 580 & 5,229 & 24,042 \\
\hline 1997 & 2,300 & 1,309 & 1,027 & 581 & 5,217 & 24,404 \\
\hline 1998 & 2,371 & 1,295 & 901 & 585 & 5,153 & 24,839 \\
\hline 1999 & 2,471 & 1,435 & 912 & 607 & 5,424 & 26,034 \\
\hline 2000 & 2,549 & 1,521 & 908 & 608 & 5,586 & 26,350 \\
\hline 2001 & 2,411 & 1,155 & 889 & 615 & 5,069 & 25,899 \\
\hline \multicolumn{7}{|c|}{ Average annual percentage change } \\
\hline 1970-2001 & $2.0 \%$ & $1.4 \%$ & $-0.4 \%$ & $0.3 \%$ & $1.1 \%$ & $1.7 \%$ \\
\hline 1991-2001 & $2.2 \%$ & $-3.1 \%$ & $0.3 \%$ & $2.4 \%$ & $0.4 \%$ & $2.0 \%$ \\
\hline
\end{tabular}

Source:

See Appendix A for Nonhighway Energy Use.

a These data have been revised slightly. See Appendix A for detailed methodologies.

'Total transportation figures do not include military and off-highway energy use and may not include all possible uses of fuel for transportation (e.g. snowmobiles). 
The use of diesel for off-highway purposes has grown from 1985 to 2001. The use of gasoline has declined in agriculture. The construction gasoline 2001 data cannot be compared due to changes in methodology (see footnote $b$ ).

Table 2.8

Off-Highway Use of Gasoline and Diesel, 1985-2001

(trillion Btu)

\begin{tabular}{|c|c|c|c|c|c|c|}
\hline \multirow[b]{2}{*}{ Year } & \multicolumn{2}{|c|}{ Agriculture } & \multicolumn{2}{|c|}{ Construction } & \multicolumn{2}{|c|}{ Total } \\
\hline & Gasoline & Diesel $^{a}$ & Gasoline & Diesel $^{\mathrm{a}}$ & Gasoline & Diesel $^{a}$ \\
\hline 1985 & 135 & 430 & 31 & 211 & 166 & 641 \\
\hline 1986 & 121 & 463 & 34 & 230 & 155 & 693 \\
\hline 1987 & 115 & 416 & 35 & 216 & 150 & 632 \\
\hline 1988 & 101 & 439 & 34 & 232 & 135 & 671 \\
\hline 1989 & 103 & 466 & 37 & 234 & 140 & 700 \\
\hline 1990 & 85 & 472 & 40 & 251 & 125 & 723 \\
\hline 1991 & 97 & 438 & 35 & 228 & 132 & 666 \\
\hline 1992 & 101 & 485 & 34 & 244 & 135 & 729 \\
\hline 1993 & 106 & 473 & 31 & 292 & 137 & 765 \\
\hline 1994 & 113 & 454 & 33 & 299 & 146 & 753 \\
\hline 1995 & 116 & 482 & 35 & 301 & 151 & 783 \\
\hline 1996 & 115 & 498 & 35 & 312 & 150 & 810 \\
\hline 1997 & 123 & 492 & 38 & 316 & 161 & 808 \\
\hline 1998 & 113 & 473 & 29 & 344 & 142 & 817 \\
\hline 1999 & 88 & 473 & 22 & 345 & 110 & 818 \\
\hline 2000 & 82 & 479 & 24 & 359 & 106 & 838 \\
\hline \multirow[t]{2}{*}{2001} & 100 & 497 & $63^{\mathrm{b}}$ & 376 & $163^{\mathrm{b}}$ & 873 \\
\hline & \multicolumn{6}{|c|}{ Average annual percentage change } \\
\hline 1985-2001 & $-1.9 \%$ & $0.9 \%$ & & $3.7 \%$ & b & $1.9 \%$ \\
\hline 1991-2001 & $0.3 \%$ & $1.3 \%$ & & $5.1 \%$ & b & $2.7 \%$ \\
\hline
\end{tabular}

\section{Source:}

Gasoline: U.S. Department of Transportation, Federal Highway Administration, Highway Statistics 2001, Washington, DC, Table MF-24, and annual. (Additional resources: www.fhwa.dot.gov)

Diesel: U.S. Department of Energy, Energy Information Administration, Fuel Oil and Kerosene Sales 2001, Washington, DC, Table 1, and annual. (Additional resources: www.eia.doe.gov)

${ }^{a}$ Unadjusted sales of distillate fuel oil.

' The FHWA methodology for calculating construction gasoline use changed in 2001. Previous years' data are likely underestimated. 
The Federal Highway Administration cautions that data from 1993 on may not be directly comparable to earlier years. Some states have improved reporting procedures in recent years, and the estimation procedures were revised in 1994. Prior to the Energy Policy Act of 1992, gasohol was defined as a blend of gasoline and at least 10\%, by volume, alcohol. Effective January 1, 1993, three types of gasohol were defined: 10\% gasohol-containing at least 10\% alcohol; $7.7 \%$ gasohol-containing 7.7\% alcohol but less than 10\%; and 5.7\% gasohol-containing at least 5.7\% alcohol but less than 7.7\%. See Table 2.3 for details on oxygenate usage.

Table 2.9

Highway Usage of Gasoline and Special Fuels, 1973-2001 (billion gallons)

\begin{tabular}{|c|c|c|c|c|c|c|c|}
\hline Year & Gasoline & Gasohol & $\begin{array}{c}\text { Ethanol used } \\
\text { in gasohol }^{\mathrm{a}}\end{array}$ & $\begin{array}{c}\text { Total gasoline } \\
\text { and gasohol }\end{array}$ & Diesel $^{\mathrm{b}}$ & $\begin{array}{c}\text { Percent } \\
\text { diesel }\end{array}$ & $\begin{array}{c}\text { Total highway } \\
\text { fuel use }\end{array}$ \\
\hline 1973 & $c$ & $\mathrm{c}$ & $c$ & 100.6 & 9.8 & $8.9 \%$ & 110.5 \\
\hline 1975 & c & c & c & 99.4 & 9.6 & $8.8 \%$ & 109.0 \\
\hline 1980 & 100.7 & 0.5 & 0.0 & 101.2 & 13.8 & $12.0 \%$ & 115.0 \\
\hline 1981 & 98.9 & 0.7 & 0.1 & 99.6 & 14.9 & $13.0 \%$ & 114.5 \\
\hline 1982 & 96.2 & 2.3 & 0.2 & 98.5 & 14.9 & $13.1 \%$ & 113.4 \\
\hline 1983 & 95.9 & 4.3 & 0.4 & 100.1 & 16.0 & $13.8 \%$ & 116.1 \\
\hline 1984 & 96.0 & 5.4 & 0.5 & 101.4 & 17.3 & $14.6 \%$ & 118.7 \\
\hline 1985 & 95.6 & 8.0 & 0.8 & 103.6 & 17.8 & $14.6 \%$ & 121.3 \\
\hline 1986 & 98.6 & 8.1 & 0.8 & 106.8 & 18.4 & $14.7 \%$ & 125.2 \\
\hline 1987 & 101.8 & 6.9 & 0.8 & 108.7 & 19.0 & $14.9 \%$ & 127.7 \\
\hline 1988 & 101.7 & 8.1 & 0.8 & 109.8 & 20.1 & $15.5 \%$ & 129.9 \\
\hline 1989 & 103.7 & 6.9 & 0.7 & 110.6 & 21.2 & $16.1 \%$ & 131.9 \\
\hline 1990 & 102.6 & 7.5 & 0.8 & 110.2 & 21.4 & $16.3 \%$ & 131.6 \\
\hline 1991 & 99.3 & 8.6 & 0.9 & 107.9 & 20.7 & $16.1 \%$ & 128.6 \\
\hline 1992 & 102.1 & 8.8 & 0.9 & 111.0 & 22.0 & $16.5 \%$ & 132.9 \\
\hline 1993 & 103.4 & 10.3 & 1.0 & 113.7 & 23.5 & $17.1 \%$ & 137.2 \\
\hline 1994 & 104.0 & 11.0 & 1.0 & 115.0 & 25.1 & $17.9 \%$ & 140.1 \\
\hline 1995 & 104.0 & 13.1 & 1.2 & 117.1 & 26.2 & $18.3 \%$ & 143.3 \\
\hline 1996 & 107.4 & 12.1 & 1.1 & 119.5 & 27.2 & $18.5 \%$ & 146.7 \\
\hline 1997 & 106.2 & 14.7 & 1.3 & 120.9 & 29.4 & $19.6 \%$ & 150.3 \\
\hline 1998 & 110.7 & 14.0 & 1.3 & 124.7 & 30.2 & $19.5 \%$ & 154.9 \\
\hline 1999 & 114.6 & 14.2 & 1.3 & 128.7 & 31.9 & $19.9 \%$ & 160.7 \\
\hline 2000 & 112.6 & 16.3 & 1.5 & 128.9 & 33.4 & $20.6 \%$ & 162.3 \\
\hline 2001 & 112.3 & 17.4 & 1.5 & 129.7 & 33.4 & $20.5 \%$ & 163.1 \\
\hline & \multicolumn{7}{|c|}{ Average annual percentage change } \\
\hline 1973-2001 & d & d & & $0.9 \%$ & $4.5 \%$ & & $1.4 \%$ \\
\hline 1991-2001 & $1.2 \%$ & $7.3 \%$ & $5.2 \%$ & $1.9 \%$ & $4.9 \%$ & & $2.4 \%$ \\
\hline
\end{tabular}

Source:

U.S. Department of Transportation, Federal Highway Administration, Highway Statistics 2001, Washington, DC, 2002, Tables MF-21 and MF-33E, and annual. (Additional resources: www.fhwa.dot.gov)

\footnotetext{
${ }^{\text {a }}$ Estimated for $1980-92$ as $10 \%$ of gasohol consumption.

${ }^{\mathrm{b}}$ Consists primarily of diesel fuel, with small quantities of liquified petroleum gas.

${ }^{\mathrm{c}}$ Data for gasoline and gasohol cannot be separated in this year.

${ }^{\mathrm{d}}$ Data are not available.
} 
Nearly all of the fuel ethanol used in the U.S. is made domestically. Twenty-eight percent of MTBE was imported in 2002.

Table 2.10

U.S. Production and Imports of $\mathrm{MTBE}^{\mathrm{a}}$ and Fuel Ethanol, 1985-2002 (million gallons)

\begin{tabular}{|c|c|c|c|c|}
\hline \multirow[b]{2}{*}{ Year } & \multicolumn{2}{|c|}{ Production } & \multicolumn{2}{|c|}{ Imports } \\
\hline & Fuel ethanol & $\mathrm{MTBE}^{\mathrm{a}}$ & Fuel ethanol & $\mathrm{MTBE}^{\mathrm{a}}$ \\
\hline 1985 & 793 & 302 & $\mathrm{~b}$ & $\mathrm{~b}$ \\
\hline 1990 & 756 & $\mathrm{~b}$ & b" & $\dddot{\mathrm{b}}$ \\
\hline 1991 & 875 & $\mathrm{~b}$ & $\mathrm{~b}$ & $\mathrm{~b}$ \\
\hline 1992 & 1,080 & 1,542 & $\mathrm{~b}$ & b \\
\hline 1993 & 1,156 & 2,081 & 10 & 306 \\
\hline 1994 & 1,280 & 2,205 & 12 & 595 \\
\hline 1995 & 1,355 & 2,506 & 16 & 692 \\
\hline 1996 & 974 & 2,846 & 13 & 733 \\
\hline 1997 & 1,274 & 3,011 & 4 & 918 \\
\hline 1998 & 1,387 & 3,151 & 3 & 1,040 \\
\hline 1999 & 1,472 & 3,315 & 4 & 1,146 \\
\hline 2000 & 1,633 & 3,253 & 5 & 1,176 \\
\hline 2001 & 1,765 & 3,257 & 13 & 1,146 \\
\hline \multirow[t]{2}{*}{2002} & 2,132 & 3,133 & 13 & 907 \\
\hline & \multicolumn{4}{|c|}{ Average annual percentage change } \\
\hline 1985-2002 & $6.0 \%$ & $14.8 \%$ & $\mathrm{~b}$ & $\mathrm{~b}$ \\
\hline 1992-2002 & $9.3 \%$ & b & $\mathrm{b}$ & $\mathrm{b}$ \\
\hline
\end{tabular}

Source:

Production - 1992-2002 Ethanol and MTBE: U.S. Department of Energy, Energy Information Administration, Petroleum Supply Monthly, Washington, DC, January 2003, Table D1. 1985-91 Ethanol: Information Resources, Inc., Washington, DC, 1991. 1985 MTBE: EA-Mueller,Inc., Baltimore, MD, 1992. Imports - U.S. Department of Energy, Energy Information Administration, Petroleum Supply Annual, 2002, Volume 1, Washington, DC, June 2003, Table 20, and annual. (Additional resources: www.eia.doe.gov)

Note:

Table 2.3 displays gasoline-equivalent gallons, which differ from these gallons.

\footnotetext{
${ }^{\text {a }}$ Methyl tertiary-butyl ether.
}

${ }^{\mathrm{b}}$ Data are not available. 
Great care should be taken when comparing modal energy intensity data among modes. Because of the inherent differences between the transportation modes in the nature of services, routes available, and many additional factors, it is not possible to obtain truly comparable national energy intensities among modes. These values are averages, and there is a great deal of variability even within a mode.

\section{Table 2.11}

Passenger Travel and Energy Use, 2001

\begin{tabular}{|c|c|c|c|c|c|c|c|}
\hline & \multirow[b]{2}{*}{$\begin{array}{c}\text { Number of vehicles } \\
\text { (thousands) }\end{array}$} & \multirow{2}{*}{$\begin{array}{l}\text { Vehicle- } \\
\text { miles } \\
\text { (millions) }\end{array}$} & \multirow{2}{*}{$\begin{array}{l}\text { Passenger- } \\
\text { miles } \\
\text { (millions) }\end{array}$} & \multirow[b]{2}{*}{$\begin{array}{c}\text { Load factor } \\
\text { (persons/vehicle) }\end{array}$} & \multicolumn{2}{|c|}{ Energy intensities } & \multirow[b]{2}{*}{$\begin{array}{l}\text { Energy use } \\
\text { (trillion Btu }\end{array}$} \\
\hline & & & & & $\begin{array}{c}\text { (Btu per } \\
\text { vehicle-mile) }\end{array}$ & $\begin{array}{c}\text { (Btu per } \\
\text { passenger-mile) }\end{array}$ & \\
\hline Automobiles & $137,633.5$ & $1,619,422$ & $2,542,493$ & 1.57 & 5,634 & 3,588 & $9,123.5$ \\
\hline Personal trucks & $64,637.0$ & 677,798 & $1,165,812$ & 1.72 & 6,989 & 4,063 & $4,736.8$ \\
\hline Motorcycles & $4,903.1$ & 9,529 & 11,625 & 1.22 & 2,500 & 2,049 & 23.8 \\
\hline Demand response & 34.6 & 789 & 855 & 1.1 & 14,375 & 13,271 & 11.3 \\
\hline Vanpool & 5.4 & 71 & 490 & 6.9 & 8,738 & 1,273 & 0.6 \\
\hline Buses & a & a & a & a & a & a & 203.2 \\
\hline Transit & 76.7 & 2,389 & 22,209 & 9.3 & 38,342 & 4,124 & 91.6 \\
\hline Intercity $^{\mathrm{b}}$ & $\mathrm{a}$ & a & 37,900 & a & a & 852 & 32.3 \\
\hline School $^{\mathrm{b}}$ & 607.8 & a & a & a & a & a & 79.3 \\
\hline Air & a & a & 581,888 & a & a & 4,143 & $2,411.0$ \\
\hline Certificated route $^{c}$ & $\mathrm{a}$ & 5,925 & 565,988 & 95.5 & 379,055 & 3,968 & $2,245.9$ \\
\hline General aviation & 211.5 & a & 15,900 & $\mathbf{a}$ & a & 10,384 & 165.1 \\
\hline Recreational boats & $12,876.3$ & a & a & a & a & a & 313.5 \\
\hline Rail & 17.6 & 1,317 & 30,734 & 23.3 & 74,032 & 3,172 & 97.5 \\
\hline Intercity $^{\mathrm{d}}$ & 0.4 & 378 & 5,571 & 14.7 & 60,847 & 4,137 & 23.0 \\
\hline Transit $^{\mathrm{e}}$ & 12.1 & 662 & 15,615 & 23.6 & 73,414 & 3,114 & 48.6 \\
\hline Commuter & 5.1 & 277 & 9,548 & 34.5 & 93,502 & 2,717 & 25.9 \\
\hline
\end{tabular}

Source:

See Appendix A for Passenger Travel and Energy Use.

${ }^{a}$ Data are not available.

${ }^{\text {b }} 2000$ energy use data. 2001 data are not available.

${ }^{\mathrm{c}}$ Includes domestic scheduled services and $1 / 2$ of international scheduled services (Table 2.13 shows only domestic services). These energy intensities may be inflated because all energy use is attributed to passengers - cargo energy use is not taken into account.

${ }^{\mathrm{d}}$ Amtrak only.

${ }^{\mathrm{e}}$ Light and heavy rail. 
Great care should be taken when comparing modal energy intensity data among modes. Because of the inherent differences between the transportation modes in the nature of services, routes available, and many additional factors, it is not possible to obtain truly comparable national energy intensities among modes. These values are averages, and there is a great deal of variability even within a mode.

Table 2.12

Energy Intensities of Highway Passenger Modes, 1970-2001

\begin{tabular}{|c|c|c|c|c|c|c|}
\hline \multirow[b]{3}{*}{ Year } & \multirow{2}{*}{\multicolumn{2}{|c|}{ Automobiles }} & \multirow{3}{*}{$\begin{array}{l}\text { Light truck }^{\mathrm{a}} \\
\text { (Btu per } \\
\text { vehicle-mile) }\end{array}$} & \multicolumn{3}{|c|}{ Buses } \\
\hline & & & & \multicolumn{2}{|c|}{ Transit $^{\mathrm{b}}$} & \multirow{2}{*}{$\begin{array}{c}\text { Intercity } \\
\text { (Btu per } \\
\text { passenger-mile }\end{array}$} \\
\hline & $\begin{array}{c}\text { (Btu per } \\
\text { vehicle-mile) }\end{array}$ & $\begin{array}{c}\text { (Btu per } \\
\text { passenger-mile) }\end{array}$ & & $\begin{array}{c}\text { (Btu per } \\
\text { vehicle-mile) }\end{array}$ & $\begin{array}{c}\text { (Btu per } \\
\text { passenger-mile) }\end{array}$ & \\
\hline 1970 & 9,250 & 4,868 & 12,479 & 31,796 & 2,472 & 1,674 \\
\hline 1975 & 8,993 & 4,733 & 11,879 & 33,748 & 2,814 & 988 \\
\hline 1976 & 9,113 & 4,796 & 11,523 & 34,598 & 2,896 & 1,007 \\
\hline 1977 & 8,950 & 4,710 & 11,160 & 35,120 & 2,889 & 970 \\
\hline 1978 & 8,839 & 4,693 & 10,807 & 36,603 & 2,883 & 976 \\
\hline 1979 & 8,647 & 4,632 & 10,467 & 36,597 & 2,795 & 1,028 \\
\hline 1980 & 7,916 & 4,279 & 10,224 & 36,553 & 2,813 & 1,082 \\
\hline 1981 & 7,670 & 4,184 & 9,997 & 37,745 & 3,027 & 1,051 \\
\hline 1982 & 7,465 & 4,109 & 9,268 & 38,766 & 3,237 & 1,172 \\
\hline 1983 & 7,365 & 4,092 & 9,124 & 37,962 & 3,177 & 1,286 \\
\hline 1984 & 7,202 & 4,066 & 8,931 & 38,705 & 3,307 & 954 \\
\hline 1985 & 7,164 & 4,110 & 8,730 & 38,876 & 3,423 & 964 \\
\hline 1986 & 7,194 & 4,197 & 8,560 & 37,889 & 3,545 & 870 \\
\hline 1987 & 6,959 & 4,128 & 8,359 & 36,247 & 3,594 & 940 \\
\hline 1988 & 6,683 & 4,033 & 8,119 & 36,673 & 3,706 & 963 \\
\hline 1989 & 6,589 & 4,046 & 7,746 & 36,754 & 3,732 & 964 \\
\hline 1990 & 6,169 & 3,856 & 7,746 & 37,374 & 3,794 & 962 \\
\hline 1991 & 5,912 & 3,695 & 7,351 & 37,732 & 3,877 & 963 \\
\hline 1992 & 5,956 & 3,723 & 7,239 & 40,243 & 4,310 & 964 \\
\hline 1993 & 6,087 & 3,804 & 7,182 & 39,043 & 4,262 & 962 \\
\hline 1994 & 6,024 & 3,765 & 7,212 & 37,313 & 4,268 & 964 \\
\hline 1995 & 5,902 & 3,689 & 7,208 & 37,277 & 4,310 & 964 \\
\hline 1996 & 5,874 & 3,683 & 7,247 & 37,450 & 4,340 & 963 \\
\hline 1997 & 5,797 & 3,646 & 7,251 & 38,832 & 4,431 & 963 \\
\hline 1998 & 5,767 & 3,638 & 7,261 & 41,182 & 4,387 & 963 \\
\hline 1999 & 5,821 & 3,684 & 7,330 & 40,460 & 4,332 & 964 \\
\hline 2000 & 5,687 & 3,611 & 7,162 & 41,548 & 4,515 & 932 \\
\hline 2001 & 5,634 & 3,588 & 7,095 & 38,341 & 4,125 & c \\
\hline \multicolumn{7}{|c|}{ Average annual percentage change } \\
\hline 1970-2001 & $-1.6 \%$ & $-1.0 \%$ & $-1.8 \%$ & $0.6 \%$ & $1.7 \%$ & c \\
\hline 1991-2001 & $-0.5 \%$ & $-0.3 \%$ & $-0.4 \%$ & $0.2 \%$ & $0.6 \%$ & c \\
\hline
\end{tabular}

Source:

See Appendix A for Highway Passenger Mode Energy Intensities.

${ }^{a}$ All two-axle, four-tire trucks.

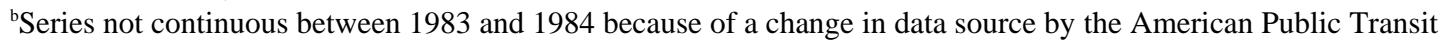
Association (APTA).

'2001data are not yet available. 
Great care should be taken when comparing modal energy intensity data among modes. Because of the inherent differences between the transportation modes in the nature of services, routes available, and many additional factors, it is not possible to obtain truly comparable national energy intensities among modes.

Table 2.13

Energy Intensities of Nonhighway Passenger Modes, 1970-2001

\begin{tabular}{|c|c|c|c|c|c|}
\hline \multirow[b]{2}{*}{ Year } & \multicolumn{2}{|c|}{ Air } & \multicolumn{2}{|c|}{ Rail } & \multirow[b]{2}{*}{$\begin{array}{c}\text { Commuter } \\
\text { rail } \\
\text { (Btu per } \\
\text { passenger-mile }\end{array}$} \\
\hline & $\begin{array}{c}\text { Certificated } \\
\text { air carriers }^{\mathrm{a}} \\
\text { (Btu per } \\
\text { passenger-mile) }\end{array}$ & $\begin{array}{c}\text { General } \\
\text { aviation } \\
\text { (Btu per } \\
\text { passenger-mile) }\end{array}$ & $\begin{array}{c}\text { Intercity } \\
\text { Amtrak } \\
\text { (Btu per } \\
\text { passenger-mile) }\end{array}$ & $\begin{array}{c}\text { Rail } \\
\text { transit } \\
\text { (Btu per } \\
\text { passenger-mile) }\end{array}$ & \\
\hline 1970 & 10,282 & 10,374 & b & 2,453 & \\
\hline 1975 & 7,826 & 10,658 & 3,677 & 2,962 & "a" \\
\hline 1976 & 7,511 & 10,769 & 3,397 & 2,971 & a \\
\hline 1977 & 6,990 & 11,695 & 3,568 & 2,691 & a \\
\hline 1978 & 6,144 & 11,305 & 3,683 & 2,210 & $\mathrm{a}$ \\
\hline 1979 & 5,607 & 10,787 & 3,472 & 2,794 & a \\
\hline 1980 & 5,561 & 11,497 & 3,176 & 3,008 & a \\
\hline 1981 & 5,774 & 11,123 & 2,957 & 2,946 & a \\
\hline 1982 & 5,412 & 13,015 & 3,156 & 3,069 & a \\
\hline 1983 & 5,133 & 11,331 & 2,957 & 3,212 & a \\
\hline 1984 & 5,298 & 11,454 & 3,027 & 3,732 & 3,011 \\
\hline 1985 & 5,053 & 11,707 & 2,800 & 3,461 & 3,053 \\
\hline 1986 & 5,011 & 11,935 & 2,574 & 3,531 & 3,174 \\
\hline 1987 & 4,827 & 11,496 & 2,537 & 3,534 & 3,043 \\
\hline 1988 & 4,861 & 11,794 & 2,462 & 3,585 & 3,075 \\
\hline 1989 & 4,844 & 10,229 & 2,731 & 3,397 & 3,120 \\
\hline 1990 & 4,875 & 10,146 & 2,609 & 3,453 & 3,068 \\
\hline 1991 & 4,662 & 9,869 & 2,503 & 3,710 & 3,011 \\
\hline 1992 & 4,516 & 9,785 & 2,610 & 3,575 & 2,848 \\
\hline 1993 & 4,490 & 9,653 & 2,646 & 3,687 & 3,222 \\
\hline 1994 & 4,397 & 9,163 & 2,357 & 3,828 & 2,904 \\
\hline 1995 & 4,349 & 9,870 & 2,590 & 3,818 & 2,849 \\
\hline 1996 & 4,172 & 9,258 & 2,792 & 3,444 & 2,796 \\
\hline 1997 & 4,166 & 9,688 & 2,918 & 3,253 & 2,946 \\
\hline 1998 & 4,146 & 11,252 & 2,900 & 3,216 & 2,859 \\
\hline 1999 & 4,061 & 12,206 & 3,062 & 3,168 & 2,929 \\
\hline 2000 & 3,952 & 11,526 & 3,356 & 3,105 & 2,759 \\
\hline \multirow[t]{2}{*}{2001} & 3,968 & 10,384 & 4,137 & 3,114 & 2,717 \\
\hline & \multicolumn{5}{|c|}{ Average annual percentage change } \\
\hline 1970-2001 & $-3.0 \%$ & $0.0 \%$ & $0.4 \%^{\mathrm{c}}$ & $0.8 \%$ & $-0.3 \%{ }^{\mathrm{b}}$ \\
\hline 1991-2001 & $-1.6 \%$ & $0.5 \%$ & $5.2 \%$ & $-1.7 \%$ & $-1.0 \%$ \\
\hline
\end{tabular}

\section{Source:}

See Appendix A for Nonhighway Passenger Mode Energy Intensities.

${ }^{\text {a }}$ These data differ from the data on Table 2.11 because they do not include any international services. These energy intensities may be inflated because all energy use is attributed to passengers - cargo energy use is not taken into account.

${ }^{\text {b}}$ Data are not available.

${ }^{c}$ Average annual percentage change begins with the earliest year possible. 
Figure 2.2. Energy Intensities for Transit Rail, 2001
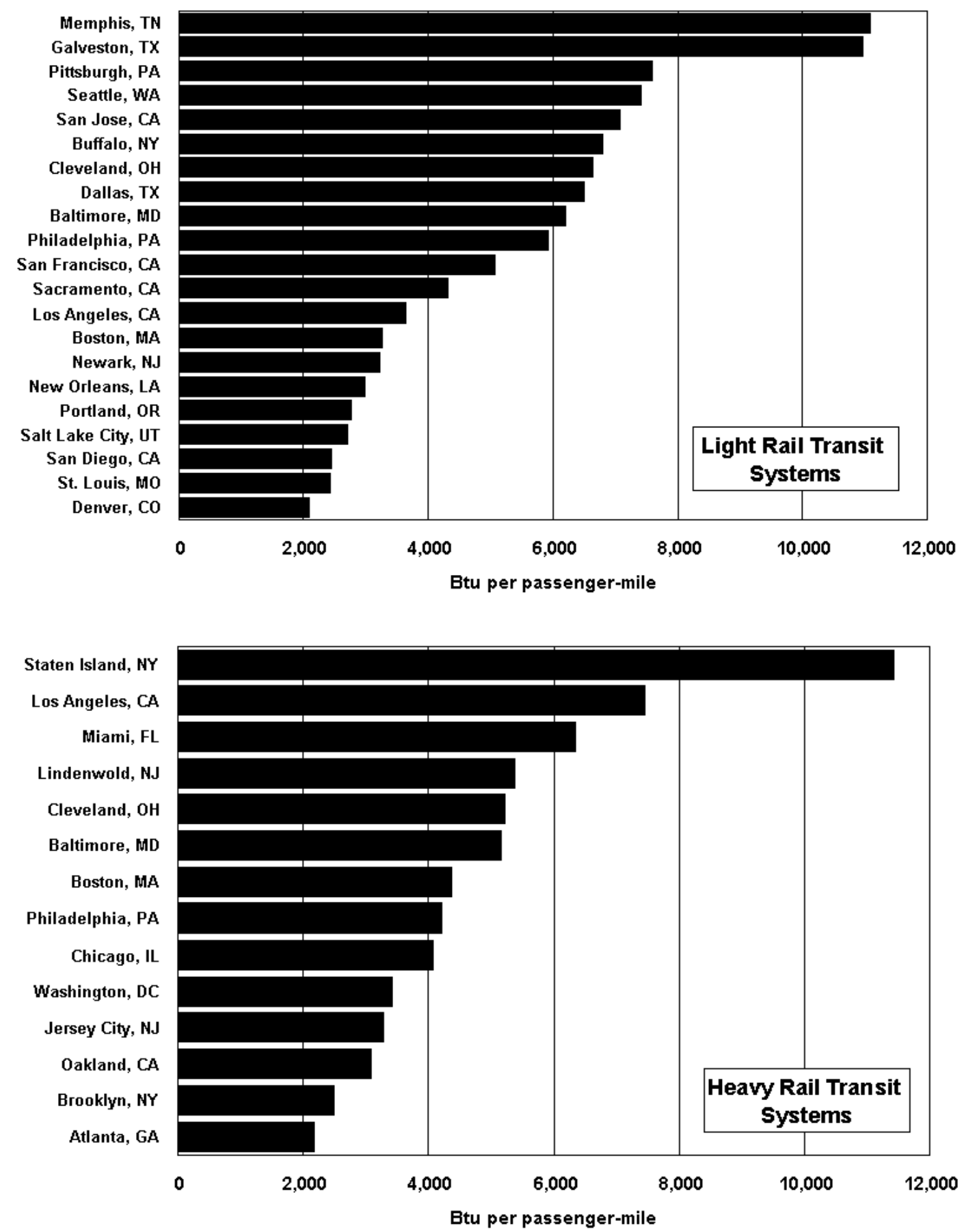

Source:

U.S. Department of Transportation, Federal Transit Administration, 2001 National Transit Databases, Washington, DC. (Additional resources: www.fta.dot.gov/ntl) 
Figure 2.3. Energy Intensities for Selected Transit Bus Systems, 2001

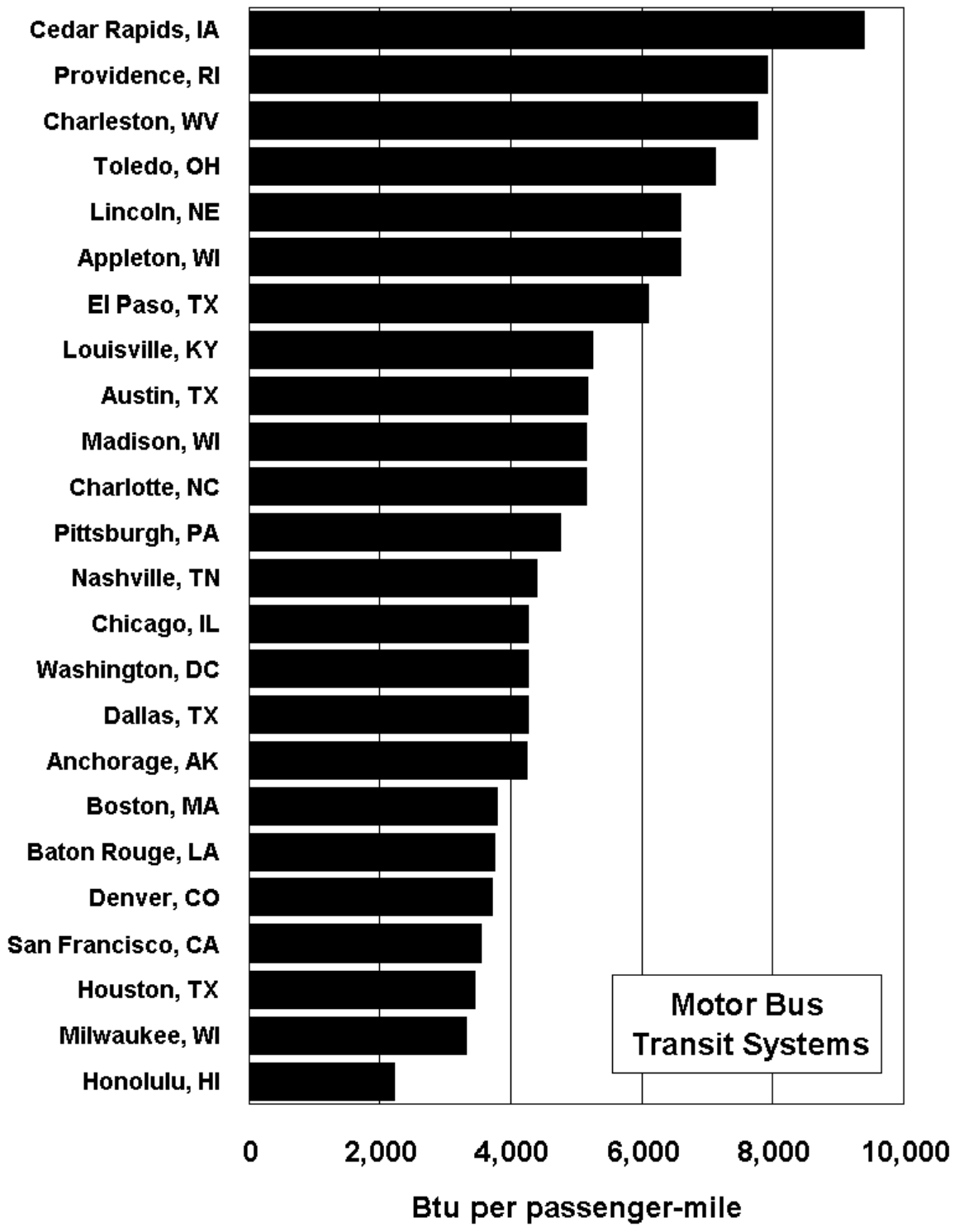

\section{Source:}

U.S. Department of Transportation, Federal Transit Administration, 2001 National Transit Databases, Washington, DC. (Additional resources: www.fta.dot.gov/ntl) 
Great care should be taken when comparing modal energy intensity data among modes. Because of the inherent differences between the transportation modes in the nature of services, routes available, and many additional factors, it is not possible to obtain truly comparable national energy intensities among modes.

Table 2.14

Intercity Freight Movement and Energy Use in the United States, 2001

\begin{tabular}{lccc}
\hline & Trucks & $\begin{array}{c}\text { Waterborne } \\
\text { commerce }\end{array}$ & $\begin{array}{c}\text { Class I } \\
\text { railroads }\end{array}$ \\
\hline Number of vehicles (thousands) & 2,615 & 42 & $20^{\mathrm{a}}$ \\
Ton-miles (billions) & 1,051 & 622 & 1,495 \\
Tons shipped (millions) & 4,122 & 1,037 & 1,742 \\
Average length of haul (miles) & $752^{\mathrm{b}}$ & 600 & 859 \\
Energy intensity (Btu/ton-mile) & 3,337 & 444 & 346 \\
Energy use (trillion Btu) & 3,507 & 276 & 517 \\
\hline
\end{tabular}

Source:

See Appendix A for Freight Movement and Energy Use.

${ }^{\text {a }}$ Number of locomotives.

b 717 miles is for general freight (less than truckload). Based on data from the Eno Transportation Foundation, the average length of haul for specialized freight (truckload) is 294 miles. 
Great care should be taken when comparing modal energy intensity data among modes. Because of the inherent differences between the transportation modes in the nature of services, routes available, and many additional factors, it is not possible to obtain truly comparable national energy intensities among modes.

Table 2.15

Energy Intensities of Freight Modes, 1970-2001

\begin{tabular}{|c|c|c|c|c|}
\hline \multirow[b]{2}{*}{ Year } & \multirow{2}{*}{$\begin{array}{l}\text { Heavy single-unit and } \\
\text { combination trucks } \\
\text { (Btu per vehicle-mile) }\end{array}$} & \multicolumn{2}{|c|}{ Class I freight railroad } & \multirow{2}{*}{$\begin{array}{c}\text { Domestic waterborne } \\
\text { commerce } \\
\text { (Btu per ton-mile) }\end{array}$} \\
\hline & & (Btu per freight car-mile) & (Btu per ton-mile) & \\
\hline 1970 & 24,960 & 17,669 & 691 & 545 \\
\hline 1971 & 24,485 & 18,171 & 717 & 506 \\
\hline 1972 & 24,668 & 18,291 & 714 & 522 \\
\hline 1973 & 24,777 & 18,468 & 677 & 576 \\
\hline 1974 & 24,784 & 18,852 & 681 & 483 \\
\hline 1975 & 24,631 & 18,739 & 687 & 549 \\
\hline 1976 & 24,566 & 18,938 & 680 & 468 \\
\hline 1977 & 24,669 & 19,226 & 669 & 458 \\
\hline 1978 & 24,655 & 18,928 & 641 & 383 \\
\hline 1979 & 24,745 & 19,188 & 618 & 436 \\
\hline 1980 & 24,757 & 18,742 & 597 & 358 \\
\hline 1981 & 25,058 & 18,629 & 572 & 360 \\
\hline 1982 & 24,296 & 18,404 & 553 & 310 \\
\hline 1983 & 23,852 & 17,864 & 525 & 286 \\
\hline 1984 & 23,585 & 17,795 & 510 & 346 \\
\hline 1985 & 23,343 & 17,500 & 497 & 446 \\
\hline 1986 & 23,352 & 17,265 & 486 & 463 \\
\hline 1987 & 22,922 & 16,790 & 456 & 402 \\
\hline 1988 & 22,596 & 16,758 & 443 & 361 \\
\hline 1989 & 22,411 & 16,894 & 437 & 403 \\
\hline 1990 & 22,795 & 16,619 & 420 & 387 \\
\hline 1991 & 22,749 & 15,835 & 391 & 386 \\
\hline 1992 & 22,608 & 16,043 & 393 & 398 \\
\hline 1993 & 22,373 & 16,056 & 389 & 389 \\
\hline 1994 & 22,193 & 16,340 & 388 & 369 \\
\hline 1995 & 22,096 & 15,992 & 372 & 374 \\
\hline 1996 & 22,109 & 15,747 & 368 & 412 \\
\hline 1997 & 21,340 & 15,784 & 370 & 415 \\
\hline 1998 & 21,514 & 15,372 & 365 & 435 \\
\hline 1999 & 22,880 & 15,363 & 363 & 457 \\
\hline 2000 & 23,443 & 14,917 & 352 & 508 \\
\hline 2001 & 23,237 & 15,107 & 346 & 444 \\
\hline \multicolumn{5}{|c|}{ Average annual percentage change } \\
\hline 1970-2001 & $-0.2 \%$ & $-0.5 \%$ & $-2.2 \%$ & $-0.7 \%$ \\
\hline 1991-2001 & $0.2 \%$ & $-0.6 \%$ & $-1.2 \%$ & $1.4 \%$ \\
\hline
\end{tabular}

Source:

See Appendix A for Freight Mode Energy Intensities. 
Nearly all of the fuel ethanol used in the U.S. is made domestically. Twenty-eight percent of MTBE was imported in 2002.

Table 2.10

U.S. Production and Imports of $\mathrm{MTBE}^{\mathrm{a}}$ and Fuel Ethanol, 1985-2002 (million gallons)

\begin{tabular}{|c|c|c|c|c|}
\hline \multirow[b]{2}{*}{ Year } & \multicolumn{2}{|c|}{ Production } & \multicolumn{2}{|c|}{ Imports } \\
\hline & Fuel ethanol & $\mathrm{MTBE}^{\mathrm{a}}$ & Fuel ethanol & $\mathrm{MTBE}^{\mathrm{a}}$ \\
\hline 1985 & 793 & 302 & $\mathrm{~b}$ & $\mathrm{~b}$ \\
\hline 1990 & 756 & $\mathrm{~b}$ & b" & $\dddot{\mathrm{b}}$ \\
\hline 1991 & 875 & $\mathrm{~b}$ & $\mathrm{~b}$ & $\mathrm{~b}$ \\
\hline 1992 & 1,080 & 1,542 & $\mathrm{~b}$ & b \\
\hline 1993 & 1,156 & 2,081 & 10 & 306 \\
\hline 1994 & 1,280 & 2,205 & 12 & 595 \\
\hline 1995 & 1,355 & 2,506 & 16 & 692 \\
\hline 1996 & 974 & 2,846 & 13 & 733 \\
\hline 1997 & 1,274 & 3,011 & 4 & 918 \\
\hline 1998 & 1,387 & 3,151 & 3 & 1,040 \\
\hline 1999 & 1,472 & 3,315 & 4 & 1,146 \\
\hline 2000 & 1,633 & 3,253 & 5 & 1,176 \\
\hline 2001 & 1,765 & 3,257 & 13 & 1,146 \\
\hline \multirow[t]{2}{*}{2002} & 2,132 & 3,133 & 13 & 907 \\
\hline & \multicolumn{4}{|c|}{ Average annual percentage change } \\
\hline 1985-2002 & $6.0 \%$ & $14.8 \%$ & $\mathrm{~b}$ & $\mathrm{~b}$ \\
\hline 1992-2002 & $9.3 \%$ & b & $\mathrm{b}$ & $\mathrm{b}$ \\
\hline
\end{tabular}

Source:

Production - 1992-2002 Ethanol and MTBE: U.S. Department of Energy, Energy Information Administration, Petroleum Supply Monthly, Washington, DC, January 2003, Table D1. 1985-91 Ethanol: Information Resources, Inc., Washington, DC, 1991. 1985 MTBE: EA-Mueller,Inc., Baltimore, MD, 1992. Imports - U.S. Department of Energy, Energy Information Administration, Petroleum Supply Annual, 2002, Volume 1, Washington, DC, June 2003, Table 20, and annual.

Note:

Table 2.3 displays gasoline-equivalent gallons, which differ from these gallons.

\footnotetext{
${ }^{\text {a }}$ Methyl tertiary-butyl ether.
}

${ }^{\mathrm{b}}$ Data are not available. 
Great care should be taken when comparing modal energy intensity data among modes. Because of the inherent differences between the transportation modes in the nature of services, routes available, and many additional factors, it is not possible to obtain truly comparable national energy intensities among modes. These values are averages, and there is a great deal of variability even within a mode.

Table 2.11

Passenger Travel and Energy Use, 2001

\begin{tabular}{|c|c|c|c|c|c|c|c|}
\hline & \multirow[b]{2}{*}{$\begin{array}{l}\text { Number of vehicles } \\
\text { (thousands) }\end{array}$} & \multirow{2}{*}{$\begin{array}{c}\text { Vehicle- } \\
\text { miles } \\
\text { (millions) }\end{array}$} & \multirow{2}{*}{$\begin{array}{l}\text { Passenger- } \\
\text { miles } \\
\text { (millions) }\end{array}$} & \multirow[b]{2}{*}{$\begin{array}{c}\text { Load factor } \\
\text { (persons/vehicle) }\end{array}$} & \multicolumn{2}{|c|}{ Energy intensities } & \multirow[b]{2}{*}{$\begin{array}{l}\text { Energy use } \\
\text { (trillion Btu) }\end{array}$} \\
\hline & & & & & $\begin{array}{c}\text { (Btu per } \\
\text { vehicle-mile) }\end{array}$ & $\begin{array}{c}\text { (Btu per } \\
\text { passenger-mile) }\end{array}$ & \\
\hline Automobiles & $137,633.5$ & $1,619,422$ & $2,542,493$ & 1.57 & 5,634 & 3,588 & $9,123.5$ \\
\hline Personal trucks & $64,637.0$ & 677,798 & $1,165,812$ & 1.72 & 6,989 & 4,063 & $4,736.8$ \\
\hline Motorcycles & $4,903.1$ & 9,529 & 11,625 & 1.22 & 2,500 & 2,049 & 23.8 \\
\hline Demand response & 34.6 & 789 & 855 & 1.1 & 14,375 & 13,271 & 11.3 \\
\hline Vanpool & 5.4 & 71 & 490 & 6.9 & 8,738 & 1,273 & 0.6 \\
\hline Buses & a & a & $\mathrm{a}$ & $\mathbf{a}$ & $\mathbf{a}$ & a & 203.2 \\
\hline Transit & 76.7 & 2,389 & 22,209 & 9.3 & 38,342 & 4,124 & 91.6 \\
\hline Intercity $^{\mathrm{b}}$ & $\mathrm{a}$ & a & 37,900 & a & a & 852 & 32.3 \\
\hline School $^{\mathrm{b}}$ & 607.8 & a & a & a & a & a & 79.3 \\
\hline Air & a & a & 581,888 & a & a & 4,143 & $2,411.0$ \\
\hline Certificated route $^{\mathrm{c}}$ & a & 5,925 & 565,988 & 95.5 & 379,055 & 3,968 & $2,245.9$ \\
\hline General aviation & 211.5 & a & 15,900 & a & a & 10,384 & 165.1 \\
\hline Recreational boats & $128,876.0$ & a & a & a & a & a & 313.5 \\
\hline Rail & 17.6 & 1,317 & 30,734 & 23.3 & 74,032 & 3,172 & 97.5 \\
\hline Intercity $^{\mathrm{d}}$ & 0.4 & 378 & 5,571 & 14.7 & 60,847 & 4,137 & 23.0 \\
\hline Transit $^{\mathrm{e}}$ & 12.1 & 662 & 15,615 & 23.6 & 73,414 & 3,114 & 48.6 \\
\hline Commuter & 5.1 & 277 & 9,548 & 34.5 & 93,502 & 2,717 & 25.9 \\
\hline
\end{tabular}

Source:

See Appendix A for Passenger Travel and Energy Use.

\footnotetext{
a Data are not available.

2000 energy use data. 2001 data are not available.

c Includes domestic scheduled services and $1 / 2$ of international scheduled services.

${ }^{\mathrm{d}}$ Amtrak only.
}

${ }^{\mathrm{e}}$ Light and heavy rail. 
Great care should be taken when comparing modal energy intensity data among modes. Because of the inherent differences between the transportation modes in the nature of services, routes available, and many additional factors, it is not possible to obtain truly comparable national energy intensities among modes. These values are averages, and there is a great deal of variability even within a mode.

Table 2.12

Energy Intensities of Highway Passenger Modes, 1970-2001

\begin{tabular}{|c|c|c|c|c|c|c|}
\hline \multirow[b]{3}{*}{ Year } & \multirow{2}{*}{\multicolumn{2}{|c|}{ Automobiles }} & \multirow{3}{*}{$\begin{array}{l}\text { Light truck }^{\mathrm{a}} \\
\text { (Btu per } \\
\text { vehicle-mile) }\end{array}$} & \multicolumn{3}{|c|}{ Buses } \\
\hline & & & & \multicolumn{2}{|c|}{ Transit $^{\mathrm{b}}$} & \multirow{2}{*}{$\begin{array}{c}\text { Intercity } \\
\text { (Btu per } \\
\text { passenger-mile }\end{array}$} \\
\hline & $\begin{array}{c}\text { (Btu per } \\
\text { vehicle-mile) }\end{array}$ & $\begin{array}{c}\text { (Btu per } \\
\text { passenger-mile) }\end{array}$ & & $\begin{array}{c}\text { (Btu per } \\
\text { vehicle-mile) }\end{array}$ & $\begin{array}{c}\text { (Btu per } \\
\text { passenger-mile) }\end{array}$ & \\
\hline 1970 & 9,250 & 4,868 & 12,479 & 31,796 & 2,472 & 1,674 \\
\hline 1975 & 8,993 & 4,733 & 11,879 & 33,748 & 2,814 & 988 \\
\hline 1976 & 9,113 & 4,796 & 11,523 & 34,598 & 2,896 & 1,007 \\
\hline 1977 & 8,950 & 4,710 & 11,160 & 35,120 & 2,889 & 970 \\
\hline 1978 & 8,839 & 4,693 & 10,807 & 36,603 & 2,883 & 976 \\
\hline 1979 & 8,647 & 4,632 & 10,467 & 36,597 & 2,795 & 1,028 \\
\hline 1980 & 7,916 & 4,279 & 10,224 & 36,553 & 2,813 & 1,082 \\
\hline 1981 & 7,670 & 4,184 & 9,997 & 37,745 & 3,027 & 1,051 \\
\hline 1982 & 7,465 & 4,109 & 9,268 & 38,766 & 3,237 & 1,172 \\
\hline 1983 & 7,365 & 4,092 & 9,124 & 37,962 & 3,177 & 1,286 \\
\hline 1984 & 7,202 & 4,066 & 8,931 & 38,705 & 3,307 & 954 \\
\hline 1985 & 7,164 & 4,110 & 8,730 & 38,876 & 3,423 & 964 \\
\hline 1986 & 7,194 & 4,197 & 8,560 & 37,889 & 3,545 & 870 \\
\hline 1987 & 6,959 & 4,128 & 8,359 & 36,247 & 3,594 & 940 \\
\hline 1988 & 6,683 & 4,033 & 8,119 & 36,673 & 3,706 & 963 \\
\hline 1989 & 6,589 & 4,046 & 7,746 & 36,754 & 3,732 & 964 \\
\hline 1990 & 6,169 & 3,856 & 7,746 & 37,374 & 3,794 & 962 \\
\hline 1991 & 5,912 & 3,695 & 7,351 & 37,732 & 3,877 & 963 \\
\hline 1992 & 5,956 & 3,723 & 7,239 & 40,243 & 4,310 & 964 \\
\hline 1993 & 6,087 & 3,804 & 7,182 & 39,043 & 4,262 & 962 \\
\hline 1994 & 6,024 & 3,765 & 7,212 & 37,313 & 4,268 & 964 \\
\hline 1995 & 5,902 & 3,689 & 7,208 & 37,277 & 4,310 & 964 \\
\hline 1996 & 5,874 & 3,683 & 7,247 & 37,450 & 4,340 & 963 \\
\hline 1997 & 5,797 & 3,646 & 7,251 & 38,832 & 4,431 & 963 \\
\hline 1998 & 5,767 & 3,638 & 7,261 & 41,182 & 4,387 & 963 \\
\hline 1999 & 5,821 & 3,684 & 7,330 & 40,460 & 4,332 & 964 \\
\hline 2000 & 5,687 & 3,611 & 7,162 & 41,548 & 4,515 & 932 \\
\hline 2001 & 5,634 & 3,588 & 7,095 & 38,341 & 4,125 & c \\
\hline \multicolumn{7}{|c|}{ Average annual percentage change } \\
\hline 1970-2001 & $-1.6 \%$ & $-1.0 \%$ & $-1.8 \%$ & $0.6 \%$ & $1.7 \%$ & c \\
\hline 1991-2001 & $-0.5 \%$ & $-0.3 \%$ & $-0.4 \%$ & $0.2 \%$ & $0.6 \%$ & c \\
\hline
\end{tabular}

Source:

See Appendix A for Highway Passenger Mode Energy Intensities.

${ }^{a}$ All two-axle, four-tire trucks.

beries not continuous between 1983 and 1984 because of a change in data source by the American Public Transit Association (APTA).

'2001data are not yet available. 
Great care should be taken when comparing modal energy intensity data among modes. Because of the inherent differences between the transportation modes in the nature of services, routes available, and many additional factors, it is not possible to obtain truly comparable national energy intensities among modes.

Table 2.13

Energy Intensities of Nonhighway Passenger Modes, 1970-2001

\begin{tabular}{|c|c|c|c|c|c|}
\hline \multirow[b]{2}{*}{ Year } & \multicolumn{2}{|c|}{ Air } & \multicolumn{2}{|c|}{ Rail } & \multirow[b]{2}{*}{$\begin{array}{c}\text { Commuter } \\
\text { rail } \\
\text { (Btu per } \\
\text { passenger-mile }\end{array}$} \\
\hline & $\begin{array}{c}\text { Certificated } \\
\text { air carriers } \\
\text { (Btu per } \\
\text { passenger-mile) }\end{array}$ & $\begin{array}{c}\text { General } \\
\text { aviation } \\
\text { (Btu per } \\
\text { passenger-mile) }\end{array}$ & $\begin{array}{c}\text { Intercity } \\
\text { Amtrak } \\
\text { (Btu per } \\
\text { passenger-mile) }\end{array}$ & $\begin{array}{c}\text { Rail } \\
\text { transit } \\
\text { (Btu per } \\
\text { passenger-mile) }\end{array}$ & \\
\hline 1970 & 10,282 & 10,374 & a & 2,453 & \\
\hline 1975 & 7,826 & 10,658 & 3,677 & 2,962 & $a$ \\
\hline 1976 & 7,511 & 10,769 & 3,397 & 2,971 & a \\
\hline 1977 & 6,990 & 11,695 & 3,568 & 2,691 & a \\
\hline 1978 & 6,144 & 11,305 & 3,683 & 2,210 & a \\
\hline 1979 & 5,607 & 10,787 & 3,472 & 2,794 & a \\
\hline 1980 & 5,561 & 11,497 & 3,176 & 3,008 & a \\
\hline 1981 & 5,774 & 11,123 & 2,957 & 2,946 & a \\
\hline 1982 & 5,412 & 13,015 & 3,156 & 3,069 & a \\
\hline 1983 & 5,133 & 11,331 & 2,957 & 3,212 & a \\
\hline 1984 & 5,298 & 11,454 & 3,027 & 3,732 & 3,011 \\
\hline 1985 & 5,053 & 11,707 & 2,800 & 3,461 & 3,053 \\
\hline 1986 & 5,011 & 11,935 & 2,574 & 3,531 & 3,174 \\
\hline 1987 & 4,827 & 11,496 & 2,537 & 3,534 & 3,043 \\
\hline 1988 & 4,861 & 11,794 & 2,462 & 3,585 & 3,075 \\
\hline 1989 & 4,844 & 10,229 & 2,731 & 3,397 & 3,120 \\
\hline 1990 & 4,875 & 10,146 & 2,609 & 3,453 & 3,068 \\
\hline 1991 & 4,662 & 9,869 & 2,503 & 3,710 & 3,011 \\
\hline 1992 & 4,516 & 9,785 & 2,610 & 3,575 & 2,848 \\
\hline 1993 & 4,490 & 9,653 & 2,646 & 3,687 & 3,222 \\
\hline 1994 & 4,397 & 9,163 & 2,357 & 3,828 & 2,904 \\
\hline 1995 & 4,349 & 9,870 & 2,590 & 3,818 & 2,849 \\
\hline 1996 & 4,172 & 9,258 & 2,792 & 3,444 & 2,796 \\
\hline 1997 & 4,166 & 9,688 & 2,918 & 3,253 & 2,946 \\
\hline 1998 & 4,146 & 11,252 & 2,900 & 3,216 & 2,859 \\
\hline 1999 & 4,061 & 12,206 & 3,062 & 3,168 & 2,929 \\
\hline 2000 & 3,952 & 11,526 & 3,356 & 3,105 & 2,759 \\
\hline \multirow[t]{2}{*}{2001} & 3,968 & 10,384 & 4,137 & 3,114 & 2,717 \\
\hline & \multicolumn{5}{|c|}{ Average annual percentage change } \\
\hline 1970-2001 & $-3.0 \%$ & $0.0 \%$ & $0.4 \%^{\mathrm{b}}$ & $0.8 \%$ & $-0.3 \%^{\mathrm{b}}$ \\
\hline 1991-2001 & $-1.6 \%$ & $0.5 \%$ & $5.2 \%$ & $-1.7 \%$ & $-1.0 \%$ \\
\hline
\end{tabular}

Source:

See Appendix A for Nonhighway Passenger Mode Energy Intensities.

${ }^{a}$ Data are not available.

${ }^{\mathrm{b}}$ Average annual percentage change begins with the earliest year possible. 
Figure 2.2. Energy Intensities for Transit Rail, 2001
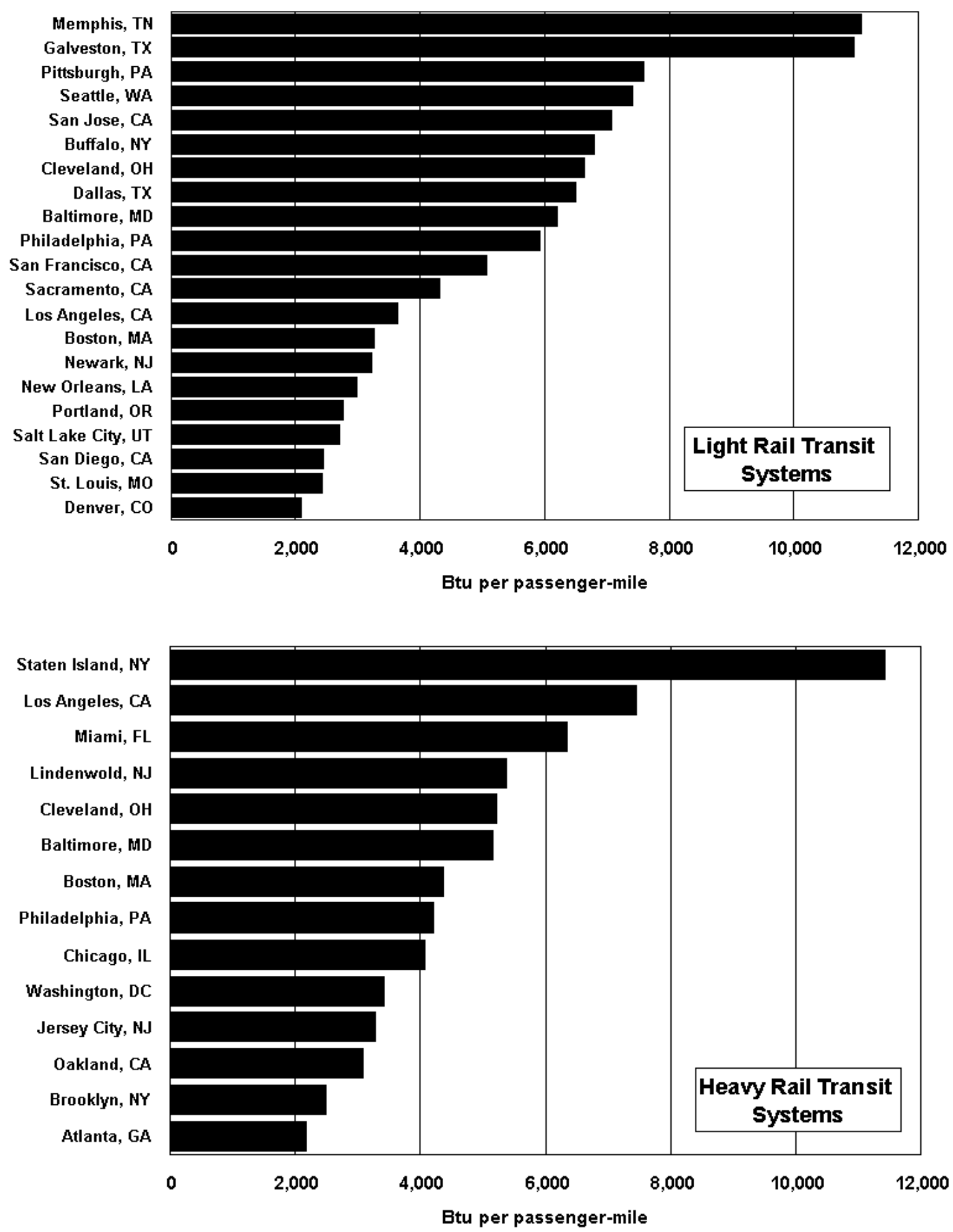

\section{Source:}

U.S. Department of Transportation, Federal Transit Administration, 2001 National Transit Databases, Washington, DC.

(Additional resources: www.fta.dot.gov/ntl) 
Figure 2.3. Energy Intensities for Selected Transit Bus Systems, 2001

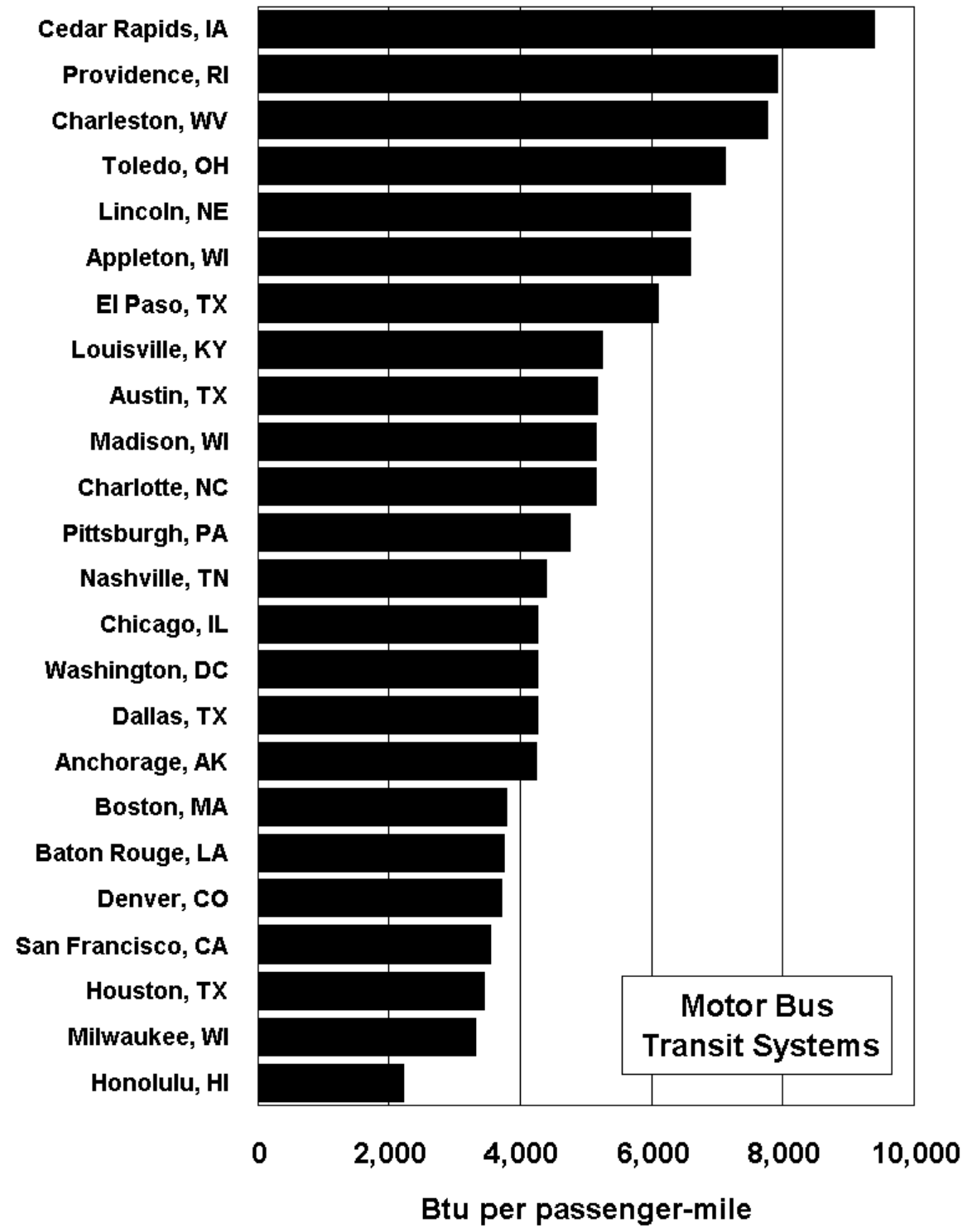

\section{Source:}

U.S. Department of Transportation, Federal Transit Administration, 2001 National Transit Databases, Washington, DC.

(Additional resources: www.fta.dot.gov/ntl) 
Great care should be taken when comparing modal energy intensity data among modes. Because of the inherent differences between the transportation modes in the nature of services, routes available, and many additional factors, it is not possible to obtain truly comparable national energy intensities among modes.

Table 2.14

Intercity Freight Movement and Energy Use in the United States, 2001

\begin{tabular}{lccc}
\hline & Trucks & $\begin{array}{c}\text { Waterborne } \\
\text { commerce }\end{array}$ & $\begin{array}{c}\text { Class I } \\
\text { railroads }\end{array}$ \\
\hline Number of vehicles (thousands) & 2,615 & 42 & $20^{\mathrm{a}}$ \\
Ton-miles (billions) & 1,051 & 622 & 1,495 \\
Tons shipped (millions) & 4,122 & 1,037 & 1,742 \\
Average length of haul (miles) & $752^{\mathrm{b}}$ & 600 & 859 \\
Energy intensity (Btu/ton-mile) & 3,337 & 444 & 346 \\
Energy use (trillion Btu) & 3,507 & 276 & 517 \\
\hline
\end{tabular}

Source:

See Appendix A for Freight Movement and Energy Use.

${ }^{a}$ Number of locomotives.

b 717 miles is for general freight (less than truckload). Based on data from the Eno Transportation Foundation, the average length of haul for specialized freight (truckload) is 294 miles. 
Great care should be taken when comparing modal energy intensity data among modes. Because of the inherent differences between the transportation modes in the nature of services, routes available, and many additional factors, it is not possible to obtain truly comparable national energy intensities among modes.

Table 2.15

Energy Intensities of Freight Modes, 1970-2001

\begin{tabular}{|c|c|c|c|c|}
\hline \multirow[b]{2}{*}{ Year } & \multirow{2}{*}{$\begin{array}{l}\text { Heavy single-unit and } \\
\text { combination trucks } \\
\text { (Btu per vehicle-mile) }\end{array}$} & \multicolumn{2}{|c|}{ Class I freight railroad } & \multirow{2}{*}{$\begin{array}{c}\text { Domestic waterborne } \\
\text { commerce } \\
\text { (Btu per ton-mile) }\end{array}$} \\
\hline & & $\begin{array}{l}\text { (Btu per freight car- } \\
\text { mile) }\end{array}$ & $\begin{array}{l}\text { (Btu per ton- } \\
\text { mile) }\end{array}$ & \\
\hline 1970 & 24,960 & 17,669 & 691 & 545 \\
\hline 1971 & 24,485 & 18,171 & 717 & 506 \\
\hline 1972 & 24,668 & 18,291 & 714 & 522 \\
\hline 1973 & 24,777 & 18,468 & 677 & 576 \\
\hline 1974 & 24,784 & 18,852 & 681 & 483 \\
\hline 1975 & 24,631 & 18,739 & 687 & 549 \\
\hline 1976 & 24,566 & 18,938 & 680 & 468 \\
\hline 1977 & 24,669 & 19,226 & 669 & 458 \\
\hline 1978 & 24,655 & 18,928 & 641 & 383 \\
\hline 1979 & 24,745 & 19,188 & 618 & 436 \\
\hline 1980 & 24,757 & 18,742 & 597 & 358 \\
\hline 1981 & 25,058 & 18,629 & 572 & 360 \\
\hline 1982 & 24,296 & 18,404 & 553 & 310 \\
\hline 1983 & 23,852 & 17,864 & 525 & 286 \\
\hline 1984 & 23,585 & 17,795 & 510 & 346 \\
\hline 1985 & 23,343 & 17,500 & 497 & 446 \\
\hline 1986 & 23,352 & 17,265 & 486 & 463 \\
\hline 1987 & 22,922 & 16,790 & 456 & 402 \\
\hline 1988 & 22,596 & 16,758 & 443 & 361 \\
\hline 1989 & 22,411 & 16,894 & 437 & 403 \\
\hline 1990 & 22,795 & 16,619 & 420 & 387 \\
\hline 1991 & 22,749 & 15,835 & 391 & 386 \\
\hline 1992 & 22,608 & 16,043 & 393 & 398 \\
\hline 1993 & 22,373 & 16,056 & 389 & 389 \\
\hline 1994 & 22,193 & 16,340 & 388 & 369 \\
\hline 1995 & 22,096 & 15,992 & 372 & 374 \\
\hline 1996 & 22,109 & 15,747 & 368 & 412 \\
\hline 1997 & 21,340 & 15,784 & 370 & 415 \\
\hline 1998 & 21,514 & 15,372 & 365 & 435 \\
\hline 1999 & 22,880 & 15,363 & 363 & 457 \\
\hline 2000 & 23,443 & 14,917 & 352 & 508 \\
\hline 2001 & 23,237 & 15,107 & 346 & 444 \\
\hline \multicolumn{5}{|c|}{ Average annual percentage change } \\
\hline 1970-2001 & $-0.2 \%$ & $-0.5 \%$ & $-2.2 \%$ & $-0.7 \%$ \\
\hline 1991-2001 & $0.2 \%$ & $-0.6 \%$ & $-1.2 \%$ & $1.4 \%$ \\
\hline
\end{tabular}

Source:

See Appendix A for Freight Mode Energy Intensities. 


\section{Chapter 3}

\section{All Highway Vehicles and Characteristics}

Summary Statistics from Tables in this Chapter

\begin{tabular}{|c|c|c|}
\hline Source & & \\
\hline Table 3.1 & U.S. share of world automobile registrations, 2001 & $22.9 \%$ \\
\hline Table 3.2 & U.S. share of world truck \& bus registrations, 2001 & $42.7 \%$ \\
\hline Table 3.3 & Number of U.S. automobiles, 2001 (thousands) & 128,714 \\
\hline Table 3.3 & Number of U.S. trucks, 2001 (thousands) & 87,969 \\
\hline \multirow[t]{7}{*}{ Table 3.5} & Vehicle miles traveled, 2001 (million miles) & $2,746,925$ \\
\hline & Automobiles & $58.2 \%$ \\
\hline & Motorcycles & $0.3 \%$ \\
\hline & Two-axle, four-tire trucks & $33.7 \%$ \\
\hline & Other single-unit trucks & $2.6 \%$ \\
\hline & Combination trucks & $4.9 \%$ \\
\hline & Buses & $0.3 \%$ \\
\hline \multirow[t]{4}{*}{ Table 3.8} & Average age of vehicles, 2001 & \\
\hline & Automobiles (years) & 9.0 \\
\hline & Trucks (years) & 7.9 \\
\hline & Median lifetime of vehicles & \\
\hline Table 3.9 & Automobiles (years) & 16.9 \\
\hline Table 3.10 & Light trucks (years) & 15.5 \\
\hline
\end{tabular}


The 1997 data in this series were never published. Use caution comparing historical data because of disconnects in data series, such as China in

1998. Also, the U.S. is unique in how many light trucks (SUVs, minivans, pickups) are used for personal travel. Those light trucks are not included

on this table. The U.S. share of world automobiles has been declining since 1998.

Table 3.1

Automobile Registrations for Selected Countries, 1950-2001

\begin{tabular}{|c|c|c|c|c|c|c|c|c|c|c|}
\hline Year & China & India & Japan & France & $\begin{array}{c}\text { United } \\
\text { Kingdom }\end{array}$ & Germany $^{\mathrm{a}}$ & Canada $^{\mathrm{b}}$ & $\begin{array}{l}\text { United } \\
\text { States }^{c}\end{array}$ & $\begin{array}{l}\text { U.S. percentage } \\
\text { of world }\end{array}$ & $\begin{array}{l}\text { World } \\
\text { total }\end{array}$ \\
\hline 1950 & d & d & 43 & d & 2,307 & $\mathrm{~d}$ & 1,913 & 40,339 & $76.0 \%$ & 53,051 \\
\hline 1955 & d & d & 153 & $\mathrm{~d}$ & 360 & & $2,961$. & 52,145 & $71.4 \%$ & 73,036 \\
\hline 1960 & $\dddot{\mathrm{d}}$ & d" & 457 & 4,950 & 5,650 & 4,856 & 4,104 & 61,671 & $62.7 \%$ & 98,305 \\
\hline 1965 & d & d & 2,181 & 8,320 & 9,131 & 9,719 & 5,279 & 75,258 & $53.8 \%$ & 139,776 \\
\hline 1970 & $\dddot{d}$ & d & 8,779 & 11,860 & 11,802 & 14,376 & 6,602 & 89,244 & $46.1 \%$ & 193,479 \\
\hline 1975 & d & d & 17,236 & 15,180 & 14,061 & 18,161 & 8,870 & 106,706 & $41.0 \%$ & 260,201 \\
\hline 1980 & 351 & $\mathrm{~d}$ & 23,660 & 18,440 & 15,438 & 23,236 & 10,256 & 121,601 & $38.0 \%$ & 320,390 \\
\hline 1985 & 795 & 1,607 & 27,845 & 20,800 & 18,953 & 26,099 & 11,118 & 127,885 & $34.5 \%$ & 370,504 \\
\hline 1990 & 1,622 & 2,694 & 34,924 & 23,010 & 22,528 & 30,695 & 12,622 & 133,700 & $30.7 \%$ & 435,050 \\
\hline 1991 & 1,852 & 2,954 & 37,076 & 23,550 & 22,744 & 31,309 & 12,578 & 128,300 & $29.1 \%$ & 441,377 \\
\hline 1992 & 2,262 & 3,205 & 38,963 & 24,020 & 23,008 & 37,579 & 12,781 & 126,581 & $28.0 \%$ & 452,311 \\
\hline 1993 & 2,860 & 3,361 & 40,772 & 24,385 & 23,402 & 39,202 & 12,927 & 127,327 & $28.3 \%$ & 450,473 \\
\hline 1994 & 3,497 & 3,569 & 42,678 & 24,900 & 23,832 & 39,918 & 13,122 & 127,883 & $27.0 \%$ & 473,487 \\
\hline 1995 & 4,179 & 3,837 & 44,680 & 25,100 & 24,307 & 40,499 & 13,183 & 128,387 & $26.9 \%$ & 477,010 \\
\hline 1996 & 4,700 & 4,246 & 46,868 & 25,500 & 24,864 & 41,045 & 13,300 & 129,728 & $26.7 \%$ & 485,954 \\
\hline 1997 & \multicolumn{10}{|c|}{ Data are not available. } \\
\hline 1998 & $2,940^{\mathrm{e}}$ & 4,820 & 49,896 & 26,800 & 22,115 & 41,674 & 13,887 & 131,839 & $27.5 \%$ & 478,625 \\
\hline 1999 & 3,400 & 5,200 & 51,164 & 27,480 & 27,539 & 42,423 & 16,538 & 126,869 & $26.7 \%$ & 496,059 \\
\hline 2000 & 3,750 & 5,150 & 52,437 & 28,060 & 27,185 & 43,772 & 16,832 & 127,721 & $23.3 \%$ & 547,147 \\
\hline 2001 & 4,325 & 5,750 & 53,300 & 28,700 & 27,790 & 44,383 & 17,055 & 128,714 & $22.9 \%$ & 561,687 \\
\hline \multicolumn{11}{|c|}{ Average annual percentage change } \\
\hline 1950-2001 & d & d & $15.0 \%$ & & $5.0 \%$ & & $4.4 \%$ & $2.3 \%$ & & $4.7 \%$ \\
\hline $1970-2001$ & d & d & $6.0 \%$ & $2.9 \%$ & $2.8 \%$ & $3.7 \%$ & $3.1 \%$ & $1.2 \%$ & & $3.5 \%$ \\
\hline 1991-2001 & $8.9 \%$ & $6.9 \%$ & $3.7 \%$ & $2.0 \%$ & $2.0 \%$ & $3.6 \%$ & $3.1 \%$ & $0.0 \%$ & & $2.4 \%$ \\
\hline
\end{tabular}

Source:

Ward's Communications, Ward's World Motor Vehicle Data, 2002 Edition, Southfield, MI, 2002, pp. 232-235 and annual. (Additional resources: www.wardsauto.com)

\footnotetext{
${ }^{\text {a }}$ Data for 1991 and prior include West Germany only. Kraftwagen are included with automobiles.

${ }^{\mathrm{b}}$ Data from 1991 and later are not comparable to prior data and data from 1999 and later are not comparable to prior data.

c Data from 1985 and later are not comparable to prior data.

${ }^{\mathrm{d}}$ Data are not available.

e Data are not comparable to prior data due to reclassification of autos and trucks.
} 
The 1997 data in this series were never published. Use caution comparing historical data because of disconnects in data series, such as China in 1998. The U.S. totals include SUVs, minivans, and light trucks, many of which are used for personal travel.

Table 3.2

Truck and Bus Registrations for Selected Countries, 1950-2001 (thousands)

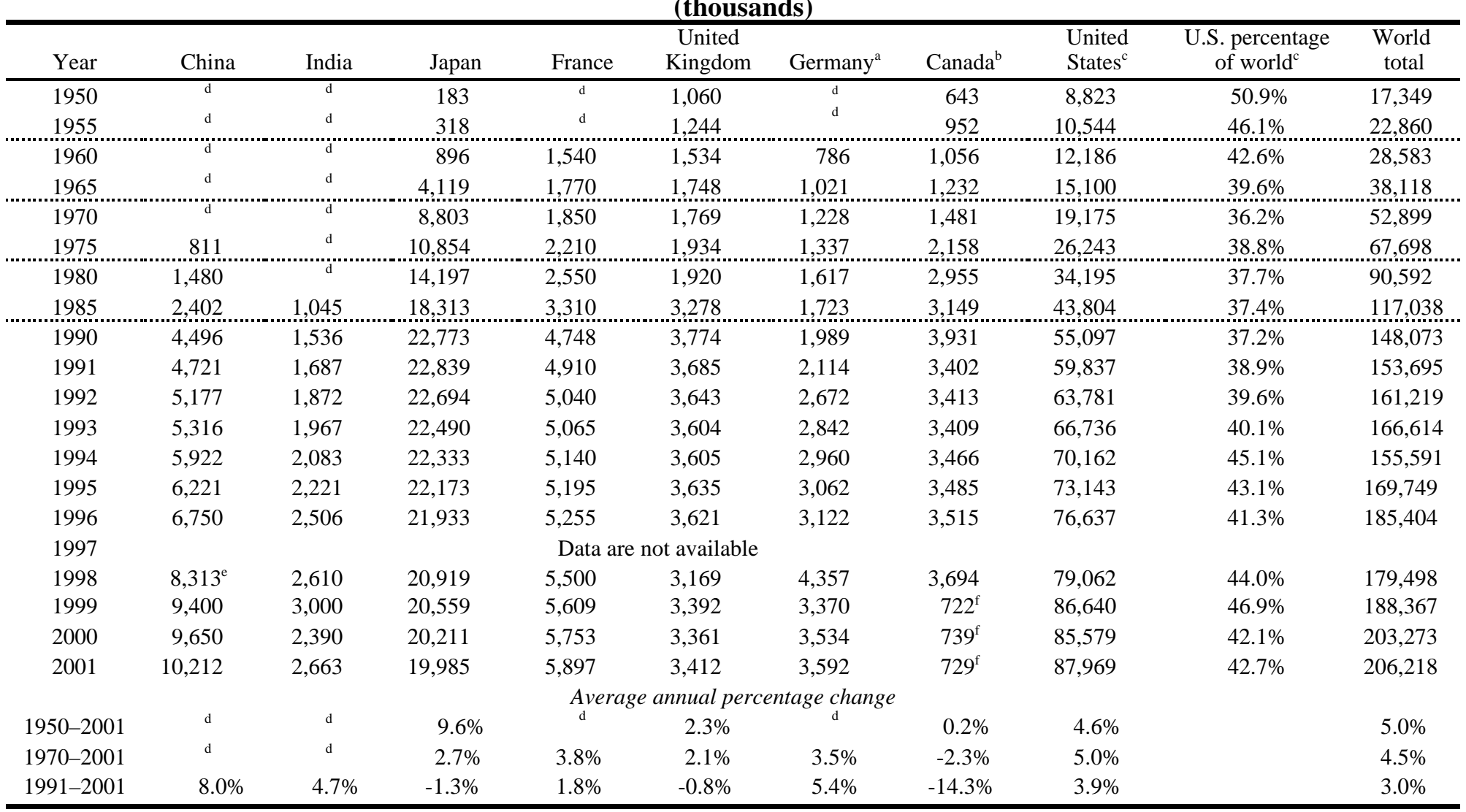

\section{Source:}

Ward's Communications, Ward's World Motor Vehicle Data, 2002 Edition, Southfield, MI, 2002, pp. 232-235 and annual. (Additional resources: www.wardsauto.com)

\footnotetext{
${ }^{\text {a }}$ Data for 1991 and prior include West Germany only. Kraftwagen are included with automobiles. Data from 1999 and later are not comparable to prior data.

${ }^{\mathrm{b}}$ Data from 1991 and later are not comparable to prior data.

${ }^{\text {c }}$ Data from 1985 and later are not comparable to prior data.

${ }^{\mathrm{d}}$ Data are not available.

${ }^{\mathrm{e}}$ Data not comparable to prior data due to reclassification of autos and trucks.

${ }^{\mathrm{f}}$ Canada
} 


\section{VEHICLES IN USE}

Both the Federal Highway Administration (FHWA) and The Polk Company report figures on the automobile and truck population each year. The two estimates, however, differ by as much as 11.2\% (1981). The differences can be attributed to several factors:

- The FHWA data include all vehicles which have been registered at any time throughout the calendar year. Therefore, the data include vehicles which were retired during the year and may double count vehicles which have been registered in different states or the same states to different owners. The Polk Company data include only those vehicles which are registered on July 1 of the given year.

- The classification of mini-vans, station wagons on truck chasses, and utility vehicles as passenger cars or trucks causes important differences in the two estimates. The Polk Company data included passenger vans in the automobile count until 1980; since 1980 all vans have been counted as trucks. Recently, the Federal Highway Administration adjusted their definition of automobiles and trucks. Starting in 1993, some minivans and sport utility vehicles that were previously included with automobiles were included with trucks. This change produced a dramatic change in the individual percentage differences of cars and trucks. The difference in total vehicles has been less than 5\% each year since 1990 and does not appear to be significantly affected by the FHWA reclassifications.

- The FHWA data include all non-military Federal vehicles, while The Polk Company data include only those Federal vehicles which are registered within a state. Federal vehicles are not required to have State registrations, and, according to the General Services Administration, most Federal Vehicles are not registered.

According to The Polk Company statistics, the number of passenger cars in use in the U.S. declined from 1991 to 1992. This is the first decline in vehicle stock since the figures were first reported in 1924. However, the data should be viewed with caution. A redesign of Polk's approach in 1992 allowed a national check for duplicate registrations, which was not possible in earlier years. Polk estimates that, due to processing limitations, its vehicle population counts may have been inflated by as much as $1 \frac{1}{2}$ percent. Assuming that percentage is correct, the number of passenger cars in use would have declined from 1991 to 1992 under the previous Polk method. The growing popularity of light trucks being used as passenger vehicles could also have had an impact on these figures. 
Table 3.3

U.S. Automobiles and Trucks in Use, 1970-2001

(thousands)

\begin{tabular}{|c|c|c|c|c|c|c|c|c|c|}
\hline \multirow[b]{2}{*}{ Year } & \multicolumn{3}{|c|}{ Automobiles } & \multicolumn{3}{|c|}{ Trucks } & \multicolumn{3}{|c|}{ Total } \\
\hline & FHWA & $\begin{array}{l}\text { The Polk } \\
\text { Company }\end{array}$ & $\begin{array}{c}\text { Percentage } \\
\text { difference }\end{array}$ & FHWA & $\begin{array}{l}\text { The Polk } \\
\text { Company }\end{array}$ & $\begin{array}{c}\text { Percentage } \\
\text { difference }\end{array}$ & FHWA & $\begin{array}{l}\text { The Polk } \\
\text { Company }\end{array}$ & $\begin{array}{r}\text { Percentage } \\
\text { difference } \\
\end{array}$ \\
\hline 1970 & 89,243 & 80,448 & $10.9 \%$ & 18,797 & 17,688 & $6.3 \%$ & 108,040 & 98,136 & $10.1 \%$ \\
\hline 1975 & 106,706 & 95,241 & $12.0 \%$ & 25,781 & 24,813 & $3.9 \%$ & 132,487 & 120,054 & $10.4 \%$ \\
\hline 1980 & 121,601 & 104,564 & $16.3 \%$ & 33,667 & 35,268 & $-4.5 \%$ & 155,267 & 139,832 & $11.0 \%$ \\
\hline 1981 & 123,098 & 105,839 & $16.3 \%$ & 34,644 & 36,069 & $-4.0 \%$ & 157,743 & 141,908 & $11.2 \%$ \\
\hline 1982 & 123,702 & 106,867 & $15.8 \%$ & 35,382 & 36,987 & $-4.3 \%$ & 159,084 & 143,854 & $10.6 \%$ \\
\hline 1983 & 126,444 & 108,961 & $16.0 \%$ & 36,723 & 38,143 & $-3.7 \%$ & 163,166 & 147,104 & $10.9 \%$ \\
\hline 1984 & 128,158 & 112,019 & $14.4 \%$ & 37,507 & 40,143 & $-6.6 \%$ & 165,665 & 152,162 & $8.9 \%$ \\
\hline 1985 & 127,885 & 114,662 & $11.5 \%$ & 43,210 & 42,387 & $1.9 \%$ & 171,095 & 157,049 & $8.9 \%$ \\
\hline 1986 & 130,004 & 117,268 & $10.9 \%$ & 45,103 & 44,826 & $0.6 \%$ & 175,106 & 162,094 & $8.0 \%$ \\
\hline 1987 & 131,482 & 119,849 & $9.7 \%$ & 46,826 & 47,344 & $-1.1 \%$ & 178,308 & 167,193 & $6.6 \%$ \\
\hline 1988 & 133,836 & 121,519 & $10.1 \%$ & 49,941 & 50,221 & $-0.6 \%$ & 183,777 & 171,740 & $7.0 \%$ \\
\hline 1989 & 134,559 & 122,758 & $9.6 \%$ & 52,172 & 53,202 & $-1.9 \%$ & 186,731 & 175,960 & $6.1 \%$ \\
\hline 1990 & 133,700 & 123,276 & $8.5 \%$ & 54,470 & 56,023 & $-2.8 \%$ & 188,171 & 179,299 & $4.9 \%$ \\
\hline 1991 & 128,300 & 123,268 & $4.1 \%$ & 59,206 & 58,179 & $1.8 \%$ & 187,505 & 181,447 & $3.3 \%$ \\
\hline 1992 & 126,581 & 120,347 & $5.2 \%$ & 63,136 & 61,172 & $3.2 \%$ & 189,717 & 181,519 & $4.5 \%$ \\
\hline 1993 & 127,327 & 121,055 & $5.2 \%$ & 66,082 & 65,260 & $1.3 \%$ & 193,409 & 186,315 & $3.8 \%$ \\
\hline 1994 & 127,883 & 121,997 & $4.8 \%$ & 69,491 & 66,717 & $4.2 \%$ & 197,375 & 188,714 & $4.6 \%$ \\
\hline 1995 & 128,387 & 123,242 & $4.2 \%$ & 72,458 & 70,199 & $3.2 \%$ & 200,845 & 193,441 & $3.8 \%$ \\
\hline 1996 & 129,728 & 124,613 & $4.1 \%$ & 75,940 & 73,681 & $3.1 \%$ & 205,669 & 198,294 & $3.7 \%$ \\
\hline 1997 & 129,749 & 124,673 & $4.1 \%$ & 77,307 & 76,398 & $1.2 \%$ & 207,056 & 201,071 & $3.0 \%$ \\
\hline 1998 & 131,839 & 125,966 & $4.7 \%$ & 79,062 & 79,077 & $0.0 \%$ & 210,901 & 205,043 & $2.9 \%$ \\
\hline 1999 & 132,432 & 126,869 & $4.4 \%$ & 83,148 & 82,640 & $0.6 \%$ & 215,580 & 209,509 & $2.9 \%$ \\
\hline 2000 & 133,621 & 127,721 & $4.6 \%$ & 87,108 & 85,579 & $1.8 \%$ & 220,729 & 213,300 & $3.5 \%$ \\
\hline 2001 & 137,633 & 128,714 & $6.9 \%$ & 92,045 & 87,969 & $4.6 \%$ & 229,678 & 216,683 & $6.0 \%$ \\
\hline
\end{tabular}

Source:

FHWA - U.S. Department of Transportation, Federal Highway Administration, Highway Statistics 2001, Washington, DC, 2002, Table VM-1, p. V-57, and annual. (Additional resources: www.fhwa.dot.gov)

Polk - The Polk Company, Detroit, Michigan. FURTHER REPRODUCTION PROHIBITED. (Additional resources: www.polk.com) 
The line on this graph shows the trend of vehicles per thousand people for the U.S. from 1900 to 2001. The symbols on the graph show the 2001 vehicles per thousand people for other countries or regions around the world. Canada in 2001 had about the same ratio of vehicles to people as the U.S. did in 1972 and Western Europe had about the same ratio as the U.S. did in 1969. On the other end of the scale, China had about the same ratio of vehicles per thousand people in 2001 as the U.S. had in 1913 and Africa had about the same ratio as the U.S. did in 1915.

Figure 3.1. Vehicles per Thousand People: U.S. Compared to Other Countries

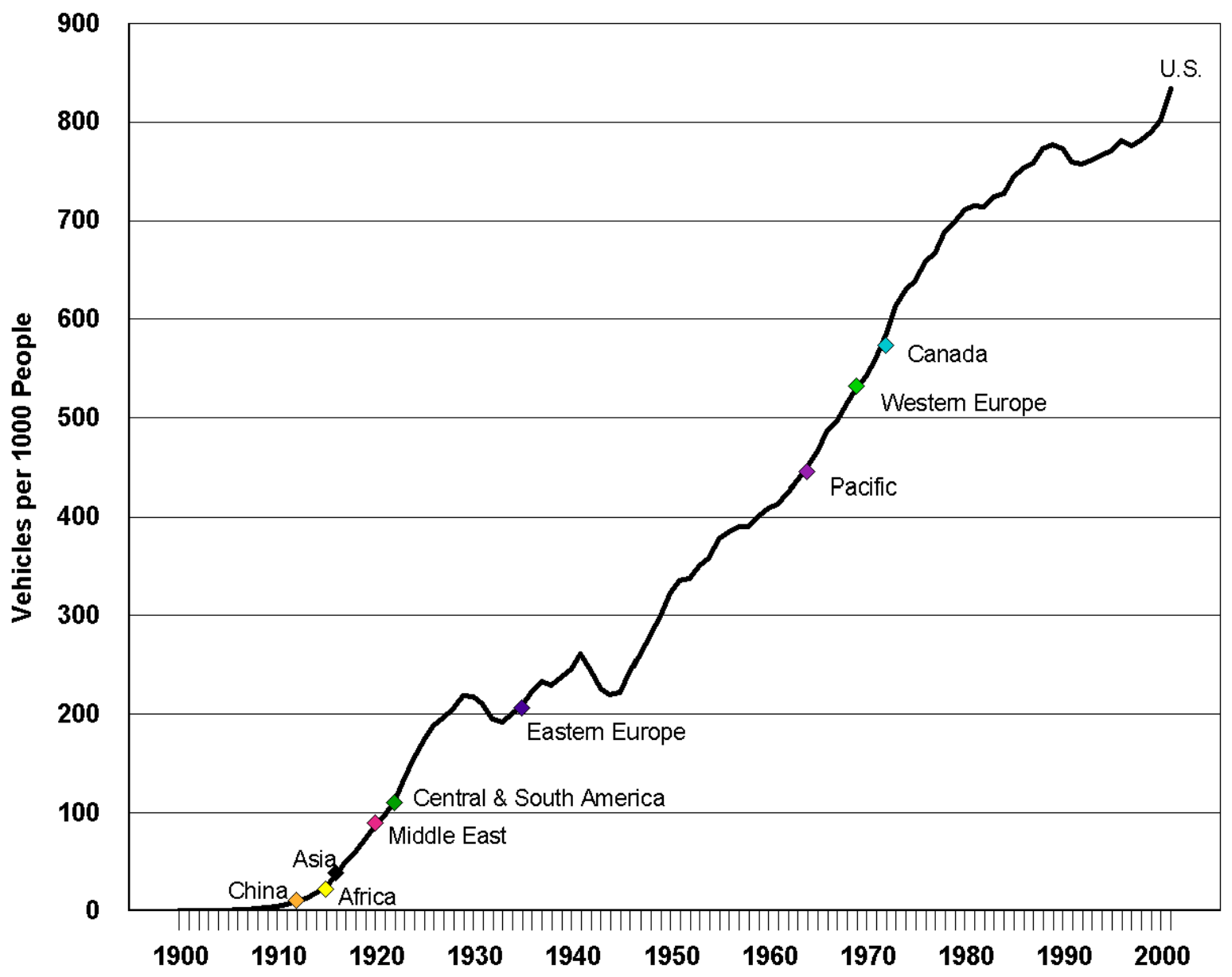

Source:

Population - U.S.: U.S. Bureau of the Census, Statistical Abstract of the United States: 2002, Table No. 2. All others: United Nations Secretariat, Population Division, World Urbanization Prospects, The 2001 Revision, March 20, 2002.

(Additional resources: www.un.org/esa/population/unpop.htm

Vehicles - U.S.: U.S. Department of Transportation, Federal Highway Administration, Highway Statistics 2001, Washington, DC, 2003. All others: Ward's Communications, Ward's Motor Vehicle Data 2002, pp. 232-235.

(Additional resources: www.fhwa.dot.gov, www.wardsauto.com) 
The data on automobile and light truck stock by size class are estimations based on historical sales data. This method assumes a constant scrappage rate for all size classes. The definitions for the size classes are in the Glossary. The data on trucks by weight class are based on estimates from the 1997 Vehicle Inventory and Use Survey (latest available survey) and a 2002 report entitled "Investigation of Class $2 b$ Trucks (Vehicles of 8,500 to 10,000 lbs GVWR)."

Table 3.4

Vehicle Stock and New Sales in the United States, 2001 Calendar Year

\begin{tabular}{|c|c|c|c|c|c|c|c|}
\hline \multirow[b]{3}{*}{ Autos } & \multicolumn{2}{|c|}{ Vehicle stock $^{a}$} & \multicolumn{5}{|c|}{ New sales (in thousands) } \\
\hline & \multirow{2}{*}{$\begin{array}{c}\text { Thousands } \\
128,714\end{array}$} & \multirow{2}{*}{$\begin{array}{l}\text { Share } \\
100.0 \%\end{array}$} & \multicolumn{2}{|c|}{ Domestic } & \multicolumn{2}{|c|}{ Import $^{\mathrm{b}}$} & \multirow{2}{*}{$\begin{array}{c}\text { Total } \\
8,423\end{array}$} \\
\hline & & & 6,325 & $(75.1 \%)$ & 2,098 & $(24.9 \%)$ & \\
\hline Two-seaters & 1,908 & $1.5 \%$ & 51 & $(42.5 \%)$ & 69 & (57.5\%) & 120 \\
\hline Minicompact & 1,260 & $1.0 \%$ & $\mathbf{0}$ & $(0.0 \%)$ & 34 & $(100.0$ & 34 \\
\hline Subcompact & 26,294 & $20.4 \%$ & 715 & $(76.5 \%)$ & 220 & $(23.5 \%)$ & 935 \\
\hline Compact & 41,480 & $32.2 \%$ & 2,150 & $(69.3 \%)$ & 954 & $(30.7 \%)$ & 3,104 \\
\hline Midsize & 39,032 & $30.3 \%$ & 1,961 & $(72.3 \%)$ & 751 & $(27.7 \%)$ & 2,712 \\
\hline Large & 18,740 & $14.6 \%$ & 1,448 & $(95.5 \%)$ & 69 & $(4.5 \%)$ & 1,517 \\
\hline Autos & 128,714 & $100.0 \%$ & $\mathrm{c}$ & $\mathrm{c}$ & c & c & $\mathrm{c}^{\mathrm{c}}$ \\
\hline Business fleet autos & 6,640 & $5.2 \%$ & c & c & c & c & c \\
\hline Personal autos & 122,074 & $94.8 \%$ & c & c & c & c & c \\
\hline Trucks & 87,969 & $100.0 \%$ & 8,048 & $(88.9 \%)$ & 1,002 & $(11.1 \%)$ & 9,050 \\
\hline Less than 8,500 lbs. & 75,835 & $86.2 \%$ & 7,137 & $(88.0 \%)$ & 978 & $(12.0 \%)$ & 8,115 \\
\hline Small pickup & 13,151 & $14.9 \%$ & 821 & $(100.0 \%)$ & $\mathbf{0}$ & $(0.0 \%)$ & 821 \\
\hline Large pickup & 21,880 & $24.9 \%$ & 1,998 & $(100.0 \%)$ & $\mathbf{0}$ & $(0.0 \%)$ & 1,998 \\
\hline Small van & 13,141 & $14.9 \%$ & 1,113 & $(96.7 \%)$ & 38 & $(3.3 \%)$ & 1,151 \\
\hline Large van & 5,497 & $6.2 \%$ & 321 & $(97.6 \%)$ & 8 & $(2.4 \%)$ & 329 \\
\hline Small SUV & 6,302 & $7.2 \%$ & 578 & $(62.9 \%)$ & 340 & (37.1\%) & 918 \\
\hline Medium SUV & 12,266 & $13.9 \%$ & 1,542 & $(73.2 \%)$ & 565 & $(26.8 \%)$ & 2,107 \\
\hline Large SUV & 3,599 & $4.1 \%$ & 764 & $(96.7 \%)$ & 26 & $(3.3 \%)$ & 790 \\
\hline 8,500 - 10,000 lbs. & 6,416 & $7.3 \%$ & 492 & $(100.0 \%)$ & $\mathbf{0}$ & $(0.0 \%)$ & 492 \\
\hline Pickup & 4,728 & $5.4 \%$ & 404 & $(100.0 \%)$ & $\mathbf{0}$ & $(0.0 \%)$ & 404 \\
\hline Van/SUV & 1,687 & $1.9 \%$ & 88 & $(100.0 \%)$ & $\mathbf{0}$ & $(0.0 \%)$ & 88 \\
\hline 10,000 - 26,000 lbs. & 2,639 & $3.0 \%$ & 188 & $(88.8 \%)$ & 24 & $(11.2 \%)$ & 212 \\
\hline 26,000 lbs. and over & 3,079 & $3.5 \%$ & 231 & $(99.7 \%)$ & 1 & $(0.3 \%)$ & 231 \\
\hline Trucks & 87,969 & $100.0 \%$ & 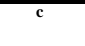 & $\mathrm{c}$ & c & $\mathrm{c}^{\mathrm{c}}$ & c \\
\hline Business fleet trucks $<=19,500$ lbs. GVW & 7,002 & $8.0 \%$ & c & c & c & c & c \\
\hline Personal trucks $<=19,500$ lbs. GVW & 77,008 & $87.5 \%$ & c & c & c & c & c \\
\hline Trucks $>19,500$ lbs. GVW & 3,959 & $4.5 \%$ & c & c & c & c & c \\
\hline
\end{tabular}

Source:

See Appendix A Highway Vehicle Stock and New Sales for detailed methodology and sources.

(Additional resources: www.polk.com)

${ }^{\text {a }}$ Total auto and truck vehicle stocks as of July 1, 2001 from The Polk Company (FURTHER REPRODUCTION PROHIBITED).

${ }^{\mathrm{b}}$ Includes domestic-sponsored imports.

${ }^{\mathrm{c}}$ Data are not available.

${ }^{\mathrm{d}}$ In fleets of four or more vehicles. 
The trend of using two-axle, four-tire trucks, such as pickups, vans, and sport-utility vehicles, for personal travel is evident in these data; two-axle, four-tire trucks account for 23\% more travel in 2001 than in 1970, and automobiles account for $24 \%$ less travel in that time period.

Table 3.5

Shares of Highway Vehicle-Miles Traveled by Vehicle Type, 1970-2001

\begin{tabular}{|c|c|c|c|c|c|c|c|}
\hline Year & Automobiles & $\begin{array}{c}\text { Motorcycle } \\
\text { s } \\
\end{array}$ & $\begin{array}{c}\text { Two-axle, } \\
\text { four-tire } \\
\text { trucks }\end{array}$ & $\begin{array}{l}\text { Other } \\
\text { single-unit } \\
\text { trucks }\end{array}$ & $\begin{array}{l}\text { Combinatio } \\
\text { n trucks } \\
\end{array}$ & Buses $^{a}$ & $\begin{array}{c}\text { Total } \\
\text { vehicle-miles } \\
\text { traveled } \\
\text { (million miles) }\end{array}$ \\
\hline 1970 & $82.6 \%$ & $0.3 \%$ & $11.1 \%$ & $2.4 \%$ & $3.2 \%$ & $0.4 \%$ & $1,109,724$ \\
\hline 1975 & $77.9 \%$ & $0.4 \%$ & $15.1 \%$ & $2.6 \%$ & $3.5 \%$ & $0.5 \%$ & $1,327,664$ \\
\hline 1980 & $72.8 \%$ & $0.7 \%$ & $19.0 \%$ & $2.6 \%$ & $4.5 \%$ & $0.4 \%$ & $1,527,295$ \\
\hline 1981 & $72.9 \%$ & $0.7 \%$ & $19.1 \%$ & $2.5 \%$ & $4.4 \%$ & $0.4 \%$ & $1,555,308$ \\
\hline 1982 & $72.8 \%$ & $0.6 \%$ & $19.2 \%$ & $2.5 \%$ & $4.4 \%$ & $0.4 \%$ & $1,595,010$ \\
\hline 1983 & $72.3 \%$ & $0.5 \%$ & $19.8 \%$ & $2.6 \%$ & $4.5 \%$ & $0.3 \%$ & $1,652,788$ \\
\hline 1984 & $71.3 \%$ & $0.5 \%$ & $20.8 \%$ & $2.6 \%$ & $4.5 \%$ & $0.3 \%$ & $1,720,269$ \\
\hline 1985 & $70.2 \%$ & $0.5 \%$ & $22.0 \%$ & $2.6 \%$ & $4.4 \%$ & $0.3 \%$ & $1,774,826$ \\
\hline 1986 & $69.2 \%$ & $0.5 \%$ & $23.1 \%$ & $2.5 \%$ & $4.4 \%$ & $0.3 \%$ & 1,834,872 \\
\hline 1987 & $68.5 \%$ & $0.5 \%$ & $23.8 \%$ & $2.5 \%$ & $4.5 \%$ & $0.3 \%$ & 1,921,204 \\
\hline 1988 & $67.6 \%$ & $0.5 \%$ & $24.8 \%$ & $2.4 \%$ & $4.4 \%$ & $0.3 \%$ & $2,025,962$ \\
\hline 1989 & $66.8 \%$ & $0.5 \%$ & $25.6 \%$ & $2.4 \%$ & $4.4 \%$ & $0.3 \%$ & $2,096,487$ \\
\hline 1990 & $65.7 \%$ & $0.4 \%$ & $26.8 \%$ & $2.4 \%$ & $4.4 \%$ & $0.3 \%$ & $2,144,362$ \\
\hline 1991 & $62.5 \%$ & $0.4 \%$ & $29.9 \%$ & $2.4 \%$ & $4.4 \%$ & $0.3 \%$ & $2,172,050$ \\
\hline 1992 & $61.0 \%$ & $0.4 \%$ & $31.5 \%$ & $2.4 \%$ & $4.4 \%$ & $0.3 \%$ & $2,247,151$ \\
\hline 1993 & $59.9 \%$ & $0.4 \%$ & $32.5 \%$ & $2.5 \%$ & $4.5 \%$ & $0.3 \%$ & $2,296,378$ \\
\hline 1994 & $59.6 \%$ & $0.4 \%$ & $32.4 \%$ & $2.6 \%$ & $4.6 \%$ & $0.3 \%$ & $2,357,588$ \\
\hline 1995 & $59.4 \%$ & $0.4 \%$ & $32.6 \%$ & $2.6 \%$ & $4.8 \%$ & $0.3 \%$ & $2,422,696$ \\
\hline 1996 & $59.1 \%$ & $0.4 \%$ & $32.8 \%$ & $2.6 \%$ & $4.8 \%$ & $0.3 \%$ & $2,485,848$ \\
\hline 1997 & $58.7 \%$ & $0.4 \%$ & $33.2 \%$ & $2.6 \%$ & $4.9 \%$ & $0.3 \%$ & $2,561,695$ \\
\hline 1998 & $58.9 \%$ & $0.4 \%$ & $33.0 \%$ & $2.6 \%$ & $4.9 \%$ & $0.3 \%$ & $2,631,522$ \\
\hline 1999 & $58.3 \%$ & $0.4 \%$ & $33.5 \%$ & $2.6 \%$ & $4.9 \%$ & $0.3 \%$ & $2,691,056$ \\
\hline 2000 & $58.3 \%$ & $0.4 \%$ & $33.6 \%$ & $2.6 \%$ & $4.9 \%$ & $0.3 \%$ & $2,746,925$ \\
\hline 2001 & $58.2 \%$ & $0.3 \%$ & $33.7 \%$ & $2.6 \%$ & $4.9 \%$ & $0.3 \%$ & $2,781,462$ \\
\hline \multicolumn{8}{|c|}{ Average annual percentage change } \\
\hline 1970-2001 & & & & & & & $3.0 \%$ \\
\hline 1991-2001 & & & & & & & $2.5 \%$ \\
\hline
\end{tabular}

Source:

U.S. Department of Transportation, Federal Highway Administration, Highway Statistics 2001, Washington, DC, 2002, Table VM-1, p. V-57, and annual. (Additional resources: www.fhwa.dot.gov)

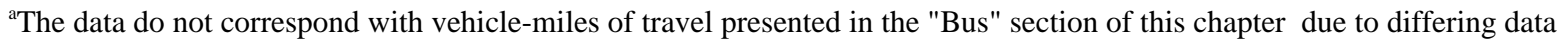
sources. 
Table 3.6

Automobiles in Operation and Vehicle Travel by Age, 1970 and 2001

\begin{tabular}{|c|c|c|c|c|c|c|c|c|c|}
\hline \multirow[b]{2}{*}{$\begin{array}{c}\text { Age } \\
\text { (years) }\end{array}$} & \multicolumn{3}{|c|}{1970} & \multicolumn{3}{|c|}{2001} & \multicolumn{2}{|c|}{$\begin{array}{c}2001 \text { Estimated } \\
\text { vehicle travel }\end{array}$} & \multirow{2}{*}{$\begin{array}{c}\text { Average } \\
\text { annual miles } \\
\text { per vehicle } \\
\end{array}$} \\
\hline & $\begin{array}{c}\text { Vehicles } \\
\text { (thousands) }\end{array}$ & Percentage & $\begin{array}{l}\text { Cumulative } \\
\text { percentage }\end{array}$ & $\begin{array}{c}\text { Vehicles } \\
\text { (thousands) }\end{array}$ & Percentage & $\begin{array}{l}\text { Cumulative } \\
\text { percentage }\end{array}$ & Percentage & $\begin{array}{l}\text { Cumulative } \\
\text { percentage }\end{array}$ & \\
\hline Under $\mathbf{1}^{\mathrm{a}}$ & 6,288 & $7.8 \%$ & $7.8 \%$ & 6,183 & $4.8 \%$ & $4.8 \%$ & $6.9 \%$ & $6.9 \%$ & 15,000 \\
\hline 1 & 9,299 & $11.6 \%$ & $19.4 \%$ & 8,882 & $6.9 \%$ & $11.7 \%$ & $9.4 \%$ & $16.3 \%$ & 14,300 \\
\hline 2 & 8,816 & $11.0 \%$ & $30.3 \%$ & 8,093 & $6.3 \%$ & $18.0 \%$ & $8.2 \%$ & $24.6 \%$ & 13,700 \\
\hline 3 & 7,878 & $9.8 \%$ & $40.1 \%$ & 7,555 & $5.9 \%$ & $23.9 \%$ & $7.2 \%$ & $31.8 \%$ & 12,900 \\
\hline 4 & 8,538 & $10.6 \%$ & $50.8 \%$ & 7,860 & $6.1 \%$ & $30.0 \%$ & $7.2 \%$ & $39.1 \%$ & 12,400 \\
\hline 5 & 8,506 & $10.6 \%$ & $61.3 \%$ & 7,337 & $5.7 \%$ & $35.7 \%$ & $6.5 \%$ & $45.6 \%$ & 12,000 \\
\hline 6 & 7,116 & $8.8 \%$ & $70.2 \%$ & 8,555 & $6.6 \%$ & $42.3 \%$ & $7.4 \%$ & $53.1 \%$ & 11,700 \\
\hline 7 & 6,268 & $7.8 \%$ & $78.0 \%$ & 7,471 & $5.8 \%$ & $48.1 \%$ & $6.3 \%$ & $59.4 \%$ & 11,400 \\
\hline 8 & 5,058 & $6.3 \%$ & $84.3 \%$ & 7,420 & $5.8 \%$ & $53.9 \%$ & $6.1 \%$ & $65.5 \%$ & 11,100 \\
\hline 9 & 3,267 & $4.1 \%$ & $88.3 \%$ & 6,807 & $5.3 \%$ & $59.2 \%$ & $5.4 \%$ & $71.0 \%$ & 10,700 \\
\hline 10 & 2,776 & $3.5 \%$ & $91.8 \%$ & 6,810 & $5.3 \%$ & $64.5 \%$ & $5.0 \%$ & $76.0 \%$ & 9,900 \\
\hline 11 & 1,692 & $2.1 \%$ & $93.9 \%$ & 6,692 & $5.2 \%$ & $69.7 \%$ & $4.5 \%$ & $80.5 \%$ & 9,000 \\
\hline 12 & 799 & $1.0 \%$ & $94.9 \%$ & 6,742 & $5.2 \%$ & $74.9 \%$ & $4.7 \%$ & $85.2 \%$ & 9,400 \\
\hline 13 & 996 & $1.2 \%$ & $96.1 \%$ & 6,189 & $4.8 \%$ & $79.7 \%$ & $3.8 \%$ & $88.9 \%$ & 8,200 \\
\hline 14 & 794 & $1.0 \%$ & $97.1 \%$ & 5,345 & $4.2 \%$ & $83.9 \%$ & $2.9 \%$ & $91.8 \%$ & 7,200 \\
\hline \multirow{2}{*}{$\begin{array}{l}15 \text { and older } \\
\text { Subtotal }\end{array}$} & 2,336 & $2.9 \%$ & $100.0 \%$ & 20,773 & $16.1 \%$ & $100.0 \%$ & $8.2 \%$ & $100.0 \%$ & 5,300 \\
\hline & 80,427 & $100.0 \%$ & & 128,714 & $100.0 \%$ & & & & \\
\hline \multirow{2}{*}{$\begin{array}{l}\text { Age not given } \\
\text { Total }\end{array}$} & 22 & & & $\mathbf{0}$ & & & & & \\
\hline & 80,449 & & & 128,714 & & & & & \\
\hline $\begin{array}{l}\text { Average age } \\
\text { Median age }\end{array}$ & & $\begin{array}{l}5.6 \\
4.9\end{array}$ & & & $\begin{array}{l}9.0 \\
8.1\end{array}$ & & & & \\
\hline
\end{tabular}

Source:

The Polk Company, Detroit, MI. FURTHER REPRODUCTION PROHIBITED.

Vehicle travel - Average annual miles per auto by age were multiplied by the number of vehicles in operation by age to estimate the vehicle travel. Average annual miles per auto by age - generated by ORNL from the National Household Travel Survey website: nhts.ornl.gov.

(Additional resources: www.polk.com, nhts.ornl.gov)

ancludes automobiles from model year 2002 and 2001 which were sold prior to July 1, 2002, and similarly, model years 1971 and 1970 sold prior to July 1 , 1970. 
Table 3.7

Trucks in Operation and Vehicle Travel by Age, 1970 and 2001

\begin{tabular}{|c|c|c|c|c|c|c|c|c|c|}
\hline \multirow[b]{2}{*}{$\begin{array}{c}\text { Age } \\
\text { (years) }\end{array}$} & \multicolumn{3}{|c|}{1970} & \multicolumn{3}{|c|}{2001} & \multicolumn{2}{|c|}{$\begin{array}{c}2001 \text { Estimated } \\
\text { vehicle travel }\end{array}$} & \multirow{2}{*}{$\begin{array}{c}\text { Average annual } \\
\text { miles per } \\
\text { vehicle }\end{array}$} \\
\hline & $\begin{array}{c}\text { Vehicles } \\
\text { (thousands } \\
\text { ) }\end{array}$ & $\begin{array}{c}\text { Percentag } \\
\text { e } \\
\end{array}$ & $\begin{array}{l}\text { Cumulative } \\
\text { percentage }\end{array}$ & $\begin{array}{c}\text { Vehicles } \\
\text { (thousands) }\end{array}$ & $\begin{array}{c}\text { Percentag } \\
\text { e } \\
\end{array}$ & $\begin{array}{l}\text { Cumulative } \\
\text { percentage }\end{array}$ & $\begin{array}{c}\text { Percentag } \\
\text { e } \\
\end{array}$ & $\begin{array}{l}\text { Cumulative } \\
\text { percentage }\end{array}$ & \\
\hline Under $1^{\mathrm{a}}$ & 1,262 & $7.1 \%$ & $7.1 \%$ & 6,213 & $7.1 \%$ & $7.1 \%$ & $8.5 \%$ & $8.5 \%$ & 17,500 \\
\hline 1 & 1,881 & $10.6 \%$ & $17.8 \%$ & 7,958 & $9.0 \%$ & $16.1 \%$ & $12.0 \%$ & $20.6 \%$ & 19,200 \\
\hline 2 & 1,536 & $8.7 \%$ & $26.5 \%$ & 7,522 & $8.6 \%$ & $24.7 \%$ & $11.7 \%$ & $32.3 \%$ & 19,800 \\
\hline 3 & 1,428 & $8.1 \%$ & $34.6 \%$ & 6,398 & $7.3 \%$ & $31.9 \%$ & $9.0 \%$ & $41.3 \%$ & 17,900 \\
\hline 4 & 1,483 & $8.4 \%$ & $43.0 \%$ & 6,109 & $6.9 \%$ & $38.9 \%$ & $8.4 \%$ & $49.7 \%$ & 17,500 \\
\hline 5 & 1,339 & $7.6 \%$ & $50.5 \%$ & 5,122 & $5.8 \%$ & $44.7 \%$ & $6.8 \%$ & $56.6 \%$ & 17,000 \\
\hline 6 & 1,154 & $6.5 \%$ & $57.1 \%$ & 5,574 & $6.3 \%$ & $51.0 \%$ & $6.8 \%$ & $63.4 \%$ & 15,600 \\
\hline 7 & 975 & $5.5 \%$ & $62.6 \%$ & 5,042 & $5.7 \%$ & $56.8 \%$ & $6.1 \%$ & $69.5 \%$ & 15,400 \\
\hline 8 & 826 & $4.7 \%$ & $67.3 \%$ & 4,148 & $4.7 \%$ & $61.5 \%$ & $4.9 \%$ & $74.4 \%$ & 15,100 \\
\hline 9 & 621 & $3.5 \%$ & $70.8 \%$ & 3,395 & $3.9 \%$ & $65.3 \%$ & $3.5 \%$ & $77.9 \%$ & 13,200 \\
\hline 10 & 658 & $3.7 \%$ & $74.5 \%$ & 3,221 & $3.7 \%$ & $69.0 \%$ & $2.3 \%$ & $80.3 \%$ & 9,200 \\
\hline 11 & 583 & $3.3 \%$ & $77.8 \%$ & 3,039 & $3.5 \%$ & $72.5 \%$ & $2.2 \%$ & $82.5 \%$ & 9,200 \\
\hline 12 & 383 & $2.2 \%$ & $80.0 \%$ & 3,345 & $3.8 \%$ & $76.3 \%$ & $2.4 \%$ & $84.9 \%$ & 9,200 \\
\hline 13 & 417 & $2.4 \%$ & $82.3 \%$ & 3,112 & $3.5 \%$ & $79.8 \%$ & $2.3 \%$ & $89.1 \%$ & 9,200 \\
\hline 14 & 414 & $2.3 \%$ & $84.7 \%$ & 2,544 & $2.9 \%$ & $82.7 \%$ & $1.8 \%$ & $89.0 \%$ & 9,200 \\
\hline 15 and older & 2,710 & $15.3 \%$ & $100.0 \%$ & 15,227 & $17.3 \%$ & $100.0 \%$ & $11.0 \%$ & $100.0 \%$ & 9,200 \\
\hline Subtotal & 17,670 & $100.0 \%$ & & 87,969 & $100.0 \%$ & & $100.0 \%$ & & \\
\hline Age not given & 15 & & & 0 & & & & & \\
\hline Total & 17,685 & & & 87,969 & & & & & \\
\hline Average age & & 7.3 & & & 7.9 & & & & \\
\hline Median age & & 5.9 & & & 6.8 & & & & \\
\hline
\end{tabular}

Source:

The Polk Company, Detroit, MI. FURTHER REPRODUCTION PROHIBITED.

Vehicle travel-The average annual vehicle-miles per truck by age were multiplied by the number of trucks in operation by age to estimate the vehicle travel. Average annual miles per truck by age were generated by ORNL from the 1997 Truck Inventory and Use Survey public use tape provided by U.S. Department of Commerce, Bureau of the Census, Washington, DC, 2000. (Additional resources: www.polk.com, www.census.gov)

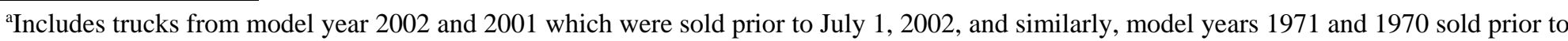
July 1,1970 
The average age of automobiles was lower than the average age of trucks until 1995. Since then, the average automobile age continues to grow, while the average truck age has held about the same. The increasing popularity of light trucks as personal passenger vehicles may have had an influence on the average age of trucks.

Table 3.8

Average Age of Automobiles and Trucks in Use, 1970-2001

(years)

\begin{tabular}{|c|c|c|c|c|}
\hline \multirow{2}{*}{$\begin{array}{c}\text { Calendar } \\
\text { year }\end{array}$} & \multicolumn{2}{|c|}{ Automobiles } & \multicolumn{2}{|c|}{ Trucks } \\
\hline & Mean $^{a}$ & Median $^{b}$ & Mean $^{\mathrm{a}}$ & Median $^{\mathrm{b}}$ \\
\hline 1970 & 5.6 & 4.9 & 7.3 & 5.9 \\
\hline 1971 & 5.7 & 5.1 & 7.4 & 6.1 \\
\hline 1972 & 5.7 & 5.1 & 7.2 & 6.0 \\
\hline 1973 & 5.7 & 5.1 & 6.9 & 5.8 \\
\hline 1974 & 5.7 & 5.2 & 7.0 & 5.6 \\
\hline 1975 & 6.0 & 5.4 & 6.9 & 5.8 \\
\hline 1976 & 6.2 & 5.5 & 7.0 & 5.8 \\
\hline 1977 & 6.2 & 5.6 & 6.9 & 5.7 \\
\hline 1978 & 6.3 & 5.7 & 6.9 & 5.8 \\
\hline 1979 & 6.4 & 5.9 & 6.9 & 5.9 \\
\hline 1980 & 6.6 & 6.0 & 7.1 & 6.3 \\
\hline 1981 & 6.9 & 6.0 & 7.5 & 6.5 \\
\hline 1982 & 7.2 & 6.2 & 7.8 & 6.8 \\
\hline 1983 & 7.4 & 6.5 & 8.1 & 7.2 \\
\hline 1984 & 7.5 & 6.7 & 8.2 & 7.4 \\
\hline 1985 & 7.6 & 6.9 & 8.1 & 7.6 \\
\hline 1986 & 7.6 & 7.0 & 8.0 & 7.7 \\
\hline 1987 & 7.6 & 6.9 & 8.0 & 7.8 \\
\hline 1988 & 7.6 & 6.8 & 7.9 & 7.1 \\
\hline 1989 & 7.6 & 6.5 & 7.9 & 6.7 \\
\hline 1990 & 7.6 & 6.5 & 8.0 & 6.5 \\
\hline 1991 & 7.8 & 6.7 & 8.1 & 6.8 \\
\hline 1992 & 7.9 & 7.0 & 8.4 & 7.2 \\
\hline 1993 & 8.1 & 7.3 & 8.6 & 7.5 \\
\hline 1994 & 8.3 & 7.5 & 8.4 & 7.5 \\
\hline 1995 & 8.4 & 7.7 & 8.4 & 7.6 \\
\hline 1996 & 8.5 & 7.9 & 8.3 & 7.7 \\
\hline 1997 & 8.6 & 8.1 & 8.3 & 7.8 \\
\hline 1998 & 8.8 & 8.3 & 8.3 & 7.5 \\
\hline 1999 & 8.9 & 8.3 & 8.2 & 7.2 \\
\hline 2000 & 9.0 & 8.1 & 8.0 & 6.9 \\
\hline 2001 & 9.0 & 8.1 & 7.9 & 6.8 \\
\hline
\end{tabular}

Source:

The Polk Company, Detroit, MI. FURTHER REPRODUCTION PROHIBITED.

(Additional resources: www.polk.com)

${ }^{\mathrm{a}}$ Mean is the sum of the products of units multiplied by age, divided by the total units.

${ }^{\mathrm{b}}$ Median is a value in an ordered set of values below and above which there are an equal number of values. 
The average age of trucks (classes 1-8) has historically been higher than the average age of automobiles. In 1995, however, this trend reversed, with average automobile age higher than average truck age for the first time. The recent boom in the sales of minivans, sport-utility vehicles, and pickups, which are classified as trucks, is influencing the average age of trucks. So many new light trucks are being added into the truck population, that the average age of trucks has been declining since 1993, while the average age of automobiles continues an upward trend.

Figure 3.2. Average Age and Registrations of Automobiles and Trucks, 1970-2001

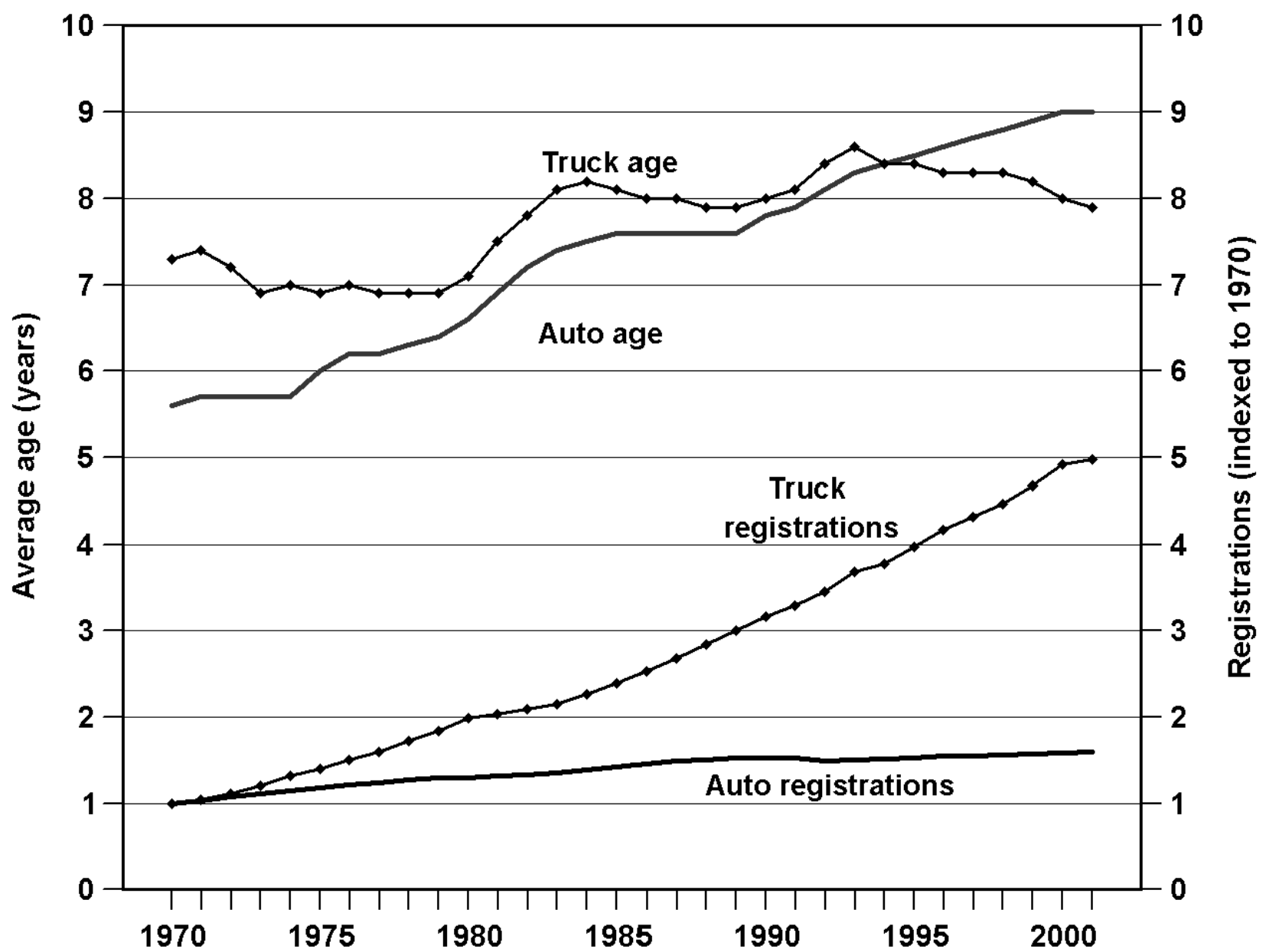

Source:

See Tables 3.3 and 3.8 . 
Using current registration data and a scrappage model by Greenspan and Cohen, [1996 paper:

http://www.federalreserve.gov/pubs/feds/1996/199640/199640pap.pdf], ORNL calculated new automobile scrappage rates. The expected median lifetime for a 1990 model year automobile is 16.9 years. These data are fitted model values which assume constant economic conditions.

Table 3.9

Automobile Scrappage and Survival Rates 1970, 1980 and 1990 Model Years

\begin{tabular}{|c|c|c|c|c|c|c|}
\hline \multirow{2}{*}{$\begin{array}{c}\text { Vehicle } \\
\text { age }^{a} \\
\text { (years) }\end{array}$} & \multicolumn{2}{|c|}{1970 model year } & \multicolumn{2}{|c|}{1980 model year } & \multicolumn{2}{|c|}{1990 model year } \\
\hline & $\begin{array}{c}\text { Survival } \\
\text { rate }^{\mathrm{b}}\end{array}$ & $\begin{array}{l}\text { Scrappag } \\
\text { e rate }^{c}\end{array}$ & $\begin{array}{c}\text { Survival } \\
\text { rate }^{\mathbf{b}}\end{array}$ & $\begin{array}{l}\text { Scrappag } \\
\text { e rate }^{c}\end{array}$ & $\begin{array}{c}\text { Survival } \\
\text { rate }^{\mathrm{b}}\end{array}$ & $\begin{array}{l}\text { Scrappag } \\
\text { e rate }^{c}\end{array}$ \\
\hline 4 & 99.0 & 1.0 & 100.0 & 0.0 & 100.0 & 0.0 \\
\hline 5 & 94.1 & 5.0 & 96.3 & 3.7 & 100.0 & 0.0 \\
\hline 6 & 88.4 & 6.1 & 91.3 & 5.1 & 99.4 & 0.6 \\
\hline 7 & 82.0 & 7.2 & 85.7 & 6.1 & 96.3 & 3.2 \\
\hline 8 & 75.2 & 8.3 & 79.7 & 7.1 & 92.7 & 3.7 \\
\hline 9 & 68.1 & 9.5 & 73.3 & 8.1 & 88.7 & 4.3 \\
\hline 10 & 60.9 & 10.6 & 66.6 & 9.0 & 84.4 & 4.9 \\
\hline 11 & 53.8 & 11.7 & 60.0 & 10.0 & 79.8 & 5.5 \\
\hline 12 & 46.9 & 12.8 & 53.3 & 11.0 & 75.0 & 6.1 \\
\hline 13 & 40.3 & 14.0 & 46.9 & 12.0 & 70.0 & 6.7 \\
\hline 14 & 34.2 & 15.1 & 40.8 & 13.0 & 64.9 & 7.3 \\
\hline 15 & 28.7 & 16.2 & 35.1 & 14.0 & 59.7 & 7.9 \\
\hline 16 & 23.7 & 17.4 & 29.8 & 15.0 & 54.6 & 8.6 \\
\hline 17 & 19.3 & 18.5 & 25.0 & 16.1 & 49.5 & 9.3 \\
\hline 18 & 15.5 & 19.6 & 20.8 & 17.1 & 44.6 & 9.9 \\
\hline 19 & 12.3 & 20.8 & 17.0 & 18.1 & 39.9 & 10.6 \\
\hline 20 & 9.6 & 21.9 & 13.8 & 19.1 & 35.4 & 11.3 \\
\hline 21 & 7.4 & 23.0 & 11.0 & 20.1 & 31.1 & 12.0 \\
\hline 22 & 5.6 & 24.2 & 8.7 & 21.2 & 27.2 & 12.7 \\
\hline 23 & 4.2 & 25.3 & 6.7 & 22.2 & 23.5 & 13.5 \\
\hline 24 & 3.1 & 26.4 & 5.2 & 23.2 & 20.2 & 14.2 \\
\hline 25 & 2.2 & 27.5 & 3.9 & 24.2 & 17.1 & 15.0 \\
\hline 26 & 1.6 & 28.6 & 2.9 & 25.3 & 14.5 & 15.7 \\
\hline 27 & 1.1 & 29.7 & 2.2 & 26.3 & 12.1 & 16.5 \\
\hline 28 & 0.8 & 30.8 & 1.6 & 27.3 & 10.0 & 17.2 \\
\hline 29 & 0.5 & 31.9 & 1.1 & 28.4 & 8.2 & 18.0 \\
\hline 30 & 0.4 & 33.0 & 0.8 & 29.4 & 6.6 & 18.8 \\
\hline $\begin{array}{l}\text { Median } \\
\text { lifetime }\end{array}$ & \multicolumn{2}{|c|}{11.5 years } & \multicolumn{2}{|c|}{12.5 years } & \multicolumn{2}{|c|}{16.9 years } \\
\hline
\end{tabular}

Source:

Schmoyer, Richard L., unpublished study on scrappage rates, Oak Ridge National Laboratory, Oak Ridge, TN, 2001.

${ }^{\text {a It }}$ was assumed that scrappage for vehicles less than 4 years old is 0 .

${ }^{b}$ The percentage of automobiles which will be in use at the end of the year.

'The percentage of automobiles which will be retired from use during the year. 
Figure 3.3. Automobile Survival Rates

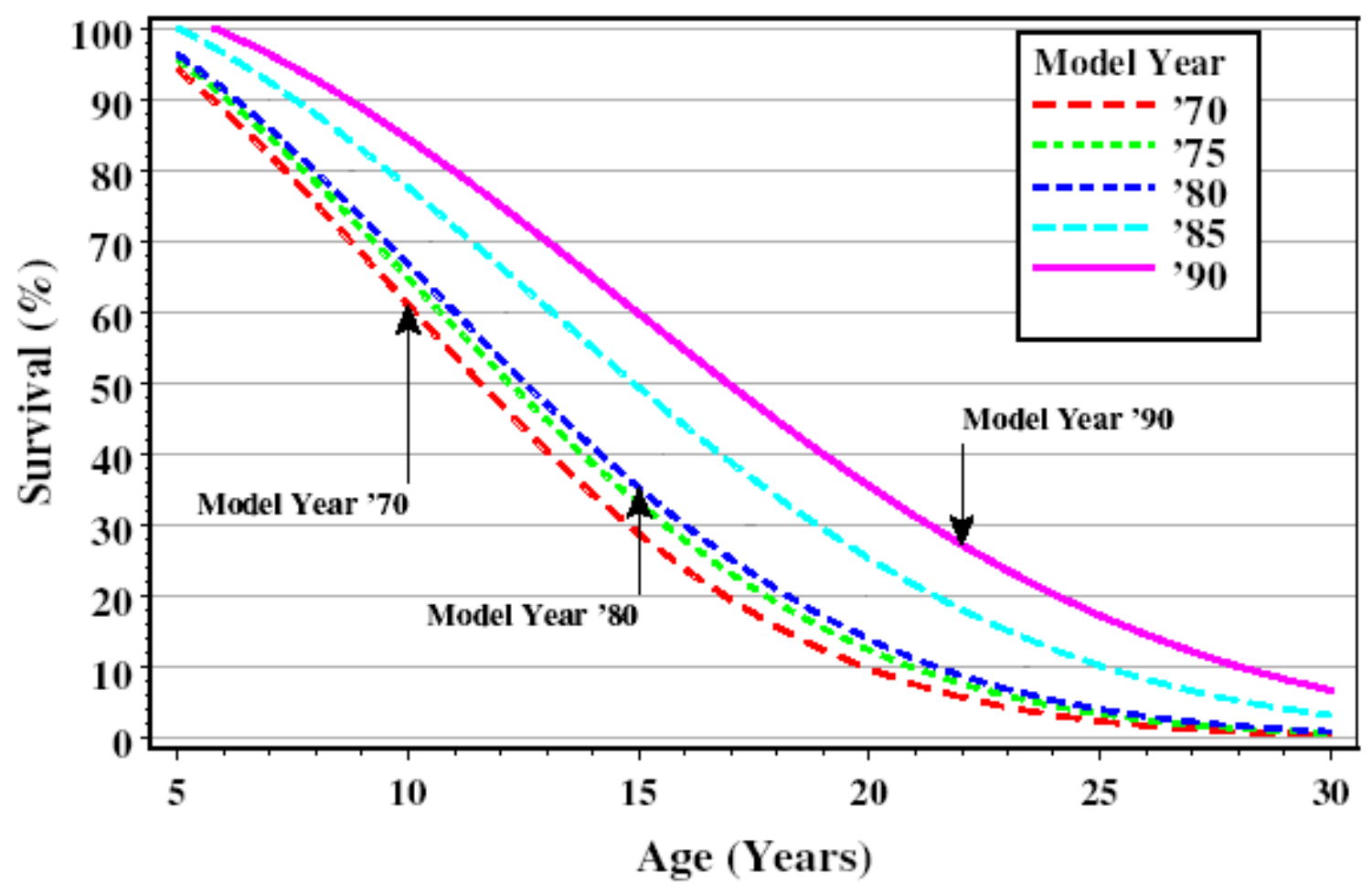

Source: See Table 3.9. 
Using current registration data and a scrappage model by Greenspan and Cohen [1996 paper:

http://www.federalreserve.gov/pubs/feds/1996/199640/199640pap.pdf], ORNL calculated new light truck scrappage rates. The expected median lifetime for a 1990 model year light truck is 15.5 years. These data are fitted model values which assume constant economic conditions.

Table 3.10

Light Truck ${ }^{\mathrm{a}}$ Scrappage and Survival Rates

\begin{tabular}{|c|c|c|c|c|c|c|}
\hline \multirow{2}{*}{$\begin{array}{c}\text { Vehicle } \\
\text { age }^{\mathrm{b}} \\
\text { (years) }\end{array}$} & \multicolumn{2}{|c|}{1970 model year } & \multicolumn{2}{|c|}{1980 model year } & \multicolumn{2}{|c|}{1990 model year } \\
\hline & $\begin{array}{c}\text { Survival } \\
\text { rate }^{c}\end{array}$ & $\begin{array}{c}\text { Scrappag } \\
\text { e rate }^{\mathrm{d}}\end{array}$ & $\begin{array}{c}\text { Survival } \\
\text { rate }^{\mathbf{b}}\end{array}$ & $\begin{array}{c}\text { Scrappag } \\
\text { e rate }^{\mathrm{c}}\end{array}$ & $\begin{array}{c}\text { Survival } \\
\text { rate }^{\mathbf{b}}\end{array}$ & $\begin{array}{c}\text { Scrappag } \\
\text { e rate }^{\mathrm{c}}\end{array}$ \\
\hline 4 & 99.7 & 0.3 & 99.1 & 0.9 & 99.3 & 0.7 \\
\hline 5 & 97.5 & 2.2 & 96.6 & 2.5 & 96.9 & 2.4 \\
\hline 6 & 94.9 & 2.7 & 93.7 & 3.1 & 94.1 & 3.0 \\
\hline 7 & 91.8 & 3.2 & 90.2 & 3.7 & 90.7 & 3.6 \\
\hline 8 & 88.3 & 3.8 & 86.3 & 4.3 & 86.9 & 4.2 \\
\hline 9 & 84.4 & 4.4 & 82.0 & 5.0 & 82.7 & 4.8 \\
\hline 10 & 80.2 & 5.0 & 77.3 & 5.7 & 78.2 & 5.5 \\
\hline 11 & 75.7 & 5.6 & 72.4 & 6.4 & 73.4 & 6.1 \\
\hline 12 & 70.9 & 6.3 & 67.3 & 7.1 & 68.4 & 6.8 \\
\hline 13 & 66.0 & 6.9 & 62.1 & 7.8 & 63.3 & 7.5 \\
\hline 14 & 61.0 & 7.6 & 56.8 & 8.5 & 58.0 & 8.2 \\
\hline 15 & 55.9 & 8.3 & 51.5 & 9.3 & 52.8 & 9.0 \\
\hline 16 & 50.8 & 9.0 & 46.3 & 10.1 & 47.7 & 9.7 \\
\hline 17 & 45.9 & 9.8 & 41.3 & 10.8 & 42.7 & 10.5 \\
\hline 18 & 41.1 & 10.5 & 36.5 & 11.6 & 37.9 & 11.3 \\
\hline 19 & 36.4 & 11.3 & 32.0 & 12.4 & 33.3 & 12.1 \\
\hline 20 & 32.1 & 12.0 & 27.7 & 13.3 & 29.0 & 12.9 \\
\hline 21 & 28.0 & 12.8 & 23.8 & 14.1 & 25.0 & 13.7 \\
\hline 22 & 24.2 & 13.6 & 20.3 & 14.9 & 21.4 & 14.5 \\
\hline 23 & 20.7 & 14.4 & 17.1 & 15.8 & 18.1 & 15.4 \\
\hline 24 & 17.5 & 15.2 & 14.2 & 16.7 & 15.2 & 16.2 \\
\hline 25 & 14.7 & 16.1 & 11.7 & 17.5 & 12.6 & 17.1 \\
\hline 26 & 12.2 & 16.9 & 9.6 & 18.4 & 10.3 & 18.0 \\
\hline 27 & 10.1 & 17.8 & 7.7 & 19.3 & 8.4 & 18.8 \\
\hline 28 & 8.2 & 18.6 & 6.2 & 20.2 & 6.7 & 19.7 \\
\hline 29 & 6.6 & 19.5 & 4.9 & 21.1 & 5.3 & 20.6 \\
\hline 30 & 5.2 & 20.4 & 3.8 & 22.1 & 4.2 & 21.5 \\
\hline $\begin{array}{l}\text { Median } \\
\text { lifetime }\end{array}$ & \multicolumn{2}{|c|}{16.2 years } & \multicolumn{2}{|c|}{15.3 years } & \multicolumn{2}{|c|}{15.5 years } \\
\hline
\end{tabular}

Source:

Schmoyer, Richard L., unpublished study on scrappage rates, Oak Ridge National Laboratory, Oak Ridge, TN, 2001.

${ }^{a}$ Light trucks are trucks less than 10,000 lbs. gross vehicle weight.

bIt was assumed that scrappage for vehicles less than 4 years old is 0 .

'The percentage of light trucks which will be in use at the end of the year.

${ }^{\mathrm{d}}$ The percentage of light trucks which will be retired from use during the year. 
Figure 3.4. Light Truck Survival Rates

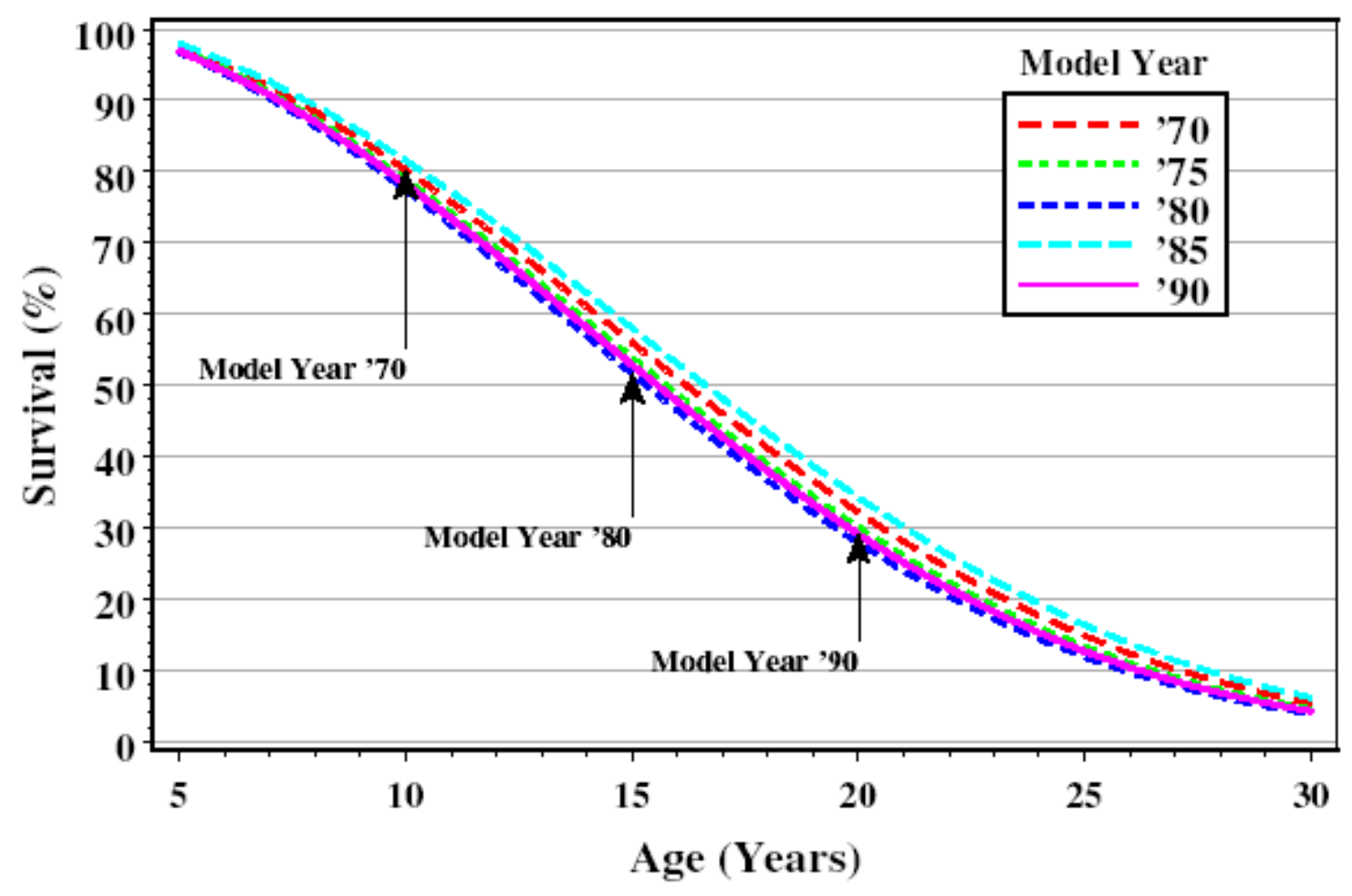

Source: See Table 3.10. 
Using current registration data and a scrappage model by Greenspan and Cohen [1996 paper:

http://www.federalreserve.gov/pubs/feds/1996/199640/199640pap.pdf], ORNL calculated heavy truck (trucks over 26,000 lbs. gross vehicle weight) scrappage rates. The expected median lifetime for a 1990 model year heavy truck is 29 years. These data are fitted model values which assume constant economic conditions.

Table 3.11

Heavy Truck ${ }^{\mathrm{a}}$ Scrappage and Survival Rates

\begin{tabular}{|c|c|c|c|c|c|c|}
\hline \multirow{2}{*}{$\begin{array}{c}\text { Vehicle } \\
\text { age }^{\mathrm{b}} \\
\text { (years) }\end{array}$} & \multicolumn{2}{|c|}{1970 model year } & \multicolumn{2}{|c|}{1980 model year } & \multicolumn{2}{|c|}{1990 model year } \\
\hline & $\begin{array}{c}\text { Survival } \\
\text { rate }^{\mathrm{c}}\end{array}$ & $\begin{array}{c}\text { Scrappag } \\
\text { e rate }^{\mathrm{d}}\end{array}$ & $\begin{array}{c}\text { Survival } \\
\text { rate }^{\mathbf{b}}\end{array}$ & $\begin{array}{c}\text { Scrappag } \\
\text { e rate }^{\mathrm{c}}\end{array}$ & $\begin{array}{c}\text { Survival } \\
\text { rate }^{\mathrm{b}}\end{array}$ & $\begin{array}{c}\text { Scrappag } \\
\text { e rate }^{\mathrm{c}}\end{array}$ \\
\hline 4 & 98.8 & 1.2 & 98.5 & 1.5 & 99.4 & 0.6 \\
\hline 5 & 97.2 & 1.6 & 96.7 & 1.9 & 98.6 & 0.8 \\
\hline 6 & 95.3 & 1.9 & 94.5 & 2.3 & 97.6 & 1.0 \\
\hline 7 & 93.2 & 2.3 & 92.0 & 2.7 & 96.5 & 1.2 \\
\hline 8 & 90.7 & 2.6 & 89.1 & 3.1 & 95.2 & 1.3 \\
\hline 9 & 88.1 & 3.0 & 86.0 & 3.5 & 93.8 & 1.5 \\
\hline 10 & 85.2 & 3.3 & 82.7 & 3.9 & 92.2 & 1.7 \\
\hline 11 & 82.1 & 3.6 & 79.1 & 4.3 & 90.5 & 1.9 \\
\hline 12 & 78.8 & 4.0 & 75.4 & 4.7 & 88.6 & 2.0 \\
\hline 13 & 75.4 & 4.3 & 71.6 & 5.1 & 86.7 & 2.2 \\
\hline 14 & 71.9 & 4.7 & 67.7 & 5.5 & 84.6 & 2.4 \\
\hline 15 & 68.3 & 5.0 & 63.7 & 5.9 & 82.4 & 2.6 \\
\hline 16 & 64.6 & 5.3 & 59.7 & 6.3 & 80.2 & 2.7 \\
\hline 17 & 61.0 & 5.7 & 55.7 & 6.7 & 77.9 & 2.9 \\
\hline 18 & 57.3 & 6.0 & 51.8 & 7.1 & 75.5 & 3.1 \\
\hline 19 & 53.7 & 6.3 & 47.9 & 7.4 & 73.0 & 3.3 \\
\hline 20 & 50.1 & 6.7 & 44.2 & 7.8 & 70.5 & 3.4 \\
\hline 21 & 46.6 & 7.0 & 40.6 & 8.2 & 68.0 & 3.6 \\
\hline 22 & 43.2 & 7.3 & 37.1 & 8.6 & 65.4 & 3.8 \\
\hline 23 & 39.9 & 7.6 & 33.7 & 9.0 & 62.8 & 3.9 \\
\hline 24 & 36.7 & 8.0 & 30.6 & 9.4 & 60.3 & 4.1 \\
\hline 25 & 33.7 & 8.3 & 27.6 & 9.7 & 57.7 & 4.3 \\
\hline 26 & 30.8 & 8.6 & 24.8 & 10.1 & 55.1 & 4.5 \\
\hline 27 & 28.0 & 8.9 & 22.2 & 10.5 & 52.6 & 4.6 \\
\hline 28 & 25.4 & 9.3 & 19.8 & 10.9 & 50.0 & 4.8 \\
\hline 29 & 23.0 & 9.6 & 17.6 & 11.2 & 47.6 & 5.0 \\
\hline 30 & 20.7 & 9.9 & 15.5 & 11.6 & 45.1 & 5.1 \\
\hline $\begin{array}{l}\text { Median } \\
\text { lifetime }\end{array}$ & \multicolumn{2}{|c|}{20.0 years } & \multicolumn{2}{|c|}{18.5 years } & \multicolumn{2}{|c|}{ 28.0 years } \\
\hline
\end{tabular}

Source:

Schmoyer, Richard L., unpublished study on scrappage rates, Oak Ridge National Laboratory, Oak Ridge, TN, 2001.

${ }^{a}$ Heavy trucks are trucks more than 26,000 lbs. gross vehicle weight.

bIt was assumed that scrappage for vehicles less than 4 years old is 0 .

'The percentage of heavy trucks which will be in use at the end of the year.

${ }^{\mathrm{d}}$ The percentage of heavy trucks which will be retired from use during the year. 
Figure 3.5. Heavy Truck Survival Rates

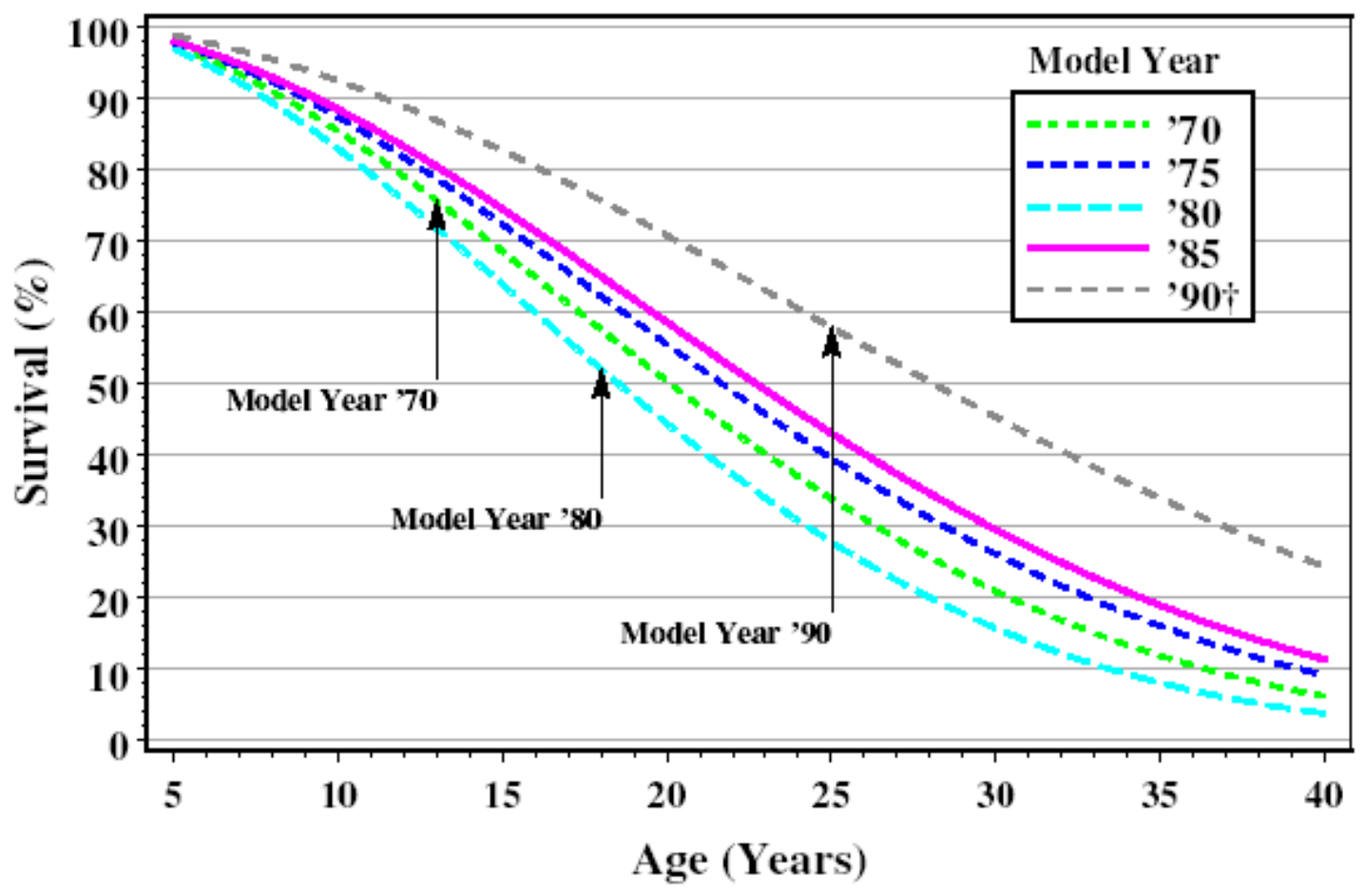

Source: See Table 3.11.

Model year ' 90 estimates are based on minimal preliminary data. 


\section{Chapter 4 \\ Light Vehicles and Characteristics}

\section{Summary Statistics from Tables in this Chapter}

\begin{tabular}{|c|c|c|}
\hline Source & & \\
\hline \multirow[t]{4}{*}{ Table 4.1} & Passenger cars, 2001 & \\
\hline & Registrations (thousands) & 137,633 \\
\hline & Vehicle miles (million miles) & $1,600,287$ \\
\hline & Fuel economy (miles per gallon) & 21.9 \\
\hline \multirow[t]{4}{*}{ Table 4.2} & Two-axle, four-tire trucks, 2001 & \\
\hline & Registrations (thousands) & 84,188 \\
\hline & Vehicle miles (million miles) & 937,839 \\
\hline & Fuel economy (miles per gallon) & 17.6 \\
\hline \multirow[t]{3}{*}{ Table 4.5} & Light truck share of total light vehicle sales & \\
\hline & 1970 calendar year & $14.8 \%$ \\
\hline & 2001 calendar year & $50.5 \%$ \\
\hline \multirow[t]{7}{*}{ Table 4.7} & Automobile sales, 2002 sales period & $8,336,459$ \\
\hline & Minicompact & 53,840 \\
\hline & Subcompact & 636,397 \\
\hline & Compact & $3,217,151$ \\
\hline & Midsize & 2,917,527 \\
\hline & Large & $1,377,357$ \\
\hline & Two-seater & 134,187 \\
\hline \multirow[t]{8}{*}{ Table 4.8} & Light truck sales, 2002 sales period & $8,673,079$ \\
\hline & Small pickup & 761,802 \\
\hline & Large pickup & $2,209,671$ \\
\hline & Small van & $1,165,202$ \\
\hline & Large van & 349,706 \\
\hline & Small SUV & 877,777 \\
\hline & Medium SUV & $2,448,269$ \\
\hline & Large SUV & 860,652 \\
\hline Tables 4.18 & Corporate average fuel economy & (mpg) \\
\hline \multirow[t]{4}{*}{ and 4.19} & Automobile standard, MY 2002 & 27.5 \\
\hline & Automobile fuel economy, MY 2002 & 29.5 \\
\hline & Light truck standard, MY 2002 & 20.7 \\
\hline & Light truck fuel economy, MY 2002 & 21.8 \\
\hline Table 4.24 & Average fuel economy loss from 55 to $70 \mathrm{mph}$ & $17.1 \%$ \\
\hline
\end{tabular}


The Federal Highway Administration released revised historical data back to 1985 in their "Highway Statistics Summary to 1995" report. As a result, the data in this table have been revised. The data in this table from 1985-on DO NOT include minivans, pickups, or sport utility vehicles.

Table 4.1

Summary Statistics for Passenger Cars, 1970-2001

\begin{tabular}{|c|c|c|c|c|}
\hline Year & $\begin{array}{l}\text { Registrations }{ }^{\mathrm{a}} \\
\text { (thousands) }\end{array}$ & $\begin{array}{c}\text { Vehicle travel } \\
\text { (million miles) }\end{array}$ & $\begin{array}{c}\text { Fuel use } \\
\text { (million gallons) }\end{array}$ & $\begin{array}{l}\text { Fuel economy } \\
\text { (miles per gallon) }\end{array}$ \\
\hline 1970 & 89,244 & 916,700 & 67,820 & 13.5 \\
\hline 1971 & 92,718 & 966,330 & 71,346 & 13.5 \\
\hline 1972 & 97,082 & $1,021,365$ & 75,937 & 13.5 \\
\hline 1973 & 101,985 & $1,045,981$ & 78,233 & 13.4 \\
\hline 1974 & 104,856 & $1,007,251$ & 74,229 & 13.6 \\
\hline 1975 & 106,706 & $1,033,950$ & 74,140 & 13.9 \\
\hline 1976 & 110,189 & $1,078,215$ & 78,297 & 13.8 \\
\hline 1977 & 112,288 & $1,109,243$ & 79,060 & 14.0 \\
\hline 1978 & 116,573 & $1,146,508$ & 80,652 & 14.2 \\
\hline 1979 & 118,429 & $1,113,640$ & 76,588 & 14.5 \\
\hline 1980 & 121,601 & $1,111,596$ & 69,981 & 15.9 \\
\hline 1981 & 123,098 & $1,133,332$ & 69,112 & 16.4 \\
\hline 1982 & 123,702 & $1,161,713$ & 69,116 & 16.8 \\
\hline 1983 & 126,444 & $1,195,054$ & 70,322 & 17.0 \\
\hline 1984 & 128,158 & $1,227,043$ & 70,663 & 17.4 \\
\hline $1985^{c}$ & 127,885 & $1,246,798$ & 71,518 & 17.4 \\
\hline 1986 & 130,004 & $1,270,167$ & 73,174 & 17.4 \\
\hline 1987 & 131,482 & $1,315,982$ & 73,308 & 18.0 \\
\hline 1988 & 133,836 & $1,370,271$ & 73,345 & 18.7 \\
\hline 1989 & 134,559 & $1,401,221$ & 73,913 & 19.0 \\
\hline 1990 & 133,700 & $1,408,266$ & 69,568 & 20.2 \\
\hline 1991 & 128,300 & $1,358,185$ & 64,318 & 21.1 \\
\hline 1992 & 126,581 & $1,371,569$ & 65,436 & 21.0 \\
\hline 1993 & 127,327 & $1,374,709$ & 67,047 & 20.5 \\
\hline 1994 & 127,883 & $1,406,089$ & 67,874 & 20.7 \\
\hline 1995 & 128,387 & $1,438,294$ & 68,072 & 21.1 \\
\hline 1996 & 129,728 & $1,469,854$ & 69,221 & 21.2 \\
\hline 1997 & 129,749 & $1,502,556$ & 69,892 & 21.5 \\
\hline 1998 & 131,839 & $1,549,577$ & 71,695 & 21.4 \\
\hline 1999 & 132,432 & $1,569,100$ & 73,283 & 21.4 \\
\hline 2000 & 133,621 & $1,600,287$ & 73,065 & 21.9 \\
\hline 2001 & 137,633 & $1,619,422$ & 73,261 & 22.1 \\
\hline \multicolumn{5}{|c|}{ Average annual percentage change } \\
\hline 1970-2001 & $1.4 \%$ & $1.9 \%$ & $0.2 \%$ & $1.6 \%$ \\
\hline 1991-2001 & $0.7 \%$ & $1.8 \%$ & $1.3 \%$ & $0.5 \%$ \\
\hline
\end{tabular}

Source:

U.S. Department of Transportation, Federal Highway Administration, Highway Statistics 2001, Washington, DC, 2002, Table VM-1, p. V-57, and annual.

(Additional resources: www.fhwa.dot.gov)

${ }^{a}$ This number differs from R.L. Polk’s estimates of “number of automobiles in use.” See Table 3.3.

${ }^{\mathrm{b}}$ Fuel economy for automobile population.

${ }^{\mathrm{c}}$ Beginning in this year the data were revised to exclude minivans, pickups and sport utility vehicles which may have been previously included. 
The Federal Highway Administration released revised historical data back to 1985 which better reflected two-axle, four-tire trucks. The definition of this category includes vans, pickup trucks, and sport utility vehicles.

Table 4.2 Summary Statistics for Two-Axle, Four-Tire Trucks, 1970-2001

\begin{tabular}{ccccc}
\hline Year & $\begin{array}{c}\text { Registrations } \\
\text { (thousands) }\end{array}$ & $\begin{array}{c}\text { Vehicle travel } \\
\text { (million miles) }\end{array}$ & $\begin{array}{c}\text { Fuel use } \\
\text { (million gallons) }\end{array}$ & $\begin{array}{c}\text { Fuel economy } \\
\text { (miles per gallon) }\end{array}$ \\
\hline 1970 & 14,211 & 123,286 & 12,313 & 10.0 \\
1971 & 15,181 & 137,870 & 13,484 & 10.2 \\
1972 & 16,428 & 156,622 & 15,150 & 10.3 \\
1973 & 18,083 & 176,833 & 16,828 & 10.5 \\
1974 & 19,335 & 182,757 & 16,657 & 11.0 \\
1975 & 20,418 & 200,700 & 19,081 & 10.5 \\
1976 & 22,301 & 225,834 & 20,828 & 10.8 \\
1977 & 23,624 & 250,591 & 22,383 & 11.2 \\
1978 & 25,476 & 279,414 & 24,162 & 11.6 \\
1979 & 27,022 & 291,905 & 24,445 & 11.9 \\
1980 & 27,876 & 290,935 & 23,796 & 12.2 \\
1981 & 28,928 & 296,343 & 23,697 & 12.5 \\
1982 & 29,792 & 306,141 & 22,702 & 13.5 \\
1983 & 31,214 & 327,643 & 23,945 & 13.7 \\
1984 & 32,106 & 358,006 & 25,604 & 14.0 \\
$1985^{\mathrm{a}}$ & 37,214 & 390,961 & 27,363 & 14.3 \\
1986 & 39,382 & 423,915 & 29,074 & 14.6 \\
1987 & 41,107 & 456,870 & 30,598 & 14.9 \\
1988 & 43,805 & 502,207 & 32,653 & 15.4 \\
1989 & 45,945 & 536,475 & 33,271 & 16.1 \\
1990 & 48,275 & 574,571 & 35,611 & 16.1 \\
1991 & 53,033 & 649,394 & 38,217 & 17.0 \\
1992 & 57,091 & 706,863 & 40,929 & 17.3 \\
1993 & 59,994 & 745,750 & 42,851 & 17.4 \\
1994 & 62,904 & 764,634 & 44,112 & 17.3 \\
1995 & 65,738 & 790,029 & 45,605 & 17.3 \\
1996 & 69,134 & 816,540 & 47,354 & 17.2 \\
1997 & 70,224 & 850,739 & 49,389 & 17.2 \\
1998 & 71,330 & 868,275 & 50,462 & 17.4 \\
1999 & 75,356 & 901,022 & 52,859 & 17.6 \\
2000 & 79,085 & 923,059 & 52,939 & \\
2001 & 84,188 & 937,839 & 53,294 & \\
$1970-2001$ & $5.9 \%$ & Average annual percentage change & \\
$1991-2001$ & $4.7 \%$ & $6.8 \%$ & $4.8 \%$ & \\
\hline & & $3.7 \%$ & & \\
\hline
\end{tabular}

\section{Source:}

U.S. Department of Transportation, Federal Highway Administration, Highway Statistics 2001, Washington, DC, 2002, Table VM-1, p. V-57, and annual. (Additional resources: www.fhwa.dot.gov) utility vehicles.

${ }^{\text {a }}$ Beginning in this year the data were revised to include all vans (including mini-vans), pickups and sport 
Because data on Class $2 b$ trucks are scarce, the U.S. DOE funded a study to investigate available sources of data. In the final report, four methodologies are described to estimate the sales of Class $2 b$ trucks.

Table 4.3

Summary Statistics on Class 1, Class 2a, and Class 2b Light Trucks

\begin{tabular}{|c|c|c|c|c|c|c|}
\hline & $\begin{array}{c}\text { CY } 1999 \\
\text { truck } \\
\text { sales } \\
\text { (millions) }\end{array}$ & $\begin{array}{l}\text { MY } 2000 \\
\text { truck } \\
\text { population } \\
\text { (millions) }\end{array}$ & $\begin{array}{c}\text { Percent } \\
\text { diesel } \\
\text { trucks in } \\
\text { population }\end{array}$ & $\begin{array}{c}\text { Average } \\
\text { age } \\
\text { (years) }\end{array}$ & $\begin{array}{l}\text { Estimated } \\
\text { annual } \\
\text { miles }^{\mathrm{a}} \\
\text { (billions) }\end{array}$ & $\begin{array}{l}\text { Estimated } \\
\text { fuel use } \\
\text { (billion }^{\mathrm{a}} \\
\text { gallons) }\end{array}$ \\
\hline Class 1 & 5.7 & 49.7 & $0.3 \%$ & 7.3 & 672.7 & 37.4 \\
\hline Class 2a & 1.8 & 19.2 & $2.5 \%$ & 7.4 & 251.9 & 18.0 \\
\hline Class 2b & 0.5 & 5.8 & $24.0 \%$ & 8.6 & 76.7 & 5.5 \\
\hline
\end{tabular}

Source: Davis, S.C. and L.F. Truett, Investigation of Class $2 b$ Trucks (Vehicles of 8,500 to 10,000 lbs GVWR), ORNL/TM-2002/49, March 2002, Table 16.

Note: CY - calendar year. MY - model year.

Table 4.4

Sales Estimates of Class 1, Class 2a, and Class 2b Light Trucks, 1989-1999

\begin{tabular}{ccccc}
\hline & \multicolumn{4}{c}{ Sales estimates (thousands) } \\
\cline { 2 - 5 } Calendar Year & $\begin{array}{c}\text { Class } 1 \\
(6,000 \mathrm{lbs}\end{array}$ & $\begin{array}{c}\text { Class 2a } \\
(6,001-\end{array}$ & $\begin{array}{c}\text { Class } 2 \mathrm{~b} \\
(8,5001-\end{array}$ \\
and under $)$ & $8,500 \mathrm{lbs})$ & $10,000 \mathrm{lbs})$ & Total \\
\hline 1989 & 3,313 & 918 & 379 & 4,610 \\
1990 & 3,451 & 829 & 268 & 4,548 \\
1991 & 3,246 & 670 & 206 & 4,122 \\
1992 & 3,608 & 827 & 194 & 4,629 \\
1993 & 4,119 & 975 & 257 & 5,351 \\
1994 & 4,527 & 1,241 & 265 & 6,033 \\
1995 & 4,422 & 1,304 & 327 & 6,053 \\
1996 & 4,829 & 1,356 & 334 & 6,519 \\
1997 & 5,085 & 1,315 & 397 & 7,797 \\
1998 & 5,263 & 1,694 & 342 & 8,073 \\
1999 & 5,707 & 1,845 & 521 & $75.1 \%$ \\
\hline
\end{tabular}

Source: Davis, S.C. and L.F. Truett, Investigation of Class $2 b$ Trucks (Vehicles of 8,500 to 10,000 lbs GVWR), ORNL/TM-2002/49, March 2002, Table 1.

Note: These data were calculated using Methodology 4 from the report.

aEstimates derived using 2000 population data and 1997 usage data. See source for details. 
Over one-quarter of autos sold in 2002 were transplants-autos built in the U.S. by a foreign firm.

Table 4.5

New Retail Automobile Sales in the United States, 1970-2002

\begin{tabular}{|c|c|c|c|c|c|c|c|}
\hline \multirow[b]{2}{*}{$\begin{array}{c}\text { Calendar } \\
\text { year }\end{array}$} & Domestic $^{\mathrm{a}}$ & Import $^{\mathrm{b}}$ & Total & \multirow[b]{2}{*}{$\begin{array}{l}\text { Percentage } \\
\text { imports }\end{array}$} & \multirow{2}{*}{$\begin{array}{c}\text { Percentage } \\
\text { transplants }^{c} \\
\text { on model } \\
\text { year basis }\end{array}$} & \multirow{2}{*}{$\begin{array}{l}\text { Percentage } \\
\text { imports and } \\
\text { transplants }\end{array}$} & \multirow[b]{2}{*}{$\begin{array}{c}\text { Percentage } \\
\text { diesel }\end{array}$} \\
\hline & \multicolumn{2}{|c|}{ (thousands) } & & & & & \\
\hline 1970 & 7,119 & 1,285 & 8,404 & $15.3 \%$ & $\mathrm{~d}$ & d & d \\
\hline 1975 & 7,053 & 1,571 & 8,624 & $18.2 \%$ & d & d & $0.31 \%$ \\
\hline 1980 & 6,581 & 2,398 & 8,979 & $26.7 \%$ & $2.1 \%$ & $28.8 \%$ & $4.31 \%$ \\
\hline 1981 & 6,209 & 2,327 & 8,536 & $27.3 \%$ & $1.8 \%$ & $29.1 \%$ & $6.10 \%$ \\
\hline 1982 & 5,759 & 2,223 & 7,982 & $27.9 \%$ & $1.4 \%$ & $29.3 \%$ & $4.44 \%$ \\
\hline 1983 & 6,795 & 2,387 & 9,182 & $26.0 \%$ & $1.3 \%$ & $27.3 \%$ & $2.09 \%$ \\
\hline 1984 & 7,952 & 2,439 & 10,391 & $23.5 \%$ & $2.0 \%$ & $25.5 \%$ & $1.45 \%$ \\
\hline 1985 & 8,205 & 2,838 & 11,043 & $25.7 \%$ & $2.2 \%$ & $27.9 \%$ & $0.82 \%$ \\
\hline 1986 & 8,215 & 3,238 & 11,453 & $28.3 \%$ & $2.8 \%$ & $31.1 \%$ & $0.37 \%$ \\
\hline 1987 & 7,081 & 3,197 & 10,278 & $31.1 \%$ & $5.2 \%$ & $36.3 \%$ & $0.16 \%$ \\
\hline 1988 & 7,526 & 3,099 & 10,626 & $29.2 \%$ & $5.8 \%$ & $35.0 \%$ & $0.02 \%$ \\
\hline 1989 & 7,073 & 2,825 & 9,898 & $28.5 \%$ & $7.3 \%$ & $35.8 \%$ & $0.13 \%$ \\
\hline 1990 & 6,897 & 2,404 & 9,301 & $25.8 \%$ & $11.2 \%$ & $37.0 \%$ & $0.08 \%$ \\
\hline 1991 & 6,137 & 2,038 & 8,175 & $24.9 \%$ & $13.7 \%$ & $38.6 \%$ & $0.10 \%$ \\
\hline 1992 & 6,277 & 1,937 & 8,213 & $23.6 \%$ & $14.1 \%$ & $37.7 \%$ & $0.06 \%$ \\
\hline 1993 & 6,742 & 1,776 & 8,518 & $20.9 \%$ & $14.9 \%$ & $35.8 \%$ & $0.03 \%$ \\
\hline 1994 & 7,255 & 1,735 & 8,990 & $19.3 \%$ & $16.5 \%$ & $35.8 \%$ & $0.04 \%$ \\
\hline 1995 & 7,129 & 1,506 & 8,635 & $17.4 \%$ & $18.9 \%$ & $36.3 \%$ & $0.04 \%$ \\
\hline 1996 & 7,255 & 1,271 & 8,526 & $14.9 \%$ & $22.3 \%$ & $37.2 \%$ & $0.10 \%$ \\
\hline 1997 & 6,917 & 1,355 & 8,272 & $16.4 \%$ & $23.7 \%$ & $40.1 \%$ & $0.09 \%$ \\
\hline 1998 & 6,762 & 1,380 & 8,142 & $16.9 \%$ & $25.1 \%$ & $42.0 \%$ & $0.13 \%$ \\
\hline 1999 & 6,979 & 1,719 & 8,698 & $19.8 \%$ & $24.6 \%$ & $44.4 \%$ & $0.16 \%$ \\
\hline 2000 & 6,831 & 2,016 & 8,847 & $22.8 \%$ & $24.4 \%$ & $47.2 \%$ & $0.26 \%$ \\
\hline 2001 & 6,325 & 2,098 & 8,423 & $24.9 \%$ & $26.0 \%$ & $50.9 \%$ & $0.18 \%$ \\
\hline \multirow[t]{2}{*}{2002} & 5,878 & 2,226 & 8,104 & $27.5 \%$ & $26.7 \%$ & $54.2 \%$ & $0.39 \%$ \\
\hline & \multicolumn{7}{|c|}{ Average annual percentage change } \\
\hline 1970-2002 & $-0.6 \%$ & $1.7 \%$ & $-0.1 \%$ & & & & \\
\hline 1992-2002 & $-0.7 \%$ & $1.4 \%$ & $-0.1 \%$ & & & & \\
\hline
\end{tabular}

Source:

Domestic and import data - 1970-97: American Automobile Manufacturers Association, Motor Vehicle Facts and

Figures 1998, Detroit, MI, 1998, p. 15, and annual. 1997 data from Economic Indicators, 4th Quarter 1997. 1998-2002: Ward's Communication, Ward's Motor Vehicle Facts and Figures, Detroit, MI, 2000, p. 15.

Diesel data - Ward's Communications, Ward's Automotive Yearbook, Detroit, MI, 2003, p. 52, and annual.

Transplant data - Oak Ridge National Laboratory, Light Vehicle MPG and Market Shares Data System, Oak Ridge,

TN, 2002. (Additional resources: www.aama.com, www.wardsauto.com)

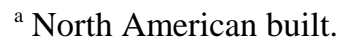

${ }^{\mathrm{b}}$ Does not include import tourist deliveries.

${ }^{\mathrm{c}}$ A transplant is an automobile which was built in the U.S. by a foreign firm. Also included are joint ventures which are built in the U.S.

${ }^{\mathrm{d}}$ Data are not available. 
In 2000, light trucks, which include pick-ups, minivans, sport-utility vehicles, and other trucks less than 10,000 pounds gross vehicle weight (GVW), accounted for $48.7 \%$ of light vehicle sales.

Table 4.6

New Retail Sales of Trucks 10,000 Pounds GVW and Less in the United States, 1970-2002

\begin{tabular}{|c|c|c|c|c|c|c|c|}
\hline \multirow[b]{2}{*}{$\begin{array}{c}\text { Calendar } \\
\text { year }\end{array}$} & \multirow[b]{2}{*}{$\begin{array}{l}\text { Light truck } \\
\text { sales }^{\mathrm{a}} \\
\text { (thousands) }\end{array}$} & \multicolumn{6}{|c|}{ Percentages } \\
\hline & & Import $^{\mathrm{b}}$ & Transplants $^{\mathrm{c}}$ & Diesel $^{\mathrm{d}}$ & $\begin{array}{c}\text { Four-wheel drive } \\
\text { of domestic } \\
\text { light trucks }\end{array}$ & $\begin{array}{l}\text { Light trucks of } \\
\text { light-duty } \\
\text { vehicle sales }\end{array}$ & $\begin{array}{c}\text { Light trucks } \\
\text { of total } \\
\text { truck sales }\end{array}$ \\
\hline 1970 & 1,463 & $4.5 \%$ & $\mathrm{f}$ & $\mathrm{g}$ & $\mathrm{f}$ & $14.8 \%$ & $80.4 \%$ \\
\hline 1975 & 2,281 & $10.0 \%$ & f & $\mathrm{g}$ & $23.4 \%$ & $20.9 \%$ & $87.9 \%$ \\
\hline 1980 & 2,440 & $19.7 \%$ & $0.9 \%$ & $3.6 \%$ & $20.7 \%$ & $21.4 \%$ & $88.9 \%$ \\
\hline 1981 & 2,189 & $20.3 \%$ & $0.0 \%$ & $3.1 \%$ & $18.6 \%$ & $20.4 \%$ & $89.8 \%$ \\
\hline 1982 & 2,470 & $16.5 \%$ & $0.0 \%$ & $8.5 \%$ & $16.8 \%$ & $23.6 \%$ & $92.8 \%$ \\
\hline 1983 & 2,984 & $15.6 \%$ & $0.0 \%$ & $6.7 \%$ & $28.5 \%$ & $24.5 \%$ & $93.6 \%$ \\
\hline 1984 & 3,863 & $15.7 \%$ & $2.0 \%$ & $4.8 \%$ & $27.0 \%$ & $27.1 \%$ & $93.0 \%$ \\
\hline 1985 & 4,458 & $17.2 \%$ & $2.6 \%$ & $3.8 \%$ & $29.1 \%$ & $28.8 \%$ & $93.6 \%$ \\
\hline 1986 & 4,594 & $20.1 \%$ & $2.3 \%$ & $3.7 \%$ & $27.0 \%$ & $28.6 \%$ & $94.3 \%$ \\
\hline 1987 & 4,610 & $17.9 \%$ & $1.7 \%$ & $2.3 \%$ & $32.0 \%$ & $31.0 \%$ & $93.9 \%$ \\
\hline 1988 & 4,800 & $12.6 \%$ & $2.4 \%$ & $2.3 \%$ & $32.1 \%$ & $31.1 \%$ & $93.2 \%$ \\
\hline 1989 & 4,610 & $10.9 \%$ & $2.6 \%$ & $2.9 \%$ & $31.4 \%$ & $31.8 \%$ & $93.3 \%$ \\
\hline 1990 & 4,548 & $13.2 \%$ & $3.4 \%$ & $3.1 \%$ & $31.6 \%$ & $32.8 \%$ & $93.9 \%$ \\
\hline 1991 & 4,123 & $12.8 \%$ & $4.5 \%$ & $3.2 \%$ & $34.4 \%$ & $33.5 \%$ & $94.5 \%$ \\
\hline 1992 & 4,629 & $8.6 \%$ & $5.5 \%$ & $3.3 \%$ & $31.6 \%$ & $36.0 \%$ & $94.4 \%$ \\
\hline 1993 & 5,351 & $6.8 \%$ & $7.1 \%$ & $3.7 \%$ & $32.6 \%$ & $38.6 \%$ & $94.2 \%$ \\
\hline 1994 & 6,033 & $6.5 \%$ & $8.1 \%$ & $3.9 \%$ & $34.4 \%$ & $40.2 \%$ & $94.0 \%$ \\
\hline 1995 & 6,053 & $6.5 \%$ & $7.5 \%$ & $4.1 \%$ & $39.1 \%$ & $41.2 \%$ & $93.4 \%$ \\
\hline 1996 & 6,519 & $6.6 \%$ & $8.4 \%$ & $3.7 \%$ & $35.7 \%$ & $43.3 \%$ & $94.1 \%$ \\
\hline 1997 & 6,797 & $8.4 \%$ & $7.0 \%$ & $4.8 \%$ & $39.6 \%$ & $46.6 \%$ & $94.1 \%$ \\
\hline 1998 & 7,299 & $8.9 \%$ & $7.6 \%$ & $1.7 \%$ & $43.8 \%$ & $47.3 \%$ & $93.3 \%$ \\
\hline 1999 & 8,073 & $9.5 \%$ & $8.7 \%$ & $5.9 \%$ & $43.3 \%$ & $48.1 \%$ & $92.6 \%$ \\
\hline 2000 & 8,387 & $9.9 \%$ & $11.3 \%$ & $4.8 \%$ & $41.7 \%$ & $48.7 \%$ & $93.9 \%$ \\
\hline 2001 & 8,700 & $11.3 \%$ & $12.8 \%$ & $5.3 \%$ & $42.2 \%$ & $50.8 \%$ & $96.1 \%$ \\
\hline 2002 & 8,713 & $12.2 \%$ & $12.1 \%$ & $4.9 \%$ & $46.4 \%$ & $51.8 \%$ & $96.4 \%$ \\
\hline \multicolumn{8}{|c|}{ Average annual percentage change } \\
\hline 1970-2002 & $5.7 \%$ & & & & & & \\
\hline 1992-2002 & $6.5 \%$ & & & & & & \\
\hline
\end{tabular}

Source:

Four-wheel drive and diesel - 1970-88: Ward's Communications, Ward's Automotive Yearbook, Detroit, MI, 1989, p. 168, and annual. 1989-on: Ward's Communications, Ward's Automotive Yearbook, Factory Installation Reports, Detroit, MI, 2003, and annual.

Transplants - Oak Ridge National Laboratory, Light-Duty Vehicle MPG and Market Shares System, Oak Ridge, TN, 1996. All other - 1970-97: American Automobile Manufacturers Association, Motor Vehicle Facts and Figures 1998, Detroit, MI, 1998, pp. 8, 15, 24, and annual. 1998-on: Ward's Communications, Ward's 2003 Motor Vehicle Facts and Figures, Detroit, MI, p. 24, and annual. (Additional resources: www.aama.com, www.wardsauto.com)

${ }^{\text {a }}$ Includes all trucks of 10,000 pounds gross vehicle weight and less sold in the U.S.

${ }^{\mathrm{b}}$ Excluding transplants.

${ }^{\text {c }}$ Based on model year data. A transplant is a light truck which was built in the U.S. by a foreign firm. Also included are joint ventures built in the U.S.

${ }^{\mathrm{d}}$ Based on model year factory installations. Column was revised.

${ }^{\mathrm{e}}$ Light-duty vehicles include automobiles and light trucks.

${ }^{\mathrm{f}}$ Data are not available.

${ }^{\mathrm{g}}$ Indicates less than 1 percent. 
The sales-weighted fuel economy of automobiles increased dramatically from 1976 (17.2 mpg) to 1990 (27.6 mpg),

but has risen only about $1 \mathrm{mpg}$ since then.

Table 4.7

Period Sales, Market Shares, and Sales-Weighted Fuel Economies of New Domestic and Import Automobiles, Selected Sales Periods ${ }^{\mathrm{a}}$ 1976-2002

\begin{tabular}{|c|c|c|c|c|c|c|c|c|}
\hline Sales Period $^{\mathrm{a}}$ & 1976 & 1980 & 1985. & 1990 & 1995 & 2000 & 2001. & 2002 \\
\hline \multicolumn{9}{|l|}{ MINICOMPACT } \\
\hline Total sales, units & - & 428,346 & 52,295 & 76,698 & 44,752 & 19,245 & 33,206 & 53,840 \\
\hline Market share, \% & - & 4.7 & 0.5 & 0.8 & 0.5 & 0.2 & 0.4 & 0.6 \\
\hline Fuel economy, mpg & - & 29.4 & 32.7 & 26.4 & 27.0 & 25.6 & 24.6 & 26.2 \\
\hline \multicolumn{9}{|l|}{ SUBCOMPACT } \\
\hline Total sales, units & $2,625,929$ & $3,441,480$ & 2,382,339 & $2,030,226$ & $1,518,209$ & $1,789,350$ & 922,287 & 636,397 \\
\hline Market share, \% & 27.1 & 37.8 & 21.7 & 22.0 & 17.4 & 19.9 & 11.1 & 7.6 \\
\hline Fuel economy, mpg & 23.5 & 27.3 & 30.1 & 31.3 & 31.7 & 31.1 & 29.6 & 27.6 \\
\hline \multicolumn{9}{|l|}{ СОМРАCT } \\
\hline Total sales, units & $2,839,603$ & 599,423 & $3,526,118$ & $3,156,481$ & 3,289,735 & $2,397,813$ & $3,058,389$ & $3,217,151$ \\
\hline Market share, \% & 29.3 & 6.6 & 32.1 & 34.2 & 37.7 & 26.7 & 36.8 & 38.6 \\
\hline Fuel economy, mpg & 17.1 & 22.3 & 29.6 & 28.9 & 30.2 & 30.4 & 31.3 & 31.5 \\
\hline \multicolumn{9}{|l|}{ MIDSIZE } \\
\hline Total sales, units & $1,815,505$ & $3,073,103$ & $3,117,817$ & $2,511,503$ & $2,498,521$ & 3,352,198 & $2,669,116$ & 2,917,527 \\
\hline Market share, \% & 18.7 & 33.8 & 28.4 & 27.2 & 28.6 & 37.3 & 32.1 & 35.0 \\
\hline Fuel economy, mpg & 15.3 & 21.3 & 24.9 & 25.9 & 25.9 & 26.8 & 27.2 & 27.4 \\
\hline \multicolumn{9}{|l|}{ LARGE } \\
\hline Total sales, units & $2,206,102$ & $1,336,190$ & $1,516,249$ & $1,279,092$ & $1,320,608$ & $1,297,237$ & $1,506,890$ & $1,377,357$ \\
\hline Market share, \% & 22.8 & 14.7 & 13.8 & 13.9 & 15.1 & 14.4 & 18.1 & 16.5 \\
\hline Fuel economy, mpg & 13.9 & 19.3 & 22.3 & 23.5 & 24.1 & 25.3 & 25.4 & 25.5 \\
\hline \multicolumn{9}{|l|}{ TWO SEATER } \\
\hline Total sales, units & 199,716 & 215,964 & 373,697 & 170,465 & 53,045 & 122,259 & 118,097 & 134,187 \\
\hline Market share, \% & 2.1 & 2.4 & 3.4 & 1.8 & 0.6 & 1.4 & 1.4 & 1.6 \\
\hline Fuel economy, mpg & 20.1 & 21.0 & 27.6 & 28.0 & 24.7 & 25.8 & 26.5 & 25.2 \\
\hline \multicolumn{9}{|l|}{ TOTAL } \\
\hline Total sales, units & $9,686,855$ & $9,094,506$ & $10,968,51$ & $9,224,465$ & $8,724,870$ & $8,978,102$ & $8,307,985$ & $8,336,459$ \\
\hline Market share, \% & 100 & 100 & 100 & 100 & 100 & 100 & 100 & 100 \\
\hline Fuel economy, mpg & 17.2 & 23.2 & 27.0 & 27.6 & 28.0 & 28.2 & 28.5 & 28.5 \\
\hline
\end{tabular}

Source:

Oak Ridge National Laboratory, Light Vehicle MPG and Market Shares System, Oak Ridge, TN, 2003.

(Additional resources: www-cta.ornl.gov)

${ }^{a}$ Sales period is October 1 of the previous year through September 30 of the current year. These figures represent only those sales that could be matched to corresponding EPA fuel economy values. 
Light truck sales have more than tripled from 1976 to 2001. Similar to the automobile trend, the sales-weighted fuel economy of light trucks increased during the late '70's and ' 80 's, but has remained fairly constant since then.

Table 4.8

Period Sales, Market Shares, and Sales-Weighted Fuel Economies of New Domestic and Import Light Trucks, Selected Sales Periods ${ }^{\mathrm{a}}$ 1976-2002

\begin{tabular}{|c|c|c|c|c|c|c|c|c|}
\hline Sales Period $^{\mathrm{a}}$ & 1976 & 1980 & 1985 & 1990 & 1995 & 2000 & 2001 & 2002 \\
\hline \multicolumn{9}{|l|}{ SMALL PICKUP } \\
\hline Total sales, units & 170,351 & 516,412 & 863,584 & $1,135,727$ & $1,067,764$ & $1,071,730$ & 819,033 & 761,802 \\
\hline Market share, \% & 7.1 & 23.3 & 20.4 & 25.2 & 18.0 & 12.9 & 10.2 & 8.8 \\
\hline Fuel economy, mpg & 23.9 & 25.5 & 26.8 & 24.5 & 24.4 & 22.0 & 21.3 & 21.3 \\
\hline \multicolumn{9}{|l|}{ LARGE PICKUP } \\
\hline Total sales, units & $1,586,020$ & $1,115,248$ & $1,690,931$ & $1,116,490$ & $1,472,885$ & $1,968,710$ & $1,987,833$ & $2,209,671$ \\
\hline Market share, \% & 65.8 & 50.3 & 39.9 & 24.7 & 24.8 & 23.7 & 24.8 & 25.5 \\
\hline Fuel economy, mpg & 15.1 & 17.0 & 19.0 & 17.5 & 17.8 & 18.7 & 19.0 & 18.4 \\
\hline \multicolumn{9}{|l|}{ SMALL VAN } \\
\hline Total sales, units & 18,651 & 13,649 & 437,660 & $1,012,141$ & $1,330,586$ & $1,272,070$ & $1,141,109$ & $1,165,202$ \\
\hline Market share, \% & 0.8 & 0.6 & 10.3 & 22.4 & 22.4 & 15.3 & 14.2 & 13.4 \\
\hline Fuel economy, mpg & 19.5 & 19.6 & 23.9 & 22.3 & 22.4 & 23.0 & 23.2 & 23.0 \\
\hline \multicolumn{9}{|l|}{ LARGE VAN } \\
\hline Total sales, units & 574,745 & 328,065 & 536,242 & 319,429 & 327,586 & 368,820 & 323,806 & 349,706 \\
\hline Market share, \% & 23.9 & 14.8 & 12.7 & 7.1 & 5.5 & 4.4 & 4.0 & 4.0 \\
\hline Fuel economy, mpg & 15.4 & 16.3 & 16.4 & 17.1 & 17.2 & 18.2 & 18.3 & 18.5 \\
\hline \multicolumn{9}{|l|}{ SMALL SUV } \\
\hline Total sales, units & 0 & 51,684 & 441,966 & 402,354 & 509,737 & 756,142 & 894,788 & 877,777 \\
\hline Market share, \% & 0.0 & 2.3 & 10.4 & 8.9 & 8.6 & 9.1 & 11.2 & 10.1 \\
\hline Fuel economy, mpg & & 17.7 & 22.1 & 22.5 & 22.0 & 23.8 & 24.3 & 25.3 \\
\hline \multicolumn{9}{|l|}{ MEDIUM SUV } \\
\hline Total sales, units & 50,763 & 151,929 & 187,447 & 434,491 & $1,076,686$ & $2,167,329$ & $2,067,855$ & $2,448,269$ \\
\hline Market share, \% & 2.1 & 6.9 & 4.4 & 9.6 & 18.1 & 26.1 & 25.8 & 28.2 \\
\hline Fuel economy, mpg & 15.1 & 14.9 & 17.2 & 19.7 & 19.2 & 20.4 & 20.5 & 20.5 \\
\hline \multicolumn{9}{|l|}{ LARGE SUV } \\
\hline Total sales, units & 9,228 & 39,550 & 77,535 & 93,993 & 148,622 & 702,152 & 785,094 & 860,652 \\
\hline Market share, \% & 0.4 & 1.8 & 1.8 & 2.1 & 2.5 & 8.5 & 9.8 & 9.9 \\
\hline Fuel economy, mpg & 14.2 & 13.7 & 17.1 & 16.5 & 16.1 & 17.5 & 17.6 & 17.5 \\
\hline \multicolumn{9}{|l|}{ TOTAL } \\
\hline Total sales, units & $2,409,758$ & $2,216,537$ & $4,235,365$ & $4,514,625$ & $5,933,866$ & $8,306,953$ & $8,019,518$ & $8,673,079$ \\
\hline Market share, \% & 100 & 100 & 100 & 100 & 100 & 100 & 100 & 100 \\
\hline Fuel economy, mpg & 15.6 & 18.1 & 20.4 & 20.5 & 20.2 & 20.4 & 20.5 & 20.2 \\
\hline
\end{tabular}

Source:

Oak Ridge National Laboratory, Light Vehicle MPG and Market Shares System, Oak Ridge, TN, 2003.

(Additional resources: www-cta.ornl.gov)

Note:

Revised definitions of light trucks are based on vehicle curb weight as follows:

Small pickup $=<3,500$ lbs. $\quad$ Large pickup $=3,500-8,500$ lbs.

Small van $=<4,500$ lbs. $\quad$ Large van $=4,500-8,500$ lbs.

Small utility $=<3,500$ lbs. Medium utility=3,500-4,799 lbs. Large utility=4,800-8,500 lbs.

\footnotetext{
${ }^{\text {a }}$ Sales period is October 1 of the previous year through September 30 of the current year. These figures represent only those sales that could be matched to corresponding EPA fuel economy values.
} 
Back in 1976 only 20\% of new light vehicle sales were light trucks. Because of the boom in sales of minivans, sport utility vehicles, and pick-up trucks, today more than half of light vehicle sales are light trucks.

Table 4.9

Light Vehicle Market Shares by Size Class, Sales Periods ${ }^{\mathrm{a}}$ 1976-2002

\begin{tabular}{|c|c|c|c|c|c|c|c|c|}
\hline Sales period ${ }^{\mathrm{a}}$ & 1976 & 1980 & 1985 & 1990 & 1995 & 2000 & 2001 & 2002 \\
\hline Minicompact & $0.0 \%$ & $3.8 \%$ & $0.3 \%$ & $0.6 \%$ & $0.3 \%$ & $0.1 \%$ & $0.2 \%$ & $0.3 \%$ \\
\hline Subcompact & $21.7 \%$ & $30.4 \%$ & $15.7 \%$ & $14.8 \%$ & $10.4 \%$ & $10.4 \%$ & $5.6 \%$ & $3.7 \%$ \\
\hline Compact & $23.5 \%$ & $5.3 \%$ & $23.2 \%$ & $23.0 \%$ & $22.4 \%$ & $13.9 \%$ & $18.7 \%$ & $18.8 \%$ \\
\hline Midsize & $15.0 \%$ & $27.2 \%$ & $20.5 \%$ & $18.3 \%$ & $17.0 \%$ & $19.4 \%$ & $16.3 \%$ & $17.2 \%$ \\
\hline Large & $18.2 \%$ & $11.8 \%$ & $10.0 \%$ & $9.3 \%$ & $9.0 \%$ & $7.5 \%$ & $9.2 \%$ & $8.1 \%$ \\
\hline Two seater & $1.7 \%$ & $1.9 \%$ & $2.5 \%$ & $1.2 \%$ & $0.4 \%$ & $0.7 \%$ & $0.7 \%$ & $0.8 \%$ \\
\hline Small pickup & $1.4 \%$ & $4.6 \%$ & $5.7 \%$ & $8.3 \%$ & $7.3 \%$ & $6.2 \%$ & $5.0 \%$ & $4.5 \%$ \\
\hline Large pickup & $13.1 \%$ & $9.9 \%$ & $11.1 \%$ & $8.1 \%$ & $10.0 \%$ & $11.4 \%$ & $12.2 \%$ & $13.0 \%$ \\
\hline Small van & $0.2 \%$ & $0.1 \%$ & $2.9 \%$ & $7.4 \%$ & $8.6 \%$ & $7.4 \%$ & $6.4 \%$ & $6.9 \%$ \\
\hline Large van & $4.8 \%$ & $2.9 \%$ & $3.5 \%$ & $2.3 \%$ & $9.1 \%$ & $2.1 \%$ & $2.0 \%$ & $2.1 \%$ \\
\hline Small utility & $0.0 \%$ & $0.5 \%$ & $2.9 \%$ & $2.9 \%$ & $3.5 \%$ & $4.4 \%$ & $5.5 \%$ & $5.2 \%$ \\
\hline Medium utility & $0.4 \%$ & $1.3 \%$ & $1.2 \%$ & $3.2 \%$ & $7.3 \%$ & $12.5 \%$ & $13.2 \%$ & $14.3 \%$ \\
\hline Large utility & $0.1 \%$ & $0.3 \%$ & $0.5 \%$ & $0.7 \%$ & $1.0 \%$ & $4.1 \%$ & $4.8 \%$ & $5.1 \%$ \\
\hline Total light vehicles sold & $12,096,613$ & $11,311,043$ & $15,203,880$ & $13,739,090$ & $14,658,736$ & $17,285,055$ & $16,327,503$ & $17,009,538$ \\
\hline Cars & $80.1 \%$ & $80.4 \%$ & $72.1 \%$ & $67.1 \%$ & $59.5 \%$ & $51.9 \%$ & $50.9 \%$ & $49.0 \%$ \\
\hline Light trucks & $19.9 \%$ & $19.6 \%$ & $27.9 \%$ & $32.9 \%$ & $40.5 \%$ & $48.1 \%$ & $49.1 \%$ & $51.0 \%$ \\
\hline
\end{tabular}

Source:

Oak Ridge National Laboratory, Light Vehicle MPG and Market Shares System, Oak Ridge, TN, 2003.

(Additional resources: www-cta.ornl.gov)

\footnotetext{
${ }^{\text {a }}$ Sales period is October 1 of the current year through September 30 of the next year.
} 
This graph shows the emergence of the mini-van in the early 1980's and the rising popularity of sport utility vehicles in the 1990's.

Figure 4.1. Light Vehicle Market Shares, Sales Periods 1976-2002

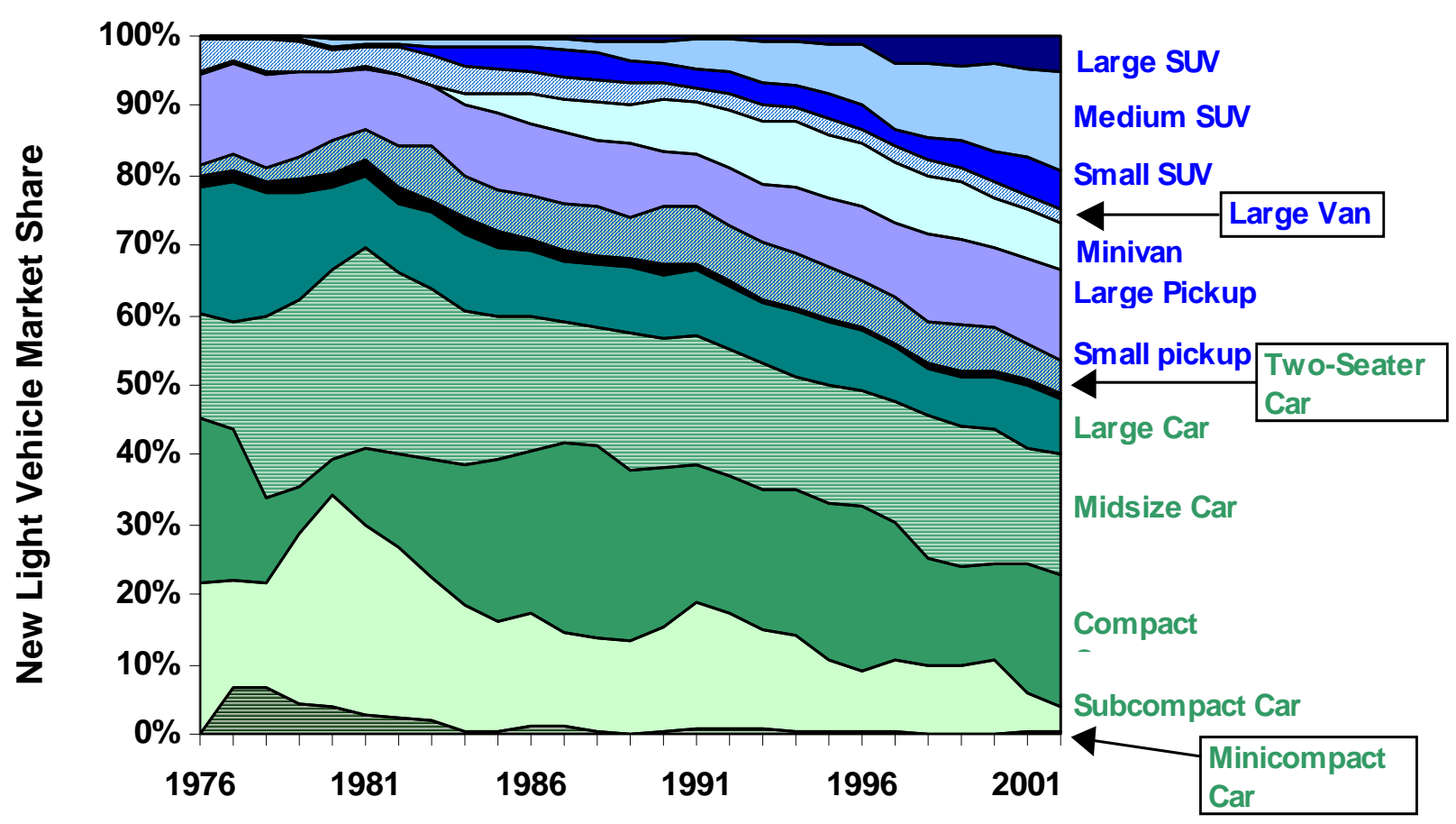

Source:

See Table 4.9 
The compact, midsize, and large automobile sales-weighted engine sizes declined dramatically in the late '70's and early ' 80 's.

Table 4.10

Sales-Weighted Engine Size of New Domestic and Import Automobiles by Size Class, Sales Periods ${ }^{\mathrm{a}}$ 1976-2002

( liters ${ }^{\mathrm{b}}$ )

\begin{tabular}{|c|c|c|c|c|c|c|c|}
\hline Sales period $^{\mathrm{a}}$ & Minicompact & Subcompact & Compact & Midsize & Large & Two seater & All \\
\hline 1976 & & 2.67 & 5.00 & 5.85 & 6.79 & 2.89 & 4.89 \\
\hline 1977 & 1.98 & 2.73 & 4.79 & 5.47 & 6.02 & 2.81 & 4.56 \\
\hline 1978 & 2.06 & 2.67 & 3.95 & 4.89 & 6.17 & 3.01 & 4.33 \\
\hline 1979 & 1.86 & 2.39 & 3.74 & 4.41 & 5.56 & 2.77 & 3.78 \\
\hline 1980 & 1.90 & 2.10 & 3.03 & 3.90 & 5.12 & 2.79 & 3.22 \\
\hline 1981 & 1.57 & 2.04 & 2.20 & 3.63 & 5.00 & 2.49 & 2.98 \\
\hline 1982 & 1.53 & 2.08 & 2.12 & 3.47 & 4.73 & 2.41 & 2.89 \\
\hline 1983 & 1.60 & 2.19 & 2.20 & 3.45 & 4.95 & 2.52 & 2.98 \\
\hline 1984 & 2.17 & 2.22 & 2.21 & 3.40 & 4.87 & 2.50 & 2.97 \\
\hline 1985 & 1.95 & 2.29 & 2.27 & 3.37 & 4.65 & 2.47 & 2.92 \\
\hline 1986 & 1.45 & 2.19 & 2.21 & 3.19 & 4.38 & 2.83 & 2.76 \\
\hline 1987 & 1.48 & 2.19 & 2.20 & 2.99 & 4.36 & 2.57 & 2.68 \\
\hline 1988 & 1.52 & 2.05 & 2.21 & 3.00 & 4.32 & 2.75 & 2.66 \\
\hline 1989 & 2.54 & 2.08 & 2.11 & 3.01 & 4.31 & 2.81 & 2.68 \\
\hline 1990 & 2.42 & 1.96 & 2.25 & 3.13 & 4.33 & 2.57 & 2.72 \\
\hline 1991 & 2.17 & 1.97 & 2.23 & 3.16 & 4.40 & 2.67 & 2.72 \\
\hline 1992 & 1.89 & 2.01 & 2.33 & 3.16 & 4.34 & 3.01 & 2.76 \\
\hline 1993 & 1.96 & 2.07 & 2.28 & 3.16 & 4.27 & 3.47 & 2.78 \\
\hline 1994 & 2.21 & 2.27 & 2.23 & 3.15 & 4.17 & 3.82 & 2.79 \\
\hline 1995 & 2.42 & 2.26 & 2.23 & 3.12 & 4.12 & 3.76 & 2.79 \\
\hline 1996 & 2.49 & 2.23 & 2.19 & 2.98 & 4.09 & 3.67 & 2.71 \\
\hline 1997 & 2.62 & 2.13 & 2.28 & 3.02 & 4.03 & 3.08 & 2.74 \\
\hline 1998 & 3.15 & 2.29 & 2.17 & 2.94 & 3.98 & 3.51 & 2.75 \\
\hline 1999 & 2.86 & 2.31 & 2.25 & 2.91 & 3.91 & 3.62 & 2.76 \\
\hline 2000 & 2.55 & 2.30 & 2.23 & 2.85 & 3.88 & 3.45 & 2.73 \\
\hline 2001 & 3.01 & 2.66 & 2.16 & 2.85 & 3.69 & 3.48 & 2.74 \\
\hline 2002 & 2.90 & 3.01 & 2.14 & 2.87 & 3.69 & 3.74 & 2.75 \\
\hline \multicolumn{8}{|c|}{ Average annual percentage change } \\
\hline 1976-2002 & $1.6 \%^{\mathrm{d}}$ & $0.5 \%$ & $-3.3 \%$ & $-2.9 \%$ & $-2.5 \%$ & $1.1 \%$ & $-2.4 \%$ \\
\hline 1992-2002 & $4.4 \%$ & $4.1 \%$ & $-0.8 \%$ & $-1.0 \%$ & $-1.6 \%$ & $2.2 \%$ & $0.0 \%$ \\
\hline
\end{tabular}

Source:

Oak Ridge National Laboratory, Light Vehicle MPG and Market Shares System, Oak Ridge, TN, 2003.

(Additional resources: www-cta.ornl.gov)

\footnotetext{
a Sales period is October 1 of the previous year through September 30 of the current year.

b 1 liter $=61.02$. cubic inches.

c There were no minicompact automobiles sold in 1976.

${ }^{\mathrm{d}}$ Average annual percentage change begins with 1977.
} 
Pickups and vans have been increasing over the years while utility vehicles engine sizes are smaller in 2002 than in the 1970's.

Table 4.11

Sales-Weighted Engine Size of New Domestic and Import Light Trucks by Size Class Sales Periods ${ }^{\mathrm{a}}$ 1976-2002

(liters ${ }^{\mathrm{b}}$ )

\begin{tabular}{ccccccccc}
\hline $\begin{array}{c}\text { Sales } \\
\text { period }^{\text {a }}\end{array}$ & $\begin{array}{c}\text { Small } \\
\text { pickup }\end{array}$ & $\begin{array}{c}\text { Large } \\
\text { pickup }\end{array}$ & $\begin{array}{c}\text { Small } \\
\text { van }\end{array}$ & $\begin{array}{c}\text { Large } \\
\text { van }\end{array}$ & $\begin{array}{c}\text { Small } \\
\text { utility }\end{array}$ & $\begin{array}{c}\text { Medium } \\
\text { utility }\end{array}$ & $\begin{array}{c}\text { Large } \\
\text { utility }\end{array}$ & All \\
\hline 1976 & 1.92 & 4.41 & 1.97 & 4.27 & $c$ & 4.21 & 5.74 & 4.18 \\
1977 & 1.95 & 4.41 & 1.97 & 4.37 & c & 4.21 & 5.74 & 4.11 \\
1978 & 1.96 & 4.39 & 1.97 & 4.25 & 3.80 & 4.48 & 5.74 & 4.09 \\
1979 & 2.00 & 5.15 & 1.97 & 4.24 & 4.23 & 4.67 & 5.74 & 4.41 \\
1980 & 1.99 & 4.41 & 1.97 & 4.85 & 2.47 & 4.51 & 5.74 & 3.88 \\
1981 & 2.08 & 4.16 & 1.97 & 4.34 & 2.47 & 4.55 & 5.00 & 3.67 \\
1982 & 2.06 & 4.02 & 1.59 & 4.33 & 2.47 & 4.54 & 5.00 & 3.55 \\
1983 & 2.04 & 4.05 & 1.59 & 4.32 & 2.28 & 4.84 & 5.59 & 3.37 \\
1984 & 2.05 & 4.17 & 2.13 & 4.33 & 2.33 & 4.14 & 5.65 & 3.40 \\
1985 & 2.09 & 4.02 & 2.22 & 4.43 & 2.60 & 4.44 & 4.96 & 3.38 \\
1986 & 2.13 & 3.79 & 2.29 & 4.41 & 2.28 & 4.33 & 4.95 & 3.12 \\
1987 & 2.17 & 3.71 & 2.29 & 4.46 & 2.39 & 3.83 & 4.95 & 3.07 \\
1988 & 2.56 & 4.68 & 3.15 & 5.21 & 3.23 & 4.19 & 5.55 & 3.82 \\
1989 & 2.64 & 4.70 & 3.11 & 5.22 & 3.77 & 3.77 & 5.58 & 3.93 \\
1990 & 2.90 & 5.14 & 3.43 & 5.24 & 3.68 & 3.55 & 5.56 & 3.93 \\
1991 & 2.93 & 5.22 & 3.36 & 5.26 & 3.60 & 3.85 & 5.46 & 3.92 \\
1992 & 3.09 & 5.15 & 3.43 & 5.31 & 3.62 & 3.94 & 5.45 & 4.00 \\
1993 & 3.15 & 5.15 & 3.41 & 5.24 & 3.60 & 4.06 & 5.58 & 4.02 \\
1994 & 3.05 & 5.26 & 3.58 & 5.37 & 3.53 & 4.01 & 5.54 & 4.10 \\
1995 & 2.99 & 5.13 & 3.50 & 5.16 & 3.56 & 4.04 & 5.41 & 4.06 \\
1996 & 2.93 & 5.17 & 3.51 & 5.25 & 3.43 & 4.29 & 5.35 & 4.12 \\
1997 & 3.00 & 5.05 & 3.47 & 5.04 & 2.75 & 3.96 & 5.33 & 4.09 \\
1998 & 2.89 & 5.01 & 3.45 & 4.99 & 2.84 & 4.15 & 5.39 & 4.16 \\
1999 & 3.36 & 5.02 & 3.48 & 5.05 & 2.87 & 4.12 & 5.46 & 4.19 \\
2000 & 3.42 & 4.94 & 3.43 & 5.00 & 2.78 & 4.03 & 5.21 & 4.11 \\
2001 & 3.50 & 4.79 & 3.59 & 4.96 & 2.70 & 3.84 & 5.13 & 4.05 \\
2002 & 3.54 & 4.88 & 3.61 & 4.89 & 2.60 & 3.80 & 5.18 & 4.09 \\
& & & Average annual percentage change & & & \\
$1976-2002$ & $2.5 \%$ & $0.4 \%$ & $2.5 \%$ & $0.5 \%$ & $c$ & $-0.4 \%$ & $-0.4 \%$ & $-0.1 \%$ \\
$1992-2002$ & $1.4 \%$ & $-0.5 \%$ & $0.5 \%$ & $-0.8 \%$ & $-3.3 \%$ & $-0.4 \%$ & $-0.5 \%$ & $0.2 \%$ \\
\hline
\end{tabular}

Source:

Oak Ridge National Laboratory, Light Vehicle MPG and Market Shares System, Oak Ridge, TN, 2003.

(Additional resources: www-cta.ornl.gov)

Note:

Revised definitions of light trucks are based on vehicle curb weight as follows:

Small pickup $=<3,500$ lbs. $\quad$ Large pickup $=3,500-8,500$ lbs.

Small van $=<4,500$ lbs. $\quad$ Large van $=4,500-8,500$ lbs.

Small utility $=<3,500 \mathrm{lbs} . \quad$ Medium utility=3,500-4,799 lbs. Large utility=4,800-8,500 lbs.

${ }^{\text {a }}$ Sales period is October 1 of the previous year through September 30 of the current year.

${ }^{\mathrm{b}} 1$ liter $=61.02$ cubic inches.

${ }^{\mathrm{c}}$ Data are not available. 
The large car size class is the only class that showed a decline in curb weight from 1992 to 2002.

Table 4.12

Sales-Weighted Curb Weight of New Domestic and Import Automobiles by Size Class, Sales Periods ${ }^{\mathrm{a}}$ 1976-2002

(pounds)

\begin{tabular}{|c|c|c|c|c|c|c|c|}
\hline $\begin{array}{c}\text { Sales } \\
\text { period }^{\mathrm{a}}\end{array}$ & Minicompact & Subcompact & Compact & Midsize & Large & $\begin{array}{c}\text { Two } \\
\text { seater }\end{array}$ & All \\
\hline 1976 & $\mathrm{~b}$ & 2,577 & 3,609 & 4,046 & 4,562 & 2,624 & 3,608 \\
\hline 1977 & 2,228 & 2,586 & 3,550 & 3,900 & 4,026 & 2,608 & 3,424 \\
\hline 1978 & 2,200 & 2,444 & 3,138 & 3,427 & 3,956 & 2,763 & 3,197 \\
\hline 1979 & 2,120 & 2,367 & 3,048 & 3,287 & 3,763 & 2,699 & 3,000 \\
\hline 1980 & 2,154 & 2,270 & 2,813 & 3,081 & 3,667 & 2,790 & 2,790 \\
\hline 1981 & 1,920 & 2,370 & 2,382 & 2,996 & 3,672 & 2,744 & 2,744 \\
\hline 1982 & 2,002 & 2,302 & 2,422 & 2,992 & 3,703 & 2,525 & 2,730 \\
\hline 1983 & 2,072 & 2,334 & 2,441 & 3,027 & 3,779 & 2,663 & 2,788 \\
\hline 1984 & 2,376 & 2,380 & 2,454 & 2,990 & 3,734 & 2,559 & 2,788 \\
\hline 1985 & 2,211 & 2,392 & 2,464 & 2,954 & 3,575 & 2,539 & 2,743 \\
\hline 1986 & 2,120 & 2,415 & 2,432 & 2,857 & 3,451 & 2,575 & 2,675 \\
\hline 1987 & 1,960 & 2,423 & 2,474 & 2,857 & 3,483 & 2,602 & 2,689 \\
\hline 1988 & 1,933 & 2,346 & 2,558 & 2,880 & 3,487 & 2,693 & 2,717 \\
\hline 1989 & 2,576 & 2,357 & 2,517 & 2,985 & 3,496 & 2,735 & 2,760 \\
\hline 1990 & 2,651 & 2,368 & 2,637 & 3,065 & 3,594 & 2,656 & 2,828 \\
\hline 1991 & 2,584 & 2,406 & 2,652 & 3,085 & 3,650 & 2,707 & 2,848 \\
\hline 1992 & 2,395 & 2,444 & 2,674 & 3,131 & 3,670 & 2,770 & 2,879 \\
\hline 1993 & 2,449 & 2,478 & 2,659 & 3,142 & 3,615 & 2,967 & 2,894 \\
\hline 1994 & 2,719 & 2,571 & 2,639 & 3,171 & 3,657 & 3,035 & 2,921 \\
\hline 1995 & 2,831 & 2,552 & 2,647 & 3,179 & 3,648 & 2,947 & 2,937 \\
\hline 1996 & 2,847 & 2,533 & 2,667 & 3,203 & 3,671 & 2,985 & 2,950 \\
\hline 1997 & 2,997 & 2,489 & 2,737 & 3,241 & 3,653 & 2,863 & 2,977 \\
\hline 1998 & 3,004 & 2,584 & 2,703 & 3,198 & 3,675 & 2,956 & 3,002 \\
\hline 1999 & 2,835 & 2,626 & 2,755 & 3,198 & 3,689 & 3,007 & 3,034 \\
\hline 2000 & 2,906 & 2,635 & 2,800 & 3,215 & 3,680 & 2,943 & 3,052 \\
\hline 2001 & 3,332 & 2,803 & 2,720 & 3,197 & 3,606 & 2,849 & 3,047 \\
\hline 2002 & 3,068 & 2,928 & 2,731 & 3,218 & 3,587 & 3,086 & 3,066 \\
\hline \multicolumn{8}{|c|}{ Average annual percentage change } \\
\hline 1976-2002 & $1.3 \%^{\mathrm{c}}$ & $0.5 \%$ & $-1.1 \%$ & $-0.9 \%$ & $-0.9 \%$ & $0.6 \%$ & $-0.6 \%$ \\
\hline 1992-2002 & $2.5 \%$ & $1.8 \%$ & $0.2 \%$ & $0.3 \%$ & $-0.2 \%$ & $1.1 \%$ & $0.6 \%$ \\
\hline
\end{tabular}

Source:

Oak Ridge National Laboratory, Light Vehicle MPG and Market Shares System, Oak Ridge, TN, 2003.

(Additional resources: www-cta.ornl.gov)

\footnotetext{
${ }^{\text {a }}$ Sales period is October 1 of the previous year through September 30 of the current year.

${ }^{\mathrm{b}}$ There were no minicompact automobiles sold in 1976.

${ }^{c}$ Average annual percentage change begins with 1977.
} 
The sales-weighted interior space has not changed much for midsize automobiles over the last two decades, but has increased for subcompact autos and decreased for compact and large autos.

Table 4.13

Sales-Weighted Interior Space of New Domestic and Import Automobiles by Size Class, Sales Periods ${ }^{\mathrm{a}}$ 1976-2002

(cubic feet)

\begin{tabular}{|c|c|c|c|c|c|c|}
\hline Sales period ${ }^{\mathrm{a}}$ & $\begin{array}{c}\begin{array}{c}\text { Minicompact } \\
(<85)\end{array} \\
\end{array}$ & $\begin{array}{c}\text { Subcompact } \\
(85-99)\end{array}$ & $\begin{array}{c}\text { Compact } \\
(100-109) \\
\end{array}$ & $\begin{array}{c}\text { Midsize } \\
(110-119)\end{array}$ & $\begin{array}{c}\text { Large } \\
(>120)\end{array}$ & $\mathrm{All}^{\mathrm{b}}$ \\
\hline 1977 & 78.8 & 89.8 & 107.1 & 113.0 & 128.0 & 107.9 \\
\hline 1978 & 79.4 & 89.8 & 105.3 & 112.9 & 128.5 & 107.9 \\
\hline 1979 & 80.0 & 90.2 & 105.8 & 113.4 & 130.1 & 106.9 \\
\hline 1980 & 82.4 & 89.9 & 105.4 & 113.5 & 130.8 & 104.9 \\
\hline 1981 & 83.3 & 90.2 & 103.6 & 113.7 & 130.6 & 105.5 \\
\hline 1982 & 83.1 & 91.3 & 102.9 & 113.9 & 130.4 & 106.0 \\
\hline 1983 & 82.7 & 93.3 & 103.0 & 113.1 & 131.3 & 107.3 \\
\hline 1984 & 77.0 & 93.8 & 103.0 & 113.3 & 130.4 & 108.0 \\
\hline 1985 & 77.8 & 94.1 & 103.1 & 113.5 & 129.7 & 107.9 \\
\hline 1986 & 80.1 & 94.5 & 102.8 & 113.8 & 127.6 & 107.0 \\
\hline 1987 & 81.6 & 93.1 & 103.0 & 113.9 & 127.5 & 106.9 \\
\hline 1988 & 81.0 & 93.5 & 103.3 & 113.6 & 127.2 & 107.0 \\
\hline 1989 & 75.0 & 93.3 & 102.7 & 113.8 & 127.4 & 107.5 \\
\hline 1990 & 79.9 & 93.9 & 103.2 & 113.8 & 127.8 & 107.3 \\
\hline 1991 & 79.6 & 94.4 & 103.2 & 113.8 & 128.3 & 107.1 \\
\hline 1992 & 79.1 & 94.0 & 104.2 & 114.0 & 129.2 & 107.5 \\
\hline 1993 & 79.2 & 94.5 & 104.0 & 114.0 & 128.9 & 108.0 \\
\hline 1994 & 79.4 & 94.4 & 103.8 & 113.8 & 128.8 & 108.0 \\
\hline 1995 & 78.5 & 93.8 & 103.9 & 114.3 & 128.1 & 108.7 \\
\hline 1996 & 76.7 & 94.9 & 103.4 & 114.2 & 128.0 & 108.8 \\
\hline 1997 & 77.2 & 95.6 & 103.2 & 114.6 & 128.0 & 108.7 \\
\hline 1998 & 66.9 & 97.0 & 102.2 & 114.4 & 127.7 & 109.2 \\
\hline 1999 & 76.3 & 96.7 & 103.3 & 114.1 & 127.1 & 109.5 \\
\hline 2000 & 76.3 & 96.6 & 103.1 & 114.2 & 126.4 & 109.3 \\
\hline 2001 & 78.2 & 94.6 & 103.2 & 113.5 & 125.2 & 109.4 \\
\hline 2002 & 80.3 & 94.7 & 103.7 & 114.8 & 125.0 & 110.1 \\
\hline \multicolumn{7}{|c|}{ Average annual percentage change } \\
\hline 1977-2002 & $0.1 \%$ & $0.2 \%$ & $-0.1 \%$ & $0.1 \%$ & $-0.1 \%$ & $0.1 \%$ \\
\hline 1992-2002 & $0.2 \%$ & $0.1 \%$ & $0.0 \%$ & $0.1 \%$ & $-0.3 \%$ & $0.2 \%$ \\
\hline
\end{tabular}

Source:

Oak Ridge National Laboratory, Light Vehicle MPG and Market Shares System, Oak Ridge, TN, 2003.

(Additional resources: www-cta.ornl.gov)

${ }^{a}$ Sales period is October 1 of the previous year through September 30 of the current year.

${ }^{\mathrm{b}}$ Interior volumes of two-seaters are not reported to EPA. 
The sales-weighted wheelbase of new automobiles and light trucks was rising in the 1990's. In this decade, automobile wheel-base has not varied much while light truck wheel-base has declined slightly.

Table 4.14

Sales-Weighted Wheelbase of New Automobiles and Light Trucks, Sales Periods ${ }^{\mathrm{a}}$ 1976-2002 (inches)

\begin{tabular}{cccc}
\hline $\begin{array}{c}\text { Sales } \\
\text { period }^{\text {a }}\end{array}$ & Automobiles & $\begin{array}{c}\text { Light } \\
\text { trucks }\end{array}$ & $\begin{array}{c}\text { Automobiles and } \\
\text { light trucks } \\
\text { combined }\end{array}$ \\
\hline 1976 & 110.78 & 118.87 & 112.03 \\
1977 & 109.75 & 117.79 & 111.05 \\
1978 & 107.67 & 116.23 & 108.65 \\
1979 & 105.77 & 116.27 & 107.93 \\
1980 & 103.61 & 114.54 & 105.76 \\
1981 & 102.97 & 114.86 & 105.10 \\
1982 & 103.01 & 114.87 & 105.60 \\
1983 & 103.76 & 113.73 & 106.10 \\
1984 & 103.50 & 113.87 & 106.21 \\
1985 & 102.96 & 113.98 & 106.02 \\
1986 & 102.27 & 113.40 & 105.48 \\
1987 & 102.11 & 113.27 & 105.52 \\
1988 & 102.21 & 111.79 & 105.21 \\
1989 & 102.66 & 112.23 & 105.71 \\
1990 & 103.13 & 111.41 & 105.85 \\
1991 & 103.27 & 111.09 & 105.82 \\
1992 & 103.60 & 112.68 & 106.78 \\
1993 & 104.03 & 112.57 & 107.21 \\
1994 & 104.31 & 113.23 & 107.75 \\
1995 & 104.95 & 113.37 & 108.31 \\
1996 & 105.04 & 113.36 & 108.53 \\
1997 & 105.36 & 113.36 & 108.89 \\
1998 & 105.55 & 114.53 & 109.76 \\
1999 & 105.77 & 114.70 & 110.06 \\
2000 & 105.89 & 114.05 & 109.81 \\
2001 & 105.66 & 113.04 & 109.64 \\
2002 & 105.87 & 112.91 & 109.84 \\
& Average annual percentage change \\
$1976-2002$ & $-0.2 \%$ & $-0.2 \%$ & $-0.1 \%$ \\
$1992-2002$ & $0.2 \%$ & $0.0 \%$ & $0.3 \%$ \\
\hline & & & \\
\hline
\end{tabular}

Source:

Oak Ridge National Laboratory, Light Vehicle MPG and Market Shares System, Oak Ridge, TN, 2003.

(Additional resources: www-cta.ornl.gov)

${ }^{\text {a }}$ Sales period is October 1 of the current year through September 30 of the next year. 
The average auto lost over 300 pounds from 1978 to 1985, but gained a few pounds back since then. Much of the weight reduction was due to the declining use of conventional steel and iron and the increasing use of aluminum and plastics. Conventional steel, however, remained the predominant component of automobiles in 2001 with a $40.8 \%$ share of total materials. As conventional steel use has been decreasing, use of high-strength steel has increased.

Table 4.15

Average Material Consumption for a Domestic Automobile, 1978, 1985, and 2001

\begin{tabular}{|c|c|c|c|c|c|c|}
\hline \multirow[b]{2}{*}{ Material } & \multicolumn{2}{|c|}{1978} & \multicolumn{2}{|c|}{1985} & \multicolumn{2}{|c|}{2001} \\
\hline & Pounds & Percentage & Pounds & Percentage & Pounds & Percentage \\
\hline Conventional steel $^{\mathrm{a}}$ & $1,880.0$ & $53.8 \%$ & $1,481.5$ & $46.5 \%$ & $1,349.0$ & $40.8 \%$ \\
\hline High-strength steel & 127.5 & $3.6 \%$ & 217.5 & $6.8 \%$ & 351.5 & $10.6 \%$ \\
\hline Stainless steel & 25.0 & $0.7 \%$ & 29.0 & $0.9 \%$ & 54.5 & $1.6 \%$ \\
\hline Other steels & 56.0 & $1.6 \%$ & 54.5 & $1.7 \%$ & 25.5 & $0.8 \%$ \\
\hline Iron & 503.0 & $14.4 \%$ & 468.0 & $14.7 \%$ & 345.0 & $10.4 \%$ \\
\hline Aluminum & 112.0 & $3.2 \%$ & 138.0 & $4.3 \%$ & 256.5 & $7.8 \%$ \\
\hline Rubber & 141.5 & $4.1 \%$ & 136.0 & $4.3 \%$ & 145.5 & $4.4 \%$ \\
\hline Plastics/composites & 176.0 & $5.0 \%$ & 211.5 & $6.6 \%$ & 253.0 & $7.6 \%$ \\
\hline Glass & 88.0 & $2.5 \%$ & 85.0 & $2.7 \%$ & 98.5 & $3.0 \%$ \\
\hline Copper & 39.5 & $1.1 \%$ & 44.0 & $1.4 \%$ & 46.0 & $1.4 \%$ \\
\hline Zinc die castings & 28.0 & $0.8 \%$ & 18.0 & $0.5 \%$ & 11.0 & $0.3 \%$ \\
\hline Powder metal parts & 16.0 & $0.5 \%$ & 19.0 & $0.6 \%$ & 37.5 & $1.1 \%$ \\
\hline Fluids \& lubricants & 189.0 & $5.4 \%$ & 184.0 & $5.8 \%$ & 196.0 & $5.9 \%$ \\
\hline Other materials & 112.5 & $3.2 \%$ & 101.5 & $3.2 \%$ & 139.5 & $4.2 \%$ \\
\hline Total & $3,494.0$ & $100.0 \%$ & $3,187.5$ & $100.0 \%$ & $3,309.0$ & $100.0 \%$ \\
\hline
\end{tabular}

Source:

American Metal Market, www.amm.com/ref/carmat98.htm, New York, NY, 2000. (Additional resources: www.amm.com)

${ }^{a}$ Includes cold-rolled and pre-coated steel. 
The number of franchised dealerships which sell new light-duty vehicles (cars and light trucks) has declined 27\% since 1970, though new vehicle sales have increased. The average number of vehicles sold per dealer in 2000 was 774 vehicles per dealer - more than double the 1970 number.

Table 4.16

New Light Vehicle Dealerships and Sales, 1970-2000

\begin{tabular}{|c|c|c|c|}
\hline $\begin{array}{c}\text { Calendar } \\
\text { year }\end{array}$ & $\begin{array}{l}\text { Number of } \\
\text { franchised new } \\
\text { light vehicle } \\
\text { dealerships }^{\mathrm{a}}\end{array}$ & $\begin{array}{c}\text { New } \\
\text { light vehicle } \\
\text { sales } \\
\text { (thousands) }\end{array}$ & $\begin{array}{c}\text { Light vehicle } \\
\text { sales } \\
\text { per dealer } \\
\end{array}$ \\
\hline 1970 & 30,800 & 9,867 & 320 \\
\hline 1971 & 30,300 & 12,006 & 396 \\
\hline 1972 & 30,100 & 13,189 & 438 \\
\hline 1973 & 30,100 & 14,184 & 471 \\
\hline 1974 & 30,000 & 11,191 & 373 \\
\hline 1975 & 29,600 & 10,905 & 368 \\
\hline 1976 & 29,300 & 13,066 & 446 \\
\hline 1977 & 29,100 & 14,613 & 502 \\
\hline 1978 & 29,000 & 15,122 & 521 \\
\hline 1979 & 28,500 & 13,984 & 491 \\
\hline 1980 & 27,900 & 11,419 & 409 \\
\hline 1981 & 26,350 & 10,725 & 407 \\
\hline 1982 & 25,700 & 10,452 & 407 \\
\hline 1983 & 24,725 & 12,166 & 492 \\
\hline 1984 & 24,725 & 14,254 & 577 \\
\hline 1985 & 24,725 & 15,501 & 627 \\
\hline 1986 & 24,825 & 16,047 & 646 \\
\hline 1987 & 25,150 & 14,888 & 592 \\
\hline 1988 & 25,025 & 15,426 & 616 \\
\hline 1989 & 25,000 & 14,508 & 580 \\
\hline 1990 & 24,825 & 13,849 & 558 \\
\hline 1991 & 24,200 & 12,298 & 508 \\
\hline 1992 & 23,500 & 12,842 & 546 \\
\hline 1993 & 22,950 & 13,869 & 604 \\
\hline 1994 & 22,850 & 15,023 & 657 \\
\hline 1995 & 22,800 & 14,688 & 644 \\
\hline 1996 & 22,750 & 15,046 & 661 \\
\hline 1997 & 22,700 & 15,069 & 664 \\
\hline 1998 & 22,600 & 15,441 & 683 \\
\hline 1999 & 22,400 & 16,771 & 748 \\
\hline 2000 & 22,250 & 17,234 & 774 \\
\hline & \multicolumn{3}{|c|}{ Average annual percentage change } \\
\hline $1970-2000$ & $-1.1 \%$ & $1.9 \%$ & $3.0 \%$ \\
\hline $1990-2000$ & $-1.1 \%$ & $2.2 \%$ & $3.3 \%$ \\
\hline
\end{tabular}

Source:

Number of dealers - National Automobile Dealers Association, Automotive Executive Magazine, 2001. (Additional resources: www.nada.org) Light-duty vehicle sales - See tables 4.5 and 4.6.

\footnotetext{
${ }^{\mathrm{a}}$ As of the beginning of the year.
} 
The number of conventional refueling stations is declining while the number of vehicles fueling at those stations continues to rise. In 2001, there were 0.79 fueling stations per thousand vehicles. Data for alternative fuels in 2001 indicate that there was an average of 10.91 stations per thousand alternative fuel vehicles.

Table 4.17

Conventional and Alternative Fuel Refueling Stations

\begin{tabular}{lccc}
\hline & $\begin{array}{c}\text { Number of } \\
\text { retail outlets }\end{array}$ & $\begin{array}{c}\text { Vehicles } \\
\text { in operation } \\
\text { (thousands) }\end{array}$ & $\begin{array}{c}\text { Stations per } \\
\text { thousand vehicles }\end{array}$ \\
\cline { 2 - 4 } trear & \multicolumn{3}{c}{ Conventional fuels } \\
1993 & 207,416 & 186,315 & 1.11 \\
1994 & 202,878 & 188,714 & 1.08 \\
1995 & 195,455 & 193,441 & 1.01 \\
1996 & 190,246 & 198,294 & 0.96 \\
1998 & 187,892 & 201,071 & 0.93 \\
1999 & 182,596 & 205,043 & 0.89 \\
2000 & 180,567 & 209,509 & 0.86 \\
2001 & 175,941 & 213,300 & 0.82 \\
2002 & 171,169 & 216,683 & 0.79 \\
\hline \multicolumn{5}{c}{170,678} & a & \\
\hline LPG & \multicolumn{3}{c}{ Alternative fuels, 2002 } \\
CNG & 3,431 & 281 & 12.21 \\
Electricity & 1,166 & 126 & 9.25 \\
M85/M100 & 872 & 20 & 43.60 \\
LNG & 0 & 6 & 0.00 \\
E85/E95 & 35 & 3 & 11.67 \\
Total & 149 & 82 & 1.82 \\
\hline
\end{tabular}

\section{Source:}

Conventional refueling stations: National Petroleum News Survey, 2002.

Alternative fuel refueling stations: Alternative Fuels Data Center, www.afdc.doe.gov.

Conventional vehicles: The Polk Company, Detroit, MI, FURTHER REPRODUCTION PROHIBITED.

Alternative fuels vehicles: U.S. Department of Energy, Energy Information Administration, Alternatives to Traditional

Transportation Fuels web site, www.eia.doe.gov/cneaf/alternate/page/datatables/atf01-13_00.html

Note:

The County Business Patterns (CBP) data published by the Bureau of the Census tells the number of establishments by North American Industry Classification System (NAICS). NAICS is an industry classification system that groups establishments into industries based on the activities in which they are primarily engaged. NAICS 447 represents gasoline stations. However, the CBP gasoline station data differ from the National Petroleum News Survey data; the CBP may not include every gasoline retail outlet due to the classification of the primary activity of the business.

\footnotetext{
${ }^{\mathrm{a}}$ Data are not available.
} 
The Corporate Average Fuel Economy standards were established by the U.S. Energy Policy and Conservation Act of 1975 (PL94-163). These standards must be met at the manufacturer level. Though the averages shown here indicate the standards were met in most years, some manufacturers fell short of meeting the standards while others exceeded them.

Table 4.18

Automobile Corporate Average Fuel Economy (CAFE) Standards versus Sales-Weighted Fuel Economy Estimates, 1978-2003 (miles per gallon)

\begin{tabular}{|c|c|c|c|c|c|}
\hline \multirow{3}{*}{$\begin{array}{l}\text { Model } \\
\text { year }^{\mathrm{b}}\end{array}$} & \multicolumn{4}{|c|}{ Automobiles } & \multirow{3}{*}{$\begin{array}{l}\text { CAFE estimates } \\
\text { Autos and light } \\
\text { trucks combined }\end{array}$} \\
\hline & \multirow{2}{*}{$\begin{array}{c}\text { CAFE } \\
\text { standards }\end{array}$} & \multicolumn{3}{|c|}{ CAFE estimates $^{c}$} & \\
\hline & & Domestic & Import & Combined & \\
\hline 1978 & 18.0 & 18.7 & 27.3 & 19.9 & 19.9 \\
\hline 1979 & 19.0 & 19.3 & 26.1 & 20.3 & 20.1 \\
\hline 1980 & 20.0 & 22.6 & 29.6 & 24.3 & 23.1 \\
\hline 1981 & 22.0 & 24.2 & 31.5 & 25.9 & 24.6 \\
\hline 1982 & 24.0 & 25.0 & 31.1 & 26.6 & 25.1 \\
\hline 1983 & 26.0 & 24.4 & 32.4 & 26.4 & 24.8 \\
\hline 1984 & 27.0 & 25.5 & 32.0 & 26.9 & 25.0 \\
\hline 1985 & 27.5 & 26.3 & 31.5 & 27.6 & 25.4 \\
\hline 1986 & 26.0 & 26.9 & 31.6 & 28.2 & 25.9 \\
\hline 1987 & 26.0 & 27.0 & 31.2 & 28.4 & 26.2 \\
\hline 1988 & 26.0 & 27.4 & 31.5 & 28.0 & 26.0 \\
\hline 1989 & 26.5 & 27.2 & 30.8 & 28.4 & 25.6 \\
\hline 1990 & 27.5 & 26.9 & 29.9 & 27.9 & 25.4 \\
\hline 1991 & 27.5 & 27.3 & 30.1 & 28.4 & 25.6 \\
\hline 1992 & 27.5 & 27.0 & 29.2 & 27.9 & 25.1 \\
\hline 1993 & 27.5 & 27.8 & 29.6 & 28.4 & 25.2 \\
\hline 1994 & 27.5 & 27.5 & 29.7 & 28.3 & 24.7 \\
\hline 1995 & 27.5 & 27.7 & 30.3 & 28.6 & 24.9 \\
\hline 1996 & 27.5 & 28.1 & 29.6 & 28.5 & 24.9 \\
\hline 1997 & 27.5 & 27.8 & 30.1 & 28.7 & 24.6 \\
\hline 1998 & 27.5 & 28.6 & 29.2 & 28.8 & 24.7 \\
\hline 1999 & 27.5 & 28.0 & 29.0 & 28.3 & 24.5 \\
\hline 2000 & 27.5 & 28.7 & 28.3 & 28.5 & 24.8 \\
\hline 2001 & 27.5 & 28.7 & 29.0 & 28.8 & 24.6 \\
\hline 2002 & 27.5 & 29.0 & 28.7 & 28.9 & 24.6 \\
\hline 2003 & 27.5 & 29.7 & 29.1 & 29.5 & 25.1 \\
\hline
\end{tabular}

Source:

U.S. Department of Transportation, NHTSA, "Summary of Fuel Economy Performance," Washington, DC, March 2003. (Additional resources: www.nhtsa.dot.gov) manufacturer.

aOnly vehicles with at least 75 percent domestic content can be counted in the average domestic fuel economy for a

\footnotetext{
${ }^{\mathrm{b}}$ Model year as determined by the manufacturer on a vehicle by vehicle basis.
}

${ }^{c}$ All CAFE calculations are sales-weighted. 
The Corporate Average Fuel Economy standards for light trucks are lower than the automobile standards. Light trucks include pickups, minivans, sport utility vehicles and vans.

T able 4.19

Light Truck Corporate Average Fuel Economy (CAFE) Standards versus Sales-Weighted Fuel Economy Estimates, 1978-2003 (miles per gallon)

\begin{tabular}{|c|c|c|c|c|c|}
\hline \multirow{3}{*}{$\begin{array}{l}\text { Model } \\
\text { year }^{\mathrm{b}}\end{array}$} & \multicolumn{4}{|c|}{ Light trucks ${ }^{\mathrm{c}}$} & \multirow{3}{*}{$\begin{array}{l}\text { CAFE estimates } \\
\text { Autos and light } \\
\text { trucks combined }\end{array}$} \\
\hline & \multirow{2}{*}{$\begin{array}{l}\text { CAFE } \\
\text { standards }\end{array}$} & \multicolumn{3}{|c|}{ CAFE estimates ${ }^{\mathrm{d}}$} & \\
\hline & & Domestic & Import & Combined & \\
\hline 1978 & \multirow{4}{*}{$\begin{array}{l}\mathrm{e} \\
\mathrm{e}\end{array}$} & $\mathrm{f}$ & $\mathrm{f}$ & $\mathrm{g}$ & 19.9 \\
\hline 1979 & & 17.7 & 20.8 & 18.2 & 20.1 \\
\hline 1980 & & 16.8 & 24.3 & 18.5 & 23.1 \\
\hline 1981 & & 18.3 & 27.4 & 20.1 & 24.6 \\
\hline 1982 & 17.5 & 19.2 & 27.0 & 20.5 & 25.1 \\
\hline 1983 & 19.0 & 19.6 & 27.1 & 20.7 & 24.8 \\
\hline 1984 & 20.0 & 19.3 & 26.7 & 20.6 & 25.0 \\
\hline 1985 & 19.5 & 19.6 & 26.5 & 20.7 & 25.4 \\
\hline 1986 & 20.0 & 20.0 & 25.9 & 21.5 & 25.9 \\
\hline 1987 & 20.5 & 20.5 & 25.2 & 21.7 & 26.2 \\
\hline 1988 & 20.5 & 20.6 & 24.6 & 21.3 & 26.0 \\
\hline 1989 & 20.5 & 20.4 & 23.5 & 21.0 & 25.6 \\
\hline 1990 & 20.0 & 20.3 & 23.0 & 20.8 & 25.4 \\
\hline 1991 & 20.2 & 20.9 & 23.0 & 21.3 & 25.6 \\
\hline 1992 & 20.2 & 20.5 & 22.7 & 20.8 & 25.1 \\
\hline 1993 & 20.4 & 20.7 & 22.8 & 21.0 & 25.2 \\
\hline 1994 & 20.5 & 20.5 & 22.0 & 20.8 & 24.7 \\
\hline 1995 & 20.6 & 20.3 & 21.5 & 20.5 & 24.9 \\
\hline 1996 & 20.7 & 20.5 & 22.1 & 20.8 & 24.9 \\
\hline 1997 & 20.7 & 20.1 & 22.1 & 20.6 & 24.6 \\
\hline 1998 & 20.7 & 20.4 & 23.0 & 21.1 & 24.7 \\
\hline 1999 & 20.7 & & & 20.9 & 24.5 \\
\hline 2000 & 20.7 & $\mathrm{f}$ & $\mathrm{f}$ & 21.3 & 24.8 \\
\hline 2001 & 20.7 & f & f & 20.9 & 24.6 \\
\hline 2002 & 20.7 & f & f & 21.3 & 24.6 \\
\hline 2003 & 20.7 & $f$ & $\mathrm{f}$ & 21.8 & 25.1 \\
\hline
\end{tabular}

Source:

U.S. Department of Transportation, NHTSA, "Summary of Fuel Economy Performance," Washington, DC, March 2003. (Additional resources: www.nhtsa.dot.gov) manufacturer.

${ }^{a}$ Only vehicles with at least 75 percent domestic content can be counted in the average domestic fuel economy for a

${ }^{\mathrm{b}}$ Model year as determined by the manufacturer on a vehicle by vehicle basis.

'Represents two- and four-wheel drive trucks combined. Gross vehicle weight of 0-6,000 pounds for model year 1978-1979 and 0-8,500 pounds for subsequent years.

${ }^{\mathrm{d}}$ All CAFE calculations are sales-weighted.

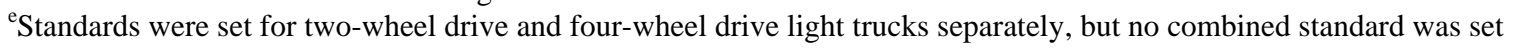
in this year.

${ }^{\mathrm{f}}$ Data are not available. 
Manufacturers of autos and light trucks whose vehicles do not meet the CAFE standards are fined. Data from the National Highway Traffic Safety Administration show that \$34 million has been collected from the manufacturers for model year (MY) 2001 and \$51 million for MY 2000.

Table 4.20

Corporate Average Fuel Economy (CAFE) Fines Collected, 1983-2001 (thousands)

\begin{tabular}{ccc}
\hline $\begin{array}{c}\text { Model } \\
\text { year }\end{array}$ & $\begin{array}{c}\text { Current } \\
\text { dollars }\end{array}$ & $\begin{array}{c}\text { 2001 constant } \\
\text { dollars }^{\mathrm{b}}\end{array}$ \\
\hline 1983 & 58 & 103 \\
1984 & 5,958 & 10,156 \\
1985 & 15,565 & 25,618 \\
1986 & 29,872 & 48,269 \\
1987 & 31,261 & 48,735 \\
1988 & 44,519 & 66,647 \\
1989 & 47,381 & 67,670 \\
1990 & 48,429 & 65,621 \\
1991 & 42,243 & 54,928 \\
1992 & 38,287 & 48,329 \\
1993 & 28,688 & 35,161 \\
1994 & 31,499 & 37,641 \\
1995 & 40,787 & 47,398 \\
1996 & 19,302 & 21,787 \\
1997 & 36,212 & 39,957 \\
1998 & 21,740 & 23,620 \\
1999 & 27,516 & 29,251 \\
2000 & 51,067 & 52,520 \\
2001 & 33,974 & 33,974 \\
\hline & &
\end{tabular}

\section{Source:}

U.S. Department of Transportation, National Highway Traffic Safety Administration, Office of Vehicle Safety Compliance, Washington, DC, January 2003.

(Additional resources: www.nhtsa.dot.gov)

${ }^{\mathrm{a}}$ These are fines which are actually collected. Fines which are assessed in certain year may not have been collected in that year.

\footnotetext{
${ }^{\mathrm{b}}$ Adjusted using the Consumer Price Inflation Index.
} 
Consumers must pay the Gas Guzzler Tax when purchasing an automobile that has an Environmental Protection Agency (EPA) fuel economy rating less than that stipulated in the table below. The Gas Guzzler Tax doubled in 1991 after remaining constant from 1986 to 1990. The tax has not changed since 1991. This tax does not apply to light trucks such as pickups, minivans, sport utility vehicles, and vans.

Table 4.21

The Gas Guzzler Tax on New Cars (dollars per vehicle)

\begin{tabular}{crrrrrrrr}
\hline $\begin{array}{c}\text { Vehicle fuel } \\
\text { economy } \\
\text { (mpg) }\end{array}$ & 1980 & 1981 & \multicolumn{1}{c}{1982} & 1983 & 1984 & 1985 & $1986-90$ & $1991+$ \\
\hline Over 22.5 & 0 & 0 & 0 & 0 & 0 & 0 & 0 & 0 \\
$22.0-22.5$ & 0 & 0 & 0 & 0 & 0 & 0 & 500 & 1,000 \\
$21.5-22.0$ & 0 & 0 & 0 & 0 & 0 & 0 & 500 & 1,000 \\
$21.0-21.5$ & 0 & 0 & 0 & 0 & 0 & 0 & 650 & 1,300 \\
$20.5-21.0$ & 0 & 0 & 0 & 0 & 0 & 500 & 650 & 1,300 \\
$20.0-20.5$ & 0 & 0 & 0 & 0 & 0 & 500 & 850 & 1,700 \\
$19.5-20.0$ & 0 & 0 & 0 & 0 & 0 & 600 & 850 & 1,700 \\
$19.0-19.5$ & 0 & 0 & 0 & 0 & 450 & 600 & 1,050 & 2,100 \\
$18.5-19.0$ & 0 & 0 & 0 & 350 & 450 & 800 & 1,050 & 2,100 \\
$18.0-18.5$ & 0 & 0 & 200 & 350 & 600 & 800 & 1,300 & 2,600 \\
$17.5-18.0$ & 0 & 0 & 200 & 500 & 600 & 1,000 & 1,300 & 2,600 \\
$17.0-17.5$ & 0 & 0 & 350 & 500 & 750 & 1,000 & 1,500 & 3,000 \\
$16.5-17.0$ & 0 & 200 & 350 & 650 & 750 & 1,200 & 1,500 & 3,000 \\
$16.0-16.5$ & 0 & 200 & 450 & 650 & 950 & 1,200 & 1,850 & 3,700 \\
$15.5-16.0$ & 0 & 350 & 450 & 800 & 950 & 1,500 & 1,850 & 3,700 \\
$15.0-15.5$ & 0 & 350 & 600 & 800 & 1,150 & 1,500 & 2,250 & 4,500 \\
$14.5-15.0$ & 200 & 450 & 600 & 1,000 & 1,150 & 1,800 & 2,250 & 4,500 \\
$14.0-14.5$ & 200 & 450 & 750 & 1,000 & 1,450 & 1,800 & 2,700 & 5,400 \\
$13.5-14.0$ & 300 & 550 & 750 & 1,250 & 1,450 & 2,200 & 2,700 & 5,400 \\
$13.0-13.5$ & 300 & 550 & 950 & 1,250 & 1,750 & 2,200 & 3,200 & 6,400 \\
$12.5-13.0$ & 550 & 650 & 950 & 1,550 & 1,750 & 2,650 & 3,200 & 6,400 \\
Under 12.5 & 550 & 650 & 1,200 & 1,550 & 2,150 & 2,650 & 3,850 & 7,700 \\
\hline
\end{tabular}

\section{Source:}

Internal Revenue Service, Form 6197, (Rev. 1-91), "Gas Guzzler Tax."

(Additional resources: www.irs.ustreas.gov) 
Consumers continue to demand gas guzzling automobiles. The IRS collected over $\$ 78$ million in 2001 from those buying autos with fuel economy less than 22.5 miles per gallon. This tax does not apply to light trucks such as pickups, minivans, sport utility vehicles, and vans.

Table 4.22

Tax Receipts from the Sale of Gas Guzzlers, 1980-2001 (thousands)

\begin{tabular}{crr}
\hline $\begin{array}{c}\text { Model } \\
\text { year }\end{array}$ & $\begin{array}{c}\text { Current } \\
\text { dollars }\end{array}$ & $\begin{array}{c}\text { 2001 constant } \\
\text { dollars }^{\mathrm{a}}\end{array}$ \\
\hline 1980 & 740 & 1,590 \\
1981 & 780 & 1,520 \\
1982 & 1,720 & 3,157 \\
1983 & 4,020 & 7,148 \\
1984 & 8,820 & 15,034 \\
1985 & 39,790 & 65,491 \\
1986 & 147,660 & 238,600 \\
1987 & 145,900 & 227,455 \\
1988 & 116,780 & 174,824 \\
1989 & 109,640 & 156,591 \\
1990 & 103,200 & 139,837 \\
1991 & 118,400 & 153,955 \\
1992 & 144,200 & 182,023 \\
1993 & 111,600 & 136,778 \\
1994 & 64,100 & 76,600 \\
1995 & 73,500 & 85,412 \\
1996 & 52,600 & 59,372 \\
1997 & 48,200 & 53,185 \\
1998 & 47,700 & 51,826 \\
1999 & 68,300 & 72,605 \\
2000 & 70,800 & 72,815 \\
2001 & 78,200 & 78,200 \\
\hline & & \\
\hline & & 1900 \\
\hline
\end{tabular}

\section{Source:}

Ward's Communications, Motor Vehicle Facts and Figures, 2002, Detroit, MI, 2002, p. 85. Original data source: Internal Revenue Service.

\footnotetext{
${ }^{\mathrm{a}}$ Adjusted using the Consumer Price Inflation Index.
} 


\section{Fuel Economy by Vehicle Speed}

ORNL has developed fuel consumption and emissions lookup tables for the Federal Highway Administration, for use in their TRAF series of traffic models (NETSIM, CORSIM, FRESIM), although more generic uses are also possible. To develop the databased models, vehicles are tested both on-road and on a chassis dynamometer. Engine parameters are measured on-road under real-world driving conditions that cover the vehicle's entire operating envelope. Emissions and fuel consumption are then measured on the chassis dynamometer as functions of engine conditions. The two data sets are merged to produce the final three-dimensional maps as functions of vehicle speed and acceleration. Eight wellfunctioning, late-model vehicles, and one 1997 model vehicle, have been tested thus far in fully warmed-up conditions.

Similar continuing work is planned for the Department of Energy as well as FHWA, which will include more well-functioning, late-model vehicles, pre-control (1960's) vehicles, malfunctioning high-emitter vehicles, light-duty diesel vehicles (cars and pickup trucks), alternative fuel vehicles, and possibly heavy-duty diesel vehicles. ORNL will also be developing cold-start algorithms to enhance the existing models, since emissions and fuel economy generally improve as vehicles warm up to normal operating temperatures.

For further information regarding this study please contact:

Scott Sluder

Fuels, Combustion, and Engine Technology

P.O. Box 2009, Building 9108

Oak Ridge, TN 37831-8087
Phone: 865-241-9133

Fax: 865-241-1747

email: sluders@ornl.gov 
Table 4.23

Vehicle Specifications for Vehicles Tested in the 1997 Study

\begin{tabular}{|c|c|c|c|c|c|c|}
\hline \multirow[b]{2}{*}{ Vehicle } & \multirow[b]{2}{*}{$\begin{array}{c}\text { Curb } \\
\text { weight }\end{array}$} & \multirow[b]{2}{*}{ Engine } & \multirow{2}{*}{$\begin{array}{c}\text { Fuel } \\
\text { delivery } \\
\text { system }^{\mathrm{a}}\end{array}$} & \multirow[b]{2}{*}{$\begin{array}{l}\text { Trans- } \\
\text { mission }\end{array}$} & \multicolumn{2}{|c|}{ EPA fuel economy } \\
\hline & & & & & City & Highway \\
\hline 1988 Chevrolet Corsica & 2,665 & 2.8 liter V6 & PFI & M5 & 19 & 29 \\
\hline 1994 Olds Cutlass Supreme & 3,290 & 3.4 liter V6 & PFI & L4 & 17 & 26 \\
\hline 1994 Oldsmobile 88 & 3,433 & 3.8 literV6 & PFI & L4 & 19 & 29 \\
\hline 1994 Mercury Villager & 4,020 & 3.0 liter V6 & PFI & L4 & 17 & 23 \\
\hline 1995 Geo Prizm & 2,359 & 1.6 liter I-4 & PFI & L3 & 26 & 30 \\
\hline 1994 Jeep Grand Cherokee & 3,820 & 4.0 liter I-6 & PFI & L4 & 15 & 20 \\
\hline 1994 Chevrolet Pickup & 4,020 & 5.7 liter V8 & TBI & L4 & 14 & 18 \\
\hline 1993 Subaru Legacy & 2,800 & 2.2 liter $\mathrm{H} 4$ & PFI & L4 & 22 & 29 \\
\hline 1997 Toyota Celica & 2,395 & 1.8 liter I4 & PFI & L4 & 27 & 34 \\
\hline
\end{tabular}

\section{Source:}

West, B.H., R.N. McGill, J.W. Hodgson, S.S. Sluder, and D.E. Smith, Development and Verification of Light-Duty Modal Emissions and Fuel Consumption Values for Traffic Models, Washington, DC, April 1997 and additional project data, April 1998.

${ }^{\mathrm{a}} \mathrm{PFI}=$ port fuel injection. $\mathrm{TBI}=$ throttle- body fuel injection . 
The two earlier studies by the Federal Highway Administration (FHWA) indicate maximum fuel efficiency was achieved at speeds of 35 to $40 \mathrm{mph}$. The recent FHWA study indicates greater fuel efficiency at higher speeds. Note that the 1973 study did not include light trucks.

Table 4.24

Fuel Economy by Speed, 1973, 1984, and 1997 Studies (miles per gallon)

\begin{tabular}{cccc}
\hline $\begin{array}{c}\text { Speed } \\
\text { (miles per hour) }\end{array}$ & $\begin{array}{c}1973^{\mathrm{a}} \\
(13 \text { vehicles })\end{array}$ & $\begin{array}{c}1984^{\mathrm{b}} \\
(15 \text { vehicles })\end{array}$ & $\begin{array}{c}1997^{\mathrm{c}} \\
(9 \text { vehicles })\end{array}$ \\
\hline 15 & $\mathrm{~d}$ & 21.1 & 24.4 \\
20 & $\mathrm{~d}$ & 25.5 & 27.9 \\
25 & $\mathrm{~d}$ & 30.0 & 30.5 \\
30 & 21.1 & 31.8 & 31.7 \\
35 & 21.1 & 33.6 & 31.2 \\
40 & 21.1 & 33.6 & 31.0 \\
45 & 20.3 & 33.5 & 31.6 \\
50 & 19.5 & 31.9 & 32.4 \\
55 & 18.5 & 30.3 & 32.4 \\
60 & 17.5 & 27.6 & 31.4 \\
65 & 16.2 & 24.9 & 29.2 \\
70 & 14.9 & 22.5 & 26.8 \\
75 & $\mathrm{~d}$ & 20.0 & 24.8 \\
& & Fuel economy loss & \\
$55-65 \mathrm{mph}$ & $12.4 \%$ & $17.8 \%$ & $9.7 \%$ \\
$65-70 \mathrm{mph}$ & $8.0 \%$ & $9.6 \%$ & $8.2 \%$ \\
$55-70 \mathrm{mph}$ & $19.5 \%$ & $25.7 \%$ & $17.1 \%$ \\
\hline
\end{tabular}

\section{Source:}

1973- U.S. Department of Transportation, Federal Highway Administration, Office of Highway Planning, The Effect of Speed on Automobile Gasoline Consumption Rates, Washington, DC, October 1973.

1984 - U.S. Department of Transportation, Federal Highway Administration, Fuel Consumption and Emission Values for Traffic Models, Washington, DC, May 1985.

1997 - West, B.H., R.N. McGill, J.W. Hodgson, S.S. Sluder, and D.E. Smith, Development and Verification of Light-Duty Modal Emissions and Fuel Consumption Values for Traffic Models, FHWA Report (in press), Washington, DC, April 1997, and additional project data, April 1998.

(Additional resources: www.fhwa-tsis.com)

${ }^{a}$ Model years 1970 and earlier automobiles.

${ }^{b}$ Model years 1981-84 automobiles and light trucks.

${ }^{\mathrm{c}}$ Model years 1988-97 automobiles and light trucks.

${ }^{\mathrm{d}}$ Data are not available. 
Figure 4.2. Fuel Economy by Speed, 1973, 1984, and 1997 Studies

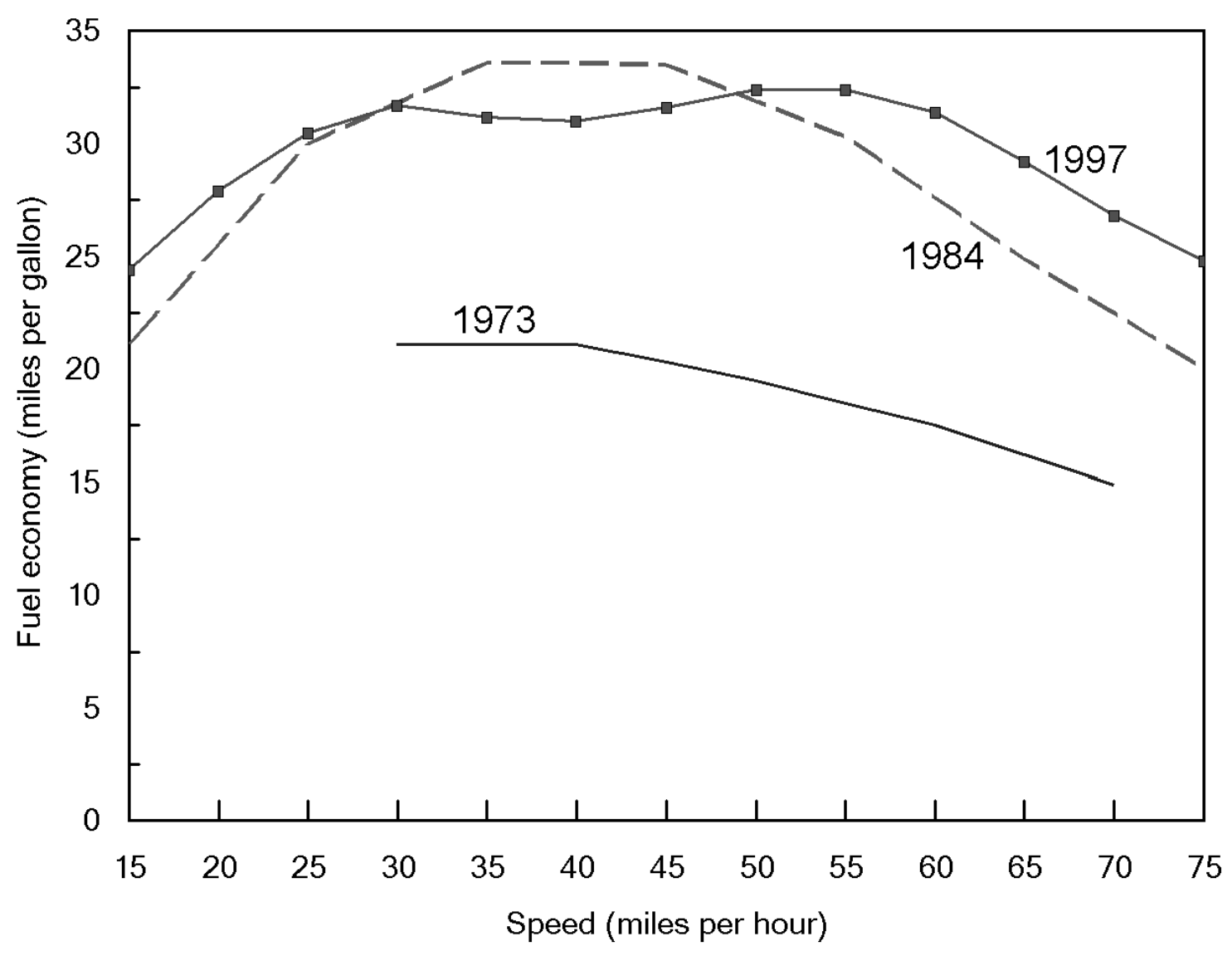

Source: See Table 4.23 . 
Of the tested vehicles, the 1994 Oldsmobile Olds 88 had the greatest fuel economy loss from 55 mph to $75 \mathrm{mpg}$. The 1997 Toyota Celica tested fuel economy was slightly better at $65 \mathrm{mph}$ than at $55 \mathrm{mph}$.

Table 4.25

Steady Speed Fuel Economy for Vehicles Tested in the 1997 Study (miles per gallon)

\begin{tabular}{|c|c|c|c|c|c|c|c|c|c|}
\hline $\begin{array}{l}\text { Speed } \\
(\mathrm{mph})\end{array}$ & $\begin{array}{c}1988 \\
\text { Chevrolet } \\
\text { Corsica }\end{array}$ & $\begin{array}{c}1993 \\
\text { Subaru } \\
\text { Legacy }\end{array}$ & $\begin{array}{c}1994 \\
\text { Oldsmobile } \\
\text { Olds } 88\end{array}$ & $\begin{array}{c}1994 \\
\text { Oldsmobile } \\
\text { Cutlass }\end{array}$ & $\begin{array}{c}1994 \\
\text { Chevrolet } \\
\text { Pickup }\end{array}$ & $\begin{array}{l}1994 \text { Jeep } \\
\text { Grand } \\
\text { Cherokee }\end{array}$ & $\begin{array}{c}1994 \\
\text { Mercury } \\
\text { Villager }\end{array}$ & $\begin{array}{c}1995 \\
\text { Geo } \\
\text { Prizm }\end{array}$ & $\begin{array}{c}1997 \\
\text { Toyota } \\
\text { Celica }\end{array}$ \\
\hline 5 & 10.0 & 14.5 & 10.5 & 5.1 & 7.9 & 8.2 & 12.3 & 18.1 & 19.1 \\
\hline 10 & 16.8 & 24.7 & 14.9 & 7.9 & 16.0 & 11.2 & 19.0 & 23.1 & 34.1 \\
\hline 15 & 17.7 & 31.9 & 22.2 & 11.4 & 16.3 & 17.5 & 22.4 & 38.9 & 41.7 \\
\hline 20 & 21.7 & 34.4 & 26.3 & 12.5 & 19.9 & 24.7 & 25.8 & 39.4 & 46.0 \\
\hline 25 & 23.9 & 37.4 & 28.3 & 15.6 & 22.7 & 21.8 & 30.8 & 41.7 & 52.6 \\
\hline 30 & 28.7 & 39.7 & 29.0 & 19.0 & 26.3 & 21.6 & 30.3 & 40.0 & 50.8 \\
\hline 35 & 28.6 & 38.0 & 30.9 & 21.2 & 24.3 & 25.0 & 26.1 & 39.1 & 47.6 \\
\hline 40 & 29.2 & 37.0 & 33.2 & 23.0 & 26.7 & 25.5 & 29.0 & 38.9 & 36.2 \\
\hline 45 & 28.8 & 33.7 & 32.4 & 23.0 & 27.3 & 25.4 & 27.8 & 42.3 & 44.1 \\
\hline 50 & 31.2 & 33.7 & 34.2 & 27.3 & 26.3 & 24.8 & 30.1 & 39.1 & 44.8 \\
\hline 55 & 29.1 & 37.7 & 34.6 & 29.1 & 25.1 & 24.0 & 31.7 & 37.7 & 42.5 \\
\hline 60 & 28.2 & 35.9 & 32.5 & 28.2 & 22.6 & 23.2 & 27.3 & 36.7 & 48.4 \\
\hline 65 & 28.7 & 33.4 & 30.0 & 25.0 & 21.8 & 21.3 & 25.3 & 34.1 & 43.5 \\
\hline 70 & 26.1 & 31.0 & 26.7 & 22.9 & 20.1 & 20.0 & 23.9 & 31.7 & 39.2 \\
\hline 75 & 23.7 & 28.8 & 24.0 & 21.6 & 18.1 & 19.1 & 22.4 & 28.3 & 36.8 \\
\hline \multicolumn{10}{|c|}{ Fuel economy loss } \\
\hline 55-65 mph & $1.4 \%$ & $11.4 \%$ & $13.3 \%$ & $14.1 \%$ & $13.1 \%$ & $11.3 \%$ & $20.2 \%$ & $9.5 \%$ & $-2.4 \%$ \\
\hline 65-75 mph & $17.4 \%$ & $13.8 \%$ & $20.0 \%$ & $13.6 \%$ & $17.0 \%$ & $10.3 \%$ & $11.5 \%$ & $17.0 \%$ & $15.4 \%$ \\
\hline 55-75 mph & $18.6 \%$ & $23.6 \%$ & $30.6 \%$ & $25.8 \%$ & $27.9 \%$ & $20.4 \%$ & $29.3 \%$ & $24.9 \%$ & $13.4 \%$ \\
\hline
\end{tabular}

Source:

B.H. West, R.N. McGill, J.W. Hodgson, S.S. Sluder, D.E. Smith, Development and Verification of Light-Duty Modal Emissions and Fuel Consumption Values for Traffic Models, Washington, DC, April 1997, and additional project data, April 1998.

Note:

(Additional resources: www.fhwa-tsis.com)

For specifications of the tested vehicles, please see Table 4.21 . 
The Environmental Protection Agency (EPA) tests new vehicles to determine fuel economy ratings. The city and highway fuel economies that are posted on the windows of new vehicles are determined by testing the vehicle during these driving cycles. The driving cycles simulate the performance of an engine while driving in the city and on the highway. Once the urban cycle is completed, the engine is stopped, then started again for the 8.5 minute hot start cycle.

Figure 4.3. Urban Driving Cycle

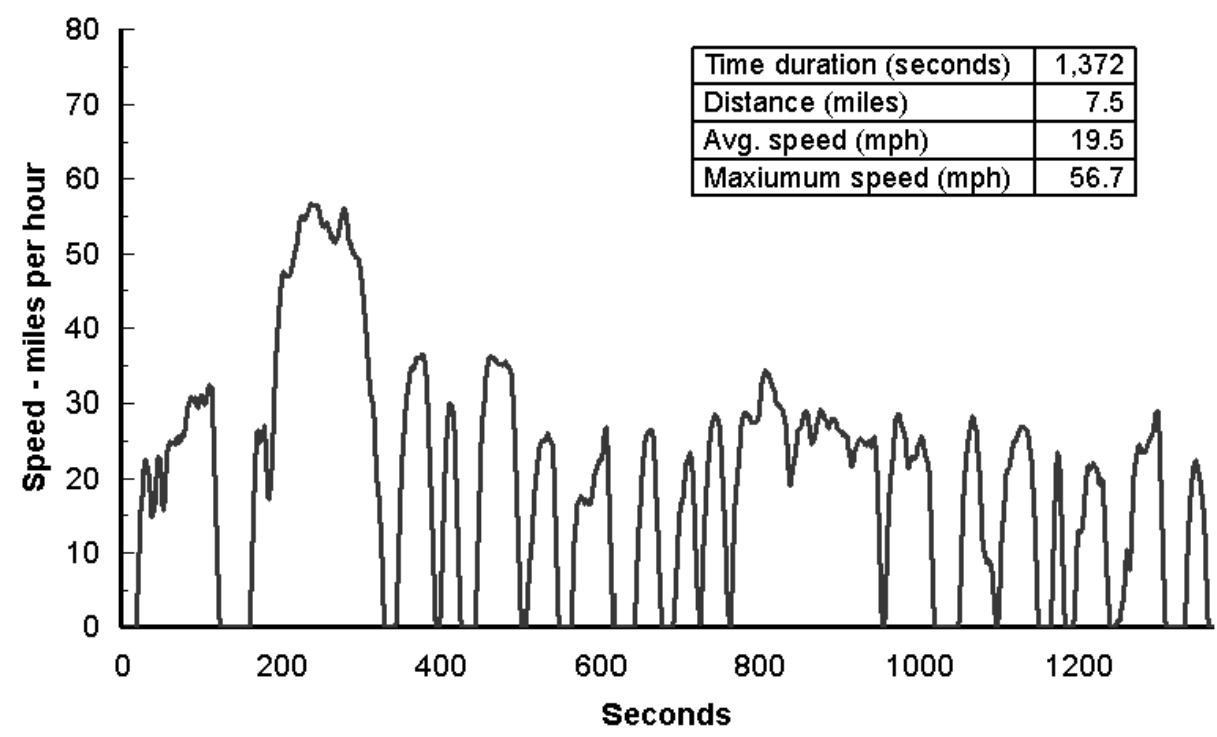

Figure 4.4. Highway Driving Cycle

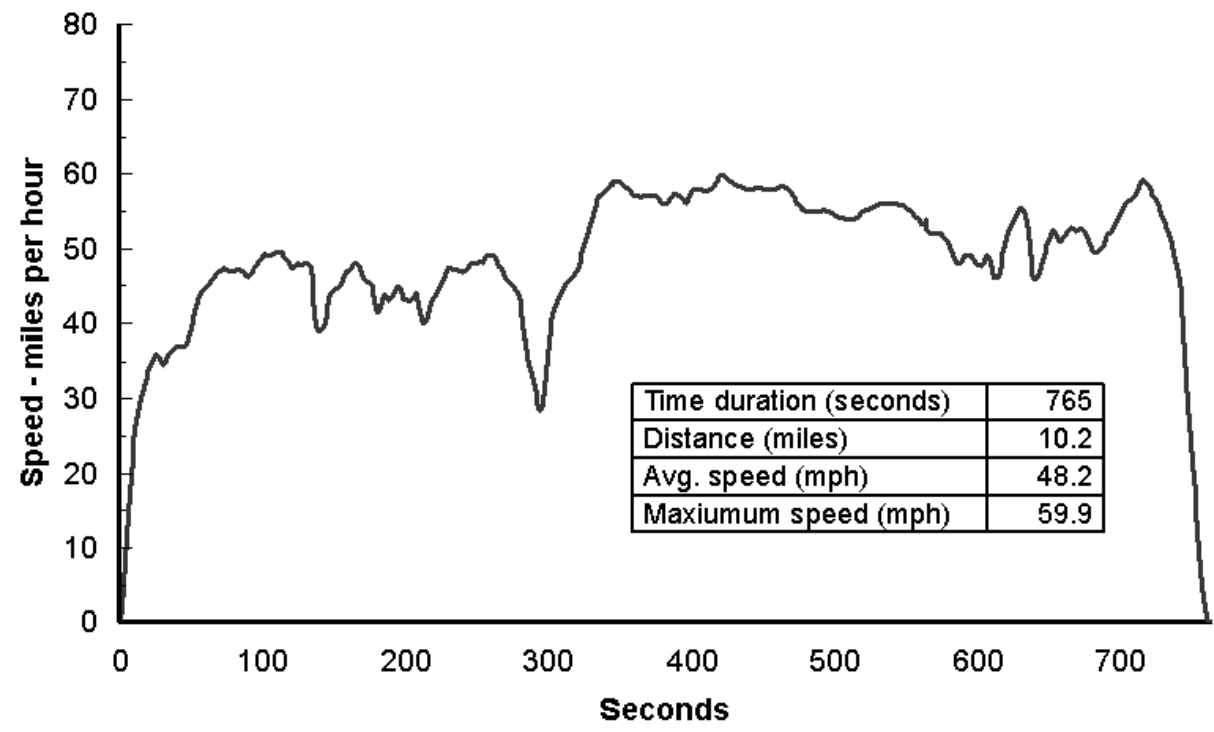

Source: Seconds

Code of Federal Regulations, 40CFR, "Subpart B - Fuel Economy Regulations for 1978 and Later Model Year Automobiles - Test Procedures," July 1, 1988 edition, p. 676. 
The New York Test Cycle was developed in the 1970's in order to simulate driving in downtown congested areas. The Representative Number Five Test Cycle was developed recently to better represent actual on-road driving by combining modern urban and freeway driving.

Figure 4.5. New York City Driving Cycle

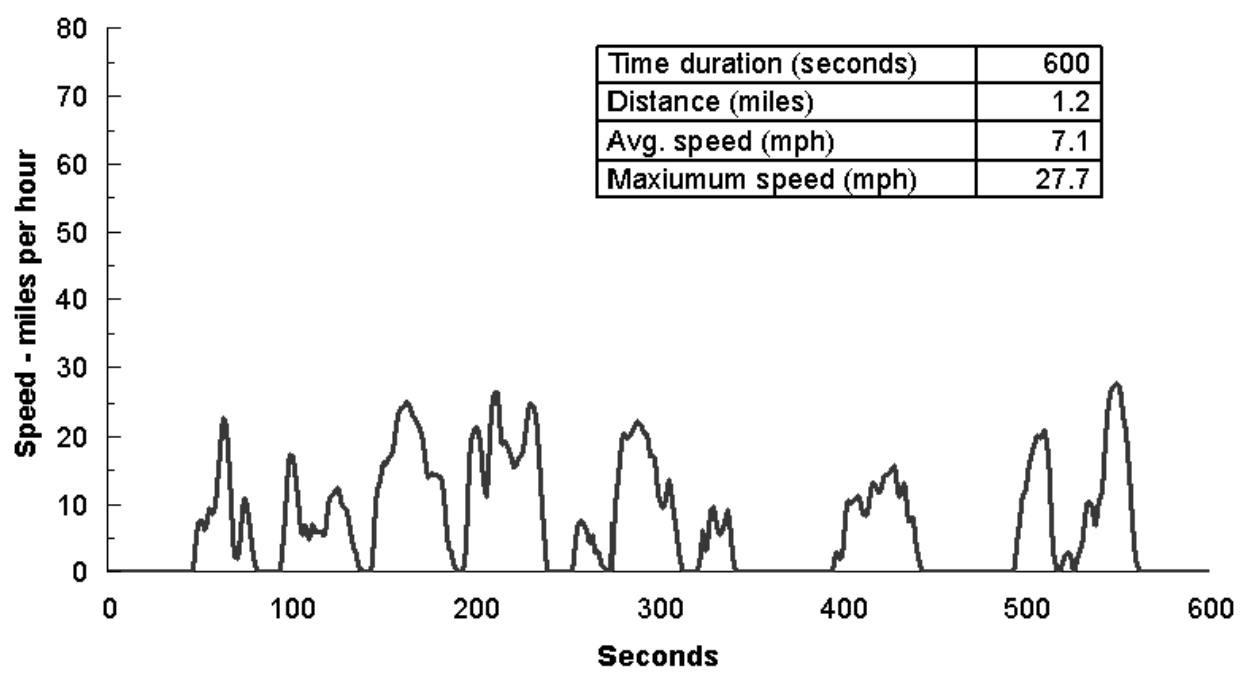

Figure 4.6. Representative Number Five Driving Cycle

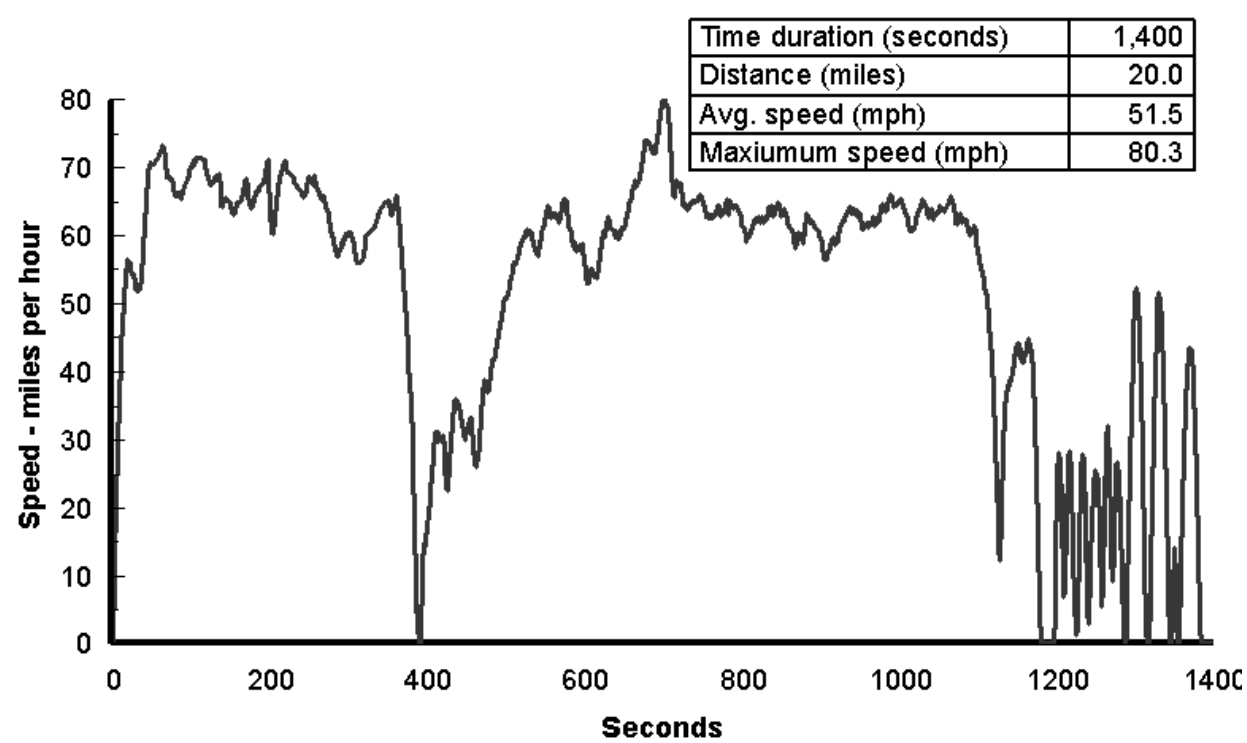

Source:

Data obtained from Michael Wang, Argonne National Laboratory, Argonne, IL, 1997. 
The US06 driving cycle was developed as a supplement to the Federal Test Procedure. It is a short-duration cycle (600 seconds) which represents hard-acceleration driving.

Figure 4.7. US06 Driving Cycle

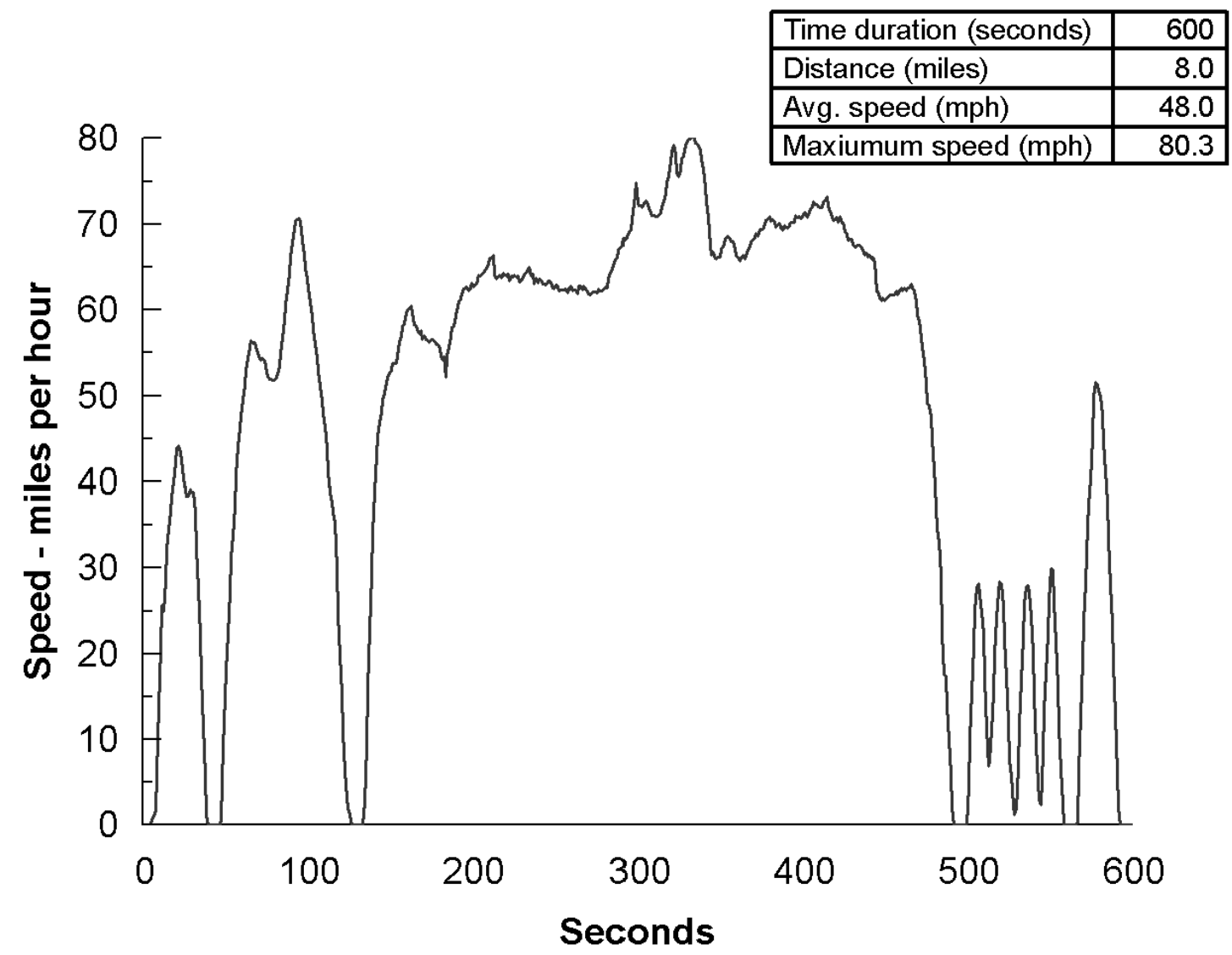

Source:

Data obtained from Michael Wang, Argonne National Laboratory, Argonne, IL, 1997. 
Researchers at Argonne National Laboratory have estimated the fuel economy of a midsize car using driving cycles from different countries. These results illustrate the difference in fuel economy which can be obtained from the same vehicle using different test cycles.

Table 4.26

Projected Fuel Economies from U.S., European, and Japanese Driving Cycles

\begin{tabular}{lc}
\hline Driving Cycle & $\begin{array}{c}\text { Projected fuel economy } \\
\text { for a } 1995 \text { composite }^{\text {midsize vehicle }}\end{array}$ \\
\hline Japanese 10/15 mode test cycle & $17.5 \mathrm{mpg}$ \\
New European Driving Cycle (NEDC) & $22.0 \mathrm{mpg}$ \\
U.S. EPA city cycle (LA4) & $19.8 \mathrm{mpg}$ \\
U.S. EPA highway cycle & $32.1 \mathrm{mpg}$ \\
U.S. Corporate Average Fuel Economy cycle & $23.9 \mathrm{mpg}$ \\
\hline
\end{tabular}

Source:

Santini, D., A. Vyas, J. Anderson, and F. An, Estimating Trade-Offs along the Path to the PNGV $3 X$ Goal, presented at the Transportation Research Board 80 ${ }^{\text {th }}$ Annual Meeting, Washington, DC, January 2001.

${ }^{a}$ The 1995 composite midsize vehicle is an average of a Chevrolet Lumina, Chrysler Concord, and Ford Taurus. The fuel economies were projected using the National Renewable Energy Laboratory's Advanced Vehicle Simulator (ADVISOR) model. 
When comparing data between countries, one must realize that different countries have different testing cycles to determine fuel economy and emissions. This table compares various statistics on the European, Japanese, and U.S. testing cycles [for fuel economy measurements, the U.S. uses the formula, 1/fuel economy $=(0.55 /$ city fuel economy $)+(0.45 /$ highway fuel economy $)]$. Most vehicles will achieve higher fuel economy on the U.S. test cycle than on the European or Japanese cycles.

Table 4.27

Comparison of U.S., European, and Japanese Driving Cycles

\begin{tabular}{lcccccc}
\hline & $\begin{array}{c}\text { Percent of } \\
\text { time } \\
\text { stopped } \\
\text { or }\end{array}$ & $\begin{array}{c}\text { Time } \\
\text { (seconds) }\end{array}$ & $\begin{array}{c}\text { Distance } \\
\text { decelerating } \\
\text { (miles) }\end{array}$ & $\begin{array}{c}\text { Average } \\
\text { speed } \\
\text { (mph) }\end{array}$ & $\begin{array}{c}\text { Maximum } \\
\text { speed } \\
\text { (mph) }\end{array}$ & $\begin{array}{c}\text { Maximum } \\
\text { acceleration } \\
\text { (mph/s) }\end{array}$ \\
\hline $\begin{array}{c}\text { Japanese 10/15 mode } \\
\text { test cycle }\end{array}$ & 631 & 52.3 & 2.6 & 14.8 & 43.5 & 1.78 \\
$\begin{array}{c}\text { New European Driving } \\
\text { Cycle (NEDC) }\end{array}$ & 1,181 & 24.9 & 6.84 & 20.9 & 74.6 & 2.4 \\
$\begin{array}{c}\text { U.S. EPA city cycle } \\
\text { (LA4) }\end{array}$ & 1,372 & 43.2 & 7.5 & 19.5 & 56.7 & 3.3 \\
$\begin{array}{l}\text { U.S. EPA highway cycle } \\
\text { U.S. Corporate Average }\end{array}$ & 765 & 9.3 & 17.8 & 48.2 & 59.9 & 3.3 \\
$\quad$ Fuel Economy cycle & 2,137 & 27.9 & 10.3 & 29.9 & 59.9 & 3.3 \\
\hline
\end{tabular}

Source:

Santini, D., A. Vyas, J. Anderson, and F. An, Estimating Trade-Offs along the Path to the PNGV $3 X$ Goal, presented at the Transportation Research Board 80 ${ }^{\text {th }}$ Annual Meeting, Washington, DC, January 2001.

${ }^{\mathrm{a}}$ The actual Federal Procedure (FTP), which is also the test for emissions certification, repeats the first 505 seconds of the Federal Urban Driving Simulation cycle, hot started, after a 10 minute hot soak. Starting with Model Year 2001, the emissions test-but not the fuel economy test-incorporates a supplemental cycle that simulates aggressive urban driving, coupled with an added air conditioning load. 
Total traffic fatalities were lower in 2001 than in 1975. Fourteen percent of traffic fatalities in 2001 were not vehicle occupants (pedestrians, cyclists, etc.).

Table 4.28

Occupant Fatalities by Vehicle Type and Nonoccupant Fatalities, 1975-2001

\begin{tabular}{|c|c|c|c|c|c|c|c|c|}
\hline & 1975 & 1980 & 1985 & 1990 & 1995 & 2000 & 2001 & $\begin{array}{l}2001 \\
\text { share }\end{array}$ \\
\hline \multicolumn{9}{|c|}{$\begin{array}{l}\text { Vehicle occupant fatalities by } \\
\text { vehicle type }\end{array}$} \\
\hline \multicolumn{9}{|c|}{ Passenger car } \\
\hline Subcompact & 3,834 & 7,299 & 7,993 & 8,309 & 6,791 & 4,718 & 4,417 & $10.5 \%$ \\
\hline Compact & 614 & 927 & 2,635 & 5,310 & 6,899 & 6,933 & 6,718 & $16.0 \%$ \\
\hline Intermediate & 1,869 & 3,878 & 4,391 & 4,849 & 4,666 & 5,131 & 5,401 & $12.8 \%$ \\
\hline Full & 10,800 & 11,580 & 6,586 & 4,635 & 3,413 & 2,259 & 2,304 & $5.5 \%$ \\
\hline Unknown & 8,812 & 3,765 & 1,607 & 989 & 654 & 1,451 & 1,393 & $3.3 \%$ \\
\hline Total & 25,929 & 27,449 & 23,212 & 24,092 & 22,423 & 20,492 & 20,233 & $48.1 \%$ \\
\hline \multicolumn{9}{|l|}{ Truck } \\
\hline Light & 4,856 & 7,486 & 7 & 8,601 & 9,568 & 11,418 & 11,677 & $27.7 \%$ \\
\hline Large & 961 & 1,262 & 977 & 705 & 648 & 741 & 704 & $1.7 \%$ \\
\hline Total & 5,817 & 8,748 & 7,666 & 9,306 & 10,216 & 12,159 & 12,381 & $29.4 \%$ \\
\hline \multicolumn{9}{|l|}{ Other Vehicles } \\
\hline Motorcycle & 3,189 & 5,144 & 4,564 & 3,244 & 2,227 & 2,862 & 3,181 & $7.6 \%$ \\
\hline Bus & 53 & 46 & 57 & 32 & 33 & 22 & 34 & $0.1 \%$ \\
\hline $\begin{array}{l}\text { Other/unknown vehicle } \\
\text { type }\end{array}$ & 937 & 540 & 544 & 460 & 392 & 714 & 557 & $1.3 \%$ \\
\hline Total & 4,179 & 5,730 & 5,165 & 3,736 & 2,652 & 3,598 & 3,772 & $9.0 \%$ \\
\hline $\begin{array}{l}\text { TOTAL vehicle } \\
\text { occupant fatalities }\end{array}$ & 35,925 & 41,927 & 36,043 & 37,134 & 35,291 & 36,249 & 36,386 & $86.4 \%$ \\
\hline \multicolumn{9}{|l|}{ Nonoccupant fatalities } \\
\hline Pedestrian & 7,516 & 8,070 & 6,808 & 6,482 & 5,584 & 4,739 & 4,882 & $11.6 \%$ \\
\hline Pedalcyclist & 1,003 & 965 & 890 & 859 & 833 & 690 & 728 & $1.7 \%$ \\
\hline Other & 81 & 129 & 84 & 124 & 109 & 143 & 105 & $0.2 \%$ \\
\hline Total & 8,600 & 9,164 & 7,782 & 7,465 & 6,526 & 5,572 & 5,715 & $13.6 \%$ \\
\hline $\begin{array}{l}\text { TOTAL traffic } \\
\text { fatalities }\end{array}$ & 44,525 & 51,091 & 43,825 & 44,599 & 41,817 & 41,821 & 42,101 & $100.0 \%$ \\
\hline
\end{tabular}

Source:

Traffic Safety Facts 2001, Washington, DC, December 2002, pp. 86, 101 and 110.

(Additional resources: www.nhtsa.dot.gov) 
In 2001, the fatality rate for vehicle occupants per 100 million vehicle miles are surprisingly similar for passenger cars and light trucks-1.3 and 1.2 fatalities per 100 million vehicle miles, respectively. However, the injury rate per 100 million vehicle miles is much lower for light trucks (88) than for passenger cars (122).

Table 4.29

Light Vehicle Occupant Safety Data, 1975-2001

\begin{tabular}{|c|c|c|c|c|c|c|c|}
\hline & 1975 & 1980 & 1985 & 1990 & 1995 & 2000 & 2001 \\
\hline & \multicolumn{7}{|c|}{ Passenger cars } \\
\hline Fatalities & 25,929 & 27,449 & 23,212 & 24,092 & 22,423 & 20,699 & 20,233 \\
\hline Injuries (thousands) & $\mathrm{a}$ & a & a & 2,376 & 2,469 & 2,052 & 1,927 \\
\hline Vehicle-miles (billions) ${ }^{\mathrm{b}}$ & 1,030 & 1,107 & 1,249 & 1,427 & 1,478 & 1,580 & 1,585 \\
\hline \multicolumn{8}{|c|}{ Rates per 100 million vehicle miles } \\
\hline Fatalities & 2.5 & 2.5 & 1.9 & 1.7 & 1.5 & 1.3 & 1.3 \\
\hline \multirow[t]{2}{*}{ Injuries } & a & a & a & 167 & 167 & 130 & 122 \\
\hline & \multicolumn{7}{|c|}{ Light trucks (10,000 lbs. or less) } \\
\hline Fatalities & 4,856 & 7,486 & 6,689 & 8,601 & 9,568 & 11,526 & 11,677 \\
\hline Injuries (thousands) & a & a & a & 505 & 722 & 887 & 861 \\
\hline Vehicle-miles (billions) ${ }^{\mathrm{b}}$ & 204 & 295 & 389 & 556 & 750 & 943 & 973 \\
\hline \multicolumn{8}{|c|}{ Rates per 100 million vehicle-miles } \\
\hline Fatalities & 2.4 & 2.5 & 1.7 & 1.5 & 1.3 & 1.2 & 1.2 \\
\hline Injuries & a & a & a & 91 & 96 & 94 & 88 \\
\hline
\end{tabular}

Source:

U.S. DOT, National Highway Traffic Safety Administration, Traffic Safety Facts 2001, Washington, DC, December 2002, pp. 22, 24. (Additional resources: www.nhtsa.dot.gov)

${ }^{\mathrm{a}}$ Data are not available.

${ }^{b}$ Vehicle-miles are estimated by the National Highway Traffic Safety Administration and do not match Federal Highway data. 
In 2001,38\% of all passenger car and light truck fatal crashes were single-vehicle crashes. Because there are so many passenger cars on the roads compared to the other vehicle types, total passenger car crashes are half of total crashes. Most crashes are multiple-vehicle crashes with property damage only.

Table 4.30

Crashes by Crash Severity, Crash Type, and Vehicle Type, 2001

\begin{tabular}{|c|c|c|c|c|c|c|c|}
\hline \multirow[b]{2}{*}{ Vehicle type } & \multicolumn{2}{|c|}{ Fatal } & \multicolumn{2}{|c|}{ Injury } & \multicolumn{2}{|c|}{ Property damage only } & \multirow[b]{2}{*}{$\begin{array}{c}\text { Total } \\
\text { crashes }\end{array}$} \\
\hline & $\begin{array}{c}\text { Single- } \\
\text { vehicle } \\
\text { crash }\end{array}$ & $\begin{array}{l}\text { Multiple- } \\
\text { vehicle } \\
\text { crash }\end{array}$ & $\begin{array}{l}\text { Single- } \\
\text { vehicle } \\
\text { crash }\end{array}$ & $\begin{array}{c}\text { Multiple- } \\
\text { vehicle } \\
\text { crash }\end{array}$ & $\begin{array}{l}\text { Single- } \\
\text { vehicle } \\
\text { crash }\end{array}$ & $\begin{array}{c}\text { Multiple- } \\
\text { vehicle } \\
\text { crash }\end{array}$ & \\
\hline Passenger cars & 10,314 & 17,115 & 344,000 & $1,935,000$ & 725,000 & $3,674,000$ & $6,705,429$ \\
\hline Light trucks ${ }^{\mathrm{a}}$ & 8,114 & 12,608 & 201,000 & $1,017,000$ & 473,000 & $2,206,000$ & 3,917,722 \\
\hline Large trucks ${ }^{\mathrm{b}}$ & 810 & 3,983 & 14,000 & 76,000 & 81,000 & 253,000 & 428,793 \\
\hline Buses & 100 & 192 & 1,000 & 11,000 & 8,000 & 35,000 & 55,292 \\
\hline Motorcycles & 1,454 & 1,795 & 26,000 & 31,000 & 7,000 & 8,000 & 75,249 \\
\hline Total & 20,792 & 35,693 & 586,000 & $3,070,000$ & $1,294,000$ & $6,176,000$ & $11,182,485$ \\
\hline Share & $0.2 \%$ & $0.3 \%$ & $5.2 \%$ & $27.5 \%$ & $11.6 \%$ & $55.2 \%$ & $100 \%$ \\
\hline
\end{tabular}

Source:

U.S. Department of Transportation, National Highway Traffic Safety Administration, Traffic Safety Facts 2001, Washington, DC, December 2002, pp. 72, 74, 76, 80, 82.

(Additional resources: www.nhtsa.dot.gov)

Note:

Multiple-vehicle crashes cannot be totaled over vehicle type due to duplication of accidents between vehicle types.

${ }^{\mathrm{a}}$ Trucks 10,000 lbs. gross vehicle weight rating or less, including pickups, vans, and utility vehicles.

b Trucks over 10,000 pounds gross vehicle weight rating including single-unit trucks and truck tractors. 
For fatal crashes in 2001, sport-utility vehicles (SUVs) had the highest rollover rate (35.2\%) while other light trucks had the lowest (13.8\%). This does not mean that the rollover caused the fatality, just that a vehicle in the crash rolled over.

Figure 4.8. Percent Rollover Occurrence in Fatal Crashes by Vehicle Type, 2001

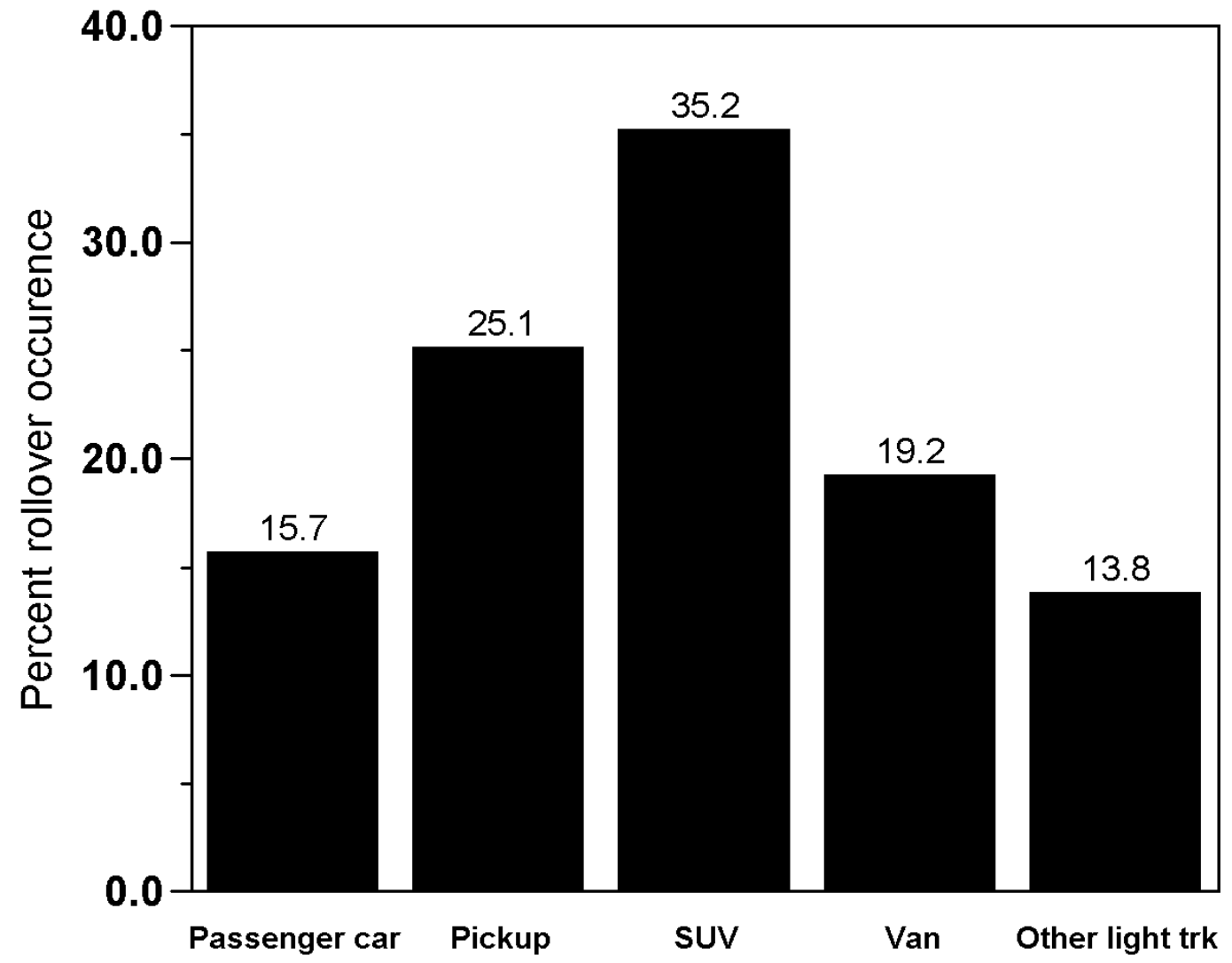

\section{Source:}

U.S. Department of Transportation, National Highway Traffic Safety Administration, Traffic Safety Facts 2001 Washington, DC, December 2002, p. 64.

(Additional resources: www.nhtsa.dot.gov) 
Demand response (also called paratransit or dial-a-ride) and public vanpools are widely used by transit agencies. There were over 40 thousand of these vehicles active in 2001.

Table 4.31

Summary Statistics on Light Transit Vehicles, 1994-2001

\begin{tabular}{rcccc}
\hline Year & $\begin{array}{c}\text { Number of } \\
\text { active vehicles }\end{array}$ & $\begin{array}{c}\text { Vehicle-miles } \\
\text { (millions) }\end{array}$ & $\begin{array}{c}\text { Passenger-miles } \\
\text { (millions) }\end{array}$ & $\begin{array}{c}\text { Energy use } \\
\text { (trillion Btu) }\end{array}$ \\
\hline 1994 & 31,090 & 490 & 781 & 9.8 \\
1995 & 31,773 & 538 & 856 & 9.6 \\
1996 & 33,472 & 588 & 958 & 10.2 \\
1997 & 35,657 & 627 & 1,075 & 10.3 \\
1998 & 33,481 & 721 & 1,103 & 10.9 \\
1999 & 36,651 & 784 & 1,258 & 11.2 \\
2000 & 37,957 & 826 & 1,274 & 11.4 \\
2001 & 40,049 & 861 & 1,345 & 12.0 \\
& & Average annual percentage change & \\
$1994-2001$ & $3.7 \%$ & $8.4 \%$ & $8.1 \%$ & $2.9 \%$ \\
\hline
\end{tabular}

\section{Source:}

American Public Transit Association, 2003 Public Transportation Fact Book, Washington, DC, February 2003, Tables 6, 18, 22, 77 and 95. Historical van pool data are from earlier editions. (Additional resources: www.apta.com)

\section{Note:}

See Glossary for detailed definitions of demand response and vanpool.

\footnotetext{
${ }^{a}$ Includes demand response service and public van pools.
} 


\section{Chapter 5}

\section{Heavy Vehicles and Characteristics}

Summary Statistics from Tables in this Chapter

Source

Table 5.1 Heavy single-unit trucks, 2001

Registration (thousands) $\quad 5,704$

$\begin{array}{ll}\text { Vehicle miles (millions) } & 72,286\end{array}$

Fuel economy (miles per gallon) $\quad 7.4$

Table 5.2 Combination trucks, 2001

Registration (thousands) 2,154

$\begin{array}{ll}\text { Vehicle miles (millions) } & 135,400\end{array}$

Table 5.6 Trucks by size, 1997 Vehicle Inventory \& Use Survey

Light (0-10,000 lbs average weight) $\quad 92.88 \%$

Medium (10,001-26,000 lbs average weight) 3.80\%

Heavy (26,001 lbs and over average weight) 3.32\%

Tables 5.10 Freight Shipments,1997 Commodity Flow Survey

$\begin{array}{lll}\text { and } 5.11 \quad \text { Value (billion dollars) } & 6,944\end{array}$

Tons (millions) $\quad 11,089$

Ton-miles (billions) $\quad$ 2,661

Tables 5.12 Buses in operation, 2001

$\begin{array}{llr}\text { and } 5.13 \quad \text { Transit } & \text { 75,013 }\end{array}$

$\begin{array}{ll}\text { School } & 607,835\end{array}$ 
Heavy single-unit trucks include all single-unit trucks which have more than two axles or more than four tires. Most of these trucks would be used for business or for individuals with heavy hauling or towing needs.

Table 5.1 Summary Statistics for Heavy Single-Unit Trucks, 1970-2001

\begin{tabular}{|c|c|c|c|c|}
\hline Year & $\begin{array}{c}\text { Registrations } \\
\text { (thousands) }\end{array}$ & $\begin{array}{l}\text { Vehicle travel } \\
\text { (million miles) }\end{array}$ & $\begin{array}{c}\text { Fuel use } \\
\text { (million gallons) }\end{array}$ & $\begin{array}{c}\text { Fuel economy } \\
\text { (miles per gallon) }\end{array}$ \\
\hline 1970 & 3,681 & 27,081 & 3,968 & 6.8 \\
\hline 1975 & 4,232 & 34,606 & 5,420 & 6.4 \\
\hline 1980 & 4,374 & 39,813 & 6,923 & 5.8 \\
\hline 1981 & 4,455 & 39,568 & 6,867 & 5.8 \\
\hline 1982 & 4,325 & 40,658 & 6,803 & 6.0 \\
\hline 1983 & 4,204 & 42,546 & 6,965 & 6.1 \\
\hline 1984 & 4,061 & 44,419 & 7,240 & 6.1 \\
\hline 1985 & 4,593 & 45,441 & 7,399 & 6.1 \\
\hline 1986 & 4,313 & 45,637 & 7,386 & 6.2 \\
\hline 1987 & 4,188 & 48,022 & 7,523 & 6.4 \\
\hline 1988 & 4,470 & 49,434 & 7,701 & 6.4 \\
\hline 1989 & 4,519 & 50,870 & 7,779 & 6.5 \\
\hline 1990 & 4,487 & 51,901 & 8,357 & 6.2 \\
\hline 1991 & 4,481 & 52,898 & 8,172 & 6.5 \\
\hline 1992 & 4,370 & 53,874 & 8,237 & 6.5 \\
\hline 1993 & 4,408 & 56,772 & 8,488 & 6.7 \\
\hline 1994 & 4,906 & 61,284 & 9,032 & 6.8 \\
\hline 1995 & 5,024 & 62,705 & 9,216 & 6.8 \\
\hline 1996 & 5,266 & 64,072 & 9,409 & 6.8 \\
\hline 1997 & 5,293 & 66,893 & 9,576 & 7.0 \\
\hline 1998 & 5,414 & 67,894 & 9,741 & 7.0 \\
\hline 1999 & 5,763 & 70,304 & 9,372 & 7.5 \\
\hline 2000 & 5,926 & 70,500 & 9,563 & 7.4 \\
\hline 2001 & 5,704 & 72,286 & 9,732 & 7.4 \\
\hline \multicolumn{5}{|c|}{ Average annual percentage change } \\
\hline 1970-2001 & $1.4 \%$ & $3.2 \%$ & $2.9 \%$ & $0.3 \%$ \\
\hline $1991-2001$ & $2.4 \%$ & $3.2 \%$ & $1.8 \%$ & $1.3 \%$ \\
\hline
\end{tabular}

\section{Source:}

U. S. Department of Transportation, Federal Highway Administration, Highway Statistics 2001, Washington, DC, 2002, Table VM1 and annual.

(Additional resources: www.fhwa.dot.gov)

\section{Note:}

Highway Statistics 1999 data were not used. 
Combination trucks include all trucks designed to be used in combination with one or more trailers. The average vehicle travel of these trucks (on a per truck basis) far surpasses the travel of other trucks due to long-haul freight movement.

Table 5.2

Summary Statistics for Combination Trucks, 1970-2001 ${ }^{1}$

\begin{tabular}{|c|c|c|c|c|}
\hline Year & $\begin{array}{c}\text { Registrations } \\
\text { (thousands) }\end{array}$ & $\begin{array}{l}\text { Vehicle travel } \\
\text { (million miles) }\end{array}$ & $\begin{array}{c}\text { Fuel use } \\
\text { (million gallons) }\end{array}$ & $\begin{array}{c}\text { Fuel economy } \\
\text { (miles per gallon) }\end{array}$ \\
\hline 1970 & 905 & 35,134 & 7,348 & 4.8 \\
\hline 1975 & 1,131 & 46,724 & 9,177 & 5.1 \\
\hline 1980 & 1,417 & 68,678 & 13,037 & 5.3 \\
\hline 1981 & 1,261 & 69,134 & 13,509 & 5.1 \\
\hline 1982 & 1,265 & 70,765 & 13,583 & 5.2 \\
\hline 1983 & 1,304 & 73,586 & 13,796 & 5.3 \\
\hline 1984 & 1,340 & 77,377 & 14,188 & 5.5 \\
\hline 1985 & 1,403 & 78,063 & 14,005 & 5.6 \\
\hline 1986 & 1,408 & 81,038 & 14,475 & 5.6 \\
\hline 1987 & 1,530 & 85,495 & 14,990 & 5.7 \\
\hline 1988 & 1,667 & 88,551 & 15,224 & 5.8 \\
\hline 1989 & 1,707 & 91,879 & 15,733 & 5.8 \\
\hline 1990 & 1,709 & 94,341 & 16,133 & 5.8 \\
\hline 1991 & 1,691 & 96,645 & 16,809 & 5.7 \\
\hline 1992 & 1,675 & 99,510 & 17,216 & 5.8 \\
\hline 1993 & 1,680 & 103,116 & 17,748 & 5.8 \\
\hline 1994 & 1,681 & 108,932 & 18,653 & 5.8 \\
\hline 1995 & 1,696 & 115,451 & 19,777 & 5.8 \\
\hline 1996 & 1,747 & 118,899 & 20,192 & 5.9 \\
\hline 1997 & 1,790 & 124,584 & 20,302 & 6.1 \\
\hline 1998 & 1,831 & 128,159 & 21,100 & 6.1 \\
\hline 1999 & 2,029 & 132,384 & 24,537 & 5.4 \\
\hline 2000 & 2,097 & 135,020 & 25,666 & 5.3 \\
\hline 2001 & 2,154 & 135,400 & 25,555 & 5.3 \\
\hline \multicolumn{5}{|c|}{ Average annual percentage change } \\
\hline 1970-2001 & $2.8 \%$ & $4.4 \%$ & $4.1 \%$ & $0.3 \%$ \\
\hline 1991-2001 & $2.4 \%$ & $3.4 \%$ & $4.3 \%$ & $-0.7 \%$ \\
\hline
\end{tabular}

\section{Source:}

U. S. Department of Transportation, Federal Highway Administration, Highway Statistics 2001, Washington, DC, 2002, Table VM1 and annual.

Note:

(Additional resources: www.fhwa.dot.gov)

Highway Statistics 1999 data were not used.

\footnotetext{
a The Federal Highway Administration changed the combination truck travel methodology in 1993.
} 
Sales of the medium trucks, classes 3-6 rose substantially in 1998. Light trucks under 10,000 lbs., continue to dominate truck sales.

Table 5.3

New Retail Truck Sales by Gross Vehicle Weight, 1970-2002 (thousands)

\begin{tabular}{|c|c|c|c|c|c|c|c|c|c|}
\hline & & & & & & & & & \\
\hline Calendar year & $\begin{array}{c}\text { Class } 1 \\
6,000 \text { lbs. } \\
\text { or less }\end{array}$ & $\begin{array}{c}\text { Class } 2 \\
6,001- \\
10,000 \text { lbs. }\end{array}$ & $\begin{array}{c}\text { Class } 3 \\
10,001- \\
14,000 \mathrm{lbs} .\end{array}$ & $\begin{array}{c}\text { Class } 4 \\
14,001- \\
16,000 \text { lbs. }\end{array}$ & $\begin{array}{c}\text { Class } 5 \\
16,001- \\
19,500 \text { lbs. }\end{array}$ & $\begin{array}{c}\text { Class 6 } \\
19,501- \\
26,000 \text { lbs. }\end{array}$ & $\begin{array}{c}\text { Class } 7 \\
26,001- \\
33,000 \text { lbs. }\end{array}$ & $\begin{array}{c}\text { Class } 8 \\
33,001 \text { lbs. } \\
\text { and over }\end{array}$ & Total \\
\hline \multicolumn{10}{|c|}{ Domestic sales (import data are not available) } \\
\hline $1970^{\mathrm{b}}$ & 1,049 & 408 & 6 & 12 & 58 & 133 & 36 & 89 & 1,791 \\
\hline 1975 & 1,101 & 952 & 23 & 1 & 9 & 159 & 23. & 83 & 2,351 \\
\hline 1980 & 985 & 975 & 4 & c' & 2 & 90 & 58 & 117 & 2,231 \\
\hline 1981 & 896 & 850 & 1 & c & 2 & 72 & 51 & 100 & 1,972 \\
\hline 1982 & 1,102 & 961 & 1 & c & 1 & 44 & 62 & 76 & 2,248 \\
\hline 1983 & 1,314 & 1,207 & c & c & 1 & 47 & 59 & 82 & 2,710 \\
\hline 1984 & 2,031 & 1,224 & 6 & c & 5 & 55 & 78 & 138 & 3,538 \\
\hline 1985 & 2,408 & 1,280 & 11 & c & 5 & 48 & 97 & 134 & 3,983 \\
\hline \multicolumn{10}{|c|}{ Domestic and import sales } \\
\hline 1986 & 3,380 & 1,214 & 12 & $\mathrm{c}$ & 6 & 45 & 101 & 113 & 4,870 \\
\hline 1987 & 3,435 & 1,175 & 14 & 2 & 8 & 44 & 103 & 131 & 4,912 \\
\hline 1988 & 3,467 & 1,333 & 14 & 21 & 8 & 54 & 103 & 148 & 5,149 \\
\hline 1989 & 3,313 & 1,297 & 19 & 27 & 7 & 39 & 93 & 145 & 4,942 \\
\hline 1990 & 3,451 & 1,097 & 21 & 27 & 5 & 38 & 85 & 121 & 4,846 \\
\hline 1991 & 3,246 & 876 & 21 & 24 & 3 & 22 & 73 & 99 & 4,365 \\
\hline 1992 & 3,608 & 1,021 & 26 & 26 & 4 & 28 & 73 & 119 & 4,903 \\
\hline 1993 & 4,119 & 1,232 & 27 & 33 & 4 & 27 & 81 & 158 & 5,681 \\
\hline 1994 & 4,527 & 1,506 & 35 & 44 & 4 & 20 & 98 & 186 & 6,421 \\
\hline 1995 & 4,422 & 1,631 & 40 & 53 & 4 & 23 & 107 & 201 & 6,481 \\
\hline 1996 & 4,829 & 1,690 & 52 & 59 & 7 & 19 & 104 & 170 & 6,930 \\
\hline 1997 & 5,085 & 1,712 & 53 & 57 & 9 & 18 & 114 & 179 & 7,226 \\
\hline 1998 & 5,263 & 2,036 & 102 & 43 & 25 & 32 & 115 & 209 & 7,826 \\
\hline 1999 & 5,707 & 2,366 & 122 & 49 & 30 & 48 & 130 & 262 & 8,716 \\
\hline 2000 & 5,965 & 2,421 & 117 & 47 & 29 & 51 & 123 & 212 & 8,965 \\
\hline 2001 & 6,073 & 2,525 & 102 & 52 & 24 & 42 & 92 & 140 & 9,050 \\
\hline \multirow[t]{2}{*}{2002} & 6,068 & 2,565 & 80 & 38 & 24 & 45 & 69 & 146 & 9,035 \\
\hline & \multicolumn{9}{|c|}{ Average annual percentage change } \\
\hline 1970-1985 & $5.7 \%$ & $7.9 \%$ & $4.1 \%$ & - & $-15.1 \%$ & $-6.6 \%$ & $6.8 \%$ & $2.8 \%$ & $5.5 \%$ \\
\hline 1986-2002 & $3.7 \%$ & $4.8 \%$ & $12.6 \%$ & - & $9.1 \%$ & $0.0 \%$ & $-2.4 \%$ & $1.6 \%$ & $3.9 \%$ \\
\hline
\end{tabular}

Source:

Ward's Communication's, Motor Vehicle Facts and Figures 2000, Southfield, MI, 2000, p. 24, and annual. (Additional resources: www.wardsauto.com)

\footnotetext{
a Sales include domestic-sponsored imports.

${ }^{\mathrm{b}}$ Data for 1970 is based on new truck registrations.

'Data are not available.
} 


\section{Vehicle Inventory and Use Survey}

The Vehicle Inventory and Use Survey (VIUS), which was formerly the Truck Inventory and Use Survey (TIUS), provides data on the physical and operational characteristics of the Nation's truck population. It is based on a probability sample of private and commercial trucks registered (or licensed) in each state. The name of the 1997 survey was changed to the Vehicle Inventory and Use Survey due to future possibilities of including additional vehicle types. The 2002 VIUS, however, will only include trucks. Data from the 2002 VIUS is expected in 2004. Copies of the 1997 VIUS report or CD may be obtained by contacting the U.S. Bureau of the Census, Transportation Characteristics Surveys Branch (301) 457-2797. Internet site: www. census.gov/svsd/www/tiusview.html

Since 1987, the survey has included minivans, vans, station wagons on truck chassis, and sport utility vehicles in addition to the bigger trucks. The 1977 and 1982 surveys did not include those vehicle types. The estimated number of trucks that were within the scope of the 1997 VIUS and registered in the U.S. as of July 1, 1997, was 72.8 million. These trucks were estimated to have been driven a total of 1,044 billion miles during 1997, an increase of 32.8\% from 1992. The average annual miles traveled per truck was estimated at 14,300 miles.

In the 1997 VIUS, there are several ways to classify a truck by weight. The survey respondent was asked the average weight of the vehicle or vehicle-trailer combination when carrying a typical payload; the empty weight (truck minus cargo) of the vehicle as it was usually operated; and the maximum gross weight at which the vehicle or vehicle-trailer combination was operated. The Census Bureau also collected information on the Gross Vehicle Weight Class of the vehicles (decoded from the vehicle identification number) and the registered weight of the vehicles from the State registration files. Some of these weights are only provided in categories, while others are exact weights. Since all these weights could be quite different for a single truck, the tabulations by weight can be quite confusing. In most tables, the Gross Vehicle Weight Class was used. 
Table 5.4

Truck Statistics by Gross Vehicle Weight Class, 1997

\begin{tabular}{lrrrrrr}
\hline $\begin{array}{c}\text { Manufacturer's } \\
\text { gross vehicle weight } \\
\text { class }\end{array}$ & $\begin{array}{c}\text { Number of } \\
\text { trucks }\end{array}$ & $\begin{array}{c}\text { Percentage of } \\
\text { trucks }\end{array}$ & $\begin{array}{c}\text { Average } \\
\text { annual miles } \\
\text { per truck }\end{array}$ & $\begin{array}{c}\text { Average } \\
\text { fuel } \\
\text { economy }\end{array}$ & $\begin{array}{c}\text { Gallons of } \\
\text { fuel used } \\
\text { (millions) }\end{array}$ & $\begin{array}{c}\text { Percentage of } \\
\text { fuel use }\end{array}$ \\
\hline 1) 6,000 lbs and less & $\begin{array}{r}45,240,632 \\
\text { 2) } 6,001-10,000 \text { lbs }\end{array}$ & $62.14 \%$ & 13,328 & 17.82 & 35,184 & $44.34 \%$ \\
3) $10,001-14,000$ & $510,473,167$ & $30.73 \%$ & 12,952 & 14.11 & 21,226 & $26.75 \%$ \\
4) $14,001-16,000$ & 194,951 & $0.70 \%$ & 15,650 & 10.83 & 771 & $0.97 \%$ \\
5) $16,001-19,500$ & 178,111 & $0.24 \%$ & 16,390 & 10.11 & 320 & $0.40 \%$ \\
6) $19,501-26,000$ & $1,884,246$ & $2.59 \%$ & 13,637 & 8.616 & 117 & $0.15 \%$ \\
7) 26,001 - 33,000 & 207,386 & $0.28 \%$ & 35,588 & 7.07 & 1,096 & $1.38 \%$ \\
8) 33,001 lbs and up & $2,211,283$ & $3.04 \%$ & 48,095 & 6.69 & 17,427 & $21.96 \%$ \\
\hline Total & $\mathbf{7 2 , 8 0 0 , 2 5 2}$ & $\mathbf{1 0 0 . 0 0 \%}$ & $\mathbf{1 4 , 3 4 7}$ & $\mathbf{1 6 . 0 2}$ & $\mathbf{7 9 , 3 4 4}$ & $\mathbf{1 0 0 . 0 0 \%}$ \\
\hline
\end{tabular}

Source:

U.S. Department of Commerce, Bureau of the Census, 1997 Vehicle Inventory and Use Survey, Microdata File on CD, 2000. (Additional resources: www.census.gov/svsd/www.tiusview.html)

Table 5.5

Truck Harmonic Mean Fuel Economy by Size Class, 1992 and 1997 (miles per gallon)

\begin{tabular}{lrr}
\hline \multicolumn{1}{c}{ Manufacturer's } & 1992 & 1997 \\
gross vehicle weight class & TIUS & VIUS \\
\hline 1) 6,000 lbs and less & 17.2 & 17.1 \\
2) $6,001-10,000 \mathrm{lbs}$ & 13.0 & 13.6 \\
3) $10,000-14,000 \mathrm{lbs}$ & 8.8 & 9.4 \\
4) $14,001-16,000 \mathrm{lbs}$ & 8.8 & 9.3 \\
5) $16,001-19,500 \mathrm{lbs}$ & 7.4 & 8.7 \\
6) $19,501-26,000 \mathrm{lbs}$ & 6.9 & 7.3 \\
7) $26,001-33,000 \mathrm{lbs}$ & 6.5 & 6.4 \\
8) $33,001 \mathrm{lbs}$ and over & 5.5 & 5.7 \\
\hline
\end{tabular}

\section{Source:}

Estimates are based on data provided on the following public use files: U.S. Department of Commerce, Bureau of the Census, Census of Transportation, Washington, DC, 1992 Truck Inventory and Use Survey, 1995; 1997 Vehicle Inventory and Use Survey, 2000. (Additional resources: www.census.gov/svsd/www/tiusview.html)

Note:

Based on average fuel economy as reported by respondent. 
As expected, most light trucks travel within 50 miles of their home base and refuel at public stations. Sixty percent of heavy trucks travel over 50 miles from their home base and 36\% of them refuel at central company-owned refueling stations.

Table 5.6

Truck Statistics by Size, 1997

\begin{tabular}{|c|c|c|c|c|}
\hline & \multicolumn{3}{|c|}{ Manufacturer's gross vehicle weight class } & \multirow[b]{2}{*}{ Total } \\
\hline & $\begin{array}{c}\text { Light } \\
(<10,000 \mathrm{lbs})\end{array}$ & $\begin{array}{c}\text { Medium } \\
(10,001- \\
26,000 \mathrm{lbs}) \\
\end{array}$ & $\begin{array}{c}\text { Heavy } \\
(>26,000 \mathrm{lbs})\end{array}$ & \\
\hline Trucks & $67,613,799$ & $2,767,784$ & $2,418,669$ & $72,800,252$ \\
\hline Trucks (\%) & $92.88 \%$ & $3.80 \%$ & $3.32 \%$ & $100 \%$ \\
\hline Miles per truck & 13,204 & 13,712 & 47,022 & 14,347 \\
\hline Total miles (\%) & $86.35 \%$ & $3.35 \%$ & $10.31 \%$ & $100 \%$ \\
\hline Fuel use (\%) & $71.10 \%$ & $5.56 \%$ & $23.35 \%$ & $100 \%$ \\
\hline \multirow[t]{2}{*}{ Fuel economy (mpg) } & 15.81 & 7.84 & 5.75 & 13.02 \\
\hline & \multicolumn{4}{|c|}{ Range of operation } \\
\hline Under 50 miles & $75.11 \%$ & $64.45 \%$ & $39.37 \%$ & 73.53\% \\
\hline 51-100 miles & $12.83 \%$ & $16.53 \%$ & $16.44 \%$ & $13.09 \%$ \\
\hline 101-200 miles & $3.86 \%$ & $5.64 \%$ & $10.54 \%$ & $4.15 \%$ \\
\hline 201-500 miles & $2.09 \%$ & $4.65 \%$ & $12.19 \%$ & $2.52 \%$ \\
\hline Over 500 miles & $2.31 \%$ & $1.25 \%$ & $16.80 \%$ & $2.75 \%$ \\
\hline Off-road & $3.81 \%$ & $7.49 \%$ & $4.66 \%$ & $3.97 \%$ \\
\hline \multirow[t]{2}{*}{ Total } & $100 \%$ & $100 \%$ & $100 \%$ & $100 \%$ \\
\hline & \multicolumn{4}{|c|}{ Primary refueling facility } \\
\hline Central company-owned & $11.52 \%$ & $27.32 \%$ & $35.94 \%$ & $29.20 \%$ \\
\hline Single off-site contract & $3.61 \%$ & $5.84 \%$ & $7.00 \%$ & $6.08 \%$ \\
\hline Pubic station & $82.49 \%$ & $61.96 \%$ & $53.25 \%$ & $60.56 \%$ \\
\hline Other & $2.38 \%$ & $4.88 \%$ & $3.80 \%$ & $4.16 \%$ \\
\hline Total & $100 \%$ & $100 \%$ & $100 \%$ & $100 \%$ \\
\hline
\end{tabular}

Source:

U.S. Department of Commerce, Bureau of the Census, 1997 Vehicle Inventory and Use Survey, Microdata File on CD, 2000. (Additional resources: www.census.gov/svsd/www/tiusview.html) 
More medium truck owners listed construction as the truck's major use than any other major use category.

Construction was the second highest major use for light trucks and heavy trucks.

Table 5.7

Percentage of Trucks by Size Ranked by Major Use, 1997

\begin{tabular}{|c|c|c|c|}
\hline Rank & $\begin{array}{c}\text { Light } \\
(<\mathbf{1 0 , 0 0 0} \text { lbs } \\
\text { average weight }) \\
\end{array}$ & $\begin{array}{c}\text { Medium } \\
(10,001-26,000 \text { lbs } \\
\text { average weight }) \\
\end{array}$ & $\begin{array}{c}\text { Heavy } \\
(>26,000 \text { lbs } \\
\text { average weight }) \\
\end{array}$ \\
\hline \multirow[t]{2}{*}{1} & Personal & Construction & For Hire \\
\hline & $74.56 \%$ & $20.19 \%$ & $31.48 \%$ \\
\hline \multirow[t]{2}{*}{2} & Construction & Agriculture & Construction \\
\hline & $7.56 \%$ & $19.54 \%$ & $17.56 \%$ \\
\hline \multirow[t]{2}{*}{3} & Services $^{\mathrm{a}}$ & Services $^{\mathrm{a}}$ & Agriculture \\
\hline & $5.57 \%$ & $11.64 \%$ & $14.01 \%$ \\
\hline \multirow[t]{2}{*}{4} & Agriculture & Retail & Wholesale \\
\hline & $3.82 \%$ & $9.28 \%$ & $7.81 \%$ \\
\hline \multirow[t]{2}{*}{5} & Retail & Wholesale & Services $^{\mathrm{a}}$ \\
\hline & $2.79 \%$ & $7.31 \%$ & $7.39 \%$ \\
\hline \multirow[t]{2}{*}{6} & Not in Use & Personal & Retail \\
\hline & $1.61 \%$ & $7.00 \%$ & $5.67 \%$ \\
\hline \multirow[t]{2}{*}{7} & Wholesale & For Hire & Manufacturing \\
\hline & $1.33 \%$ & $5.47 \%$ & $5.61 \%$ \\
\hline \multirow[t]{2}{*}{8} & Utilities & Utilities & Forestry \\
\hline & $0.75 \%$ & $4.40 \%$ & $2.56 \%$ \\
\hline \multirow[t]{2}{*}{9} & Manufacturing & Daily Rental & Utilities \\
\hline & $0.74 \%$ & $4.21 \%$ & $2.18 \%$ \\
\hline \multirow[t]{2}{*}{10} & Daily Rental & Manufacturing & Mining \\
\hline & $0.53 \%$ & $3.72 \%$ & $2.18 \%$ \\
\hline \multirow[t]{2}{*}{11} & Forestry & Not in Use & Daily Rental \\
\hline & $0.26 \%$ & $3.21 \%$ & $2.11 \%$ \\
\hline \multirow[t]{2}{*}{12} & Mining & Forestry & Not in Use \\
\hline & $0.25 \%$ & $1.64 \%$ & $1.11 \%$ \\
\hline \multirow[t]{2}{*}{13} & For Hire & One-Way Rental & Personal \\
\hline & $0.21 \%$ & $1.24 \%$ & $0.31 \%$ \\
\hline \multirow[t]{2}{*}{14} & One-Way Rental & Mining & One-Way Rental \\
\hline & $0.01 \%$ & $1.14 \%$ & $0.01 \%$ \\
\hline
\end{tabular}

Source:

U.S. Department of Commerce, Bureau of the Census, 1997 Vehicle Inventory and Use Survey, Micro data File on CD, 2000. (Additional resources: www.census.gov/svsd/www/tiusview.html)

${ }^{a}$ Business and personal services. 
In 1997 nearly 60\% of all truck fleets use public fueling stations as their primary refueling facility. As expected, larger fleets use central company-owned facilities more than smaller fleets. Mid-size fleets (10-500 vehicles) use off-site contract facilities more than the smaller or larger fleets.

Table 5.8

Percentage of Trucks by Fleet Size and Primary Fueling Facility, 1997

\begin{tabular}{lccccc}
\hline & \multicolumn{4}{c}{ Primary refueling facility } & \\
\cline { 2 - 5 } \multicolumn{1}{c}{ Truck } & $\begin{array}{c}\text { Central } \\
\text { fleet size }\end{array}$ & $\begin{array}{c}\text { Single contract fueling } \\
\text { facility } \\
\text { fueling-owned }\end{array}$ & $\begin{array}{c}\text { Public fueling } \\
\text { stations }\end{array}$ & Other & Total \\
\hline 1 & $5.94 \%$ & $2.70 \%$ & $87.26 \%$ & $4.09 \%$ & $100 \%$ \\
$2-5$ & $13.80 \%$ & $4.56 \%$ & $76.12 \%$ & $5.52 \%$ & $100 \%$ \\
$6-9$ & $25.77 \%$ & $7.32 \%$ & $62.02 \%$ & $4.88 \%$ & $100 \%$ \\
$10-24$ & $37.08 \%$ & $10.43 \%$ & $49.70 \%$ & $2.79 \%$ & $100 \%$ \\
$25-99$ & $48.48 \%$ & $9.65 \%$ & $39.29 \%$ & $2.59 \%$ & $100 \%$ \\
$100-499$ & $48.76 \%$ & $10.62 \%$ & $38.40 \%$ & $2.22 \%$ & $100 \%$ \\
$500-999$ & $46.39 \%$ & $7.46 \%$ & $44.38 \%$ & $1.77 \%$ & $100 \%$ \\
$1,000-4,999$ & $45.24 \%$ & $4.93 \%$ & $45.94 \%$ & $3.89 \%$ & $100 \%$ \\
$5,000-9,999$ & $35.77 \%$ & $6.01 \%$ & $53.36 \%$ & $4.87 \%$ & $100 \%$ \\
10,000 \& up & $71.72 \%$ & $2.56 \%$ & $19.27 \%$ & $6.45 \%$ & $100 \%$ \\
\hline Overall & $\mathbf{3 0 . 0 8 \%}$ & $\mathbf{6 . 3 9 \%}$ & $\mathbf{5 9 . 3 7 \%}$ & $\mathbf{4 . 1 6 \%}$ & $\mathbf{1 0 0 \%}$ \\
\hline
\end{tabular}

Source:

U.S. Department of Commerce, Bureau of the Census, 1997 Vehicle Inventory and Use Survey, Microdata File on CD, 2000. (Additional resources: www.census.gov/svsd/www/tiusview.html) 
Most trucks are fueled at public fueling stations but one-way rental trucks are more often fueled at company-owned central fueling facilities or contract fueling facilities than at public stations. Mining and quarrying activities use central fueling facilities more than $40 \%$ of the time.

Table 5.9

Percentage of Trucks by Major Use and Primary Fueling Facility, 1997

\begin{tabular}{|c|c|c|c|c|c|}
\hline \multirow[b]{2}{*}{ Major Use } & \multicolumn{4}{|c|}{ Primary fueling facility } & \multirow[b]{2}{*}{ Total } \\
\hline & $\begin{array}{c}\text { Central } \\
\text { company-owned } \\
\text { fueling facility }\end{array}$ & $\begin{array}{l}\text { Single contract } \\
\text { fueling facility } \\
\text { located off-site }\end{array}$ & $\begin{array}{c}\text { Public fueling } \\
\text { stations }\end{array}$ & Other & \\
\hline Agricultural services & $32.09 \%$ & $2.99 \%$ & $53.92 \%$ & $11.00 \%$ & $100 \%$ \\
\hline Forestry or lumbering activities & $22.49 \%$ & $4.50 \%$ & $70.33 \%$ & $2.68 \%$ & $100 \%$ \\
\hline Construction work & $33.40 \%$ & $5.39 \%$ & $58.79 \%$ & $2.42 \%$ & $100 \%$ \\
\hline Contractor activities or special trades & $12.09 \%$ & $4.38 \%$ & $81.18 \%$ & $2.36 \%$ & $100 \%$ \\
\hline Manufacturing, refining or processing activities & $35.47 \%$ & $9.48 \%$ & $53.69 \%$ & $1.36 \%$ & $100 \%$ \\
\hline Wholesale trade & $32.56 \%$ & $11.90 \%$ & $53.62 \%$ & $1.92 \%$ & $100 \%$ \\
\hline Retail trade & $28.21 \%$ & $10.25 \%$ & $59.41 \%$ & $2.12 \%$ & $100 \%$ \\
\hline Business and personal services & $26.40 \%$ & $6.33 \%$ & $65.42 \%$ & $1.85 \%$ & $100 \%$ \\
\hline Utilities & $40.56 \%$ & $5.09 \%$ & $52.25 \%$ & $2.09 \%$ & $100 \%$ \\
\hline Mining or quarrying activities & $43.82 \%$ & $9.32 \%$ & $44.44 \%$ & $2.42 \%$ & $100 \%$ \\
\hline Daily rental & $39.42 \%$ & $13.29 \%$ & $45.12 \%$ & $2.17 \%$ & $100 \%$ \\
\hline Not in use for more than six months & $10.56 \%$ & $2.37 \%$ & $53.12 \%$ & $33.94 \%$ & $100 \%$ \\
\hline For-hire transportation & $32.87 \%$ & $4.90 \%$ & $59.53 \%$ & $2.70 \%$ & $100 \%$ \\
\hline One-way rental & $48.47 \%$ & $3.10 \%$ & $48.43 \%$ & $0.00 \%$ & $100 \%$ \\
\hline Personal transportation & $2.02 \%$ & $0.56 \%$ & $94.46 \%$ & $2.96 \%$ & $100 \%$ \\
\hline Overall & $29.20 \%$ & $6.08 \%$ & $60.56 \%$ & $4.16 \%$ & $100 \%$ \\
\hline
\end{tabular}

\section{Source:}

U.S. Department of Commerce, Bureau of the Census, 1997 Vehicle Inventory and Use Survey, Microdata File on CD, 2000. (Additional resources: www.census.gov/svsd/www/tiusview.html) 


\section{Commodity Flow Survey}

The Commodity Flow Survey (CFS) is designed to provide data on the flow of goods and materials by mode of transport. The 1993 and 1997 CFS are a continuation of statistics collected in the Commodity Transportation Survey from 1963 through 1977, and include major improvements in methodology, sample size, and scope. In 1997, CFS used a sample of 100,000 domestic establishments randomly selected from a universe of about 800,000 establishments engaged in mining, manufacturing, wholesale, auxiliary establishments (warehouses) of multi-establishment companies, and some selected activities in retail and service was used. Each selected establishment reported a sample of approximately 25 outbound shipments for a oneweek period in each of the four calendar quarters of 1997. This produced a total sample of over 5 million shipments. For each sampled shipment, zip codes of origin and destination, 5-digit Standard Classification of Transported Goods (SCTG) code, weight, value, and modes of transport, were provided. Establishments also reported whether the shipment was containerized, a hazardous material, or an export.

The 1993 and 1997 CFS differ from previous surveys in their greatly expanded coverage of intermodalism (i.e., shipments which travel by at least two different modes, such as rail and truck). Earlier surveys reported only the principal mode. The 1993 and 1997 surveys report all modes used for the shipment (for-hire truck, private truck, rail, inland water, deep sea water, pipeline, air, parcel delivery or U.S. Postal Service, other mode, unknown). Route distance for each mode for each shipment as imputed from a mode-distance table was developed by Oak Ridge National Laboratory. Distance, in turn, was used to compute ton-mileage by mode of transport.

For more information about the CFS, contact the Commodity Flow Survey Branch, Department of Commerce, Bureau of the Census, Services Division at (301) 457-2108, or visit the following Internet site: www.bts.gov/cfs . 
Industries covered by the 1997 Commodity Flow Survey (CFS) shipped over 11 billion tons of goods worth almost $\$ 7$ trillion. Compared to the 1993 CFS, the value of shipments is up 2.2\% per year and ton shipped are up $3.4 \%$ per year. By value, intermodal shipments increased 7.0\% per year from 1993 to 1997.

Table 5.10

Growth of Freight in the United States: Comparison of the 1997 and 1993 Commodity Flow Surveys (Detail may not add to total because of rounding)

\begin{tabular}{|c|c|c|c|c|c|c|}
\hline \multirow[b]{2}{*}{ Mode of Transportation } & \multicolumn{3}{|c|}{ Value of goods shipped } & \multicolumn{3}{|c|}{ Tons } \\
\hline & $\begin{array}{c}1997 \text { (billion } \\
1997 \\
\text { dollars) }\end{array}$ & $\begin{array}{c}1993 \\
\text { (billion } \\
1997 \\
\text { dollars) }\end{array}$ & $\begin{array}{l}\text { Average } \\
\text { annual } \\
\text { percent } \\
\text { change }\end{array}$ & $\begin{array}{c}1997 \\
\text { (millions) }\end{array}$ & $\begin{array}{c}1993 \\
\text { (millions) }\end{array}$ & $\begin{array}{l}\text { Average } \\
\text { annual } \\
\text { percent } \\
\text { change }\end{array}$ \\
\hline All modes & $6,944.0$ & $6,360.8$ & $2.2 \%$ & $11,089.7$ & $9,688.5$ & $3.4 \%$ \\
\hline Single modes & $5,719.6$ & $5,376.3$ & $1.6 \%$ & $10,436.5$ & $8,922.3$ & $4.0 \%$ \\
\hline $\begin{array}{l}\text { Truck }^{\mathrm{a}} \\
\text { For-hire truck } \\
\text { Private truck }\end{array}$ & $\begin{array}{l}4981.5 \\
2901.3 \\
2036.5\end{array}$ & $\begin{array}{l}4791.0 \\
2856.1 \\
1910.4\end{array}$ & $\begin{array}{l}1.0 \% \\
0.4 \% \\
1.6 \%\end{array}$ & $\begin{array}{l}7700.7 \\
3402.6 \\
4137.3\end{array}$ & $\begin{array}{l}6385.9 \\
2808.3 \\
3543.5\end{array}$ & $\begin{array}{l}4.8 \% \\
4.9 \% \\
3.9 \%\end{array}$ \\
\hline Rail & 319.6 & 269.2 & $4.4 \%$ & $1,549.8$ & $1,544.1$ & $0.1 \%$ \\
\hline $\begin{array}{l}\text { Water } \\
\text { Shallow draft } \\
\text { Great Lakes } \\
\text { Deep draft }\end{array}$ & $\begin{array}{r}75.8 \\
53.9 \\
1.5 \\
20.4\end{array}$ & $\begin{array}{c}67.1 \\
44.3 \\
c \\
21.5\end{array}$ & $\begin{array}{l}3.1 \% \\
5.0 \% \\
c \\
-1.3 \%\end{array}$ & $\begin{array}{r}563.4 \\
414.8 \\
38.4 \\
110.2\end{array}$ & $\begin{array}{r}505.4 \\
362.5 \\
33.0 \\
109.9\end{array}$ & $\begin{array}{l}2.8 \% \\
3.4 \% \\
3.9 \% \\
0.1 \%\end{array}$ \\
\hline Air (includes truck and air) & 229.1 & 151.3 & $10.9 \%$ & 4.5 & 3.1 & $9.8 \%$ \\
\hline Pipeline $^{\mathrm{b}}$ & 113.5 & 97.8 & $3.8 \%$ & 618.2 & 483.6 & $6.3 \%$ \\
\hline Multiple modes & 945.9 & 720.9 & $7.0 \%$ & 216.7 & 225.7 & $-1.0 \%$ \\
\hline $\begin{array}{l}\text { Parcel, U.S. Postal Service } \\
\quad \text { or courier } \\
\text { Truck and rail } \\
\text { Truck and water } \\
\text { Rail and water } \\
\text { Other multiple modes }\end{array}$ & $\begin{array}{r}855.9 \\
75.7 \\
8.2 \\
1.8 \\
4.3\end{array}$ & $\begin{array}{r}612.8 \\
90.4 \\
10.2 \\
4.0 \\
3.5\end{array}$ & $\begin{array}{r}8.7 \% \\
-4.3 \% \\
-5.3 \% \\
-18.1 \% \\
5.3 \%\end{array}$ & $\begin{array}{l}23.7 \\
54.2 \\
33.2 \\
79.3 \\
26.2\end{array}$ & $\begin{array}{l}18.9 \\
40.6 \\
68.0 \\
79.2 \\
18.9\end{array}$ & $\begin{array}{r}5.8 \% \\
7.5 \% \\
-16.4 \% \\
0.0 \% \\
8.5 \%\end{array}$ \\
\hline Other and unknown modes & 278.6 & 263.6 & $1.4 \%$ & 436.5 & 540.5 & $-5.2 \%$ \\
\hline
\end{tabular}

Source:

U.S. Department of Transportation, Bureau of Transportation Statistics, Freight USA, Washington, DC, 2000. (Additional resources: www.bts.gov/cfs)

\footnotetext{
a "Truck" as a single mode includes shipments which went by private truck only, for-hire truck only, or a combination of private truck and for-hire truck.

b CFS data for pipeline lack most shipments of crude oil.

${ }^{c}$ Denotes data do not meet publication standards because of high sampling variability or other reasons. Some unpublished estimates can be derived from other data published in this table. However, figures obtained in this manner are subject to these same limitations.
} 
Industries covered by the 1997 Commodity Flow Survey (CFS) accounted for about 2.7 trillion ton-miles on the nation's highways, railways, waterways, pipelines, and aviation system. Ton-miles increased an average of $2.4 \%$ per year from 1993 to 1997.

Table 5.11

Growth of Freight Miles in the United States: Comparison of the 1997 and 1993 Commodity Flow Surveys (Detail may not add to total because of rounding)

\begin{tabular}{|c|c|c|c|c|c|c|}
\hline \multirow[b]{2}{*}{ Mode of Transportation } & \multicolumn{3}{|c|}{ Ton-miles } & \multicolumn{3}{|c|}{ Average miles per shipment } \\
\hline & $\begin{array}{c}1997 \\
\text { (billions) }\end{array}$ & $\begin{array}{c}1993 \\
\text { (billions) }\end{array}$ & $\begin{array}{l}\text { Average annual } \\
\text { percent change }\end{array}$ & 1997 & 1993 & $\begin{array}{l}\text { Average annual } \\
\text { percent change }\end{array}$ \\
\hline All modes & $2,661.4$ & $2,420.9$ & $2.4 \%$ & 472 & 424 & $2.7 \%$ \\
\hline Single modes & $2,383.5$ & $2,136.9$ & $2.8 \%$ & 184 & 197 & $-1.7 \%$ \\
\hline $\begin{array}{l}\text { Truck }^{\mathrm{a}} \\
\text { For-hire truck } \\
\text { Private truck }\end{array}$ & $\begin{array}{r}1023.5 \\
741.1 \\
268.6\end{array}$ & $\begin{array}{l}869.5 \\
629.0 \\
235.9\end{array}$ & $\begin{array}{l}4.2 \% \\
4.2 \% \\
3.3 \%\end{array}$ & $\begin{array}{r}144 \\
485 \\
53\end{array}$ & $\begin{array}{r}144 \\
472 \\
52\end{array}$ & $\begin{array}{l}0.0 \% \\
0.7 \% \\
0.5 \%\end{array}$ \\
\hline Rail & $1,022.5$ & 942.6 & $2.1 \%$ & 769 & 766 & $0.1 \%$ \\
\hline $\begin{array}{l}\text { Water } \\
\text { Shallow draft } \\
\text { Great Lakes } \\
\text { Deep draft }\end{array}$ & $\begin{array}{r}261.7 \\
189.3 \\
13.4 \\
59.0\end{array}$ & $\begin{array}{r}272.0 \\
164.4 \\
12.4 \\
95.2\end{array}$ & $\begin{array}{c}-1.0 \% \\
3.6 \% \\
2.0 \% \\
-11.3 \%\end{array}$ & $\begin{array}{r}482 \\
177 \\
204 \\
1,024\end{array}$ & $\begin{array}{r}c \\
c \\
534 \\
1,861\end{array}$ & $\begin{array}{c}c \\
c \\
-21.4 \% \\
-13.9 \%\end{array}$ \\
\hline $\begin{array}{l}\text { Air (includes truck and air) } \\
\text { Pipeline }^{\mathrm{b}}\end{array}$ & $\begin{array}{l}6.2 \\
c\end{array}$ & $\begin{array}{l}4.0 \\
c\end{array}$ & $\underset{c}{11.6 \%}$ & $\begin{array}{r}1,380 \\
\mathrm{c}\end{array}$ & $\begin{array}{r}1,415 \\
\text { c }\end{array}$ & $\underset{c}{-0.6 \%}$ \\
\hline Multiple modes & 204.5 & 191.5 & $1.7 \%$ & 813 & 736 & $2.5 \%$ \\
\hline $\begin{array}{l}\text { Parcel, U.S. Postal Service } \\
\quad \text { or courier } \\
\text { Truck and rail } \\
\text { Truck and water } \\
\text { Rail and water } \\
\text { Other multiple modes }\end{array}$ & $\begin{array}{l}18.0 \\
55.6 \\
34.8 \\
77.6 \\
18.6\end{array}$ & $\begin{array}{l}13.2 \\
37.7 \\
40.6 \\
70.2\end{array}$ & $\begin{array}{c}8.1 \% \\
10.2 \% \\
-3.8 \% \\
2.5 \%\end{array}$ & $\begin{array}{r}813 \\
1,347 \\
1,265 \\
1,092\end{array}$ & $\begin{array}{r}734 \\
1,403 \\
1,417 \\
627 \\
1,082\end{array}$ & $\begin{array}{r}2.6 \% \\
-1.0 \% \\
-2.8 \% \\
14.9 \%\end{array}$ \\
\hline Other and unknown modes & 73.4 & 92.6 & $-5.6 \%$ & 122 & 229 & $-14.6 \%$ \\
\hline
\end{tabular}

Source:

U.S. Department of Transportation, Bureau of Transportation Statistics, Freight USA, Washington, DC, 2000. (Additional resources: www.bts.gov/cfs)

a "Truck" as a single mode includes shipments which went by private truck only, for-hire truck only, or a combination of private truck and for-hire truck.

b CFS data for pipeline lack most shipments of crude oil.

c Denotes data do not meet publication standards because of high sampling variability or other reasons. Some unpublished estimates can be derived from other data published in this table. However, figures obtained in this manner are subject to these same limitations. 
The American Public Transportation Association recently published data by mode and fuel type that were not previously available. Transit bus energy use had been overestimated in previous editions due to this lack of data.

Table 5.12

Summary Statistics on Transit Buses and Trolleybuses, 1994-2001

\begin{tabular}{cccccc}
\hline Year & $\begin{array}{c}\text { Number of } \\
\text { active buses }\end{array}$ & $\begin{array}{c}\text { Vehicle-miles } \\
\text { (millions) }\end{array}$ & $\begin{array}{c}\text { Passenger- } \\
\text { miles } \\
\text { (millions) }\end{array}$ & $\begin{array}{c}\text { Btu/ } \\
\text { passenger-mile }\end{array}$ & $\begin{array}{c}\text { Energy use } \\
\text { (trillion Btu) }\end{array}$ \\
\hline 1994 & 69,000 & 2,176 & 19,019 & 4,268 & 81.2 \\
1995 & 67,992 & 2,198 & 19,005 & 4,310 & 81.9 \\
1996 & 72,549 & 2,234 & 19,280 & 4,340 & 83.7 \\
1997 & 73,629 & 2,259 & 19,793 & 4,431 & 87.7 \\
1998 & 73,022 & 2,188 & 20,542 & 4,387 & 90.1 \\
1999 & 75,087 & 2,290 & 21,391 & 4,332 & 92.7 \\
2000 & 75,964 & 2,329 & 21,433 & 4,515 & 96.8 \\
2001 & 76,675 & 2,389 & 22,209 & 4,125 & 91.6 \\
$1994-2001$ & $1.5 \%$ & Average annual percentage change & \\
\hline
\end{tabular}

\section{Source:}

American Public Transportation Association, 2003 Public Transportation Fact Book, Washington, DC, February 2003, Tables 6, 18, 22, and 77. (Additional resources: www.apta.com)

\footnotetext{
${ }^{\text {a }}$ Comparisons cannot be made with data before 1992. Beginning in 1992, data were available on nondiesel fuel consumption (i.e. propane, compressed natural gas, methanol).
} 
There are currently not many sources of data on intercity and school buses. The Eno Foundation for Transportation publishes petroleum use for intercity and school buses, and passenger-miles for intercity buses. The Federal Highway Administration publishes an estimate of the total number of school buses. School Bus Fleet magazine also contains statistics on school buses (www.schoolbusfleet.com/stats.cfm).

Table 5.13

Summary Statistics on Intercity and School Buses, 1970-2001

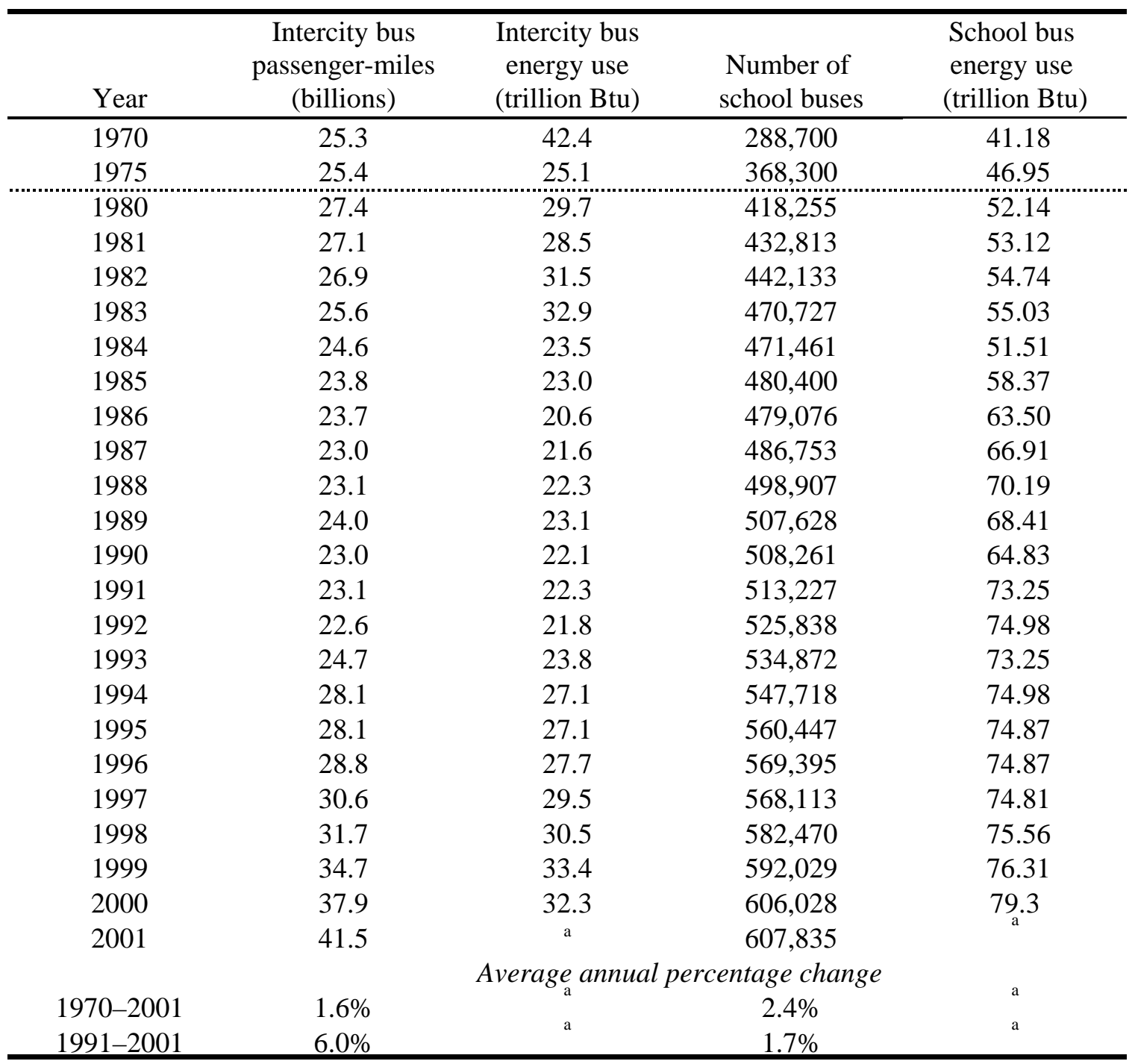

Source:

Intercity bus data and school bus energy use - Eno Foundation for Transportation, Transportation in America 2001, Nineteenth edition, Washington, DC, pp. 13 and 45. See Appendix A Energy Use Sources for detailed methodology on energy use conversion. (Additional resources: www.enotrans.com)

School buses - Federal Highway Administration, Highway Statistics 2001, Washington, DC, 2002, Table MV-10, and annual. (Additional resources: www.fhwa.dot.gov/policy/ohpi)

\footnotetext{
${ }^{a}$ Data are not yet available.
} 


\section{Chapter 6 \\ Alternative Fuel and Advanced Technology \\ Vehicles and Characteristics}

Summary Statistics from Tables in this Chapter

\begin{tabular}{ccc}
\hline Source & & \\
\hline Table 6.1 & Alternative fuel vehicles in use, 2002 estimates & 518,919 \\
& LPG & 281,286 \\
& CNG & 126,341 \\
& E85 & \\
& Electric & 82,477 \\
& M85 & 19,755 \\
Table 6.4 & NomG & 5,873 \\
& LPG of alternative fuel refuel sites, 2002 & 3,187 \\
& CNG & 5,739 \\
& Electric & 3,431 \\
Table 6.5 & U.S. sales of advanced technology vehicles, 1999-2002 & 1,166 \\
& Honda Insight & 872 \\
& Toyota Prius & 10,747 \\
& Honda Civic Hybrid & 41,237 \\
& & $\sim 12,000$ \\
\hline
\end{tabular}

Fuel type abbreviations are used throughout this chapter.

$B 20=20 \%$ biodiesel, $80 \%$ petroleum diesel

CNG $=$ compressed natural gas

E-85 $=85 \%$ ethanol, $15 \%$ gasoline

E-95 $=95 \%$ ethanol, $5 \%$ gasoline

$\mathrm{H}_{2}=$ hydrogen

$L N G=$ liquified natural gas

$L P G=$ liquified petroleum gas

$M-85=85 \%$ methanol, $15 \%$ gasoline

$M-100=100 \%$ methanol

${ }^{\mathrm{a} D o e s}$ not include flex-fuel vehicles. 


\begin{abstract}
Alternative Fuels
The U.S. Department of Energy (DOE) defines alternative fuels as fuels which are substantially non-petroleum and yield energy security and environmental benefits. DOE currently recognizes the following as alternative fuels:

- methanol and denatured ethanol as alcohol fuels (alcohol mixtures that contain no less than $70 \%$ of the alcohol fuel),

- natural gas (compressed or liquefied),

- liquefied petroleum gas,

- hydrogen,

- coal-derived liquid fuels

- fuels derived from biological materials, and

- electricity (including solar energy).
\end{abstract}

DOE has established the Alternative Fuels Data Center (AFDC) in support of its work aimed at fulfilling the Alternative Motor Fuels Act (AMFA) directives. The AFDC is operated and managed by the National Renewable Energy Laboratory (NREL) in Golden, Colorado.

The purposes of the AFDC are:

- to gather and analyze information on the fuel consumption, emissions, operation, and durability of alternative fuel vehicles, and

- to provide unbiased, accurate information on alternative fuels and alternative fuel vehicles to government agencies, private industry, research institutions, and other interested organizations.

The data are collected for three specific vehicle types: (1) light vehicles, including automobiles, light trucks, and mini-vans; (2) heavy vehicles such as tractor-trailers and garbage trucks; and (3) urban transit buses. Much of the AFDC data can be obtained through their web site: www.afdc.doe.gov. Several tables and graphs in this chapter contain statistics which were generated by the AFDC.

DOE is sponsoring the National Alternative Fuels Hotline for Transportation Technologies in order to assist the general public and interested organizations in improving their understanding of alternative transportation fuels. The Hotline can be reached by dialing 1800-423-1DOE, or on the Internet at www.afdc.doe.gov/hotline.html. 
There are more LPG vehicles in use than any other alternative fuel vehicle. The population of E85 vehicles, however, has grown the most since 1993. For details on alternative fuel use by fuel type, see Table 2.3.

Table 6.1

Estimates of Alternative Fuel Vehicles in Use, 1993-2002

\begin{tabular}{|c|c|c|c|c|c|c|c|}
\hline Fuel type & 1993 & 1995 & 1998 & 2000 & $2001^{a}$ & $2002^{\mathrm{a}}$ & $\begin{array}{l}\text { Average annual } \\
\text { percentage } \\
\text { change } \\
\text { 1993-2002 }\end{array}$ \\
\hline LPG & 269,000 & 259,000 & 266,000 & 272,193 & 276,597 & 281,286 & $0.5 \%$ \\
\hline CNG & 32,714 & 50,218 & 78,782 & 100,738 & 113,835 & 126,341 & $16.2 \%$ \\
\hline LNG & 299 & 603 & 1,172 & 2,090 & 2,576 & 3,187 & $30.1 \%$ \\
\hline M85 & 10,263 & 18,319 & 19,648 & 10,426 & 7,827 & 5,873 & $-6.0 \%$ \\
\hline M100 & 414 & 386 & 200 & 0 & 0 & 0 & $-100.0 \%$ \\
\hline $\mathrm{E} 85^{\mathrm{b}}$ & 441 & 1,527 & 12,788 & 58,621 & 71,336 & 82,477 & $78.8 \%$ \\
\hline E95 & 27 & 136 & 14 & 4 & 0 & 0 & $-100.0 \%$ \\
\hline Electricity & 1,690 & 2,860 & 5,243 & 11,834 & 17,848 & 19,755 & $31.4 \%$ \\
\hline Total & 314,848 & 333,049 & 383,847 & 455,906 & 490,019 & 518,919 & $5.7 \%$ \\
\hline
\end{tabular}

Source:

U. S. Department of Energy, Energy Information Administration, Alternatives to Traditional Transportation Fuels, 2000, Washington, DC, 2002, web site www.eia.doe.gov/cneaf/alternate/page/datatables.html. (Additional resources: www.eia.doe.gov)

a2001 data are preliminary. 2002 data are based on plans or projections.

${ }^{\mathrm{b}}$ Does not include flex-fuel vehicles. 
Nearly $90 \%$ of private alternative fuel vehicles are fueled by LPG and CNG. The Federal Government does not own many LPG vehicles; its alternative fuel vehicle fleet is 30\% CNG and 65\% E-85 vehicles in 2002.

Table 6.2

Estimates of Alternative Fuel Vehicles by Ownership, 1998 and 2002

\begin{tabular}{|c|c|c|c|c|c|c|}
\hline \multirow[b]{2}{*}{ Fuel type } & \multicolumn{2}{|c|}{ Private } & \multicolumn{2}{|c|}{$\begin{array}{l}\text { State and local } \\
\text { government }\end{array}$} & \multicolumn{2}{|c|}{ Federal Government } \\
\hline & 1998 & $2002^{\mathrm{a}}$ & 1998 & $2002^{\mathrm{a}}$ & 1998 & $2002^{\mathrm{a}}$ \\
\hline LPG & 213,000 & 222,727 & 53,000 & 56,999 & 175 & 1,560 \\
\hline CNG & 43,329 & 66,866 & 22,291 & 38,619 & 13,162 & 20,856 \\
\hline LNG & 279 & 876 & 879 & 2,216 & 14 & 95 \\
\hline M-85 & 10,773 & 3,061 & 8,332 & 2,809 & 543 & 3 \\
\hline M-100 & 0 & 0 & 200 & 0 & 0 & 0 \\
\hline E-85 & 2,595 & 25,294 & 5,906 & 12,571 & 4,287 & 44,612 \\
\hline E-95 & 0 & 0 & 14 & 0 & 0 & 0 \\
\hline Electricity & 3,461 & 8,362 & 1,621 & 9,629 & 161 & 1,764 \\
\hline Total & 273,437 & 327,186 & 92,243 & 122,843 & 18,342 & 68,890 \\
\hline
\end{tabular}

\section{Source:}

U. S. Department of Energy, Energy Information Administration, Alternatives to Traditional Transportation Fuels, 2000, Washington, DC, 2002, web site www.eia.doe.gov/cneaf/alternate/page/datatables.html. (Additional resources: www.eia.doe.gov)

${ }^{\mathrm{a} B a s e d}$ on plans or projections. 
Table 6.3

Alternative Fuel Vehicles Available by Manufacturer, Model Year 2001

\begin{tabular}{|c|c|c|c|}
\hline Model & Fuel & Type & Emission class \\
\hline \multicolumn{4}{|c|}{ Daimler Chrysler: 1-800-999-FLEET } \\
\hline Chrysler Sebring Sedan & E-85 flex fuel & Mid-Size Sedan & LEV \\
\hline Chrysler Sebring Convertible & E-85 flex fuel & Convertible & LEV \\
\hline Dodge Stratus Sedan & E-85 flex fuel & Sedan & LEV \\
\hline Chrysler Town and Country & E-85 flex fuel & Minivan & LEV \\
\hline Dodge Caravan & E-85 flex fuel & Minivan & LEV \\
\hline Dodge Grand Caravan & E-85 flex fuel & Minivan & LEV \\
\hline Dodge Ram Maxi Van & CNG dedicated & Van & ILEV/ULEV/CA-SULEV \\
\hline Dodge Ram Van & CNG dedicated & Van & ILEV/ULEV/CA-SULEV \\
\hline \multicolumn{4}{|l|}{ Ford: 1-877-ALT-FUEL } \\
\hline Ford F-150 & CNG Bi-Fuel & Light-duty pickup & ULEV \\
\hline Ford E-Series Van & CNG Dedicated & Van & SULEV \\
\hline Ford E-Series Wagon & CNG Dedicated & Wagon & CA-SULEV \\
\hline Ford F-150 & CNG Dedicated & Light-duty pickup & ILEV/SULEV/CA-SULEV \\
\hline Ford Crown Victoria & CNG dedicated & Large sedan & ULEV \\
\hline Ford Taurus & E-85 flex fuel & Mid-size sedan & ULEV \\
\hline Mercury Sable & E-85 flex fuel & Wagon & ULEV \\
\hline Ford Explorer & E-85 flex fuel & Sport utility vehicle & LEV \\
\hline Mercury Mountaineer & E-85 flex fuel & Sport utility vehicle & LEV \\
\hline Ford Explorer Sport & E-85 flex-fuel & Sport utility vehicle & LEV \\
\hline Ford Ranger & E-85 flex-fuel & Light-duty pickup & LEV \\
\hline Ford F-150 & LPG Bi-fuel & Light-duty pickup & ULEV \\
\hline \multicolumn{4}{|c|}{ General Motors: 1-800-25Electric, 313-556-7723 or 1-888-GM-AFT-4U (CNG) } \\
\hline Chevy Silverado & CNG Bi-fuel & Light-duty pickup & LEV \\
\hline GMC Sierra & CNG Bi-fuel & Light-duty pickup & LEV \\
\hline Chevy Express & CNG Bi-fuel/CNG Dedicated & Cargo or passenger van & ULEV/(Dedicated CA-SULEV) \\
\hline GMC Savana & CNG Bi-fuel/CNG Dedicated & Cargo or passenger van & ULEV/(Dedicated CA-SULEV) \\
\hline Chevrolet Cavalier & CNG Bi-fuel & Compact sedan & LEV \\
\hline GMC Yukon & E-85 flex-fuel & Sport utility vehicle & Tier 1 \\
\hline Chevrolet Suburban & E-85 flex fuel & Sport utility vehicle & Tier 1 \\
\hline GMC Yukon XL & E-85 flex fuel & Sport utility vehicle & Tier 1 \\
\hline Chevrolet Silverado & E-85 flex fuel & Light-duty pickup & Tier 1 \\
\hline GMC Sierra & E-85 flex fuel & Light-duty pickup & Tier 1 \\
\hline \multicolumn{4}{|l|}{ Honda: 1-888-CCHonda } \\
\hline Civic GX & CNG dedicated & Compact sedan & ILEV/SULEV (Tier II Bin II) \\
\hline \multicolumn{4}{|l|}{ Mazda: 1-800-222-5500 } \\
\hline B3000 & E-85 flex fuel & Light-duty pickup & LEV \\
\hline \multicolumn{4}{|l|}{ Nissan: 1-310-771-3422 } \\
\hline Altra EV (CA fleets only) & Electric-lithium ion & Mid-size wagon & ZEV \\
\hline Hypermini (CA fleets only) & Electric-lithium ion & Two-seater & ZEV \\
\hline \multicolumn{4}{|c|}{ Solectria Corporation: 1-508-658-2231 } \\
\hline Civitan & Electric-lead acid & Service van & ZEV \\
\hline \multicolumn{4}{|c|}{$\begin{array}{l}\text { Source: } \\
\text { U.S. Department of Energy, National Alternative Fuels Data Center, web site, www.afdc.doe.gov/afvehicles.htm, May } 2003 . \\
\text { (Additional resources: www.afdc.nrel.gov) }\end{array}$} \\
\hline \multicolumn{4}{|c|}{ Note: } \\
\hline
\end{tabular}


This list includes public and private refuel sites; therefore, not all of these sites are available to the public.

Table 6.4

Number of Alternative Refuel Sites by State and Fuel Type, 2002

\begin{tabular}{|c|c|c|c|c|c|c|c|c|}
\hline State & $\begin{array}{l}\text { CNG } \\
\text { sites }\end{array}$ & $\begin{array}{l}\text { E85 } \\
\text { sites }\end{array}$ & $\begin{array}{l}\text { LPG } \\
\text { sites }\end{array}$ & $\begin{array}{l}\text { LNG } \\
\text { sites }\end{array}$ & $\begin{array}{l}\text { Electric } \\
\text { sites }\end{array}$ & $\begin{array}{l}\text { Biodiesel } \\
\text { sites }\end{array}$ & $\begin{array}{l}\text { Hydrogen } \\
\text { sites }\end{array}$ & Total \\
\hline Alabama & 9 & 0 & 77 & 2 & 34 & 0 & 0 & 122 \\
\hline Alaska & 0 & 0 & 9 & 0 & 0 & 0 & 0 & 9 \\
\hline Arizona & 27 & 1 & 109 & 1 & 63 & 2 & 1 & 204 \\
\hline Arkansas & 4 & 0 & 85 & 0 & 0 & 0 & 0 & 89 \\
\hline California & 188 & 0 & 345 & 5 & 545 & 9 & 5 & 1,097 \\
\hline Colorado & 35 & 8 & 83 & 1 & 6 & 1 & 0 & 134 \\
\hline Connecticut & 25 & 0 & 29 & 0 & 5 & 0 & 0 & 59 \\
\hline Delaware & 4 & 0 & 4 & 0 & 0 & 0 & 0 & 8 \\
\hline District of Columbia & 2 & 0 & 0 & 0 & 0 & 0 & 0 & 2 \\
\hline Florida & 42 & 0 & 154 & 1 & 3 & 0 & 0 & 199 \\
\hline Georgia & 65 & 0 & 54 & 1 & 83 & 0 & 0 & 203 \\
\hline Hawaii & 0 & 0 & 7 & 0 & 11 & 3 & 0 & 21 \\
\hline Idaho & 9 & 1 & 33 & 0 & 1 & 0 & 0 & 43 \\
\hline Illinois & 21 & 13 & 91 & 0 & 0 & 2 & 0 & 127 \\
\hline Indiana & 32 & 1 & 54 & 3 & 0 & 1 & 0 & 91 \\
\hline Iowa & 0 & 11 & 44 & 0 & 0 & 0 & 0 & 55 \\
\hline Kansas & 5 & 1 & 67 & 1 & 0 & 0 & 0 & 74 \\
\hline Kentucky & 6 & 7 & 26 & 0 & 0 & 0 & 0 & 39 \\
\hline Louisiana & 14 & 0 & 45 & 0 & 0 & 0 & 0 & 59 \\
\hline Maine & 0 & 0 & 20 & 0 & 0 & 2 & 0 & 22 \\
\hline Maryland & 28 & 2 & 28 & 1 & 1 & 3 & 0 & 63 \\
\hline Massachusetts & 12 & 0 & 44 & 0 & 41 & 1 & 0 & 98 \\
\hline Michigan & 25 & 4 & 138 & 1 & 5 & 6 & 0 & 179 \\
\hline Minnesota & 11 & 69 & 58 & 1 & 0 & 0 & 0 & 139 \\
\hline Mississippi & 3 & 0 & 34 & 0 & 0 & 0 & 0 & 37 \\
\hline Missouri & 7 & 5 & 151 & 0 & 0 & 1 & 0 & 164 \\
\hline Montana & 9 & 1 & 40 & 1 & 0 & 1 & 0 & 52 \\
\hline Nebraska & 5 & 5 & 27 & 0 & 0 & 1 & 0 & 38 \\
\hline Nevada & 20 & 0 & 34 & 0 & 0 & 6 & 1 & 61 \\
\hline New Hampshire & 1 & 0 & 30 & 0 & 12 & 2 & 0 & 45 \\
\hline New Jersey & 30 & 0 & 29 & 0 & 0 & 0 & 0 & 59 \\
\hline New Mexico & 15 & 1 & 81 & 1 & 0 & 1 & 0 & 99 \\
\hline New York & 60 & 0 & 95 & 0 & 16 & 0 & 0 & 171 \\
\hline N. Carolina & 11 & 0 & 75 & 0 & 6 & 21 & 0 & 113 \\
\hline N. Dakota & 4 & 2 & 18 & 0 & 0 & 0 & 0 & 24 \\
\hline Ohio & 35 & 2 & 73 & 1 & 0 & 1 & 0 & 112 \\
\hline Oklahoma & 58 & 0 & 93 & 0 & 0 & 0 & 0 & 151 \\
\hline Oregon & 16 & 0 & 49 & 1 & 4 & 2 & 0 & 72 \\
\hline Pennsylvania & 55 & 0 & 104 & 1 & 0 & 1 & 0 & 161 \\
\hline Rhode Island & 6 & 0 & 7 & 0 & 2 & 0 & 0 & 15 \\
\hline S. Carolina & 4 & 1 & 62 & 0 & 0 & 1 & 0 & 68 \\
\hline S. Dakota & 2 & 6 & 26 & 0 & 0 & 0 & 0 & 34 \\
\hline Tennessee & 2 & 0 & 59 & 0 & 0 & 0 & 0 & 61 \\
\hline Texas & 67 & 0 & 423 & 7 & 7 & 1 & 0 & 505 \\
\hline Utah & 62 & 2 & 38 & 1 & 0 & 0 & 0 & 103 \\
\hline Vermont & 0 & 0 & 16 & 0 & 11 & 0 & 0 & 27 \\
\hline Virginia & 24 & 1 & 58 & 3 & 11 & 1 & 0 & 98 \\
\hline Washington & 23 & 0 & 83 & 1 & 6 & 7 & 0 & 120 \\
\hline W. Virginia & 43 & 0 & 9 & 0 & 0 & 0 & 0 & 52 \\
\hline Wisconsin & 22 & 4 & 77 & 0 & 0 & 0 & 0 & 103 \\
\hline Wyoming & 18 & 1 & 36 & 1 & 0 & 2 & 0 & 58 \\
\hline Total & 1,166 & 149 & 3,431 & 35 & 872 & 79 & 7 & 5,739 \\
\hline
\end{tabular}

Source:

U.S. Department of Energy, Alternative Fuels Data Center web site, www.afdc.doe.gov/refuel/state_tot.shtml, April 2003. 
Clean Cities is a locally-based government/industry partnership, coordinated by the U.S. Department of Energy to expand the use of alternatives to gasoline and diesel fuel. By combining the decision-making with voluntary action by partners, the "grass-roots" approach of Clean Cities departs from traditional "top-down" Federal programs.

\section{Figure 6.1. Clean Cities Coalitions}

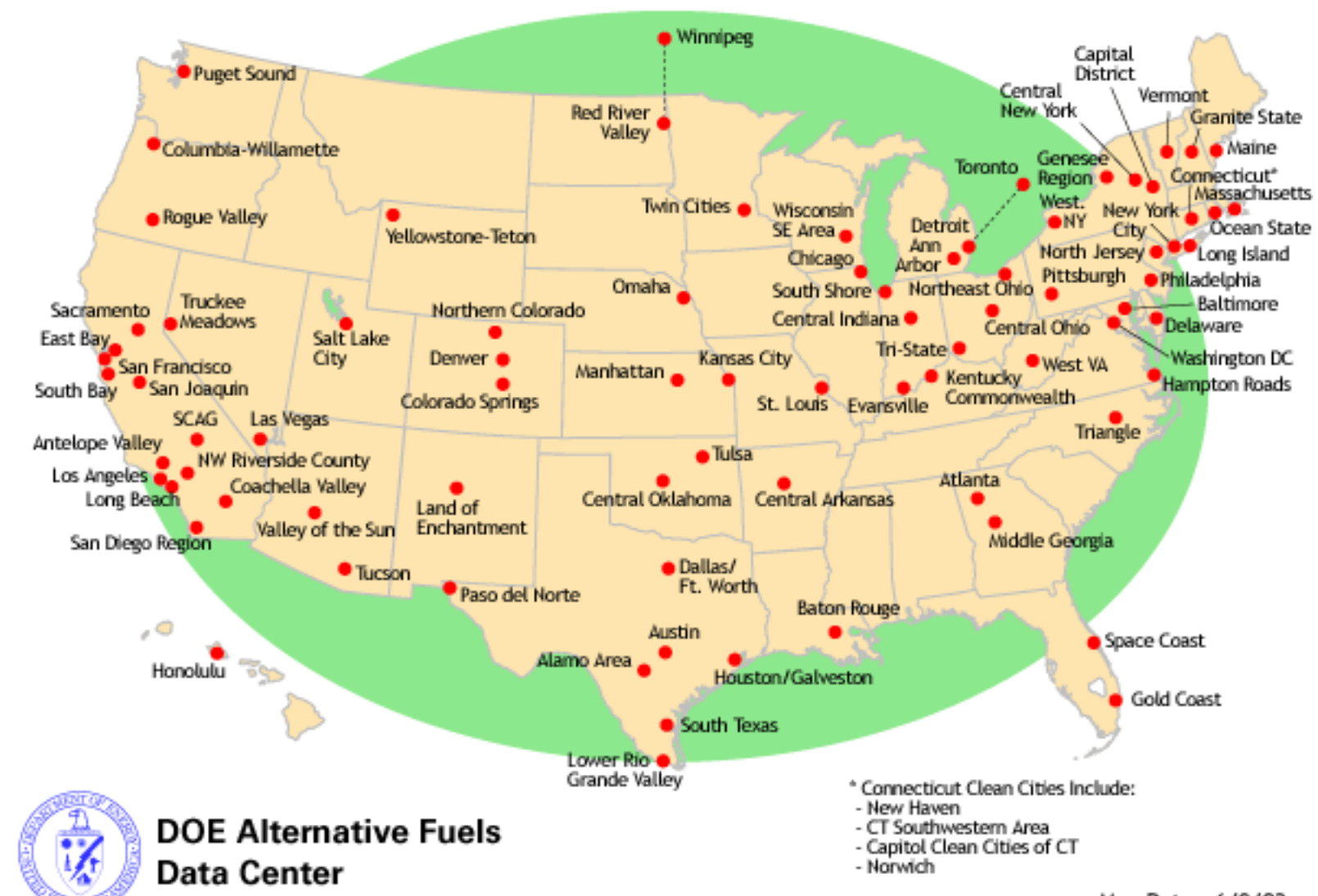

Map Date: 6/9/03

\section{Source:}

U.S. Department of Energy, Alternative Fuel Data Center, July 2003.

(Additional resources: www.ccities.doe.gov) 
The Honda Insight, Civic Hybrid and Toyota Prius are the three advanced technology vehicles which are currently available to the public in the U.S. They are hybrid vehicles, using both electricity (from batteries) and mechanical power (from a small internal combustion engine). Learn more about DOE's hybrid vehicle program at: www.ott.doe.gov/hev.

Table 6.5

Sales and Specifications of Available Advanced Technology Vehicles

\begin{tabular}{|c|c|c|c|}
\hline & $\begin{array}{c}\text { Honda } \\
\text { Insight } \mathbf{C V T}^{\mathrm{a}} \\
\end{array}$ & $\begin{array}{c}\text { Toyota } \\
\text { Prius }^{\text {CVT }}{ }^{\mathrm{a}} \\
\end{array}$ & $\begin{array}{c}\text { Honda } \\
\text { Civic Hybrid CVT SULEV } \\
\end{array}$ \\
\hline Fuel economy (city/hwy) & 57/56 mpg & 52/45 mpg & $47 / 48 \mathrm{mpg}$ \\
\hline Fuel tank capacity & 10.6 gal. & 11.9 gal. & 11.9 gal. \\
\hline Acceleration (0-60 mph) & 11.5 sec. & 12.3 sec. & 11.6 sec. \\
\hline Emissions rating & SULEV & SULEV & SULEV \\
\hline Aerodynamics & $0.25 \mathrm{Cd}$ & $0.29 \mathrm{Cd}$ & $0.28 \mathrm{Cd}$ \\
\hline Curb weight & 1,967 lbs. & 2,765 lbs. & 2,740 lbs. \\
\hline Passenger capacity & 2 & 5 & 5 \\
\hline $\begin{array}{l}\text { Dimensions: } \\
\text { Length } \\
\text { Width }\end{array}$ & $\begin{array}{l}155.1 \mathrm{in.} \\
66.7 \mathrm{in.}\end{array}$ & $\begin{array}{l}169.6 \text { in. } \\
66.7 \text { in. }\end{array}$ & $\begin{array}{c}174.8 \mathrm{in} . \\
67.5 \mathrm{in.}\end{array}$ \\
\hline Cargo Capacity & $16.3 \mathrm{ft}^{3}$ & $11.8 \mathrm{ft}^{3}$ & $10.1 \mathrm{ft}^{3}$ \\
\hline Price & $\$ 21,280$ & $\$ 20,480$ & $\$ 20,550$ \\
\hline \multicolumn{4}{|c|}{ Calendar year sales in the U.S. } \\
\hline 1999 & 17 & 0 & 0 \\
\hline 2000 & 3,788 & 5,562 & 0 \\
\hline 2001 & 4,726 & 15,556 & 0 \\
\hline 2002 & 2,216 & 20,119 & $\sim 12,000^{\mathrm{b}}$ \\
\hline Total & 10,747 & 41,237 & $\sim 12,000^{\mathrm{b}}$ \\
\hline
\end{tabular}

Source:

Manufacturer's web sites: www.hondacars.com and www.toyota.com.

Insight and Prius sales data - Ward's Communications, Inc., Wards Automotive Reports, Southfield, MI, 2003.

Civic sales data - Crain Communications, Automotive News, December 23, 2002.

Note:

SULEV = Super ultra low emission vehicle. See Chapter 12 for details on emissions.

${ }^{a}$ Specifications are for the model containing a continuously variable transmission (CVT).

bSales for the Civic Hybrid are not shown separately from other Civic models, but estimates of 2002 sales are approximately 12,000 vehicles since its March 2002 debut. 


\section{FreedomCAR and Fuel Initiative}

\section{www.eere.energy.gov/hydrogenfuel \\ www.eere.energy.gov/vehiclesandfuels \\ www.eere.energy.gov/hydrogenandfuelcells}

Freedom Cooperative Automotive Research (FreedomCAR) is a government-industry partnership for the advancement of high-efficiency vehicles, focused on fuel cells and hydrogen produced from renewable energy sources. The U.S. Department of Energy and the U.S. Council for Automotive Research (composed of automakers Ford, General Motors, and DaimlerChrysler) began this effort in January 2002 with the long-term goal of developing technologies for hydrogenpowered fuel cell cars and trucks that will require no foreign oil and emit no harmful pollutants or greenhouse gases.

But, successful marketing of hydrogen cars may depend on the development of a hydrogen infrastructure, like today's petroleum infrastructure, at the same time automakers are perfecting hydrogen vehicles. That means the creation of everything from hydrogen manufacturing plants, to distribution and storage networks, to convenient hydrogen fueling stations.

The FreedomCAR and Hydrogen Fuel Initiative is aimed at coordinating the efforts of the energy companies, automakers, utilities, state and local governments, foreign interests and other appropriate players. By working on parallel tracks, developing the hydrogen vehicles and infrastructure concurrently instead of consecutively, a decision to go forward with the commercialization of hydrogen automobiles could be made as early as 2015, 15 years ahead of current projections.

For additional information about the FreedomCAR and Hydrogen Fuel Initiative, visit the websites listed above or call 1-800-DOE-3732. 
The relative efficiencies of seven different hydrogen production methods are summarized here as a result of research done by Argonne National Laboratory. The study indicates that:

- Steam methane reforming is a very efficient and cost-effective way to manufacture hydrogen, but there are issues with natural gas supply and carbon sequestration.

- Electrolysis is well understood but its overall efficiency depends largely on efficient electrical generation.

- Thermochemical cycles have the potential to produce hydrogen from any high-temperature heat source with high efficiency in very high volumes. New research into lower-temperature cycles should increase the applicability of this method.

Table 6.6

Hydrogen Production Methods

\begin{tabular}{lcccc}
\hline & $\begin{array}{c}\text { Maximum process } \\
\text { temperature } \\
\text { Method }\end{array}$ & $\begin{array}{c}\text { Overall } \\
\text { efficiency (\%) }\end{array}$ & Status \\
\hline Sulfur-iodine thermo-chemical cycle & 850 & $45 ! 49$ & Calculation $^{\mathrm{a}}$ \\
Calcium-bromine thermo-chemical cycle & 760 & $36 ! 40$ & Pilot plant $^{\mathrm{b}}$ \\
Copper-chlorine thermo-chemical cycle & 500 & 41 & Bench $^{\mathrm{c}}$ \\
Electrolysis & 90 & $20 ! 30^{\mathrm{d}}$ & Commercial $^{\mathrm{e}}$ \\
High-temperature electrolysis & 900 & 40 & Experiment $^{\mathrm{f}}$ \\
Steam methane reforming (SMR) & 900 & 77 & Commercial $^{\mathrm{e}}$ \\
SMR with $\mathrm{CO}_{2}$ sequestration & 900 & 58 & Calculation $^{\mathrm{g}}$ \\
\hline
\end{tabular}

\section{Source:}

Argonne National Laboratory (ANL), Meeting U.S. Transportation Needs in the Hydrogen Economy, http://www.hydrogen.anl.gov/pdfs/meeting_transportation_needs.pdf, May 2003, and updates from ANL.

\section{Note:}

The efficiency is the ratio of the energy value of the hydrogen produced to that of the heat used in the process, except in the case of steam methane reforming, where it is the ratio of the energy of the hydrogen produced to that of the methane consumed.

${ }^{\mathrm{a}}$ Calculated from laboratory experiments and thermodynamic data. A full-scale pilot plant has not yet been built.

${ }^{\mathrm{b}}$ A pilot plant has been constructed.

${ }^{\mathrm{c}}$ The efficiency calculation is based on thermodynamics.

${ }^{d}$ Takes electricity generation efficiency into account.

${ }^{\text {e }}$ Commercial Data.

${ }^{\mathrm{f}}$ Calculated from commercial electrolysis data and thermodynamic data. No pilot plant data are yet available.

${ }^{\mathrm{g}}$ Calculated from SMR commercial plant data and estimates of the energy required to sequester the $\mathrm{CO}_{2}$. 
According to recent data compiled by Argonne National Laboratory, there are more than 200 hydrogen production plants in existence today. Many of the plants that produce hydrogen are part of other processes, like petroleum refining, ammonia production, and methanol production.

Table 6.7

U.S. Hydrogen Production Plants and Storage Terminals

\begin{tabular}{lcc}
\hline & Number of production plants & Number of storage terminals \\
\hline Gaseous hydrogen & 81 & 14 \\
Liquid hydrogen & 10 & 3 \\
Gaseous and liquid hydrogen & Not applicable & 3 \\
Petroleum refineries & 61 & Not available \\
Ammonia producers & 54 & Not available \\
Methanol producers & 15 & 4 \\
\hline Total & 221 & 24 \\
\hline
\end{tabular}

Source:

Mintz, Marianne, Argonne National Laboratory, September 2003. 
In 1999 (the latest year for which data are available) the U.S. accounted for about 20\% of world hydrogen consumption. Ammonia producers made up $61 \%$ of World hydrogen consumption, but only $38 \%$ of U.S. hydrogen consumption.

Table 6.8

U.S. and World Hydrogen Consumption by End-Use Category, 1999

\begin{tabular}{|c|c|c|c|c|c|}
\hline & \multicolumn{2}{|c|}{ United States } & \multicolumn{2}{|c|}{ World total } & \multirow{2}{*}{$\begin{array}{c}\text { U.S. share } \\
\text { of World } \\
\text { total }\end{array}$} \\
\hline & $\begin{array}{l}\text { (trillion } \\
\text { cubic feet) }\end{array}$ & (share) & $\begin{array}{l}\text { (trillion } \\
\text { cubic feet) }\end{array}$ & (share) & \\
\hline \multicolumn{6}{|l|}{ Captive users: } \\
\hline Ammonia producers & 1.185 & $38 \%$ & 9.662 & $61 \%$ & $12 \%$ \\
\hline Oil refiners ${ }^{\mathrm{a}}$ & 1.164 & $37 \%$ & 3.721 & $23 \%$ & $31 \%$ \\
\hline Methanol producers & 0.303 & $10 \%$ & 1.428 & $9 \%$ & $21 \%$ \\
\hline Other & 0.121 & $4 \%$ & 0.482 & $3 \%$ & $25 \%$ \\
\hline Merchant users & 0.379 & $12 \%$ & 0.570 & $4 \%$ & $67 \%$ \\
\hline Total & 3.153 & $100 \%$ & 15.864 & $100 \%$ & $20 \%$ \\
\hline
\end{tabular}

\section{Source:}

Hydrogen, Chemical Economics Handbook program, SRI Consulting, Menlo Park, CA, July 2001.

\section{Note:}

Captive users consume hydrogen at the site where it is produced. Merchant users consume hydrogen at sites other than where it is produced.

${ }^{a}$ Excluding byproduct hydrogen. 
The Department of Energy is currently developing systems which will store hydrogen on-board a light vehicle. Below is a list of storage technologies and the advantages/disadvantages of each The DOE goals for on-board hydrogen storage systems are listed at the bottom of the table.

Table 6.9

Hydrogen Storage Systems for On-Board Light Vehicles

\begin{tabular}{|c|c|c|}
\hline Storage technology & System status & Advantages/disadvantages \\
\hline Chemical hydride & $\begin{array}{l}1.6 \mathrm{kWh} / \mathrm{kg}, 1.4 \mathrm{kWh} / \mathrm{L} \text {, } \\
\$ 8 / \mathrm{kWh}\end{array}$ & $\begin{array}{l}\square \text { Low pressure } \\
\text { 囚 Low cost, energy-efficient regeneration } \\
\text { processes have not been developed } \\
\text { 囚 By-product removal }\end{array}$ \\
\hline Complex metal hydride & $\begin{array}{l}0.8 \mathrm{kWh} / \mathrm{kg}, 0.6 \mathrm{kWh} / \mathrm{L} \text {, } \\
\$ 16 / \mathrm{kWh}\end{array}$ & $\begin{array}{l}\square \text { Low pressure } \\
\square \text { Reversible } H_{2} \text { uptake and release } \\
\text { 囚 Insufficient storage capacity at practical } \\
\text { temperature and pressure }\end{array}$ \\
\hline Liquid hydrogen & $\begin{array}{l}2.0 \mathrm{kWh} / \mathrm{kg}, 1.6 \mathrm{kWh} / \mathrm{L} \text {, } \\
\$ 6 / \mathrm{kWh}\end{array}$ & $\begin{array}{l}\square \text { Lowest capital cost } \\
\square \text { Highest gravimetric and volumetric } \\
\text { capacities } \\
\square \text { Most energy intensive } \\
\square \text { Boil-off requires venting, and presents an } \\
\text { energy penalty and a potential safety hazard }\end{array}$ \\
\hline $\begin{array}{l}\text { 10,000 psi compressed } \\
\text { hydrogen tanks } \\
\text { 5,000 psi compressed } \\
\text { hydrogen tanks }\end{array}$ & $\begin{array}{l}1.9 \mathrm{kWh} / \mathrm{kg}, 1.3 \mathrm{kWh} / \mathrm{L} \text {, } \\
\$ 16 / \mathrm{kWh}\end{array}$ & $\begin{array}{l}\square \text { Near-term solution to hydrogen storage } \\
\square \text { Most energy efficient method to densify } \mathrm{H}_{2} \\
\text { Ð High pressure } \\
\text { 邓 Cost is high due to high pressure } \\
\text { containment materials }\end{array}$ \\
\hline \multicolumn{3}{|c|}{ Department of Energy 2010 and 2015 System Goals ${ }^{\mathrm{a}}$} \\
\hline & $\begin{aligned} & \text { Year } 2010 \\
\text { - } & 2.0 \mathrm{kWh} / \mathrm{kg}(6 \mathrm{wt} \%) \\
\text { - } & 1.5 \mathrm{kWh} / \mathrm{L} \\
\text { - } & \$ 4 / \mathrm{kWh}\end{aligned}$ & $\begin{aligned} & \text { Year } 2015 \\
\text { - } & 3.0 \mathrm{kWh} / \mathrm{kg}(9 \mathrm{wt} \%) \\
\cdot & 2.7 \mathrm{kWh} / \mathrm{L} \\
\text { - } & \$ 2 / \mathrm{kWh}\end{aligned}$ \\
\hline
\end{tabular}

\section{Source:}

U.S. Department of Energy, Hydrogen, Fuel Cells \& Infrastructure Technologies Program, 2003.

${ }^{a}$ Goals apply to all storage technologies and are for the complete system including storage material, packaging, regulators, valves, and any thermal management or other ancillary equipment; cost goals and status are based on high-volume production. 
Table 6.10

Properties of Conventional and Alternative Fuels

\begin{tabular}{|c|c|c|c|c|}
\hline Property & Gasoline & No. 2 diesel & Methanol & Ethanol \\
\hline Chemical formula & $\mathrm{C}_{4}$ to $\mathrm{C}_{12}$ & $\mathrm{C}_{10}$ to $\mathrm{C}_{20}$ & $\mathrm{CH}_{3} \mathrm{OH}$ & $\mathrm{C}_{2} \mathrm{H}_{5} \mathrm{OH}$ \\
\hline Physical state & Liquid & Liquid & Liquid & Liquid \\
\hline Molecular weight & $100-105$ & .200 & 32.04 & 46.07 \\
\hline \multicolumn{5}{|l|}{ Composition (weight \%) } \\
\hline Carbon & $85-88$ & $84-87$ & 37.5 & 52.2 \\
\hline Hydrogen & $12-15$ & $33-16$ & 12.6 & 13.1 \\
\hline Oxygen & 0 & 0 & 49.9 & 34.7 \\
\hline Main fuel source(s) & Crude oil & Crude oil & $\begin{array}{l}\text { Natural gas, coal, or } \\
\text { woody biomass }\end{array}$ & $\begin{array}{l}\text { Corn, grains, or } \\
\text { agricultural waste }\end{array}$ \\
\hline Specific gravity $(60 \varangle \mathrm{F} / 60 \varangle \mathrm{F})$ & $0.72-0.78$ & $0.81-0.89$ & 0.796 & 0.796 \\
\hline Density (lb/gal @ 60× F) & $6.0-6.5$ & $6.7-7.4$ & 6.63 & 6.61 \\
\hline Boiling temperature (F & $80-437$ & $370-650$ & 149 & 172 \\
\hline Freezing point (F女 & -40 & $-40-30$ & -143.5 & -173.2 \\
\hline Autoiginition temperature (F & 495 & .600 & 867 & 793 \\
\hline Reid vapor pressure (psi) & $8-15$ & 0.2 & 4.6 & 2.3 \\
\hline
\end{tabular}

\begin{tabular}{|c|c|c|c|}
\hline Property & Propane & $\mathrm{CNG}$ & Hydrogen \\
\hline Chemical formula & $\mathrm{C}_{3} \mathrm{H}_{8}$ & $\mathrm{CH}_{4}$ & $\mathrm{H}_{2}$ \\
\hline Physical state & Compressed gas & Compressed gas & $\begin{array}{c}\text { Compressed gas or } \\
\text { liquid }\end{array}$ \\
\hline Molecular weight & 44.1 & 16.04 & 2.02 \\
\hline \multicolumn{4}{|l|}{ Composition (weight \%) } \\
\hline Carbon & 82 & 75 & 0 \\
\hline Hydrogen & 18 & 25 & 100 \\
\hline Oxygen & $\mathrm{n} / \mathrm{a}$ & $\mathrm{n} / \mathrm{a}$ & 0 \\
\hline Main fuel source & Underground reserves & Underground reserves & $\begin{array}{l}\text { Natural gas, methanol, } \\
\text { and other energy } \\
\text { sources }\end{array}$ \\
\hline Specific gravity $(60 \varangle / 60 \varangle)$ & 0.508 & 0.424 & 0.07 \\
\hline Density (lb/gal @ 60× F) & 4.22 & 1.07 & $\mathrm{n} / \mathrm{a}$ \\
\hline Boiling temperature (F $\Varangle$ & -44 & -259 & -423 \\
\hline Freezing point (F女 & -305.8 & -296 & -435 \\
\hline Autoiginition temperature (F女 & 850-950 & 1,004 & $1,050-1,080$ \\
\hline Reid vapor pressure (psi) & 208 & 2,400 & $\mathrm{n} / \mathrm{a}$ \\
\hline
\end{tabular}

\section{Source:}

Alternative Fuels Data Center, "Properties of Fuel," www.afdc.doe.gov/pdfs/fueltable.pdf and "Fuel Comparison," www.afdc.doe.gov/fuel_comp.html, August 2003.

Note:

$\mathrm{n} / \mathrm{a}=$ not applicable. 
There are many types of fuel cells which can be used in many different applications. The Proton Exchange Membrane Fuel Cells (PEMFCs) are the best candidates for transportation-related applications, such as cars, trucks, buses and small portable devices, due to their relatively low operating temperatures and their ability to vary their output to meet changing power demands.

Table 6.11

Fuel Cell Type Comparison

\begin{tabular}{lccccc}
\hline & Electrolyte & $\begin{array}{c}\text { Operating } \\
\text { Temperature }\end{array}$ & Efficiency & $\begin{array}{c}\text { Electrical } \\
\text { Power }\end{array}$ & $\begin{array}{c}\text { Possible } \\
\text { Applications }\end{array}$ \\
\hline Alkaline & Potassium Hydroxide & $60-90^{\circ} \mathrm{C}$ & $45-60 \%$ & Up to $20 \mathrm{~kW}$ & Submarines, spacecraft \\
$\begin{array}{l}\text { Direct } \\
\text { Methanol }\end{array}$ & Polymer Membrane & $60-130^{\circ} \mathrm{C}$ & $40 \%$ & $<1 \mathrm{~kW}$ & Portable applications \\
$\begin{array}{l}\text { Molten } \\
\text { Carbonate }\end{array}$ & $\begin{array}{c}\text { Immobilized Liquid } \\
\text { Molten Carbonate }\end{array}$ & $650^{\circ} \mathrm{C}$ & $45-60 \%$ & $>1 \mathrm{MW}$ & Power stations \\
$\begin{array}{l}\text { Phosphoric } \\
\text { Acid }\end{array}$ & $\begin{array}{c}\text { Immobilized Liquid } \\
\text { Phosphoric Acid }\end{array}$ & $200^{\circ} \mathrm{C}$ & $36-38 \%$ & $>50 \mathrm{~kW}$ & Power stations \\
$\begin{array}{l}\text { Proton } \\
\begin{array}{l}\text { Exchange } \\
\text { Membrane }\end{array}\end{array}$ & $\begin{array}{c}\text { Ion Exchange } \\
\text { Membrane }\end{array}$ & $80^{\circ} \mathrm{C}$ & $35-60 \%$ & $\mathrm{Up} \mathrm{to} 250 \mathrm{~kW}$ & $\begin{array}{c}\text { Cars, buses, residential } \\
\text { energy supply }\end{array}$ \\
\begin{tabular}{l} 
Solid Oxide \\
\hline
\end{tabular} & Ceramic & $1,000^{\circ} \mathrm{C}$ & $50-65 \%$ & Up to $1 \mathrm{MW}$ & Small power stations \\
\hline
\end{tabular}

\section{Source:}

Fuel Cell Today, http://www.fuelcelltoday.com . 


\section{Chapter 7 \\ Fleet Vehicles and Characteristics}

Summary Statistics from Tables/Figures in this Chapter

\begin{tabular}{|c|c|c|}
\hline Source & & \\
\hline Figure 7.1 & Fleet automobiles, 2002 & $5,350,000$ \\
\hline Figure 7.1 & Fleet trucks \#19,500 lbs. GVW, 2002 & $5,127,000$ \\
\hline \multirow[t]{4}{*}{ Table 7.4} & Average annual miles per automobile & \\
\hline & Business fleets & 22,780 \\
\hline & Utility fleets & 13,399 \\
\hline & Government fleets & 12,895 \\
\hline \multirow[t]{4}{*}{ Table 7.4} & $\begin{array}{l}\text { Average annual miles per light truck } \\
(<8,500 \text { lbs. GVW })\end{array}$ & \\
\hline & Business fleets & 26,282 \\
\hline & Utility fleets & 12,096 \\
\hline & Government fleets & 6,797 \\
\hline \multirow[t]{6}{*}{ Table 7.5} & Federal government vehicles, FY 2001 & 567,581 \\
\hline & Automobiles & 114,544 \\
\hline & Buses & 6,726 \\
\hline & Light trucks $(<8,500$ lbs. GVW) & 357,136 \\
\hline & Medium trucks (8,500-26,000 lbs. GVW) & 89,720 \\
\hline & Heavy trucks (>26,000 lbs. GVW) & 27,988 \\
\hline
\end{tabular}


Significant changes have been made in recent years to fleet vehicle estimations. Newly available data improve the accuracy of fleet vehicle estimates but, at the same time, make it impossible to compare the data historically. Therefore, only the latest data are presented here.

Figure 7.1. Fleet Vehicles in Service as of February 1, 2002

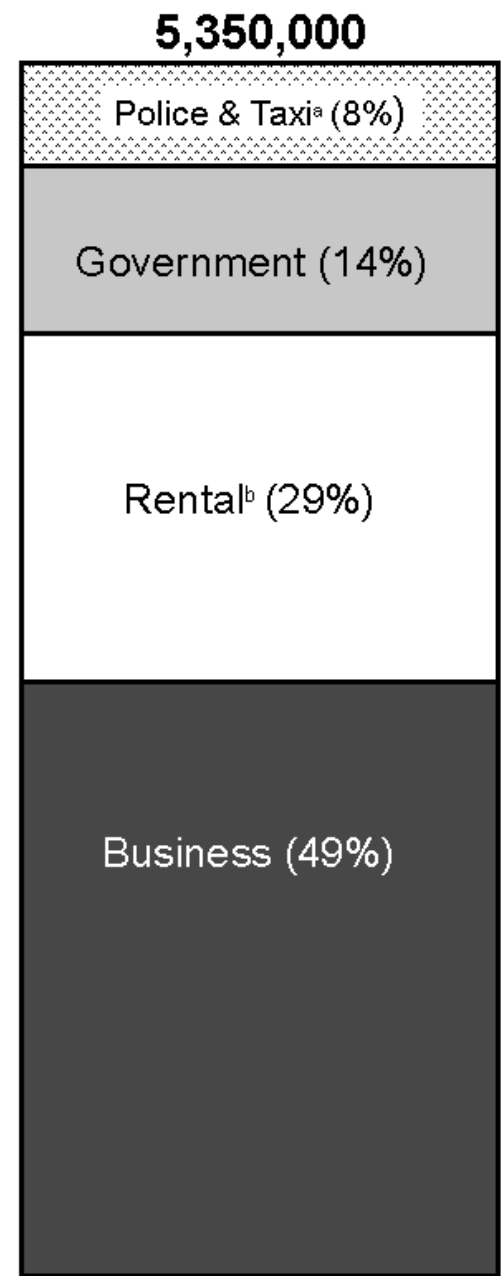

Automobiles

in fleets of
10 or more
$5,127,000$

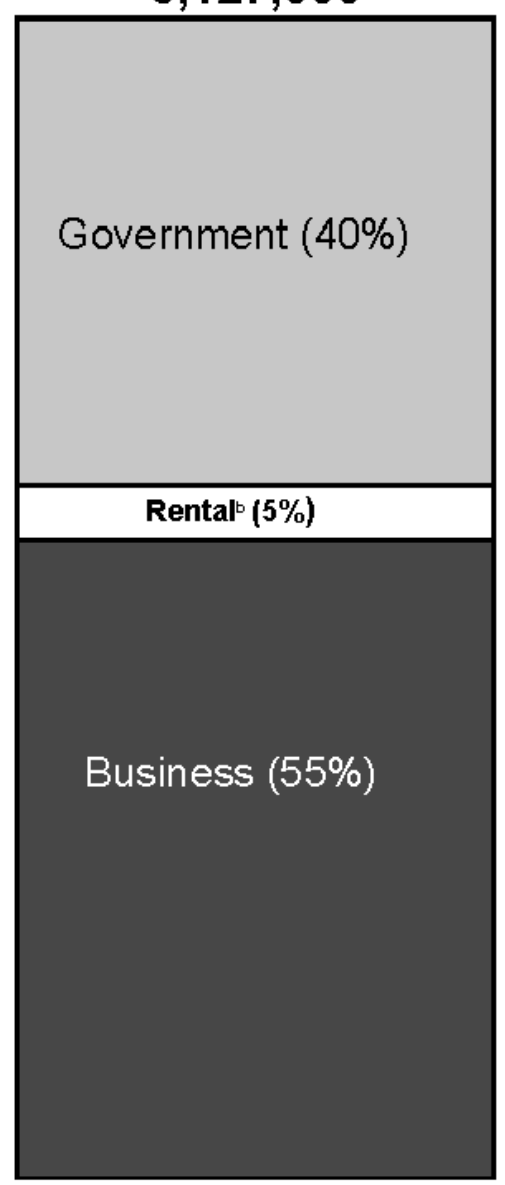

Trucks $<|9,50|$ lbs.

gross vehicle weight

in fleets of

10 or more

\section{Source:}

Bobit Publishing Company, Automotive Fleet Research Department, Automotive Fleet Factbook 2003,

Redondo Beach, CA, 2003. (Additional resources: www.fleet-central.com)

\footnotetext{
${ }^{a}$ Taxi category includes vans.

${ }^{\mathrm{b}}$ Rental category includes vans and sports utility vehicles under automobiles, not trucks.
} 
According to these estimates of light fleet vehicle population, utility and government fleets have a greater share of light trucks in their light vehicle population than business fleets do. This is also reflected in the new vehicle purchases.

Table 7.1

Light Vehicles in Fleets of 10 or More, 2000

\begin{tabular}{lccc}
\hline & Business & Utility & Government \\
\hline Cars & $60.7 \%$ & $41.5 \%$ & $37.7 \%$ \\
Light trucks ${ }^{\mathrm{a}}$ and vans & $39.3 \%$ & $58.5 \%$ & $62.3 \%$ \\
Total light vehicles & $7,694,733$ & 763,190 & $3,152,831$ \\
\hline
\end{tabular}

Source:

See Appendix A for Fleet Vehicle Data, Light Fleet Vehicle Population.

Table 7.2

New Light Fleet Vehicle Purchases by Vehicle Type, 2000

\begin{tabular}{lccc}
\hline & Business & Utility & Government \\
\hline Cars & $73.2 \%$ & $11.8 \%$ & $47.1 \%$ \\
Light trucks ${ }^{\text {a }}$ and vans & $26.8 \%$ & $88.2 \%$ & $52.9 \%$ \\
Total light vehicles & $2,146,351$ & 355,989 & 235,085 \\
\hline
\end{tabular}

Source:

See Appendix A for Fleet Vehicle Data., Light Fleet Vehicle New Sales.

an this study, light trucks are $<10,000 \mathrm{lbs}$ gross vehicle weight. 
The average length of service for an intermediate size fleet car is 30 months. Of the light vehicle types, full-size vans have the longest average months in service. Medium trucks are in service for an average of 70 months.

Table 7.3

Average Length of Time Business Fleet

Vehicles are in Service, 2001

\begin{tabular}{lc}
\hline Vehicle type & $\begin{array}{c}\text { Average months } \\
\text { in service }\end{array}$ \\
\hline Compact cars & 21.1 \\
Intermediate cars & 25.8 \\
Pickup trucks & 30.4 \\
Minivans & 27.1 \\
Sport utility vehicles & 25.1 \\
Full-size vans & 29.4 \\
Medium trucks & 70.4 \\
\hline
\end{tabular}

Source:

Bobit Publishing Company, Automotive Fleet Factbook 2002, pp. 52-60.

(Additional resources: www.fleet-central.com)

Note:

Based on data collected from four leading Fleet Management companies.

Table 7.4

Average Annual Vehicle-Miles of Travel for Fleet Vehicles, 2000

\begin{tabular}{lccc}
\hline Vehicle type & Business & Utility & Government \\
\hline Cars & 22,780 & 13,399 & 12,895 \\
Light trucks ${ }^{\mathrm{a}}$ & 26,282 & 12,096 & 6,797 \\
All light vehicles & 24,158 & 12,583 & 8,328 \\
\hline
\end{tabular}

Source:

See Appendix A for Fleet Vehicle Data, Light Fleet Vehicle Travel.

an this study, light trucks are $<10,000 \mathrm{lbs}$ gross vehicle weight. 
These data, which apply to domestic Federal fleet vehicles, indicate that sedans and station wagons have the highest average annual miles per vehicle, followed closely by buses. There is a 6,000-mile difference in the average for $4 \times 2$ light trucks as opposed to $4 \times 4$ light trucks.

Figure 7.2. Average Miles per Domestic Federal Vehicle by Vehicle Type, 2001

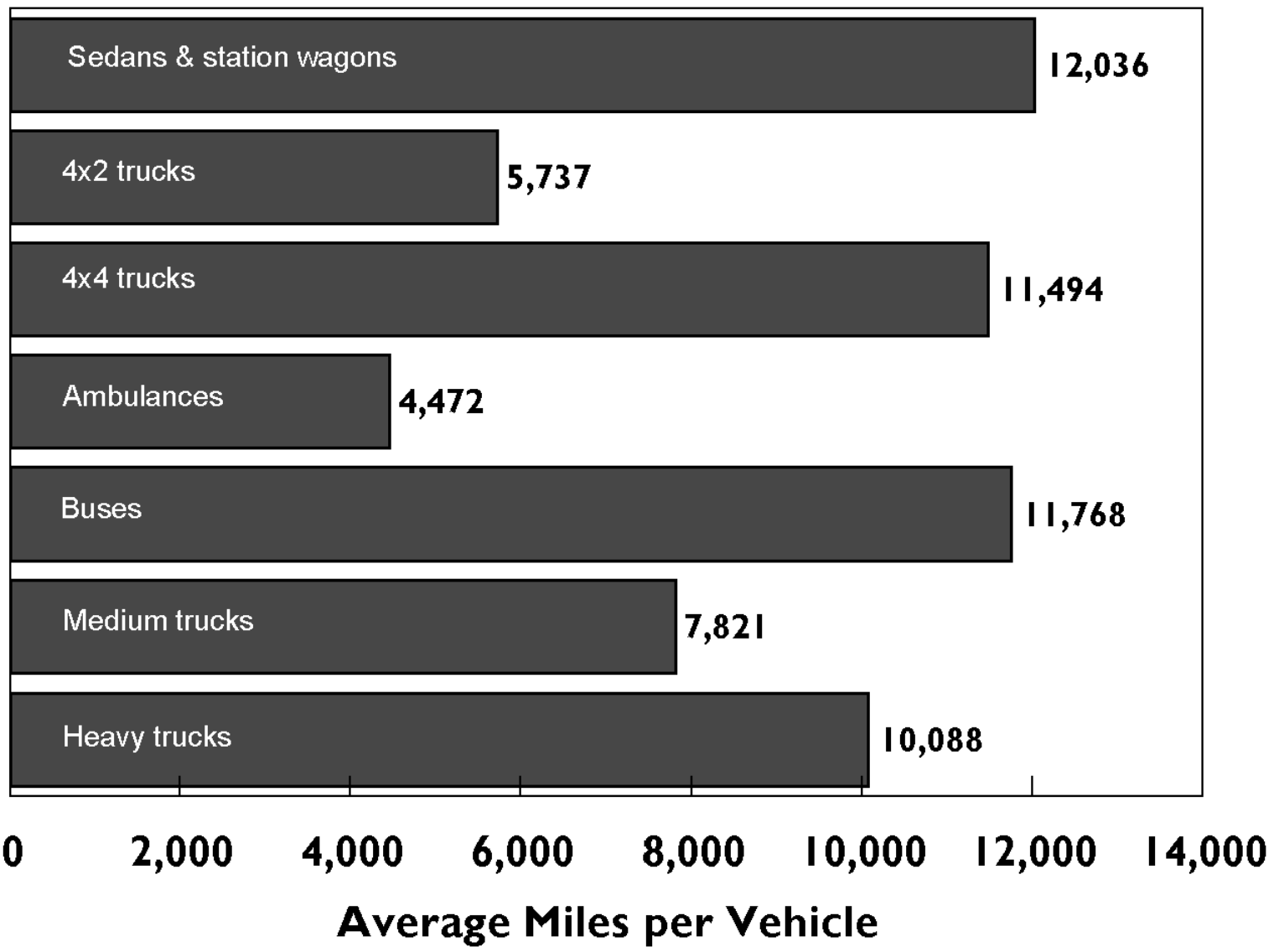

\section{Source:}

U.S. General Services Administrations, Federal Vehicle Policy Division, FY 2001 Federal Fleet Report, Washington, DC, 2002, Table 5.

(Additional resources: policyworks.gov/org/main/mt/homepage/mtv/mtvhp.htm) 
Table 7.5

Federal Government Vehicles by Agency, Fiscal Year 2001

\begin{tabular}{|c|c|c|c|c|c|c|}
\hline Department or Agency & Autos & Buses & $\begin{array}{l}\text { Light } \\
\text { trucks }\end{array}$ & $\begin{array}{c}\text { Medium } \\
\text { trucks }^{\mathrm{c}}\end{array}$ & $\begin{array}{l}\text { Heavy } \\
\text { trucks }^{\text {d }}\end{array}$ & Total \\
\hline \multicolumn{7}{|l|}{ DOMESTIC } \\
\hline General Services Administration $^{\mathrm{e}}$ & 54,039 & 3,145 & 76,593 & 30,885 & 3,902 & 168,564 \\
\hline Department of Justice & 21,414 & 373 & 14,519 & 3,958 & 504 & 40,768 \\
\hline Department of Agriculture & 3,439 & 39 & 24,762 & 5,395 & 650 & 34,285 \\
\hline Department of Treasury & 11,030 & 12 & 5,114 & 434 & 202 & 16,792 \\
\hline Department of the Interior & 1,299 & 158 & 9,502 & 3,293 & 2,014 & 16,266 \\
\hline Department of Energy & 467 & 141 & 2,321 & 960 & 1,402 & 5,291 \\
\hline Tennessee Valley Authority & 585 & 0 & 921 & 905 & 353 & 2,764 \\
\hline Department of Veterans Affairs & 121 & 106 & 1,056 & 225 & 136 & 1,644 \\
\hline National Aeronautics and Space Administration & 104 & 71 & 357 & 223 & 111 & 866 \\
\hline Department of Transportation & 73 & 11 & 415 & 96 & 198 & 793 \\
\hline Department of Health and Human Services & 155 & 6 & 351 & 68 & 102 & 682 \\
\hline Department of Commerce & 117 & 2 & 313 & 50 & 10 & 492 \\
\hline Department of State & 132 & 1 & 143 & 8 & 12 & 296 \\
\hline Department of Housing and Urban Development & 133 & 0 & 83 & 4 & 0 & 220 \\
\hline Environmental Protection Agency & 15 & 1 & 80 & 39 & 28 & 163 \\
\hline Smithsonian Institution & 9 & 5 & 122 & 21 & 5 & 162 \\
\hline Federal Communications Commission & 53 & 0 & 63 & 0 & 0 & 116 \\
\hline National Science Foundation & 14 & 5 & 66 & 8 & 16 & 109 \\
\hline All other departments or agencies & 45 & 2 & 65 & 28 & 10 & 150 \\
\hline DOMESTIC CIVILIAN AGENCIES & 93,244 & 4,078 & 136,846 & 46,600 & 9,655 & 290,423 \\
\hline Department of Air Force & 1,073 & 968 & 9,301 & 12,838 & 4,451 & 28,631 \\
\hline Department of Navy & 2,428 & 373 & 11,648 & 6,181 & 3,215 & 23,845 \\
\hline Department of Army & 471 & 205 & 2,983 & 4,603 & 1,670 & 9,932 \\
\hline United States Marine Corps & 170 & 191 & 541 & 707 & 751 & 2,360 \\
\hline Defense Agencies & 1,923 & 0 & 75 & 1 & 0 & 1,999 \\
\hline Corps of Engineers Civil & 0 & 0 & 95 & 234 & 355 & 684 \\
\hline DOMESTIC MILITARY AGENCIES & 6,065 & 1,737 & 24,643 & 24,564 & 10,442 & 67,451 \\
\hline U.S. POSTAL SERVICE & 9,214 & $\mathbf{0}$ & 181,504 & 10,874 & 4,713 & 206,305 \\
\hline TOTAL DOMESTIC FLEETS & 108,523 & 5,815 & 342,993 & 82,038 & 24,810 & 564,179 \\
\hline \multicolumn{7}{|l|}{ FOREIGN } \\
\hline Department of State & 1,743 & 20 & 2,113 & 1,843 & 99 & 5,818 \\
\hline General Services Administration $^{\mathrm{e}}$ & 1,753 & 160 & 2,260 & 402 & 150 & 4,725 \\
\hline Department of Justice & 312 & 0 & 512 & 14 & 0 & 838 \\
\hline U.S. Agency for International Development & 99 & 9 & 635 & 64 & 15 & 822 \\
\hline Department of Agriculture & 23 & 0 & 160 & 1 & 1 & 185 \\
\hline Department of Commerce & 87 & 0 & 90 & 0 & 0 & 177 \\
\hline Department of Health and Human Services & 24 & 0 & 151 & 0 & 0 & 175 \\
\hline All other departments or agencies & 88 & 1 & 61 & 4 & 0 & 154 \\
\hline FOREIGN CIVILIAN AGENCIES & 4,129 & 190 & 5,982 & 2,328 & 265 & 12,894 \\
\hline Department of Air Force & 865 & 482 & 3,830 & 4,471 & 1,848 & 11,496 \\
\hline Department of Navy & 465 & 163 & 2,572 & 662 & 850 & 4,712 \\
\hline Department of Army & 368 & 62 & 1,156 & 166 & 176 & 1,928 \\
\hline United States Marine Corps & 194 & 14 & 603 & 55 & 39 & 905 \\
\hline FOREIGN MILITARY AGENCIES & 1,892 & 721 & 8,161 & 5,354 & 2,913 & 19,041 \\
\hline TOTAL FOREIGN FLEETS & 6,021 & 911 & 14,143 & 7,682 & 3,178 & 31,935 \\
\hline GRAND TOTAL OF ALL FLEETS & 114,544 & 6,726 & 357,136 & 89,720 & 27,988 & 596,114 \\
\hline
\end{tabular}

Source:

U.S. General Services Administration, Federal Supply Service, FY 2001 Federal Fleet Report, Washington, DC, 2003, Table 14.

(Additional resources: policyworks.gov/org/main/mt/homepage/mtv/mtvhp.htm)

\footnotetext{
${ }^{\text {a }}$ Federally-owned and commercially-leased domestic vehicles.

${ }^{\mathrm{b}}$ Less than 8,500 lbs GVWR. Includes ambulances.

${ }^{c} 8,501-23,999$ lbs GVWR.

d 24,000 lbs. or more GVWR.

${ }^{\mathrm{e}}$ Most are leased by other Federal agencies.
} 
Table 7.6

Federal Fleet Vehicle Acquisitions

by Fuel Type, FY 1998-2001

\begin{tabular}{lrrrr}
\hline & FY98 & FY99 & FY00 & \multicolumn{1}{c}{ FY01 } \\
\hline Gasoline & 48,338 & 54,625 & 38,561 & 18,886 \\
Diesel & 2,503 & 3,100 & 1,700 & 2,569 \\
Natural gas & 1,139 & 1,836 & 1,469 & 371 \\
Ethanol/E-85 & 3,015 & 3,886 & 5,615 & 1,466 \\
Electricity & 36 & 11 & 620 & 8 \\
Other & 0 & 107 & 0 & 0 \\
Methanol/M-85 & 104 & 33 & 10 & 3 \\
LPG & 91 & 33 & 63 & 22 \\
Biodiesel & 0 & 5 & 0 & 0 \\
Hydrogen & 0 & 0 & 0 & 0 \\
\hline Total & 55,226 & 63,636 & 48,038 & 23,325 \\
\hline
\end{tabular}

Source:

U.S. General Services Administrations, Federal Vehicle Policy Division, FY 2001 Federal Fleet Report, Washington, DC, 2003, Chart 16.

(Additional resources: policyworks.gov/org/main/mt/homepage/mtv/mtvhp.htm)

Table 7.7

Fuel Consumed by Federal Government Fleets, FY 1998-2001

(thousand gasoline equivalent gallons)

\begin{tabular}{lrrrr}
\hline & FY98 & \multicolumn{1}{c}{ FY99 } & FY00 & \multicolumn{1}{c}{ FY01 } \\
\hline Gasoline & 251,478 & 275,879 & 284,480 & 281,791 \\
Diesel & 55,188 & 63,942 & 70,181 & 70,761 \\
CNG & 5,510 & 4,019 & 865 & 2,387 \\
Electricity & 63 & 25 & 1 & 35 \\
Biodiesel & 11 & 128 & 569 & 1,315 \\
Methanol/M-85 & 232 & 13 & 14 & 5 \\
LPG & 43 & 26 & 34 & 102 \\
Ethanol/E-85 & 3,708 & 130 & 347 & 5,900 \\
LNG & 0 & 1 & 0 & 0 \\
Other & 195 & 2,143 & 0 & 0 \\
\hline Total & 316,428 & 346,306 & 356,491 & 362,296 \\
\hline
\end{tabular}

Source:

U.S. General Services Administrations, Federal Vehicle Policy Division, FY 2001 Federal Fleet Report, Washington, DC, 2003, Charts 8 and 9.

(Additional resources: policyworks.gov/org/main/mt/homepage/mtv/mtvhp.htm) 
The Energy Policy Act of 1992 (EPACT) set alternative fuel vehicle acquisition requirements for Federal and State Governments, alternative fuel providers and the private sector. Additional rule making has adjusted the original purchase requirements. State government and alternative fuel providers requirements began in 1997.

Table 7.8

Energy Policy Act Purchase Requirements of Light Alternative Fuel Vehicles

\begin{tabular}{lrccc}
\hline Year & Federal & State & $\begin{array}{c}\text { Alternative fuel } \\
\text { providers }\end{array}$ & Private $^{\mathrm{a}}$ \\
\hline 1993 & 5,000 & - & - & - \\
1994 & 7,500 & - & - & - \\
1995 & 10,000 & - & - & - \\
1996 & $25 \%$ & - & - & - \\
1997 & $33 \%$ & $10 \%$ & $30 \%$ & - \\
1998 & $50 \%$ & $15 \%$ & $50 \%$ & - \\
1999 & $75 \%$ & $25 \%$ & $70 \%$ & - \\
2000 & $75 \%$ & $50 \%$ & $90 \%$ & - \\
2001 & $75 \%$ & $75 \%$ & $90 \%$ & - \\
2002 & $75 \%$ & $75 \%$ & $90 \%$ & $20 \%$ \\
2003 & $75 \%$ & $75 \%$ & $90 \%$ & $40 \%$ \\
2004 & $75 \%$ & $75 \%$ & $90 \%$ & $60 \%$ \\
2005 & $75 \%$ & $75 \%$ & $90 \%$ & $70 \%$ \\
$2006-$ on & $75 \%$ & $75 \%$ & $90 \%$ & $70 \%$ \\
\hline
\end{tabular}

\section{Source:}

Final rule for the alternative fuels transportation programs, Federal Register, Vol. 61, p. 10622, March 14, 1996.

Private alternative fueled vehicle acquisition requirements for private and local government fleets, Federal Register, vol. 62, p. 19701, April 23, 1997.

aThe Department of Energy is presently considering implementation of private and municipal fleet rule making. 


\section{Chapter 8 \\ Household Vehicles and Characteristics}

Summary Statistics from Tables/Figures in this Chapter

\begin{tabular}{|c|c|c|}
\hline Source & & \\
\hline Table 8.2 & Vehicles per licensed driver, 2001 & 1.13 \\
\hline Table 8.3 & Average household transportation expense, 2000 & $19.1 \%$ \\
\hline \multirow[t]{6}{*}{ Table 8.4} & Share of households owning 3 or more vehicles & \\
\hline & 1960 & $2.5 \%$ \\
\hline & 1970 & $5.5 \%$ \\
\hline & 1980 & $17.5 \%$ \\
\hline & 1990 & $17.3 \%$ \\
\hline & 2000 & $18.3 \%$ \\
\hline \multirow[t]{5}{*}{ Figure 8.1} & Average occupancy rates by vehicle type, 2001 & \\
\hline & Automobile & 1.57 \\
\hline & Pickup truck & 1.48 \\
\hline & Sports Utility & 1.76 \\
\hline & Van & 2.22 \\
\hline Table 8.12 & Average annual miles per household vehicle, 2001 & 11,000 \\
\hline Table 8.14 & Share of workers who car pooled, 2000 & $11.2 \%$ \\
\hline \multirow[t]{3}{*}{ Figure 8.4} & Long-distance trips in the U.S., 1995 & \\
\hline & Trips & 1,001 million \\
\hline & Person-miles & 827 billion \\
\hline
\end{tabular}


Vehicle-miles are growing at a faster rate than vehicles and more than twice the rate of population. See Table 8.2 for vehicles per capita and vehicle-miles per capita.

Table 8.1

Population and Vehicle Profile, 1950-2001

\begin{tabular}{|c|c|c|c|c|c|c|}
\hline Year & $\begin{array}{c}\text { Resident } \\
\text { population }^{\mathrm{a}} \\
\text { (thousands) }\end{array}$ & $\begin{array}{c}\text { Total } \\
\text { households } \\
\text { (thousands) }\end{array}$ & $\begin{array}{l}\text { Number of } \\
\text { vehicles in } \\
\text { operation } \\
\text { (thousands) }\end{array}$ & $\begin{array}{c}\text { Total } \\
\text { vehicle-miles } \\
\text { (millions) }\end{array}$ & $\begin{array}{l}\text { Number of } \\
\text { licensed } \\
\text { drivers } \\
\text { (thousands) }\end{array}$ & $\begin{array}{l}\text { Number of } \\
\text { civilian } \\
\text { employed } \\
\text { persons } \\
\text { (thousands) }\end{array}$ \\
\hline 1950 & 151,868 & 43,554 & 43,256 & 458,246 & 62,194 & 58,918 \\
\hline 1955 & 165,069 & 47,874 & 55,804 & 605,646 & 74,686 & 62,170 \\
\hline 1960 & 179,979 & 52,799 & 66,582 & 718,762 & 87,253 & 65,778 \\
\hline 1965 & 193,526 & 57,251 & 82,067 & 887,812 & 98,502 & 71,088 \\
\hline 1970 & 203,984 & 63,401 & 98,136 & $1,109,724$ & 111,543 & 78,678 \\
\hline 1975 & 215,465 & 71,120 & 120,054 & $1,327,664$ & 129,791 & 85,846 \\
\hline 1980 & 227,225 & 80,776 & 139,832 & $1,527,295$ & 145,295 & 99,303 \\
\hline 1985 & 237,924 & 86,789 & 157,048 & $1,774,826$ & 156,868 & 107,150 \\
\hline 1986 & 240,133 & 88,458 & 162,094 & $1,834,872$ & 159,487 & 109,597 \\
\hline 1987 & 242,289 & 89,479 & 167,193 & $1,921,204$ & 161,975 & 112,440 \\
\hline 1988 & 244,499 & 91,061 & 171,741 & $2,025,962$ & 162,853 & 114,968 \\
\hline 1989 & 246,819 & 92,830 & 175,960 & $2,096,487$ & 165,555 & 117,342 \\
\hline 1990 & 249,623 & 93,347 & 179,299 & $2,144,362$ & 167,015 & 118,793 \\
\hline 1991 & 252,981 & 94,312 & 181,438 & $2,172,050$ & 168,995 & 117,718 \\
\hline 1992 & 256,514 & 95,689 & 181,519 & $2,247,151$ & 173,125 & 118,492 \\
\hline 1993 & 259,916 & 96,391 & 186,315 & $2,296,378$ & 173,149 & 120,259 \\
\hline 1994 & 263,126 & 97,107 & 188,714 & $2,357,588$ & 175,403 & 123,060 \\
\hline 1995 & 266,278 & 98,990 & 193,441 & $2,422,696$ & 176,628 & 124,900 \\
\hline 1996 & 269,394 & 99,627 & 198,294 & $2,485,848$ & 179,539 & 126,708 \\
\hline 1997 & 272,647 & 101,018 & 201,071 & $2,561,695$ & 182,709 & 129,558 \\
\hline 1998 & 275,854 & 102,528 & 205,043 & $2,631,522$ & 184,980 & 131,463 \\
\hline 1999 & 279,040 & 103,874 & 209,509 & $2,691,056$ & 187,170 & 133,488 \\
\hline 2000 & 282,797 & 104,705 & 213,300 & $2,746,925$ & 190,625 & 135,208 \\
\hline 2001 & 284,797 & b & 216,683 & $2,781,462$ & 191,276 & 135,073 \\
\hline & \multicolumn{6}{|c|}{ Average annual percentage change } \\
\hline 1950-2001 & $1.2 \%$ & b & $3.2 \%$ & $3.6 \%$ & $2.2 \%$ & $1.6 \%$ \\
\hline 1991-2001 & $1.2 \%$ & b & $1.8 \%$ & $2.5 \%$ & $1.2 \%$ & $1.4 \%$ \\
\hline
\end{tabular}

Source:

Resident population, total households, and civilian employed persons - U.S. Department of Commerce, Bureau of the Census, Statistical Abstract of the United States-2002, 122nd edition, Washington, DC, 2002, pp. 8, 49, 367, and annual. (Additional resources: www.census.gov)

Vehicles in operation - The Polk Company. FURTHER REPRODUCTION PROHIBITED. (Additional resources: www.polk.com)

Licensed drivers and vehicle-miles - U.S. Department of Transportation, Federal Highway Administration, Highway Statistics 2001, Tables DL-20 and VM-1, and annual. (Additional resources: www.fhwa.dot.gov)

${ }^{\text {a } E s t i m a t e s ~ a s ~ o f ~ J u l y ~ 1 . ~ I n c l u d e s ~ A r m e d ~ F o r c e s ~ s t a t i o n e d ~ i n ~ t h e ~ U n i t e d ~ S t a t e s . ~}$

${ }^{\mathrm{b}}$ Data is not available. 
Vehicle-miles per capita have nearly reached 10,000 miles. There were 1.6 vehicles for every employed civilian in the U.S. in 2001.

Table 8.2

Population and Vehicle Ratios, 1950-2001

\begin{tabular}{|c|c|c|c|}
\hline Year & $\begin{array}{l}\text { Vehicles per } \\
\text { capita }\end{array}$ & $\begin{array}{l}\text { Vehicle-miles } \\
\text { per capita }\end{array}$ & $\begin{array}{c}\text { Vehicles per } \\
\text { civilian employed } \\
\text { persons }\end{array}$ \\
\hline 1950 & 0.285 & 3,029 & 0.73 \\
\hline 1955 & 0.338 & 3,656 & 0.90 \\
\hline 1960 & 0.370 & 3,994 & 1.01 \\
\hline 1965 & 0.424 & 4,587 & 1.15 \\
\hline 1970 & 0.481 & 5,440 & 1.25 \\
\hline 1975 & 0.557 & 6,162 & 1.40 \\
\hline 1980 & 0.615 & 6,722 & 1.41 \\
\hline 1985 & 0.660 & 7,460 & 1.47 \\
\hline 1986 & 0.675 & 7,641 & 1.48 \\
\hline 1987 & 0.690 & 7,929 & 1.49 \\
\hline 1988 & 0.702 & 8,286 & 1.49 \\
\hline 1989 & 0.713 & 8,494 & 1.50 \\
\hline 1990 & 0.718 & 8,590 & 1.51 \\
\hline 1991 & 0.717 & 8,586 & 1.54 \\
\hline 1992 & 0.708 & 8,760 & 1.53 \\
\hline 1993 & 0.717 & 8,835 & 1.55 \\
\hline 1994 & 0.717 & 8,960 & 1.53 \\
\hline 1995 & 0.726 & 9,098 & 1.55 \\
\hline 1996 & 0.736 & 9,228 & 1.56 \\
\hline 1997 & 0.737 & 9,396 & 1.55 \\
\hline 1998 & 0.743 & 9,540 & 1.56 \\
\hline 1999 & 0.751 & 9,644 & 1.57 \\
\hline 2000 & 0.754 & 9,713 & 1.58 \\
\hline 2001 & 0.761 & 9,766 & 1.60 \\
\hline \multicolumn{4}{|c|}{ Average annual percentage change } \\
\hline 1950-2001 & $2.0 \%$ & $2.3 \%$ & $1.6 \%$ \\
\hline 1991-2001 & $0.5 \%$ & $1.3 \%$ & $0.4 \%$ \\
\hline
\end{tabular}

Source:

Resident population and civilian employed persons - U.S. Department of Commerce, Bureau of the Census, Statistical Abstract of the United States-2002, 122nd edition, Washington, DC, 2002, pp. 8, 367, and annual. (Additional resources: www.census.gov)

Vehicles in operation - The Polk Company. FURTHER REPRODUCTION PROHIBITED. (Additional resources: www.polk.com)

Vehicle-miles - U.S. Department of Transportation, Federal Highway Administration, Highway Statistics 2001, Table VM-1 and annual.

(Additional resources: www.fhwa.dot.gov) 
Transportation (19.1\%) is second only to housing (31.7\%) as the largest expenditure for the average household. In 2001, approximately 16.3\% of transportation

expenditures were for purchasing gasoline and motor oil. There is an average of two vehicles per household.

Table 8.3

Average Annual Expenditures of Households by Income, 2001

\begin{tabular}{|c|c|c|c|c|c|c|c|c|c|c|}
\hline & \multirow[b]{2}{*}{$\begin{array}{c}\text { All } \\
\text { households }\end{array}$} & \multicolumn{9}{|c|}{ Income before taxes } \\
\hline & & $\begin{array}{l}\text { Less than } \\
\$ 5,000\end{array}$ & $\begin{array}{c}\$ 5,000- \\
\$ 9999\end{array}$ & $\begin{array}{c}\$ 10,000- \\
\$ 14999\end{array}$ & $\begin{array}{l}\$ 15,000- \\
\$ 19,999\end{array}$ & $\begin{array}{l}\$ 20,000- \\
\$ 29,999\end{array}$ & $\begin{array}{c}\$ 30,000- \\
\$ 39,999\end{array}$ & $\begin{array}{c}\$ 40,000- \\
\$ 49,999\end{array}$ & $\begin{array}{l}\$ 50,000- \\
\$ 69,999\end{array}$ & $\begin{array}{c}\$ 70,000 \text { and } \\
\text { over }\end{array}$ \\
\hline \multirow[t]{2}{*}{ Total expenditures } & $\$ 41,395$ & $\$ 20,517$ & $\$ 16,625$ & $\$ 20,642$ & $\$ 25,028$ & $\$ 28,623$ & $\$ 35,430$ & $\$ 40,900$ & $\$ 50,136$ & $\$ 76,124$ \\
\hline & \multicolumn{10}{|c|}{ Percentage of total expenditures ${ }^{\mathrm{b}}$} \\
\hline Food $^{c}$ & $14.6 \%$ & $18.9 \%$ & $19.5 \%$ & $17.6 \%$ & $16.0 \%$ & $16.5 \%$ & $15.2 \%$ & $15.4 \%$ & $14.5 \%$ & $12.8 \%$ \\
\hline Housing & $31.7 \%$ & $35.6 \%$ & $36.2 \%$ & $36.2 \%$ & $33.2 \%$ & $33.3 \%$ & $31.1 \%$ & $29.9 \%$ & $30.6 \%$ & $31.0 \%$ \\
\hline Apparel and services & $4.5 \%$ & $5.4 \%$ & $4.5 \%$ & $4.2 \%$ & $5.1 \%$ & $3.7 \%$ & $4.6 \%$ & $4.6 \%$ & $4.3 \%$ & $4.6 \%$ \\
\hline Transportation & $19.1 \%$ & $16.7 \%$ & $16.4 \%$ & $17.1 \%$ & $18.5 \%$ & $19.7 \%$ & $21.3 \%$ & $21.2 \%$ & $19.7 \%$ & $18.2 \%$ \\
\hline Vehicle purchases (net outlay) & $9.1 \%$ & $8.3 \%$ & $8.1 \%$ & $8.2 \%$ & $8.6 \%$ & $9.4 \%$ & $10.9 \%$ & $10.2 \%$ & $9.2 \%$ & $8.5 \%$ \\
\hline Gasoline and motor oil & $3.1 \%$ & $3.1 \%$ & $3.1 \%$ & $3.1 \%$ & $3.4 \%$ & $3.7 \%$ & $3.5 \%$ & $3.6 \%$ & $3.2 \%$ & $2.7 \%$ \\
\hline Other vehicle expenditures & $5.9 \%$ & $4.1 \%$ & $4.3 \%$ & $4.9 \%$ & $5.4 \%$ & $5.8 \%$ & $6.1 \%$ & $6.8 \%$ & $6.4 \%$ & $5.8 \%$ \\
\hline Public transportation & $1.0 \%$ & $1.0 \%$ & $0.8 \%$ & $0.9 \%$ & $0.9 \%$ & $0.8 \%$ & $0.8 \%$ & $0.6 \%$ & $0.9 \%$ & $1.2 \%$ \\
\hline Health care & $5.4 \%$ & $5.6 \%$ & $7.7 \%$ & $8.7 \%$ & $8.6 \%$ & $7.3 \%$ & $6.2 \%$ & $5.5 \%$ & $5.0 \%$ & $3.8 \%$ \\
\hline Entertainment & $4.9 \%$ & $4.5 \%$ & $3.8 \%$ & $3.8 \%$ & $5.2 \%$ & $4.1 \%$ & $4.6 \%$ & $4.8 \%$ & $5.3 \%$ & $5.2 \%$ \\
\hline Personal Insurance \& pensions & $10.9 \%$ & $1.7 \%$ & $2.0 \%$ & $3.4 \%$ & $4.8 \%$ & $6.2 \%$ & $8.5 \%$ & $9.9 \%$ & $11.8 \%$ & $15.2 \%$ \\
\hline Others $^{d}$ & $9.0 \%$ & $11.6 \%$ & $10.0 \%$ & $8.9 \%$ & $8.7 \%$ & $9.1 \%$ & $8.5 \%$ & $8.6 \%$ & $8.7 \%$ & $9.1 \%$ \\
\hline Households ${ }^{\mathrm{e}}$ (thousands) & 88,735 & 4,100 & 6,829 & 8,099 & 7,014 & 12,075 & 10,508 & 8,737 & 12,480 & 18,892 \\
\hline Percentage of households & $100 \%$ & $4.6 \%$ & $7.7 \%$ & $9.1 \%$ & $7.9 \%$ & $13.6 \%$ & $11.8 \%$ & $9.8 \%$ & $14.1 \%$ & $21.3 \%$ \\
\hline Average number of vehicles in $\mathrm{HH}$ & 2.0 & 1.0 & 0.9 & 1.1 & 1.5 & 1.7 & 1.9 & 2.2 & 2.5 & 2.9 \\
\hline
\end{tabular}

Source:

U.S. Department of Labor, Bureau of Labor Statistics, web site: www.bls.gov/pub/special.requests/ce/share/2001/income.txt, April 2003. (Additional resources: www.bls.gov)

${ }^{\text {a }}$ Public assistance monies are included in reported income. Data for those reporting income.

${ }^{\mathrm{b}}$ Percentages may not sum to totals due to rounding.

${ }^{\mathrm{c}}$ Includes alcoholic beverages.

${ }^{\mathrm{d}}$ Includes personal care, reading, education, tobacco and smoking supplies, cash contributions, and miscellaneous items.

e The term household refers to a "consumer unit," which is defined differently than households on Table 8.1 
Household vehicle ownership shows a dramatic increase from 1960 to 1990. In 1960, nearly 79\% of households owned less than two vehicles; by 1990, it declined to 45\%. Census data prior to 1990 indicated that the majority of households owned one vehicle; in 1990 that changed to two vehicles.

Table 8.4

Household Vehicle Ownership, 1960-2000 Census

(percentage)

\begin{tabular}{cccccc}
\hline & $\begin{array}{c}\text { No } \\
\text { vehicles }\end{array}$ & $\begin{array}{c}\text { One } \\
\text { vehicle }\end{array}$ & $\begin{array}{c}\text { Two } \\
\text { vehicles }\end{array}$ & $\begin{array}{c}\text { Three or } \\
\text { more } \\
\text { vehicles }\end{array}$ & $\begin{array}{c}\text { Total } \\
\text { vehicles }^{\mathrm{a}}\end{array}$ \\
\hline 1960 & $21.53 \%$ & $56.94 \%$ & $19.00 \%$ & $2.53 \%$ & $54,766,718$ \\
1970 & $17.47 \%$ & $47.71 \%$ & $29.32 \%$ & $5.51 \%$ & $79,002,052$ \\
1980 & $12.92 \%$ & $35.53 \%$ & $34.02 \%$ & $17.52 \%$ & $129,747,911$ \\
1990 & $11.53 \%$ & $33.74 \%$ & $37.35 \%$ & $17.33 \%$ & $152,380,479$ \\
2000 & $9.35 \%$ & $33.79 \%$ & $38.55 \%$ & $18.31 \%$ & $179,417,526$ \\
\hline
\end{tabular}

\section{Source:}

U. S. Department of Transportation, Volpe National Transportation Systems Center, Journey-toWork Trends in the United States and its Major Metropolitan Area, 1960-1990, Cambridge, MA, 1994, p. 2-2.

2000 data - U.S. Bureau of the Census, American Fact Finder, factfinder.census.gov, Table QT-04, August 2001. (Additional resources: www.census.gov)

${ }^{a}$ Estimates using Census Bureau data; these data on the total number of vehicles do not match the figures on Table 8.1. The figures on Table 8.1, from R.L. Polk and Company, are the preferred data. 


\section{National Household Travel Survey}

The Department of Transportation (DOT) colleted data on daily trips in 1969, 1977, 1983, 1990 and 1995 via the Nationwide Personal Transportation Survey (NPTS). Data on longer trips were collected in 1977 and 1995 via the American Travel Survey (ATS). For 2001, the DOT combined the collection of long trip and daily trip data into one survey - the 2001 National Travel Household Travel Survey (NHTS).

The NHTS is the nation's inventory of daily and long-distance travel. The survey includes demographic characteristics of households, people, vehicles, and detailed information on daily and longer-distance travel for all purposes by all modes. NHTS survey data are collected from a sample of U.S. households and expanded to provide national estimates of trips and miles by travel mode, trip purpose, and a host of household attributes.

The NHTS was designed to continue the NPTS and ATS series, but as with all data surveys, caution should be used when comparing statistics from one survey to another due to changes in terminology, survey procedures, and target population. The 2001 survey collected data on trips of children under 5 years of age, while the previous NPTS did not. Improved methodologies first used in the collection of trip information in the 1995 NPTS make it impossible to compare these data with past NPTS survey data. Thus, the 1990 NPTS trip data have been adjusted to make it comparable with the later surveys.

Version 1 of the NHTS data containing the daily trip data were released in January 2003 and are available at the Internet site: nhts.ornl.gov. Data in this report are all generated from the Version 1 NHTS databases. Subsequent versions of the databases will contain long trip data and revisions to the daily trip data.

Table 8.5

Demographic Statistics from the 1969, 1977, 1983, 1990, 1995 NPTS and 2001 NHTS

\begin{tabular}{lccccccc}
\hline & & & & & & & $\begin{array}{c}\text { Percent } \\
\text { change } \\
1969-2001\end{array}$ \\
\hline Persons per household & 1969 & 1977 & 1983 & 1990 & 1995 & 2001 & $-18 \%$ \\
Vehicles per household & 3.16 & 2.83 & 2.69 & 2.56 & 2.63 & 2.58 & $64 \%$ \\
Workers per household & 1.16 & 1.59 & 1.68 & 1.77 & 1.78 & 1.90 & $12 \%$ \\
Licensed drivers per household & 1.21 & 1.23 & 1.21 & 1.27 & 1.33 & 1.35 & $8 \%$ \\
Vehicles per worker & 1.65 & 1.69 & 1.72 & 1.75 & 1.78 & 1.77 & $46 \%$ \\
Vehicles per licensed driver & 0.96 & 1.29 & 1.39 & 1.40 & 1.34 & 1.41 & $52 \%$ \\
Average vehicle trip length (miles) & 0.70 & 0.94 & 0.98 & 1.01 & 1.00 & 1.07 & $10 \%$ \\
\hline
\end{tabular}

Source:

U.S. Department of Transportation, Federal Highway Administration, 1990 Nationwide Personal Transportation Survey: Summary of Travel Trends, FHWA-PL-92-027, Washington, DC, March 1992, Table 2. Data for 1995 and 2001 were generated from the Internet sites www-cta.ornl.gov/npts, and nhts.ornl.gov.

(Additional resources: www.fhwa.dot.gov)

Note:

Average vehicle trip length for 1990 and 1995 is calculated using only those records with trip mileage information present. The 1969 survey does not include pickups and other light trucks as household vehicles. 
Due to methodology improvements in collecting trip information, the 2001 and 1995 data should be compared only to the 1990 adjusted data. The original 1990 data are comparable to all previous surveys; however, comparisons should always be made with caution because of differing survey methodologies.

Table 8.6

Average Annual Vehicle-Miles, Vehicle Trips and Trip Length per Household

1969, 1977, 1983, 1990, 1995 NPTS and 2001 NHTS

\begin{tabular}{lcc}
\hline & Journey-to-work $^{\mathrm{a}}$ & All trips \\
\hline & Average annual vehicle-miles per household \\
1969 & 4,183 & 12,423 \\
1977 & 3,815 & 12,036 \\
1983 & 3,538 & 11,739 \\
1990 original & 4,853 & 15,100 \\
\hline \hline 1990 adjusted & 4,853 & 18,161 \\
1995 & 6,492 & 20,895 \\
2001 & 5,783 & 21,253 \\
\hline \hline
\end{tabular}

Average annual vehicle trips per household

\begin{tabular}{lcc}
1969 & 445 & 1,396 \\
1977 & 423 & 1,442 \\
1983 & 414 & 1,486 \\
1990 original & 448 & 1,702 \\
\hline \hline 1990 adjusted & 448 & 2,077 \\
1995 & 553 & 2,321 \\
2001 & 479 & 2,189 \\
\hline \hline & Average vehicle trip length (miles) & \\
1969 & 9.4 & 8.9 \\
1977 & 9.0 & 8.4 \\
1983 & 8.5 & 7.9 \\
1990 original & 11.0 & 9.0 \\
\hline \hline 1990 adjusted & 11.0 & 8.9 \\
1995 & 11.8 & 9.1 \\
2001 & 12.2 & 9.8 \\
\hline
\end{tabular}

\section{Source:}

U.S. Department of Transportation, Federal Highway Administration, 1990 Nationwide Personal Transportation Survey: Summary of Travel Trends, FHWA-PL-92-027, Washington, DC, March 1992, Table 7. Data for 1995 were generated from the Internet site $w w w-$ cta.ornl.gov/npts. 1990 adjusted data - Oak Ridge National Laboratory, Oak Ridge, TN, August 1998. 2001 NHTS data were generated from the Internet site nhts.ornl.gov. (Additional resources: www.fhwa.dot.gov, www-cta.ornl.gov/npts)

${ }^{a}$ It is believed that the methodology changes in the 1995 NPTS did not affect journey-to-work trips; therefore, no adjustment is necessary. 
Due to methodology improvements in collecting trip information, the 2001 and 1995 data should be compared only to the 1990 adjusted data. The original 1990 data are comparable to all previous surveys; however, comparisons should always be made with caution because of differing survey methodologies.

Table 8.7

Average Annual Person-Miles Traveled (PMT), Person Trips and Trip Length per Household by Selected Trip Purposes 1983, 1990, 1995 NPTS and 2001 NHTS

\begin{tabular}{|c|c|c|c|c|}
\hline & Journey-to-work ${ }^{\mathrm{a}}$ & Shopping & $\begin{array}{l}\text { Social and } \\
\text { recreational }\end{array}$ & $\begin{array}{c}\text { All } \\
\text { purposes }^{\mathrm{b}}\end{array}$ \\
\hline \multicolumn{5}{|c|}{ Average annual PMT per household } \\
\hline 1983 & $4,586(20 \%)$ & $2,567(11 \%)$ & $8,964(39 \%)$ & $22,802(100 \%)$ \\
\hline 1990 original & $5,637(23 \%)$ & $2,674(11 \%)$ & $8,567(35 \%)$ & $24,803(100 \%)$ \\
\hline 1990 adjusted & $\overline{5,637(19 \%)}$ & 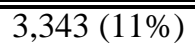 & $11,308(37 \%)$ & $30,316(100 \%)$ \\
\hline 1995 & $7,740(22 \%)$ & $4,659(14 \%)$ & $10,571(31 \%)$ & $34,459(100 \%)$ \\
\hline 2001 & $6,770(18 \%)$ & $5,086(14 \%)$ & $11,215(30 \%)$ & $37,498(100 \%)$ \\
\hline \multicolumn{5}{|c|}{ Average annual person trips per household } \\
\hline 1983 & $537(20 \%)$ & $474(18 \%)$ & $728(28 \%)$ & $2,628(100 \%)$ \\
\hline 1990 original & $539(20 \%)$ & $504(19 \%)$ & $662(25 \%)$ & $2,673(100 \%)$ \\
\hline 1990 adjusted & $539(17 \%)$ & $630(19 \%)$ & $874(27 \%)$ & $3,262(100 \%)$ \\
\hline 1995 & $676(18 \%)$ & $775(20 \%)$ & $953(25 \%)$ & $3,828(100 \%)$ \\
\hline 2001 & $567(15 \%)$ & $742(19 \%)$ & $1,031(27 \%)$ & $\underline{3,828(100 \%)}$ \\
\hline \multicolumn{5}{|c|}{ Average person trip length (miles) } \\
\hline 1983 & 8.5 & 5.4 & 12.3 & 8.7 \\
\hline 1990 original & 10.7 & 5.4 & 13.2 & 9.5 \\
\hline 1990 adjusted & 10.7 & 5.4 & 13.2 & 9.5 \\
\hline 1995 & 11.6 & 6.1 & 11.3 & 9.1 \\
\hline 2001 & 12.2 & 7.0 & 11.1 & 10.0 \\
\hline
\end{tabular}

Source:

U.S. Department of Transportation, Federal Highway Administration, Nationwide Personal Transportation Study, Public Use Tapes, Washington, DC. Data for 1995 and 2001 were generated from the Internet sites www-cta.ornl.gov/npts and nhts.ornl.gov. 1990 adjusted data - Oak Ridge National Laboratory, Oak Ridge, TN, August 1998. (Additional resources: www.fhwa.dot.gov, www-cta.ornl.gov/npts)

Note:

Average person trip length for 1990 and 1995 is calculated using only those records with trip mileage information present. "All purposes" includes unreported trip purposes.

aIt is believed that the methodology changes in the 1995 NPTS did not affect journey-to-work trips; therefore, no adjustment is necessary.

${ }^{\mathrm{b}}$ Includes trip purposes not shown on this table. 
In 2001 vehicle-miles traveled (vmt) for a three-person household is over 28,000 miles. The number of drivers in a household makes a big difference in vmt, as does the presence of children in the household. Households with children have $74 \%$ more vmt than households without children.

Table 8.8

Average Number of Vehicles and Vehicle Travel per Household, 1990 NPTS and 2001 NHTS

\begin{tabular}{lllll}
\hline & \multicolumn{2}{c}{$\begin{array}{c}\text { Average } \\
\text { number of vehicles } \\
\text { per household }\end{array}$} & $\begin{array}{c}\text { Average } \\
\text { vehicle-miles traveled } \\
\text { per household }\end{array}$ \\
\hline $\begin{array}{l}\text { Number of Licenced } \\
\text { Drivers }\end{array}$ & $\mathbf{1 9 9 0}$ & $\mathbf{2 0 0 1}$ & $\mathbf{1 9 9 0}$ & $\mathbf{2 0 0 1}$ \\
\hline 1 & 1.5 & 1.2 & 15,200 & 9,800 \\
2 & 2.1 & 2.2 & 22,900 & 26,000 \\
3 & 2.9 & 3.0 & 29,400 & 36,400 \\
4 or more & 3.8 & 3.9 & 40,500 & 48,400 \\
\hline Household size & & & & \\
\hline 1 person & 1.2 & 1.0 & 11,400 & 7,800 \\
2 persons & 1.9 & 2.0 & 19,300 & 21,000 \\
3 persons & 2.2 & 2.3 & 23,700 & 28,200 \\
4 persons & 2.4 & 2.4 & 25,300 & 29,400 \\
5 persons & 2.4 & 2.5 & 24,900 & 32,600 \\
6 or more persons & 2.7 & 2.6 & 29,200 & 35,000 \\
\hline Household urban status & & & & \\
\hline Urban & 1.9 & 1.8 & 19,000 & 19,400 \\
Rural & 2.1 & 2.3 & 22,200 & 28,500 \\
\hline Household composition & & & & \\
\hline With children & 2.2 & 2.3 & 24,100 & 28,700 \\
Without children & 1.8 & 1.7 & 17,600 & 16,500 \\
\hline All households & $\mathbf{1 . 8}$ & $\mathbf{1 . 9}$ & $\mathbf{1 8 , 3 0 0}$ & $\mathbf{2 1 , 3 0 0}$ \\
\hline & & & & \\
\hline & & & & \\
\hline
\end{tabular}

\section{Source:}

Generated from the Department of Transportation, Federal Highway Administration, Nationwide Personal Transportation Survey Public Use Files, Washington, DC, 2000 and the National Household Travel Survey Internet site: nhts.ornl.gov. (Additional resources: www-cta.ornl.gov/npts) 
While automobile occupancy declined slightly from 1995 to 2001, all other vehicle types showed increased occupancy. Vans and sport utility vehicles have higher vehicle occupancies than automobiles.

Figure 8.1. Average Vehicle Occupancy by Vehicle Type, 1995 NPTS and 2001 NHTS

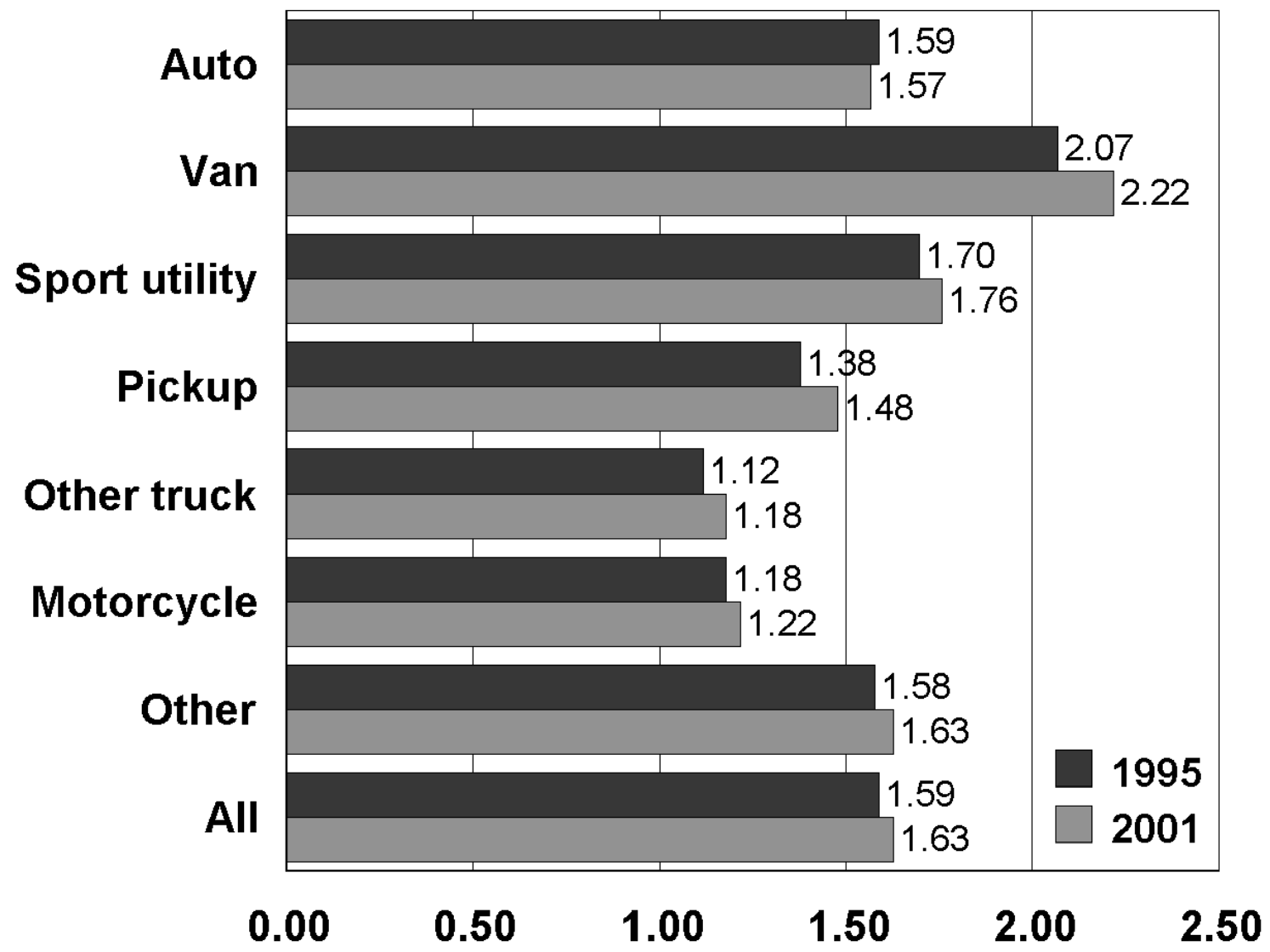

Source:

U.S. Department of Transportation, Federal Highway Administration, Nationwide Personal Transportation Survey, Washington, DC, 1997.

(Additional resources: www.fhwa.dot.gov, www-cta.ornl.gov/npts, nhts.ornl.gov) 
The average vehicle occupancy, calculated as person-miles per vehicle-mile, is highest for social and recreational purposes. The highest vehicle occupancy levels for all purposes were in 1977. The increase in number of vehicles per household and the decrease in average household size could have contributed to the decline since then.

Figure 8.2. Average Vehicle Occupancy by Trip Purpose 1977 NPTS and 2001 NHTS

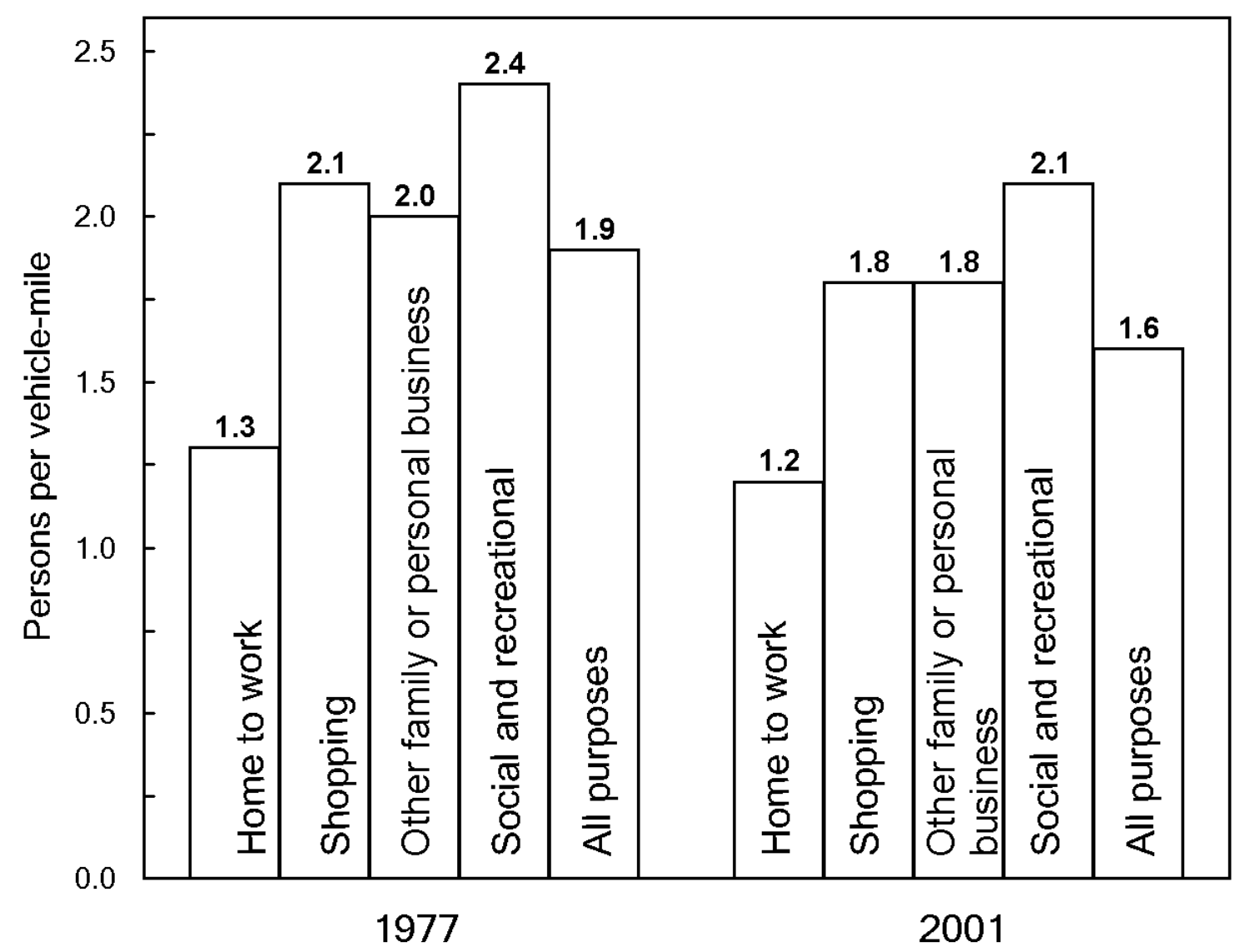

Source:

U.S. Department of Transportation, Federal Highway Administration, 1990 Nationwide Personal Transportation Survey: Summary of Travel Trends, FHWA-PL-92027, Washington, DC, March 1992, Figure 6. Data from 2001 NHTS were generated from the Internet site nhts.ornl.gov, June 2003.

(Additional resources: www.fhwa.dot.gov, nhts.ornl.gov) 
As households owned more vehicles, the average annual miles for the most frequently driven vehicle increased.

For example, the most frequently driven vehicle in five-vehicle households was driven $36 \%$ more per year than the one in two-vehicle households (15,019 miles vs. 20,467 miles).

Table 8.9

Average Annual Miles per Vehicle by Household Vehicle Ownership, 2001 NHTS

\begin{tabular}{cccccc}
\hline Vehicle $^{\text {a }}$ & $\begin{array}{c}\text { One-vehicle } \\
\text { household }\end{array}$ & $\begin{array}{c}\text { Two-vehicle } \\
\text { household }\end{array}$ & $\begin{array}{c}\text { Three-vehicle } \\
\text { household }\end{array}$ & $\begin{array}{c}\text { Four-vehicle } \\
\text { household }\end{array}$ & $\begin{array}{c}\text { Five-vehicle } \\
\text { household }\end{array}$ \\
\hline$\# 1$ & 10,306 & 15,019 & 16,951 & 19,092 & 20,467 \\
$\# 2$ & - & 7,505 & 8,951 & 10,212 & 11,433 \\
$\# 3$ & - & - & 4,101 & 5,673 & 6,825 \\
$\# 4$ & - & - & - & 2,851 & 3,883 \\
$\# 5$ & - & - & - & - & 2,000 \\
Average & $\mathbf{1 0 , 3 0 6}$ & $\mathbf{1 1 , 7 7 5}$ & $\mathbf{1 1 , 0 0 0}$ & $\mathbf{1 0 , 7 1 1}$ & $\mathbf{1 0 , 3 2 7}$ \\
\hline
\end{tabular}

Source:

Generated from the National Household Travel Survey Internet site nhts.ornl.gov.

Table 8.10

Average Age of Vehicles by Household Vehicle Ownership, 2001 NHTS

\begin{tabular}{cccccc}
\hline Vehicle $^{\mathrm{a}}$ & $\begin{array}{c}\text { One-vehicle } \\
\text { household }\end{array}$ & $\begin{array}{c}\text { Two-vehicle } \\
\text { household }\end{array}$ & $\begin{array}{c}\text { Three-vehicle } \\
\text { household }\end{array}$ & $\begin{array}{c}\text { Four-vehicle } \\
\text { household }\end{array}$ & $\begin{array}{c}\text { Five-vehicle } \\
\text { household }\end{array}$ \\
\hline$\# 1$ & 8.1 & 6.8 & 7.1 & 7.2 & 7.6 \\
$\# 2$ & - & 8.9 & 9.1 & 9.2 & 9.1 \\
$\# 3$ & - & - & 12.5 & 11.7 & 11.4 \\
$\# 4$ & - & - & - & 15.0 & 14.8 \\
$\# 5$ & - & - & - & - & 16.3 \\
Average & $\mathbf{8 . 1}$ & $\mathbf{7 . 7}$ & $\mathbf{9 . 1}$ & $\mathbf{1 0 . 1}$ & $\mathbf{1 1 . 0}$ \\
\hline
\end{tabular}

Source:

Generated from the National Household Travel Survey Internet site nhts.ornl.gov.

${ }^{\text {a}}$ Vehicles are ranked by descending annual miles driven. 
The average annual miles per vehicle declined from 1995 to 2001. With households having more and more vehicles at their disposal, each vehicle is being driven fewer miles.

Table 8.11

Average Annual Miles Per Household Vehicle by Vehicle Age

\begin{tabular}{ccccc}
\hline $\begin{array}{c}\text { Vehicle age } \\
\text { (years) }\end{array}$ & $\begin{array}{c}1983 \\
\text { self-reported }\end{array}$ & $\begin{array}{c}1990 \\
\text { self-reported }\end{array}$ & $\begin{array}{c}1995 \\
\text { self-reported }\end{array}$ & $\begin{array}{c}2001 \\
\text { self-reported }\end{array}$ \\
\hline Under 1 & 8,200 & 19,600 & 15,900 & 15,000 \\
1 & 15,200 & 16,800 & 16,800 & 14,300 \\
2 & 16,800 & 16,600 & 15,500 & 13,700 \\
3 & 14,500 & 14,700 & 14,400 & 12,900 \\
4 & 13,000 & 13,600 & 14,100 & 12,400 \\
5 & 12,100 & 12,900 & 13,500 & 12,000 \\
6 & 11,300 & 13,200 & 13,200 & 11,700 \\
7 & 10,000 & 12,400 & 12,800 & 11,400 \\
8 & 9,800 & 12,600 & 12,200 & 11,100 \\
9 & 9,000 & 11,500 & 12,200 & 10,700 \\
10 and older & 7,300 & 9,200 & 8,900 & 7,400 \\
All household & & & & \\
vehicles & $\mathbf{1 0 , 4 0 0}$ & $\mathbf{1 2 , 5 0 0}$ & $\mathbf{1 2 , 2 0 0}$ & $\mathbf{1 1 , 0 0 0}$ \\
\hline
\end{tabular}

Source:

Nationwide Personal Transportation Study-1983: D. Klinger and J. Richard Kuzmyak, COMSIS Corporation, Personal Travel in the United States, Volume 1: 1983-84 Nationwide Personal Travel Study, prepared for the U.S. Department of Transportation, Washington, DC, August 1986, Table 4-22, p.4-21. 1990: Generated from the 1990 Nationwide Personal Transportation Study Public Use Tape, March 1992. 1995: Generated from the Internet site: wwwcta.ornl.gov/npts.

(Additional resources: www.fhwa.dot.gov, www.eia.doe.gov)

Note:

Data include all household vehicles, and have been rounded to the nearest hundred. 
Historically, the data from the Nationwide Personal Transportation Survey (NPTS) are based on estimates reported by survey respondents. For the 1995 survey, odometer data was also collected. These data indicate that respondents overestimate the number of miles driven in a year.

Table 8.12

Self-Reported vs. Odometer Average Annual Miles, 1995 NPTS

\begin{tabular}{ccc}
\hline $\begin{array}{c}\text { Vehicle age } \\
\text { (years) }\end{array}$ & $\begin{array}{c}1995 \\
\text { self-reported }\end{array}$ & $\begin{array}{c}1995 \\
\text { odometer }\end{array}$ \\
\hline Under 1 & 15,900 & 15,600 \\
1 & 16,800 & 14,500 \\
2 & 15,500 & 14,800 \\
3 & 14,400 & 13,800 \\
4 & 14,100 & 12,900 \\
5 & 13,500 & 12,700 \\
6 & 13,200 & 12,400 \\
7 & 12,800 & 11,600 \\
8 & 12,200 & 11,300 \\
9 & 12,200 & 11,200 \\
10 and older & 8,900 & 9,000 \\
All household & & \\
vehicles & 12,200 & 11,800 \\
\hline
\end{tabular}

Source:

Generated from the Internet site www-cta.ornl.gov/npts. 
According to the U.S. Census data, the percentage of workers who car pooled has dropped from $19.7 \%$ in 1980 to $11.2 \%$ in 2000. The percent of workers using public transit declined from $6.4 \%$ to $5.3 \%$ in the ten year period between 1980 and 1990, but stayed relatively the same from 1990 to 2000 (5.2\%). The average travel time increased by 2.6 minutes from 1980 to 2000.

Table 8.13

Means of Transportation to Work, 1980, 1990 and 2000 Census

\begin{tabular}{|c|c|c|c|c|c|c|}
\hline \multirow[b]{2}{*}{ Means of transportation } & \multicolumn{2}{|c|}{1980 Census } & \multicolumn{2}{|c|}{1990 Census } & \multicolumn{2}{|c|}{2000 Census } \\
\hline & $\begin{array}{l}\text { Number } \\
\text { of workers } \\
\text { (thousands) }\end{array}$ & Share & $\begin{array}{l}\text { Number } \\
\text { of workers } \\
\text { (thousands) }\end{array}$ & Share & $\begin{array}{l}\text { Number of } \\
\text { workers } \\
\text { (thousands) }\end{array}$ & Share \\
\hline Private vehicle & 81,258 & $84.1 \%$ & 99,593 & $86.5 \%$ & 111,554 & $87.5 \%$ \\
\hline Drove alone & 62,193 & $64.4 \%$ & 84,215 & $73.2 \%$ & 97,247 & $76.3 \%$ \\
\hline Car pooled & 19,065 & $19.7 \%$ & 15,378 & $13.4 \%$ & 14,307 & $11.2 \%$ \\
\hline Public transportation & 6,175 & $6.4 \%$ & 6,070 & $5.3 \%$ & 6,575 & $5.2 \%$ \\
\hline Bus or trolley bus ${ }^{a}$ & 3,925 & $4.1 \%$ & 3,445 & $3.0 \%$ & 3,572 & $2.8 \%$ \\
\hline Streetcar or trolley car ${ }^{a}$ & $b$ & & 78 & $0.1 \%$ & 88 & $0.1 \%$ \\
\hline Subway or elevated & 1,529 & $1.6 \%$ & 1,755 & $1.5 \%$ & 1,981 & $1.6 \%$ \\
\hline Railroad & 554 & $0.6 \%$ & 574 & $0.5 \%$ & 696 & $0.5 \%$ \\
\hline Ferryboat & $b$ & $b$ & 37 & $0.0 \%$ & 43 & $0.0 \%$ \\
\hline Taxicab & 167 & $0.2 \%$ & 179 & $0.2 \%$ & 194 & $0.2 \%$ \\
\hline Motorcycle & 419 & $0.4 \%$ & 237 & $0.2 \%$ & 158 & $0.1 \%$ \\
\hline Bicycle & 468 & $0.5 \%$ & 467 & $0.4 \%$ & 563 & $0.4 \%$ \\
\hline Walked only & 5,413 & $5.6 \%$ & 4,489 & $3.9 \%$ & 3,413 & $2.7 \%$ \\
\hline Other means & 703 & $0.7 \%$ & 809 & $0.7 \%$ & 1,099 & $0.9 \%$ \\
\hline Worked at home & 2,180 & $2.3 \%$ & 3,406 & $3.0 \%$ & 4,075 & $3.2 \%$ \\
\hline Total workers & 96,617 & $100.0 \%$ & 115,070 & $100.0 \%$ & 127,437 & $100.0 \%$ \\
\hline Average travel time (minutes) & 21.7 & & 22.4 & & 24.3 & \\
\hline
\end{tabular}

\section{Source:}

1980-1990 data - Provided by the Journey-to-Work and Migration Statistics Branch, Population Division, U.S. Bureau of the Census

2000 data - U.S. Bureau of the Census, American Fact Finder, factfinder.census.gov, Tables QT-03 and P047, August 2001. (Additional resources: www.census.gov)

\footnotetext{
a This category was "Bus or streetcar" in 1980.

${ }^{\mathrm{b}}$ Data are not available.
} 
More than half of workers had 15-29 minute commutes in 1990, but that dropped to $35 \%$ by 2000. The share of workers commuting less than 15 minutes increased the most in the ten-year period (14 percentage points), but the share of workers commuting 30 minutes or more also saw small increases.

Table 8.14

Workers by Commute Time, 1990 and 2000 Census

\begin{tabular}{lcc}
\hline \multicolumn{1}{c}{ Commute time } & 1990 & 2000 \\
\hline Less than 15 minutes & $15.9 \%$ & $30.1 \%$ \\
$15-29$ minutes & $51.6 \%$ & $36.3 \%$ \\
$30-39$ minutes & $14.7 \%$ & $15.7 \%$ \\
$40-59$ minutes & $9.0 \%$ & $10.7 \%$ \\
60 minutes or more & $5.9 \%$ & $7.3 \%$ \\
\hline Average travel time (minutes) & 22.4 & 24.3 \\
\hline
\end{tabular}

\section{Source:}

1990 - U. S. Department of Transportation, Volpe National Transportation

Systems Center, Journey-to-Work Trends in the United States and its Major Metropolitan Area, 1960-1990, FHWA-PL-94-012, Cambridge, MA, 1994, p. 2-6.

2000 - U.S. Bureau of the Census, American Fact Finder, factfinder.census.gov, Tables QT-03 and P048, August 2001.

(Additional resources: www.census.gov) 
Sales of bicycles with wheel sizes of 20-inches and over have grown at an average annual rate of 2\%

from 1981 to 2002. The largest growth in bicycle sales, however, were bicycles with wheel sizes under 20 inches which grew at an average annual rate of $4.8 \%$.

Table 8.15

Bicycle Sales, 1981-2002

(millions)

\begin{tabular}{cccc}
\hline & $\begin{array}{c}\text { Wheel sizes } \\
\text { under } \\
\text { 20 inches }\end{array}$ & $\begin{array}{c}\text { Wheel sizes } \\
\text { of 20 inches } \\
\text { and over }\end{array}$ & $\begin{array}{c}\text { All } \\
\text { wheel sizes }\end{array}$ \\
\hline 1981 & a & 8.9 & a \\
1982 & a & 6.8 & a \\
1983 & a & 9.0 & a \\
1984 & a & 10.1 & a \\
1985 & a & 11.4 & a \\
1986 & a & 12.3 & a \\
1987 & a & 12.6 & a \\
1988 & a & 9.9 & a \\
1989 & a & 10.7 & a \\
1990 & a & 10.8 & a \\
1991 & a & 11.6 & a \\
1992 & 3.7 & 11.6 & 15.3 \\
1993 & 3.8 & 13.0 & 16.8 \\
1994 & 4.2 & 12.5 & 16.7 \\
1995 & 4.1 & 12.0 & 16.1 \\
1996 & 4.5 & 10.9 & 15.4 \\
1997 & 4.2 & 11.0 & 15.2 \\
1998 & 4.7 & 11.1 & 15.8 \\
1999 & 5.9 & 11.6 & 17.5 \\
2000 & 9.0 & 11.9 & 20.9 \\
2001 & 5.4 & 11.3 & 16.7 \\
2002 & 5.9 & 13.6 & 19.5 \\
& Average annual percentage change & \\
$1981-2002$ & & $2.0 \%$ & a \\
$1992-2002$ & $4.8 \%$ & $1.6 \%$ & \\
\hline
\end{tabular}

\section{Source:}

1981-1996: Bicycle Manufacturers Association. 1997-on: The Bicycle Council. (Additional resources: www.nbda.com)

${ }^{\text {a }}$ Data are not available. 
One-third of bicycle sales in 2002 were mountain bikes, which sold for an average of $\$ 450$ per bike. Road bicycles, which list the most expensive average price, have been slowly gaining market share from 2000 to 2002. Youth bicycles account for $28 \%$ of the bicycle market.

Table 8.16

Specialty Bicycle Sales by Year, 2000-2002

\begin{tabular}{lcccc}
\hline Category & 2000 & 2001 & 2002 & $\begin{array}{c}\text { Average 2002 } \\
\text { price }\end{array}$ \\
\hline Mountain & $41.0 \%$ & $36.8 \%$ & $33.8 \%$ & $\$ 450.30$ \\
Youth & $28.5 \%$ & $26.1 \%$ & $28.3 \%$ & $\$ 197.49$ \\
Comfort & $13.6 \%$ & $20.8 \%$ & $20.6 \%$ & $\$ 339.15$ \\
Hybrid & $10.2 \%$ & $8.8 \%$ & $9.4 \%$ & $\$ 404.66$ \\
Road & $3.6 \%$ & $4.4 \%$ & $5.3 \%$ & $\$ 1,194.68$ \\
Cruiser & $2.8 \%$ & $2.8 \%$ & $2.2 \%$ & $\$ 275.17$ \\
Tandem & $0.12 \%$ & $0.11 \%$ & $0.15 \%$ & $\$ 887.91$ \\
\hline
\end{tabular}

Source:

National Bicycle Dealers Association Retail Data Capture Program.

${ }^{\text {a }}$ Sales of top 19 bicycle brands through panel of retailers. 
In 2001, 5\% of walk trips and 8\% of bike trips were to/from work. More than half of all bike trips were for social/recreational purposes. Thirteen-percent of walk trips were shopping trips.

Figure 8.3 Walk and Bike Trips by Trip Purpose, 2001 NHTS

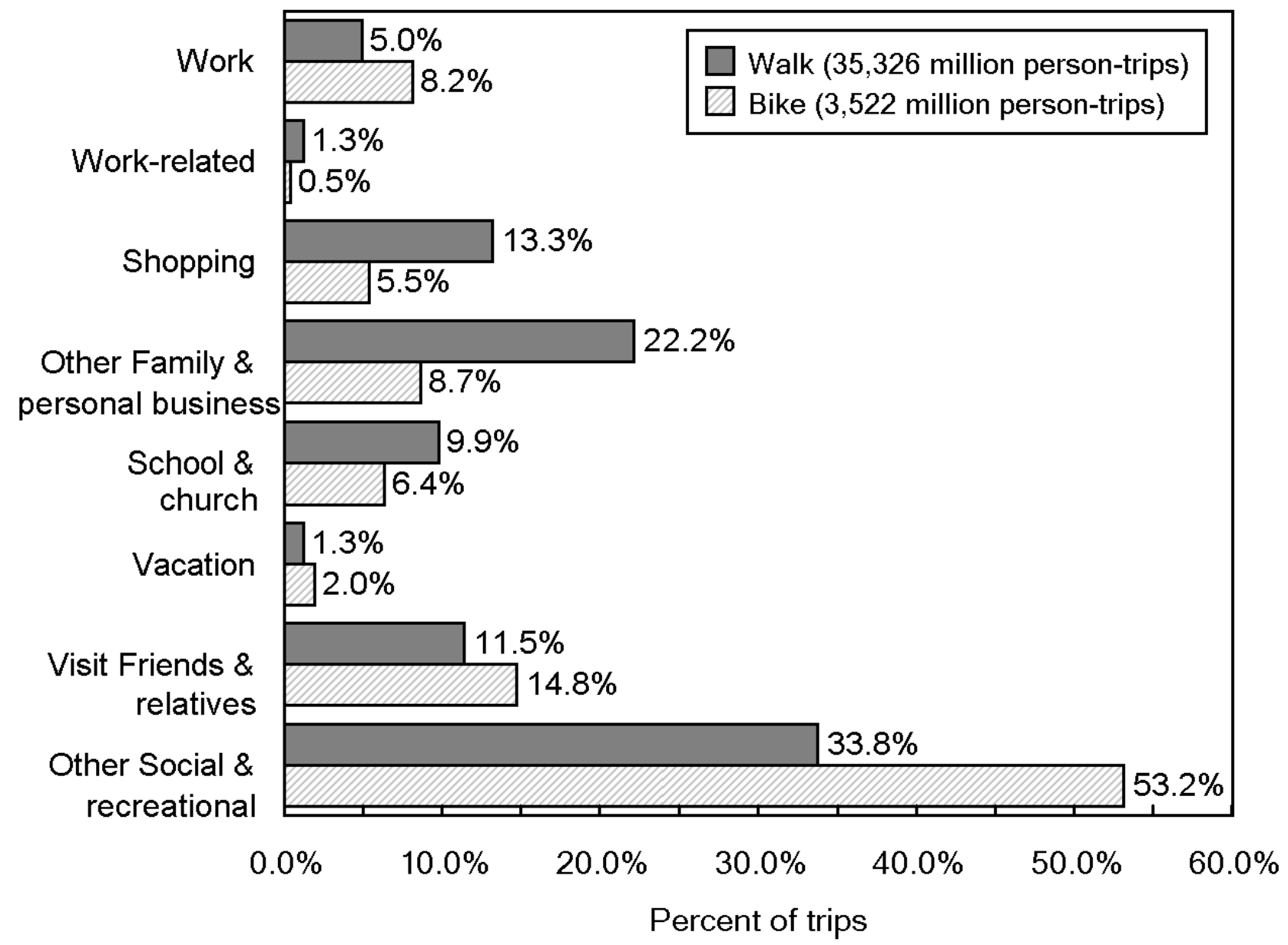

\section{Source:}

U.S. Department of Transportation, Federal Highway Administration, National Household Travel Survey web site: nhts.ornl.gov. 


\section{American Travel Survey}

The American Travel Survey (ATS) was conducted by the Bureau of Transportation Statistics, U.S. Department of Transportation, to obtain information about the long-distance travel of persons living in the United States. Approximately 80,000 randomly selected households were interviewed for the survey, which collected information about all trips of 100 miles or more, one-way, taken by household members in 1995. The ATS data provide detailed information on state-to-state travel, as well as travel to and from metropolitan areas by mode of transportation.

For additional information about the American Travel Survey, contact the Bureau of Transportation Statistics at (202) 366-3282 or visit the following Internet site: www.bts.gov/ats. New data on long-distance travel will be available in late 2003 from the 2001 National Household Travel Survey at nhts.ornl.gov.

Figure 8.4 Long-Distance Trips by Destination, 1995

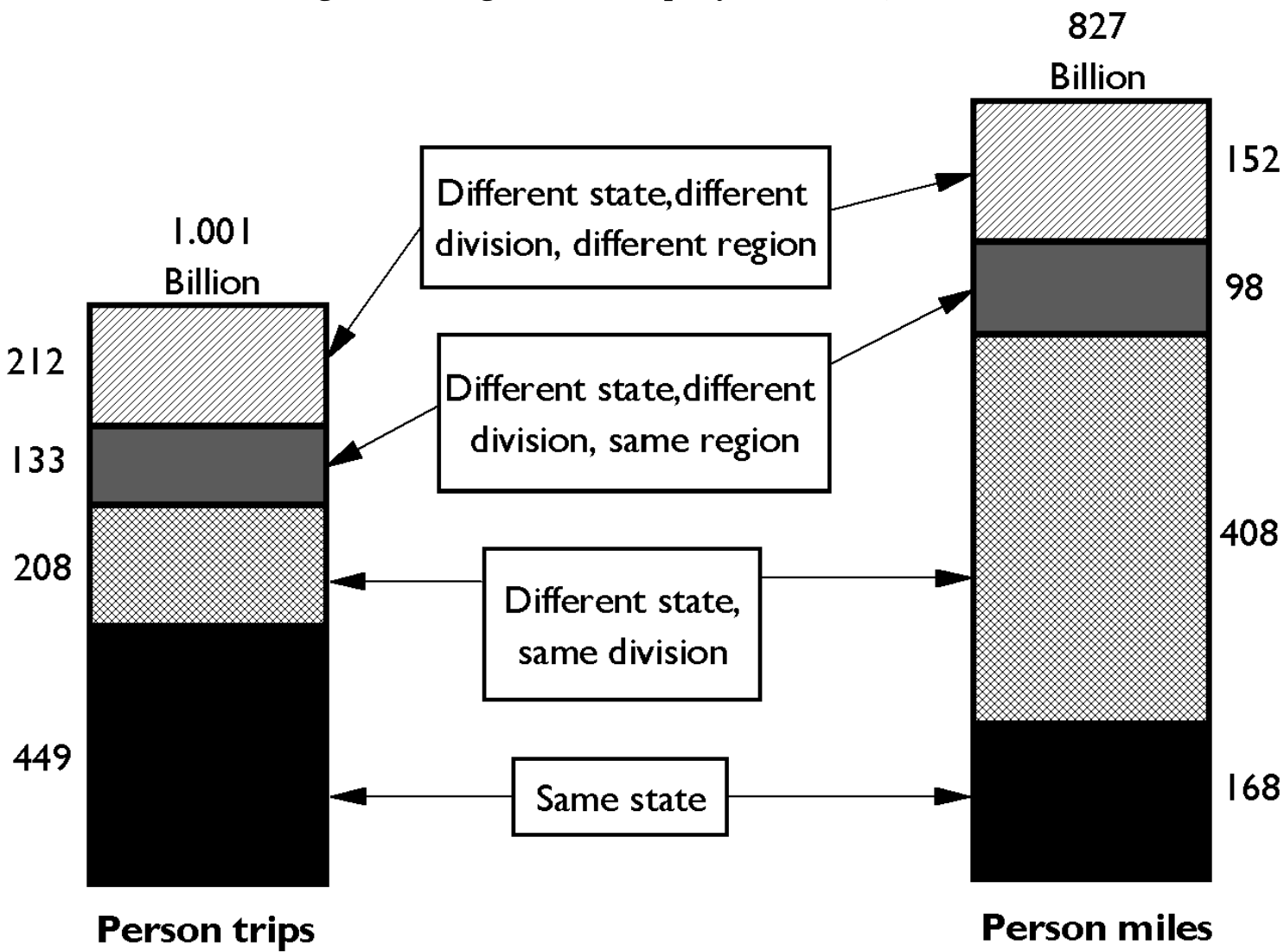

Source:

U.S. Department of Transportation, Bureau of Transportation Statistics, 1995 American Travel Survey Profile, Washington, DC, October 1997, p. 2. (Additional resources: www.bts.gov/ats)

Note:

Definitions of divisions and regions are in Appendix C. 
Personal-use vehicles are by far the most predominant means of transportation on long-distance trips (100 miles or more, one way); two-thirds of those personal vehicle trips are pleasure trips.

Table 8.17

Long-Distance Trips ${ }^{\mathrm{a}}$ by Mode and Purpose, 1995

\begin{tabular}{|c|c|c|c|c|c|c|}
\hline \multirow[b]{3}{*}{$\begin{array}{l}\text { Principal means of } \\
\text { transportation }\end{array}$} & \multicolumn{6}{|c|}{ Main purpose of trip } \\
\hline & \multirow[b]{2}{*}{ Business } & \multicolumn{3}{|c|}{ Pleasure } & \multirow[b]{2}{*}{$\begin{array}{l}\text { Personal } \\
\text { business }\end{array}$} & \multirow[b]{2}{*}{ Total } \\
\hline & & $\begin{array}{l}\text { Visit friends } \\
\text { or relatives }\end{array}$ & Leisure & $\begin{array}{c}\text { Total } \\
\text { pleasure }\end{array}$ & & \\
\hline & \multicolumn{6}{|c|}{ Person trips (thousands) } \\
\hline Personal use vehicle & 151,697 & 283,153 & 254,186 & 537,339 & 124,791 & 813,858 \\
\hline Commercial airplane & 67,083 & 41,881 & 31,581 & 73,462 & 15,386 & 155,936 \\
\hline Intercity bus & 286 & 1,830 & 690 & 2,519 & 439 & 3,244 \\
\hline Charter or tour bus & 1,281 & 1,198 & 9,253 & 10,451 & 2,514 & 14,247 \\
\hline Train & 1,342 & 2,004 & 944 & 2,948 & 704 & 4,994 \\
\hline Ship, boat, or ferry & 68 & 43 & 483 & 525 & 20 & 614 \\
\hline \multirow[t]{2}{*}{ Total person-trips } & 224,835 & 330,755 & 299,355 & 630,110 & 146,338 & $1,001,31$ \\
\hline & \multicolumn{6}{|c|}{ Percentage } \\
\hline Personal use vehicle & 18.6 & 34.8 & 31.2 & 66.0 & 15.3 & 100.0 \\
\hline Commercial airplane & 43.0 & 26.9 & 20.3 & 47.1 & 9.9 & 100.0 \\
\hline Intercity bus & 8.8 & 56.4 & 21.3 & 77.7 & 13.5 & 100.0 \\
\hline Charter or tour bus & 9.0 & 8.4 & 64.9 & 73.4 & 17.6 & 100.0 \\
\hline Train & 26.9 & 40.1 & 18.9 & 59.0 & 14.1 & 100.0 \\
\hline Ship, boat, or ferry & 11.1 & 7.0 & 78.7 & 85.5 & 3.3 & 100.0 \\
\hline Total & 22.5 & 33.0 & 29.9 & 62.9 & 14.6 & 100.0 \\
\hline
\end{tabular}

Source:

U.S. Department of Transportation, Bureau of Transportation Statistics, 1995 American Travel Survey Profile, Washington, DC, October 1997, p. 13. (Additional resources: www.bts.gov/ats)

${ }^{\mathrm{a} A}$ long-distance trip is any trip of 100 miles or more, one way. 
Those with a household income of less than \$25,000 account for more than half (54\%) of intercity bus person-trips. Those with a household income of $\$ 50,000$ or more account for two-thirds (66\%) of commercial airplane person-trips.

Figure 8.5. Shares of Long-Distance Person Trips by Mode and Household Income, 1995

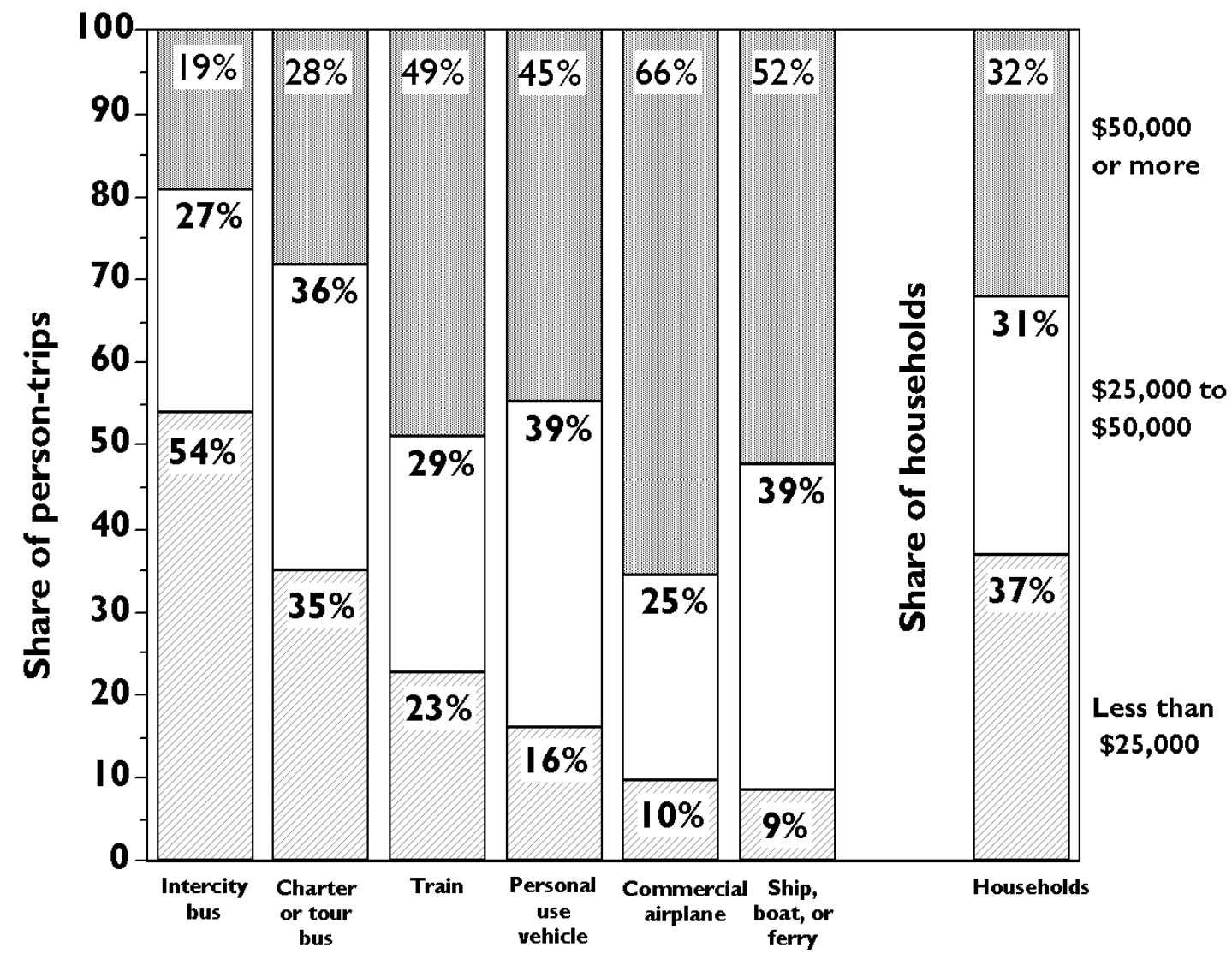

Source:

U.S. Department of Transportation, Bureau of Transportation Statistics, 1995 American Travel Survey Profile, Washington, DC, October 1997, p. 8.

U.S. Department of Commerce, Bureau of the Census, Statistical Abstract of the United States, $117^{\text {th }}$ Edition, Washington, DC, 1997, p. 465.

(Additional resources: www.bts.gov/ats, www.census.gov) 


\section{Chapter 9 \\ Nonhighway Modes}

Summary Statistics from Tables in this Chapter

\begin{tabular}{|c|c|c|}
\hline \multicolumn{3}{|l|}{ Source } \\
\hline & Passenger-miles, 2001 & (millions) \\
\hline Table 9.2 & Domestic and international air carrier & 664,841 \\
\hline Table 9.3 & General aviation & 16 \\
\hline Table 9.12 & Amtrak & 5,571 \\
\hline Table 9.13 & Commuter rail & 9,548 \\
\hline \multirow[t]{2}{*}{ Table 9.14} & Transit rail & 15,615 \\
\hline & Freight ton-miles, 2001 & (millions) \\
\hline Table 9.5 & Domestic waterborne commerce & 622,000 \\
\hline \multirow[t]{2}{*}{ Table 9.9} & Class I railroad & $1,495,472$ \\
\hline & Passenger energy use, 2001 & (trillion Btus) \\
\hline Table 9.2 & Domestic and international air carrier & 2,599.4 \\
\hline Table 9.3 & General aviation & 165.1 \\
\hline Table 9.7 & Recreational boats & 313.5 \\
\hline Table 9.12 & Amtrak & 19.8 \\
\hline Table 9.13 & Commuter rail & 25.9 \\
\hline \multirow[t]{2}{*}{ Table 9.14} & Transit rail & 48.6 \\
\hline & Freight energy use, 2001 & (trillion Btus) \\
\hline Table 9.5 & Domestic waterborne commerce & 276.2 \\
\hline Table 9.9 & Class I railroad & 517.3 \\
\hline
\end{tabular}


Nonhighway transportation modes accounted for about 20\% of total transportation energy use in 2001.

Table 9.1

Nonhighway Energy Use Shares, 1970-2001

\begin{tabular}{|c|c|c|c|c|c|c|}
\hline \multirow[b]{2}{*}{ Year } & \multicolumn{6}{|c|}{ Share of transportation energy use } \\
\hline & Air & Water & Pipeline & Rail & $\begin{array}{c}\text { Nonhighway } \\
\text { total }\end{array}$ & $\begin{array}{l}\text { Transportation } \\
\text { total (trillion Btu) }\end{array}$ \\
\hline 1970 & $8.5 \%$ & $4.9 \%$ & $6.5 \%$ & $3.6 \%$ & $23.6 \%$ & 15,321 \\
\hline 1971 & $8.2 \%$ & $4.4 \%$ & $6.4 \%$ & $3.5 \%$ & $22.4 \%$ & 15,945 \\
\hline 1972 & $7.7 \%$ & $4.2 \%$ & $6.1 \%$ & $3.4 \%$ & $21.5 \%$ & 16,969 \\
\hline 1973 & $7.7 \%$ & $4.6 \%$ & $5.6 \%$ & $3.5 \%$ & $21.4 \%$ & 17,824 \\
\hline 1974 & $7.3 \%$ & $4.7 \%$ & $5.5 \%$ & $3.6 \%$ & $21.1 \%$ & 17,104 \\
\hline 1975 & $7.3 \%$ & $4.9 \%$ & $4.9 \%$ & $3.2 \%$ & $20.4 \%$ & 17,356 \\
\hline 1976 & $7.2 \%$ & $5.5 \%$ & $4.4 \%$ & $3.2 \%$ & $20.3 \%$ & 18,426 \\
\hline 1977 & $7.0 \%$ & $6.3 \%$ & $4.1 \%$ & $3.1 \%$ & $20.5 \%$ & 19,157 \\
\hline 1978 & $7.1 \%$ & $7.0 \%$ & $3.9 \%$ & $2.9 \%$ & $20.9 \%$ & 20,126 \\
\hline 1979 & $7.4 \%$ & $8.1 \%$ & $4.3 \%$ & $3.0 \%$ & $22.8 \%$ & 20,135 \\
\hline 1980 & $7.6 \%$ & $7.5 \%$ & $4.7 \%$ & $3.1 \%$ & $22.9 \%$ & 18,979 \\
\hline 1981 & $7.6 \%$ & $8.6 \%$ & $4.8 \%$ & $3.0 \%$ & $23.9 \%$ & 19,120 \\
\hline 1982 & $7.8 \%$ & $7.4 \%$ & $4.6 \%$ & $2.6 \%$ & $22.5 \%$ & 18,560 \\
\hline 1983 & $7.7 \%$ & $6.8 \%$ & $4.0 \%$ & $2.6 \%$ & $21.1 \%$ & 18,677 \\
\hline 1984 & $8.3 \%$ & $6.8 \%$ & $4.1 \%$ & $2.8 \%$ & $22.0 \%$ & 19,323 \\
\hline 1985 & $8.5 \%$ & $6.7 \%$ & $3.9 \%$ & $2.6 \%$ & $21.6 \%$ & 19,659 \\
\hline 1986 & $9.0 \%$ & $6.5 \%$ & $3.6 \%$ & $2.4 \%$ & $21.5 \%$ & 20,277 \\
\hline 1987 & $9.2 \%$ & $6.5 \%$ & $3.7 \%$ & $2.4 \%$ & $21.8 \%$ & 20,742 \\
\hline 1988 & $9.3 \%$ & $6.4 \%$ & $4.1 \%$ & $2.4 \%$ & $22.2 \%$ & 21,280 \\
\hline 1989 & $9.2 \%$ & $6.5 \%$ & $4.1 \%$ & $2.4 \%$ & $22.2 \%$ & 21,580 \\
\hline 1990 & $9.6 \%$ & $7.0 \%$ & $4.3 \%$ & $2.4 \%$ & $23.2 \%$ & 21,689 \\
\hline 1991 & $9.1 \%$ & $7.5 \%$ & $4.1 \%$ & $2.3 \%$ & $22.9 \%$ & 21,279 \\
\hline 1992 & $9.0 \%$ & $7.6 \%$ & $3.9 \%$ & $2.3 \%$ & $22.7 \%$ & 21,939 \\
\hline 1993 & $8.9 \%$ & $6.7 \%$ & $4.0 \%$ & $2.3 \%$ & $21.8 \%$ & 22,393 \\
\hline 1994 & $9.0 \%$ & $6.3 \%$ & $4.2 \%$ & $2.4 \%$ & $21.8 \%$ & 22,997 \\
\hline 1995 & $9.1 \%$ & $6.5 \%$ & $4.1 \%$ & $2.4 \%$ & $22.1 \%$ & 23,536 \\
\hline 1996 & $9.2 \%$ & $6.1 \%$ & $4.1 \%$ & $2.4 \%$ & $21.8 \%$ & 24,042 \\
\hline 1997 & $9.4 \%$ & $5.4 \%$ & $4.2 \%$ & $2.4 \%$ & $21.4 \%$ & 24,404 \\
\hline 1998 & $9.5 \%$ & $5.2 \%$ & $3.6 \%$ & $2.4 \%$ & $20.7 \%$ & 24,839 \\
\hline 1999 & $9.5 \%$ & $5.5 \%$ & $3.5 \%$ & $2.3 \%$ & $20.8 \%$ & 26,034 \\
\hline 2000 & $9.7 \%$ & $5.8 \%$ & $3.4 \%$ & $2.3 \%$ & $21.2 \%$ & 26,350 \\
\hline 2001 & $9.3 \%$ & $4.5 \%$ & $3.4 \%$ & $2.4 \%$ & $19.6 \%$ & 25,899 \\
\hline
\end{tabular}

Source:

Table 2.7. 
These data include all international and domestic certificated route air carrier statistics; therefore, the data are different than those in Chapter 2. All of the air carrier statistics declined in the year 2001, most likely due to the events of September 11, 2001, which caused air travel to decline drastically in the last quarter of the year.

Table 9.2

Summary Statistics for U.S. Domestic and International Certificated Route Air Carriers (Combined Totals), 1970-2001

\begin{tabular}{|c|c|c|c|c|c|c|c|c|}
\hline Year & $\begin{array}{l}\text { Revenue } \\
\text { aircraft-miles } \\
\text { (millions) }\end{array}$ & $\begin{array}{c}\text { Average } \\
\text { passenger trip } \\
\text { length }^{\mathrm{b}} \text { (miles) }\end{array}$ & $\begin{array}{c}\text { Revenue } \\
\text { passenger-miles } \\
\text { (millions) }\end{array}$ & $\begin{array}{l}\text { Available } \\
\text { seat-miles } \\
\text { (millions) }\end{array}$ & $\begin{array}{l}\text { Available } \\
\text { seats per } \\
\text { aircraft } \\
\end{array}$ & $\begin{array}{c}\text { Passenger } \\
\text { load factor } \\
{\text { (percentage })^{\mathrm{d}}}\end{array}$ & $\begin{array}{c}\text { Revenue cargo } \\
\text { ton-miles } \\
\text { (millions) }\end{array}$ & $\begin{array}{c}\text { Energy use } \\
(\text { trillion Btu) }\end{array}$ \\
\hline 1970 & 2,383 & 678 & $131,719^{\mathrm{f}}$ & $264,904^{\mathrm{f}}$ & 111 & $49.7 \%^{\mathrm{f}}$ & 4,994 & $1,363.4$ \\
\hline 1975 & 2,241 & 698 & 173,324 & 315,823 & 135 & $54.9 \%$ & 5,944 & $1,283.4$ \\
\hline 1980 & 2,924 & 736 & 267,722 & 448,479 & 148 & $59.7 \%$ & 7,515 & $1,386.0$ \\
\hline 1985 & 3,462 & 758 & 351,073 & 565,677 & 163 & $62.1 \%$ & 9,048 & $1,701.4$ \\
\hline 1986 & 3,873 & 767 & 378,923 & 623,073 & 161 & $60.8 \%$ & 10,987 & $1,847.1$ \\
\hline 1987 & 4,182 & 779 & 417,830 & 670,871 & 160 & $62.3 \%$ & 13,130 & $1,945.9$ \\
\hline 1988 & 4,355 & 786 & 437,649 & 696,337 & 160 & $62.9 \%$ & 14,633 & $2,049.4$ \\
\hline 1989 & 4,442 & 792 & 447,480 & 703,888 & 158 & $63.6 \%$ & 16,347 & $2,087.4$ \\
\hline 1990 & 4,724 & 803 & 472,236 & 753,211 & 159 & $62.7 \%$ & 16,411 & $2,213.0$ \\
\hline 1991 & 4,661 & 806 & 463,296 & 738,030 & 158 & $62.8 \%$ & 16,149 & $2,085.2$ \\
\hline 1992 & 4,899 & 806 & 493,715 & 772,869 & 158 & $63.9 \%$ & 17,306 & $2,144.2$ \\
\hline 1993 & 5,118 & 799 & 505,996 & 793,959 & 155 & $63.7 \%$ & 19,083 & $2,169.7$ \\
\hline 1994 & 5,360 & 787 & 537,506 & 809,240 & 151 & $66.4 \%$ & 21,773 & $2,266.2$ \\
\hline 1995 & 5,627 & 791 & 558,757 & 845,012 & 150 & $66.1 \%$ & 23,375 & $2,338.6$ \\
\hline 1996 & 5,855 & 802 & 596,164 & 859,720 & 147 & $69.3 \%$ & 24,892 & $2,409.1$ \\
\hline 1997 & 6,025 & 814 & 619,969 & 880,607 & 146 & $70.4 \%$ & 27,610 & $2,514.2$ \\
\hline 1998 & 6,227 & 812 & 635,517 & 899,851 & 145 & $70.6 \%$ & 28,102 & $2,573.4$ \\
\hline 1999 & 6,558 & 824 & 668,626 & 942,311 & 144 & $71.0 \%$ & 28,984 & $2,653.1$ \\
\hline 2000 & 6,944 & 833 & 708,419 & 980,379 & 141 & $72.3 \%$ & 30,863 & $2,743.1$ \\
\hline 2001 & 6,807 & 842 & 664,841 & 950,530 & 140 & $69.9 \%$ & 27,882 & $2,599.4$ \\
\hline \multicolumn{9}{|c|}{ Average annual percentage change } \\
\hline 1970-2001 & $3.4 \%$ & $0.7 \%$ & $5.4 \%$ & $4.2 \%$ & $0.8 \%$ & & $5.7 \%$ & $2.1 \%$ \\
\hline $1991-2001$ & $3.9 \%$ & $0.4 \%$ & $3.7 \%$ & $2.6 \%$ & $-1.2 \%$ & & $5.6 \%$ & $2.2 \%$ \\
\hline
\end{tabular}

Source:

U.S. Department of Transportation, Bureau of Transportation Statistics, Air Carrier Traffic Statistics Monthly, December 2001/2000, Washington, DC, pp. 1-2, and annual.

1970-76 Energy Use - Department of Transportation, Civil Aeronautics Board, Fuel Cost and Consumption, Washington, DC, 1981, and annual.

1977-2001 Energy Use - Department of Transportation, Bureau of Transportation Statistics, "Fuel Cost and Consumption Table," Washington, DC. (Additional resources: www.bts.gov, www.faa.gov)

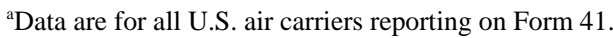

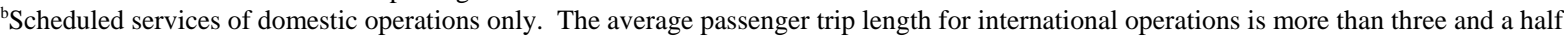
times longer than for domestic operations.

${ }^{c}$ Available seats per aircraft is calculated as the ratio of available seat-miles to revenue aircraft-miles.

${ }^{\mathrm{d}}$ Passenger load factor is calculated as the ratio of revenue passenger-miles to available seat-miles for scheduled and nonscheduled services.

energy use includes fuel purchased abroad for international flights.

${ }^{\text {f }}$ Scheduled services only. 
General aviation includes: (1) aircraft operating under general operating and flight rules; (2) not-for-hire airplanes with a seating capacity of 20 or more or a maximum payload capacity of 6,000 lbs. or more; (3) rotocraft external load operations; (4) on-demand and commuter operations not covered under Federal Aviation Regulations Part 121; and (5) agricultural aircraft operations.

Table 9.3

Summary Statistics for General Aviation, 1970-2001

\begin{tabular}{|c|c|c|c|c|}
\hline Calendar year & $\begin{array}{c}\text { Total number } \\
\text { of aircraft }\end{array}$ & $\begin{array}{c}\text { Aircraft } \\
\text { hours flown } \\
\text { (thousands) }\end{array}$ & $\begin{array}{l}\text { Intercity passenger travel } \\
\text { (billion passenger-miles) }\end{array}$ & $\begin{array}{l}\text { Energy use } \\
\text { (trillion btu) }\end{array}$ \\
\hline 1970 & $131,700^{\mathrm{a}}$ & $26,030^{\mathrm{b}}$ & 9.1 & 94.4 \\
\hline 1975 & 168,475 & 30,298 & 11.4 & 121.5 \\
\hline 1976 & 177,964 & 31,950 & 12.1 & 130.3 \\
\hline 1977 & 184,294 & 33,679 & 12.8 & 149.7 \\
\hline 1978 & 199,178 & 36,844 & 14.1 & 159.4 \\
\hline 1979 & 210,339 & 40,432 & 15.5 & 167.2 \\
\hline 1980 & 211,045 & 41,016 & 14.7 & 169.0 \\
\hline 1981 & 213,226 & 40,704 & 14.6 & 162.4 \\
\hline 1982 & 209,779 & 36,457 & 13.1 & 170.5 \\
\hline 1983 & 213,293 & 35,249 & 12.7 & 143.9 \\
\hline 1984 & 220,943 & 36,119 & 13.0 & 148.9 \\
\hline 1985 & 196,500 & 31,456 & 12.3 & 144.0 \\
\hline 1986 & 205,300 & 31,782 & 12.4 & 148.0 \\
\hline 1987 & 202,700 & 30,883 & 12.1 & 139.1 \\
\hline 1988 & 196,200 & 31,114 & 12.6 & 148.6 \\
\hline 1989 & 205,000 & 32,332 & 13.1 & 134.0 \\
\hline 1990 & 198,000 & 32,096 & 13.0 & 131.9 \\
\hline 1991 & 196,874 & 29,862 & 12.1 & 120.4 \\
\hline 1992 & 185,650 & 26,747 & 10.8 & 104.7 \\
\hline 1993 & 177,120 & 24,455 & 9.9 & 97.5 \\
\hline 1994 & 172,935 & 24,092 & 9.8 & 95.3 \\
\hline 1995 & 188,089 & 26,612 & 10.8 & 106.6 \\
\hline 1996 & 191,129 & 26,909 & 12.0 & 111.1 \\
\hline 1997 & 192,414 & 27,713 & 12.5 & 121.1 \\
\hline 1998 & 204,710 & 28,100 & 13.1 & 147.4 \\
\hline 1999 & 219,464 & 31,756 & 14.1 & 172.1 \\
\hline 2000 & 217,533 & 30,975 & 15.2 & 175.2 \\
\hline 2001 & 211,446 & 29,133 & 15.9 & 165.1 \\
\hline \multicolumn{5}{|c|}{ Average annual percentage change } \\
\hline 1970-2001 & $1.5 \%$ & $0.4 \%$ & $1.8 \%$ & $1.8 \%$ \\
\hline 1991-2001 & $0.7 \%$ & $-0.2 \%$ & $2.8 \%$ & $3.2 \%$ \\
\hline
\end{tabular}

Sources:

Intercity passenger-miles - Eno Foundation for Transportation, Transportation in America 2001, Nineteenth edition, Lansdowne, VA, 2002, p. 45, and annual.

All other- U.S. Department of Transportation, Federal Aviation Administration, General Aviation Activity and Avionics Survey: Calendar Year 2001, Tables 1.2, 1.5, 5.1, and annual. (Additional resources: apo.faa.gov/pubs.asp)

${ }^{a}$ Active fixed-wing general aviation aircraft only.

bincludes rotocraft. 
In the early seventies, domestic waterborne commerce accounted for over $60 \%$ of total tonnage, but by 1994 foreign tonnage grew to more than half of all waterborne tonnage and has continued to grow each year since.

Table 9.4

Tonnage Statistics for Domestic and International Waterborne Commerce, 1970-2001 (million tons shipped)

\begin{tabular}{ccccc}
\hline Year & $\begin{array}{c}\text { Foreign and } \\
\text { domestic total }\end{array}$ & Foreign total $^{\mathrm{a}}$ & Domestic total $^{\mathrm{b}}$ & $\begin{array}{c}\text { Percent domestic } \\
\text { of total }^{-1,532}\end{array}$ \\
\hline 1970 & 581 & 951 & $62.1 \%$ \\
\hline 1975 & 1,695 & 749 & 946 & $55.8 \%$ \\
1976 & 1,835 & 856 & 979 & $53.4 \%$ \\
1977 & 1,908 & 935 & 973 & $51.0 \%$ \\
1978 & 2,021 & 946 & 1,075 & $53.2 \%$ \\
1979 & 2,073 & 993 & 1,080 & $52.1 \%$ \\
1980 & 1,999 & 921 & 1,077 & $53.9 \%$ \\
1981 & 1,942 & 887 & 1,054 & $54.3 \%$ \\
1982 & 1,777 & 820 & 957 & $53.9 \%$ \\
1983 & 1,708 & 751 & 957 & $56.0 \%$ \\
1984 & 1,836 & 803 & 1,033 & $56.3 \%$ \\
1985 & 1,788 & 774 & 1,014 & $56.7 \%$ \\
1986 & 1,874 & 837 & 1,037 & $55.3 \%$ \\
1987 & 1,967 & 891 & 1,076 & $54.7 \%$ \\
1988 & 2,088 & 976 & 1,112 & $53.3 \%$ \\
1989 & 2,140 & 1,038 & 1,103 & $51.5 \%$ \\
1990 & 2,164 & 1,042 & 1,122 & $51.8 \%$ \\
1991 & 2,092 & 1,014 & 1,079 & $51.6 \%$ \\
1992 & 2,132 & 1,037 & 1,095 & $51.4 \%$ \\
1993 & 2,128 & 1,060 & 1,068 & $50.2 \%$ \\
1994 & 2,215 & 1,116 & 1,099 & $49.6 \%$ \\
1995 & 2,240 & 1,147 & 1,093 & $48.8 \%$ \\
1996 & 2,284 & 1,183 & 1,101 & $48.2 \%$ \\
1997 & 2,334 & 1,221 & 1,113 & $47.7 \%$ \\
1998 & 2,339 & 1,245 & 1,094 & $46.8 \%$ \\
1999 & 2,323 & 1,261 & 1,062 & $45.6 \%$ \\
2000 & 2,425 & 1,355 & 1,070 & $44.1 \%$ \\
2001 & 2,387 & 1,344 & 1,042 & $43.7 \%$ \\
& Average annual percentage change & & \\
$1970-2001$ & $1.4 \%$ & $2.7 \%$ & $0.3 \%$ & \\
$1991-2001$ & $1.3 \%$ & $2.9 \%$ & $-0.3 \%$ & \\
\hline
\end{tabular}

\section{Source:}

U.S. Department of the Army, Corps of Engineers, Waterborne Commerce of the United States, Calendar Year 2001, Part 5: National Summaries, New Orleans, Louisiana, 2002, Table 1-1, p. 1-3, and annual. (Additional resources: www.wrc-ndc.usace.army.mil/ndc)

\footnotetext{
${ }^{a}$ All movements between the U.S. and foreign countries and between Puerto Rico and the Virgin Islands and foreign countries are classified as foreign trade.

${ }^{b}$ All movements between U.S. ports, continental and noncontiguous, and on the inland rivers, canals, and connecting channels of the U.S., Puerto Rico, and the Virgin Islands, excluding the Panama Canal. Beginning in 1996, fish was excluded for internal and intra port domestic traffic.
} 
Table 9.5

Summary Statistics for Domestic Waterborne Commerce, 1970-2001

\begin{tabular}{|c|c|c|c|c|c|c|}
\hline Year & $\begin{array}{c}\text { Number of } \\
\text { vessels }^{\mathrm{a}}\end{array}$ & $\begin{array}{l}\text { Ton-miles } \\
\text { (billions) }\end{array}$ & $\begin{array}{c}\text { Tons shipped }^{\mathrm{b}} \\
\text { (millions) }\end{array}$ & $\begin{array}{c}\text { Average } \\
\text { length of haul } \\
\text { (miles) }\end{array}$ & $\begin{array}{c}\text { Energy } \\
\text { intensity } \\
\text { (Btu/ton-mile) }\end{array}$ & $\begin{array}{l}\text { Energy use } \\
\text { (trillion Btu) }\end{array}$ \\
\hline 1970 & 25,832 & 596 & 949 & 628.2 & 545 & 324.8 \\
\hline 1975 & 31,666 & 566 & $944^{\circ}$ & 599.9 & 549 & 311.0 \\
\hline 1976 & 33,204 & 592 & 976 & 606.3 & 468 & 277.3 \\
\hline 1977 & 35,333 & 599 & 969 & 618.0 & 458 & 274.3 \\
\hline 1978 & 35,723 & 827 & 1,072 & 771.6 & 383 & 316.6 \\
\hline 1979 & 36,264 & 829 & 1,076 & 770.0 & 457 & 378.7 \\
\hline 1980 & 38,792 & 922 & 1,074 & 856.4 & 358 & 329.8 \\
\hline 1981 & 42,079 & 929 & 1,051 & 884.0 & 360 & 334.5 \\
\hline 1982 & 42,079 & 886 & 954 & 929.0 & 310 & 274.9 \\
\hline 1983 & 41,784 & 920 & 953 & 964.6 & 319 & 293.7 \\
\hline 1984 & 41,784 & 888 & 1,029 & 862.5 & 346 & 307.3 \\
\hline 1985 & 41,672 & 893 & 1,011 & 883.5 & 446 & 398.6 \\
\hline 1986 & 40,308 & 873 & 1,033 & 845.3 & 463 & 404.0 \\
\hline 1987 & 40,000 & 895 & 1,072 & 835.0 & 402 & 370.7 \\
\hline 1988 & 39,192 & 890 & 1,106 & 804.3 & 361 & 321.3 \\
\hline 1989 & 39,209 & 816 & 1,097 & 743.2 & 403 & 328.6 \\
\hline 1990 & 39,233 & 834 & 1,118 & 745.7 & 388 & 323.2 \\
\hline 1991 & 39,233 & 848 & 1,074 & 789.9 & 386 & 327.5 \\
\hline 1992 & 39,210 & 857 & 1,090 & 785.7 & 398 & 341.0 \\
\hline 1993 & 39,064 & 790 & 1,063 & 742.7 & 389 & 307.0 \\
\hline 1994 & 39,064 & 815 & 1,093 & 745.5 & 369 & 300.7 \\
\hline 1995 & 39,641 & 808 & 1,086 & 743.6 & 374 & 302.2 \\
\hline 1996 & 41,104 & 765 & 1,093 & 699.4 & 412 & 314.9 \\
\hline 1997 & 41,419 & 707 & 1,106 & 639.5 & 415 & 293.2 \\
\hline 1998 & 42,032 & 673 & 1,087 & 619.0 & 436 & 293.1 \\
\hline 1999 & 41,766 & 656 & 1,056 & 621.1 & 457 & 299.9 \\
\hline 2000 & 41,354 & 646 & 1,064 & 606.8 & 473 & 305.6 \\
\hline 2001 & 41,588 & 622 & 1,037 & 599.7 & 444 & 276.2 \\
\hline \multicolumn{7}{|c|}{ Average annual percentage change } \\
\hline 1970-2001 & $1.5 \%$ & $0.1 \%$ & $0.3 \%$ & $-0.1 \%$ & $-0.7 \%$ & $-0.5 \%$ \\
\hline 1991-2001 & $0.6 \%$ & $-3.1 \%$ & $-0.3 \%$ & $-2.7 \%$ & $1.4 \%$ & $-1.7 \%$ \\
\hline
\end{tabular}

Source:

Number of vessels -

1970-92, 1995-2001 - U.S. Department of the Army, Corps of Engineers, "Summary of U.S. Flag

Passenger and cargo vessels, 2001," New Orleans, LA, 2002, and annual.

1993-94 - U.S. Dept of the Army, Corps of Engineers, The U.S. Waterway System-Facts,

Navigation Data Center, New Orleans, Louisiana, January 1996.

Ton-miles, tons shipped, average length of haul - U.S. Department of the Army, Corps of Engineers,

Waterborne Commerce of the United States, Calendar Year 2001 Part 5: National Summaries,

New Orleans, LA, 2002, Table 1-4, pp. 1-6, 1-7, and annual.

Energy use - See Appendix A for Water Energy Use.

(Additional resources: www.wrc-ndc.usace.army.mil/ndc)

${ }^{\mathrm{a}} \mathrm{Grand}$ total for self-propelled and non-self-propelled.

${ }^{b}$ These figures are not consistent with the figures on Table 9.3 because intra-territory tons are not included in this table. Intra-territory traffic is traffic between ports in Puerto Rico and the Virgin Islands. 
Fifty-six percent of all domestic marine cargo in 2001 were energy-related products (petroleum, coal, coke). The majority of the energy-related products were shipped internally and locally (62\%). Barge traffic accounted for $96.3 \%$ of all internal and local waterborne commerce.

Table 9.6

Breakdown of Domestic Marine Cargo by Commodity Class, 2001

\begin{tabular}{|c|c|c|c|c|c|c|c|c|c|}
\hline \multirow[b]{2}{*}{ Commodity class } & \multicolumn{2}{|c|}{ Coastwise } & \multicolumn{2}{|c|}{ Lakewise } & \multicolumn{2}{|c|}{ Internal and local } & \multicolumn{3}{|c|}{ Total domestic $^{\mathrm{a}}$} \\
\hline & $\begin{array}{c}\text { Tons } \\
\text { shipped } \\
\text { (millions) }\end{array}$ & $\begin{array}{c}\text { Average } \\
\text { haul }^{\mathrm{b}} \\
\text { (miles) }\end{array}$ & $\begin{array}{c}\text { Tons } \\
\text { shipped } \\
\text { (millions) }\end{array}$ & $\begin{array}{c}\text { Average } \\
\text { haul }^{\mathrm{b}} \\
\text { (miles) }^{\text {mile }}\end{array}$ & $\begin{array}{c}\text { Tons } \\
\text { shipped } \\
\text { (millions) }\end{array}$ & $\begin{array}{c}\text { Average } \\
\text { haul }^{\mathrm{b}} \\
\text { (miles) }\end{array}$ & $\begin{array}{c}\text { Tons } \\
\text { shipped } \\
\text { (millions) }\end{array}$ & Percentage & $\begin{array}{c}\text { Average } \\
\text { haul }^{\mathrm{b}} \\
\text { (miles) }^{\text {mile }}\end{array}$ \\
\hline Petroleum and products & 165 & 1,260 & 2 & 328 & 154 & 265 & 321 & $34.0 \%$ & 777 \\
\hline Chemicals and related products & 13 & 1,906 & c & 349 & 48 & 632 & 61 & $6.4 \%$ & 899 \\
\hline Crude materials & 11 & 503 & 75 & 507 & 116 & 408 & 202 & $21.4 \%$ & 450 \\
\hline Coal and coke & 13 & 638 & 19 & 561 & 174 & 365 & 206 & $21.8 \%$ & 400 \\
\hline Primary manufactured goods & 8 & 511 & 3 & 323 & 27 & 823 & 38 & $4.0 \%$ & 712 \\
\hline Food and farm products & 6 & 1,646 & & 981 & 90 & 1,002 & 96 & $10.2 \%$ & 1,041 \\
\hline Manufactured equipment & 9 & 1,771 & c & ${ }^{c}$ & 10 & 85 & 19 & $2.0 \%$ & 862 \\
\hline Waste and scrap & c & 0 & 0 & 0 & 1 & 198 & 1 & $0.1 \%$ & 198 \\
\hline Unknown & c & 2,167 & c & 1,000 & c & $\mathrm{c}$ & c & $0.0 \%$ & 2,135 \\
\hline Total & 224 & 1,228 & 100 & 509 & 620 & 476 & 943 & $100.0 \%$ & 658 \\
\hline Barge traffic (million tons) & 102 & & 13 & & 597 & & 712 & & \\
\hline Percentage by barge & $45.6 \%$ & & $13.4 \%$ & & $96.3 \%$ & & $75.5 \%$ & & \\
\hline
\end{tabular}

Source:

U.S. Department of the Army, Corps of Engineers, Waterborne Commerce of the United States, Calendar Year 2001, Part 5: National Summaries, New Orleans, Louisiana, 2002, Tables 2-1, 2-2, and 2-3, pp. 2-1-2-8, and annual.

Note: (Additional resources: www.wrc-ndc.usace.army.mil/ndc)

Coastwise applies to domestic traffic receiving a carriage over the ocean or between the Great Lakes ports and seacoast ports when having a carriage over the ocean. Lakewise applies to traffic between United States ports on the Great Lakes. Internal applies to traffic between ports or landings wherein the entire movement takes place on inland waterways. Local applies to movements of freight within the confines of a port.

${ }^{a}$ Does not include intra-territory tons.

${ }^{\mathrm{b}}$ Calculated as ton-miles divided by tons shipped.

${ }^{c}$ Negligible. 
According to the U.S. Coast Guard there are 4,900 more recreational boats in 2001 than in 1977. Even so, recreational boat fatalities are on the decline. There were only 5.3 fatalities per 100,000 boats in 2001.

Table 9.7

Recreational Boating Statistics, 1977-2001

\begin{tabular}{|c|c|c|c|c|}
\hline Year & $\begin{array}{l}\text { Number of } \\
\text { boats } \\
\text { (thousands) }\end{array}$ & Fatalities & $\begin{array}{c}\text { Fatalities per } \\
100,000 \\
\text { numbered boats }\end{array}$ & $\begin{array}{l}\text { Energy use }^{\mathrm{a}} \\
\text { (trillion btu) }\end{array}$ \\
\hline 1977 & 7,976 & 1,312 & 16.5 & 194.2 \\
\hline 1978 & 8,036 & 1,321 & 16.4 & 195.6 \\
\hline 1979 & 8,279 & 1,400 & 16.9 & 201.5 \\
\hline 1980 & 8,578 & 1,360 & 15.9 & 208.8 \\
\hline 1981 & 8,905 & 1,280 & 14.4 & 216.8 \\
\hline 1982 & 9,074 & 1,178 & 13.0 & 220.9 \\
\hline 1983 & 9,165 & 1,241 & 13.5 & 223.1 \\
\hline 1984 & 9,420 & 1,063 & 11.3 & 229.3 \\
\hline 1985 & 9,589 & 1,116 & 11.6 & 233.4 \\
\hline 1986 & 9,876 & 1,066 & 10.8 & 240.4 \\
\hline 1987 & 9,964 & 1,036 & 10.4 & 242.6 \\
\hline 1988 & 10,363 & 946 & 9.1 & 252.3 \\
\hline 1989 & 10,777 & 896 & 8.3 & 262.4 \\
\hline 1990 & 10,996 & 865 & 7.8 & 267.7 \\
\hline 1991 & 11,068 & 924 & 8.3 & 269.4 \\
\hline 1992 & 11,132 & 816 & 7.3 & 271.0 \\
\hline 1993 & 11,283 & 800 & 7.1 & 274.7 \\
\hline 1994 & 11,430 & 784 & 6.9 & 278.2 \\
\hline 1995 & 11,735 & 829 & 7.1 & 285.7 \\
\hline 1996 & 11,878 & 709 & 5.9 & 289.2 \\
\hline 1997 & 12,313 & 821 & 6.7 & 299.7 \\
\hline 1998 & 12,566 & 815 & 6.5 & 305.9 \\
\hline 1999 & 12,738 & 734 & 5.8 & 310.1 \\
\hline 2000 & 12,782 & 701 & 5.5 & 311.2 \\
\hline 2001 & 12,876 & 681 & 5.3 & 313.5 \\
\hline & \multicolumn{4}{|c|}{ Average annual percentage change } \\
\hline 1977-2001 & $2.0 \%$ & $-2.7 \%$ & $-4.6 \%$ & $2.0 \%$ \\
\hline $1991-2001$ & $1.5 \%$ & $-3.0 \%$ & $-4.4 \%$ & $1.5 \%$ \\
\hline
\end{tabular}

Source:

U.S. Department of Transportation, United States Coast Guard, Boating Statistics - 2001, pp. 5 and annual.

${ }^{a}$ Energy use estimated using the methodology developed by D.L. Greene in the report Off-Highway Gasoline in the United States, (DOT, FHWA, July 1986, p. 3-22) [0.95 x 205 gallons/boat x number of boats]. 
The Interstate Commerce Commission designates Class I railroads on the basis of annual gross revenues. In 2001, eight railroads were given this designation. The number of railroads designated as Class I has changed considerably in the last 25 years; in 1976 there were 52 railroads given Class I designation.

Table 9.8

Class I Railroad Freight Systems in the United States Ranked by Revenue Ton-Miles, 2001

\begin{tabular}{lcc}
\hline \multicolumn{1}{c}{ Railroad } & $\begin{array}{c}\text { Revenue ton-miles } \\
\text { (billions) }\end{array}$ & Percent \\
\hline Union Pacific Railroad Company & 504 & $33.7 \%$ \\
Burlington Northern and Sante Fe Railway Company & 502 & $33.6 \%$ \\
CSX Transportation & 228 & $15.2 \%$ \\
Norfolk Southern Corporation & 182 & $12.2 \%$ \\
Illinois Central Railroad Company & 25 & $1.7 \%$ \\
Soo Line Railroad Company & 23 & $1.5 \%$ \\
Kansas City Southern Railway Company & 20 & $1.3 \%$ \\
Grand Trunk Western Railroad Inc. & 12 & $0.8 \%$ \\
& & $\mathbf{1 0 0 . 0 \%}$ \\
Total & $\mathbf{1 , 4 9 6}$ & \\
\hline
\end{tabular}

\section{Source:}

Association of American Railroads, Railroad Facts, 2002 Edition, Washington, DC, October 2002, p. 66. (Additional resources: www.aar.org) 
Revenue ton-miles for Class I freight railroads was nearly 1.5 trillion in 2001. Though there are many regional and local freight railroads, the Class I freight railroads accounted for 92\% of the railroad industry's freight revenue in 2001 and 68\% of the industry's mileage operated. The energy intensity of Class I railroads hit an all-time low of 346 btu/ton-mile in 2001.

Table 9.9

Summary Statistics for Class I Freight Railroads, 1970-2001

\begin{tabular}{|c|c|c|c|c|c|c|c|c|c|}
\hline Year & $\begin{array}{c}\text { Number of } \\
\text { locomotives } \\
\text { in service }\end{array}$ & $\begin{array}{l}\text { Number of } \\
\text { freight cars } \\
\text { (thousands) }^{\mathrm{b}}\end{array}$ & $\begin{array}{c}\text { Train- } \\
\text { miles } \\
\text { (millions) }\end{array}$ & $\begin{array}{l}\text { Car-miles } \\
\text { (millions) }\end{array}$ & $\begin{array}{c}\text { Tons } \\
\text { originated }^{\mathrm{c}} \\
\text { (millions) }^{-}\end{array}$ & $\begin{array}{l}\text { Average } \\
\text { length of } \\
\text { haul } \\
\text { (miles) }\end{array}$ & $\begin{array}{l}\text { Revenue } \\
\text { ton-miles } \\
\text { (millions) }\end{array}$ & $\begin{array}{l}\text { Energy } \\
\text { intensity } \\
\text { (Btu/ton- } \\
\text { mile) }\end{array}$ & $\begin{array}{c}\text { Energy } \\
\text { use } \\
\text { (trillion } \\
\text { Btu) } \\
\end{array}$ \\
\hline 1970 & $27,077^{\mathrm{d}}$ & 1,424 & 427 & 29,890 & 1,485 & 515 & 764,809 & 691 & 528.1 \\
\hline 1975 & 27,846 & 1,359 & 403 & 27,656 & 1,395 & 541 & 754,252 & 687 & 518.3 \\
\hline 1980 & 28,094 & 1,168 & 428 & 29,277 & 1,492 & 616 & 918,958 & 597 & 548.7 \\
\hline 1981 & 27,421 & 1,111 & 408 & 27,968 & 1,453 & 626 & 910,169 & 572 & 521.0 \\
\hline 1982 & 26,795 & 1,039 & 345 & 23,952 & 1,269 & 629 & 797,759 & 553 & 440.8 \\
\hline 1983 & 25,448 & 1,007 & 346 & 24,358 & 1,293 & 641 & 828,275 & 525 & 435.1 \\
\hline 1984 & 24,117 & 948 & 369 & 26,409 & 1,429 & 645 & 921,542 & 510 & 469.9 \\
\hline 1985 & 22,548 & 867 & 347 & 24,920 & 1,320 & 665 & 876,984 & 497 & 436.1 \\
\hline 1986 & 20,790 & 799 & 347 & 24,414 & 1,306 & 664 & 867,722 & 486 & 421.5 \\
\hline 1987 & 19,647 & 749 & 361 & 25,627 & 1,372 & 688 & 943,747 & 456 & 430.3 \\
\hline 1988 & 19,364 & 725 & 379 & 26,339 & 1,430 & 697 & 996,182 & 443 & 441.4 \\
\hline 1989 & 19,015 & 682 & 383 & 26,196 & 1,403 & 723 & $1,013,841$ & 437 & 442.6 \\
\hline 1990 & 18,835 & 659 & 380 & 26,159 & 1,425 & 726 & $1,033,969$ & 420 & 434.7 \\
\hline 1991 & 18,344 & 633 & 375 & 25,628 & 1,383 & 751 & $1,038,875$ & 391 & 405.8 \\
\hline 1992 & 18,004 & 605 & 390 & 26,128 & 1,399 & 763 & $1,066,781$ & 393 & 419.2 \\
\hline 1993 & 18,161 & 587 & 405 & 26,883 & 1,397 & 794 & $1,109,309$ & 389 & 431.6 \\
\hline 1994 & 18,505 & 591 & 441 & 28,485 & 1,470 & 817 & $1,200,701$ & 388 & 465.4 \\
\hline 1995 & 18,812 & 583 & 458 & 30,383 & 1,550 & 843 & $1,305,688$ & 372 & 485.9 \\
\hline 1996 & 19,269 & 571 & 469 & 31,715 & 1,611 & 842 & $1,355,975$ & 368 & 499.4 \\
\hline 1997 & 19,684 & 568 & 475 & 31,660 & 1,585 & 851 & $1,348,926$ & 370 & 499.7 \\
\hline 1998 & 20,261 & 576 & 475 & 32,657 & 1,649 & 835 & $1,376,802$ & 365 & 502.0 \\
\hline 1999 & 20,256 & 579 & 490 & 33,851 & 1,717 & 835 & $1,433,461$ & 363 & 520.0 \\
\hline 2000 & 20,028 & 560 & 504 & 34,590 & 1,738 & 843 & $1,465,960$ & 352 & 516.0 \\
\hline 2001 & 19,745 & 500 & 500 & 34,243 & 1,742 & 859 & $1,495,472$ & 346 & 517.3 \\
\hline & \multicolumn{9}{|c|}{ Average annual percentage change } \\
\hline 1970-2001 & $-1.0 \%$ & $-3.3 \%$ & $0.5 \%$ & $0.4 \%$ & $0.5 \%$ & $1.7 \%$ & $2.1 \%$ & $-2.2 \%$ & $-0.1 \%$ \\
\hline $1991-2001$ & $0.7 \%$ & $-2.3 \%$ & $2.9 \%$ & $2.9 \%$ & $2.3 \%$ & $1.4 \%$ & $3.7 \%$ & $-1.2 \%$ & $2.5 \%$ \\
\hline
\end{tabular}

Source:

Association of American Railroads, Railroad Facts, 2002 Edition, Washington, DC, October 2002, pp. 27, 28, 33, 34, 36, 49 , 51, 61. (Additional resources: www.aar.org)

${ }^{\mathrm{a} D}$ Does not include self-powered units.

${ }^{\mathrm{b}}$ Does not include private or shipper-owned cars.

${ }^{c}$ Tons originated is a more accurate representation of total tonnage than revenue tons. Revenue tons often produces double-counting of loads switched between rail companies.

${ }^{\mathrm{d}}$ Data represent total locomotives used in freight and passenger service. Separate estimates are not available. 
The "other" category, which consists primarily of intermodal traffic, has grown $146 \%$ in carloads from 1974 to 2001. Coal now accounts for more than one quarter of all carloads.

Table 9.10

Railroad Revenue Carloads by Commodity Group, 1974 and 2001

\begin{tabular}{lrrrrrrr}
\hline & \multicolumn{2}{c}{$\begin{array}{c}\text { Carloads } \\
\text { (thousands) }\end{array}$} & & Percent distribution & $\begin{array}{c}\text { Percentage } \\
\text { change }\end{array}$ \\
\cline { 2 - 3 } Commodity group & 1974 & 2001 & & 1974 & 2001 & $1974-2001$ \\
\hline Coal & 4,544 & 7,295 & & $17.0 \%$ & $26.8 \%$ & $60.5 \%$ \\
Farm products & 3,021 & 1,461 & & $11.3 \%$ & $5.4 \%$ & $-51.6 \%$ \\
Chemicals and allied products & 1,464 & 1,801 & & $5.5 \%$ & $6.6 \%$ & $23.0 \%$ \\
Nonmetallic minerals & 821 & 1,280 & & $3.1 \%$ & $4.7 \%$ & $55.9 \%$ \\
Food and kindred products & 1,777 & 1,446 & & $6.6 \%$ & $5.3 \%$ & $-18.6 \%$ \\
Lumber and wood products & 1,930 & 603 & & $7.2 \%$ & $2.2 \%$ & $-68.8 \%$ \\
Metallic ores & 1,910 & 251 & & $7.1 \%$ & $0.9 \%$ & $-86.9 \%$ \\
Stone, clay and glass & 2,428 & 528 & & $9.1 \%$ & $1.9 \%$ & $-78.3 \%$ \\
Pulp, paper, and allied products & 1,180 & 601 & & $4.4 \%$ & $2.2 \%$ & $-49.1 \%$ \\
Petroleum products & 877 & 523 & & $3.3 \%$ & $1.9 \%$ & $-40.4 \%$ \\
Primary metal products & 1,366 & 692 & & $5.1 \%$ & $2.5 \%$ & $-49.3 \%$ \\
Waste and scrap material & 889 & 591 & & $3.3 \%$ & $2.2 \%$ & $-33.5 \%$ \\
Transportation equipment & 1,126 & 1,650 & & $4.2 \%$ & $6.1 \%$ & $46.5 \%$ \\
Others & 3,451 & 8,483 & & $12.9 \%$ & $31.2 \%$ & $145.8 \%$ \\
& & & & & \\
Total & 26,784 & 27,205 & & $100.0 \%$ & $100.0 \%$ & $1.6 \%$ \\
\hline
\end{tabular}

Source:

1974 - Association of American Railroads, Railroad Facts, 1976 Edition, Washington, DC, 1975, p. 26.

2001 - Association of American Railroads, Railroad Facts, 2002 Edition, Washington, DC,

October 2002, p. 25.

((Additional resources: www.aar.org) 
According to the 1997 Commodity Flow Survey, 5\% of all freight ton-miles are rail intermodal shipments (truck/rail or rail/water). See Table 5.11 for details. The number of trailers and containers moved by railroads has increased more than five-fold from 1965 to 2001. Containerization has increased in recent years, evidenced by the $175 \%$ increase in the number of containers from 1988 to 2001.

Table 9.11

Intermodal Rail Traffic, 1965-2001

\begin{tabular}{cccc}
\hline Year & $\begin{array}{c}\text { Trailers \& } \\
\text { containers }\end{array}$ & Trailers & Containers \\
\hline 1965 & $1,664,929$ & $\mathrm{a}$ & $\mathrm{a}$ \\
\hdashline 1970 & $2,363,200$ & $\mathrm{a}$ & $\mathrm{a}$ \\
1975 & $2,238,117$ & $\mathrm{a}$ & $\mathrm{a}$ \\
\hdashline 1980 & $3,059,402$ & $\mathrm{a}$ & $\mathrm{a}$ \\
\hdashline 1985 & $4,590,952$ & $\mathrm{a}$ & $\ldots \ldots \ldots$ \\
1986 & $4,997,229$ & $\mathrm{a}$ & $\mathrm{a}$ \\
1987 & $5,503,819$ & $\mathrm{a}$ & $\mathrm{a}$ \\
1988 & $5,779,547$ & $3,481,020$ & $2,298,527$ \\
1989 & $5,987,355$ & $3,496,262$ & $2,491,093$ \\
1990 & $6,206,782$ & $3,451,953$ & $2,754,829$ \\
1991 & $6,246,134$ & $3,201,560$ & $3,044,574$ \\
1992 & $6,627,841$ & $3,264,597$ & $3,363,244$ \\
1993 & $7,156,628$ & $3,464,126$ & $3,692,502$ \\
1994 & $8,128,228$ & $3,752,502$ & $4,375,726$ \\
$1995^{\mathrm{b}}$ & $7,936,172$ & $3,492,463$ & $4,443,709$ \\
$1996^{\mathrm{b}}$ & $8,143,258$ & $3,302,128$ & $4,841,130$ \\
$1997^{\mathrm{b}}$ & $8,698,308$ & $3,453,907$ & $5,244,401$ \\
$1998^{\mathrm{b}}$ & $8,772,663$ & $3,353,032$ & $5,419,631$ \\
$1999^{\mathrm{c}}$ & $8,907,626$ & $3,207,407$ & $5,700,219$ \\
$2000^{\mathrm{c}}$ & $9,176,890$ & $2,888,630$ & $6,288,260$ \\
2001 & $8,935,444$ & $2,603,423$ & $6,332,021$ \\
$1965-2001$ & $4.8 \%$ & $\mathrm{a}$ & $\mathrm{a}$ \\
\hline $1991-2001$ & $3.6 \%$ & $-2.0 \%$ & $7.6 \%$ \\
\hline
\end{tabular}

Source:

Association of American Railroads, Railroad Facts, 2002 edition, Washington, DC, October 2002 p. 26. (Additional resources: www.aar.org)

\footnotetext{
${ }^{\mathrm{a}}$ Data are not available.

${ }^{\mathrm{b}}$ The Grand Trunk Western Railroad and the Soo Line Railroad Company data are excluded.

${ }^{c}$ The Illinois Central, Grand Trunk Western Railroad and the Soo Line Railroad Company data are excluded.
} 
The National Railroad Passenger Corporation, known as Amtrak, began operation in 1971. Though Amtrak revenue passenger-miles have grown at an average annual rate of 3.5\% from 1971 to 2001, they showed a small decline in annual percentage change from 1991 to 2001.

Table 9.12

Summary Statistics for the National Railroad Passenger Corporation (Amtrak), 1971-2001

\begin{tabular}{|c|c|c|c|c|c|c|c|c|}
\hline Year & $\begin{array}{l}\text { Number of } \\
\text { locomotives } \\
\text { in service } \\
\end{array}$ & $\begin{array}{c}\text { Number of } \\
\text { passenger } \\
\text { cars }\end{array}$ & $\begin{array}{l}\text { Train-miles } \\
\text { (thousands) }\end{array}$ & $\begin{array}{c}\text { Car-miles } \\
\text { (thousands) }\end{array}$ & $\begin{array}{l}\text { Revenue } \\
\text { passenger- } \\
\text { miles } \\
\text { (millions) } \\
\end{array}$ & $\begin{array}{l}\text { Average } \\
\text { trip length } \\
\text { (miles) } \\
\end{array}$ & $\begin{array}{l}\text { Energy intensity } \\
\text { (Btu per revenue } \\
\text { passenger-mile) }\end{array}$ & $\begin{array}{c}\text { Energy } \\
\text { use } \\
\text { (trillion } \\
\text { Btu) } \\
\end{array}$ \\
\hline 1971 & $\begin{array}{l}\text { a } \\
\ldots \ldots . . .\end{array}$ & 1,165 & 16,537 & 140,147 & 1,993 & 188 & a & a \\
\hline 1975 & 355 & 1,913 & 30,166 & 253,898 & 3,753 & 224 & 3,677 & 13.8 \\
\hline 1980 & 448 & 2,128 & 29,487 & 235,235 & 4,503 & 217 & 3,176 & 14.3 \\
\hline 1981 & 398 & 1,830 & 30,380 & 222,753 & 4,397 & 226 & 2,979 & 13.1 \\
\hline 1982 & 396 & 1,929 & 28,833 & 217,385 & 3,993 & 220 & 3,156 & 12.6 \\
\hline 1983 & 388 & 1,880 & 28,805 & 223,509 & 4,227 & 223 & 2,957 & 12.5 \\
\hline 1984 & 387 & 1,844 & 29,133 & 234,557 & 4,427 & 227 & 3,027 & 13.4 \\
\hline 1985 & 382 & 1,818 & 30,038 & 250,642 & 4,785 & 238 & 2,800 & 13.4 \\
\hline 1986 & 369 & 1,793 & 28,604 & 249,665 & 5,011 & 249 & 2,574 & 12.9 \\
\hline 1987 & 381 & 1,850 & 29,515 & 261,054 & 5,361 & 259 & 2,537 & 13.6 \\
\hline 1988 & 391 & 1,845 & 30,221 & 277,774 & 5,686 & 265 & 2,462 & 14.0 \\
\hline 1989 & 312 & 1,742 & 31,000 & 285,255 & 5,859 & 274 & 2,731 & 16.0 \\
\hline 1990 & 318 & 1,863 & 33,000 & 300,996 & 6,057 & 273 & 2,609 & 15.8 \\
\hline 1991 & 316 & 1,786 & 34,000 & 312,484 & 6,273 & 285 & 2,503 & 15.7 \\
\hline 1992 & 336 & 1,796 & 34,000 & 307,282 & 6,091 & 286 & 2,610 & 15.9 \\
\hline 1993 & 360 & 1,853 & 34,936 & 302,739 & 6,199 & 280 & 2,646 & 16.4 \\
\hline 1994 & 411 & 1,874 & 34,940 & 305,600 & 5,869 & 276 & 2,357 & $13.8^{\mathrm{b}}$ \\
\hline 1995 & 422 & 1,907 & 31,579 & 282,579 & 5,401 & 266 & 2,590 & 14.0 \\
\hline 1996 & 348 & 1,501 & 30,542 & 277,750 & 5,066 & 257 & 2,792 & 14.1 \\
\hline 1997 & 292 & 1,572 & 32,000 & 287,760 & 5,166 & 255 & 2,918 & 15.1 \\
\hline 1998 & 362 & 1,347 & 32,926 & 315,823 & 5,325 & 251 & 2,900 & 15.4 \\
\hline 1999 & 385 & 1,285 & 34,080 & 349,337 & 5,289 & 245 & 3,062 & 16.2 \\
\hline 2000 & 385 & 1,891 & 35,404 & 371,215 & 5,574 & 243 & 3,356 & 18.7 \\
\hline 2001 & 401 & 2,084 & 36,512 & 377,705 & 5,571 & 238 & 4,137 & 23.0 \\
\hline \multicolumn{9}{|c|}{ Average annual percentage change } \\
\hline 1971-2001 & a & $2.0 \%$ & $2.7 \%$ & $3.4 \%$ & $3.5 \%$ & $0.8 \%$ & a & a \\
\hline 1991-2001 & $2.4 \%$ & $1.6 \%$ & $0.7 \%$ & $1.9 \%$ & $-1.2 \%$ & $-1.8 \%$ & $5.2 \%$ & $3.9 \%$ \\
\hline
\end{tabular}

Source:

1971-83- Association of American Railroads, Economics and Finance Department, Statistics of Class I Railroads, Washington, DC, and annual.

1984-88- Association of American Railroads, Railroad Facts, 1988 Edition, Washington, DC, December 1989, p. 61, and annual. 1989-93- Personal communication with the Corporate Accounting Office of Amtrak, Washington, D.C.

1994-2001 - Number of locomotives in service, number of passenger cars, train-miles, car-miles, revenue passenger-miles, and average trip length - Association of American Railroads, Railroad Facts, 2002 Edition, Washington, DC, 2002, p. 77.

Energy use - Personal communication with the Amtrak, Washington, DC.

(Additional resources: www.amtrak.com, www.aar.org)

\footnotetext{
${ }^{\text {a }}$ Data are not available.

${ }^{\mathrm{b}}$ Energy use for 1994 on is not directly comparable to earlier years. Some commuter rail energy use may have been inadvertently included in earlier years.
} 
Commuter rail, which is also known as regional rail or suburban rail, is long-haul rail passenger service operating between metropolitan and suburban areas, whether within or across state lines. Commuter rail lines usually have reduced fares for multiple rides and commutation tickets for regular, recurring riders. In 2001, commuter rail operations showed higher vehicle-miles, passenger-miles, and passenger trips than any other year in this series.

Table 9.13

Summary Statistics for Commuter Rail Operations, 1984-2001

\begin{tabular}{|c|c|c|c|c|c|c|c|}
\hline Year & $\begin{array}{c}\text { Number of } \\
\text { passenger } \\
\text { vehicles }\end{array}$ & $\begin{array}{l}\text { Vehicle- } \\
\text { miles } \\
\text { (millions) }\end{array}$ & $\begin{array}{l}\text { Passenger } \\
\text { trips } \\
\text { (millions) }\end{array}$ & $\begin{array}{l}\text { Passenger- } \\
\text { miles } \\
\text { (millions) }\end{array}$ & $\begin{array}{l}\text { Average } \\
\text { trip length } \\
\text { (miles) }\end{array}$ & $\begin{array}{l}\text { Energy } \\
\text { intensity } \\
\text { (Btu/ } \\
\text { passenger- } \\
\text { mile) }\end{array}$ & $\begin{array}{l}\text { Energy use } \\
\text { (trillion Btu) }\end{array}$ \\
\hline 1984 & 4,075 & 167.9 & 267 & 6,207 & 23.2 & 3,011 & 18.7 \\
\hline 1985 & 4,035 & 182.7 & 275 & 6,534 & 23.8 & 3,053 & 20.0 \\
\hline 1986 & 4,440 & 188.6 & 306 & 6,723 & 22.0 & 3,174 & 21.3 \\
\hline 1987 & 4,686 & 188.9 & 311 & 6,818 & 21.9 & 3,043 & 20.7 \\
\hline 1988 & 4,649 & 202.2 & 325 & 6,964 & 21.4 & 3,075 & 21.4 \\
\hline 1989 & 4,472 & 209.6 & 330 & 7,211 & 21.9 & 3,120 & 22.5 \\
\hline 1990 & 4,415 & 212.7 & 328 & 7,082 & 21.6 & 3,068 & 21.7 \\
\hline 1991 & 4,370 & 214.9 & 318 & 7,344 & 23.1 & 3,011 & 22.1 \\
\hline 1992 & 4,413 & 218.8 & 314 & 7,320 & 23.3 & 2,848 & 20.8 \\
\hline 1993 & 4,494 & 223.9 & 322 & 6,940 & 21.6 & 3,222 & 22.4 \\
\hline 1994 & 4,517 & 230.8 & 339 & 7,996 & 23.6 & 2,904 & 23.2 \\
\hline 1995 & 4,565 & 237.7 & 344 & 8,244 & 24.0 & 2,849 & 23.5 \\
\hline 1996 & 4,665 & 241.9 & 352 & 8,351 & 23.7 & 2,796 & 23.3 \\
\hline 1997 & 4,943 & 250.7 & 357 & 8,038 & 22.5 & 2,949 & 23.7 \\
\hline 1998 & 4,963 & 259.5 & 381 & 8,704 & 22.8 & 2,859 & 24.9 \\
\hline 1999 & 4,883 & 265.9 & 396 & 8,766 & 22.1 & 2,929 & 25.7 \\
\hline 2000 & 5,073 & 270.9 & 413 & 9,402 & 22.8 & 2,759 & 25.9 \\
\hline \multirow[t]{2}{*}{2001} & 5,124 & 277.3 & 419 & 9,548 & 22.8 & 2,717 & 25.9 \\
\hline & \multicolumn{7}{|c|}{ Average annual percentage change } \\
\hline 1984-2001 & $1.4 \%$ & $3.0 \%$ & $2.7 \%$ & $2.6 \%$ & $-0.1 \%$ & $-0.6 \%$ & $1.9 \%$ \\
\hline 1991-2001 & $1.6 \%$ & $2.6 \%$ & $2.8 \%$ & $2.7 \%$ & $-0.1 \%$ & $-1.0 \%$ & $1.6 \%$ \\
\hline
\end{tabular}

Source:

American Public Transportation Association, 2003 Public Transportation Fact Book, Washington, DC, February 2003, pp. 31, 32, 37, 41, and 46. (Additional resources: www.apta.com) 
This table on transit rail operations includes data on light rail and heavy rail systems. Light rail vehicles are usually single vehicles driven electrically with power drawn from overhead wires. Heavy rail is characterized by high speed and rapid acceleration of rail cars operating on a separate right-of-way. Transit rail operations were hitting all-time highs in vehicle-miles, passenger-miles, and passenger trips in 2001.

Table 9.14

Summary Statistics for Rail Transit Operations, 1970-2001

\begin{tabular}{|c|c|c|c|c|c|c|c|}
\hline Year & $\begin{array}{c}\text { Number of } \\
\text { passenger } \\
\text { vehicles }\end{array}$ & $\begin{array}{l}\text { Vehicle- } \\
\text { miles } \\
\text { (millions) }\end{array}$ & $\begin{array}{l}\text { Passenger } \\
\text { trips } \\
\text { (millions) }^{\mathrm{b}}\end{array}$ & $\begin{array}{c}\text { Passenger-miles } \\
\left(^{(m i l l i o n s}\right)^{c}\end{array}$ & $\begin{array}{l}\text { Average trip } \\
\text { length } \\
(\text { miles })^{\mathrm{d}}\end{array}$ & $\begin{array}{l}\text { Energy intensity } \\
(\text { Btu/ } \\
\text { passenger-mile) }^{\mathrm{e}}\end{array}$ & $\begin{array}{l}\text { Energy use } \\
\text { (trillion Btu) }\end{array}$ \\
\hline 1970 & 10,548 & 440.8 & 2,116 & 12,273 & $\mathrm{f}$ & 2,453 & 30.1 \\
\hline 1975 & 10,617 & 446.9 & 1,797 & 10,423 & f & 2,962 & 31.1 \\
\hline 1980 & 10,654 & 402.2 & 2,241 & 10,939 & 4.9 & 3,008 & 32.9 \\
\hline 1981 & 10,824 & 436.6 & 2,217 & 10,590 & 4.8 & 2,946 & 31.2 \\
\hline 1982 & 10,831 & 445.2 & 2,201 & 10,428 & 4.7 & 3,069 & 32.0 \\
\hline 1983 & 10,904 & 423.5 & 2,304 & 10,741 & 4.7 & 3,212 & 34.5 \\
\hline 1984 & 10,848 & 452.7 & 2,388 & 10,531 & 4.4 & 3,732 & 39.3 \\
\hline 1985 & 11,109 & 467.8 & 2,422 & 10,777 & 4.4 & 3,461 & 37.3 \\
\hline 1986 & 11,083 & 492.8 & 2,467 & 11,018 & 4.5 & 3,531 & 38.9 \\
\hline 1987 & 10,934 & 508.6 & 2,535 & 11,603 & 4.6 & 3,534 & 41.0 \\
\hline 1988 & 11,370 & 538.3 & 2,462 & 11,836 & 4.8 & 3,565 & 42.2 \\
\hline 1989 & 11,261 & 553.4 & 2,704 & 12,539 & 4.6 & 3,397 & 42.6 \\
\hline 1990 & 11,332 & 560.9 & 2,521 & 12,046 & 4.8 & 3,453 & 41.6 \\
\hline 1991 & 11,426 & 554.8 & 2,356 & 11,190 & 4.7 & 3,727 & 41.7 \\
\hline 1992 & 11,303 & 554.0 & 2,395 & 11,438 & 4.8 & 3,575 & 40.9 \\
\hline 1993 & 11,286 & 549.8 & 2,234 & 10,936 & 4.9 & 3,687 & 42.2 \\
\hline 1994 & 11,192 & 565.8 & 2,453 & 11,501 & 4.7 & 3,828 & 44.0 \\
\hline 1995 & 11,156 & 571.8 & 2,284 & 11,419 & 5.0 & 3,818 & 43.6 \\
\hline 1996 & 11,341 & 580.7 & 2,418 & 12,487 & 5.2 & 3,444 & 43.0 \\
\hline 1997 & 11,471 & 598.9 & 2,692 & 13,091 & 4.9 & 3,253 & 42.6 \\
\hline 1998 & 11,521 & 609.5 & 2,669 & 13,412 & 5.0 & 3,216 & 43.1 \\
\hline 1999 & 11,603 & 626.4 & 2,813 & 14,108 & 5.0 & 3,168 & 44.7 \\
\hline 2000 & 12,168 & 648.0 & 2,952 & 15,200 & 5.1 & 3,105 & 47.2 \\
\hline 2001 & 12,084 & 662.4 & 3,064 & 15,615 & 5.1 & 3,114 & 48.6 \\
\hline \multicolumn{8}{|c|}{ Average annual percentage change } \\
\hline 1970-2001 & $0.6 \%$ & $2.4 \%$ & $1.5 \%$ & $1.7 \%$ & $0.2 \%^{\mathrm{g}}$ & $0.8 \%$ & $1.6 \%$ \\
\hline 1991-2001 & $0.6 \%$ & $1.8 \%$ & $2.7 \%$ & $3.4 \%$ & $0.8 \%$ & $-1.8 \%$ & $1.5 \%$ \\
\hline
\end{tabular}

Source:

American Public Transit Association, 2003 Public Transportation Fact Book, Washington, DC, February 2003, pp. 31, 32 , 41, and 46. (Additional resources: www.apta.com)

Energy use - See Appendix A for Rail Transit Energy Use.

${ }^{a}$ Heavy rail and light rail. Series not continuous between 1983 and 1984 because of a change in data source by the American Public Transit Association (APTA). Beginning in 1984, data provided by APTA are taken from mandatory reports filed with the Urban Mass Transit Administration (UMTA). Data for prior years were provided on a voluntary basis by APTA members and expanded statistically.

${ }^{\mathrm{b}}$ 1970-79 data represents total passenger rides; after 1979, data represents unlinked passenger trips.

'Estimated for years 1970-76 based on an average trip length of 5.8 miles.

${ }^{\mathrm{d}}$ Calculated as the ratio of passenger-miles to passenger trips.

${ }^{\mathrm{e}}$ Large system-to-system variations exist within this category.

fData are not available.

${ }^{g}$ Average annual percentage change is calculated for years 1980-2001. 


\section{Chapter 10}

\section{Transportation and the Economy}

Summary Statistics from Tables/Figures in this Chapter

Source

Figure 10.1 Share of gasoline cost attributed to taxes, 2002

Canada

France

Germany

Japan

United Kingdom

United States

Table 10.11 Average price of a new car, 2001 (current dollars)

Domestic

Import

Table 10.12 Automobile operating costs, 2002

Variable costs (constant 2002 dollars per 10,000 miles)

Fixed costs (constant 2002 dollars per 10,000 miles)

Table 10.18 Transportation sector share of total employment

1960

1980

2001 1,180

$41 \%$

$73 \%$

$73 \%$

$57 \%$

$77 \%$

$27 \%$

21,605

19,654

27,477

$$
4,874
$$

$13.5 \%$

$11.4 \%$

$11.0 \%$ 
Table 10.1

Gasoline Prices for Selected Countries, 1978-2002

\begin{tabular}{|c|c|c|c|c|c|c|c|c|c|c|}
\hline & \multicolumn{8}{|c|}{ Current dollars per gallon } & \multicolumn{2}{|c|}{$\begin{array}{l}\text { Average annual } \\
\text { percentage change }\end{array}$} \\
\hline & $1978^{\mathrm{a}}$ & $1982^{\mathrm{a}}$ & $1986^{\mathrm{a}}$ & $1990^{\mathrm{b}}$ & $1994^{\mathrm{b}}$ & $1996^{\mathrm{b}}$ & $2000^{\mathrm{b}}$ & $2002^{\mathrm{b}}$ & 1978-2002 & 1990-2002 \\
\hline China & c & c & c & $\mathrm{c}$ & c & 0.93 & 1.21 & c & $\mathrm{c}$ & c \\
\hline India & $\mathrm{c}$ & $\mathrm{c}$ & $\mathrm{c}$ & 1.92 & 2.28 & 2.25 & $\mathrm{c}$ & c & c & c \\
\hline Japan & 2.00 & 2.60 & 2.79 & 3.05 & 4.14 & 3.77 & 3.65 & 2.94 & $1.6 \%$ & $-0.3 \%$ \\
\hline France & 2.15 & 2.56 & 2.58 & 3.40 & 3.31 & 4.41 & 4.01 & 3.31 & $1.8 \%$ & $-0.2 \%$ \\
\hline United Kingdom & 1.22 & 2.42 & 2.07 & 2.55 & 2.86 & 3.47 & 5.13 & 4.16 & $5.2 \%$ & $4.2 \%$ \\
\hline Germany & 1.75 & 2.17 & 1.88 & 2.72 & 3.34 & 4.32 & 3.78 & 3.49 & $2.9 \%$ & $2.1 \%$ \\
\hline Canada & 0.69 & 1.37 & 1.31 & 1.92 & 1.57 & 1.80 & 2.04 & 1.73 & $3.9 \%$ & $-0.9 \%$ \\
\hline \multirow[t]{3}{*}{ United States $^{\mathrm{d}}$} & 0.66 & 1.32 & 0.93 & 1.04 & 1.24 & 1.28 & 1.47 & 1.29 & $2.8 \%$ & $1.8 \%$ \\
\hline & \multicolumn{8}{|c|}{ Constant 2002 dollars $^{\mathrm{e}}$ per gallon } & \multicolumn{2}{|c|}{$\begin{array}{l}\text { Average annual } \\
\text { percentage change }\end{array}$} \\
\hline & $1978^{\mathrm{a}}$ & $1982^{\mathrm{a}}$ & $1986^{\mathrm{a}}$ & $1990^{b}$ & $1994^{\mathrm{b}}$ & $1996^{\mathrm{b}}$ & $2000^{\mathrm{b}}$ & $2002^{\mathrm{b}}$ & 1978-2002 & 1990-2002 \\
\hline China & $\mathrm{c}$ & $\mathrm{c}$ & $\mathrm{c}$ & $\mathrm{c}$ & $\mathrm{c}$ & 1.07 & 1.26 & c & $\mathrm{c}$ & $\mathrm{c}$ \\
\hline India & $\mathrm{c}$ & c & c & 2.64 & 2.77 & 2.58 & $\mathrm{c}$ & c & $\mathrm{c}$ & $\mathrm{c}$ \\
\hline Japan & 5.52 & 4.85 & 4.58 & 4.20 & 5.03 & 4.32 & 3.81 & 2.94 & $-2.6 \%$ & $-2.9 \%$ \\
\hline France & 5.93 & 4.77 & 4.23 & 4.68 & 4.02 & 5.06 & 4.19 & 3.31 & $-2.4 \%$ & $-2.8 \%$ \\
\hline United Kingdom & 3.37 & 4.51 & 3.40 & 3.51 & 3.47 & 3.98 & 5.36 & 4.16 & $0.9 \%$ & $1.4 \%$ \\
\hline Germany & 4.83 & 4.05 & 3.09 & 3.74 & 4.05 & 4.95 & 3.95 & 3.49 & $-1.3 \%$ & $-0.6 \%$ \\
\hline Canada & 1.90 & 2.55 & 2.15 & 2.64 & 1.91 & 2.06 & 2.13 & 1.73 & $-0.4 \%$ & $-3.5 \%$ \\
\hline United States $^{\mathrm{d}}$ & 1.82 & 2.46 & 1.53 & 1.43 & 1.51 & 1.47 & 1.54 & 1.29 & $-1.4 \%$ & $-0.9 \%$ \\
\hline
\end{tabular}

Source:

U.S. Department of Energy, Energy Information Administration, International Energy Annual 2001, Washington, DC, March 2003, Table 7.2 and annual.

(Additional resources: ww.eia.doe.gov)

Comparisons between prices and price trends in different countries require care. They are of limited validity because of fluctuations in exchange rates; differences in product quality, marketing practices, and market structures; and the extent to which the standard categories of sales are representative of total national sales for a given period.

${ }^{a}$ Prices represent the retail prices (including taxes) for premium leaded gasoline. Prices are representative for each country based on quarterly data averaged for the year.

${ }^{\mathrm{b}}$ Regular gasoline

Data are not available.

${ }^{d}$ These estimates are for international comparisons only and do not necessarily correspond to gasoline price estimates in other sections of the book.

${ }^{\mathrm{e}}$ Adjusted by the U.S. Consumer Price Inflation Index. 
In 2002 more than seventy percent of the cost of gasoline in France, Germany, and the United Kingdom went for taxes. Of the listed countries, the U.S. has the lowest percentage of taxes.

Figure 10.1. Gasoline Prices for Selected Countries, 1990 and 2002

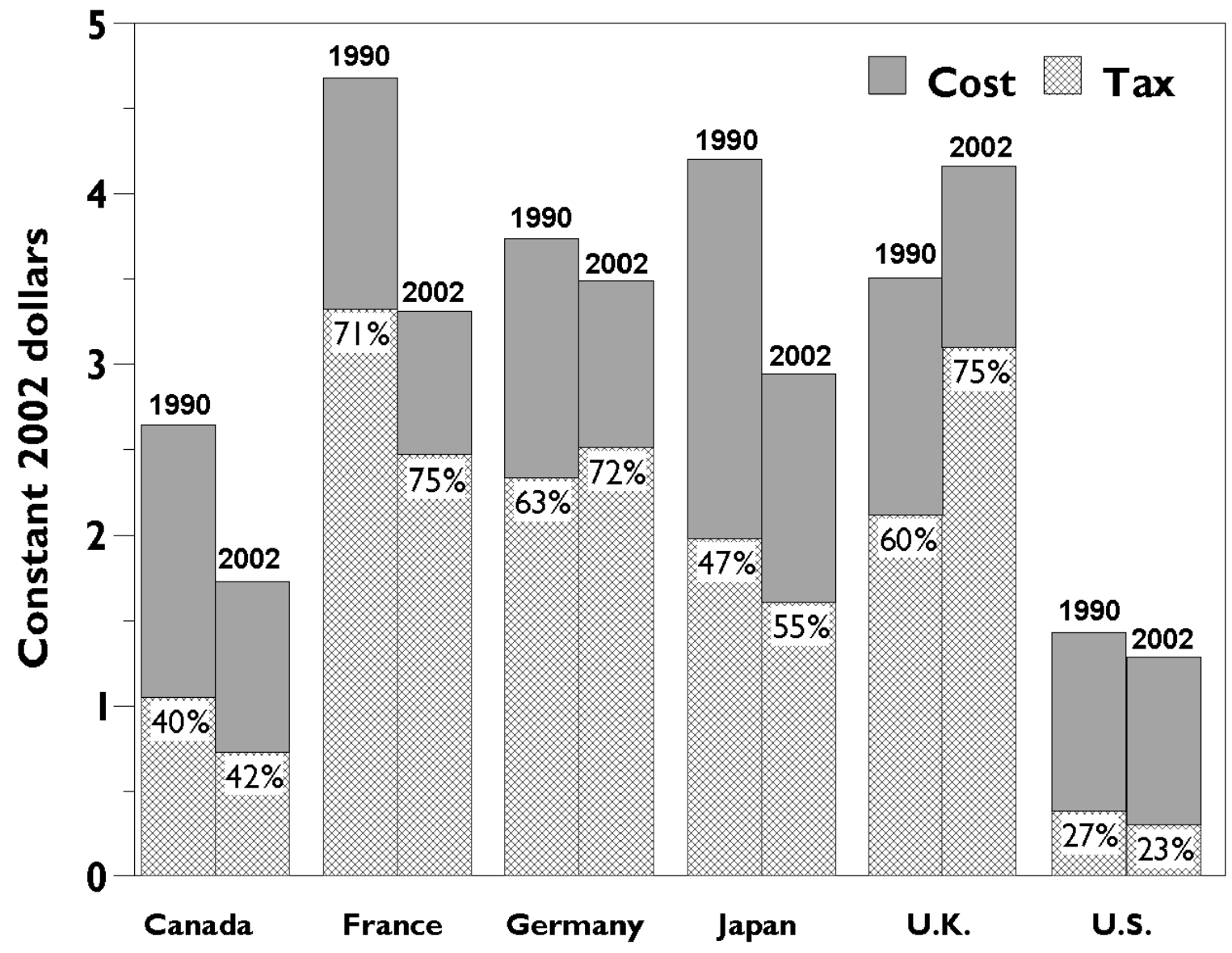

Source:

Table 10.1 and International Energy Agency, Energy Prices and Taxes, Fourth Quarter 2002, Paris, France, 2003. (Additional resources: www.iea.org) 
Table 10.2

Diesel Fuel Prices for Selected Countries, 1978-2002

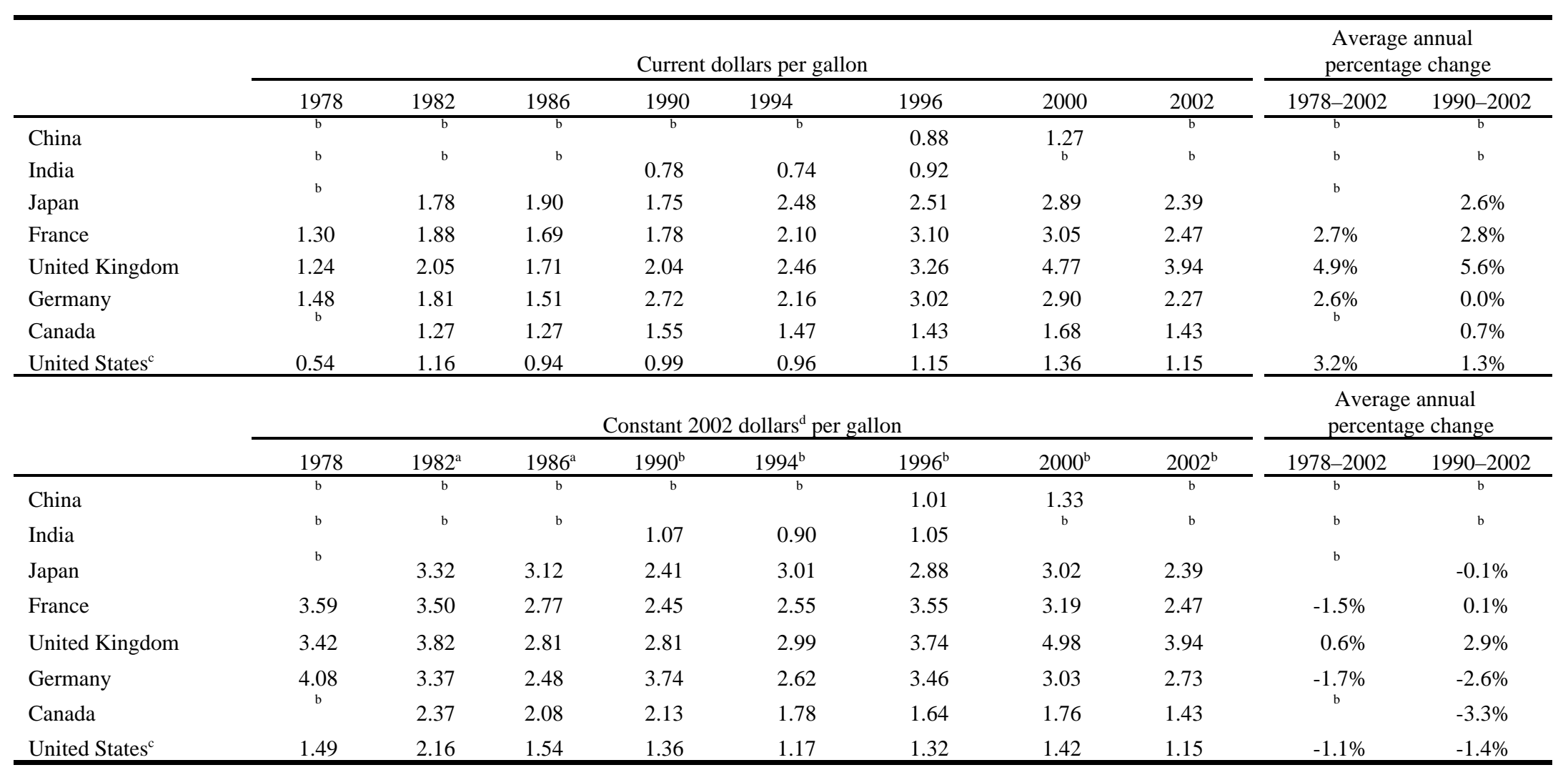

Source:

U.S. Department of Energy, Energy Information Administration, International Energy Annual 2001, Washington, DC, March 2003, Table 7.2 and annual.

Note: (Additional resources: www.eia.doe.gov)

Comparisons between prices and price trends in different countries require care. They are of limited validity because of fluctuations in exchange rates; differences in product quality, marketing practices, and market structures; and the extent to which the standard categories of sales are representative of total national sales for a given period.

\footnotetext{
a Prices represent the retail prices (including taxes) for diesel fuel. Prices are representative for each country based on quarterly data averaged for the year or on data as of January 1. ${ }^{6}$ Data are not available.

These estimates are for international comparisons only and do not necessarily correspond to gasoline price estimates in other sections of the book.

${ }^{\mathrm{d}}$ Adjusted by the U.S. Consumer Price Inflation Index.
} 
Diesel fuel is taxed heavily in the European countries shown here. The U.S. diesel fuel tax share is the lowest of the listed countries.

Figure 10.2. Diesel Prices for Selected Countries, 1990 and 2002

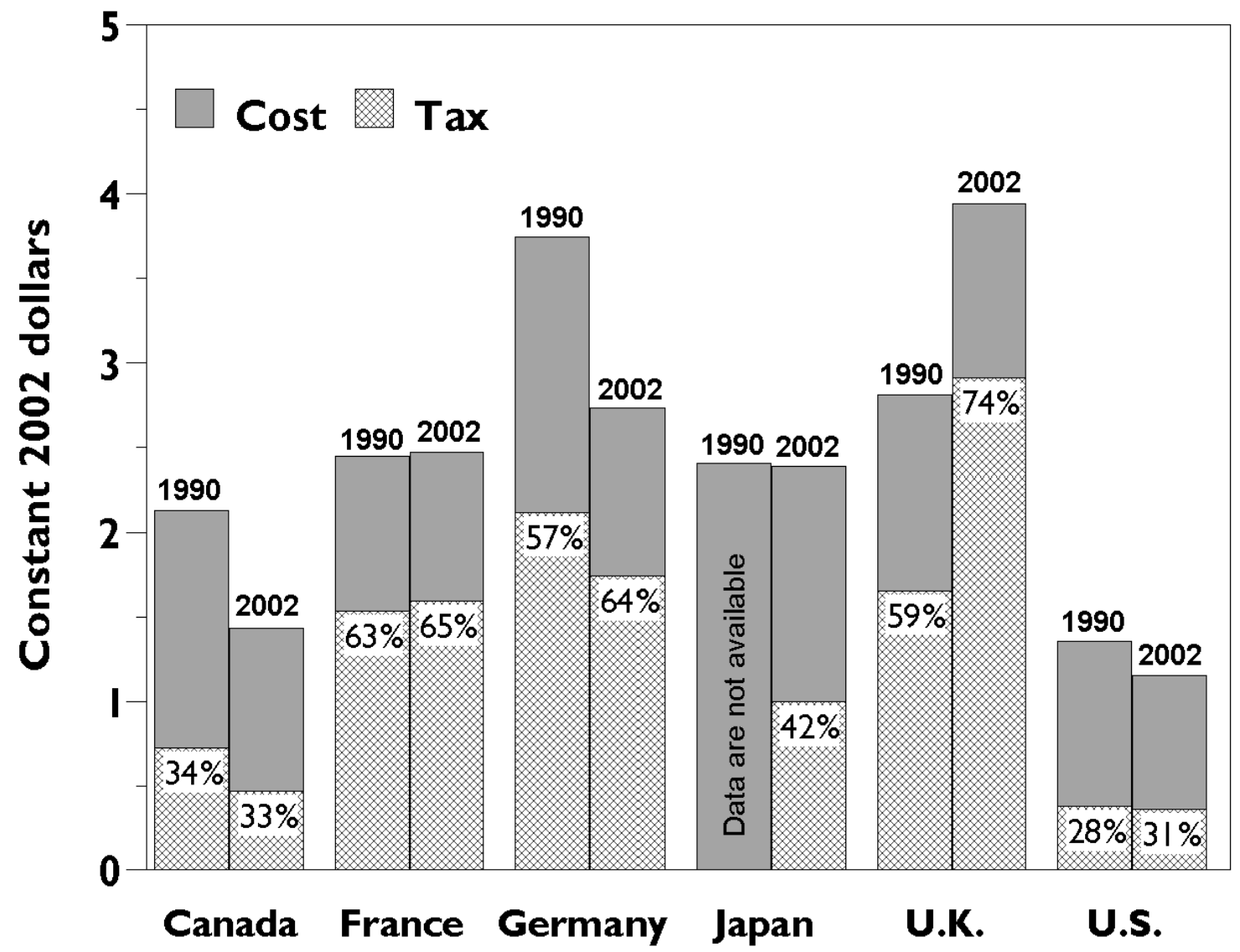

Source:

Table 10.2 and International Energy Agency, Energy Prices and Taxes, Fourth Quarter 2002, Paris, France, 2003. (Additional resources: www.iea.org) 
Though the cost of crude oil certainly influences the price of gasoline, it is not the only factor which determines the price at the pump. Processing cost, transportation cost, and taxes also play a major part of the cost of a gallon of gasoline. The average price of a barrel of crude oil (in constant 2002 dollars) rose by 75\% from 1998 to 2002, while the average price of a gallon of gasoline increased only 17\% in this same time period.

Table 10.3

Prices for a Barrel of Crude Oil and a Gallon of Gasoline, 1978-2002

\begin{tabular}{|c|c|c|c|c|c|}
\hline \multirow[b]{2}{*}{ Year } & \multicolumn{2}{|c|}{$\begin{array}{c}\text { Crude oil }{ }^{\mathrm{a}} \\
\text { (dollars per barrel) }\end{array}$} & \multicolumn{2}{|c|}{$\begin{array}{c}\text { Gasoline }{ }^{\mathrm{b}} \\
\text { (cents per gallon) }\end{array}$} & \multirow{2}{*}{$\begin{array}{l}\text { Ratio of } \\
\text { gasoline } \\
\text { to } \\
\text { crude oil }\end{array}$} \\
\hline & Current & Constant $2002^{c}$ & Current & Constant $2002^{c}$ & \\
\hline 1978 & 12.5 & 34.4 & 65.2 & 179.9 & 219.8 \\
\hline 1979 & 17.7 & 43.9 & 88.2 & 218.6 & 209.1 \\
\hline 1980 & 28.1 & 61.3 & 122.1 & 266.6 & 182.7 \\
\hline 1981 & 35.2 & 69.7 & 135.3 & 267.8 & 161.3 \\
\hline 1982 & 31.9 & 59.4 & 128.1 & 238.6 & 168.8 \\
\hline 1983 & 29.0 & 52.4 & 122.5 & 221.3 & 177.5 \\
\hline 1984 & 28.6 & 49.6 & 119.8 & 207.4 & 175.7 \\
\hline 1985 & 26.8 & 44.7 & 119.6 & 200.0 & 187.8 \\
\hline 1986 & 14.6 & 23.9 & 93.1 & 152.8 & 268.7 \\
\hline 1987 & 17.9 & 28.1 & 95.7 & 151.6 & 224.5 \\
\hline 1988 & 14.7 & 22.3 & 96.3 & 146.4 & 275.7 \\
\hline 1989 & 18.0 & 26.1 & 106.0 & 153.8 & 247.7 \\
\hline 1990 & 22.2 & 30.6 & 121.7 & 167.5 & 230.0 \\
\hline 1991 & 19.1 & 25.2 & 119.6 & 158.0 & 263.5 \\
\hline 1992 & 18.4 & 23.6 & 119.0 & 152.6 & 271.2 \\
\hline 1993 & 16.4 & 20.4 & 117.3 & 146.0 & 300.2 \\
\hline 1994 & 15.6 & 18.9 & 117.4 & 142.5 & 316.3 \\
\hline 1995 & 17.2 & 20.3 & 120.5 & 142.2 & 293.7 \\
\hline 1996 & 20.7 & 23.7 & 128.8 & 147.7 & 261.2 \\
\hline 1997 & 19.0 & 21.3 & 129.1 & 144.7 & 284.8 \\
\hline 1998 & 12.5 & 13.8 & 111.5 & 123.1 & 374.0 \\
\hline 1999 & 17.5 & 18.9 & 122.1 & 131.8 & 292.9 \\
\hline 2000 & 28.3 & 29.5 & 156.3 & 163.3 & 232.3 \\
\hline 2001 & 23.1 & 23.3 & 153.1 & 155.5 & 280.2 \\
\hline 2002 & 24.1 & 24.1 & 144.1 & 144.1 & 251.2 \\
\hline \multicolumn{6}{|c|}{ Average annual percentage change } \\
\hline 1978-2001 & $2.8 \%$ & $-1.5 \%$ & $3.4 \%$ & $-0.9 \%$ & \\
\hline 1992-2002 & $2.7 \%$ & $0.2 \%$ & $1.9 \%$ & $-0.6 \%$ & \\
\hline
\end{tabular}

Sources:

Crude oil - U.S. Department of Energy, Energy Information Administration, Monthly Energy Review, April 2003, Washington, DC, Table 9.1.

Gasoline - U.S. Department of Energy, Energy Information Administration, Monthly Energy Review, April 2003, Washington, DC, Table 9.4.

(Additional resources: www.eia.doe.gov)

${ }^{a}$ Refiner acquisition cost of composite (domestic and imported) crude oil.

${ }^{b}$ Average for all types. These prices were collected from a sample of service stations in 85 urban areas selected to represent all urban consumers. Urban consumers make up about $80 \%$ of the total U.S. population.

${ }^{c}$ Adjusted by the Consumer Price Inflation Index. 
Diesel fuel price is generally lower than gasoline; however, in 2001 the price of gasoline and diesel fuel were almost equal.

Table 10.4

Retail Prices for Motor Fuel, 1978-2002

(cents per gallon, including tax)

\begin{tabular}{|c|c|c|c|c|}
\hline \multirow[b]{2}{*}{ Year } & \multicolumn{2}{|c|}{ Diesel fuel $^{\mathrm{a}}$} & \multicolumn{2}{|c|}{$\begin{array}{l}\text { Average for all } \\
\text { gasoline types }\end{array}$} \\
\hline & Current & $\begin{array}{c}\text { Constant } \\
2002^{c}\end{array}$ & Current & $\begin{array}{c}\text { Constant } \\
2002^{c}\end{array}$ \\
\hline 1978 & $\mathrm{~d}$ & d & 65 & 180 \\
\hline 1979 & d & $\mathrm{d}$ & 88 & 219 \\
\hline 1980 & 101 & 221 & 122 & 267 \\
\hline 1981 & 118 & 234 & 135 & 268 \\
\hline 1982 & 116 & 216 & 128 & 239 \\
\hline 1983 & 120 & 217 & 123 & 221 \\
\hline 1984 & 122 & 211 & 120 & 207 \\
\hline 1985 & 122 & 204 & 120 & 200 \\
\hline 1986 & 94 & 154 & 93 & 153 \\
\hline 1987 & 96 & 152 & 96 & 152 \\
\hline 1988 & 95 & 144 & 96 & 146 \\
\hline 1989 & 102 & 148 & 106 & 154 \\
\hline 1990 & 107 & 147 & 122 & 168 \\
\hline 1991 & 91 & 120 & 120 & 158 \\
\hline 1992 & 106 & 136 & 119 & 153 \\
\hline 1993 & 98 & 122 & 117 & 146 \\
\hline 1994 & 96 & 117 & 117 & 143 \\
\hline 1995 & 97 & 115 & 121 & 142 \\
\hline 1996 & 115 & 132 & 129 & 148 \\
\hline 1997 & 129 & 145 & 129 & 145 \\
\hline 1998 & 112 & 124 & 112 & 123 \\
\hline 1999 & 97 & 105 & 122 & 132 \\
\hline 2000 & 136 & 142 & 156 & 163 \\
\hline 2001 & 152 & 154 & 153 & 156 \\
\hline 2002 & 115 & 115 & 144 & 144 \\
\hline \multicolumn{5}{|c|}{ Average annual percentage change } \\
\hline 1978-2002 & $0.5 \%{ }^{\mathrm{e}}$ & $-2.7 \% \mathrm{e}^{\mathrm{e}}$ & $3.4 \%$ & $2.5 \%$ \\
\hline 1992-2002 & $0.8 \%$ & $-1.7 \%$ & $1.9 \%$ & $-0.6 \%$ \\
\hline
\end{tabular}

\section{Source:}

Gasoline - U.S. Department of Energy, Energy Information Administration, Monthly Energy Review, 2003, Washington, DC, Table 9.4.

Diesel - U.S. Department of Energy, Energy Information Administration, International Energy Annual 2001, Washington, DC, March 2003, Table 7.2 (Additional resources: www.eia.doe.gov)

${ }^{a}$ Collected from a survey of prices on January 1 of the current year.

${ }^{b}$ These prices were collected from a sample of service stations in 85 urban areas selected to represent all urban consumers.

Urban consumers make up about $80 \%$ of the total U.S. population.

'Adjusted by the Consumer Price Inflation Index.

${ }^{\mathrm{d}}$ Data are not available.

${ }^{\mathrm{e}}$ Average annual percentage change is from the earliest year possible to 2002 . 
The fuel prices shown here are refiner sales prices of transportation fuels to end users, excluding tax. Sales to end users are those made directly to the ultimate consumer, including bulk consumers. Bulk sales to utility, industrial, and commercial accounts previously included in the wholesale category are now counted as sales to end users.

Table 10.5

Refiner Sales Prices for Propane and No. 2 Diesel, 1978-2002 (cents per gallon, excluding tax)

\begin{tabular}{|c|c|c|c|c|}
\hline \multirow[b]{2}{*}{ Year } & \multicolumn{2}{|c|}{ Propane $^{a}$} & \multicolumn{2}{|c|}{ No. 2 diesel fuel } \\
\hline & Current & $\begin{array}{c}\text { Constant } \\
2002^{\mathrm{b}}\end{array}$ & Current & $\begin{array}{c}\text { Constant } \\
2002^{\mathrm{b}}\end{array}$ \\
\hline 1978 & 33.5 & 92.4 & 37.7 & 104.0 \\
\hline 1979 & 35.7 & 88.5 & 58.5 & 145.0 \\
\hline 1980 & 48.2 & 105.2 & 81.8 & 178.6 \\
\hline 1981 & 56.5 & 111.8 & 99.5 & 196.9 \\
\hline 1982 & 59.2 & 110.4 & 94.2 & 175.6 \\
\hline 1983 & 70.9 & 128.1 & 82.6 & 149.2 \\
\hline 1984 & 73.7 & 127.6 & 82.3 & 142.5 \\
\hline 1985 & 71.7 & 119.9 & 78.9 & 131.9 \\
\hline 1986 & 74.5 & 122.3 & 47.8 & 78.5 \\
\hline 1987 & 70.1 & 111.0 & 55.1 & 87.3 \\
\hline 1988 & 71.4 & 108.6 & 50.0 & 76.0 \\
\hline 1989 & 61.5 & 89.2 & 58.5 & 84.9 \\
\hline 1990 & 74.5 & 102.5 & 72.5 & 99.8 \\
\hline 1991 & 73.0 & 96.4 & 64.8 & 85.6 \\
\hline 1992 & 64.3 & 82.4 & 61.9 & 79.4 \\
\hline 1993 & 67.3 & 83.8 & 60.2 & 74.9 \\
\hline 1994 & 53.0 & 64.3 & 55.4 & 67.3 \\
\hline 1995 & 49.2 & 58.1 & 56.0 & 66.1 \\
\hline 1996 & 60.5 & 69.4 & 68.1 & 78.1 \\
\hline 1997 & 55.2 & 61.9 & 64.2 & 72.0 \\
\hline 1998 & 40.5 & 44.7 & 49.4 & 54.5 \\
\hline 1999 & 45.8 & 49.5 & 58.4 & 63.1 \\
\hline 2000 & 60.3 & 63.0 & 93.5 & 97.7 \\
\hline 2001 & 50.6 & 51.4 & 84.2 & 85.5 \\
\hline \multirow[t]{2}{*}{2002} & 41.9 & 41.9 & 76.2 & 76.2 \\
\hline & \multicolumn{4}{|c|}{ Average annual percentage change } \\
\hline 1978-2002 & $0.9 \%$ & $-3.2 \%$ & $3.0 \%$ & $-1.3 \%$ \\
\hline $1992-2002$ & $-4.2 \%$ & $-6.5 \%$ & $2.1 \%$ & $-0.4 \%$ \\
\hline
\end{tabular}

\section{Source:}

U.S. Department of Energy, Energy Information Administration, Monthly Energy Review, April 2003, Washington, DC, Table 9.7.

(Additional resources: www.eia.doe.gov)

${ }^{a}$ Consumer grade.

${ }^{\mathrm{b}}$ Adjusted by the Consumer Price Inflation Index. 
Average jet fuel prices jumped more than 30 cents per gallon from 1999 to 2000, but lowered again in 2001 and 2002.

Table 10.6

Refiner Sales Prices for Aviation Gasoline and Jet Fuel, 1978-2002 (cents per gallon, excluding tax)

\begin{tabular}{|c|c|c|c|c|}
\hline \multirow[b]{2}{*}{ Year } & \multicolumn{2}{|c|}{$\begin{array}{c}\text { Finished aviation } \\
\text { gasoline }\end{array}$} & \multicolumn{2}{|c|}{$\begin{array}{l}\text { Kerosene-type } \\
\text { jet fuel }\end{array}$} \\
\hline & Current & $\begin{array}{c}\text { Constant } \\
2002^{\mathrm{a}}\end{array}$ & Current & $\begin{array}{l}\text { Constant } \\
2002^{\mathrm{a}}\end{array}$ \\
\hline 1978 & 51.6 & 142.4 & 38.7 & 106.8 \\
\hline 1979 & 68.9 & 170.7 & 54.7 & 135.5 \\
\hline 1980 & 108.4 & 236.7 & 86.6 & 189.1 \\
\hline 1981 & 130.3 & 257.9 & 102.4 & 202.7 \\
\hline 1982 & 131.2 & 244.6 & 96.3 & 179.5 \\
\hline 1983 & 125.5 & 226.7 & 87.8 & 158.6 \\
\hline 1984 & 123.4 & 213.7 & 84.2 & 145.8 \\
\hline 1985 & 120.1 & 200.8 & 79.6 & 133.1 \\
\hline 1986 & 101.1 & 165.9 & 52.9 & 86.8 \\
\hline 1987 & 90.7 & 143.6 & 54.3 & 86.0 \\
\hline 1988 & 89.1 & 135.5 & 51.3 & 78.0 \\
\hline 1989 & 99.5 & 144.4 & 59.2 & 85.9 \\
\hline 1990 & 112.0 & 154.2 & 76.6 & 105.4 \\
\hline 1991 & 104.7 & 138.3 & 65.2 & 86.1 \\
\hline 1992 & 102.7 & 131.7 & 61.0 & 78.2 \\
\hline 1993 & 99.0 & 123.3 & 58.0 & 72.2 \\
\hline 1994 & 95.7 & 116.2 & 53.4 & 64.8 \\
\hline 1995 & 100.5 & 118.2 & 54.0 & 63.7 \\
\hline 1996 & 111.6 & 128.0 & 65.1 & 74.6 \\
\hline 1997 & 112.8 & 126.4 & 61.3 & 68.7 \\
\hline 1998 & 97.5 & 107.6 & 45.2 & 49.9 \\
\hline 1999 & 105.9 & 114.4 & 54.3 & 58.6 \\
\hline 2000 & 130.6 & 136.4 & 89.9 & 93.9 \\
\hline 2001 & 132.3 & 134.4 & 77.5 & 78.7 \\
\hline \multirow[t]{2}{*}{2002} & 131.7 & 131.7 & 72.2 & 72.2 \\
\hline & \multicolumn{4}{|c|}{ Average annual percentage change } \\
\hline 1978-2002 & $4.0 \%$ & $-0.3 \%$ & $2.6 \%$ & $-1.6 \%$ \\
\hline 1992-2002 & $2.5 \%$ & $0.0 \%$ & $1.7 \%$ & $-0.8 \%$ \\
\hline
\end{tabular}

\section{Source:}

U.S. Department of Energy, Energy Information Administration, Monthly Energy Review, April 2003, Washington, DC, Table 9.7.

(Additional resources: www.eia.doe.gov)

${ }^{a}$ Adjusted by the Consumer Price Inflation Index. 
Table 10.7

State Taxes on Motor Fuels, 2000

(dollars per gallon or gasoline equivalent gallon)

(Footnotes for this table appear on next page)

\begin{tabular}{|c|c|c|c|c|c|c|}
\hline State & Gasoline & Diesel fuel & $\mathrm{CNG}$ & Propane & Methanol & Ethanol \\
\hline Alabama & 0.18 & 0.19 & $\mathrm{a}$ & $\mathrm{a}$ & $0.16^{\mathrm{b}}$ & $0.16^{\mathrm{b}}$ \\
\hline Alaska & 0.08 & 0.08 & 0.08 & 0.00 & $0.08^{b}$ & 0.04 \\
\hline Arizona & 0.18 & 0.27 & 0.00 & 0.00 & 0.00 & 0.00 \\
\hline Arkansas & 0.186 & 0.186 & $0.05^{\mathrm{c}}$ & ${ }^{a}$ & 0.186 & 0.186 \\
\hline California & 0.18 & 0.18 & $\mathrm{a}$ & a & 0.09 & 0.09 \\
\hline Colorado & 0.22 & 0.205 & $\mathrm{a}$ & $\mathrm{a}$ & 0.205 & $0.17^{\mathrm{b}}$ \\
\hline Connecticut & 0.36 & 0.18 & 0.18 & 0.18 & $0.37^{\mathrm{b}}$ & 0.35 \\
\hline Delaware & 0.23 & 0.22 & 0.22 & 0.22 & 0.22 & 0.23 \\
\hline $\begin{array}{l}\text { District of } \\
\text { Columbia }\end{array}$ & 0.20 & 0.20 & 0.20 & 0.20 & 0.20 & 0.20 \\
\hline Florida & 0.13 & 0.25 & $\mathrm{a}$ & $\mathrm{a}$ & $0.04^{\mathrm{b}}$ & $0.04^{\mathrm{b}}$ \\
\hline Georgia & 0.075 & 0.075 & 0.075 & 0.075 & 0.075 & 0.075 \\
\hline Hawaii & 0.16 & 0.16 & 0.16 & 0.16 & 0.16 & 0.16 \\
\hline Idaho & 0.25 & 0.25 & $0.197^{\mathrm{d}}$ & 0.181 & $0.25^{\mathrm{b}}$ & $0.23^{\mathrm{b}}$ \\
\hline Illinois & 0.19 & 0.215 & 0.19 & 0.19 & $0.19^{b}$ & $0.19^{b}$ \\
\hline Indiana & 0.15 & 0.16 & $\mathrm{a}$ & $\mathrm{a}$ & 0.15 & 0.15 \\
\hline Iowa & 0.20 & 0.225 & $0.16^{\mathrm{c}}$ & 0.20 & $0.19^{b}$ & $0.19^{\mathrm{b}}$ \\
\hline Kansas & 0.18 & 0.20 & 0.17 & 0.17 & 0.20 & 0.20 \\
\hline Kentucky & 0.164 & 0.134 & 0.15 & 0.15 & 0.15 & 0.15 \\
\hline Louisiana & 0.20 & 0.20 & a & $\mathrm{a}$ & $0.20^{\mathrm{b}}$ & $0.20^{\mathrm{b}}$ \\
\hline Maine & 0.19 & 0.20 & 0.18 & 0.18 & 0.18 & 0.18 \\
\hline Maryland & 0.235 & 0.2425 & 0.235 & 0.235 & 0.235 & 0.235 \\
\hline Massachusetts & 0.21 & 0.21 & 0.10 & 0.10 & 0.21 & 0.21 \\
\hline Michigan & 0.19 & 0.15 & 0.0 & 0.15 & $0.15^{\mathrm{b}}$ & $0.025^{\mathrm{b}}$ \\
\hline Minnesota & 0.20 & 0.20 & 0.174 & 0.15 & 0.114 & 0.142 \\
\hline Mississippi & 0.184 & 0.184 & $0.184^{\mathrm{c}}$ & 0.17 & $0.18^{b}$ & $0.18^{\mathrm{b}}$ \\
\hline Missouri & 0.17 & 0.17 & & & $0.17^{b}$ & $0.17^{\mathrm{b}}$ \\
\hline Montana & 0.27 & 0.2775 & $0.07^{\mathrm{e}}$ & $\mathrm{a}$ & 0.27 & 0.27 \\
\hline Nebraska & 0.246 & 0.246 & a & a & a & $\mathrm{a}$ \\
\hline Nevada & 0.2475 & 0.2775 & 0.21 & $0.2475^{\mathrm{c}}$ & 0.2475 & 0.2475 \\
\hline New Hampshire & 0.195 & 0.195 & 0.195 & 0.195 & $0.195^{\mathrm{b}}$ & $0.195^{\mathrm{b}}$ \\
\hline New Jersey & 0.105 & 0.135 & 0.0525 & 0.0525 & $0.105^{\mathrm{b}}$ & $0.105^{b}$ \\
\hline New Mexico & 0.188 & 0.198 & $\mathrm{a}$ & $\mathrm{a}$ & $0.22^{\mathrm{b}}$ & $0.22^{\mathrm{b}}$ \\
\hline New York & $0.10^{\mathrm{f}}$ & $0.10^{\mathrm{f}}$ & $0.08^{f}$ & $0.08^{\mathrm{f}}$ & $0.08^{f}$ & $0.08^{\mathrm{f}}$ \\
\hline North Carolina & 0.223 & 0.223 & 0.223 & 0.223 & 0.223 & 0.223 \\
\hline North Dakota & 0.20 & 0.20 & 0.20 & 0.20 & $0.20^{\mathrm{b}}$ & $0.20^{\mathrm{b}}$ \\
\hline Ohio & 0.22 & 0.22 & 0.22 & 0.22 & $0.22^{\mathrm{b}}$ & $0.21^{\mathrm{b}}$ \\
\hline Oklahoma & 0.17 & 0.14 & 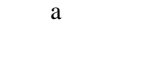 & $\mathrm{a}$ & $0.16^{\mathrm{b}}$ & $0.16^{\mathrm{b}}$ \\
\hline Oregon & 0.24 & 0.24 & 0.24 & 0.24 & 0.24 & 0.24 \\
\hline Pennsylvania & $0.12^{\mathrm{g}}$ & $0.12^{\mathrm{g}}$ & $0.12^{\mathrm{g}}$ & $0.12^{\mathrm{g}}$ & $0.12^{\mathrm{g}}$ & $0.12^{\mathrm{g}}$ \\
\hline Rhode Island & 0.29 & 0.29 & 0.0 & 0.29 & 0.29 & 0.29 \\
\hline
\end{tabular}


Table 10.7 (continued)

State Taxes on Motor Fuels, 2000

(dollars per gallon or gasoline equivalent gallon)

\begin{tabular}{lcccccc}
\hline State & Gasoline & Diesel fuel & CNG & Propane & Methanol & Ethanol \\
\hline South Carolina & 0.16 & 0.16 & 0.16 & 0.16 & 0.16 & 0.16 \\
South Dakota & 0.21 & 0.21 & 0.06 & 0.16 & 0.06 & 0.19 \\
Tennessee & 0.20 & 0.17 & 0.13 & 0.17 & 0.17 & 0.17 \\
Texas & 0.20 & 0.20 & $\mathrm{a}$ & $\mathrm{a}$ & $0.20^{\mathrm{b}}$ & $0.20^{\mathrm{b}}$ \\
Utah & 0.245 & 0.245 & 0.04 & 0.04 & 0.04 & 0.04 \\
\hline Vermont & 0.20 & 0.17 & 0.20 & $\mathrm{a}$ & 0.20 & 0.20 \\
Virginia & 0.18 & 0.16 & 0.10 & 0.10 & $0.18^{\mathrm{b}}$ & $0.18^{\mathrm{b}}$ \\
Washington & 0.23 & 0.23 & $\mathrm{a}$ & $\mathrm{a}$ & 0.23 & 0.23 \\
West Virginia & 0.2535 & 0.2535 & 0.2535 & 0.2535 & 0.2535 & 0.2535 \\
Wisconsin & 0.238 & 0.238 & 0.203 & 0.186 & 0.238 & 0.238 \\
Wyoming & 0.09 & 0.09 & 0.00 & 0.00 & $0.09^{\mathrm{b}}$ & $0.09^{\mathrm{b}}$ \\
\hline
\end{tabular}

Source:

Energy Futures, Inc., The Clean Fuels and Electric Vehicles Report, Boulder, CO, December 2000, pp. 154-155.

${ }^{\mathrm{a}}$ Annual flat fee.

${ }^{\mathrm{b}}$ Blends with gasoline only.

${ }^{\mathrm{c}}$ Per $100 \mathrm{ft}^{3}$.

${ }^{\mathrm{d}}$ Per therm.

${ }^{\mathrm{e}}$ Per $120 \mathrm{ft}^{3}$.

${ }^{\mathrm{f}}$ Plus a petroleum business tax; the amount varies but is usually in the ballpark of $\$ 0.12-\$ 0.14$.

${ }^{\mathrm{g}}$ Plus 0.1035 oil franchise tax. 
At the end of 2001, only four states offered tax exemptions to encourage the use of gasohol for transportation purposes. This list is quite short compared to the 30 states which offered gasohol tax exemptions twenty years ago. Still, the Federal Government encourages gasohol use via a difference in the Federal tax rates of gasoline and gasohol.

Table 10.8

State Tax Exemptions for Gasohol, 2001

\begin{tabular}{lc}
\hline State & $\begin{array}{c}\text { Exemption } \\
\text { (Cents/gallon of gasohol) }\end{array}$ \\
\hline Connecticut & 1.0 \\
Idaho & 2.5 \\
Iowa & 1.0 \\
South Dakota & 2.0 \\
\hline
\end{tabular}

Source:

U.S. Department of Transportation, Federal Highway Administration, "Highway Statistics," January 2003, Washington, DC, Table MF-121T. (Additional resources: www.fhwa.dot.gov)

Table 10.9

Federal Excise Taxes on Motor Fuels

\begin{tabular}{llc}
\hline Fuel & & Cents per gallon \\
\hline Gasoline & & 18.30 \\
Diesel $^{\mathrm{a}}$ & & 24.30 \\
Gasohol & $10 \%$ Ethanol & 13.00 \\
& $7.7 \%$ Ethanol & 14.24 \\
& $5.7 \%$ Ethanol & 15.32 \\
Gasohol & 10\% Methanol & 12.40 \\
& 7.7\% Methanol & 13.78 \\
& 5.7\% Methanol & 14.98 \\
Methanol & Qualified & 12.85 \\
& Partially exempt $^{\mathrm{b}}$ & 9.20 \\
Ethanol & Qualified $^{\mathrm{b}}$ & 12.85 \\
& Partially exempt $^{\mathrm{c}}$ & 9.25 \\
CNG & & $48.54 / \mathrm{mc}^{\mathrm{d}}$ \\
LNG & & 18.30 \\
Propane & & 13.60 \\
\hline
\end{tabular}

Source:

Energy Futures, Inc., The Clean Fuels and Electric Vehicles Report, Boulder, CO, December 2000, p. 155.

${ }^{a}$ Reduced diesel rates are specified for marine fleets, trains and certain intercity buses. Diesel rates are also reduced for diesel/alcohol blends. Diesel used exclusively in state and local government fleets, nonprofit organization vehicles, school buses and qualified local buses is exempt from Federal taxes.

${ }^{\mathrm{b}} \mathrm{Qualified}$ - contains at least 85 percent methanol or ethanol or other alcohol produced from a substance other than petroleum or natural gas.

'Partially exempt - 85 percent alcohol and produced from natural gas.

${ }^{\mathrm{d}}$ Thousand cubic feet. 
These states currently offer extra incentives for ethanol production or consumption (gasohol or E85). Details on these incentives can be found at

www.fleets.doe.gov/fleet_tool.cgi?27519,benefits,2,3957 .

Table $\mathbf{1 0 . 1 0}$

State Ethanol Incentives, 2003

\begin{tabular}{|c|c|c|c|}
\hline State & $\begin{array}{l}\text { Producer } \\
\text { incentives }\end{array}$ & $\begin{array}{c}\text { State tax } \\
\text { incentives }\end{array}$ & $\begin{array}{c}\text { Other } \\
\text { incentives }\end{array}$ \\
\hline Arkansas & $\mathrm{T}$ & & \\
\hline California & & $\mathrm{T}$ & \\
\hline Connecticut & & $\mathrm{T}$ & \\
\hline Florida & & & $\mathrm{T}$ \\
\hline Hawaii & $\mathrm{T}$ & $\mathrm{T}$ & \\
\hline Idaho & & & $\mathrm{T}$ \\
\hline Illinois & $\mathrm{T}$ & $\mathrm{T}$ & \\
\hline Indiana & $\mathrm{T}$ & & \\
\hline Iowa & $\mathrm{T}$ & $\mathrm{T}$ & $\mathrm{T}$ \\
\hline Kansas & T & & \\
\hline Maine & $\mathrm{T}$ & $\mathrm{T}$ & \\
\hline Minnesota & $\mathrm{T}$ & & $\mathrm{T}$ \\
\hline Missouri & $\mathrm{T}$ & & \\
\hline Montana & $\mathrm{T}$ & $\mathrm{T}$ & \\
\hline Nebraska & $\mathrm{T}$ & & \\
\hline North Carolina & T & & \\
\hline North Dakota & $\mathrm{T}$ & & \\
\hline Ohio & & $\mathrm{T}$ & \\
\hline South Dakota & T & $\mathrm{T}$ & \\
\hline Wyoming & $\mathrm{T}$ & & \\
\hline
\end{tabular}

\section{Source:}

U.S. Department of Energy, “Alternative Fuel Vehicle Fleet Buyer's Guide, Incentives and Laws," www.fleets.doe.gov/fleet_tool.cgi?27519,benefits,2,3957. 
In current dollars, import cars, on average, were less expensive than domestic cars until 1982. Since then, import prices have nearly tripled, while domestic prices have nearly doubled (current dollars).

Table 10.11

Average Price of a New Car, 1970-2001

\begin{tabular}{|c|c|c|c|c|c|c|}
\hline & \multicolumn{2}{|c|}{ Domestic $^{\mathrm{a}}$} & \multicolumn{2}{|c|}{ Import } & \multicolumn{2}{|c|}{ Total } \\
\hline Year & $\begin{array}{c}\text { Current } \\
\text { dollars }\end{array}$ & $\begin{array}{c}\text { Constan } \\
\mathrm{t} \\
2001 \\
\text { dollars } \\
\end{array}$ & $\begin{array}{c}\text { Current } \\
\text { dollars }\end{array}$ & $\begin{array}{c}\text { Constant } \\
2001 \\
\text { dollars } \\
\end{array}$ & $\begin{array}{c}\text { Current } \\
\text { dollars }\end{array}$ & $\begin{array}{c}\text { Constant } \\
2001 \\
\text { dollars } \\
\end{array}$ \\
\hline 1970 & 3,708 & 16,925 & 2,648 & 12,087 & 3,542 & 16,167 \\
\hline 1975 & 5,084 & 16,736 & 4,384 & 14,431 & 4,950 & 16,295 \\
\hline 1980 & 7,609 & 16,354 & 7,482 & 16,081 & 7,574 & 16,279 \\
\hline 1981 & 8,912 & 17,363 & 8,896 & 17,332 & 8,910 & 17,359 \\
\hline 1982 & 9,865 & 18,105 & 9,957 & 18,273 & 9,890 & 18,150 \\
\hline 1983 & 10,516 & 18,699 & 10,868 & 19,325 & 10,606 & 18,859 \\
\hline 1984 & 11,079 & 18,884 & 12,336 & 21,027 & 11,375 & 19,389 \\
\hline 1985 & 11,589 & 19,074 & 12,853 & 21,155 & 11,838 & 19,484 \\
\hline 1986 & 12,319 & 19,906 & 13,670 & 22,089 & 12,652 & 20,444 \\
\hline 1987 & 12,922 & 20,145 & 14,470 & 22,558 & 13,386 & 20,868 \\
\hline 1988 & 13,418 & 20,087 & 15,221 & 22,786 & 13,932 & 20,857 \\
\hline 1989 & 13,936 & 19,904 & 15,510 & 22,152 & 14,371 & 20,525 \\
\hline 1990 & 14,489 & 19,633 & 16,640 & 22,547 & 15,042 & 20,382 \\
\hline 1991 & 15,192 & 19,754 & 16,327 & 21,230 & 15,475 & 20,122 \\
\hline 1992 & 15,644 & 19,747 & 18,593 & 23,470 & 16,336 & 20,621 \\
\hline 1993 & 15,976 & 19,580 & 20,261 & 24,832 & 16,871 & 20,677 \\
\hline 1994 & 16,930 & 20,231 & 21,989 & 26,277 & 17,903 & 21,394 \\
\hline 1995 & 16,864 & 19,597 & 23,202 & 26,962 & 17,959 & 20,870 \\
\hline 1996 & 17,468 & 19,717 & 26,205 & 29,579 & 18,777 & 21,194 \\
\hline 1997 & 17,907 & 19,759 & 27,722 & 30,589 & 19,531 & 21,551 \\
\hline 1998 & 18,479 & 20,077 & 29,614 & 32,176 & 20,364 & 22,126 \\
\hline 1999 & 18,630 & 19,804 & 28,931 & 30,754 & 20,658 & 21,960 \\
\hline 2000 & 18,684 & 19,216 & 27,767 & 28,557 & 20,355 & 20,934 \\
\hline \multirow{2}{*}{2001} & 19,654 & 19,654 & 27,477 & 27,477 & 21,605 & 21,605 \\
\hline & \multicolumn{6}{|c|}{ Average annual percentage change } \\
\hline 1970-2001 & $5.5 \%$ & $0.5 \%$ & $7.8 \%$ & $2.7 \%$ & $6.0 \%$ & $0.9 \%$ \\
\hline 1991-2001 & $2.6 \%$ & $-0.1 \%$ & $5.3 \%$ & $2.6 \%$ & $3.4 \%$ & $0.7 \%$ \\
\hline
\end{tabular}

Source:

U.S. Department of Commerce, Bureau of Economic Analysis, National Income and Product Accounts, underlying detail estimates for Motor Vehicle Output, Washington, DC, 2002.

(Additional resources: www.stat-usa.gov)

ancludes transplants.

${ }^{\mathrm{b}}$ Adjusted by the Consumer Price Inflation Index. 
The total cost of operating an automobile is the sum of the fixed cost (depreciation, insurance, finance charge, and license fee) and the variable cost (gas and oil, tires, and maintenance), which is related to the amount of travel. The total auto operating cost declined slightly in 2001 and again in 2002. The gas and oil share of total cost in 2002 was only 9.7\%, which is the lowest in the history of this series.

Table 10.12

Automobile Operating Cost per Mile, 1985-2002

\begin{tabular}{|c|c|c|c|c|c|}
\hline \multirow{2}{*}{$\begin{array}{c}\text { Model } \\
\text { year }\end{array}$} & \multicolumn{3}{|c|}{ Constant 2002 dollars per 10,000 miles $^{\mathrm{a}}$} & \multirow{2}{*}{$\begin{array}{c}\text { Total cost per } \\
\text { mile }^{\mathrm{b}} \\
(\text { constant } \\
\left.2002 \text { cents }^{\mathrm{a}}\right) \\
\end{array}$} & \multirow{2}{*}{$\begin{array}{l}\text { Percentage } \\
\text { gas and oil of } \\
\text { total cost }\end{array}$} \\
\hline & Variable cost & Fixed cost & Total cost & & \\
\hline 1985 & 1,241 & 3,446 & 4,686 & 46.86 & $19.9 \%$ \\
\hline 1986 & 1,070 & 3,787 & 4,857 & 48.57 & $15.1 \%$ \\
\hline 1987 & 1,061 & 3,687 & 4,748 & 47.48 & $14.7 \%$ \\
\hline 1988 & 1,201 & 4,608 & 5,809 & 58.09 & $13.6 \%$ \\
\hline 1989 & 1,161 & 4,236 & 5,397 & 53.97 & $14.2 \%$ \\
\hline 1990 & 1,156 & 4,482 & 5,638 & 56.38 & $13.2 \%$ \\
\hline 1991 & 1,281 & 4,710 & 5,991 & 59.91 & $14.6 \%$ \\
\hline 1992 & 1,154 & 4,852 & 6,006 & 60.06 & $12.6 \%$ \\
\hline 1993 & 1,145 & 4,634 & 5,779 & 57.79 & $12.7 \%$ \\
\hline 1994 & 1,105 & 4,657 & 5,761 & 57.61 & $11.8 \%$ \\
\hline 1995 & 1,133 & 4,728 & 5,861 & 58.61 & $11.7 \%$ \\
\hline 1996 & 1,101 & 4,808 & 5,908 & 59.08 & $10.9 \%$ \\
\hline 1997 & 1,211 & 4,874 & 6,095 & 60.95 & $12.1 \%$ \\
\hline 1998 & 1,181 & 4,997 & 6,167 & 61.67 & $11.1 \%$ \\
\hline 1999 & 1,145 & 5,032 & 6,177 & 61.77 & $9.8 \%$ \\
\hline 2000 & 1,275 & 4,935 & 6,210 & 62.10 & $11.6 \%$ \\
\hline 2001 & 1,382 & 4,694 & 6,076 & 60.76 & $13.2 \%$ \\
\hline 2002 & 1,180 & 4,874 & 6,054 & 60.54 & $9.7 \%$ \\
\hline \multicolumn{6}{|c|}{ Average annual percentage change } \\
\hline 1985-2002 & $-0.3 \%$ & $2.1 \%$ & $1.5 \%$ & $1.8 \%$ & \\
\hline
\end{tabular}

Source:

American Automobile Association, Your Driving Costs, 2002 Edition, Heathrow, FL, and annual.

(Additional resources: www.aaa.com, www.runzheimer.com)

${ }^{a}$ Adjusted by the Consumer Price Inflation Index.

${ }^{\mathrm{b}}$ Based on 10,000 miles per year. 
While the previous table shows costs per mile, this table presents costs per year for fixed costs associated with automobile operation. For 2002

model year autos, the fixed cost is almost \$16 per day.

Table 10.13

Fixed Automobile Operating Costs per Year, 1975-2002 (constant 2002 dollars) ${ }^{\mathrm{a}}$

\begin{tabular}{|c|c|c|c|c|c|c|c|c|}
\hline Model year & Fire $\&$ theft ${ }^{\mathrm{b}}$ & Collision $^{c}$ & $\begin{array}{c}\text { Property } \\
\text { damage \& } \\
\text { liability }^{\mathrm{d}}\end{array}$ & $\begin{array}{l}\text { License, } \\
\text { registration } \\
\text { \& taxes }\end{array}$ & Depreciation & $\begin{array}{c}\text { Finance } \\
\text { charge }\end{array}$ & Total & $\begin{array}{l}\text { Average } \\
\text { fixed cost } \\
\text { per day }\end{array}$ \\
\hline 1975 & 177 & 471 & 632 & 100 & 2,585 & $\mathrm{e}$ & 3,966 & 10.87 \\
\hline $1980^{\circ}$ & 153 & 376 & 541 & 179 & 2,269 & 924 & 4,439 & 12.16 \\
\hline $1985^{\circ}$ & 125 & 296 & 356 & 184 & 2,110 & 893 & 3,964 & 10.87 \\
\hline 1986 & 141 & 314 & 381 & 213 & 2,167 & 1,046 & 4,261 & 11.67 \\
\hline 1987 & 138 & 310 & 399 & 203 & 2,366 & 833 & 4,249 & 11.64 \\
\hline 1988 & 131 & 309 & 432 & 211 & 2,713 & 859 & 4,655 & 12.76 \\
\hline 1989 & 148 & 339 & 448 & 209 & 2,928 & 853 & 4,925 & 13.49 \\
\hline 1990 & 151 & 337 & 438 & 227 & 3,244 & 936 & 5,334 & 14.62 \\
\hline 1991 & 143 & 326 & 466 & 222 & 3,307 & 1,144 & 4,816 & 13.20 \\
\hline 1992 & 164 & 367 & 478 & 223 & 3,484 & 1,021 & 5,737 & 15.72 \\
\hline 1993 & 144 & 303 & 479 & 222 & 3,523 & 834 & 5,505 & 15.09 \\
\hline 1994 & 149 & 299 & 486 & 235 & 3,569 & 787 & 5,524 & 15.14 \\
\hline 1995 & 143 & 297 & 484 & 240 & 3,628 & 810 & 5,601 & 15.35 \\
\hline 1996 & 165 & 315 & 488 & 247 & 3,635 & 823 & 5,673 & 15.55 \\
\hline 1997 & 135 & 365 & 449 & 242 & 3,667 & 861 & 5,720 & 15.67 \\
\hline 1998 & 148 & 317 & 529 & 249 & 3,713 & 897 & 5,853 & 16.04 \\
\hline 1999 & 175 & 350 & 523 & 244 & 3,710 & 894 & 5,896 & 16.15 \\
\hline 2000 & 170 & 341 & 503 & 233 & 3,648 & 887 & 5,781 & 15.84 \\
\hline 2001 & 170 & 350 & 487 & 206 & 3,604 & 880 & 5,702 & 15.62 \\
\hline 2002 & 173 & 357 & 484 & 201 & 3,721 & 828 & 5,764 & 15.79 \\
\hline \multicolumn{9}{|c|}{ Average annual percentage change } \\
\hline 1975-2002 & $-0.1 \%$ & $-1.0 \%$ & $-1.0 \%$ & $2.6 \%$ & $1.4 \%$ & e & $1.4 \%$ & $1.4 \%$ \\
\hline 1992-2002 & $0.5 \%$ & $-0.3 \%$ & $0.1 \%$ & $-1.0 \%$ & $0.7 \%$ & $-2.1 \%$ & $0.0 \%$ & $0.0 \%$ \\
\hline
\end{tabular}

Source:

American Automobile Association, "Your Driving Costs," 2002 Edition, Heathrow, FL, and annual. (Additional resources: www.aaa.com, www.runzheimer.com

a Adjusted by the Consumer Price Inflation Index.

b $\$ 50$ deductible 1975 through 1977; $\$ 100$ deductible 1978 through 1992; \$250 deductible for 1993 - on.

c $\$ 100$ deductible through 1977; \$250 deductible 1978 through 1992; \$500 deductible for 1993 - on.

${ }^{d}$ Coverage: $\$ 100,000 / \$ 300,000$

${ }^{\mathrm{e}}$ Data are not available. 
Table 10.14

Economic Indicators, 1970-2002

(billion dollars)

\begin{tabular}{|c|c|c|c|c|c|}
\hline \multirow[b]{2}{*}{ Year } & \multicolumn{2}{|c|}{$\begin{array}{l}\text { Gross National } \\
\text { Product }\end{array}$} & \multicolumn{2}{|c|}{$\begin{array}{l}\text { Total transportation } \\
\text { outlays } \\
\end{array}$} & \multirow[b]{2}{*}{$\begin{array}{c}\text { Transportation as a } \\
\text { percent of GNP }\end{array}$} \\
\hline & Current & $\begin{array}{c}\text { Constant } \\
2001^{\mathrm{a}}\end{array}$ & Current & $\begin{array}{c}\text { Constant } \\
2001^{\mathrm{a}}\end{array}$ & \\
\hline 1970 & $1,046.1$ & $3,938.8$ & 192.8 & 725.9 & $18.4 \%$ \\
\hline 1980 & $2,830.8$ & $5,427.4$ & 560.9 & $1,075.4$ & $19.8 \%$ \\
\hline 1990 & $5,832.2$ & $7,372.3$ & 975.6 & $1,233.2$ & $16.7 \%$ \\
\hline 2000 & $9,848.0$ & $10,080.2$ & $1,549.0$ & $1,586.5$ & $15.7 \%$ \\
\hline \multirow[t]{2}{*}{2001} & $10,104.1$ & $10,104.1$ & $1,572.0$ & $1,572.0$ & $15.6 \%$ \\
\hline & \multicolumn{2}{|c|}{$\begin{array}{c}\text { Personal Consumption } \\
\text { Expenditures } \\
\end{array}$} & \multicolumn{2}{|c|}{$\begin{array}{c}\text { Transportation Personal } \\
\text { Consumption Expenditures }\end{array}$} & $\begin{array}{l}\text { Transportation PCE as } \\
\text { a percent of total PCE }\end{array}$ \\
\hline 1970 & 648.9 & $2,443.3$ & 81.1 & 305.4 & $12.5 \%$ \\
\hline 1980 & $1,762.9$ & $3,379.9$ & 238.4 & 457.1 & $13.5 \%$ \\
\hline 1990 & $3,831.5$ & $4,843.3$ & 455.5 & 575.8 & $11.9 \%$ \\
\hline 2000 & $6,683.7$ & $6,841.3$ & 768.8 & 788.9 & $11.5 \%$ \\
\hline 2001 & $6,987.0$ & $6,987.0$ & 794.8 & 794.8 & $11.4 \%$ \\
\hline 2002 & $7,303.7$ & $7,221.2$ & 810.4 & 801.2 & $11.1 \%$ \\
\hline
\end{tabular}

Sources:

GNP - U.S. Department of Commerce, Bureau of Economic Analysis, Survey of Current Business, April 2003, Table 1.9, p. D-4, and annual. (Additional resources: www.bea.doc.gov)

Transportation outlays - Eno Transportation Foundation, Transportation in America 2001, Nineteenth Edition, Lansdowne, VA, 2002, p. 1.

PCE - U.S. Department of Commerce, Bureau of Economic Analysis, Survey of Current Business, April 2002, Table 2.2 and annual. (Additional resources: www.bea.doc.gov/bea/scbinf.html)

Table 10.15

Consumer Price Indices, 1970-2002

$(1970=1.000)$

\begin{tabular}{cccccc}
\hline Year & $\begin{array}{c}\text { Transportation } \\
\text { Consumer } \\
\text { Price Index }\end{array}$ & $\begin{array}{c}\text { New car } \\
\text { Price Index }\end{array}$ & $\begin{array}{c}\text { Used car } \\
\text { Price Index }\end{array}$ & $\begin{array}{c}\text { Consumer } \\
\text { Price Index }\end{array}$ & $\begin{array}{c}\text { Gross National } \\
\text { Product Index }\end{array}$ \\
\hline 1970 & 1.000 & 1.000 & 1.000 & 1.000 & 1.000 \\
1980 & 2.124 & 2.216 & 1.667 & 1.997 & 2.706 \\
1990 & 3.369 & 3.213 & 2.286 & 3.769 & 5.575 \\
2000 & 4.438 & 4.088 & 2.689 & 4.994 & 9.414 \\
2002 & 4.637 & 4.077 & 2.637 & 4.872 & 9.977 \\
\hline
\end{tabular}

Source:

Bureau of Labor Statistics, Consumer Price Index Table 1A for 2002, and annual. [GNP—see above.]

(Additional resources: stats.bls.gov/cpihome.htm)

${ }^{\mathrm{a}}$ Adjusted by the implicit GNP price deflator.

b Transportation Personal Consumption Expenditures include user operating expenses (new and used auto purchases, gas and oil, repair, greasing, washing, parking, storage, rental, other motor vehicles, insurance premiums, tires, tubes and other parts); purchased intercity transportation; and purchased local transportation.

${ }^{c}$ Transportation Consumer Price Index includes new and used cars, gasoline, auto insurance rates, intracity mass transit, intracity bus fare, and airline fares. 
Knowing the number of employees that are in transportation-related jobs is not an easy task. The data below were summarized from the Bureau of Labor Statistics (BLS) Current Employment Statistics Survey data using the North American Industry Classification System (NAICS). Employment statistics shown in previous editions used the Standard Industrial Classification System (SIC) and do not match these data due to the differences between the two classification systems and other survey revisions by the BLS.

Table 10.16

Transportation-related Employment, 1993 and 2002 (thousands)

\begin{tabular}{lrr}
\hline & 1993 & 2002 \\
\hline Truck transportation & $1,154.8$ & $1,339.1$ \\
Transit and ground transportation & 299.9 & 371.5 \\
Air transportation & 516.6 & 559.3 \\
Rail transportation & 242.2 & 218.1 \\
Water transportation & 52.8 & 51.6 \\
Pipeline transportation & 58.7 & 41.5 \\
Motor vehicle and parts - retail & $1,475.3$ & $1,879.2$ \\
Motor vehicles and parts - wholesale & 305.9 & 345.5 \\
Gasoline stations - retail & 881.2 & 903.6 \\
Automotive repair & 669.9 & 896.9 \\
Automotive equipment rental and leasing & 155.7 & 197.2 \\
Manufacturing & $1,972.0$ & $1,882.1$ \\
$\quad$ Autos and light trucks & 225.1 & 234.6 \\
$\quad$ Heavy-duty trucks & 38.6 & 32.4 \\
$\quad$ Motor vehicle bodies and trailers & 136.3 & 153.5 \\
$\quad$ Motor vehicle parts & 677.8 & 731.1 \\
$\quad$ Aerospace products and parts & 624.0 & 468.3 \\
$\quad$ Ship \& boat building & 146.5 & 146.4 \\
$\quad$ All other transportation equipment & 36.5 & 39.6 \\
Tires & 87.2 & 76.2 \\
Oil and gas pipeline construction & 66.0 & 75.7 \\
Highway street and bridge construction & 270.9 & 344.4 \\
Scenic \& sightseeing & 19.3 & 25.9 \\
Support activities for transporation & 381.8 & 526.7 \\
Couriers and messengers & 414.3 & 558.0 \\
Travel arrangement and reservation services & 255.7 & 258.0 \\
\hline Total transportation-related employment & $\mathbf{9 , 1 9 3 . 0}$ & $\mathbf{1 0 , 4 7 4 . 3}$ \\
\hline Total nonfarm employment & $110,844.0$ & $130,376.0$ \\
Transportation-related to total employment & $8.3 \%$ & $8.0 \%$ \\
\hline
\end{tabular}

\section{Source:}

Bureau of Labor Statistics web site query system: data.bls.gov/labjava/outside.jsp?survey=ce (Additional resources: www.bls.gov) 


\section{Chapter 11 \\ Greenhouse Gas Emissions}

Summary Statistics from Tables in this Chapter

\begin{tabular}{ccrr}
\hline Source & & & \\
\hline Table 11.1 & Carbon emissions (million metric tonnes) & 1990 & 2001 \\
\cline { 2 - 3 } \cline { 3 - 3 } & United States & 1,352 & 1,559 \\
& China & 617 & 832 \\
& Germany & 271 & 223 \\
& Japan & 269 & 316 \\
United Kingdom & India & 164 & 109 \\
& France & 153 & 250 \\
& Transportation share of U.S. carbon dioxide emissions from fossil fuel & 108 \\
& consumption & & \\
& 1990 & & $31.7 \%$ \\
& 1995 & & $31.9 \%$ \\
& 2001 & & $32.8 \%$ \\
\hline
\end{tabular}


The U. S. accounted for 23.0\% of the World's carbon emissions in 1990 and 23.9\% in 2001. Nearly half (48\%) of the U.S. carbon emissions are from oil use.

Table 11.1

World Carbon Emissions from Energy Consumption, 1990 and 2001

\begin{tabular}{|c|c|c|c|c|}
\hline & \multicolumn{2}{|c|}{1990} & \multicolumn{2}{|c|}{2001} \\
\hline & $\begin{array}{c}\text { Million } \\
\text { metric tons }\end{array}$ & $\begin{array}{l}\text { Percent of } \\
\text { emissions } \\
\text { from oil use }\end{array}$ & $\begin{array}{c}\text { Million } \\
\text { metric tons }\end{array}$ & $\begin{array}{l}\text { Percent of } \\
\text { emissions } \\
\text { from oil use }\end{array}$ \\
\hline Industrialized countries & 2,844 & $49 \%$ & 3,179 & $48 \%$ \\
\hline United States & 1,352 & $44 \%$ & 1,559 & $43 \%$ \\
\hline Canada & 129 & $47 \%$ & 155 & $45 \%$ \\
\hline Mexico & 84 & $77 \%$ & 96 & $71 \%$ \\
\hline United Kingdom & 164 & $40 \%$ & 153 & $41 \%$ \\
\hline France & 102 & $66 \%$ & 108 & $68 \%$ \\
\hline Germany & 271 & $38 \%$ & 223 & $43 \%$ \\
\hline Italy & 113 & $65 \%$ & 121 & $59 \%$ \\
\hline Netherlands & 58 & $47 \%$ & 68 & $40 \%$ \\
\hline Other Western Europe & 223 & $62 \%$ & 271 & $64 \%$ \\
\hline Japan & 269 & $67 \%$ & 316 & $58 \%$ \\
\hline Other industrialized countries & 80 & $46 \%$ & 109 & $34 \%$ \\
\hline Eastern Europe & 1,337 & $30 \%$ & 856 & $23 \%$ \\
\hline Developing countries & 1,691 & $40 \%$ & 2,487 & $41 \%$ \\
\hline China & 617 & $15 \%$ & 832 & $21 \%$ \\
\hline India & 153 & $29 \%$ & 250 & $30 \%$ \\
\hline Other developing countries & 921 & $58 \%$ & 1,405 & $55 \%$ \\
\hline Total World & 5,872 & $42 \%$ & 6,522 & $42 \%$ \\
\hline
\end{tabular}

\section{Source:}

U.S. Department of Energy, Energy Information Administration, International Energy Outlook 2003, Washington, DC, May 2003, Tables A10 and A11. (Additional resources: www.eia.doe.gov) 
Global Warming Potentials (GWP) were developed to allow comparison of the ability of each greenhouse gas to trap heat in the atmosphere relative to carbon dioxide. Extensive research has been performed and it has been discovered that the effects of various gases on global warming are too complex to be precisely summarized by a single number. Further understanding of the subject also causes frequent changes to estimates. Despite that, the scientific community has developed approximations, which are shown below. Most analysts use the 100-year time horizon.

Table 11.2

Numerical Estimates of Global Warming Potentials Compared With Carbon Dioxide (kilogram of gas per kilogram of carbon dioxide)

\begin{tabular}{|c|c|c|c|c|}
\hline \multirow[b]{2}{*}{ Gas } & \multirow{2}{*}{$\begin{array}{c}\text { Lifetime } \\
\text { (years) }\end{array}$} & \multicolumn{3}{|c|}{ direct effect for time horizons of } \\
\hline & & 20 years & 100 years & 500 years \\
\hline Carbon Dioxide $\left(\mathrm{CO}_{2}\right)$ & $5-200^{\mathrm{a}}$ & 1 & 1 & 1 \\
\hline Methane $\left(\mathrm{CH}_{4)}\right.$ & 12 & 62 & 23 & 7 \\
\hline Nitrous Oxide $\left(\mathrm{N}_{2} \mathrm{O}\right)$ & 114 & 275 & 296 & 156 \\
\hline \multicolumn{5}{|c|}{$\mathrm{HFCs}^{\mathrm{b}}, \mathrm{PFCs}^{\mathrm{c}}$, and Sulfur Hexafluoride } \\
\hline HFC-23 & 260 & 9,400 & 12,000 & 10,000 \\
\hline HFC-125 & 29 & 5,900 & 3,400 & 1,100 \\
\hline HFC-134a & 14 & 3,300 & 1,300 & 400 \\
\hline HFC-152a & 1 & 410 & 120 & 37 \\
\hline HFC-227ea & 33 & 5,600 & 3,500 & 1,100 \\
\hline Perfluoromethane $\left(\mathrm{CF}_{4}\right)$ & 50,000 & 3,900 & 5,700 & 8,900 \\
\hline Perfluoroethane $\left(\mathrm{C}_{2} \mathrm{~F}_{6}\right)$ & 10,000 & 8,000 & 11,900 & 18,000 \\
\hline Sulfur hexafluoride $\left(\mathrm{SF}_{6}\right)$ & 3,200 & 15,100 & 22,200 & 32,400 \\
\hline
\end{tabular}

\section{Source:}

U.S. Department of Energy, Energy Information Administration, Emissions of Greenhouse Gases in the United States 2001, Washington, DC, December 2002, Table G1. Original source:

Intergovernmental Panel on Climate Change; Climate Change 2001: The Scientific Basis

(Cambridge, UK: Cambridge University Press, 2000), pp. 38 and 388-389.

(Additional resources: www.eia.doe.gov, www.ipcc.ch)

Note:

The typical uncertainty for global warming potentials is estimated by the Intergovernmental Panel on Climate Change \pm 35 percent.

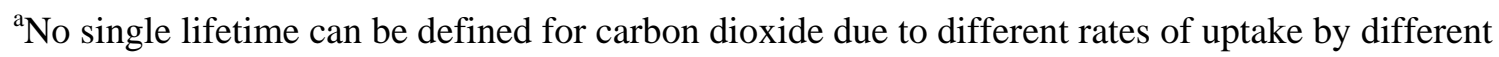
removal processes.

${ }^{\mathrm{b}}$ Hydrofluorocarbons

${ }^{c}$ Perfluorocarbons 
Carbon dioxide emissions in 2001 were 16\% higher than in 1990. Carbon dioxide accounts for the majority of greenhouse gases.

Table 11.3

Estimated U.S. Emissions of Greenhouse Gases, 1990-2001

\begin{tabular}{llrrrr}
\hline Greenhouse gas & Unit of measure $^{\mathrm{a}}$ & 1990 & 1995 & 2000 & 2001 \\
\hline Carbon dioxide & million metric tons of gas & $5,002.8$ & $5,320.9$ & $5,855.1$ & $5,788.5$ \\
& million metric tons of carbon & $1,364.0$ & $1,451.0$ & $1,597.0$ & $1,579.0$ \\
\multirow{2}{*}{ Methane } & million metric tons of gas & 31.7 & 31.1 & 28.3 & 28.0 \\
& million metric tons of carbon $(\mathrm{gwp})^{\mathrm{b}}$ & 199.0 & 195.0 & 178.0 & 176.0 \\
Nitrous oxide & million metric tons of gas & 1.2 & 1.3 & 1.2 & 1.2 \\
& million metric tons of carbon $(\mathrm{gwp})^{\mathrm{b}}$ & 94.0 & 102.0 & 98.0 & 97.0 \\
HFCs, PFCs, and $\mathrm{SF}_{6}{ }^{\mathrm{c}}$ & million metric tons of carbon $(\mathrm{gwp})^{\mathrm{b}}$ & 25.0 & 27.0 & 34.0 & 31.0 \\
\hline
\end{tabular}

\section{Source:}

U.S. Department of Energy, Energy Information Administration, Emissions of Greenhouse Gases in the United States, 2001, Washington, DC, December 2002, Tables ES1 andES2.

(Additional resources: www.eia.doe.gov)

${ }^{a}$ Gases that contain carbon can be measured either in terms of the full molecular weight of the gas or just in terms of their carbon content. See Appendix B, Table B.5 for details.

${ }^{\mathrm{b}}$ Based on global warming potential.

${ }^{c} \mathrm{HFC}$-hydrofluorocarbons. PFC-perfluorocarbons. $\mathrm{SF}_{6}$-sulfur hexaflouride. 
Gases which contain carbon can be measured in terms of the full molecular weight of the gas or just in terms of their carbon content. This table presents carbon content. The ratio of the weight of carbon to carbon dioxide is 0.2727. The transportation sector accounts for approximately one-third of carbon emissions.

Table 11.4

U.S. Carbon Emissions from Fossil Energy Consumption

by End-Use Sector, 1990-2001 ${ }^{\text {a }}$

(million metric tons of carbon)

\begin{tabular}{lcccccccc}
\hline End use sector & 1990 & 1995 & 1996 & 1997 & 1998 & 1999 & 2000 & 2001 \\
\hline Residential & 257.5 & 280.1 & 297.0 & 295.0 & 297.6 & 302.5 & 318.1 & 314.9 \\
Commercial & 212.6 & 228.5 & 237.4 & 249.3 & 253.9 & 258.0 & 274.4 & 279.7 \\
Industrial & 458.0 & 468.0 & 482.2 & 486.9 & 479.5 & 474.2 & 478.4 & 452.4 \\
Transportation & 431.4 & 457.4 & 468.5 & 473.2 & 481.3 & 495.3 & 507.3 & 511.6 \\
$\quad$ Percentage & $31.7 \%$ & $31.9 \%$ & $31.5 \%$ & $31.5 \%$ & $31.8 \%$ & $32.4 \%$ & $32.1 \%$ & $32.8 \%$ \\
Total energy & $\mathbf{1 , 3 5 9 . 5}$ & $\mathbf{1 , 4 3 4 . 0}$ & $\mathbf{1 , 4 8 5 . 1}$ & $\mathbf{1 , 5 0 4 . 4}$ & $\mathbf{1 , 5 1 2 . 3}$ & $\mathbf{1 , 5 3 0 . 0}$ & $\mathbf{1 , 5 7 8 . 2}$ & $\mathbf{1 , 5 5 8 . 6}$ \\
\hline
\end{tabular}

\section{Source:}

U.S. Department of Energy, Energy Information Administration, Emissions of Greenhouse Gases in the United States, 2001, Washington, DC, December 2002, Table 5, and annual.

(Additional resources: www.eia.doe.gov)

${ }^{a}$ Includes energy from petroleum, coal, and natural gas. Electric utility emissions are distributed across consumption sectors. 
Most U.S. transportation sector carbon emissions come from petroleum fuels (98\%). Motor gasoline has been responsible for about $60 \%$ of U.S. carbon emissions over the last twenty years.

Table 11.5

U.S. Carbon Emissions from Energy Use in the Transportation Sector, 1990-2001 (million metric tons of carbon)

\begin{tabular}{|c|c|c|c|c|c|c|}
\hline \multirow[b]{2}{*}{ Fuel } & \multicolumn{2}{|c|}{1990} & \multicolumn{2}{|c|}{1995} & \multicolumn{2}{|c|}{2001} \\
\hline & Emissions & Percentage & Emissions & Percentage & Emissions & Percentage \\
\hline & \multicolumn{6}{|c|}{ Petroleum } \\
\hline $\begin{array}{l}\text { Motor } \\
\text { gasoline }\end{array}$ & 260.5 & $60.4 \%$ & 279.0 & $61.0 \%$ & 308.0 & $60.2 \%$ \\
\hline $\mathrm{LPG}^{\mathrm{a}}$ & 0.4 & $0.1 \%$ & 0.3 & $0.1 \%$ & 0.2 & $0.0 \%$ \\
\hline Jet fuel & 60.1 & $13.9 \%$ & 60.0 & $13.1 \%$ & 65.6 & $12.8 \%$ \\
\hline Distillate fuel & 75.6 & $17.5 \%$ & 85.1 & $18.6 \%$ & 107.5 & $21.0 \%$ \\
\hline Residual fuel & 21.6 & $5.0 \%$ & 19.4 & $4.2 \%$ & 17.8 & $3.5 \%$ \\
\hline Lubricants & 1.8 & $0.4 \%$ & 1.7 & $0.4 \%$ & 1.6 & $0.3 \%$ \\
\hline Aviation gas & 0.8 & $0.2 \%$ & 0.7 & $0.2 \%$ & 0.7 & $0.1 \%$ \\
\hline \multirow[t]{2}{*}{ Subtotal } & 420.8 & $97.5 \%$ & 446.2 & $97.5 \%$ & 501.4 & $98.0 \%$ \\
\hline & \multicolumn{6}{|c|}{ Other energy } \\
\hline Natural gas & 9.8 & $2.3 \%$ & 10.4 & $2.3 \%$ & 9.2 & $1.8 \%$ \\
\hline Electricity $^{\mathrm{b}}$ & 0.7 & $0.2 \%$ & 0.9 & $0.2 \%$ & 1.0 & $0.2 \%$ \\
\hline Total & 431.3 & $100.0 \%$ & 457.5 & $100.0 \%$ & 511.6 & $100.0 \%$ \\
\hline
\end{tabular}

\section{Source:}

U.S. Department of Energy, Energy Information Administration, Emissions of Greenhouse Gases in the United States, 2001, Washington, DC, December 2002, Table 9, and annual.

(Additional resources: www.eia.doe.gov)

${ }^{a}$ Liquified petroleum gas.

${ }^{\mathrm{b}}$ Share of total electric utility carbon dioxide emissions weighted by sales to the transportation sector. 


\section{The Greenhouse Gases, Regulated Emissions, and Energy Use in Transportation (GREET) Model}

\section{greet.anl.gov}

The GREET model, which is sponsored by the Department of Energy, estimates the full fuel-cycle emissions and energy use associated with various transportation fuels and advanced transportation technologies for light-duty vehicles. It calculates fuel-cycle emissions of three greenhouse gases (carbon dioxide, methane, and nitrous oxide) and five criteria pollutants (volatile organic compounds, carbon monoxide, nitrogen oxides, sulfur oxides, and particulate matter measuring 10 microns or less). The model also calculates the total fuel-cycle energy consumption, fossil fuel consumption, and petroleum consumption using various transportation fuels. The fuel cycles that are included in the GREET model are:

- petroleum to conventional gasoline, reformulated gasoline, conventional diesel, reformulated diesel, liquefied petroleum gas, and electricity via residual oil;

- natural gas to compressed natural gas, liquefied natural gas, liquefied petroleum gas, methanol, FischerTropsch diesel, dimethyl ether, hydrogen, and electricity;

- coal to electricity;

- uranium to electricity;

- renewable energy (hydropower, solar energy, and wind) to electricity;

- corn, woody biomass, and herbaceous biomass to ethanol;

- $\quad$ soybeans to biodiesel; and

- landfill gases to methanol.

For additional information about the GREET model, see the GREET website, or contact:

Michael Q. Wang

Argonne National Laboratory

9700 South Cass Avenue, ES/362

Argonne, IL 60439-4815

phone: $630-252-2819$

fax: 630-252-3443

email: mqwang@anl.gov 


\section{Chapter 12 \\ Criteria Air Pollutants}

Summary Statistics from Tables in this Chapter

\begin{tabular}{ccc}
\hline Source & & \\
\hline Table 12.1 & Transportation's share of U.S. emissions, 2001 & \\
& $\mathrm{CO}$ & $82.4 \%$ \\
& $\mathrm{NO}_{\mathrm{X}}$ & $55.5 \%$ \\
$\mathrm{VOC}$ & $41.7 \%$ \\
& $\mathrm{PM}-10$ & $2.2 \%$ \\
& $\mathrm{PM}-2.5$ & $6.1 \%$ \\
& $\mathrm{SO}_{2}$ & $4.4 \%$ \\
$\mathrm{NH}_{3}$ & $6.3 \%$ \\
\hline
\end{tabular}


Transportation accounts for the majority of carbon monoxide and nitrogen oxide emissions. Highway vehicles are responsible for the largest share of transportation emissions.

Table 12.1

Total National Emissions of the Criteria Air Pollutants by Sector, 2001 (millions of short tons/percentage)

\begin{tabular}{|c|c|c|c|c|c|c|c|}
\hline Sector & $\mathrm{CO}$ & $\mathrm{NO}_{\mathrm{x}}$ & VOC & PM-10 & PM-2.5 & $\mathrm{SO}_{2}$ & $\mathbf{N H}_{3}$ \\
\hline \multirow[t]{2}{*}{ Highway vehicles } & 74.83 & 8.25 & 4.87 & 0.22 & 0.16 & 0.26 & 0.28 \\
\hline & $62.0 \%$ & $36.9 \%$ & $27.1 \%$ & $0.9 \%$ & $2.2 \%$ & $1.7 \%$ & $5.6 \%$ \\
\hline \multirow[t]{2}{*}{ Aircraft } & 0.26 & 0.08 & 0.02 & 0.00 & 0.00 & 0.01 & $\mathbf{0 . 0 0}$ \\
\hline & $0.2 \%$ & $0.4 \%$ & $0.1 \%$ & $0.0 \%$ & $0.0 \%$ & $0.1 \%$ & $0.0 \%$ \\
\hline \multirow[t]{2}{*}{ Railroads } & 0.10 & 1.00 & 0.04 & 0.03 & 0.02 & 0.06 & 0.00 \\
\hline & $0.1 \%$ & $4.5 \%$ & $0.2 \%$ & $0.1 \%$ & $0.3 \%$ & $0.4 \%$ & $0.0 \%$ \\
\hline \multirow[t]{2}{*}{ Vessels } & 0.13 & 1.01 & 0.03 & 0.04 & 0.04 & 0.16 & 0.00 \\
\hline & $0.1 \%$ & $4.5 \%$ & $0.2 \%$ & $0.2 \%$ & $0.5 \%$ & $1.0 \%$ & $0.0 \%$ \\
\hline \multirow[t]{2}{*}{ Other off-highway } & 24.19 & 2.07 & 2.53 & 0.24 & 0.23 & 0.22 & 0.04 \\
\hline & $20.0 \%$ & $9.2 \%$ & $14.1 \%$ & $1.0 \%$ & $3.1 \%$ & $1.4 \%$ & $0.7 \%$ \\
\hline \multirow[t]{2}{*}{ Transportation total } & 99.50 & 12.41 & 7.50 & 0.53 & 0.45 & 0.70 & 0.32 \\
\hline & $82.4 \%$ & $55.5 \%$ & $41.7 \%$ & $2.2 \%$ & $6.1 \%$ & $4.4 \%$ & $6.3 \%$ \\
\hline \multirow[t]{2}{*}{ Stationary source fuel combustion } & 4.59 & 8.60 & 1.18 & 1.50 & 1.32 & 13.63 & $\mathbf{0 . 0 7}$ \\
\hline & $3.8 \%$ & $38.5 \%$ & $6.6 \%$ & $6.2 \%$ & $17.9 \%$ & $86.3 \%$ & $1.4 \%$ \\
\hline \multirow[t]{2}{*}{ Industrial processes } & 2.74 & 0.86 & 7.45 & 0.76 & 0.52 & 1.41 & 0.17 \\
\hline & $2.3 \%$ & $3.9 \%$ & $41.5 \%$ & $3.1 \%$ & $7.1 \%$ & $8.9 \%$ & $3.4 \%$ \\
\hline \multirow[t]{2}{*}{ Waste disposal and recycling total } & 3.23 & 0.17 & 0.54 & 0.50 & 0.48 & 0.04 & 0.09 \\
\hline & $2.7 \%$ & $0.8 \%$ & $3.0 \%$ & $2.1 \%$ & $6.4 \%$ & $0.2 \%$ & $1.8 \%$ \\
\hline \multirow[t]{2}{*}{ Miscellaneous } & 10.69 & 0.31 & 1.29 & 20.80 & 4.61 & 0.01 & 4.35 \\
\hline & $8.9 \%$ & $1.4 \%$ & $7.2 \%$ & $86.3 \%$ & $62.5 \%$ & $0.1 \%$ & $87.1 \%$ \\
\hline \multirow[t]{2}{*}{ Total of all sources } & 120.76 & 22.35 & 17.96 & 24.10 & 7.38 & 15.79 & 5.00 \\
\hline & $100.0 \%$ & $100.0 \%$ & $100.0 \%$ & $100.0 \%$ & $100.0 \%$ & $100.0 \%$ & $100.0 \%$ \\
\hline
\end{tabular}

Source:

U. S. Environmental Protection Agency, National Emission Inventory Air Pollutant Emission Trends website www.epa.gov/ttn/chief/trends (Additional resources: www.epa.gov/oar/oaqps)

Note:

$\mathrm{CO}=$ Carbon monoxide $. \mathrm{NO}_{\mathrm{x}}=$ Nitrogen oxides. $\mathrm{PM}-10=$ Particulate matter less than 10 microns.

PM-2.5 = Particulate matter less than 2.5 microns. $\mathrm{SO}_{2}=$ Sulfur dioxide. $\mathrm{VOC}=$ Volatile organic compounds. $\mathrm{NH}_{3}=$ Ammonia. 
The transportation sector accounted for more than $80 \%$ of the nation's carbon monoxide (CO) emissions in 2001. Highway vehicles are by far the source of the greatest amount of CO. For details on the highway emissions of CO, see Table 12.3.

Table 12.2

Total National Emissions of Carbon Monoxide, 1980-2001

(million short tons)

\begin{tabular}{|c|c|c|c|c|c|c|c|}
\hline Source category & 1980 & 1985 & 1990 & 1995 & 2000 & 2001 & $\begin{array}{c}\text { Percent } \\
\text { of total, } \\
2001 \\
\end{array}$ \\
\hline Highway vehicles & 143.83 & 134.19 & 110.26 & 83.88 & 68.06 & 74.83 & $62.0 \%$ \\
\hline Aircraft & 0.21 & 0.22 & 0.24 & 0.25 & 0.27 & 0.26 & $0.2 \%$ \\
\hline Railroads & 0.12 & 0.10 & 0.09 & 0.10 & 0.10 & 0.10 & $0.1 \%$ \\
\hline Vessels $^{\mathrm{b}}$ & 0.13 & 0.14 & 0.13 & 0.14 & 0.13 & 0.13 & $0.1 \%$ \\
\hline Other off-highway & 16.23 & 18.58 & 20.98 & 23.39 & 23.68 & 24.19 & $20.0 \%$ \\
\hline Transportation total & 160.51 & 153.22 & 131.70 & 107.76 & 92.24 & 99.50 & $82.4 \%$ \\
\hline Stationary fuel combustion total & 7.30 & 8.49 & 5.51 & 5.93 & 4.60 & 4.59 & $3.8 \%$ \\
\hline Industrial processes total & 6.95 & 5.28 & 4.77 & 4.61 & 2.62 & 2.74 & $2.3 \%$ \\
\hline Waste disposal and recycling total & 2.30 & 1.94 & 1.08 & 1.19 & 3.23 & 3.23 & $2.7 \%$ \\
\hline Miscellaneous total & 8.34 & 7.93 & 11.12 & 7.30 & 20.90 & 10.69 & $8.9 \%$ \\
\hline Total of all sources & 185.41 & 176.85 & 154.19 & 126.78 & 123.59 & 120.76 & $100.0 \%$ \\
\hline
\end{tabular}

\section{Source:}

U. S. Environmental Protection Agency, National Emission Inventory Air Pollutant Emission Trends website www.epa.gov/ttn/chief/trends (Additional resources: www.epa.gov/oar/oaqps)

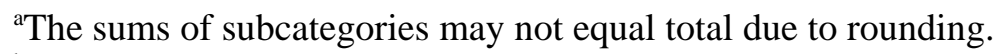

${ }^{\mathrm{b}}$ Recreational marine vessels. 
Though gasoline-powered light vehicles continue to be responsible for the majority of carbon monoxide emissions from highway vehicles, the total pollution from light vehicles in 2001 is less than half what it was in 1980. This is despite the fact that there were many more light vehicles on the road in 2001.

Table 12.3

Emissions of Carbon Monoxide from Highway Vehicles, 1980-2001 ${ }^{\mathrm{a}}$ (million short tons)

\begin{tabular}{|c|c|c|c|c|c|c|c|}
\hline Source category & 1980 & 1985 & 1990 & 1995 & 2000 & 2001 & $\begin{array}{l}\text { Percent of } \\
\text { total, } 2001\end{array}$ \\
\hline \multicolumn{8}{|c|}{ Gasoline powered } \\
\hline Light vehicles \& motorcycles & 98.21 & 87.80 & 67.24 & 46.54 & 36.40 & 41.23 & $55.1 \%$ \\
\hline Light trucks ${ }^{\mathrm{b}}$ & 28.83 & 32.11 & 32.23 & 29.81 & 27.04 & 29.33 & $39.2 \%$ \\
\hline Heavy vehicles & 15.35 & 12.40 & 8.92 & 5.96 & 3.42 & 3.13 & $4.2 \%$ \\
\hline Total & 142.39 & 132.32 & 108.39 & 82.31 & 66.86 & 73.70 & $98.5 \%$ \\
\hline \multicolumn{8}{|c|}{ Diesel powered } \\
\hline Light vehicles & 0.03 & 0.04 & 0.04 & 0.02 & 0.01 & 0.01 & $0.0 \%$ \\
\hline Light trucks ${ }^{\mathrm{b}}$ & 0.05 & 0.04 & 0.03 & 0.02 & 0.01 & 0.01 & $0.0 \%$ \\
\hline Heavy vehicles & 1.36 & 1.80 & 1.81 & 1.53 & 1.19 & 1.12 & $1.5 \%$ \\
\hline Total & 1.43 & 1.87 & 1.87 & 1.57 & 1.20 & 1.13 & $1.5 \%$ \\
\hline \multicolumn{8}{|c|}{ Total } \\
\hline Highway vehicle total & 143.83 & 134.19 & 110.26 & 83.88 & 68.06 & 74.83 & $100.0 \%$ \\
\hline Percent diesel & $1.0 \%$ & $1.4 \%$ & $1.7 \%$ & $1.9 \%$ & $1.8 \%$ & $1.5 \%$ & \\
\hline
\end{tabular}

\section{Source:}

U. S. Environmental Protection Agency, National Emission Inventory Air Pollutant Emission Trends website www.epa.gov/ttn/chief/trends (Additional resources: www.epa.gov/oar/oaqps)

${ }^{a}$ The sums of subcategories may not equal total due to rounding.

${ }^{\mathrm{b}}$ Less than 8,500 pounds. 
The transportation sector accounted for over half of the nation's nitrogen oxide (NOx) emissions in 2001, with the majority coming from highway vehicles. For details on the highway emissions of NOx, see Table 12.5 .

Table 12.4

Total National Emissions of Nitrogen Oxides, 1980-2001 ${ }^{\text {a }}$

(million short tons)

\begin{tabular}{lrrrrrrr}
\hline & & & & & & & $\begin{array}{c}\text { Percent } \\
\text { of total, }\end{array}$ \\
Source category & 1980 & 1985 & 1990 & 1995 & 2000 & 2001 & \multicolumn{1}{c}{2001} \\
\hline \multicolumn{1}{c}{ Highway vehicles } & 11.49 & 10.93 & 9.59 & 8.88 & 8.39 & 8.25 & $36.9 \%$ \\
$\quad$ Railroads & 1.19 & 0.96 & 0.95 & 1.03 & 1.00 & 1.00 & $4.5 \%$ \\
$\quad$ Other off-highway & 2.17 & 2.62 & 2.84 & 3.08 & 3.17 & 3.16 & $14.1 \%$ \\
Transportation total & 14.85 & 14.51 & 13.38 & 12.99 & 12.56 & 12.41 & $55.5 \%$ \\
Stationary fuel combustion total & 11.32 & 10.05 & 10.89 & 10.83 & 9.04 & 8.60 & $38.5 \%$ \\
Industrial processes total & 0.56 & 0.80 & 0.80 & 0.77 & 0.83 & 0.86 & $3.9 \%$ \\
Waste disposal and recycling total & 0.11 & 0.09 & 0.09 & 0.10 & 0.17 & 0.17 & $0.8 \%$ \\
Miscellaneous total & 0.25 & 0.31 & 0.37 & 0.27 & 0.61 & 0.31 & $1.4 \%$ \\
Total of all sources & $\mathbf{2 7 . 0 8}$ & $\mathbf{2 5 . 7 6}$ & $\mathbf{2 5 . 5 3}$ & $\mathbf{2 4 . 9 6}$ & $\mathbf{2 3 . 2 0}$ & $\mathbf{2 2 . 3 5}$ & $\mathbf{1 0 0 . 0 \%}$ \\
\hline
\end{tabular}

Source:

U. S. Environmental Protection Agency, National Emission Inventory Air Pollutant Emission Trends website www.epa.gov/ttn/chief/trends (Additional resources: www.epa.gov/oar/oaqps)

${ }^{a}$ The sums of subcategories may not equal total due to rounding. 
Heavy diesel-powered vehicles were responsible for nearly one-half of highway vehicle nitrogen oxide emissions in 2001, while light gasoline vehicles were responsible for the rest.

Table 12.5

Emissions of Nitrogen Oxides from Highway Vehicles, 1980-2001 (million short tons)

\begin{tabular}{|c|c|c|c|c|c|c|c|}
\hline Source category & 1980 & 1985 & 1990 & 1995 & 2000 & 2001 & $\begin{array}{l}\text { Percent of } \\
\text { total, } 2001\end{array}$ \\
\hline \multicolumn{8}{|c|}{ Gasoline powered } \\
\hline Light vehicles \& motorcycles & 6.63 & 5.68 & 4.26 & 3.05 & 2.31 & 2.39 & $28.9 \%$ \\
\hline Light trucks ${ }^{\mathrm{b}}$ & 1.58 & 1.60 & 1.50 & 1.46 & 1.44 & 1.50 & $18.2 \%$ \\
\hline Heavy vehicles & 0.62 & 0.58 & 0.57 & 0.52 & 0.45 & 0.46 & $5.5 \%$ \\
\hline Total & 8.83 & 7.85 & 6.33 & 5.03 & 4.20 & 4.35 & $52.7 \%$ \\
\hline \multicolumn{8}{|c|}{ Diesel powered } \\
\hline Light vehicles & c & 0.04 & 0.04 & 0.02 & 0.01 & 0.01 & $0.1 \%$ \\
\hline Light trucks $^{\mathrm{b}}$ & c & c & 0.02 & 0.01 & 0.01 & 0.01 & $0.1 \%$ \\
\hline Heavy vehicles & 2.59 & 3.00 & 3.19 & 3.82 & 4.18 & 3.89 & $47.2 \%$ \\
\hline Total & 2.66 & 3.08 & 3.26 & 3.85 & 4.19 & 3.90 & $47.3 \%$ \\
\hline \multicolumn{8}{|c|}{ Total } \\
\hline Highway vehicle total & 11.49 & 10.93 & 9.59 & 8.88 & 8.69 & 8.25 & $100.0 \%$ \\
\hline Percent diesel & $23.1 \%$ & $28.2 \%$ & $34.0 \%$ & $43.4 \%$ & $48.2 \%$ & $47.3 \%$ & \\
\hline
\end{tabular}

Source:

U. S. Environmental Protection Agency, National Emission Inventory Air Pollutant Emission Trends website www.epa.gov/ttn/chief/trends (Additional resources: www.epa.gov/oar/oaqps)

${ }^{\mathrm{a}}$ The sums of subcategories may not equal total due to rounding.

${ }^{\mathrm{b}}$ Less than 8,500 pounds.

${ }^{\mathrm{c}}$ Data are not available. 
The transportation sector accounted for over $40 \%$ of the nation's volatile organic compound (VOC) emissions in 2001, with the majority coming from highway vehicles. For details on the highway emissions of VOC, see Table 12.7.

Table 12.6

Total National Emissions of Volatile Organic Compounds, 1980-2001 (million short tons)

\begin{tabular}{lrrrrrrr}
\hline & & & & & & \multicolumn{3}{c}{$\begin{array}{c}\text { Percent } \\
\text { of total, }\end{array}$} \\
Source category & 1980 & 1985 & 1990 & 1995 & 2000 & 2001 & 2001 \\
\hline \multicolumn{1}{c}{ Highway vehicles } & 13.87 & 12.65 & 9.39 & 6.75 & 5.33 & 4.87 & $27.1 \%$ \\
Off-highway & 2.19 & 2.44 & 2.66 & 2.89 & 2.64 & 2.62 & $14.6 \%$ \\
Transportation total & 16.06 & 15.09 & 12.05 & 9.64 & 7.97 & 7.50 & $41.7 \%$ \\
Stationary fuel combustion total & 1.05 & 1.57 & 1.01 & 1.07 & 1.18 & 1.18 & $6.6 \%$ \\
Industrial processes total & 12.10 & 9.50 & 9.01 & 9.71 & 7.28 & 7.45 & $41.5 \%$ \\
Waste disposal and recycling total & 0.76 & 0.98 & 0.99 & 1.07 & 0.54 & 0.54 & $3.0 \%$ \\
Miscellaneous total & 1.13 & 0.57 & 1.06 & 0.55 & 2.74 & 1.29 & $7.2 \%$ \\
Total of all sources & $\mathbf{3 1 . 1 1}$ & $\mathbf{2 7 . 7 0}$ & $\mathbf{2 4 . 1 2}$ & $\mathbf{2 2 . 0 4}$ & $\mathbf{1 9 . 7 0}$ & $\mathbf{1 7 . 9 6}$ & $\mathbf{1 0 0 . 0 \%}$ \\
\hline
\end{tabular}

\section{Source:}

U. S. Environmental Protection Agency, National Emission Inventory Air Pollutant Emission Trends website www.epa.gov/ttn/chief/trends (Additional resources: www.epa.gov/oar/oaqps)

${ }^{a}$ The sum of subcategories may not equal total due to rounding. The EPA's definition of volatile organic compounds excludes methane, ethane, and certain other nonphotochemically reactive organic compounds. 
Gasoline-powered vehicles are responsible for $80 \%$ of highway vehicle emissions of volatile organic compounds. VOC emissions from highway vehicles in 2001 were less than half the 1980 level.

Table 12.7

Emissions of Volatile Organic Compounds from Highway Vehicles, 1980-2001 (thousand short tons)

\begin{tabular}{|c|c|c|c|c|c|c|c|}
\hline Source category & 1980 & 1985 & 1990 & 1995 & 2000 & 2001 & $\begin{array}{l}\text { Percent of } \\
\text { total, } 2001\end{array}$ \\
\hline \multicolumn{8}{|c|}{ Gasoline powered } \\
\hline Light vehicles \& motorcycles & 9,304 & 7,962 & 5,690 & 3,768 & 2,903 & 2,620 & $45.0 \%$ \\
\hline Light trucks ${ }^{\mathrm{b}}$ & 2,864 & 2,908 & 2,617 & 2,225 & 1,929 & 1,805 & $31.0 \%$ \\
\hline Heavy vehicles & 1,198 & 959 & 633 & 421 & 256 & 224 & $3.9 \%$ \\
\hline Total & 13,366 & 11,829 & 8,940 & 6,414 & 5,088 & 4,649 & $79.9 \%$ \\
\hline \multicolumn{8}{|c|}{ Diesel powered } \\
\hline Light vehicles & 16 & 19 & 18 & 9 & 3 & 3 & $0.1 \%$ \\
\hline Light trucks ${ }^{\mathrm{b}}$ & 28 & 22 & 15 & 10 & 4 & 4 & $0.1 \%$ \\
\hline Heavy vehicles & 459 & 483 & 415 & 315 & 230 & 218 & $3.7 \%$ \\
\hline Total & 503 & 525 & 448 & 335 & 238 & 225 & $3.9 \%$ \\
\hline \multicolumn{8}{|c|}{ Total } \\
\hline Highway vehicle total & 13,869 & 10,545 & 9,388 & 9,376 & 6,443 & 5,816 & $100.0 \%$ \\
\hline Percent diesel & $3.6 \%$ & $5.0 \%$ & $4.8 \%$ & $3.6 \%$ & $3.7 \%$ & $3.9 \%$ & \\
\hline
\end{tabular}

Source:

U. S. Environmental Protection Agency, National Emission Inventory Air Pollutant Emission Trends website www.epa.gov/ttn/chief/trends (Additional resources: www.epa.gov/oar/oaqps)

${ }^{\text {a }}$ The sums of subcategories may not equal total due to rounding.

${ }^{\mathrm{b}}$ Less than 8,500 pounds. 
The transportation sector accounted for only $2 \%$ of the nation's particulate matter (PM-10) emissions in 2001. For details on the highway emissions of PM-10, see Table 12.9.

Table 12.8

Total National Emissions of Particulate Matter (PM-10), 1980-2001 ${ }^{\mathrm{a}}$ (million short tons)

\begin{tabular}{|c|c|c|c|c|c|c|c|}
\hline Source category & 1980 & 1985 & 1990 & 1995 & 2000 & 2001 & $\begin{array}{c}\text { Percent } \\
\text { of total, } \\
2001\end{array}$ \\
\hline Highway vehicles & 0.43 & 0.41 & 0.39 & 0.30 & 0.23 & 0.22 & $0.9 \%$ \\
\hline Off-highway & 0.26 & 0.30 & 0.33 & 0.34 & 0.32 & 0.32 & $1.3 \%$ \\
\hline Transportation total & 0.69 & 0.71 & 0.72 & 0.64 & 0.55 & 0.53 & $2.2 \%$ \\
\hline $\begin{array}{l}\text { Stationary fuel combustion } \\
\text { total }\end{array}$ & 2.45 & 1.54 & 1.20 & 1.18 & 1.53 & 1.50 & $6.2 \%$ \\
\hline Industrial processes total & 2.75 & 1.06 & 1.04 & 0.95 & 0.73 & 0.76 & $3.1 \%$ \\
\hline $\begin{array}{l}\text { Waste disposal and } \\
\text { recycling total }\end{array}$ & 0.27 & 0.28 & 0.27 & 0.29 & 0.50 & 0.50 & $2.1 \%$ \\
\hline $\begin{array}{l}\text { Fugitive dust } \\
\text { Other miscellaneous }\end{array}$ & $\begin{array}{l}\mathrm{b} \\
\mathrm{b}\end{array}$ & $\begin{array}{r}29.73 \\
8.01\end{array}$ & $\begin{array}{r}18.08 \\
6.46\end{array}$ & $\begin{array}{r}17.01 \\
5.76\end{array}$ & $\begin{array}{r}14.31 \\
7.08\end{array}$ & $\begin{array}{r}14.66 \\
6.14\end{array}$ & $\begin{array}{l}60.8 \% \\
25.5 \%\end{array}$ \\
\hline Miscellaneous total & 0.85 & 37.74 & 24.54 & 22.77 & 21.39 & 20.80 & $86.3 \%$ \\
\hline Total of all sources & 7.01 & 41.32 & 27.76 & 25.82 & 24.70 & 24.10 & $100.0 \%$ \\
\hline
\end{tabular}

Source:

U. S. Environmental Protection Agency, National Emission Inventory Air Pollutant Emission Trends website www.epa.gov/ttn/chief/trends (Additional resources: www.epa.gov/oar/oaqps)

Note:

Because PM-10 is fine particle matter less than 10 microns, it also includes PM-2.5. Specific data for PM-2.5 are shown on Tables 12.10 and 12.11.

${ }^{a}$ Fine particle matter less than 10 microns. The sums of subcategories may not equal total due to rounding.

${ }^{\mathrm{b}}$ Data are not available. 
Since 1985, diesel-powered vehicles have been responsible for more than half of highway vehicle emissions of particulate matter (PM-10). Heavy vehicles are clearly the main source.

Table 12.9

Emissions of Particulate Matter (PM-10) from Highway Vehicles, 1980-2001 ${ }^{\mathrm{a}}$ (thousand short tons)

\begin{tabular}{|c|c|c|c|c|c|c|c|}
\hline Source category & 1980 & 1985 & 1990 & 1995 & 2000 & 2001 & $\begin{array}{l}\text { Percent of total, } \\
2001\end{array}$ \\
\hline \multicolumn{8}{|c|}{ Gasoline powered } \\
\hline Light vehicles \& motorcycles & 141 & 86 & 57 & 53 & 51 & 51 & $23.4 \%$ \\
\hline Light trucks ${ }^{\mathrm{b}}$ & 49 & 37 & 31 & 32 & 31 & 31 & $14.2 \%$ \\
\hline Heavy vehicles & 30 & 23 & 17 & 13 & 10 & 10 & $4.6 \%$ \\
\hline Total & 220 & 146 & 105 & 98 & 92 & 92 & $42.2 \%$ \\
\hline \multicolumn{8}{|c|}{ Diesel powered } \\
\hline Light vehicles & 9 & 13 & 11 & 4 & 1 & 1 & $0.5 \%$ \\
\hline Light trucks ${ }^{\mathrm{b}}$ & 12 & 8 & 5 & 3 & 1 & 1 & $0.5 \%$ \\
\hline Heavy vehicles & 191 & 240 & 266 & 199 & 135 & 125 & $57.3 \%$ \\
\hline Total & 212 & 262 & 282 & 206 & 137 & 127 & $58.3 \%$ \\
\hline \multicolumn{8}{|c|}{ Total } \\
\hline Highway vehicle total & 432 & 408 & 387 & 304 & 230 & 218 & $100.0 \%$ \\
\hline Percent diesel & $49.1 \%$ & $64.2 \%$ & $72.9 \%$ & $67.8 \%$ & $59.6 \%$ & $58.3 \%$ & \\
\hline
\end{tabular}

Source:

U. S. Environmental Protection Agency, National Emission Inventory Air Pollutant Emission Trends website www.epa.gov/ttn/chief/trends (Additional resources: www.epa.gov/oar/oaqps)

Note:

Because PM-10 is fine particle matter less than 10 microns, it also includes PM-2.5. Specific data for PM-2.5 are shown on Tables 12.10 and 12.11.

${ }^{\text {a }}$ The sums of subcategories may not equal total due to rounding.

${ }^{\mathrm{b}}$ Less than 8,500 pounds. 
The transportation sector accounted for only 6\% of the nation's particulate matter (PM-2.5) emissions in 2001. For details on the highway emissions of PM-2.5, see Table 12.11.

Table 12.10

Total National Emissions of Particulate Matter (PM-2.5), 1990-2001 (million short tons)

\begin{tabular}{lllllc}
\hline & & & & & $\begin{array}{c}\text { Percent } \\
\text { of total, }\end{array}$ \\
Source category & 1990 & 1995 & 2000 & 2001 & 2001 \\
\hline \multicolumn{1}{c}{$\begin{array}{l}\text { Highway vehicles } \\
\text { Off-highway }\end{array}$} & 0.32 & 0.25 & 0.17 & 0.16 & $2.2 \%$ \\
Transportation total & 0.30 & 0.31 & 0.30 & 0.29 & $3.9 \%$ \\
Stationary fuel combustion total & 0.63 & 0.56 & 0.47 & 0.45 & $6.1 \%$ \\
Industrial processes total & 0.91 & 0.90 & 1.34 & 1.32 & $17.9 \%$ \\
Waste disposal and recycling total & 0.56 & 0.50 & 0.50 & 0.52 & $7.1 \%$ \\
$\quad \begin{array}{l}\text { Fugitive dust } \\
\quad \text { Other miscellaneous }\end{array}$ & 0.23 & 0.25 & 0.47 & 0.48 & $6.4 \%$ \\
Miscellaneous total & 3.17 & 3.04 & 2.57 & 2.63 & $35.6 \%$ \\
$\quad$ Total of all sources & 2.06 & 1.69 & 2.82 & 1.98 & $26.8 \%$ \\
\hline
\end{tabular}

Source:

U. S. Environmental Protection Agency, National Emission Inventory Air Pollutant Emission Trends website www.epa.gov/ttn/chief/trends (Additional resources: www.epa.gov/oar/oaqps) 
Diesel vehicles are responsible for the majority of highway vehicle PM-2.5 emissions. More than twothirds of the highway vehicles' PM-2.5 emissions are from heavy diesel trucks.

Table 12.11

Emissions of Particulate Matter (PM-2.5) from Highway Vehicles, 1990-2001 (thousand short tons)

\begin{tabular}{|c|c|c|c|c|c|}
\hline Source category & 1990 & 1995 & 2000 & 2001 & $\begin{array}{l}\text { Percent of } \\
\text { total, } 2001\end{array}$ \\
\hline \multicolumn{6}{|c|}{ Gasoline powered } \\
\hline Light vehicles \& motorcycles & 35 & 30 & 27 & 27 & $16.7 \%$ \\
\hline Light trucks ${ }^{\mathrm{b}}$ & 21 & 20 & 18 & 17 & $10.5 \%$ \\
\hline Heavy vehicles & 11 & 9 & 7 & 7 & $4.3 \%$ \\
\hline Total & 67 & 59 & 52 & 51 & $31.5 \%$ \\
\hline \multicolumn{6}{|c|}{ Diesel powered } \\
\hline Light vehicles & 10 & 4 & 1 & 1 & $0.6 \%$ \\
\hline Light trucks ${ }^{\mathrm{b}}$ & 4 & 2 & 1 & 1 & $0.6 \%$ \\
\hline Heavy vehicles & 243 & 179 & 119 & 109 & $67.3 \%$ \\
\hline Total & 257 & 185 & 121 & 111 & $68.5 \%$ \\
\hline \multicolumn{6}{|c|}{ Total } \\
\hline Highway vehicle total & 324 & 245 & 173 & 162 & $100.0 \%$ \\
\hline Percent diesel & $79.3 \%$ & $75.5 \%$ & $69.9 \%$ & $68.5 \%$ & \\
\hline
\end{tabular}

Source:

U. S. Environmental Protection Agency, National Emission Inventory Air Pollutant Emission Trends website www.epa.gov/ttn/chief/trends (Additional resources: www.epa.gov/oar/oaqps)

${ }^{\mathrm{a}}$ The sums of subcategories may not equal total due to rounding.

${ }^{\mathrm{b}}$ Less than 8,500 pounds. 


\section{The Greenhouse Gases, Regulated Emissions, and Energy Use in Transportation (GREET) Model}

\section{greet.anl.gov}

The GREET model, which is sponsored by the Department of Energy, estimates the full fuel-cycle emissions and energy use associated with various transportation fuels and advanced transportation technologies for light vehicles. It calculates fuel-cycle emissions of five criteria pollutants (volatile organic compounds, carbon monoxide, nitrogen oxides, sulfur oxides, and particulate matter measuring 10 microns or less) and three greenhouse gases (carbon dioxide, methane, and nitrous oxide). The model also calculates the total fuel-cycle energy consumption, fossil fuel consumption, and petroleum consumption using various transportation fuels. The fuel cycles that are included in the GREET model are:

- petroleum to conventional gasoline, reformulated gasoline, conventional diesel, reformulated diesel, liquefied petroleum gas, and electricity via residual oil;

- natural gas to compressed natural gas, liquefied natural gas, liquefied petroleum gas, methanol, Fischer-Tropsch diesel, dimethyl ether, hydrogen, and electricity;

- coal to electricity;

- uranium to electricity;

- renewable energy (hydropower, solar energy, and wind) to electricity;

- corn, woody biomass, and herbaceous biomass to ethanol;

- $\quad$ soybeans to biodiesel; and

- landfill gases to methanol.

For additional information about the GREET model, see the GREET website, or contact:

Michael Q. Wang

Argonne National Laboratory

9700 South Cass Avenue, ES/362

Argonne, IL 60439-4815

phone: $630-252-2819$

fax: 630-252-3443

email: mqwang@anl.gov 
Table 12.12

Tier 2 Emission Standards for Cars and Light Trucks Effective for 2004-2009 Model Years ${ }^{\mathrm{a}}$

(grams/mile)

\begin{tabular}{|c|c|c|c|c|c|}
\hline Bin & NMOG & $\mathrm{CO}$ & NOx & PM & $\mathrm{HCHO}$ \\
\hline \multicolumn{6}{|c|}{50,000 miles } \\
\hline $10^{\mathrm{b}}$ & 0.125 & 3.4 & 0.4 & c & 0.015 \\
\hline $9^{\mathrm{b}}$ & 0.075 & 3.4 & 0.2 & a & 0.015 \\
\hline 8 & 0.100 & 3.4 & 0.14 & $\mathrm{a}$ & 0.015 \\
\hline 7 & 0.075 & 3.4 & 0.11 & a & 0.015 \\
\hline 6 & 0.075 & 3.4 & 0.08 & $\mathrm{a}$ & 0.015 \\
\hline 5 & 0.075 & 3.4 & 0.05 & a & 0.015 \\
\hline \multicolumn{6}{|c|}{120,000 miles } \\
\hline $\mathrm{MDPV}^{\mathrm{b}}$ & 0.280 & 7.3 & 0.9 & 0.12 & 0.032 \\
\hline $10^{\mathrm{b}}$ & 0.156 & 4.2 & 0.6 & 0.08 & 0.018 \\
\hline $9^{\mathrm{b}}$ & 0.090 & 4.2 & 0.3 & 0.06 & 0.018 \\
\hline 8 & 0.125 & 4.2 & 0.2 & 0.02 & 0.018 \\
\hline 7 & 0.090 & 4.2 & 0.15 & 0.02 & 0.018 \\
\hline 6 & 0.090 & 4.2 & 0.10 & 0.01 & 0.018 \\
\hline 5 & 0.090 & 4.2 & 0.07 & 0.01 & 0.018 \\
\hline 4 & 0.070 & 2.1 & 0.04 & 0.01 & 0.011 \\
\hline 3 & 0.055 & 2.1 & 0.03 & 0.01 & 0.011 \\
\hline 2 & 0.010 & 2.1 & 0.02 & 0.01 & 0.004 \\
\hline 1 & 0.000 & 0.0 & 0.00 & 0.00 & 0.000 \\
\hline
\end{tabular}

Source:

Federal Register, Vol. 65, No. 28, Thursday, February 10, 2000, pp. 6822-6870.

Acronyms Used on Tables 12.12 and 12.13

$\mathrm{CO} \quad$ Carbon monoxide

GVW Gross vehicle weight

HC Hydrocarbons

HCHO Formaldehyde

LDT Light-duty truck

LEV Low-emission vehicle

LVW Loaded vehicle weight

MDPV Medium-duty passenger vehicle (8,500-10,000 lbs. GVWR)

NMOG Non-methane organic gases

NOx Nitrogen oxides

PC Passenger car

PM Particulate matter

SULEV Super-ultra-low-emission vehicle

ULEV Ultra-low-emission vehicle

ZEV Zero-emission vehicle

${ }^{\text {a }}$ Some temporary standards are not shown.

${ }^{\mathrm{b}}$ Bin expires after 2008.

${ }^{\mathrm{c}}$ No standard. 
Table 12.13

Light Vehicle Exhaust Emission Standards in Effect in 2009

When U.S. Tier 2 Standards are Final

(grams/mile)

Vehicle fuels: Gasoline AND diesel unless noted otherwise

Vehicle size: Up to 8,500 lbs GVW unless noted otherwise

\begin{tabular}{|c|c|c|c|c|c|c|c|c|c|c|c|c|}
\hline \multirow[t]{2}{*}{ Useful life: } & \multirow[b]{2}{*}{ Bins, category, size } & \multicolumn{6}{|c|}{50,000 miles } & \multicolumn{5}{|c|}{120,000 miles } \\
\hline & & NMOG & $\mathrm{CO}$ & NOx & $\mathrm{PM}$ & $\mathrm{HCHO}$ & $\mathrm{HC}+\mathrm{NOx}$ & NMOG & $\mathrm{CO}$ & NOx & $\mathrm{PM}$ & $\mathrm{HCHO}$ \\
\hline U.S. & Bins & & & & & & & & & & & \\
\hline emission & 8 & 0.100 & 3.4 & 0.14 & - & 0.015 & - & 0.125 & 4.2 & 0.20 & 0.02 & 0.018 \\
\hline \multirow[t]{8}{*}{ standards } & 7 & 0.075 & 3.4 & 0.11 & - & 0.015 & - & 0.090 & 4.2 & 0.15 & 0.02 & 0.018 \\
\hline & 6 & 0.075 & 3.4 & 0.08 & - & 0.015 & - & 0.090 & 4.2 & 0.10 & 0.01 & 0.018 \\
\hline & 5 & 0.075 & 3.4 & 0.05 & - & 0.015 & - & 0.090 & 4.2 & 0.07 & 0.01 & 0.018 \\
\hline & 4 & - & - & - & - & - & - & 0.070 & 2.1 & 0.04 & 0.01 & 0.011 \\
\hline & 3 & - & - & - & - & - & - & 0.055 & 2.1 & 0.03 & 0.01 & 0.011 \\
\hline & 2 & - & - & - & - & - & - & 0.010 & 2.1 & 0.02 & 0.01 & 0.004 \\
\hline & 1 & - & - & - & - & - & - & 0.000 & 0.0 & 0.00 & 0.00 & 0.000 \\
\hline & Average $^{\mathrm{a}}$ & - & - & - & - & - & - & - & - & 0.07 & - & - \\
\hline California & Category & \multicolumn{6}{|c|}{ (Diesel only) } & \multicolumn{5}{|c|}{ (Diesel only) } \\
\hline LEV II & $\mathrm{LEV}^{\mathrm{b}}$ & 0.075 & 3.4 & 0.05 & - & 0.015 & - & 0.090 & 4.2 & 0.07 & 0.01 & 0.018 \\
\hline emission & ULEV & 0.04 & 1.7 & 0.05 & - & 0.08 & - & 0.055 & 2.1 & 0.07 & 0.01 & 0.011 \\
\hline \multirow[t]{4}{*}{ standards } & SULEV & - & - & - & - & - & - & 0.010 & 1.0 & 0.02 & 0.01 & 0.004 \\
\hline & $\mathrm{ZEV}^{\mathrm{c}}$ & 0.00 & 0.0 & 0.00 & - & 0.00 & - & 0.000 & 0.0 & 0.00 & 0.00 & 0.000 \\
\hline & $\begin{array}{l}\text { Avg. for all PCs + LDTs } \\
0-3,750 \mathrm{lbs} \text { LVW }\end{array}$ & 0.038 & - & - & - & - & - & - & - & - & - & - \\
\hline & $\begin{array}{l}\text { Avg. for LDTs } 3,751 \mathrm{lbs} \\
\text { LVW - } 8,500 \mathrm{lbs} \text { GVW }\end{array}$ & 0.047 & - & - & - & - & - & - & - & - & - & - \\
\hline
\end{tabular}

Source:

U.S.: Federal Register, Vol. 65, No. 28, Thursday, February 10, 2000, pp. 6822-6870.

California Exhaust Emission Standards and Test Procedures for 2001 and Subsequent Model Passenger Cars, Light-Duty Trucks and Medium-Duty Vehicles, as of December 1, 1999 (adopted August 5, 1999), incorporated by reference in section 1961(d), title 13, CCR.

Note:

See acronym list on previous page.

a Includes medium-duty passenger vehicles which are also required to meet bin standards.

${ }^{\mathrm{b}}$ A LEV Option 1 with higher NOx levels also exists for up to 4\% of LDTs above 3,750 lbs.

${ }^{c}$ Only apply to PCs and LDTs 0-3750 lbs LVW. 
Table 12.14

Federal Exhaust Emission Certification Standards for Gasoline- and Diesel-Powered Light Vehicles ${ }^{a, b}$ (grams per mile)

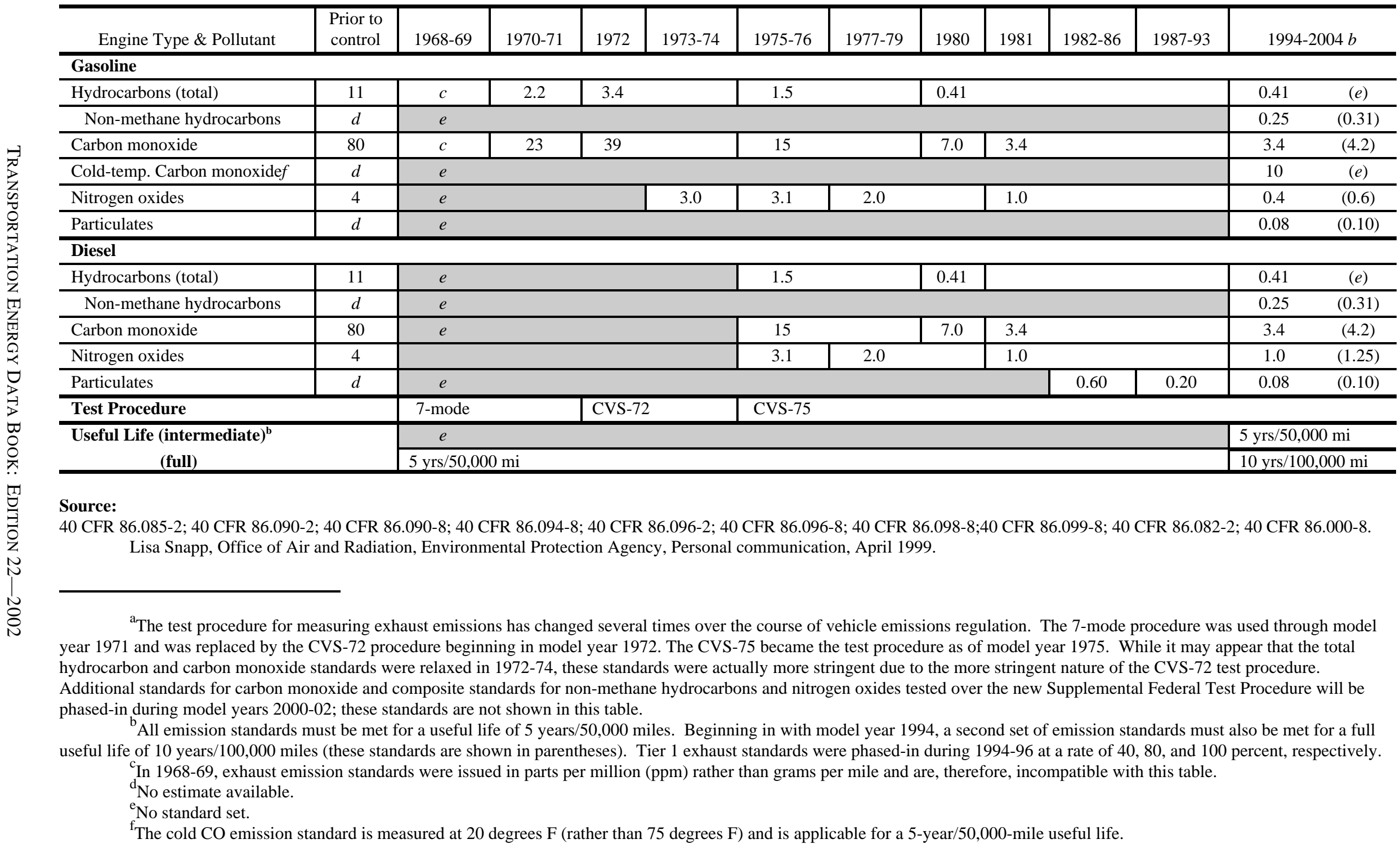


Table 12.15

Federal Exhaust Emission Certification Standards for Gasoline- and Diesel-Powered Light Trucks (Category LDT1) ${ }^{a, b, c}$ (grams per mile)

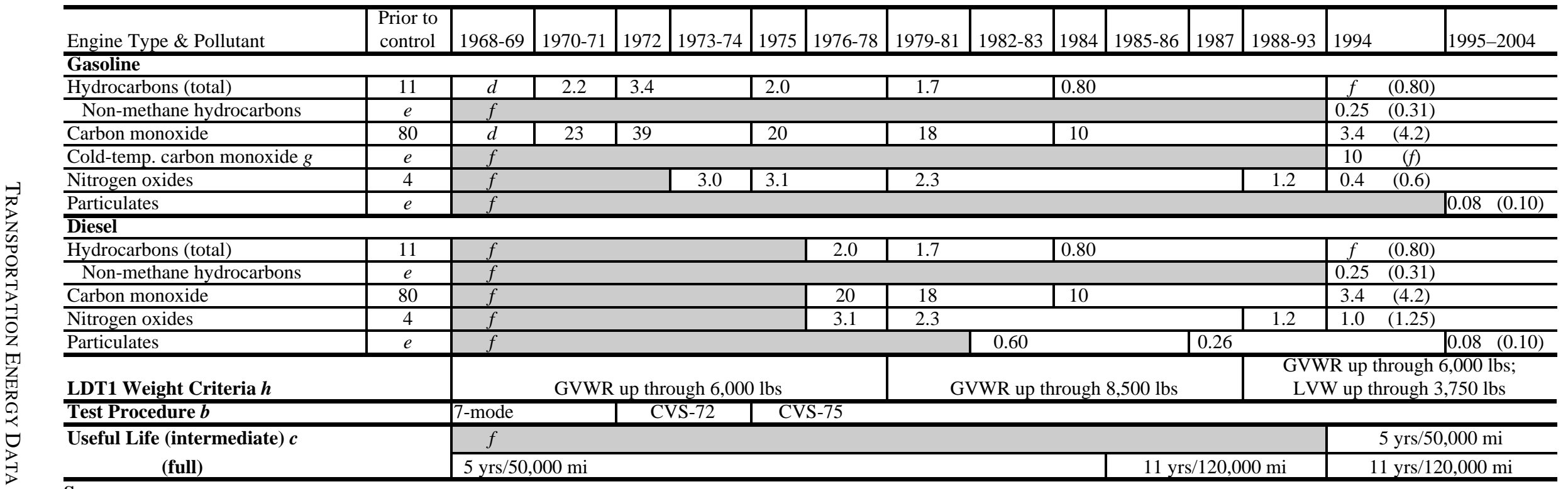

Source:

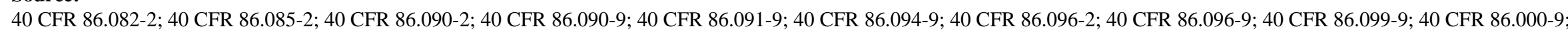

40 CFR 86.001-9; 40 CFR 86.004-9. Lisa Snapp, Office of Air and Radiation, Environmental Protection Agency, Personal communication.

${ }^{\text {a }}$ Light truck categories LDT1-LDT4 were not actually created until 1994. From 1968 to 1978 all trucks with a Gross Vehicle Weight Rating (GVWR) up to 6,000 lbs were classified as light trucks and were required to meet the same standards. As of 1979, the maximum weight was raised to 8,500 lbs GVWR. During 1988 through 1993, light trucks were divided into two subcategories that coincide with the current LDT1 and LDT2/3/4 categories.

${ }^{b}$ The test procedure for measuring exhaust emissions has changed several times over the course of vehicle emissions regulation. The 7-mode procedure was used through model year 1971 and was replaced by the CVS-72 procedure beginning in model year 1972. The CVS-75 became the test procedure as of model year 1975 . While it may appear that the total hydrocarbon and carbon monoxide standards were relaxed in 1972-74, these standards were actually more stringent due to the more stringent nature of the CVS-72 test procedure. Additional standards for carbon monoxide and composite standards for non-methane hydrocarbons and nitrogen oxides tested over the new Supplemental Federal Test Procedure will be phased-in during model years 2000-02; these standards are not shown in this table.

${ }^{c}$ Emission standards had to be met for a useful life of 5 years/50,000 miles through model year 1983, and a full useful life of 11 years 120,000 miles was defined for 1985-93

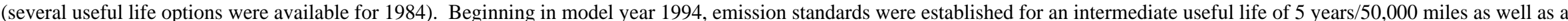
full useful life of 11 years/120,000 miles (these standards are shown in parentheses). Hydrocarbon standards, however, were established only for full useful life. Tier 1 exhaust standards, except PM standards, were phased-in during 1994-96 at a rate of 40, 80, and 100 percent, respectively. PM standards were phased-in at a rate of 40, 80, and 100 percent during $1995-97$.

${ }^{d}$ In 1968-69, exhaust emission standards were issued in parts per million (ppm) rather than grams per mile and are, therefore, incompatible with this table.

${ }^{\mathrm{e}}$ No estimate available.

fo standard set.

${ }_{\mathrm{g}}^{\mathrm{g}}$ The cold CO emission standard is measured at 20 degrees F (rather than 75 degrees F) and is applicable for a 5-year/50,000-mile useful life.

${ }^{\mathrm{h}}$ Gross vehicle weight rating (GVWR) is the maximum design loaded weight. Loaded vehicle weight (LVW) is the curb weight (nominal vehicle weight) plus 300 lbs. 
Federal Exhaust Emission Certification Standards for Gasoline- and Diesel-Powered Light Trucks (Category LDT2) ${ }^{a, b, c}$ (grams per mile)

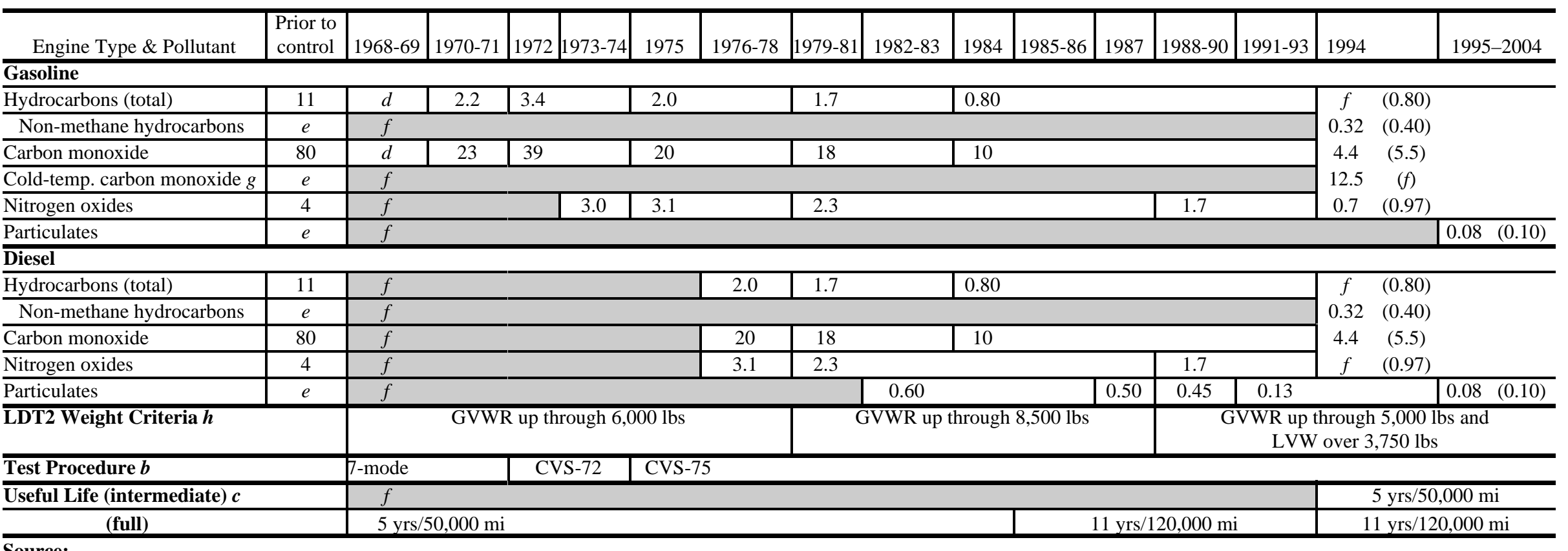

\section{Source:}

40 CFR 86.082-2; 40 CFR 86.085-2; 40 CFR 86.090-2; 40 CFR 86.090-9; 40 CFR 86.091-9; 40 CFR 86.094-9; 40 CFR 86.096-2; 40 CFR 86.096-9; 40 CFR 86.099-9; 40 CFR 86.000-9;

40 CFR 86.001-9; 40 CFR 86.004-9. Lisa Snapp, Office of Air and Radiation, Environmental Protection Agency, Personal communication, April 1999.

${ }^{a}$ Light truck categories LDT1-LDT4 were not actually created until 1994. From 1968 to 1978 all trucks with a Gross Vehicle Weight Rating (GVWR) up to 6,000 lbs were classified as light trucks and were required to meet the same standards. As of 1979, the maximum weight was raised to 8,500 lbs GVWR. During 1988-93, light trucks were divided into two subcategories that coincide with the current LDT1 and LDT2/3/4 categories.

${ }^{\mathrm{b}}$ The test procedure for measuring exhaust emissions has changed several times over the course of vehicle emissions regulation. The 7-mode procedure was used through model year 1971 and was replaced by the CVS-72 procedure beginning in model year 1972. The CVS-75 became the test procedure as of model year 1975. While it may appear that the total hydrocarbon and carbon monoxide standards were relaxed in 1972-74, these standards were actually more stringent due to the more stringent nature of the CVS-72 test procedure.

Additional standards for carbon monoxide and composite standards for non-methane hydrocarbons and nitrogen oxides tested over the new Supplemental Federal Test Procedure will be phased-in during model years 2000-02; these standards are not shown in this table.

${ }^{c}$ Emission standards had to be met for a useful life of 5 years/50,000 miles through model year 1983, and a full useful life of 11 years 120,000 miles was defined for 1985-93 (several useful life options were available for 1984). Beginning in model year 1994, emission standards were established for an intermediate useful life of 5 years/50,000 miles as well as a full useful life of 11 years/120,000 miles (these standards are shown in parentheses). Hydrocarbon standards, however, were established only for full useful life. Tier 1 exhaust standards, except PM standards, were phased-in during 1994-96 at a rate of 40, 80, and 100 percent, respectively. PM standards were phased-in at a rate of 40, 80, and 100 percent during $1995-97$.

${ }^{\mathrm{d}}$ In 1968-69, exhaust emission standards were issued in parts per million (ppm) rather than grams per mile and are, therefore, incompatible with this table.

${ }^{\mathrm{e}}$ No estimate available.

fo standard set.

${ }^{\mathrm{g}}$ The cold CO emission standard is measured at 20 degrees $\mathrm{F}$ (rather than 75 degrees $\mathrm{F}$ ) and is applicable for a 5 -year/50,000-mile useful life.

${ }^{\text {h }}$ Gross vehicle weight rating (GVWR) is the maximum design loaded weight. Loaded vehicle weight (LVW) is the curb weight (nominal vehicle weight) plus 300 lbs. 
Table 12.17

Federal Exhaust Emission Certification Standards for Gasoline- and Diesel-Powered Light Trucks (Category LDT3) ${ }^{a, b, c}$ (grams per mile)

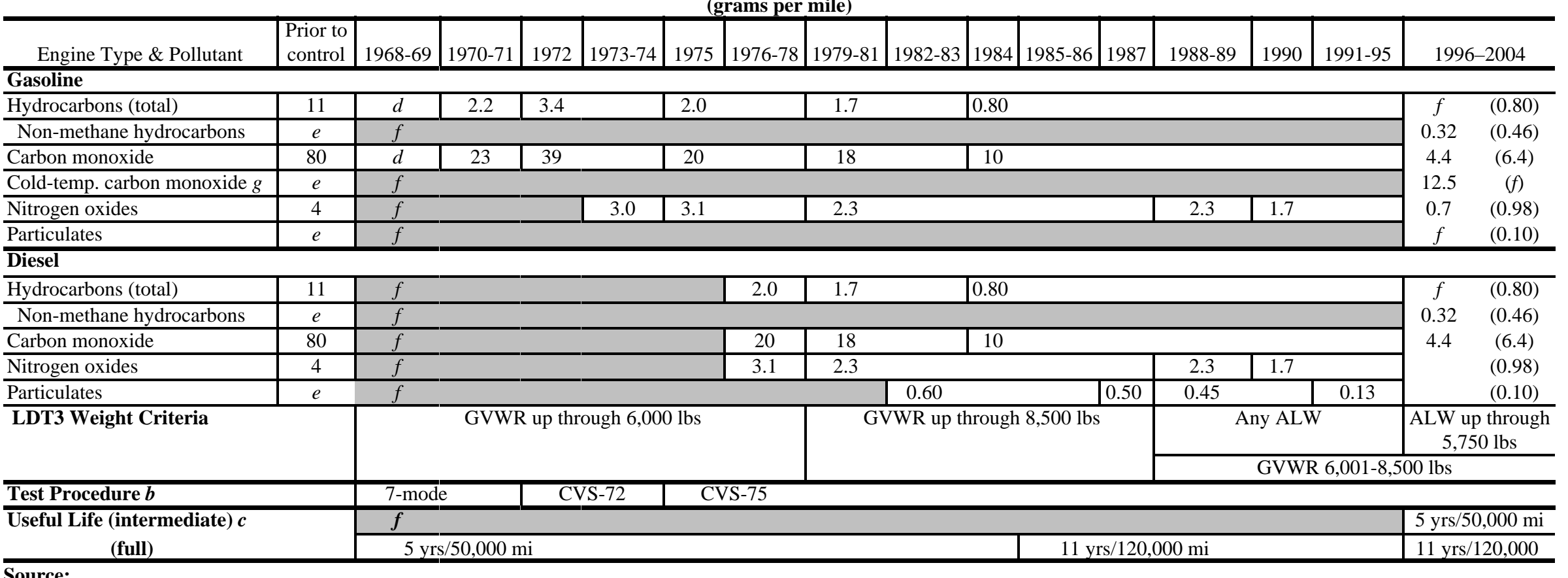

\section{Source:}

40 CFR 86.082-2; 40 CFR 86.085-2; 40 CFR 86.090-2; 40 CFR 86.090-9; 40 CFR 86.091-9; 40 CFR 86.094-9; 40 CFR 86.096-2; 40 CFR 86.096-9; 40 CFR 86.099-9; 40 CFR 86.000-9;

40 CFR 86.001-9; 40 CFR 86.004-9. Lisa Snapp, Office of Air and Radiation, Environmental Protection Agency, Personal communication, April 1999.

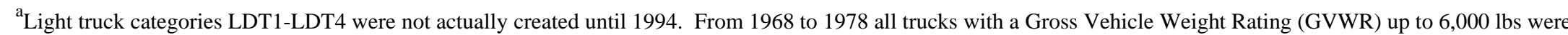
classified as light trucks and were required to meet the same standards. As of 1979, the maximum weight was raised to 8,500 lbs GVWR. During 1988-93, light trucks were divided into two subcategories that coincide with the current LDT1 and LDT2/3/4 categories.

${ }^{b}$ The test procedure for measuring exhaust emissions has changed several times over the course of vehicle emissions regulation. The 7-mode procedure was used through model year 1971 and was replaced by the CVS-72 procedure beginning in model year 1972. The CVS-75 became the test procedure as of model year 1975 . While it may appear that the total hydrocarbon and carbon monoxide standards were relaxed in 1972-74, these standards were actually more stringent due to the more stringent nature of the CVS-72 test procedure.

Additional standards for carbon monoxide and composite standards for non-methane hydrocarbons and nitrogen oxides tested over the new Supplemental Federal Test Procedure will be phased-in during model years 2002-04; these standards are not shown in this table.

${ }^{\mathrm{c}}$ Emission standards had to be met for a full useful life of 5 years/50,000 miles through model year 1983, and a full useful life of 11 years 120,000 miles was defined for 1985-93

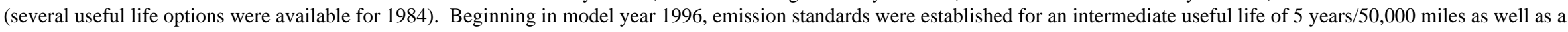

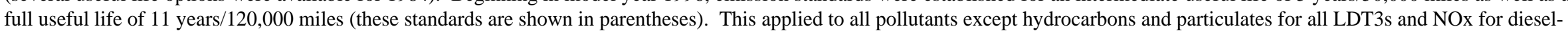
powered LDT3s, which were only required to meet full useful life standards. Tier 1 exhaust standards were phased-in during $1996-97$ at a rate of 50 and 100 percent, respectively.

${ }^{\mathrm{d}}$ In 1968-69, exhaust emission standards were issued in parts per million (ppm) rather than grams per mile and are, therefore, incompatible with this table.

No estimate available.

${ }^{\mathrm{f}}$ No standard set.

${ }^{\mathrm{g}}$ The cold CO emission standard is measured at 20 degrees $\mathrm{F}$ (rather than 75 degrees F) and is applicable for a 5-year/50,000-mile useful life.

${ }^{\mathrm{h}}$ Gross vehicle weight rating (GVWR) is the maximum design loaded weight. Loaded vehicle weight (LVW) is the curb weight (nominal vehicle weight) plus 300 lbs. 
Table 12.18

Federal Exhaust Emission Certification Standards for Gasoline- and Diesel-Powered Light Trucks (Category LDT4) ${ }^{a, b, c}$ (grams per mile)

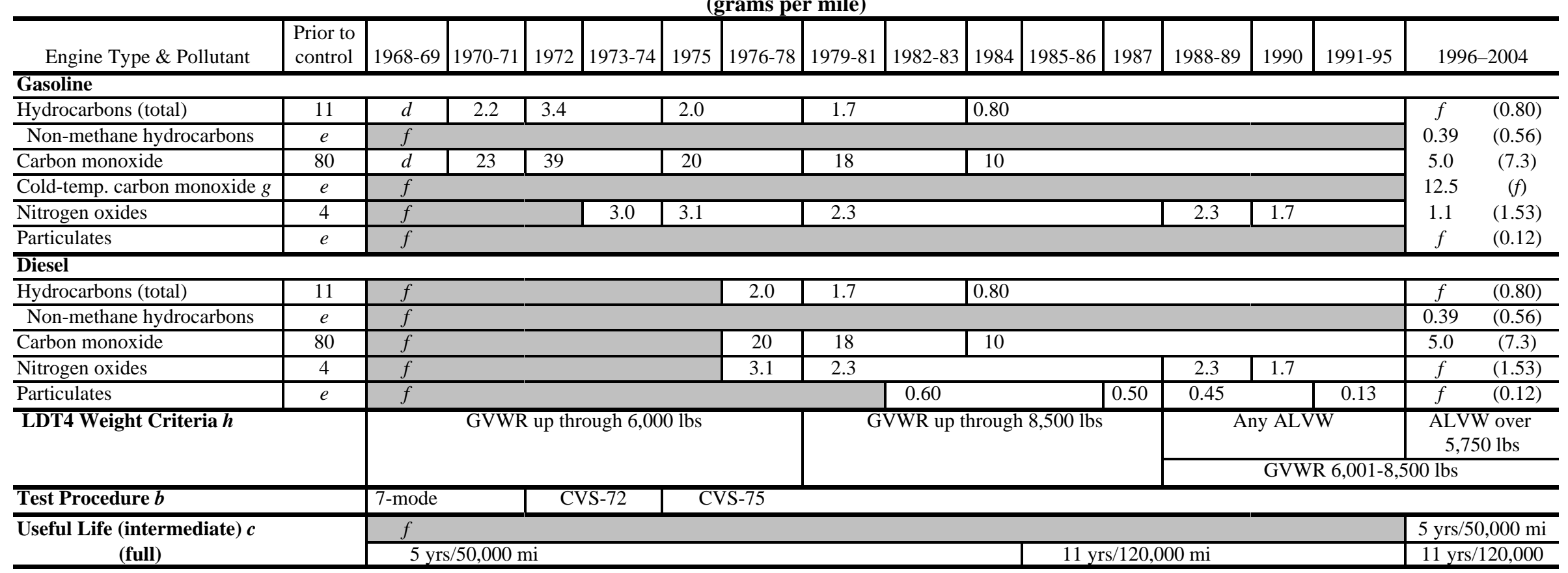

Source:

40 CFR 86.082-2; 40 CFR 86.085-2; 40 CFR 86.090-2; 40 CFR 86.090-9; 40 CFR 86.091-9; 40 CFR 86.094-9; 40 CFR 86.096-2; 40 CFR 86.096-9; 40 CFR 86.099-9; 40 CFR 86.000-9; 40 CFR 86.001-9; 40 CFR 86.004-9. Lisa Snapp, Office of Air and Radiation, Environmental Protection Agency, Personal communication, April 1999.

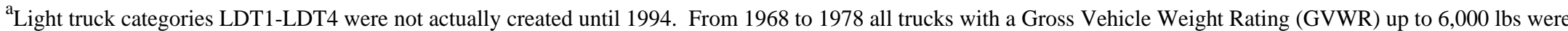
classified as light trucks and were required to meet the same standards. As of 1979, the maximum weight was raised to 8,500 lbs GVWR. During 1988-93, light trucks were divided into two subcategories that coincide with the current LDT1 and LDT2/3/4 categories.

${ }^{b}$ The test procedure for measuring exhaust emissions has changed several times over the course of vehicle emissions regulation. The 7-mode procedure was used through model year 1971 and was replaced by the CVS-72 procedure beginning in model year 1972. The CVS-75 became the test procedure as of model year 1975 . While it may appear that the total hydrocarbon and carbon monoxide standards were relaxed in 1972-74, these standards were actually more stringent due to the more stringent nature of the CVS-72 test procedure.

Additional standards for carbon monoxide and composite standards for non-methane hydrocarbons and nitrogen oxides tested over the new Supplemental Federal Test Procedure will be phased-in during model years 2002-04; these standards are not shown in this table.

Emission standards had to be met for a full useful life of 5 years/50,000 miles through model year 1983, and a full useful life of 11 years 120,000 miles was defined for 1985-93

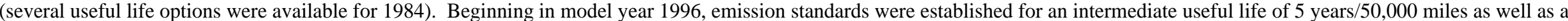
full useful life of 11 years/120,000 miles (these standards are shown in parentheses). This applied to all pollutants except hydrocarbons and particulates for all LDT3s and NOx for dieselpowered LDT3s, which were only required to meet full useful life standards. Tier 1 exhaust standards were phased-in during $1996-97$ at a rate of 50 and 100 percent, respectively.

${ }^{\mathrm{d}}$ In 1968-69, exhaust emission standards were issued in parts per million (ppm) rather than grams per mile and are, therefore, incompatible with this table.

No estimate available.

${ }^{\mathrm{f}}$ No standard set.

${ }^{\mathrm{g}}$ The cold CO emission standard is measured at 20 degrees $\mathrm{F}$ (rather than 75 degrees $\mathrm{F}$ ) and is applicable for a 5-year/50,000-mile useful life.

${ }^{\mathrm{h}}$ Gross vehicle weight rating (GVWR) is the maximum design loaded weight. Adjusted loaded vehicle weight (ALVW) is the numerical average of the GVWR and the curb weight. 
Table 12.19

Federal Exhaust Emission Certification Standards for Gasoline- and Diesel-Powered Light Heavy Trucks (Grams per brake horsepower-hour)

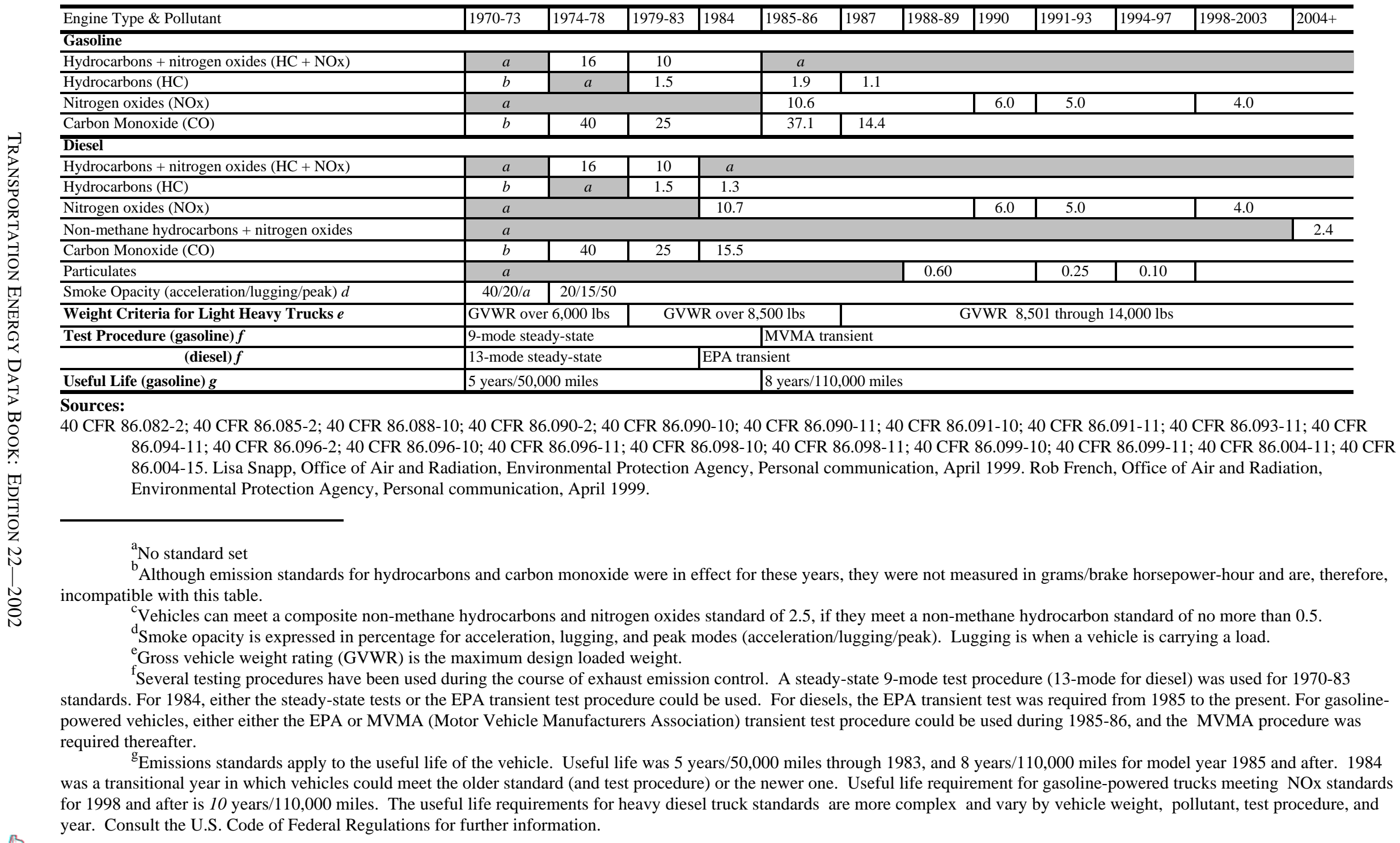


Table 12.20

Federal Exhaust Emission Certification Standards for Gasoline- and Diesel-Powered Heavy Heavy Trucks (Grams per brake horsepower-hour)

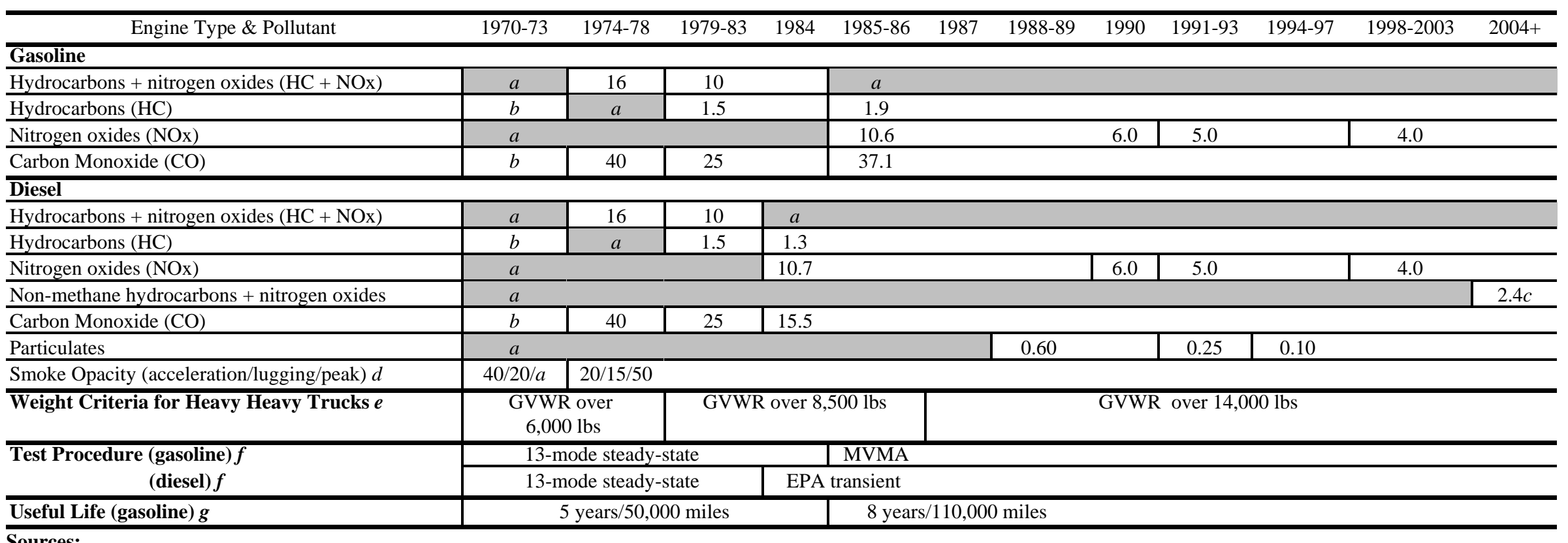

(1) 86.094-11; 40 CFR 86.096-2; 40 CFR 86.096-10; 40 CFR 86.096-11; 40 CFR 86.098-10; 40 CFR 86.098-11; 40 CFR 86.099-10; 40 CFR 86.099-11; 40 CFR 86.004-11; 40 CFR 86.004-15. Lisa Snapp, Office of Air and Radiation, Environmental Protection Agency, Personal communication, April 1999. Rob French, Office of Air and Radiation,

Environmental Protection Agency, Personal communication, April 1999.

${ }^{\mathrm{a}}$ No standard set.

${ }^{\mathrm{b}}$ Although emission standards for hydrocarbons and carbon monoxide were in effect for these years, they were not measured in grams/brake horsepower-hour and are, therefore, incompatible with this table.

${ }^{c}$ Vehicles can meet a composite non-methane hydrocarbons and nitrogen oxides standard of 2.5 , if they meet a non-methane hydrocarbon standard of no more than 0.5 .

${ }^{\mathrm{d}}$ Smoke opacity is expressed in percentage for acceleration, lugging, and peak modes (acceleration/lugging/peak). Lugging is when a vehicle is carrying a load.

${ }^{\mathrm{e}}$ Gross vehicle weight rating (GVWR) is the maximum design loaded weight.

${ }^{\mathrm{f}}$ Several testing procedures have been used during the course of exhaust emission control. A steady-state 9-mode test procedure (13-mode for diesel) was used for 1970-83

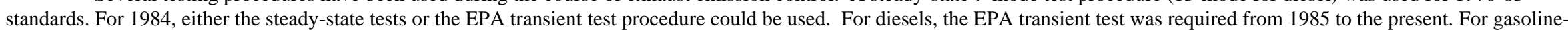
powered vehicles, either either the EPA or MVMA (Motor Vehicle Manufacturers Association) transient test procedure could be used during 1985-86, and the MVMA procedure was required thereafter.

${ }^{\mathrm{g}}$ Emissions standards apply to the useful life of the vehicle. Useful life was 5 years/50,000 miles through 1983, and 8 years/110,000 miles for model year 1985 and after. 1984

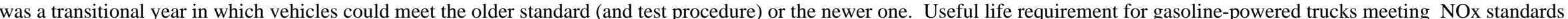
for 1998 and after is 10 years/110,000 miles. The useful life requirements for heavy diesel truck standards are more complex and vary by vehicle weight, pollutant, test procedure, and year. Consult the U.S. Code of Federal Regulations for further information. 
Table 12.21

California Passenger Cars and Light Trucks Emission Certification Standards for Model Years 2001-2006 (grams/mile)

\begin{tabular}{|c|c|c|c|c|c|c|c|c|c|c|c|c|c|c|c|}
\hline \multirow{3}{*}{$\begin{array}{l}\text { Vehicle } \\
\text { Type }\end{array}$} & \multirow{3}{*}{$\begin{array}{l}\text { Emission } \\
\text { Category }\end{array}$} & \multicolumn{14}{|c|}{ Vehicle Useful Life } \\
\hline & & \multicolumn{7}{|c|}{5 Years / 50,000 Miles } & \multicolumn{7}{|c|}{10 Years / 100,000 Miles } \\
\hline & & $\mathrm{THC}^{\mathrm{a}}$ & $\mathrm{NMHC}^{\mathrm{b}}$ & $\mathrm{NMOG}^{\mathrm{c}}$ & $\mathrm{CO}$ & $\mathrm{NO}_{\mathrm{x}}$ & PM & $\mathrm{HCHO}$ & $\mathrm{THC}^{\mathrm{a}}$ & $\mathrm{NMHC}^{\mathrm{b}}$ & $\mathrm{NMOG}^{\mathrm{c}}$ & $\mathrm{CO}$ & $\mathrm{NO}_{\mathrm{x}}$ & PM & $\mathrm{HCHO}$ \\
\hline \multirow[t]{5}{*}{ Passenger car } & Tier 1 & - & 0.25 & - & 3.4 & 0.4 & $0.08^{\mathrm{d}}$ & $0.015^{\mathrm{e}}$ & - & 0.31 & - & 4.2 & 0.6 & - & - \\
\hline & TLEV & - & - & 0.125 & 3.4 & 0.4 & - & 0.015 & - & - & 0.156 & 4.2 & 0.6 & $0.08^{\mathrm{d}}$ & 0.018 \\
\hline & LEV & - & - & 0.075 & 3.4 & 0.2 & - & 0.015 & - & - & 0.090 & 4.2 & 0.3 & $0.08^{\mathrm{d}}$ & 0.018 \\
\hline & ULEV & - & - & 0.040 & 1.7 & 0.2 & - & 0.008 & - & - & 0.055 & 2.1 & 0.3 & $0.04^{\mathrm{d}}$ & 0.011 \\
\hline & ZEV & 0.0 & 0.00 & 0.000 & 0.0 & 0.0 & 0.00 & 0.000 & 0.00 & 0.00 & 0.000 & 0.0 & 0.0 & 0.00 & 0.000 \\
\hline \multirow[t]{5}{*}{ LDT1 } & Tier 1 & - & 0.25 & - & 3.4 & 0.4 & $0.08^{\mathrm{d}}$ & $0.015^{\mathrm{e}}$ & - & 0.31 & - & 4.2 & 0.6 & - & - \\
\hline & TLEV & - & - & 0.125 & 3.4 & 0.4 & - & 0.015 & - & - & 0.156 & 4.2 & 0.6 & $0.08^{\mathrm{d}}$ & 0.018 \\
\hline & LEV & - & - & 0.075 & 3.4 & 0.2 & - & 0.015 & - & - & 0.090 & 4.2 & 0.3 & $0.08^{\mathrm{d}}$ & 0.018 \\
\hline & ULEV & - & - & 0.040 & 1.7 & 0.2 & - & 0.008 & - & - & 0.055 & 2.1 & 0.3 & $0.04^{\mathrm{d}}$ & 0.011 \\
\hline & ZEV & 0.0 & 0.00 & 0.000 & 0.0 & 0.0 & 0.00 & 0.000 & 0.00 & 0.00 & 0.000 & 0.0 & 0.0 & 0.00 & 0.000 \\
\hline \multirow[t]{4}{*}{ LDT2 } & Tier 1 & - & 0.32 & - & 4.4 & 0.7 & $0.08^{\mathrm{d}}$ & $0.018^{\mathrm{e}}$ & - & 0.40 & - & 5.5 & 0.97 & - & - \\
\hline & TLEV & - & - & 0.160 & 4.4 & 0.7 & - & 0.018 & - & - & 0.200 & 5.5 & 0.9 & $0.10^{\mathrm{d}}$ & 0.023 \\
\hline & LEV & - & - & 0.100 & 4.4 & 0.4 & - & 0.018 & - & - & 0.130 & 5.5 & 0.5 & $0.10^{\mathrm{d}}$ & 0.023 \\
\hline & ULEV & - & - & 0.050 & 2.2 & 0.4 & - & 0.009 & - & - & 0.070 & 2.8 & 0.5 & $0.05^{\mathrm{d}}$ & 0.013 \\
\hline
\end{tabular}

\section{Source:}

U.S. Environmental Protection Agency, Office of Transportation and Air Quality, EPA 420-B-00-001.

(Additional resources: www.epa.gov/otag)

Note:

After 2003, Tier 1 and TLEV standards will be eliminated.

LDT1 = light truck $(6,000 \mathrm{lbs}$. or less GVWR) up through 3,750 lbs. loaded vehicle weight; LDT2 = light truck $(6,000 \mathrm{lbs}$. or less GVWR $)$ greater than $3,750 \mathrm{lbs}$. loaded vehicle weight.

\footnotetext{
${ }^{a}$ THCE for methanol vehicles. Does not apply to CNG vehicles.

${ }^{\mathrm{b}}$ THCE for Tier 0 methanol vehicles. NMHCE for other alcohol vehicles.

${ }^{\mathrm{c}} \mathrm{NMHC}$ for diesel-fueled vehicles.

${ }^{\mathrm{d}}$ Diesel-fueled vehicles only.

${ }^{\mathrm{e}}$ Ethanol- and methanol-fueled vehicles only.
} 
California's Low-Emission Vehicle regulations provide for reduced emission vehicles to be available to consumers. Vehicles meeting these standards have even lower emissions than the basic Tier 1 standards for all new vehicles sold in California. Currently, there is a wide array of TLEVs and LEVs, and a few ULEVs, SULEVs and ZEVs on the market. For a listing of the available low emission vehicles, see the California Air Resources Board web site referenced below.

Table 12.22

\section{California Vehicle Emission Reduction for Passenger Cars and Light Trucks ${ }^{\mathrm{a}}$}

\begin{tabular}{llcc}
\hline & \multicolumn{3}{c}{$\begin{array}{c}\text { Emission reduction from Tier 1 } \\
\text { California standards }\end{array}$} \\
\cline { 2 - 4 } & $\mathrm{HC}$ & $\mathrm{CO}$ & $\mathrm{NOx}$ \\
\hline Transitional Low-Emission Vehicle (TLEV) & $50 \%$ & $=$ & $=$ \\
Low-Emission Vehicle (LEV) & $70 \%$ & $=$ & $50 \%$ \\
Ultra-Low-Emission Vehicle (ULEV) & $85 \%$ & $50 \%$ & $50 \%$ \\
Super-Ultra-Low-Emission Vehicle (SULEV) & $96 \%$ & $70 \%$ & $95 \%$ \\
Zero-Emission Vehicles (ZEV) & $100 \%$ & $100 \%$ & $100 \%$ \\
\hline
\end{tabular}

\section{Source:}

California Air Resources Board web site, www.arb.ca.gov/msprog/ccbg/ccbg.htm (Additional resources: www.arb.ca.gov)

\section{Note:}

= indicates equivalent emissions to vehicles meeting the Tier 1 California standard.

${ }^{a}$ Light trucks less than 6,000 lbs. gross vehicle weight rating.

${ }^{\mathrm{b}}$ See Table 12.23. 


\section{APPENDIX A \\ SOURCES \& METHODOLOGIES}

This appendix contains documentation of the estimation procedures used by ORNL. The reader can examine the methodology behind the estimates and form an opinion as to their utility. The appendix is arranged by subject heading. Only tables which contain ORNL estimations are documented in Appendix A; all other tables have sources listed at the bottom of the table. Since abbreviations are used throughout the appendix, a list of abbreviations is also included.

\section{Contents of Appendix A}

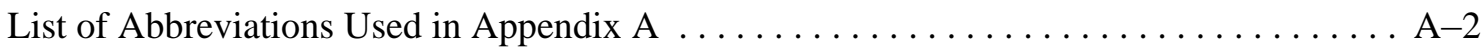

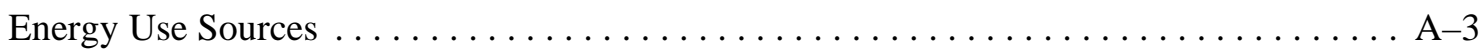

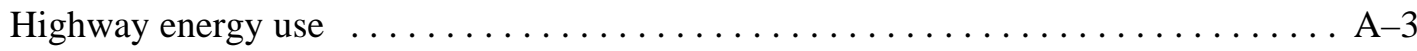

Off-highway energy use $\ldots \ldots \ldots \ldots \ldots \ldots \ldots \ldots \ldots \ldots \ldots \ldots \ldots \ldots \ldots \ldots \ldots \ldots \ldots \ldots$

Nonhighway energy use $\ldots \ldots \ldots \ldots \ldots \ldots \ldots \ldots \ldots \ldots \ldots \ldots \ldots \ldots \ldots \ldots \ldots \ldots \ldots$

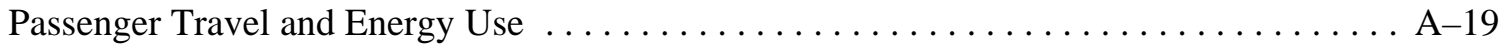

Highway Passenger Mode Energy Intensities $\ldots \ldots \ldots \ldots \ldots \ldots \ldots \ldots \ldots \ldots \ldots \ldots \ldots \ldots \ldots$

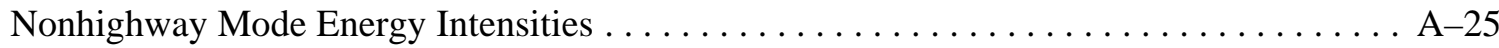

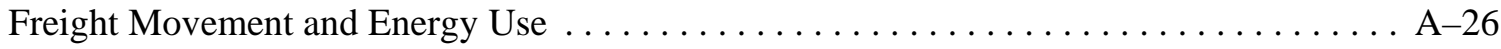

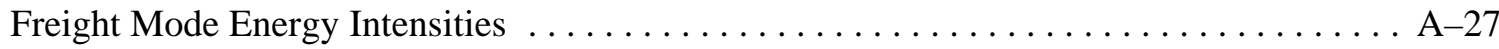

Highway Vehicle Stock and New Sales . . . . . . . . . . . . . . . . A-28

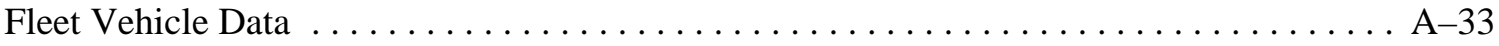




\section{List of Abbreviations Used in Appendix A}

\begin{tabular}{ll} 
AAMA & American Automobile Manufacturers Association \\
AAR & Association of American Railroads \\
APTA & American Public Transit Association \\
Amtrak & National Railroad Passenger Corporation \\
Btu & British thermal unit \\
DOC & Department of Commerce \\
DOE & Department of Energy \\
DOT & Department of Transportation \\
EIA & Energy Information Administration \\
EPA & Environmental Protection Agency \\
FAA & Federal Aviation Administration \\
FHWA & Federal Highway Administration \\
GSA & General Services Administration \\
gvw & gross vehicle weight \\
lpg & liquefied petroleum gas \\
mpg & miles per gallon \\
NHTS & National Household Travel Survey \\
NHTSA & National Highway Traffic Safety Administration \\
NPTS & Nationwide Personal Transportation Survey \\
NVPP & National Vehicle Population Profile \\
ORNL & Oak Ridge National Laboratory \\
pmt & passenger-miles traveled \\
RECS & Residential Energy Consumption Survey \\
RTECS & Residential Transportation Energy Consumption Survey \\
TIUS & Truck Inventory and Use Survey \\
vmt & Vehicle Inventory and Use Survey \\
\hline TSC & Triles traveled \\
\hline
\end{tabular}




\section{Energy Use Sources}

\section{Highway energy use}

\section{Automobiles}

Fuel use in gallons from: DOT, FHWA, Highway Statistics 2001, Table VM-1 and annual editions back to 1996; DOT, FHWA, Highway Statistics Summary to 1995. Fuel use was distributed among fuel types using the percentages shown in Table A.1.

Table A.1

Automobile Fuel Use and Fuel Type Shares for Calculation of Energy Use

\begin{tabular}{|c|c|c|c|c|c|c|}
\hline \multirow[b]{2}{*}{ Year } & \multirow{2}{*}{$\begin{array}{c}\text { Fuel use } \\
\text { (million gallons) }\end{array}$} & \multirow{2}{*}{$\begin{array}{c}\text { Source for } \\
\text { gasohol shares }\end{array}$} & \multirow{2}{*}{$\begin{array}{c}\text { Source for } \\
\text { gasoline/diesel shares }\end{array}$} & \multicolumn{3}{|c|}{ Shares by fuel type } \\
\hline & & & & Gasoline & Gasohol & Diesel \\
\hline 1970 & 67,820 & & 1984 NVPP & $99.8 \%$ & $0.0 \%$ & $0.2 \%$ \\
\hline 1971 & 71,346 & & interpolated & $99.2 \%$ & $0.0 \%$ & $0.8 \%$ \\
\hline 1972 & 75,937 & & interpolated & $98.7 \%$ & $0.0 \%$ & $1.3 \%$ \\
\hline 1973 & 78,233 & & interpolated & $98.1 \%$ & $0.0 \%$ & $1.9 \%$ \\
\hline 1974 & 74,229 & & interpolated & $97.5 \%$ & $0.0 \%$ & $2.5 \%$ \\
\hline 1975 & 74,140 & & interpolated & $97.0 \%$ & $0.0 \%$ & $3.0 \%$ \\
\hline 1976 & 78,297 & & interpolated & $96.4 \%$ & $0.0 \%$ & $3.6 \%$ \\
\hline 1977 & 79,060 & & interpolated & $95.8 \%$ & $0.0 \%$ & $4.2 \%$ \\
\hline 1978 & 80,652 & & interpolated & $95.3 \%$ & $0.0 \%$ & $4.7 \%$ \\
\hline 1979 & 76,588 & & 1979 RTECS & $94.7 \%$ & $0.0 \%$ & $5.3 \%$ \\
\hline 1980 & 69,981 & FHWA, MF-24 & interpolated & $93.9 \%$ & $0.5 \%$ & $5.6 \%$ \\
\hline 1981 & 69,112 & FHWA, MF-24 & 1981 RTECS & $93.4 \%$ & $0.7 \%$ & $5.9 \%$ \\
\hline 1982 & 69,116 & FHWA, MF-24 & interpolated & $93.5 \%$ & $2.3 \%$ & $4.2 \%$ \\
\hline 1983 & 70,322 & FHWA, MF-24 & 1983 RTECS & $93.2 \%$ & $4.3 \%$ & $2.5 \%$ \\
\hline 1984 & 70,663 & FHWA, MF-24 & interpolated & $92.7 \%$ & $5.3 \%$ & $2.0 \%$ \\
\hline 1985 & 71,518 & FHWA, MF-24 & 1985 RTECS & $90.8 \%$ & $7.7 \%$ & $1.5 \%$ \\
\hline 1986 & 73,174 & FHWA, MF-24 & interpolated & $91.0 \%$ & $7.6 \%$ & $1.4 \%$ \\
\hline 1987 & 73,308 & FHWA, MF-24 & interpolated & $92.4 \%$ & $6.3 \%$ & $1.3 \%$ \\
\hline 1988 & 73,345 & FHWA, MF-24 & 1988 RTECS & $91.4 \%$ & $7.4 \%$ & $1.2 \%$ \\
\hline 1989 & 73,913 & FHWA, MF-24 & interpolated & $92.6 \%$ & $6.2 \%$ & $1.2 \%$ \\
\hline 1990 & 69,568 & FHWA, MF-24 & interpolated & $92.0 \%$ & $6.8 \%$ & $1.2 \%$ \\
\hline 1991 & 64,318 & FHWA, MF-24 & 1991 RTECS & $90.8 \%$ & $8.0 \%$ & $1.2 \%$ \\
\hline 1992 & 65,436 & FHWA, MF-24 & interpolated & $90.8 \%$ & $7.9 \%$ & $1.2 \%$ \\
\hline 1993 & 67,047 & FHWA, MF-24 & interpolated & $89.7 \%$ & $9.1 \%$ & $1.3 \%$ \\
\hline 1994 & 67,874 & FHWA, MF-24 & 1994 RTECS & $89.1 \%$ & $9.6 \%$ & $1.3 \%$ \\
\hline 1995 & 68,072 & FHWA, MF-24 & interpolated & $87.6 \%$ & $11.2 \%$ & $1.2 \%$ \\
\hline 1996 & 69,221 & FHWA, MF-24 & interpolated & $88.8 \%$ & $10.1 \%$ & $1.0 \%$ \\
\hline 1997 & 69,892 & FHWA, MF-24 & interpolated & $86.9 \%$ & $12.2 \%$ & $0.9 \%$ \\
\hline 1998 & 71,695 & FHWA, MF-24 & interpolated & $88.0 \%$ & $11.2 \%$ & $0.8 \%$ \\
\hline 1999 & 73,283 & FHWA, MF-24 & interpolated & $88.3 \%$ & $11.0 \%$ & $0.6 \%$ \\
\hline 2000 & 73,065 & FHWA, MF-24 & 2000 NVPP & $86.9 \%$ & $12.6 \%$ & $0.5 \%$ \\
\hline 2001 & 73,261 & FHWA, MF-24 & 2001 NVPP & $86.5 \%$ & $13.0 \%$ & $0.5 \%$ \\
\hline \multicolumn{4}{|c|}{ Heat content used for conversion to btu: } & $\begin{array}{c}125,000 \\
\text { btu/gallon }\end{array}$ & $\begin{array}{c}120,900 \\
\text { btu/gallon }\end{array}$ & $\begin{array}{c}138,700 \\
\text { btu/gallon }\end{array}$ \\
\hline
\end{tabular}




\section{Motorcycles}

DOT, FHWA, Highway Statistics 2001, Table VM-1, and annual editions.

Table A.2

Motorcycle Fuel Use

\begin{tabular}{cccc}
\hline Year & $\begin{array}{c}\text { Fuel use } \\
\text { (million gallons) }\end{array}$ & Year & $\begin{array}{c}\text { Fuel use } \\
\text { (million gallons) }\end{array}$ \\
\hline 1970 & 59580000 & 1986 & $187,940,000$ \\
1971 & $72,140,000$ & 1987 & $190,120,000$ \\
1972 & $86,620,000$ & 1988 & $200,480,000$ \\
1973 & $103,880,000$ & 1989 & $207,420,000$ \\
1974 & $108,900,000$ & 1990 & $191,140,000$ \\
1975 & $112,580,000$ & 1991 & $183,560,000$ \\
1976 & $120,060,000$ & 1992 & $191,140,000$ \\
1977 & $126,980,000$ & 1993 & $198,120,000$ \\
1978 & $143,160,000$ & 1994 & $204,800,000$ \\
1979 & $172,740,000$ & 1995 & $198,262,073$ \\
1980 & $204,280,000$ & 1996 & $195,940,000$ \\
1981 & $213,800,000$ & 1997 & $201,620,000$ \\
1982 & $198,200,000$ & 1998 & $205,660,000$ \\
1983 & $175,200,000$ & 1999 & $211,680,000$ \\
1984 & $175,680,000$ & 2000 & $209,380,000$ \\
1985 & $181,720,000$ & 2001 & $190,580,000$ \\
\hline \multicolumn{2}{c}{ Heat content used for conversion to btu: } & 125,000 btu/gallon \\
\hline
\end{tabular}

\section{Buses}

\section{Transit:}

APTA, 2002 Transit Fact Book, 2002, Washington, DC. Includes motorbus and trolley bus data. This data series was detailed separately from other transit modes for the first time in 2003.

Table A.3

Transit Bus Fuel Use

\begin{tabular}{cccccccc}
\hline & $\begin{array}{c}\text { Methanol } \\
\text { thousand } \\
\text { gallons) }\end{array}$ & $\begin{array}{c}\text { LNG } \\
\text { (thousand } \\
\text { gallons) }\end{array}$ & $\begin{array}{c}\text { LPG } \\
\text { (thousand } \\
\text { gallons) }\end{array}$ & $\begin{array}{c}\text { CNG } \\
\text { (thousand } \\
\text { gal3109lons) }\end{array}$ & $\begin{array}{c}\text { Gasoline } \\
\text { (thousand } \\
\text { gallons) }\end{array}$ & $\begin{array}{c}\text { Diesel fuel } \\
\text { (thousand } \\
\text { gallons) }\end{array}$ & $\begin{array}{c}\text { Electricity } \\
\text { (thousand } \\
\text { kilowatt } \\
\text { hours) }\end{array}$ \\
\hline 1994 & 12,470 & 1,138 & 249 & 3,109 & 2,103 & 565,064 & 102,945 \\
1995 & 11,967 & 1,737 & 269 & 10,011 & 2,297 & 563,767 & 100,659 \\
1996 & 11,600 & 2,278 & 591 & 11,527 & 1,844 & 577,680 & 69,130 \\
1997 & 8,705 & 3,276 & 1,033 & 20,050 & 2,722 & 597,636 & 78,561 \\
1998 & 4,976 & 3,075 & 879 & 32,260 & 1,959 & 606,631 & 74,352 \\
1999 & 2,711 & 5,251 & 659 & 39,861 & 1,402 & 618,024 & 75,920 \\
2000 & 821 & 10,464 & 723 & 50,449 & 1,315 & 635,160 & 78,062 \\
2001 & 763 & 11,670 & 1,171 & 60,917 & 1,472 & 587,184 & 75,108 \\
\hline $\begin{array}{c}\text { Heat content used } \\
\text { for conversion }\end{array}$ & $\begin{array}{c}64,600 \\
\text { btu/gallon }\end{array}$ & $\begin{array}{c}90,800 \\
\text { btu/gallon }\end{array}$ & $\begin{array}{c}91,300 \\
\text { btu/gallon }\end{array}$ & $\begin{array}{c}129,400 \\
\text { btu/gallon }\end{array}$ & $\begin{array}{c}125,000 \\
\text { btu/gallon }\end{array}$ & $\begin{array}{c}138,700 \\
\text { btu/gallon }\end{array}$ & $\begin{array}{c}11,765 \\
\text { btu/kWhr }\end{array}$ \\
\hline
\end{tabular}

\section{Intercity and School:}


Eno Transportation Foundation, Transportation in America 2001, Nineteenth Edition, 2003, Washington, DC, pp. 20-23. School bus fuel was assumed to be $90 \%$ diesel fuel and $10 \%$ gasoline based on estimates from the National Association of State Directors of Pupil Transportation Services. Intercity bus fuel was assumed to be $100 \%$ diesel. Because the 2001 data were not available at the time this report went to press, the 2000 data were used again for 2001 .

Table A.4

Intercity and School Bus Fuel Use

\begin{tabular}{|c|c|c|}
\hline Year & $\begin{array}{c}\text { Intercity } \\
\text { (million gallons) }\end{array}$ & $\begin{array}{c}\text { School } \\
\text { (million gallons) }\end{array}$ \\
\hline 1970 & 305.34 & 299.88 \\
\hline 1971 & 296.73 & 309.75 \\
\hline 1972 & 288.12 & 319.62 \\
\hline 1973 & 252.42 & 327.04 \\
\hline 1974 & 216.72 & 334.46 \\
\hline 1975 & 181.02 & 341.88 \\
\hline 1976 & 182.28 & 389.76 \\
\hline 1977 & 181.86 & 401.52 \\
\hline 1978 & 180.18 & 406.98 \\
\hline 1979 & 205.38 & 404.88 \\
\hline 1980 & 213.78 & 379.68 \\
\hline 1981 & 205.38 & 386.82 \\
\hline 1982 & 227.22 & 398.58 \\
\hline 1983 & 237.30 & 400.68 \\
\hline 1984 & 169.26 & 375.06 \\
\hline 1985 & 165.48 & 425.04 \\
\hline 1986 & 148.68 & 462.42 \\
\hline 1987 & 155.82 & 487.20 \\
\hline 1988 & 160.44 & 511.14 \\
\hline 1989 & 166.74 & 498.12 \\
\hline 1990 & 159.60 & 472.08 \\
\hline 1991 & 160.44 & 533.40 \\
\hline 1992 & 157.08 & 546.00 \\
\hline 1993 & 171.36 & 533.40 \\
\hline 1994 & 195.30 & 546.00 \\
\hline 1995 & 195.30 & 545.16 \\
\hline 1996 & 199.92 & 545.16 \\
\hline 1997 & 212.52 & 544.74 \\
\hline 1998 & 220.08 & 550.20 \\
\hline 1999 & 241.08 & 555.66 \\
\hline 2000 & 233.10 & 577.08 \\
\hline 2001 & Not available & Not available \\
\hline Fuel type shares & $100 \%$ diesel & $\begin{array}{c}90 \% \text { diesel } \\
10 \% \text { gasoline }\end{array}$ \\
\hline $\begin{array}{l}\text { Heat content used for } \\
\text { conversion to btu: }\end{array}$ & $\begin{array}{l}\text { 138,700 } \\
\text { btu/gallon }\end{array}$ & $\begin{array}{l}138,700 \mathrm{btu} / \text { gallon } \\
125,000 \mathrm{btu} / \text { gallon }\end{array}$ \\
\hline
\end{tabular}

\section{Trucks}


Light Trucks:

DOT, FHWA, Highway Statistics 2001, Table VM-1 and annual editions back to 1996;

DOT, FHWA, Highway Statistics Summary to 1995.

Table A.5

Light Truck Fuel Use and Fuel Type Shares for Calculation of Energy Use

\begin{tabular}{|c|c|c|c|c|c|c|c|}
\hline \multirow[b]{2}{*}{ Year } & \multirow{2}{*}{$\begin{array}{l}\text { Fuel use } \\
\text { (million } \\
\text { gallons) }\end{array}$} & \multirow{2}{*}{$\begin{array}{c}\text { Source for } \\
\text { gasohol shares }\end{array}$} & \multirow{2}{*}{$\begin{array}{c}\text { Source for } \\
\text { gasoline/diesel } \\
\text { /lpg shares }\end{array}$} & \multicolumn{4}{|c|}{ Shares by fuel type } \\
\hline & & & & Gasoline & Gasohol & Diesel & Lpg \\
\hline 1970 & 12,313 & & 1977 TIUS & $97.6 \%$ & $0.0 \%$ & $1.6 \%$ & $0.8 \%$ \\
\hline 1971 & 13,484 & & 1977 TIUS & $97.6 \%$ & $0.0 \%$ & $1.6 \%$ & $0.8 \%$ \\
\hline 1972 & 15,150 & & 1977 TIUS & $97.6 \%$ & $0.0 \%$ & $1.6 \%$ & $0.8 \%$ \\
\hline 1973 & 16,828 & & 1977 TIUS & $97.6 \%$ & $0.0 \%$ & $1.6 \%$ & $0.8 \%$ \\
\hline 1974 & 16,657 & & 1977 TIUS & $97.6 \%$ & $0.0 \%$ & $1.6 \%$ & $0.8 \%$ \\
\hline 1975 & 19,081 & & 1977 TIUS & $97.6 \%$ & $0.0 \%$ & $1.6 \%$ & $0.8 \%$ \\
\hline 1976 & 20,828 & & 1977 TIUS & $97.6 \%$ & $0.0 \%$ & $1.6 \%$ & $0.8 \%$ \\
\hline 1977 & 22,383 & & 1977 TIUS & $97.6 \%$ & $0.0 \%$ & $1.6 \%$ & $0.8 \%$ \\
\hline 1978 & 24,162 & & Interpolated & $97.1 \%$ & $0.0 \%$ & $2.0 \%$ & $0.9 \%$ \\
\hline 1979 & 24,445 & & Interpolated & $96.7 \%$ & $0.0 \%$ & $2.4 \%$ & $1.0 \%$ \\
\hline 1980 & 23,796 & FHWA, MF-24 & Interpolated & $95.7 \%$ & $0.5 \%$ & $2.7 \%$ & $1.0 \%$ \\
\hline 1981 & 23,697 & FHWA, MF-24 & Interpolated & $95.1 \%$ & $0.7 \%$ & $3.1 \%$ & $1.1 \%$ \\
\hline 1982 & 22,702 & FHWA, MF-24 & 1982 TIUS & $93.0 \%$ & $2.3 \%$ & $3.5 \%$ & $1.2 \%$ \\
\hline 1983 & 23,945 & FHWA, MF-24 & Interpolated & $91.0 \%$ & $4.3 \%$ & $3.5 \%$ & $1.2 \%$ \\
\hline 1984 & 25,604 & FHWA, MF-24 & Interpolated & $90.0 \%$ & $5.3 \%$ & $3.5 \%$ & $1.2 \%$ \\
\hline 1985 & 27,363 & FHWA, MF-24 & Interpolated & $87.6 \%$ & $7.7 \%$ & $3.5 \%$ & $1.2 \%$ \\
\hline 1986 & 29,074 & FHWA, MF-24 & Interpolated & $87.7 \%$ & $7.6 \%$ & $3.5 \%$ & $1.2 \%$ \\
\hline 1987 & 30,598 & FHWA, MF-24 & 1987 TIUS & $89.0 \%$ & $6.3 \%$ & $3.5 \%$ & $1.2 \%$ \\
\hline 1988 & 32,653 & FHWA, MF-24 & Interpolated & $88.2 \%$ & $7.4 \%$ & $3.5 \%$ & $1.0 \%$ \\
\hline 1989 & 33,271 & FHWA, MF-24 & Interpolated & $89.5 \%$ & $6.2 \%$ & $3.4 \%$ & $0.8 \%$ \\
\hline 1990 & 35,611 & FHWA, MF-24 & Interpolated & $89.2 \%$ & $6.8 \%$ & $3.4 \%$ & $0.7 \%$ \\
\hline 1991 & 38,217 & FHWA, MF-24 & Interpolated & $88.1 \%$ & $8.0 \%$ & $3.3 \%$ & $0.5 \%$ \\
\hline 1992 & 40,929 & FHWA, MF-24 & 1992 TIUS & $88.5 \%$ & $7.9 \%$ & $3.3 \%$ & $0.3 \%$ \\
\hline 1993 & 42,851 & FHWA, MF-24 & Interpolated & $87.3 \%$ & $9.1 \%$ & $3.3 \%$ & $0.3 \%$ \\
\hline 1994 & 44,112 & FHWA, MF-24 & Interpolated & $86.8 \%$ & $9.6 \%$ & $3.3 \%$ & $0.3 \%$ \\
\hline 1995 & 45,605 & FHWA, MF-24 & Interpolated & $85.1 \%$ & $11.2 \%$ & $3.4 \%$ & $0.3 \%$ \\
\hline 1996 & 47,354 & FHWA, MF-24 & Interpolated & $86.2 \%$ & $10.1 \%$ & $3.4 \%$ & $0.3 \%$ \\
\hline 1997 & 49,388 & FHWA, MF-24 & 1997 VIUS & $84.2 \%$ & $12.2 \%$ & $3.4 \%$ & $0.2 \%$ \\
\hline 1998 & 50,462 & FHWA, MF-24 & 1997 VIUS & $85.2 \%$ & $11.2 \%$ & $3.4 \%$ & $0.2 \%$ \\
\hline 1999 & 52,859 & FHWA, MF-24 & 1997 VIUS & $85.4 \%$ & $11.0 \%$ & $3.4 \%$ & $0.2 \%$ \\
\hline 2000 & 52,832 & FHWA, MF-24 & 1997 VIUS & $83.8 \%$ & $12.6 \%$ & $3.4 \%$ & $0.2 \%$ \\
\hline 2001 & 53,294 & FHWA, MF-24 & 1997 VIUS & $83.4 \%$ & $13.0 \%$ & $3.4 \%$ & $0.2 \%$ \\
\hline \multicolumn{4}{|c|}{ Heat content used for conversion to btu: } & $\begin{array}{c}125,000 \\
\text { btu/gallon }\end{array}$ & $\begin{array}{c}\text { 120,900 } \\
\text { btu/gallon }\end{array}$ & $\begin{array}{c}138,700 \\
\text { btu/gallon }\end{array}$ & $\begin{array}{c}90,800 \\
\text { btu/gallon }\end{array}$ \\
\hline
\end{tabular}


Medium/Heavy Trucks:

DOT, FHWA, Highway Statistics 2001, Table VM-1 and annual editions back to 1996; DOT, FHWA, Highway Statistics Summary to 1995. Total gallons for other trucks was the difference between total trucks and 2-axle, 4-tire trucks.

Table A.6

Medium/Heavy Truck Fuel Use and Fuel Type Shares for Calculation of Energy Use

\begin{tabular}{cccccc}
\hline & & Source for & \multicolumn{3}{c}{ Shares by fuel type } \\
\cline { 4 - 6 } Year & $\begin{array}{c}\text { Fuel use } \\
\text { (million gallons) }\end{array}$ & $\begin{array}{c}\text { gasoline/diesel /lpg } \\
\text { shares }\end{array}$ & Gasoline & Diesel & Lpg \\
\hline 1970 & 11,316 & 1977 TIUS & $10.4 \%$ & $89.5 \%$ & $0.1 \%$ \\
1971 & 11,812 & 1977 TIUS & $10.4 \%$ & $89.5 \%$ & $0.1 \%$ \\
1972 & 12,964 & 1977 TIUS & $10.4 \%$ & $89.5 \%$ & $0.1 \%$ \\
1973 & 14,320 & 1977 TIUS & $10.4 \%$ & $89.5 \%$ & $0.1 \%$ \\
1974 & 14,341 & 1977 TIUS & $10.4 \%$ & $89.5 \%$ & $0.1 \%$ \\
1975 & 14,598 & 1977 TIUS & $10.4 \%$ & $89.5 \%$ & $0.1 \%$ \\
1976 & 15,408 & 1977 TIUS & $10.4 \%$ & $89.5 \%$ & $0.1 \%$ \\
1977 & 17,082 & 1977 TIUS & $10.4 \%$ & $89.5 \%$ & $0.1 \%$ \\
1978 & 19,121 & Interpolated & $16.2 \%$ & $83.5 \%$ & $0.3 \%$ \\
1979 & 19,913 & Interpolated & $22.1 \%$ & $77.5 \%$ & $0.5 \%$ \\
1980 & 19,960 & Interpolated & $27.9 \%$ & $71.4 \%$ & $0.6 \%$ \\
1981 & 20,376 & Interpolated & $33.8 \%$ & $65.4 \%$ & $0.8 \%$ \\
1982 & 20,386 & 1982 TIUS & $39.6 \%$ & $59.4 \%$ & $1.0 \%$ \\
1983 & 20,761 & Interpolated & $35.6 \%$ & $63.6 \%$ & $0.8 \%$ \\
1984 & 21,428 & Interpolated & $31.5 \%$ & $67.8 \%$ & $0.7 \%$ \\
1985 & 21,405 & Interpolated & $27.5 \%$ & $72.0 \%$ & $0.5 \%$ \\
1986 & 21,861 & Interpolated & $23.4 \%$ & $76.2 \%$ & $0.4 \%$ \\
1987 & 22,513 & 1987 TIUS & $19.4 \%$ & $80.4 \%$ & $0.2 \%$ \\
1988 & 22,925 & Interpolated & $18.8 \%$ & $81.0 \%$ & $0.3 \%$ \\
1989 & 23,512 & Interpolated & $18.1 \%$ & $81.6 \%$ & $0.3 \%$ \\
1990 & 24,490 & Interpolated & $17.5 \%$ & $82.1 \%$ & $0.4 \%$ \\
1991 & 24,981 & Interpolated & $16.8 \%$ & $82.7 \%$ & $0.4 \%$ \\
1992 & 25,453 & 1992 TIUS & $16.2 \%$ & $83.3 \%$ & $0.5 \%$ \\
1993 & 26,236 & Interpolated & $15.4 \%$ & $84.1 \%$ & $0.5 \%$ \\
1994 & 27,685 & Interpolated & $14.7 \%$ & $84.8 \%$ & $0.5 \%$ \\
1995 & 28,828 & Interpolated & $13.9 \%$ & $85.6 \%$ & $0.5 \%$ \\
1996 & 29,601 & Interpolated & $13.2 \%$ & $86.3 \%$ & $0.5 \%$ \\
1997 & 29,878 & 1997 VIUS & $12.4 \%$ & $87.1 \%$ & $0.5 \%$ \\
1998 & 30,841 & 1997 VIUS & $12.4 \%$ & $87.1 \%$ & $0.5 \%$ \\
1999 & 33,909 & 1997 VIUS & $12.4 \%$ & $87.1 \%$ & $0.5 \%$ \\
2000 & 35,193 & 1997 VIUS & $12.4 \%$ & $87.1 \%$ & $0.5 \%$ \\
2001 & 35,287 & 1997 VIUS & $12.4 \%$ & $87.1 \%$ & $0.5 \%$ \\
\hline & & & 125,000 & 138,700 & 90,800 \\
& Heat & content used for conversionyyyy & btu/gallon & btu/gallon & btu/gallon \\
\hline & & & &
\end{tabular}




\section{Off-highway energy use}

\section{Diesel:}

DOE, EIA, Fuel Oil and Kerosene Sales 2001, Table 1. Unadjusted sales of distillate.

\section{Gasoline:}

DOT, FHWA, Highway Statistics 2001, Table MF-24, and annual editions back to 1985.

Table A.7

Off-Highway Fuel Use

\begin{tabular}{cccccc}
\hline & \multicolumn{2}{c}{$\begin{array}{c}\text { Gasoline } \\
\text { (thousand gallons) }\end{array}$} & & \multicolumn{2}{c}{$\begin{array}{c}\text { Diesel } \\
\text { (thousand gallons) }\end{array}$} \\
\cline { 2 - 3 } \cline { 5 - 6 } Year & Agriculture & Construction & & Agriculture & Construction \\
\hline 1985 & $1,080,677$ & 250,935 & & $3,102,106$ & $1,522,041$ \\
1986 & 964,226 & 275,997 & & $3,340,813$ & $1,659,365$ \\
1987 & 921,692 & 278,767 & & $2,998,681$ & $1,559,873$ \\
1988 & 806,097 & 275,927 & & $3,162,575$ & $1,671,387$ \\
1989 & 821,612 & 297,577 & & $3,360,092$ & $1,689,651$ \\
1990 & 681,220 & 318,184 & & $3,403,400$ & $1,808,646$ \\
1991 & 776,217 & 278,237 & & $3,158,477$ & $1,641,560$ \\
1992 & 805,511 & 272,896 & & $3,499,518$ & $1,757,788$ \\
1993 & 845,320 & 245,299 & & $3,410,827$ & $2,104,299$ \\
1994 & 903,682 & 266,560 & & $3,270,227$ & $2,153,153$ \\
1995 & 926,732 & 280,046 & & $3,476,472$ & $2,173,054$ \\
1996 & 918,085 & 283,911 & & $3,591,383$ & $2,245,922$ \\
1997 & 984,450 & 300,491 & & $3,547,699$ & $2,276,548$ \\
1998 & 906,941 & 234,705 & & $3,410,801$ & $2,477,199$ \\
1999 & 702,700 & 177,758 & & $3,411,623$ & $2,490,492$ \\
2000 & 652,256 & 191,516 & & $3,454,861$ & $2,589,383$ \\
2001 & 801,552 & 506,682 & & $3,584,104$ & $2,708,228$ \\
\hline Heat content used & 125,000 & 125,000 & & 138,700 & 138,700 \\
for conversion & btu/gallon & btu/gallon & & btu/gallon & btu/gallon \\
to btu: & & & & & \\
\hline
\end{tabular}

Note:

The FHWA methodology for estimating construction gasoline use changed in 2001. Previous years' data are likely understated. 
Nonhighway energy use

Air

General Aviation:

DOT, FAA, General Aviation Activity and Avionics Survey: Annual Summary Report Calendar Year 2001, Table 5.1, and annual.

Table A.8

General Aviation Fuel Use

\begin{tabular}{|c|c|c|}
\hline Year & $\begin{array}{c}\text { Jet fuel } \\
\text { (million gallons) }\end{array}$ & $\begin{array}{l}\text { Aviation gasoline } \\
\text { (million gallons) }\end{array}$ \\
\hline 1970 & 208.0 & 551.0 \\
\hline 1971 & 226.0 & 508.0 \\
\hline 1972 & 245.0 & 584.0 \\
\hline 1973 & 304.0 & 411.0 \\
\hline 1974 & 357.0 & 443.0 \\
\hline 1975 & 453.0 & 412.0 \\
\hline 1976 & 495.0 & 432.0 \\
\hline 1977 & 536.0 & 456.0 \\
\hline 1978 & 763.0 & 518.0 \\
\hline 1979 & 736.0 & 570.0 \\
\hline 1980 & 766.0 & 520.0 \\
\hline 1981 & 759.0 & 489.0 \\
\hline 1982 & 887.0 & 448.0 \\
\hline 1983 & 613.0 & 428.0 \\
\hline 1984 & 738.9 & 462.4 \\
\hline 1985 & 691.0 & 421.0 \\
\hline 1986 & 732.1 & 408.6 \\
\hline 1987 & 672.7 & 401.8 \\
\hline 1988 & 746.0 & 398.0 \\
\hline 1989 & 688.0 & 342.8 \\
\hline 1990 & 662.0 & 353.0 \\
\hline 1991 & 579.0 & 348.0 \\
\hline 1992 & 496.0 & 306.0 \\
\hline 1993 & 454.1 & 268.4 \\
\hline 1994 & 470.8 & 264.1 \\
\hline 1995 & 544.0 & 276.0 \\
\hline 1996 & 567.5 & 286.5 \\
\hline 1997 & 639.4 & 289.7 \\
\hline 1998 & 814.6 & 311.4 \\
\hline 1999 & 967.2 & 345.4 \\
\hline 2000 & 998.1 & 336.3 \\
\hline 2001 & 938.7 & 319.3 \\
\hline $\begin{array}{l}\text { Heat content used for } \\
\text { conversion to btu: }\end{array}$ & $\begin{array}{c}135,000 \\
\text { btu/gallon }\end{array}$ & $\begin{array}{c}\text { 120,200 } \\
\text { btu/gallon }\end{array}$ \\
\hline
\end{tabular}




\section{Domestic and International Air Carrier:}

DOT, Bureau of Transportation Statistics, "Fuel Cost and Consumption Tables."

Because the data for international included fuel purchased abroad, the international total was divided in half to estimate domestic fuel use for international flights.

Table A.9

Air Carrier Fuel Use

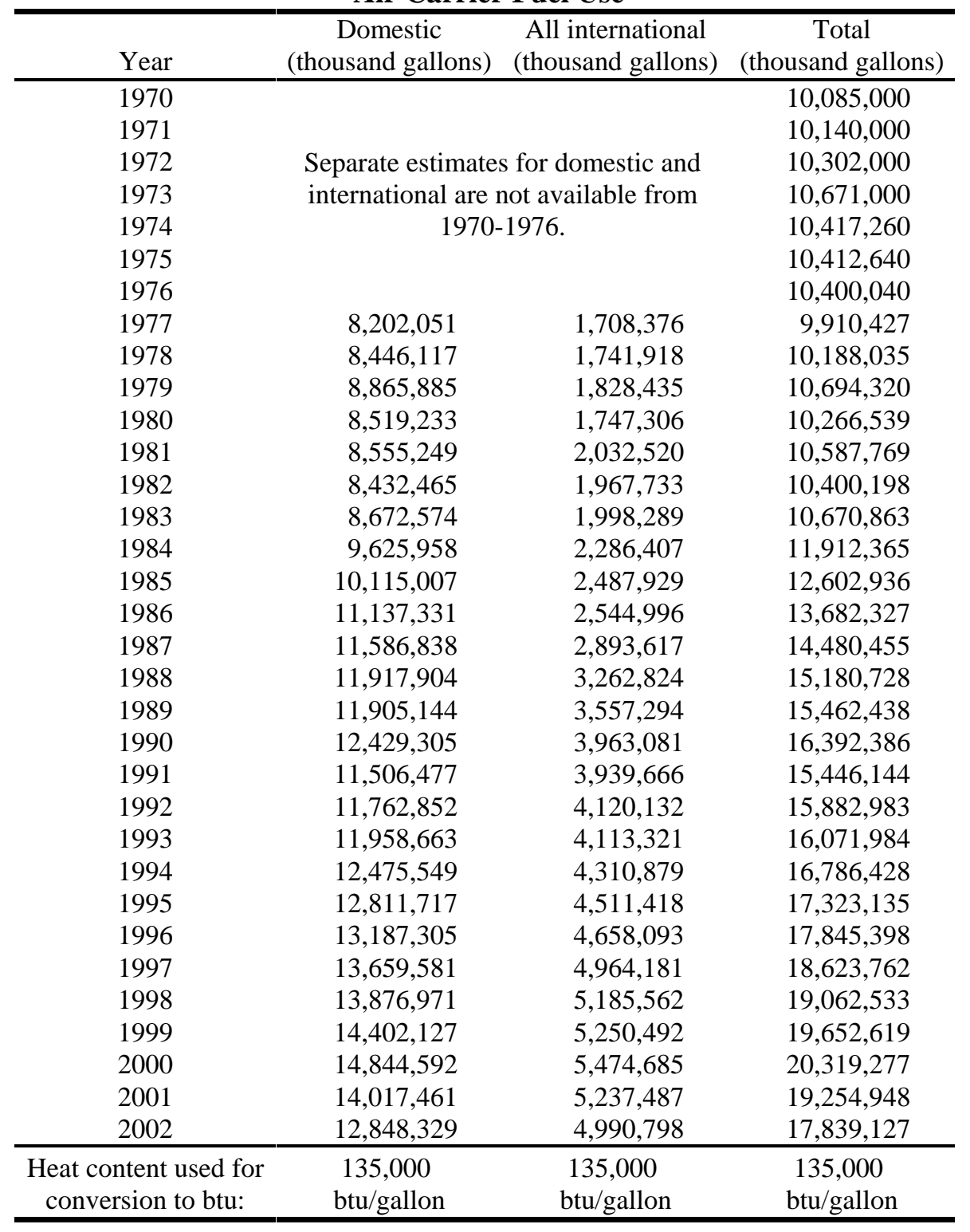


Water

Freight:

Total - DOE, EIA, Fuel Oil and Kerosene Sales 2001, Table 23. Adjusted sales of distillate and residual fuel oil for vessel bunkering. (This may include some amounts of bunker fuels used for recreational purposes.)

Table A.10

Diesel and Residual Fuel Oil for Vessel Bunkering

\begin{tabular}{ccc}
\hline Year & $\begin{array}{c}\text { Distillate fuel oil } \\
\text { (thousand gallons) }\end{array}$ & $\begin{array}{c}\text { Residual fuel oil } \\
\text { (thousand gallons) }\end{array}$ \\
\hline 1970 & 819,000 & $3,774,120$ \\
1971 & 880,000 & $3,307,000$ \\
1972 & $1,013,000$ & $3,273,000$ \\
1973 & $1,125,000$ & $3,859,000$ \\
1974 & $1,018,920$ & $3,827,040$ \\
1975 & $1,097,880$ & $4,060,140$ \\
1976 & $1,220,100$ & $4,977,000$ \\
1977 & $1,407,420$ & $5,416,740$ \\
1978 & $1,578,822$ & $6,614,790$ \\
1979 & $1,630,858$ & $8,002,672$ \\
1980 & 717,376 & $7,454,242$ \\
1981 & $1,723,143$ & $7,922,512$ \\
1982 & $1,423,216$ & $6,408,818$ \\
1983 & $1,418,890$ & $5,724,115$ \\
1984 & $1,692,141$ & $5,687,375$ \\
1985 & $1,894,016$ & $5,473,614$ \\
1986 & $2,034,215$ & $5,287,347$ \\
1987 & $2,223,258$ & $5,259,272$ \\
1988 & $2,310,367$ & $5,248,981$ \\
1989 & $2,356,444$ & $5,410,263$ \\
1990 & $2,197,004$ & $6,248,095$ \\
1991 & $2,167,640$ & $6,786,055$ \\
1992 & $2,240,170$ & $7,199,078$ \\
1993 & $2,043,745$ & $6,269,882$ \\
1994 & $2,026,899$ & $5,944,383$ \\
1995 & $1,978,105$ & $6,431,238$ \\
1996 & $2,177,608$ & $5,804,977$ \\
1997 & $2,107,561$ & $4,789,861$ \\
1998 & $2,125,568$ & $4,640,153$ \\
1999 & $2,064,590$ & $5,598,630$ \\
2000 & $2,041,433$ & $6,192,294$ \\
2001 & $2,093,252$ & $3,679,843$ \\
\hline Heat content used for & 138,700 & 149,700 \\
bonversion to btu: & btu/gallon & btu/gallon \\
\hline & & \\
& & \\
& &
\end{tabular}




\section{Recreational Boating:}

Fuel use by recreational boating from 1977-on was calculated using the methodology developed by D. L. Greene in the report, Off-Highway Use of Gasoline in the United States (DOT, FHWA, July 1986, p. 3-22). Results from Model 1 in the report indicated an average annual consumption of 205 gallons per boat. Total consumption in gallons was then calculated using the following equation: Total $=0.95$ (Gal/boat) (number of boats). An estimate of number of recreational boats in operation is from the U.S. Coast Guard (numbered boats). Fuel use for recreational boating from 1970 to 1976 was from FHWA, Highway Statistics, 1976, Table MF-24, and annual editions 1970-75.

Table A.11

Recreational Boating Fuel Use

\begin{tabular}{|c|c|c|c|}
\hline Year & $\begin{array}{c}\text { Number of } \\
\text { numbered boats }\end{array}$ & Source & $\begin{array}{c}\text { Estimated } \\
\text { gasoline use } \\
\text { (thousand gallons) }\end{array}$ \\
\hline 1970 & & FHWA, MF-24 & 598,000 \\
\hline 1971 & & FHWA, MF-24 & 645,000 \\
\hline 1972 & & FHWA, MF-24 & 687,000 \\
\hline 1973 & & FHWA, MF-24 & 717,000 \\
\hline 1974 & & FHWA, MF-24 & 696,780 \\
\hline 1975 & & FHWA, MF-24 & 729,540 \\
\hline 1976 & & FHWA, MF-24 & 763,980 \\
\hline 1977 & $7,975,587$ & & $1,553,246$ \\
\hline 1978 & $8,035,905$ & & $1,564,992$ \\
\hline 1979 & $8,278,723$ & & $1,612,281$ \\
\hline 1980 & $8,577,857$ & & $1,670,538$ \\
\hline 1981 & $8,905,097$ & & $1,734,268$ \\
\hline 1982 & $9,073,972$ & Multiply by: & $1,767,156$ \\
\hline 1983 & $9,165,094$ & $0.95 \times$ & $1,784,902$ \\
\hline 1984 & $9,420,011$ & 205 gallons/boat & $1,834,547$ \\
\hline 1985 & $9,589,483$ & & $1,867,552$ \\
\hline 1986 & $9,876,197$ & & $1,923,389$ \\
\hline 1987 & $9,963,696$ & & $1,940,430$ \\
\hline 1988 & $10,362,613$ & & $2,018,119$ \\
\hline 1989 & $10,777,370$ & & $2,098,893$ \\
\hline 1990 & $10,996,253$ & & $2,141,520$ \\
\hline 1991 & $11,068,440$ & & $2,155,579$ \\
\hline 1992 & $11,132,386$ & & $2,168,032$ \\
\hline 1993 & $11,282,736$ & & $2,197,313$ \\
\hline 1994 & $11,429,585$ & & $2,225,912$ \\
\hline 1995 & $11,734,710$ & & $2,285,335$ \\
\hline 1996 & $11,877,938$ & & $2,313,228$ \\
\hline 1997 & $12,312,982$ & & $2,397,953$ \\
\hline 1998 & $12,565,930$ & & $2,447,215$ \\
\hline 1999 & $12,738,271$ & & $2,480,778$ \\
\hline 2000 & $12,782,143$ & & $2,489,322$ \\
\hline 2001 & $12,876,346$ & & $2,507,668$ \\
\hline \multicolumn{3}{|c|}{ Heat content used for conversion to btu: } & $\begin{array}{r}125,000 \\
\text { btu/gallon }\end{array}$ \\
\hline
\end{tabular}




\section{Pipeline}

The sum of natural gas, crude petroleum and petroleum product, and coal slurry and water.

Natural Gas:

The amount of natural gas used to transport natural gas was defined as "pipeline fuel" as reported in DOE, EIA, Natural Gas Annual 2001, Table 1. Cubic feet were converted to Btu using 1,031 Btu/ $/ \mathrm{ft}^{3}$. Electricity use was estimated using the following procedure as reported on p. 5-110 of J. N. Hooker et al., End Use Energy Consumption DataBase: Transportation Sector. The energy consumption of a natural gas pipeline was taken to be the energy content of the fuel used to drive the pumps. Some $94 \%$ of the installed pumping horsepower was supplied by natural gas. The remaining $6 \%$ of the horse power was generated more efficiently, mostly by electric motors. The energy consumed by natural gas pipeline pumps that were electrically powered was not known. In order to estimate the electricity consumed, the Btu of natural gas pipeline fuel consumed was multiplied by a factor of 0.015 . From this computed value, electricity efficiency and generation loss must be taken into account. The electricity energy use in Btu must be converted to $\mathrm{kWhr}$, using the conversion factor $29.305 \times 10^{-5} \mathrm{kWhr} / \mathrm{Btu}$. Electricity generation and distribution efficiency was $29 \%$. When generation and distribution efficiency are taken into account, $1 \mathrm{kWhr}$ equals 11,765 Btu.

\section{Crude petroleum and petroleum product:}

J. N. Hooker, Oil Pipeline Energy Consumption and Efficiency, ORNL-5697, ORNL, Oak Ridge, TN, 1981. (Data held constant; Latest available data.)

\section{Coal slurry and water:}

W. F. Banks, Systems, Science and Software, Energy Consumption in the Pipeline Industry, LaJolla, CA, October 1977. (Data held constant; Latest available data.) 
Table A.12

Pipeline Fuel Use

\begin{tabular}{ccccc}
\hline & $\begin{array}{c}\text { Natural gas } \\
\text { (million } \\
\text { cubic feet) }\end{array}$ & $\begin{array}{c}\text { Formula for estimating } \\
\text { electricity use }\end{array}$ & $\begin{array}{c}\text { Estimated } \\
\text { electricity use } \\
\text { (million kWhr) }\end{array}$ & $\begin{array}{c}\text { Electricity } \\
\text { constant } \\
\text { (btu) }\end{array}$ \\
\hline 1970 & 722,166 & & $3,272.9$ & 212.1 \\
1971 & 742,592 & $3,365.4$ & 212.1 \\
1972 & 766,156 & Multiply natural gas by & $3,472.2$ & 212.1 \\
1973 & 728,177 & heat content to get btu & $3,300.1$ & 212.1 \\
1974 & 668,792 & $\times 0.015$ & $3,031.0$ & 212.1 \\
1975 & 582,963 & $\times\left(29.305 \times 10^{-5} \mathrm{kWhr} / \mathrm{btu}\right)$ & $2,642.0$ & 212.1 \\
1976 & 548,323 & & $2,485.0$ & 212.1 \\
1977 & 532,669 & $2,414.1$ & 212.1 \\
1978 & 530,451 & $2,404.0$ & 212.1 \\
1979 & 600,964 & $2,723.6$ & 212.1 \\
1980 & 634,622 & $2,876.1$ & 212.1 \\
1981 & 642,325 & $2,911.0$ & 212.1 \\
1982 & 596,411 & $2,703.0$ & 212.1 \\
1983 & 490,042 & $2,220.9$ & 212.1 \\
1984 & 528,754 & $2,396.3$ & 212.1 \\
1985 & 503,766 & $2,283.1$ & 212.1 \\
1986 & 485,041 & $2,198.2$ & 212.1 \\
1987 & 519,170 & $2,352.9$ & 212.1 \\
1988 & 613,912 & $2,782.3$ & 212.1 \\
1989 & 629,308 & $2,852.0$ & 212.1 \\
1990 & 659,816 & $2,990.3$ & 212.1 \\
1991 & 601,305 & $2,725.1$ & 212.1 \\
1992 & 587,710 & $2,663.5$ & 212.1 \\
1993 & 624,308 & $2,829.4$ & 212.1 \\
1994 & 685,362 & $3,106.1$ & 212.1 \\
1995 & 700,335 & $3,173.9$ & 212.1 \\
1996 & 711,446 & $3,224.3$ & 212.1 \\
1997 & 751,470 & $3,405.7$ & 212.1 \\
1998 & 635,477 & $2,880.0$ & 212.1 \\
1999 & 645,319 & $2,924.6$ & 212.1 \\
2000 & 642,210 & $2,910.5$ & 212.1 \\
2001 & 623,929 & $2,827.7$ & 212.1 \\
\hline Heat content used for & 1,031 btu/cubic & 11,765 & \\
conversion to btu: & foot & Btu/kWhr & \\
\hline & & & & \\
\hline
\end{tabular}


Rail

Freight:

AAR, Railroad Facts, 2002 Edition, Washington, DC, 2002.

Table A.13

Class I Freight Railroad

Fuel Use

\begin{tabular}{cc}
\hline Year & $\begin{array}{c}\text { Diesel fuel } \\
\text { (thousand gallons) }\end{array}$ \\
\hline 1970 & $3,807,663$ \\
1971 & $3,822,907$ \\
1972 & $3,996,985$ \\
1973 & $4,160,730$ \\
1974 & $4,175,375$ \\
1975 & $3,736,484$ \\
1976 & $3,895,542$ \\
1977 & $3,985,069$ \\
1978 & $3,968,007$ \\
1979 & $4,072,187$ \\
1980 & $3,955,996$ \\
1981 & $3,756,439$ \\
1982 & $3,178,116$ \\
1983 & $3,137,295$ \\
1984 & $3,388,173$ \\
1985 & $3,144,190$ \\
1986 & $3,039,069$ \\
1987 & $3,102,227$ \\
1988 & $3,182,267$ \\
1989 & $3,190,815$ \\
1990 & $3,134,446$ \\
1991 & $2,925,970$ \\
1992 & $3,022,108$ \\
1993 & $3,111,981$ \\
1994 & $3,355,802$ \\
1995 & $3,503,096$ \\
1996 & $3,600,649$ \\
1997 & $3,602,793$ \\
1998 & $3,619,341$ \\
1999 & $3,749,428$ \\
2000 & $3,720,107$ \\
2001 & $3,729,985$ \\
\hline Heat content used for & 138,700 \\
conversion to btu: & Btu/gallon \\
\hline & \\
\hline
\end{tabular}




\section{Passenger:}

Commuter - APTA, 2003 Transit Fact Book, Washington, DC, 2003.

Table A.14

Commuter Rail Fuel Use

\begin{tabular}{ccc}
\hline Year & $\begin{array}{c}\text { Diesel } \\
\text { (thousand gallons) }\end{array}$ & $\begin{array}{c}\text { Electricity } \\
\text { (million kWhr) }\end{array}$ \\
\hline 1984 & 58,320 & 901 \\
1985 & 55,372 & 1,043 \\
1986 & 54,608 & 1,170 \\
1987 & 51,594 & 1,155 \\
1988 & 53,054 & 1,195 \\
1989 & 52,516 & 1,293 \\
1990 & 52,681 & 1,226 \\
1991 & 54,315 & 1,239 \\
1992 & 54,951 & 1,124 \\
1993 & 59,766 & 1,196 \\
1994 & 61,900 & 1,244 \\
1995 & 63,064 & 1,253 \\
1996 & 61,888 & 1,255 \\
1997 & 63,195 & 1,270 \\
1998 & 69,200 & 1,299 \\
1999 & 73,005 & 1,322 \\
2000 & 70,818 & 1,370 \\
2001 & 72,204 & 1,354 \\
\hline Heat content used for & 138,700 & 11,765 \\
conversion to btu: & Btu/gallon & Btu/kWhr \\
\hline
\end{tabular}


Transit - APTA, 2003 Transit Fact Book, Washington, DC, 2003. Includes light rail and heavy rail.

\section{Table A.15}

Transit Rail Fuel Use

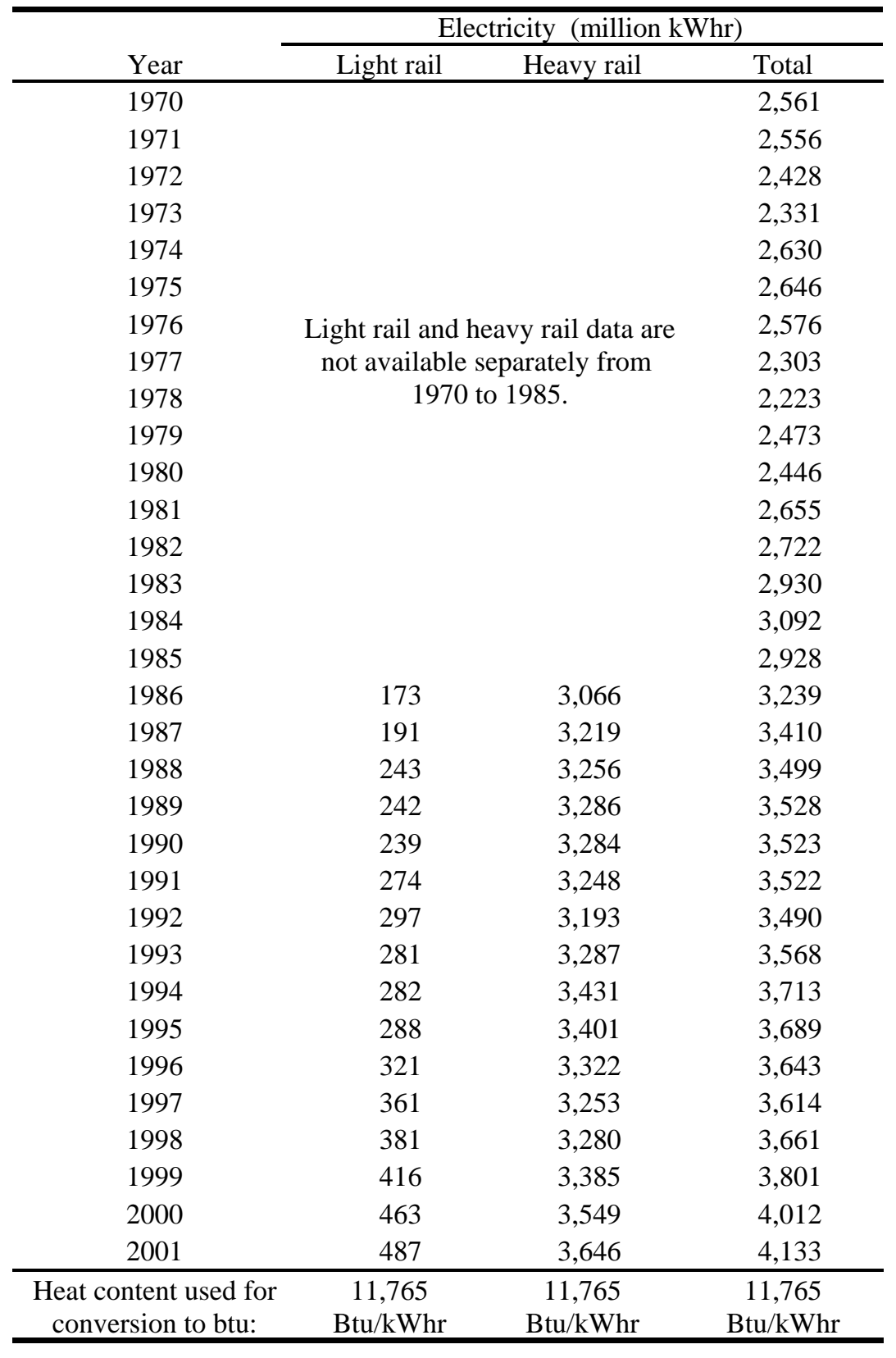


Intercity - Personal communication with Amtrak, Washington, DC.

Table A.16

Intercity Rail Fuel Use

\begin{tabular}{ccc}
\hline Year & $\begin{array}{c}\text { Diesel fuel } \\
\text { (thousand gallons) }\end{array}$ & $\begin{array}{c}\text { Electricity } \\
\text { (thousand kWhr) }\end{array}$ \\
\hline 1994 & 73,516 & 308,948 \\
1995 & 72,371 & 335,818 \\
1996 & 71,226 & 362,689 \\
1997 & 75,656 & 389,559 \\
1998 & 75,999 & 416,429 \\
1999 & 79,173 & 443,300 \\
2000 & 94,968 & 470,170 \\
2001 & 96,846 & 817,274 \\
\hline Heat content used for & 138,700 & 11,765 \\
conversion to btu: & Btu/gallon & Btu/kWhr \\
\hline
\end{tabular}

\section{Calculation of \\ Million Barrels per Day \\ Crude Oil Equivalent}

One gallon of gasoline, diesel fuel, or lpg is estimated to be the equivalent of one gallon of crude oil. Petroleum used for electricity was calculated using the following formula:

$(\{[(\mathrm{BTU} * \mathrm{~S}) / \mathrm{G}] / \mathrm{P}\} / 365) / 1000$

$\mathrm{BTU}=$ Btus of electricity from Table 2.4

$\mathrm{S}=$ Share of petroleum used in making primary electricity (Calculated from Table 2.6 from the EIA, Monthly Energy Review)

$\mathrm{G}=$ Electricity generation and distribution (assumed 29\%)

$\mathrm{P} \quad=$ Btus per barrel of petroleum product (Table A3 from the EIA, Monthly Energy Review). 


\section{Passenger Travel and Energy Use}

\section{Automobiles}

Number of vehicles, vehicle-miles - DOT, FHWA, Highway Statistics, 2001, Table VM-1. Data series shown in Table 4.1.

Passenger-miles - Vehicle-miles multiplied by an average load factor.

Load factor - 2001 NHTS shows automobile load factor as 1.1 persons per vehicle.

Energy intensities -

Btu per vehicle-mile - Automobile energy use divided by vehicle-miles.

Btu per passenger-mile - Automobile energy use divided by passenger-miles.

Energy use - See Energy Use Sources, p. A-3. Data series shown in Table 2.6.

Light trucks

Number of vehicles, vehicle-miles - DOT, FHWA, Highway Statistics 2001, Table VM-1. Data by truck type were multiplied by the shares of trucks/truck travel which are for personal use (Table A.17).

Passenger-miles - Vehicle-miles multiplied by an average load factor.

Load factor - 2001 NHTS shows personal light truck load factor as 1.72 persons per vehicle.

Energy intensities -

Btu per vehicle-mile - Personal light truck energy use divided by personal light truck vehicle-miles. Btu per passenger-mile - Personal light truck energy use divided by personal light truck passengermiles.

Energy use - See Energy Use Sources, p. A-6, A-7 (light trucks, medium/heavy trucks). Data by truck type were multiplied by the shares of truck fuel use which are for personal use (Table A.17) which were derived by ORNL from the 1997 VIUS Micro Data File on CD.

Table A.17

Share of Trucks, Truck Travel, and Fuel Use for Personal Travel
Personal trucks
$75.2 \% \quad$ 2-axle, 4-tire trucks
$16.9 \%$ Other single-unit and combination trucks

\section{Personal truck travel}
70.7\% 2-axle, 4-tire trucks
$7.1 \%$ Other single-unit and combination trucks

\section{Personal truck fuel use}
$68.5 \% \quad$ 2-axle, 4-tire trucks
$3.7 \%$ Other single-unit and combination trucks

Note:

Since these shares come from the 1997 VIUS, they may underestimate the amount of personal trucks, truck travel, and energy use for 2001. 


\section{Motorcycles}

Number of vehicles, vehicle-miles - DOT, FHWA, Highway Statistics 20001 Table VM-1.

Passenger-miles - Vehicle-miles multiplied by an average load factor.

Load factor - 2001 NHTS shows motorcycle load factor as 1.22 persons per vehicle.

Energy intensities -

Btu per vehicle-mile - Motorcycle energy use divided by vehicle-miles.

Btu per passenger-mile - Motorcycle energy use divided by passenger-miles.

Energy use - See Energy Use Sources, p. A-4. Data series shown in Table 2.6.

Demand Response

Number of vehicles, vehicle-miles, passenger-miles - APTA, 2003 Public Transportation Fact Book, Washington, DC, 2003.

Load factor - Passenger-miles divided by vehicle-miles.

Energy intensities -

Btu per vehicle-mile - Energy use divided by vehicle-miles.

Btu per passenger-mile - Energy use divided by passenger-miles.

Energy use - APTA, 2003 Public Transportation Fact Book, Washington, DC, 2003, Table 95.

Vanpool

Number of vehicles, vehicle-miles, passenger-miles - APTA, 2003 Public Transportation Fact Book, Washington, DC, 2003.

Load factor - Passenger-miles divided by vehicle-miles.

Energy intensities -

Btu per vehicle-mile - Energy use divided by vehicle-miles.

Btu per passenger-mile - Energy use divided by passenger-miles.

Energy use - APTA, 2003 Public Transportation Fact Book, Washington, DC, 2003, Table 144.

Buses

Transit

Number of vehicles, vehicle-miles, passenger-miles - APTA, 2003 Public Transportation Fact Book, Washington, DC, 2003. Data series shown on Table 5.12.

Load factor - Passenger-miles divided by vehicle-miles.

Energy intensities -

Btu per vehicle-mile - Transit bus energy use divided by transit bus vehicle-miles.

Btu per passenger-mile - Transit bus energy use divided by transit bus passenger-miles.

Energy use - See Energy Use Sources, p. A-4. Data series shown in Table 5.12. 


\section{Intercity}

Passenger-miles - Eno Foundation for Transportation, Transportation in America 2001, Nineteenth edition, Washington, DC. Data series shown in Table 5.13. Because the 2001 data were not available at the time this report went to press, the 2000 data were used again for 2001 .

\section{Energy intensities -}

Btu per passenger-mile - Intercity bus energy use divided by intercity bus passenger-miles.

Energy use - See Energy Use Sources, p. A-5 . Data series shown in Table 5.13. Because the 2001 data were not available at the time this report went to press, the 2000 data were used again for 2001.

\section{School}

Number of vehicles - DOT, FHWA, Highway Statistics 2001, Table MV-10. Data series shown in Table 5.13.

Energy use - See Energy Use Sources, p. A-5 . Data series shown in Table 5.13. Because the 2001 data were not available at the time this report went to press, the 2000 data were used again for 2001.

\section{Air}

\section{Certificated air carriers}

Aircraft-miles, passenger-miles - DOT, BTS, Air Carrier Traffic Statistics Monthly, December 2001/2000, Washington, DC.

Load factor - Passenger-miles divided by aircraft-miles.

Energy intensities -

Btu per passenger-mile - Certificated air carrier energy use divided by passenger-miles.

Energy use - See Energy Use Sources, p. A-10. All of domestic fuel use and half of international fuel use was considered to be domestic use.

Note: These data differ from the data in Table 9.1 because that table contains data on ALL domestic AND international air carrier energy use and passenger-miles.

\section{General aviation}

Number of vehicles - DOT, FAA, General Aviation Activity and Avionics Survey: Calendar Year 2001. Data series shown in Table 9.2.

Passenger-miles - Eno Foundation for Transportation, Transportation in America 2001, Nineteenth edition, Washington, DC. Data series shown in Table 9.2.

Energy intensities -

Btu per passenger-mile - General aviation energy use divided by passenger-miles.

Energy use - See Energy Use Sources, p. A-9. Data series shown in Table 9.2.

\section{$\underline{\text { Recreational boating }}$}

Number of vehicles - DOT, U.S. Coast Guard, Office of Boating Safety, Washington, DC, 2003. Energy use - See Energy Use Sources, p. A-12. 
Intercity

Number of vehicles, vehicle-miles, passenger-miles - AAR, Railroad Facts, 2002 Edition, Washington, DC, 2002.

Load factor - Passenger-miles divided by vehicle-miles.

Energy Intensities -

Btu per vehicle-mile - Intercity rail energy use divided by vehicle-miles.

Btu per passenger-mile - Intercity rail energy use divided by passenger-miles.

Energy use - See Energy Use Sources, p. A-18. Data series shown in Table 9.11.

\section{Transit}

Number of vehicles, vehicle-miles, passenger-miles - APTA, 2003 Public Transportation Fact Book, Washington, DC, 2003. Sum of light and heavy rail transit. Data series shown on Table 9.13.

Load factor - Passenger-miles divided by vehicle-miles.

Energy intensities -

Btu per vehicle-mile - Light and heavy transit rail energy use divided by vehicle-miles.

Btu per passenger-mile - Light and heavy transit rail energy use divided by passenger-miles.

Energy use - See Energy Use Sources, p. A-17. Data series shown in Table 9.13.

\section{Commuter}

Number of vehicles, vehicle-miles, passenger-miles - APTA, 2003 Public Transportation Fact Book, Washington, DC, 2003. Data series shown on Table 9.12.

Load factor - Passenger-miles divided by vehicle-miles.

Energy intensities -

Btu per vehicle-mile - Commuter rail energy use divided by vehicle-miles.

Btu per passenger-mile - Commuter rail energy use divided by passenger-miles.

Energy use - See Energy Use Sources, p. A-16. Data series shown in Table 9.12. 


\section{Highway Passenger Mode Energy Intensities}

\section{Automobiles}

Btu per vehicle-mile - Automobile energy use divided by automobile vehicle miles of travel.

Energy use - See Energy Use Sources, p. A-3. Data series shown in Table 2.6.

Vehicle-miles - DOT, FHWA, Highway Statistics 2001, Table VM-1 and annual editions back to 1996; DOT, FHWA, Highway Statistics Summary to 1995. Data series shown in Table 4.1.

Btu per passenger-mile - Automobile energy use divided by automobile passenger-miles.

Energy use - See Energy Use Sources, p. A-3. Data series shown in Table 2.6.

Passenger miles - Vehicle miles multiplied by an average load factor.

Vehicle-miles - DOT, FHWA, Highway Statistics 2001, Table VM-1 and annual editions back to 1996; DOT, FHWA, Highway Statistics Summary to 1995. Data series shown in Table 4.1.

Load factor - NPTS 1969, 1977, 1983/84, 1990, and 1995, and NHTS 2001.

Table A.18

Automobile Load Factor used to calculate Passenger-Miles

\begin{tabular}{lcc}
\hline Year & Source & Load Factor \\
\hline 1970 & 1969 NPTS & 1.90 \\
1971 & Interpolated & 1.90 \\
1972 & Interpolated & 1.90 \\
1973 & Interpolated & 1.90 \\
1974 & Interpolated & 1.90 \\
1975 & Interpolated & 1.90 \\
1976 & Interpolated & 1.90 \\
1977 & 1977 NPTS & 1.90 \\
1978 & Interpolated & 1.88 \\
1979 & Interpolated & 1.87 \\
1980 & Interpolated & 1.85 \\
1981 & Interpolated & 1.83 \\
1982 & Interpolated & 1.82 \\
1983 & 1983/84 NPTS & 1.80 \\
1984 & Interpolated & 1.77 \\
1985 & Interpolated & 1.74 \\
1986 & Interpolated & 1.71 \\
1987 & Interpolated & 1.69 \\
1988 & Interpolated & 1.66 \\
1989 & Interpolated & 1.63 \\
1990 & 1990 NPTS & 1.60 \\
1991 & Interpolated & 1.60 \\
1992 & Interpolated & 1.60 \\
1993 & Interpolated & 1.60 \\
1994 & Interpolated & 1.60 \\
1995 & 1995 NPTS & 1.60 \\
1996 & Interpolated & 1.60 \\
1997 & Interpolated & 1.59 \\
1998 & Interpolated & 1.59 \\
1999 & Interpolated & 1.58 \\
2000 & Interpolated & 1.58 \\
2001 & 2001 NHTS & 1.57 \\
\hline & & \\
\hline & &
\end{tabular}




\section{Light trucks}

Btu per vehicle-mile - Light truck energy use divided by light truck vehicle miles of travel.

Energy use - See Energy Use Sources, p. A-6. Data series shown in Table 2.6.

Vehicle-miles - DOT, FHWA, Highway Statistics 2001, Table VM-1 and annual editions back to

1996; DOT, FHWA, Highway Statistics Summary to 1995. Data series shown in Table 4.2.

Buses

Transit

Btu per vehicle-mile - Transit bus energy use divided by transit bus vehicle-miles.

Energy use - See Energy Use Sources, p. A-4. Data series shown in Table 5.12.

Vehicle-miles - APTA, 2003 Public Transportation Fact Book, Washington, DC, 2003. Data series shown on Table 5.12.

Btu per passenger-mile - Transit bus energy use divided by transit bus passenger-miles.

Energy use - See Energy Use Sources, p. A-4. Data series shown in Table 5.12.

Passenger-miles - APTA, 2003 Public Transportation Fact Book, Washington, DC, 2003. Data series shown on Table 5.12.

\section{Intercity}

Btu per passenger-mile - Intercity bus energy use divided by intercity bus passenger-miles.

Energy use - See Energy Use Sources, p. A-5 . Data series shown in Table 5.13. Because the 2001 data were not available at the time this report went to press, the 2000 data were used again for 2001.

Passenger-miles - Eno Foundation for Transportation, Transportation in America 2001, Nineteenth edition, Washington, DC. Data series shown in Table 5.13. Because the 2001 data were not available at the time this report went to press, the 2000 data were used again for 2001 . 


\section{Nonhighway Mode Energy Intensities}

Air

\section{Certificated air carriers}

Btu per passenger-mile - Certificated air carrier energy use divided by passenger-miles.

Energy use - See Energy Use Sources, p. A-10. All of domestic fuel use and half of international fuel use was considered to be domestic use.

Passenger-miles - DOT, BTS, Air Carrier Traffic Statistics Monthly, December 2001/2000, Washington, DC, and annual editions back to 1994. Pre-1994 data are from various editions of the FAA Statistical Handbook of Aviation (no longer published). Scheduled service passenger-miles of domestic air carriers and half of international air carriers were used to coincide with fuel use.

Note: These data differ from the data in Table 9.1 because that table contains data on ALL domestic AND international air carrier energy use and passenger-miles.

\section{General aviation}

Btu per passenger-mile - General aviation energy use divided by passenger-miles.

Energy use - See Energy Use Sources, p. A-9. Data series shown in Table 9.2.

Passenger-miles - Eno Foundation for Transportation, Transportation in America 2001,

Nineteenth edition, Washington, DC. Data series shown in Table 9.2.

Rail

Intercity

Btu per passenger-mile - Intercity rail energy use divided by passenger-miles.

Energy use - See Energy Use Sources, p. A-18. Data series shown in Table 9.11.

Passenger-miles - AAR, Railroad Facts, 2002 Edition, and previous annual editions.

\section{Transit}

Btu per passenger-mile - Transit rail energy use divided by passenger-miles.

Energy use - See Energy Use Sources, p. A-17. Data series shown in Table 9.13.

Passenger-miles - APTA, 2003 Public Transportation Fact Book, Washington, DC, 2003. Data series shown on Table 9.13.

\section{Commuter}

Btu per passenger-mile - Commuter rail energy use divided by passenger-miles.

Energy use - See Energy Use Sources, p. A-16. Data series shown in Table 9.12.

Passenger-miles - APTA, 2003 Public Transportation Fact Book, Washington, DC, 2003. Data series shown on Table 9.12. 


\section{Freight Movement and Energy Use}

Truck

Number of vehicles - DOT, FHWA, Highway Statistics 2001, Table VM-1. Data by truck type were multiplied by the shares of trucks engaged in intercity freight movement (Table A.19).

Ton miles, tons shipped and average length of haul - Eno Transportation Foundation, Transportation in America 2001, Nineteenth Edition, Washington, DC, 2002.

Energy intensity - Freight truck energy use divided by ton-miles.

Energy use - See Energy Use Sources (light trucks, medium/heavy trucks), pp. A-6, A-7. Data by truck type were multiplied by the shares of trucks engaged in intercity freight movement (Table A.19).

\section{Table A.19}

Share of Trucks and Truck Fuel Use

for Trucks Engaged in Intercity Freight Movement

\begin{tabular}{ll}
\hline Intercity freight trucks \\
$0.4 \%$ & 2-axle, 4-tire trucks \\
$29.0 \%$ & Other single-unit and combination trucks \\
Intercity freight truck fuel use \\
$1.0 \%$ & 2-axle, 4 -tire trucks \\
$71.3 \%$ & Other single-unit and combination trucks \\
\hline
\end{tabular}

These percentages were derived by ORNL from the 1997 VIUS Micro Data File on CD. Intercity freight trucks were defined as any truck whose:

- greatest share of miles were traveled more than 50 miles away from the vehicle's home base; and

- principal use was not personal or passenger transportation; and

- body type was not pickup, minivan, or utility vehicle.

Rail

Number of locomotives, ton-miles, tons shipped, average length of haul - AAR, Railroad Facts, 2002 Edition, Washington, DC, 2002. Data series shown in Table 9.8.

Energy intensity - Class I rail energy use divided by freight car-miles.

Energy use - See Energy Use Sources, p. A-15. Data series shown in Table 9.8.

Water

Number of vehicles - U.S. Department of the Army, Army Corps of Engineers, "Summary of U.S. Flag Passenger and Cargo Vessels, 2001," New Orleans, LA, 2002.

Ton-miles, tons shipped, average length of haul - U.S. Department of the Army, Army Corps of Engineers, Waterborne Commerce of the United States, Calendar Year 2001, Part 5: National Summaries, New Orleans, LA, 2002. Data series shown in Table 9.4.

Btu per ton-mile - Domestic waterborne commerce energy use divided by ton-miles.

Energy use - See Energy Use Sources, p. A-11. Data series shown in Table 9.4. 


\section{Freight Mode Energy Intensities}

Truck

Btu per vehicle-mile - Heavy single-unit and combination truck energy use divided by vehicle miles

Energy use - See Energy Use Sources (medium/heavy trucks), p. A-7.

Vehicle-miles - DOT, FHWA, Highway Statistics 2001, Table VM-1 and annual editions back to 1996; DOT, FHWA, Highway Statistics Summary to 1995. Data series is the total of vehicle travel data on Tables 5.1 and 5.2.

Rail

Btu per freight car-mile - Class I rail energy use divided by freight car-miles.

Energy use - See Energy Use Sources, p. A-15. Data series shown in Table 9.8.

Freight car miles - AAR, Railroad Facts, 2002 Edition, Washington, DC, 2002. Data series shown in Table 9.8 .

Btu per ton-mile - Class I rail energy use divided by ton-miles.

Energy use - See Energy Use Sources, p. A-15. Data series shown in Table 9.8.

Ton-miles - AAR, Railroad Facts, 2002 Edition, Washington, DC, 2002. Data series shown in Table 9.8.

Water

Btu per ton-mile - Domestic waterborne commerce energy use divided by ton-miles.

Energy use - See Energy Use Sources, p. A-11. Data series shown in Table 9.4.

Ton-miles - U.S. Department of the Army, Army Corps of Engineers, Waterborne Commerce of the United States, Calendar Year 2001, Part 5: National Summaries, New Orleans, LA, 2002. Data series shown in Table 9.4. 


\section{Highway Vehicle Stock and New Sales 2001 Calendar Year}

\section{Automobiles}

Stock - Vehicle registrations by model year are from The Polk Company's National Vehicle Population Profile. Vehicles were distributed into size classes using the percentages in Table A.20. This method assumed that all vehicles, large and small, were scrapped at the same rate. Shares were generated from the ORNL MPG and Market Shares Database, 2003.

Table A.20

Shares by Automobile Size Class and Model Year

\begin{tabular}{|c|c|c|c|c|c|c|c|}
\hline Year & Minicompact & Subcompact & Compact & Midsize & Large & Two-seater & Total \\
\hline Pre-1977 & $7.1 \%$ & $22.0 \%$ & $16.4 \%$ & $29.5 \%$ & $23.0 \%$ & $2.0 \%$ & $100.0 \%$ \\
\hline 1977 & $7.2 \%$ & $16.2 \%$ & $25.7 \%$ & $21.7 \%$ & $27.4 \%$ & $1.8 \%$ & $100.0 \%$ \\
\hline 1978 & $8.5 \%$ & $19.0 \%$ & $15.2 \%$ & $33.0 \%$ & $22.3 \%$ & $2.0 \%$ & $100.0 \%$ \\
\hline 1979 & $5.5 \%$ & $30.7 \%$ & $8.4 \%$ & $33.8 \%$ & $19.4 \%$ & $2.2 \%$ & $100.0 \%$ \\
\hline 1980 & $4.7 \%$ & $37.8 \%$ & $6.6 \%$ & $33.8 \%$ & $14.7 \%$ & $2.4 \%$ & $100.0 \%$ \\
\hline 1981 & $3.3 \%$ & $33.0 \%$ & $13.4 \%$ & $35.1 \%$ & $12.5 \%$ & $2.7 \%$ & $100.0 \%$ \\
\hline 1982 & $2.9 \%$ & $31.4 \%$ & $17.0 \%$ & $33.1 \%$ & $13.0 \%$ & $2.6 \%$ & $100.0 \%$ \\
\hline 1983 & $2.6 \%$ & $26.8 \%$ & $22.0 \%$ & $31.7 \%$ & $14.5 \%$ & $2.4 \%$ & $100.0 \%$ \\
\hline 1984 & $0.4 \%$ & $24.6 \%$ & $27.1 \%$ & $30.0 \%$ & $14.7 \%$ & $3.2 \%$ & $100.0 \%$ \\
\hline 1985 & $0.6 \%$ & $21.7 \%$ & $32.1 \%$ & $28.4 \%$ & $13.8 \%$ & $3.4 \%$ & $100.0 \%$ \\
\hline 1986 & $1.8 \%$ & $22.4 \%$ & $33.2 \%$ & $26.9 \%$ & $13.2 \%$ & $2.5 \%$ & $100.0 \%$ \\
\hline 1987 & $1.4 \%$ & $19.5 \%$ & $39.1 \%$ & $25.2 \%$ & $12.5 \%$ & $2.3 \%$ & $100.0 \%$ \\
\hline 1988 & $0.8 \%$ & $19.1 \%$ & $40.5 \%$ & $24.6 \%$ & $13.2 \%$ & $1.8 \%$ & $100.0 \%$ \\
\hline 1989 & $0.2 \%$ & $19.3 \%$ & $36.2 \%$ & $28.9 \%$ & $13.8 \%$ & $1.6 \%$ & $100.0 \%$ \\
\hline 1990 & $0.9 \%$ & $22.0 \%$ & $34.2 \%$ & $27.2 \%$ & $13.9 \%$ & $1.8 \%$ & $100.0 \%$ \\
\hline 1991 & $0.9 \%$ & $26.1 \%$ & $29.5 \%$ & $27.9 \%$ & $13.9 \%$ & $1.7 \%$ & $100.0 \%$ \\
\hline 1992 & $1.2 \%$ & $25.3 \%$ & $30.6 \%$ & $27.7 \%$ & $14.1 \%$ & $1.1 \%$ & $100.0 \%$ \\
\hline 1993 & $0.9 \%$ & $22.6 \%$ & $32.3 \%$ & $29.1 \%$ & $14.2 \%$ & $0.9 \%$ & $100.0 \%$ \\
\hline 1994 & $0.5 \%$ & $22.1 \%$ & $35.2 \%$ & $26.5 \%$ & $14.9 \%$ & $0.8 \%$ & $100.0 \%$ \\
\hline 1995 & $0.5 \%$ & $17.4 \%$ & $37.8 \%$ & $28.6 \%$ & $15.1 \%$ & $0.6 \%$ & $100.0 \%$ \\
\hline 1996 & $0.4 \%$ & $15.2 \%$ & $40.3 \%$ & $28.8 \%$ & $14.6 \%$ & $0.7 \%$ & $100.0 \%$ \\
\hline 1997 & $0.5 \%$ & $18.3 \%$ & $35.5 \%$ & $30.6 \%$ & $14.1 \%$ & $1.0 \%$ & $100.0 \%$ \\
\hline 1998 & $0.2 \%$ & $18.5 \%$ & $28.6 \%$ & $38.4 \%$ & $13.0 \%$ & $1.3 \%$ & $100.0 \%$ \\
\hline 1999 & $0.1 \%$ & $18.8 \%$ & $27.4 \%$ & $38.8 \%$ & $13.7 \%$ & $1.2 \%$ & $100.0 \%$ \\
\hline 2000 & $0.2 \%$ & $19.9 \%$ & $26.7 \%$ & $37.4 \%$ & $14.4 \%$ & $1.4 \%$ & $100.0 \%$ \\
\hline 2001 & $0.4 \%$ & $11.2 \%$ & $36.8 \%$ & $32.1 \%$ & $18.1 \%$ & $1.4 \%$ & $100.0 \%$ \\
\hline
\end{tabular}

Business fleet autos - Bobit Publishing Company, Automotive Fleet Research Department, Automotive Fleet Factbook 2002, Redondo Beach, CA, 2002.

Personal autos - Difference between total vehicle stock and business fleet autos. 
Sales - Domestic and import totals are from Ward's Motor Vehicle Facts and Figures 2002.

Domestic-sponsored imports (captive imports) were included in the import figure only. Domestic and import sales were distributed into size classes using the percentages in Table A.21 from the ORNL MPG and Market Shares Database, 2003.

Table A.21

Automobile Sales Shares by Size Class, 2001

\begin{tabular}{lrr}
\hline Size class & Domestic & \multicolumn{1}{c}{ Import } \\
\hline Two-seaters & $0.8 \%$ & $3.3 \%$ \\
Minicompact & $0.0 \%$ & $1.6 \%$ \\
Subcompact & $11.3 \%$ & $10.5 \%$ \\
Compact & $34.0 \%$ & $45.5 \%$ \\
Midsize & $31.0 \%$ & $35.8 \%$ \\
Large & $22.9 \%$ & $3.3 \%$ \\
\hline
\end{tabular}

See Glossary for definition of Automobile Size Classifications.

\section{Trucks}

Stock - Total truck population from The Polk Company, 2002. The trucks were distributed using shares of trucks by standard weight classes from VIUS 1997 (Table A.22).

Table A.22

Share of Trucks by Weight Class

\begin{tabular}{lc}
\hline \multicolumn{1}{c}{ Weight classes } & $\begin{array}{c}\text { Share of trucks in the } \\
\text { population }\end{array}$ \\
\hline $0-10,000 \mathrm{lbs}$ & $93.5 \%$ \\
$10,001-19,500 \mathrm{lbs}$ & $2.0 \%$ \\
$19,501-26,000 \mathrm{lbs}$ & $1.0 \%$ \\
$26,001 \mathrm{lbs}$ and over & $3.5 \%$ \\
\hline Total & $100.0 \%$ \\
\hline
\end{tabular}


Then, the number of trucks in Class $2 b$ were split from Classes 1 and 2 by model year (Polk NVPP data) using shares from ORNL's Class 2b study (Table A.23).

Table A.23

Share of Class 1 and 2 Trucks

that are Class $2 b$ Trucks $(8,500-10,000$ lbs)

\begin{tabular}{cc}
\hline Model Year & Share of class 2b trucks \\
\hline Pre-1974 & $7.35 \%$ \\
1974 & $15.64 \%$ \\
1975 & $17.15 \%$ \\
1976 & $18.29 \%$ \\
1977 & $14.60 \%$ \\
1978 & $17.90 \%$ \\
1979 & $17.79 \%$ \\
1980 & $18.20 \%$ \\
1981 & $13.87 \%$ \\
1982 & $14.05 \%$ \\
1983 & $8.13 \%$ \\
1984 & $9.74 \%$ \\
1985 & $9.56 \%$ \\
1986 & $8.77 \%$ \\
1987 & $8.91 \%$ \\
1988 & $6.90 \%$ \\
1989 & $8.34 \%$ \\
1990 & $6.73 \%$ \\
1991 & $4.91 \%$ \\
1992 & $5.04 \%$ \\
1993 & $5.60 \%$ \\
1994 & $5.60 \%$ \\
1995 & $7.05 \%$ \\
1996 & $6.71 \%$ \\
1997 & $7.86 \%$ \\
1998 & $5.01 \%$ \\
1999 & $9.36 \%$ \\
2000 & $8.94 \%$ \\
2001 & $8.61 \%$ \\
\hline
\end{tabular}


Trucks less than 8,500 lbs (Classes 1 and 2a) were distributed into size classes using the percentages in Table A.24. This method assumed that all vehicles, large and small, were scrapped at the same rate. Shares were generated from the ORNL MPG and Market Shares Database, 2003.

Table A.24

Shares by Light Truck Size Class and Model Year for Trucks under 8,500 lbs

\begin{tabular}{|c|c|c|c|c|c|c|c|c|}
\hline Sales period & Small pickup & $\begin{array}{l}\text { Large } \\
\text { pickup }\end{array}$ & Small van & $\begin{array}{l}\text { Large } \\
\text { van }\end{array}$ & Small utility & $\begin{array}{c}\text { Medium } \\
\text { utility }\end{array}$ & $\begin{array}{l}\text { Large } \\
\text { utility }\end{array}$ & Total \\
\hline Pre-1976 & $9.5 \%$ & $66.1 \%$ & $0.9 \%$ & $21.1 \%$ & $0.0 \%$ & $2.0 \%$ & $0.4 \%$ & $100.0 \%$ \\
\hline 1976 & $7.1 \%$ & $65.7 \%$ & $0.8 \%$ & $23.9 \%$ & $0.0 \%$ & $2.1 \%$ & $0.4 \%$ & $100.0 \%$ \\
\hline 1977 & $11.0 \%$ & $68.5 \%$ & $1.0 \%$ & $16.6 \%$ & $0.0 \%$ & $2.5 \%$ & $0.4 \%$ & $100.0 \%$ \\
\hline 1978 & $10.5 \%$ & $64.0 \%$ & $0.8 \%$ & $22.8 \%$ & $0.1 \%$ & $1.4 \%$ & $0.4 \%$ & $100.0 \%$ \\
\hline 1979 & $16.1 \%$ & $58.5 \%$ & $0.6 \%$ & $20.7 \%$ & $1.8 \%$ & $1.9 \%$ & $0.4 \%$ & $100.0 \%$ \\
\hline 1980 & $23.3 \%$ & $50.3 \%$ & $0.6 \%$ & $14.8 \%$ & $2.3 \%$ & $6.9 \%$ & $1.8 \%$ & $100.0 \%$ \\
\hline 1981 & $24.4 \%$ & $50.0 \%$ & $0.6 \%$ & $16.9 \%$ & $2.0 \%$ & $4.7 \%$ & $1.4 \%$ & $100.0 \%$ \\
\hline 1982 & $27.2 \%$ & $46.8 \%$ & $0.6 \%$ & $17.8 \%$ & $1.3 \%$ & $4.8 \%$ & $1.5 \%$ & $100.0 \%$ \\
\hline 1983 & $33.3 \%$ & $35.7 \%$ & $0.5 \%$ & $18.0 \%$ & $6.3 \%$ & $4.5 \%$ & $1.7 \%$ & $100.0 \%$ \\
\hline 1984 & $23.7 \%$ & $38.1 \%$ & $6.2 \%$ & $15.1 \%$ & $10.6 \%$ & $4.4 \%$ & $1.9 \%$ & $100.0 \%$ \\
\hline 1985 & $20.4 \%$ & $40.0 \%$ & $10.3 \%$ & $12.7 \%$ & $10.4 \%$ & $4.4 \%$ & $1.8 \%$ & $100.0 \%$ \\
\hline 1986 & $21.7 \%$ & $35.2 \%$ & $14.1 \%$ & $11.3 \%$ & $11.7 \%$ & $4.1 \%$ & $1.9 \%$ & $100.0 \%$ \\
\hline 1987 & $21.2 \%$ & $33.7 \%$ & $16.0 \%$ & $10.3 \%$ & $12.3 \%$ & $4.8 \%$ & $1.7 \%$ & $100.0 \%$ \\
\hline 1988 & $21.6 \%$ & $30.6 \%$ & $18.0 \%$ & $10.3 \%$ & $12.5 \%$ & $4.9 \%$ & $2.1 \%$ & $100.0 \%$ \\
\hline 1989 & $18.4 \%$ & $33.2 \%$ & $18.0 \%$ & $9.9 \%$ & $9.8 \%$ & $8.6 \%$ & $2.1 \%$ & $100.0 \%$ \\
\hline 1990 & $25.2 \%$ & $24.7 \%$ & $22.4 \%$ & $7.1 \%$ & $8.9 \%$ & $9.6 \%$ & $2.1 \%$ & $100.0 \%$ \\
\hline 1991 & $24.8 \%$ & $23.1 \%$ & $23.4 \%$ & $6.1 \%$ & $8.6 \%$ & $12.2 \%$ & $1.8 \%$ & $100.0 \%$ \\
\hline 1992 & $22.8 \%$ & $23.6 \%$ & $23.6 \%$ & $6.4 \%$ & $8.7 \%$ & $13.3 \%$ & $1.6 \%$ & $100.0 \%$ \\
\hline 1993 & $21.6 \%$ & $22.2 \%$ & $23.8 \%$ & $6.2 \%$ & $8.2 \%$ & $15.5 \%$ & $2.5 \%$ & $100.0 \%$ \\
\hline 1994 & $20.3 \%$ & $24.5 \%$ & $23.6 \%$ & $5.6 \%$ & $7.6 \%$ & $16.0 \%$ & $2.4 \%$ & $100.0 \%$ \\
\hline 1995 & $18.0 \%$ & $24.9 \%$ & $22.4 \%$ & $5.5 \%$ & $8.6 \%$ & $18.1 \%$ & $2.5 \%$ & $100.0 \%$ \\
\hline 1996 & $16.2 \%$ & $25.7 \%$ & $21.0 \%$ & $4.7 \%$ & $9.3 \%$ & $20.4 \%$ & $2.7 \%$ & $100.0 \%$ \\
\hline 1997 & $15.0 \%$ & $24.3 \%$ & $19.9 \%$ & $4.7 \%$ & $5.4 \%$ & $22.2 \%$ & $8.5 \%$ & $100.0 \%$ \\
\hline 1998 & $12.5 \%$ & $27.4 \%$ & $17.8 \%$ & $4.6 \%$ & $6.8 \%$ & $22.2 \%$ & $8.7 \%$ & $100.0 \%$ \\
\hline 1999 & $13.9 \%$ & $25.3 \%$ & $17.1 \%$ & $4.5 \%$ & $8.3 \%$ & $22.0 \%$ & $8.9 \%$ & $100.0 \%$ \\
\hline 2000 & $12.9 \%$ & $23.7 \%$ & $15.3 \%$ & $4.4 \%$ & $9.1 \%$ & $26.1 \%$ & $8.5 \%$ & $100.0 \%$ \\
\hline
\end{tabular}

The Class $2 b$ trucks were split into two truck types - pickups and van/SUV using shares from the report Investigation of Class $2 b$ Trucks, ORNL/TM-2002/49, Table 11, which are shown here in Table A.25.

Table A.25

Shares of Class $2 b$ Trucks by Truck Type

\begin{tabular}{lc}
\hline \multicolumn{1}{c}{ Truck types } & $\begin{array}{c}\text { Shares of class } 2 \mathrm{~b} \text { truck } \\
\text { population }\end{array}$ \\
\hline Pickup & $73.7 \%$ \\
Van/SUV & $26.3 \%$ \\
\hline
\end{tabular}

Business fleet trucks - Bobit Publishing Company, Automotive Fleet Research Department, Automotive Fleet Factbook 2002, Redondo Beach, CA, 2002.

Personal trucks - Difference between total stock and business fleet trucks.

Sales - Domestic and import totals are from Ward's Motor Vehicle Facts and Figures 2002. Domesticsponsored imports (captive imports) were included in the import figure only. 
According to the Investigation of Class $2 b$ Trucks, ORNL/TM-2002/49, 6.5\% of all classes 1 and 2 truck sales were Class $2 \mathrm{~b}$ trucks. Also, there were no class $2 \mathrm{~b}$ trucks which were imported into the U.S. in 2000 .

Domestic and import sales of trucks less than $8,500 \mathrm{lbs}$ were distributed into size classes using the percentages in Table A.26 from the ORNL MPG and Market Shares Database, 2003.

Table A.26

Light Truck Sales Shares by Size Class, 2001 for Trucks less than 8,500 lbs

\begin{tabular}{lrr}
\hline \multicolumn{1}{c}{ Size class } & Domestic & Import \\
\hline Small pickup & $11.5 \%$ & $0.0 \%$ \\
Large pickup & $28.0 \%$ & $0.0 \%$ \\
Small van & $15.6 \%$ & $3.9 \%$ \\
Large van & $4.5 \%$ & $0.8 \%$ \\
Small SUV & $8.1 \%$ & $34.8 \%$ \\
Medium SUV & $21.6 \%$ & $57.8 \%$ \\
Large SUV & $10.7 \%$ & $2.7 \%$ \\
\hline
\end{tabular}

The Class $2 \mathrm{~b}$ truck sales were split into two truck types - pickups and van/SUV using shares from the report Investigation of Class $2 b$ Trucks, ORNL/TM-2002/49, Table 6, which are shown here in Table A.27.

Table A.27

Shares of Class $2 b$ Truck Sales by Truck Type, 2000

\begin{tabular}{lc}
\hline \multicolumn{1}{c}{ Truck types } & $\begin{array}{c}\text { Shares of class } 2 \mathrm{~b} \text { truck } \\
\text { population }\end{array}$ \\
\hline Pickup & $82.1 \%$ \\
Van/SUV & $17.9 \%$ \\
\hline
\end{tabular}




\section{Fleet Vehicle Data}

\section{Light Fleet Vehicle Population}

Automobiles - Bobit Publishing Company, Automotive Fleet Factbook 2001, Redondo Beach, CA, 2002, p. 12. Fleets of 10 or more units. Taxi and Rental categories were considered Business fleets.

Light trucks - Bobit Publishing Company, Automotive Fleet Factbook 2001, Redondo Beach, CA, 2002, p. 12. Trucks under 19,501 lbs GVW in fleets of 10 or more units. Light trucks were split from the total using shares from the 1997 VIUS (business, rental, and utility) and the GSA Federal Fleet Factbook (government) shown in Table A.28.

Table A.28

\begin{tabular}{|c|c|}
\hline $\begin{array}{l}\text { Light Truck Shar } \\
\text { Less than } 19,\end{array}$ & ucks \\
\hline Vehicles in Fleets of & \\
\hline Business & $92.1 \%$ \\
\hline Utility & $89.6 \%$ \\
\hline Rental & $97.3 \%$ \\
\hline Gederal Government & $81.6 \%$ \\
\hline
\end{tabular}

\section{$\underline{\text { Light Fleet Vehicle New Sales }}$}

Automobiles - Bobit Publishing Company, Automotive Fleet Factbook 2001, Redondo Beach, CA, 2002, p. 40-48, Fleet 2000 Model Year registrations. New registrations are considered a proxy for new vehicle sales. Commercial and rental categories were considered Business fleets. Utility fleets were estimated as share of business fleet purchases based on data from the National Association of Fleet Administrators shown in Table A.29.

Light trucks - Bobit Publishing Company, Automotive Fleet Factbook 2001, Redondo Beach, CA, 2002, p. 48-52, Fleet 2000 Model Year registrations. New registrations are considered a proxy for new vehicle sales. Commercial and rental categories were considered Business fleets. Utility fleets were estimated as a share of business fleet purchases based on data from the National Association of Fleet Administrators shown in Table A.29.

Table A.29

Share of Business Fleet Vehicles which are Utility Fleet Vehicles

\begin{tabular}{lr}
\hline Vehicle type & \\
\hline Automobiles & $2.6 \%$ \\
Passenger vans & $7.3 \%$ \\
Cargo vans & $64.3 \%$ \\
Sport utility vehicles & $14.8 \%$ \\
Pickup trucks & $66.2 \%$ \\
\hline
\end{tabular}




\section{Light Fleet Vehicle Travel}

\section{Automobiles}

Business

Bobit Publishing Company, Automotive Fleet Factbook 2001, Redondo Beach, CA, 2002, p.5867. Average annual miles of compact and intermediate size automobiles were based on data from four leading fleet management companies. Weighted average of automobile travel was derived based on the estimated share of vehicles in the population from The Polk Company. Compact autos and smaller were assumed to travel as compact cars. Intermediate autos and larger were assumed to travel like intermediate autos. Average annual miles and weights are shown in Table A.30.

\section{Government}

The only source of data on government fleet travel was for the Federal Government fleet vehicles. Data on sedans and station wagons from the GSA Federal Fleet Factbook was used for government fleet travel and is shown in Table A.30.

\section{Utility}

The only source of data available on utility fleet vehicle travel was for the fleets of the Tennessee Valley Authority (TVA). Data on the TVA automobile fleet from the GSA Federal Fleet Factbook was used for utility fleet travel and is shown in Table A.30.

Table A.30

Average Annual Miles and Population Shares of Fleet Automobiles

\begin{tabular}{lcc}
\hline & $\begin{array}{c}\text { Average annual } \\
\text { miles, 2000 }\end{array}$ & $\begin{array}{c}\text { Estimated share of } \\
\text { vehicles in the } \\
\text { population, 2000 }\end{array}$ \\
\hline Business automobiles & 22,689 & $55.6 \%$ \\
Compact & 22,893 & $44.4 \%$ \\
Intermediate & & \\
Government automobiles & 12,895 & \\
Sedans and station wagons & & \\
& & \\
Utility automobiles & 13,399 & \\
Sedans and station wagons & &
\end{tabular}




\section{Light trucks}

Business

Bobit Publishing Company, Automotive Fleet Factbook 2001, Redondo Beach, CA, 2002, p.5867. Average annual miles of pickups, minivans, sport utility vehicles and full-size vans were based on data from four leading fleet management companies. Weighted average of light truck travel was derived based on the estimated share of vehicles in the population from The Polk Company. Average annual miles and weights are shown in Table A.31.

\section{Government}

The only source of data on government fleet travel was for the Federal Government fleet vehicles. Data on ambulances, 2x4 trucks, and 4x4 trucks from the GSA Federal Fleet Factbook were used for government fleet travel. Weighted average of light truck travel was derived based on the estimated share of vehicles in the population from the same GSA report. Average annual miles and weights are shown in Table A.31.

\section{Utility}

The only source of data available on utility fleet vehicle travel was for the fleets of the Tennessee Valley Authority (TVA). Data on the $2 \times 4$ trucks and $4 \times 4$ trucks in the TVA fleet from the GSA Federal Fleet Factbook were used for utility fleet travel. The weighted average of travel was derived based on the share of vehicles in the population from the same GSA report. Average annual miles and weights are shown in Table A.31.

Table A.31

Average Annual Miles and Population Shares of Fleet Light Trucks

\begin{tabular}{lrr}
\hline & $\begin{array}{c}\text { Average annual } \\
\text { miles, 2000 }\end{array}$ & $\begin{array}{c}\text { Estimated share of } \\
\text { vehicles in the } \\
\text { population, 2000 }\end{array}$ \\
\hline Business light trucks & 28,515 & $48.8 \%$ \\
Pickup trucks & 25,677 & $17.1 \%$ \\
Minivans & 24,003 & $28.4 \%$ \\
Sport utility vehicles & 20,412 & $5.8 \%$ \\
Full-size vans & & \\
& & \\
Government light trucks & 5,946 & $82.8 \%$ \\
Ambulances & 5,747 & $16.7 \%$ \\
$2 \times 4$ trucks & 12,022 & \\
$4 \times 4$ trucks & & \\
& & \\
Utility light trucks & 10,405 & \\
$2 \times 4$ trucks & 14,208 & \\
$4 \times 4$ trucks & & \\
\hline
\end{tabular}




\title{
APPENDIX B \\ CONVERSIONS
}

\begin{abstract}
A Note About Heating Values
The heat content of a fuel is the quantity of energy released by burning a unit amount of that fuel. However, this value is not absolute and can vary according to several factors. For example, empirical formulae for determining the heating value of liquid fuels depend on the fuels' American Petroleum Institute (API) gravity. The API gravity varies depending on the percent by weight of the chemical constituents and impurities in the fuel, both of which are affected by the combination of raw materials used to produce the fuel and by the type of manufacturing process. Temperature and climatic conditions are also factors.

Because of these variations, the heating values in Table B.1 may differ from values in other publications. The figures in this report are representative or average values, not absolute ones. The gross heating values used here agree with those used by the Energy Information Administration (EIA).

Heating values fall into two categories, usually referred to as "higher" and "lower." If the products of fuel combustion are cooled back to the initial fuel-air or fuel-oxidizer mixture temperature and the water formed during combustion is condensed, the energy released by the process is the higher (gross) heating value. If the products of combustion are cooled to the initial fuel-air temperature, but the water is considered to remain as a vapor, the energy released by the process is lower (or net) heating value. Usually the difference between the gross and net heating values for fuels used in transportation is around 5 to 8 percent; however, it is important to be consistent in their use.
\end{abstract}


Table B.1

\section{Hydrogen Heat Content}

\begin{tabular}{cc}
\hline \multicolumn{2}{c}{1 kilogram hydrogen $=$} \\
\hline Higher heating value & Lower heating value \\
$134,200 \mathrm{Btu}$ & $113,400 \mathrm{Btu}$ \\
$39.3 \mathrm{kWhr}$ & $33.2 \mathrm{kWhr}$ \\
$141,600 \mathrm{~kJ}$ & $119,600 \mathrm{~kJ}$ \\
$33,800 \mathrm{kCal}$ & $28,560 \mathrm{kCal}$ \\
\hline
\end{tabular}

Table B.2 Hydrogen Conversions

\begin{tabular}{|c|c|c|c|c|c|c|}
\hline & \multicolumn{2}{|c|}{ Weight } & \multicolumn{2}{|c|}{ Gas } & \multicolumn{2}{|c|}{ Liquid } \\
\hline & $\begin{array}{l}\text { Pounds } \\
\text { (lb) }\end{array}$ & $\begin{array}{l}\text { Kilograms } \\
\quad(\mathrm{kg})\end{array}$ & $\begin{array}{l}\text { Standard } \\
\text { cubic feet } \\
(\mathrm{SCF})\end{array}$ & $\begin{array}{c}\text { Normal } \\
\text { cubic meter } \\
\left(\mathrm{Nm}^{3}\right)\end{array}$ & $\begin{array}{l}\text { Gallons } \\
\text { (gal) }\end{array}$ & $\begin{array}{c}\text { Liters } \\
\text { (L) }\end{array}$ \\
\hline $1 \mathrm{lb}$ & 1.0 & 0.4536 & 192.00 & 5.047 & 1.6928 & 6.408 \\
\hline $1 \mathrm{~kg}$ & 2.205 & 1.0 & 423.3 & 11.126 & 3.733 & 14.128 \\
\hline $1 \mathrm{SCF}$ gas & 0.005209 & 0.002363 & 1.0 & 0.02628 & 0.008820 & 0.0339 \\
\hline $1 \mathrm{Nm}^{3}$ gas & 0.19815 & 0.08988 & 38.04 & 1.0 & 0.3355 & 1.2699 \\
\hline 1 gal liquid & 0.5906 & 0.2679 & 113.41 & 2.981 & 1.0 & 3.785 \\
\hline $1 \mathrm{~L}$ liquid & 0.15604 & 0.07078 & 29.99 & 0.77881 & 0.2642 & 1.0 \\
\hline
\end{tabular}


Table B.3

Heat Content for Various Fuels

Automotive gasoline

Diesel motor fuel

Biodiesel

Methanol

Ethanol

Gasohol

Aviation gasoline

Propane

Butane

Jet fuel (naphtha)

Jet fuel (kerosene)

Lubricants

Waxes

Asphalt and road oil

Petroleum coke

Natural gas

Wet

Dry

Compressed

Liquid

\section{Crude petroleum}

Fuel Oils

Residual

Distillate

\section{Coal}

Anthracite - Consumption Bituminous and lignite - Consumption Production average Consumption average

$$
\begin{aligned}
& 125,000 \mathrm{Btu} / \mathrm{gal}(\text { gross })=115,400 \mathrm{Btu} / \mathrm{gal}(\text { net }) \\
& 138,700 \mathrm{Btu} / \mathrm{gal}(\text { gross })=128,700 \mathrm{Btu} / \mathrm{gal}(\text { net }) \\
& 126,206 \mathrm{Btu} / \mathrm{gal}(\text { gross })=117,093 \mathrm{Btu} / \mathrm{gal}(\text { net }) \\
& \text { 64,600 Btu/gal (gross) }=56,560 \mathrm{Btu} / \mathrm{gal}(\text { net }) \\
& 84,600 \mathrm{Btu} / \mathrm{gal}(\text { gross })=75,670 \mathrm{Btu} / \mathrm{gal}(\text { net }) \\
& 120,900 \mathrm{Btu} / \mathrm{gal}(\text { gross })=112,417 \mathrm{Btu} / \mathrm{gal}(\text { net }) \\
& 120,200 \mathrm{Btu} / \mathrm{gal}(\text { gross })=112,000 \mathrm{Btu} / \mathrm{gal}(\text { net }) \\
& \text { 91,300 Btu/gal (gross) }=83,500 \mathrm{Btu} / \mathrm{gal}(\text { net }) \\
& \text { 103,000 Btu/gal (gross) = 93,000 Btu/gal (net) } \\
& 127,500 \mathrm{Btu} / \mathrm{gal}(\text { gross })=118,700 \mathrm{Btu} / \mathrm{gal}(\text { net }) \\
& 135,000 \mathrm{Btu} / \mathrm{gal}(\text { gross })=128,100 \mathrm{Btu} / \mathrm{gal}(\text { net }) \\
& 144,400 \mathrm{Btu} / \mathrm{gal}(\text { gross })=130,900 \mathrm{Btu} / \mathrm{gal}(\text { net }) \\
& 131,800 \mathrm{Btu} / \mathrm{gal}(\text { gross })=120,200 \mathrm{Btu} / \mathrm{gal}(\text { net }) \\
& 158,000 \mathrm{Btu} / \mathrm{gal}(\text { gross })=157,700 \mathrm{Btu} / \mathrm{gal}(\text { net }) \\
& 143,400 \mathrm{Btu} / \mathrm{gal}(\text { gross })=168,300 \mathrm{Btu} / \mathrm{gal}(\text { net })
\end{aligned}
$$

$$
\begin{aligned}
& \text { 1,109 Btu/ft }{ }^{3} \\
& 1,027 \mathrm{Btu} / \mathrm{ft}^{3} \\
& 20,551 \mathrm{Btu} / \text { pound } \\
& 960 \mathrm{Btu} / \text { cubic foot } \\
& \text { 90,800 Btu/gal (gross) }=87,600 \mathrm{Btu} / \text { gal (net) } \\
& 138,100 \mathrm{Btu} / \text { gal }(\text { gross })=131,800 \mathrm{Btu} / \text { gal }(\text { net })
\end{aligned}
$$

$149,700 \mathrm{Btu} / \mathrm{gal}($ gross $)=138,400 \mathrm{Btu} / \mathrm{gal}($ net $)$ $138,700 \mathrm{Btu} / \mathrm{gal}($ gross $)=131,800 \mathrm{Btu} / \mathrm{gal}($ net $)$

$$
\begin{aligned}
& 21.711 \times 10^{6} \mathrm{Btu} / \text { short ton } \\
& 21.012 \times 10^{6} \mathrm{Btu} / \text { short ton } \\
& 21.352 \times 10^{6} \mathrm{Btu} / \text { short ton } \\
& 21.015 \times 10^{6} \mathrm{Btu} / \text { short ton }
\end{aligned}
$$


Table B.4

Fuel Equivalents

1 million bbl crude oil/day

1 billion bbl crude oil/year

1 quadrillion Btu/year

1 billion short tons coal/year

1 billion metric tons coal/year

1 trillion $\mathrm{ft}^{3}$ natural gas/year

1 petajoule/year

$$
\begin{aligned}
& =0.365 \text { billion bbl crude oil/year } \\
& =2.117 \text { quadrillion Btu/year } \\
& =100.465 \text { million short tons coal/year } \\
& =91.142 \text { million metric tons coal/year } \\
& =2.065 \text { trillion } \mathrm{ft}^{3} \text { natural gas/year } \\
& =2,233.435 \text { petajoules/year }
\end{aligned}
$$

$=2.740$ million bbl crude oil/day $=5.800$ quadrillion Btu/year $=275.247$ million short tons coal/year $=249.704$ million metric tons coal/year $=5.659$ trillion $\mathrm{ft}^{3}$ natural gas/year

$=6,119$ petajoules/year

$=0.472$ million bbl crude oil/day $=172.414 \mathrm{million} \mathrm{bbl}$ crude oil/year $=47.456$ million short tons coal/year $=43.052 \mathrm{million}$ metric tons coal $/$ year $=975.610$ billion $\mathrm{ft}^{3}$ natural gas/year $=1,055$ petajoules/year

$=0.907 \mathrm{billion}$ metric tons coal $/$ year $=9.954$ million bbl crude oil $/$ day $=3.633$ billion bbl crude oil/year $=21.072$ quadrillion Btu/year $=20.558$ trillion $\mathrm{ft}^{3}$ natural gas/year $=22,230.960$ petajoules/year

$=1.102$ billion short tons coal/year $=9.030$ million bbl crude oi $1 /$ day $=3.296$ billion bbl crude oil/year $=19.117$ quadrillion btu/year $=18.650$ trillion $\mathrm{ft}^{3}$ natural gas/year $=20,167.927$ petajoules/year

$=0.484$ million bbl crude oil $/$ day $=0.177 \mathrm{billion} \mathrm{bbl}$ crude oil $/$ year $=1.025$ quadrillion Btu/year $=48.643$ million short tons coal/year $=44.129 \mathrm{million}$ metric tons coal/year $=1,081.375$ petajoules/year

$=447.741 \mathrm{bbl}$ crude oil/day $=163.425$ thousand bbl crude oil/year $=0.948$ trillion Btu/year

$=44.982$ thousand short tons coal/year $=40.808$ thousand metric tons coal/year $=0.925$ billion $\mathrm{ft}^{3}$ natural gas/year 
Table B.5

Energy Unit Conversions

\begin{tabular}{|c|c|c|c|}
\hline \multirow[t]{6}{*}{$1 \mathrm{Btu}$} & $=778.2 \mathrm{ft}-\mathrm{lb}$ & $1 \mathrm{kWhr}$ & $=3412 \mathrm{Btu}^{\mathrm{a}}$ \\
\hline & $=107.6 \mathrm{~kg}-\mathrm{m}$ & & $=2.655 \times 10^{6} \mathrm{ft}-\mathrm{lb}$ \\
\hline & $=1055 \mathrm{~J}$ & & $=3.671 \times 10^{5} \mathrm{~kg}-\mathrm{m}$ \\
\hline & $=39.30 \times 10^{-5} \mathrm{hp}-\mathrm{h}$ & & $=3.600 \times 10^{6} \mathrm{~J}$ \\
\hline & $=39.85 \times 10^{-5}$ metric hp-h & & $=1.341 \mathrm{hp}-\mathrm{h}$ \\
\hline & $=29.31 \times 10^{-5} \mathrm{kWhr}$ & & $=1.360$ metric $\mathrm{hp}-\mathrm{h}$ \\
\hline \multirow[t]{6}{*}{$1 \mathrm{~kg}-\mathrm{m}$} & $=92.95 \times 10^{-4} \mathrm{Btu}$ & 1 Joule & $=94.78 \times 10^{-5} \mathrm{Btu}$ \\
\hline & $=7.233 \mathrm{ft}-\mathrm{lb}$ & & $=0.7376 \mathrm{ft}-\mathrm{lb}$ \\
\hline & $=9.806 \mathrm{~J}$ & & $=0.1020 \mathrm{~kg}-\mathrm{m}$ \\
\hline & $=36.53 \times 10^{-7} \mathrm{hp}-\mathrm{h}$ & & $=37.25 \times 10^{-8} \mathrm{hp}-\mathrm{h}$ \\
\hline & $=37.04 \times 10^{-7}$ metric hp-h & & $=37.77 \times 10^{-8}$ metric hp-h \\
\hline & $=27.24 \times 10^{-7} \mathrm{kWhr}$ & & $=27.78 \times 10^{-8} \mathrm{kWhr}$ \\
\hline \multirow[t]{6}{*}{$1 \mathrm{hp}-\mathrm{h}$} & $=2544 \mathrm{Btu}$ & 1 metric hp-h & $=2510 \mathrm{Btu}$ \\
\hline & $=1.98 \times 10^{6} \mathrm{ft}-\mathrm{lb}$ & & $=1.953 \times 10^{6} \mathrm{ft}-\mathrm{lb}$ \\
\hline & $=2.738 \times 10^{6} \mathrm{kgm}$ & & $=27.00 \times 10^{4} \mathrm{~kg}-\mathrm{m}$ \\
\hline & $=2.685 \times 10^{6} \mathrm{~J}$ & & $=2.648 \times 10^{6} \mathrm{~J}$ \\
\hline & $=1.014$ metric $\mathrm{hp}-\mathrm{h}$ & & $=0.9863 \mathrm{hp}-\mathrm{h}$ \\
\hline & $=0.7475 \mathrm{kWhr}$ & & $=0.7355 \mathrm{kWhr}$ \\
\hline
\end{tabular}

${ }^{a}$ This figure does not take into account the fact that electricity generation and distribution efficiency is approximately $29 \%$. If generation and distribution efficiency are taken into account, $1 \mathrm{kWhr}=11,765$ Btu.

Table B.6

International Energy Conversions

\begin{tabular}{rccccc}
\hline To: & Terajoules & $\begin{array}{c}\text { Giga- } \\
\text { calories }\end{array}$ & $\begin{array}{c}\text { Million tonnes of } \\
\text { oil equivalent }\end{array}$ & $\begin{array}{c}\text { Million } \\
\text { Btu }\end{array}$ & $\begin{array}{c}\text { Gigawatt- } \\
\text { hours }\end{array}$ \\
\hline $\begin{array}{r}\text { From: } \\
\text { Terajoules }\end{array}$ & 1 & & & & \\
multiply by: & 238.8 & $2.388 \times 10^{-5}$ & 947.8 & 0.2778 \\
$\begin{array}{r}\text { Gigacalories } \\
\text { Million tonnes }\end{array}$ & $4.1868 \times 10^{-3}$ & 1 & $10^{-7}$ & 3.968 & $1.163 \times 10^{-3}$ \\
of oil equivalent & $4.1868 \times 10^{4}$ & $10^{7}$ & 1 & $3.968 \times 10^{7}$ & 11,630 \\
Million Btu & $1.0551 \times 10^{-3}$ & 0.252 & $2.52 \times 10^{-8}$ & 1 & $2.931 \times 10^{-4}$ \\
& 3.6 & 860 & $8.6 \times 10^{-5}$ & 3412 & 1 \\
\hline Gigawatthours & & & & & \\
\hline
\end{tabular}


Table B.7

Distance and Velocity Conversions

\begin{tabular}{|c|c|c|c|}
\hline \multirow[t]{5}{*}{1 in. } & $=83.33 \times 10^{-3} \mathrm{ft}$ & $1 \mathrm{ft}$ & $=12.0 \mathrm{in}$. \\
\hline & $=27.78 \times 10^{-3} \mathrm{yd}$ & & $=0.33 \mathrm{yd}$ \\
\hline & $=15.78 \times 10^{-6}$ mile & & $=189.4 \times 10^{-3} \mathrm{mile}$ \\
\hline & $=25.40 \times 10^{-3} \mathrm{~m}$ & & $=0.3048 \mathrm{~m}$ \\
\hline & $=0.2540 \times 10^{-6} \mathrm{~km}$ & & $=0.3048 \times 10^{-3} \mathrm{~km}$ \\
\hline \multirow[t]{9}{*}{1 mile } & $=63360 \mathrm{in}$. & $1 \mathrm{~km}$ & $=39370 \mathrm{in}$. \\
\hline & $=5280 \mathrm{ft}$ & & $=3281 \mathrm{ft}$ \\
\hline & $=1760 \mathrm{yd}$ & & $=1093.6 \mathrm{yd}$ \\
\hline & $=1609 \mathrm{~m}$ & & $=0.6214$ mile \\
\hline & $=1.609 \mathrm{~km}$ & & $=1000 \mathrm{~m}$ \\
\hline & \multicolumn{3}{|c|}{$1 \mathrm{ft} / \mathrm{sec}=0.3048 \mathrm{~m} / \mathrm{s}=0.6818 \mathrm{mph}=1.0972 \mathrm{~km} / \mathrm{h}$} \\
\hline & \multicolumn{3}{|c|}{$1 \mathrm{~m} / \mathrm{sec}=3.281 \mathrm{ft} / \mathrm{s}=2.237 \mathrm{mph}=3.600 \mathrm{~km} / \mathrm{h}$} \\
\hline & \multicolumn{3}{|c|}{$1 \mathrm{~km} / \mathrm{h}=0.9114 \mathrm{ft} / \mathrm{s}=0.2778 \mathrm{~m} / \mathrm{s}=0.6214 \mathrm{mph}$} \\
\hline & \multicolumn{3}{|c|}{$1 \mathrm{mph}=1.467 \mathrm{ft} / \mathrm{s}=0.4469 \mathrm{~m} / \mathrm{s}=1.609 \mathrm{~km} / \mathrm{h}$} \\
\hline
\end{tabular}

Table B.8

Alternative Measures of Greenhouse Gases

1 pound methane, measured in carbon $=1.333$ pounds methane, measured at full units $\left(\mathrm{CH}_{4}\right)$ molecular weight $\left(\mathrm{CH}_{4}\right)$

1 pound carbon dioxide, measured in $\quad=\quad 3.6667$ pounds carbon dioxide, measured at carbon units $\left(\mathrm{CO}_{2}-\mathrm{C}\right)$ full molecular weight $\left(\mathrm{CO}_{2}\right)$

1 pound carbon monoxide, measured in $\quad=\quad 2.333$ pounds carbon monoxide, measured at carbon units (CO-C) full molecular weight $(\mathrm{CO})$

1 pound nitrous oxide, measured in $\quad=\quad 1.571$ pounds nitrous oxide, measured at full nitrogen units $\left(\mathrm{N}_{2} \mathrm{O}-\mathrm{N}\right)$ molecular weight $\left(\mathrm{N}_{2} \mathrm{O}\right)$ 
Table B.9

Volume and Flow Rate Conversions ${ }^{\mathrm{a}}$

\begin{tabular}{|c|c|c|c|}
\hline \multirow[t]{6}{*}{1 U.S. gal } & $=231 \mathrm{in}^{3}$ & 1 liter & $=61.02$ in. $^{3}$ \\
\hline & $=0.1337 \mathrm{ft}^{3}$ & & $=3.531 \times 10^{-2} \mathrm{ft}^{3}$ \\
\hline & $=3.785$ liters & & $=0.2624$ U.S. gal \\
\hline & $=0.8321$ imperial gal & & $=0.2200$ imperial gal \\
\hline & $=0.0238 \mathrm{bbl}$ & & $=6.29 \times 10^{-3} \mathrm{bbl}$ \\
\hline & $=0.003785 \mathrm{~m}^{3}$ & & $=0.001 \mathrm{~m}^{3}$ \\
\hline \multicolumn{4}{|c|}{ A U.S. gallon of gasoline weighs 6.2 pounds } \\
\hline \multirow[t]{6}{*}{1 imperial gal } & $=277.4$ in. $^{3}$ & $1 \mathrm{bbl}$ & $=9702$ in. $^{3}$ \\
\hline & $=0.1606 \mathrm{ft}^{3}$ & & $=5.615 \mathrm{ft}^{3}$ \\
\hline & $=4.545$ liters & & $=158.97$ liters \\
\hline & $=1.201$ U.S.gal & & $=42$ U.S. gal \\
\hline & $=0.0286 \mathrm{bbl}$ & & $=34.97$ imperial gal \\
\hline & $=0.004546 \mathrm{~m}^{3}$ & & $=0.15897 \mathrm{~m}^{3}$ \\
\hline \multirow[t]{5}{*}{1 U.S. gal $/ \mathrm{hr}$} & $=3.209 \mathrm{ft}^{3} /$ day & & $=1171 \mathrm{ft}^{3} /$ year \\
\hline & $=90.84$ liter $/$ day & & $=33157$ liter/year \\
\hline & $=19.97 \mathrm{imperial} \mathrm{gal} /$ day & & = $7289 \mathrm{imperial} \mathrm{gal} /$ year \\
\hline & $=0.5712 \mathrm{bbl} / \mathrm{day}$ & & $=207.92 \mathrm{bbl} /$ year \\
\hline & \multicolumn{3}{|c|}{ For Imperial gallons, multiply above values by 1.201} \\
\hline \multirow[t]{4}{*}{1 liter/hr } & $=0.8474 \mathrm{ft}^{3} /$ day & & $=309.3 \mathrm{ft}^{3} /$ year \\
\hline & $=6.298$ U.S. gal/day & & $=2299$ U.S. gal/year \\
\hline & $=5.28 \mathrm{imperial} \mathrm{gal} / \mathrm{day}$ & & = $1927 \mathrm{imperial} \mathrm{gal} /$ year \\
\hline & $=0.1510 \mathrm{bbl} / \mathrm{day}$ & & $=55.10 \mathrm{bbl} / \mathrm{year}$ \\
\hline \multirow[t]{4}{*}{$1 \mathrm{bbl} / \mathrm{hr}$} & $=137.8 \mathrm{ft}^{3} /$ year & & $=49187 \mathrm{ft}^{3}$ year \\
\hline & $=1008$ U.S. gal/day & & $=3.679 \times 10^{5} \mathrm{U} . \mathrm{S} . \mathrm{gal} / \mathrm{year}$ \\
\hline & $=839.3 \mathrm{imperial} \mathrm{gal} / \mathrm{day}$ & & $=3.063 \times 10^{5} \mathrm{imperial}$ gal $/$ year \\
\hline & $=3815$ liter/day & & $=1.393 \times 10^{6}$ liter $/$ day \\
\hline
\end{tabular}

${ }^{a}$ The conversions for flow rates are identical to those for volume measures, if the time units are identical. 
Table B.10

Power Conversions

\begin{tabular}{|c|c|c|c|c|c|c|}
\hline \multirow[b]{2}{*}{ FROM } & \multicolumn{6}{|c|}{ TO } \\
\hline & Horsepower & Kilowatts & $\begin{array}{c}\text { Metric } \\
\text { horsepower }\end{array}$ & Ft-lb per sec & $\begin{array}{l}\text { Kilocalories } \\
\text { per sec }\end{array}$ & Btu per sec \\
\hline Horsepower & 1 & 0.7457 & 1.014 & 550 & 0.1781 & 0.7068 \\
\hline Kilowatts & 1.341 & 1 & 1.360 & 737.6 & 0.239 & $0=9478$ \\
\hline $\begin{array}{l}\text { Metric } \\
\text { horsepower }\end{array}$ & 0.9863 & 0.7355 & 1 & 542.5 & 0.1757 & $0=6971$ \\
\hline Ft-lb per sec & $1.36 \times 10^{-3}$ & $1.356 \times 10^{-3}$ & $1.84 \times 10^{-3}$ & 1 & $0.3238 \times 10^{-3}$ & $1.285 \times 10^{-3}$ \\
\hline $\begin{array}{l}\text { Kilocalories } \\
\text { per sec }\end{array}$ & 5.615 & 4.184 & 5.692 & 3088 & 1 & $3=968$ \\
\hline Btu per sec & 1.415 & 1.055 & 1.434 & 778.2 & 0.2520 & 1 \\
\hline
\end{tabular}


Table B.11

Mass Conversions

\begin{tabular}{llllll}
\hline & \multicolumn{5}{c}{ TO } \\
\cline { 2 - 6 } FROM & Pound & Kilogram & Short ton & Long ton & Metric ton \\
\hline Pound & 1 & 0.4536 & $5.0 \times 10^{-4}$ & $4.4643 \times 10^{-4}$ & $4.5362 \times 10^{-4}$ \\
Kilogram & 2.205 & 1 & $1.1023 \times 10^{-3}$ & $9.8425 \times 10^{-4}$ & $1.0 \times 10^{-3}$ \\
Short ton & 2000 & 907.2 & 1 & 0.8929 & 0.9072 \\
Long ton & 2240 & 1016 & 1.12 & 1 & 1.016 \\
Metric ton & 2205 & 1000 & 1.102 & 0.9842 & 1 \\
\hline
\end{tabular}


Table B.12

Fuel Efficiency Conversions ${ }^{\mathrm{a}}$

\begin{tabular}{|c|c|c|c|}
\hline MPG & Miles/liter & Kilometers/L & L/100 kilometers \\
\hline 10 & $2=64$ & $4=25$ & $23=52$ \\
\hline 15 & $3=96$ & $6=38$ & $15=68$ \\
\hline 20 & $5=28$ & $8=50$ & $11=76$ \\
\hline 25 & $6=60$ & $10=63$ & $9=41$ \\
\hline 30 & $7=92$ & $12=75$ & $7=84$ \\
\hline 35 & $9=25$ & $14=88$ & $6=72$ \\
\hline 40 & $10=57$ & $17=00$ & $5=88$ \\
\hline 45 & $11=89$ & $19=13$ & $5=23$ \\
\hline 50 & $13=21$ & $21=25$ & $4=70$ \\
\hline 55 & $14=53$ & $23=38$ & $4=28$ \\
\hline 60 & $15=85$ & $25=51$ & $3=92$ \\
\hline 65 & $17=17$ & $27=63$ & $3=62$ \\
\hline 70 & $18=49$ & $29=76$ & $3=36$ \\
\hline 75 & $19=81$ & $31=88$ & $3=14$ \\
\hline 80 & $21=13$ & $34=01$ & $2=94$ \\
\hline 85 & $22=45$ & $36=13$ & $2=77$ \\
\hline 90 & $23=77$ & $38=26$ & $2=61$ \\
\hline 95 & $25=09$ & $40=38$ & $2=48$ \\
\hline 100 & $26=42$ & $42=51$ & $2=35$ \\
\hline 105 & $27=74$ & $44=64$ & $2=24$ \\
\hline 110 & $29=06$ & $46=76$ & $2=14$ \\
\hline 115 & $30=38$ & $48=89$ & $2=05$ \\
\hline 120 & $31=70$ & $51=01$ & $1=96$ \\
\hline 125 & $33=02$ & $53=14$ & $1=88$ \\
\hline 130 & $34=34$ & $55=26$ & $1=81$ \\
\hline 135 & $35=66$ & $57=39$ & $1=74$ \\
\hline 140 & $36=98$ & $59=51$ & $1=68$ \\
\hline 145 & $38=30$ & $61=64$ & $1=62$ \\
\hline 150 & $39=62$ & $63=76$ & $1=57$ \\
\hline Formula & MPG/3.785 & MPG/[3.785/1.609] & 235.24/MPG \\
\hline
\end{tabular}


Table B.13

SI Prefixes and Their Values

\begin{tabular}{llll}
\hline & & & \\
& Value & Prefix & Symbol \\
\hline One million million millionth & $10^{-18}$ & atto & $\mathrm{a}$ \\
One thousand million millionth & $10^{-15}$ & femto & $\mathrm{f}$ \\
One million millionth & $10^{-12}$ & pico & $\mathrm{p}$ \\
One thousand millionth & $10^{-9}$ & nano & $\mathrm{n}$ \\
One millionth & $10^{-6}$ & micro & $:$ \\
One thousandth & $10^{-3}$ & milli & $\mathrm{m}$ \\
One hundredth & $10^{-2}$ & centi & $\mathrm{c}$ \\
One tenth & $10^{-1}$ & deci & \\
One & $10^{0}$ & & \\
Ten & $10^{1}$ & deca & \\
One hundred & $10^{2}$ & hecto & \\
One thousand & $10^{3}$ & kilo & $\mathrm{k}$ \\
One million & $10^{6}$ & mega & $\mathrm{M}$ \\
One billion $^{\mathrm{a}}$ & $10^{9}$ & giga & $\mathrm{G}$ \\
One trillion $^{\mathrm{a}}$ & $10^{12}$ & tera & $\mathrm{T}$ \\
One quadrillion $^{\mathrm{a}}$ & $10^{15}$ & peta & $\mathrm{P}$ \\
One quintillion $^{\mathrm{a}}$ & $10^{18}$ & exa & $\mathrm{E}$ \\
\hline
\end{tabular}

${ }^{a}$ Care should be exercised in the use of this nomenclature, especially in foreign correspondence, as it is either unknown or carries a different value in other countries. A "billion," for example, signifies a value of $10^{12}$ in most other countries. 
Table B.14

Metric Units and Abbreviations

\begin{tabular}{lll}
\hline \multicolumn{1}{c}{ Quantity } & Unit name & $\mathrm{Symbol}$ \\
\hline Energy & joule & $\mathrm{J}$ \\
Specific energy & joule/kilogram & $\mathrm{J} / \mathrm{kg}$ \\
Specific energy consumption & joule/kilogram•kilometer & $\mathrm{J} /(\mathrm{kg} \bullet \mathrm{km})$ \\
Energy consumption & joule/kilometer & $\mathrm{J} / \mathrm{km}$ \\
Energy economy & kilometer/kilojoule & $\mathrm{km} / \mathrm{kJ}$ \\
Power & kilowatt & $\mathrm{Kw}$ \\
Specific power & watt/kilogram & $\mathrm{W} / \mathrm{kg}$ \\
Power density & watt/meter & $\mathrm{W} / \mathrm{m}^{3}$ \\
Speed & kilometer/hour & $\mathrm{km} / \mathrm{h}$ \\
Acceleration & meter/second & $\mathrm{m} / \mathrm{s}^{2}$ \\
Range (distance) & kilometer & $\mathrm{km}$ \\
Weight & kilogram & $\mathrm{kg}$ \\
Torque & newton•meter & $\mathrm{N} \bullet \mathrm{m}$ \\
Volume & meter & $\mathrm{m}{ }^{3}$ \\
Mass; payload & kilogram & $\mathrm{kg}$ \\
Length; width & meter & $\mathrm{m}$ \\
Brake specific fuel consumption & kilogram/joule & $\mathrm{kg} / \mathrm{J}$ \\
Fuel economy (heat engine) & liters/100 km & $\mathrm{L} / 100 \mathrm{~km}$ \\
\hline
\end{tabular}




\section{Conversion of Constant Dollar Values}

Many types of information in this data book are expressed in dollars. Generally, constant dollars are used--that is, dollars of a fixed value for a specific year, such as 1990 dollars. Converting current dollars to constant dollars, or converting constant dollars for one year to constant dollars for another year, requires conversion factors (Table B.15 and B.16). Table B.15 shows conversion factors for the Consumer Price Index inflation factors. Table B.16 shows conversion factors using the Gross National Product inflation factors.

Due to the size of the tables, the data in Tables B.15 and B.16 were changed to two decimal places starting with Edition 17 and data for years 1971-74 were taken off in Edition 21. However, three decimal places were used to calculate all constant dollar values. 
Table B.15

Consumer Price Inflation (CPI) Index

To:

\begin{tabular}{|c|c|c|c|c|c|c|c|c|c|c|c|c|c|c|c|c|c|c|c|c|c|c|c|c|c|c|c|c|c|}
\hline & 1970 & 1975 & 1976 & 1977 & 1978 & 1979 & 1980 & 1981 & 1982 & 1983 & 1984 & 1985 & 1986 & 1987 & 1988 & 1989 & 1990 & 1991 & 1992 & 1993 & 1994 & 1995 & 1996 & 1997 & 1998 & 1999 & 2000 & 2001 & 2002 \\
\hline 1970 & 1.00 & 1.39 & 1.47 & 1.56 & 1.68 & 1.87 & 2.12 & 2.34 & 2.49 & 2.57 & 2.68 & 2.77 & 2.82 & 2.93 & & 20 & 3.37 & 3.51 & 3.62 & 3.72 & 3.82 & 3.93 & 4.04 & 4.14 & 4.20 & 4.29 & 4.44 & 4.56 & 4.64 \\
\hline 1975 & 0.72 & 1.00 & 1.06 & 1.13 & 1.21 & 1.35 & 53 & 69 & 1.79 & 1.85 & .93 & .00 & .04 & & 20 & 30 & .43 & .53 & .61 & 2.69 & 2.75 & 83 & 92 & .98 & .03 & .10 & 3.20 & .29 & 3.34 \\
\hline 1976 & .68 & 95 & 1.00 & 1.07 & 1.15 & 1.28 & 1.45 & 1.60 & 1.70 & 1.75 & 1.83 & 1.89 & 1.93 & & 2.08 & 2.18 & 2.30 & 2.39 & 2.47 & 2.54 & 2.60 & 2.68 & 2.76 & 2.82 & 2.86 & 2.93 & 3.03 & 3.11 & 3.16 \\
\hline 1977 & 0.64 & 0.89 & 0.94 & 1.00 & 1.08 & 1.20 & 1.36 & 1.50 & 1.59 & 1.64 & 1.71 & 1.78 & 1.81 & 1.87 & 1.95 & 2.05 & 2.16 & 2.25 & 2.32 & 2.38 & 2.45 & 2.51 & 2.59 & 2.65 & 2.69 & 2.75 & 2.84 & 2.92 & 2.97 \\
\hline 1978 & 0.60 & 0.83 & 0.87 & 0.93 & 1.00 & 1.11 & & 1.39 & & 1.53 & 1.59 & 1.65 & 1.68 & & & & & & & & 2.27 & 2.34 & 2.41 & 2.46 & 2.50 & 2.56 & 2.64 & 2.72 & 2.76 \\
\hline 1979 & 0.53 & 0.74 & 0.78 & 0.83 & 0.90 & 1.00 & 1.14 & 1.25 & 1.33 & 1.37 & 1.43 & 1.48 & 1.51 & 1.56 & 1.63 & 1.71 & 1.80 & 1.88 & 1.93 & 1.99 & 2.04 & 2.10 & 2.16 & 2.21 & 2.25 & .29 & 2.37 & 2.44 & 2.48 \\
\hline 1980 & 0.47 & 0.65 & 0.69 & 0.74 & 0.79 & 0.88 & & 1.10 & & 1.21 & 1.26 & 1.31 & 1.33 & 1.38 & 1.44 & 1.50 & 1.59 & 1.65 & 1.70 & 1.75 & 1.80 & 1.85 & 1.90 & 1.95 & 1.98 & 2.02 & 2.09 & 2.15 & 2.18 \\
\hline 1981 & 0.43 & 0.59 & 0.63 & 0.67 & 0.72 & 0.80 & 0.91 & 1.00 & 1.06 & 1.10 & 1.14 & 1.18 & 1.21 & 1.25 & 1.30 & 1.36 & 1.44 & 1.50 & 1.54 & 1.59 & 1.63 & 1.68 & 1.73 & 1.77 & 1.79 & 1.83 & 1.89 & 1.95 & 1.98 \\
\hline 1982 & 0.40 & 0.56 & 0.59 & 0.63 & 0.68 & 0.75 & 0.85 & 0.94 & 1.00 & 1.03 & 1.08 & 1.12 & 1.14 & 1.18 & 1.23 & 1.29 & 1.35 & 1.41 & 1.45 & 1.50 & 1.54 & 1.58 & 1.63 & 1.66 & 1.69 & 1.73 & 1.78 & 1.84 & 1.86 \\
\hline 1983 & 0.39 & 0.54 & 0.57 & 0.61 & 0.65 & 0.73 & 83 & 0.91 & 0.97 & 1.00 & 1.04 & 1.08 & 1.10 & 1.14 & 1.19 & 1.25 & 1.31 & 1.37 & 1.41 & 1.45 & 1.49 & 1.53 & 1.58 & 1.61 & 1.64 & 1.67 & 1.73 & 1.78 & 1.81 \\
\hline 1984 & 0.37 & 0.52 & 0.55 & 0.58 & 0.63 & 0.70 & 0.79 & 0.87 & 0.93 & 0.96 & 1.00 & 1.04 & 1.05 & 1.09 & 1.14 & 1.19 & 1.26 & 1.31 & 1.35 & 1.39 & 1.43 & 1.47 & 1.51 & 1.54 & 1.57 & 1.60 & 1.66 & 1.70 & 1.73 \\
\hline 1985 & 0.36 & 0.50 & 0.53 & 0.56 & 0.61 & 0.67 & 0.77 & 0.84 & 0.90 & 0.93 & 0.97 & 1.00 & 1.02 & 1.06 & 1.10 & 1.15 & 1.21 & 1.27 & 1.30 & 1.34 & 1.38 & 1.42 & 1.46 & 1.49 & 1.51 & 1.55 & 1.60 & 1.65 & 1.67 \\
\hline 1986 & 0.35 & 0.49 & 0.52 & 0.55 & 0.59 & 0.66 & & 0.83 & 0.88 & 0.91 & 0.95 & 0.98 & 1.00 & 1.04 & 1.0 & 1.13 & 1.19 & 1.24 & 1.28 & 1.32 & 1.35 & 1.39 & 1.43 & 1.46 & 1.49 & 1.52 & 1.57 & 1.62 & 1.64 \\
\hline 1987 & 0.34 & 0.47 & 0.50 & 0.53 & 0.57 & 0.64 & 0.73 & 0.80 & 0.85 & 0.88 & 0.91 & 0.95 & 0.96 & 1.00 & 1.04 & 1.09 & 1.15 & 1.20 & 1.24 & 1.27 & 1.30 & 1.34 & 1.38 & 1.41 & 1.43 & 1.47 & 1.52 & 1.56 & 1.58 \\
\hline 1988 & 0.33 & 0.45 & 0.48 & 0.51 & 0.55 & 0.61 & & 0.77 & 0.82 & 0.84 & 0.88 & 0.91 & 0.93 & 0. & 1. & 1.05 & 1.10 & 1.15 & 1.19 & 1.22 & 1.25 & 1.29 & 1.33 & 1.36 & 1.38 & 1.41 & 1.46 & 1.50 & 1.52 \\
\hline 1989 & 0.31 & 0.43 & 0.46 & 0.49 & 0.53 & 0.59 & 0.66 & 0.73 & 0.78 & 0.80 & 0.84 & 0.87 & 0.88 & 0.92 & 0.95 & 1.00 & 1.05 & 1.10 & 1.13 & 1.17 & 1.20 & 1.23 & 1.27 & 1.29 & 1.31 & 1.34 & 1.39 & 1.43 & 1.45 \\
\hline & 0.30 & & & & & & & & & 0.7 & 0.80 & 0.82 & & & & & & 04 & 07 & & 13 & & & .23 & .25 & .27 & .32 & 36 & 1.38 \\
\hline 1991 & 0.28 & 0.40 & 0.42 & 0.44 & 0.48 & 0.53 & 0.61 & 0.67 & 0.71 & 0.73 & 0.76 & 0.79 & 0.80 & 0.83 & 0.87 & 0.91 & 0.96 & 1.00 & 1.03 & 1.06 & 1.09 & 1.12 & 1.15 & 1.18 & 1.20 & 1.22 & 1.26 & 1.30 & 1.32 \\
\hline & 0.28 & 0.38 & 0.41 & 43 & 0.46 & .52 & & 0.6 & 0.69 & 0.71 & 0.74 & 0.77 & 0.78 & 0.81 & 0.84 & 0.88 & 0.93 & 0.97 & 1.00 & 1.03 & 1.06 & 1.09 & 1.12 & 1.14 & 1.16 & 1.19 & 1.23 & 1.26 & 1.28 \\
\hline 1993 & 0.27 & 0.37 & 0.39 & 0.42 & 0.45 & 0.50 & 0.57 & 0.63 & 0.67 & 0.69 & 0.72 & 0.74 & 0.76 & 0.79 & 0.82 & 0.86 & 0.90 & 0.94 & 0.97 & 1.00 & 1.03 & 1.05 & 1.09 & 1.11 & 1.13 & 1.15 & 1.19 & 1.23 & 1.24 \\
\hline 1994 & 0.26 & 0.36 & 0.38 & 0.41 & 0.44 & 0.49 & 0.56 & 0.61 & 0.65 & 0.67 & 0.70 & 0.73 & 0.74 & 0.77 & 0.80 & 0.84 & 0.88 & 0.92 & 0.95 & 0.98 & 1.00 & 1.03 & 1.06 & 1.08 & 1.10 & 1.12 & 1.16 & 1.20 & 1.21 \\
\hline 1995 & 0.25 & 0.35 & 0.37 & 0.40 & 0.43 & 0.48 & 0.54 & 0.60 & 0.63 & 0.65 & 0.68 & 0.71 & 0.72 & 0.75 & 0.7 & 0. & 0.86 & 0.89 & 0.92 & 0.95 & 0.97 & 1.00 & 1.03 & 1.05 & 1.07 & 1.09 & 1.13 & 1.16 & 1.18 \\
\hline 1996 & 0.25 & 0.34 & 0.36 & 0.39 & 0.42 & 0.46 & 0.53 & 0.58 & 0.62 & 0.63 & 0.66 & 0.69 & 0.70 & 0.72 & 0.75 & 0.79 & 0.83 & 0.87 & 0.89 & 0.92 & 0.94 & 0.97 & 1.00 & 1.02 & 1.04 & 1.06 & 1.10 & 1.13 & 1.15 \\
\hline 1997 & 0.24 & 0.34 & 0.35 & 0.38 & 0.41 & 0.45 & 0.51 & 0.57 & 0.60 & 0.62 & 0.65 & 0.67 & 0.68 & 0.71 & 0.74 & 0.77 & 0.81 & 0.85 & 0.87 & 0.90 & 0.92 & 0.95 & 0.98 & 1.00 & 1.02 & 1.04 & 1.07 & 1.10 & 1.12 \\
\hline 1998 & 0.24 & 0.33 & 0.35 & 0.37 & 0.40 & 0.45 & 0.51 & 0.56 & 0.59 & 0.61 & 0.64 & 0.66 & 0.67 & 0.70 & 0.73 & 0.76 & 0.80 & 0.84 & 0.86 & 0.89 & 0.91 & 0.94 & 0.96 & 0.98 & 1.00 & 1.02 & 1.06 & 1.09 & 1.10 \\
\hline 1999 & 0.23 & 0.32 & 0.34 & 0.36 & 0.39 & 0.44 & 0.49 & 0.55 & 0.58 & 0.60 & 0.62 & 0.65 & 0.66 & 0.68 & 0.71 & 0.74 & 0.78 & 0.82 & 0.84 & 0.87 & 0.89 & 0.91 & 0.94 & 0.96 & 0.98 & 1.00 & 1.03 & 1.06 & 1.08 \\
\hline 2000 & 0.23 & 0.31 & 0.33 & 0.35 & 0.38 & 0.42 & 0.48 & 0.53 & 0.56 & 0.58 & 0.60 & 0.62 & 0.64 & 0.66 & 0.69 & 0.72 & 0.76 & 0.79 & 0.81 & 0.84 & 0.86 & 0.89 & 0.91 & 0.93 & 0.95 & 0.97 & 1.00 & 1.03 & 1.04 \\
\hline 2001 & 0.22 & 0.30 & 0.32 & 0.34 & 0.37 & 0.41 & 0.47 & 0.51 & 0.54 & 0.56 & 0.59 & 0.61 & 0.62 & 0.64 & 0.67 & 0.70 & 0.74 & 0.77 & 0.79 & 0.82 & 0.84 & 0.86 & 0.89 & 0.91 & 0.92 & 0.94 & 0.98 & 1.00 & 1.02 \\
\hline 2002 & 0.22 & 0.30 & 0.32 & 0.34 & 0.36 & 0.40 & 0.46 & 0.51 & 0.54 & 0.55 & 0.58 & 0.60 & 0.61 & 0.63 & 0.66 & 0.69 & 0.73 & 0.76 & 0.78 & 0.80 & 0.82 & 0.85 & 0.87 & 0.89 & 0.91 & 0.93 & 0.96 & 0.98 & 1.00 \\
\hline
\end{tabular}

Source:

U.S. Bureau of Labor Statistics. 
Table B.16

Gross National Product Implicit Price Deflator

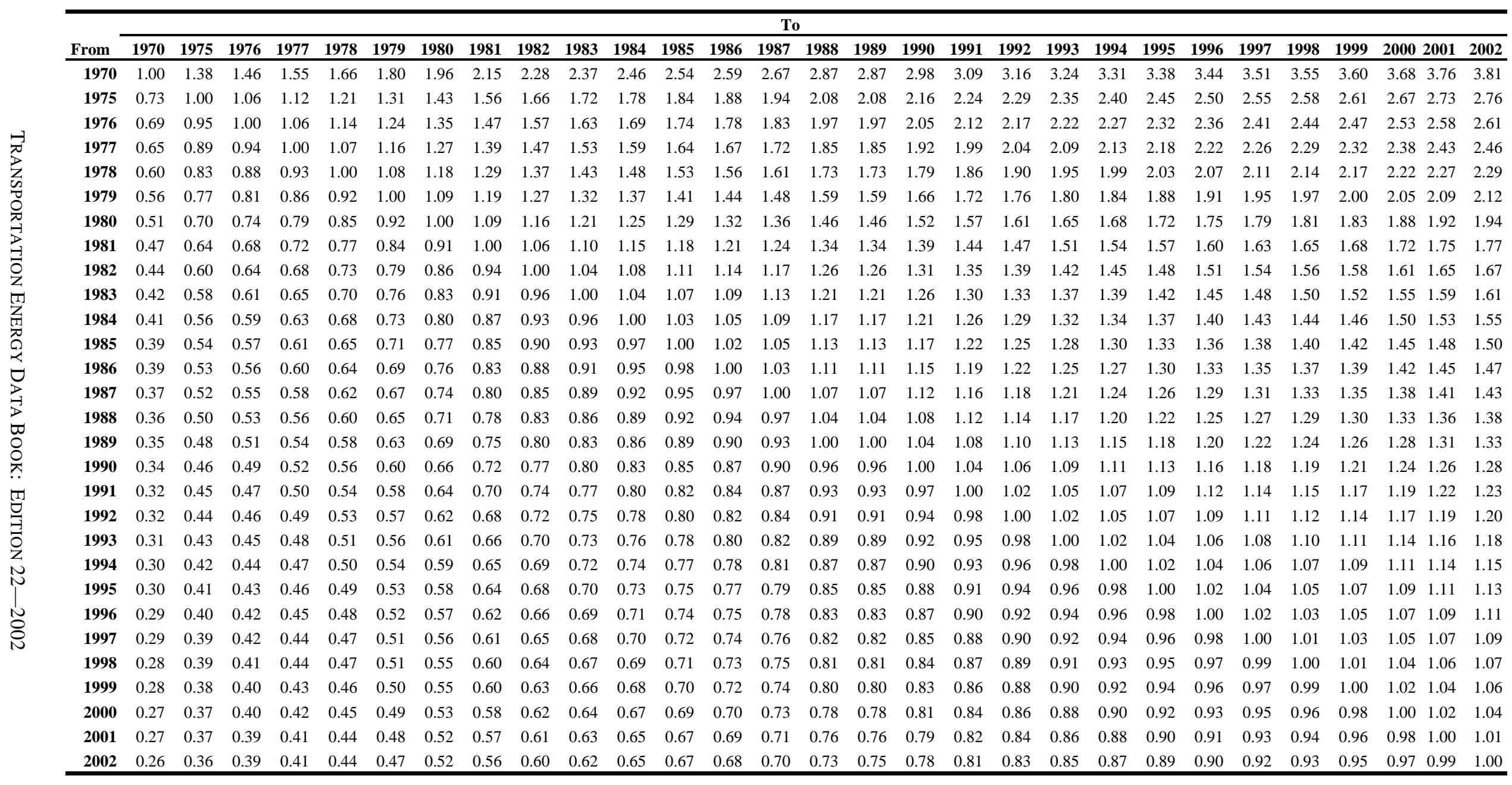

Source:

U.S. Department of Commerce, Bureau of Economic Analysis, Survey of Current Business, Washington, DC, monthly. 
APPENDIX C

MAPS 
Table C.1

Census Divisions and Regions

\begin{tabular}{|c|c|c|c|}
\hline \multicolumn{4}{|c|}{ Northeast Division } \\
\hline \multicolumn{2}{|c|}{ Mid-Atlantic region } & \multicolumn{2}{|c|}{ New England region } \\
\hline $\begin{array}{l}\text { New Jersey } \\
\text { New York }\end{array}$ & Pennsylvania & $\begin{array}{l}\text { Connecticut } \\
\text { Maine } \\
\text { Massachusetts }\end{array}$ & $\begin{array}{l}\text { New Hampshire } \\
\text { Rhode Island } \\
\text { Vermont }\end{array}$ \\
\hline \multicolumn{4}{|c|}{ South Division } \\
\hline $\begin{array}{l}\text { West South Central } \\
\text { region }\end{array}$ & $\begin{array}{l}\text { East South Central } \\
\text { region }\end{array}$ & & $\begin{array}{l}\text { Atlantic } \\
\text { ion }\end{array}$ \\
\hline $\begin{array}{l}\text { Arkansas } \\
\text { Louisiana } \\
\text { Oklahoma } \\
\text { Texas }\end{array}$ & $\begin{array}{l}\text { Alabama } \\
\text { Kentucky } \\
\text { Mississippi } \\
\text { Tennessee }\end{array}$ & $\begin{array}{l}\text { Delaware } \\
\text { Florida } \\
\text { Georgia } \\
\text { Maryland } \\
\text { North Carolina }\end{array}$ & $\begin{array}{l}\text { South Carolina } \\
\text { Virginia } \\
\text { Washington, DC } \\
\text { West Virginia }\end{array}$ \\
\hline \multicolumn{4}{|c|}{ West Division } \\
\hline \multicolumn{2}{|c|}{ Pacific region } & \multicolumn{2}{|c|}{ Mountain region } \\
\hline $\begin{array}{l}\text { Alaska } \\
\text { California } \\
\text { Hawaii }\end{array}$ & $\begin{array}{l}\text { Oregon } \\
\text { Washington }\end{array}$ & $\begin{array}{l}\text { Arizona } \\
\text { Colorado } \\
\text { Idaho } \\
\text { Montana }\end{array}$ & $\begin{array}{l}\text { Nevada } \\
\text { New Mexico } \\
\text { Utah } \\
\text { Wyoming }\end{array}$ \\
\hline \multicolumn{4}{|c|}{ Midwest Division } \\
\hline \multicolumn{2}{|c|}{ West North Central region } & \multicolumn{2}{|c|}{ East North Central region } \\
\hline $\begin{array}{l}\text { Iowa } \\
\text { Kansas } \\
\text { Minnesota } \\
\text { Missouri }\end{array}$ & $\begin{array}{l}\text { Nebraska } \\
\text { North Dakota } \\
\text { South Dakota }\end{array}$ & $\begin{array}{l}\text { Illinois } \\
\text { Indiana } \\
\text { Michigan }\end{array}$ & $\begin{array}{l}\text { Ohio } \\
\text { Wisconsin }\end{array}$ \\
\hline
\end{tabular}

Source:

U.S. Census Bureau. 
Figure C1. Census Divisions and Regions

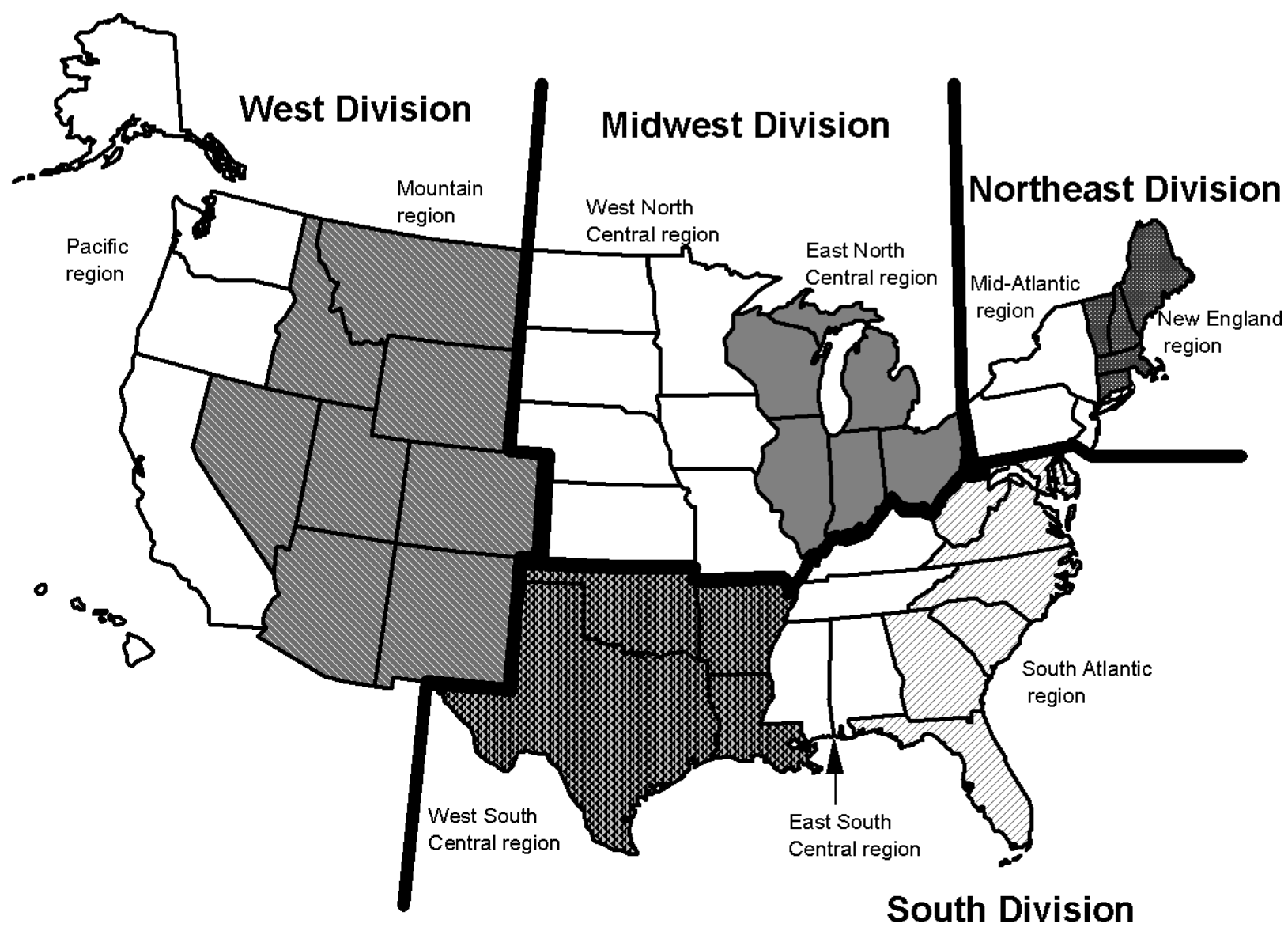

Source: See Table C.1. 
Table C.2

Petroleum Administration for Defense Districts (PADD)

\begin{tabular}{|l|l|l|}
\hline District & Subdistrict & States \\
\hline $\begin{array}{l}\text { PAD District 1 } \\
\text { East Coast }\end{array}$ & $\begin{array}{l}\text { Subdistrict 1X } \\
\text { New England }\end{array}$ & $\begin{array}{l}\text { Connecticut, Maine, Massachusetts, New } \\
\text { Hampshire, Rhode Island, Vermont }\end{array}$ \\
\hline & $\begin{array}{l}\text { Subdistrict 1Y } \\
\text { Central Atlantic }\end{array}$ & $\begin{array}{l}\text { Delaware, District of Columbia, Maryland, New } \\
\text { Jersey, New York, Pennsylvania }\end{array}$ \\
\hline $\begin{array}{l}\text { Subdistrict 1Z } \\
\text { Mower Atlantic }\end{array}$ & $\begin{array}{l}\text { Florida, Georgia, North Carolina, South Carolina, } \\
\text { Virginia, West Virginia }\end{array}$ \\
\hline $\begin{array}{l}\text { PAD District 3 } 3 \\
\text { Gulf Coast }\end{array}$ & & $\begin{array}{l}\text { Illinois, Indiana, Iowa, Kansas, Kentucky, } \\
\text { Michigan, Minnesota, Missouri, Nebraska, North } \\
\text { Dakota, South Dakota, Ohio, Oklahoma, } \\
\text { Tennessee, Wisconsin }\end{array}$ \\
\hline $\begin{array}{l}\text { PAD District 4 } \\
\text { Rocky Mountains }\end{array}$ & & $\begin{array}{l}\text { Alabama, Arkansas, Louisiana, Mississippi, New } \\
\text { Mexico, Texas }\end{array}$ \\
\hline $\begin{array}{l}\text { PAD District 5 } \\
\text { West Coast }\end{array}$ & & Colorado Idaho, Montana, Utah, Wyoming \\
\hline
\end{tabular}

\section{Source:}

Energy Information Administration web site: http://tonto.eia.doe.gov/oog/info/twip/padddef.html 
Figure C.2. Petroleum Administration for Defense Districts

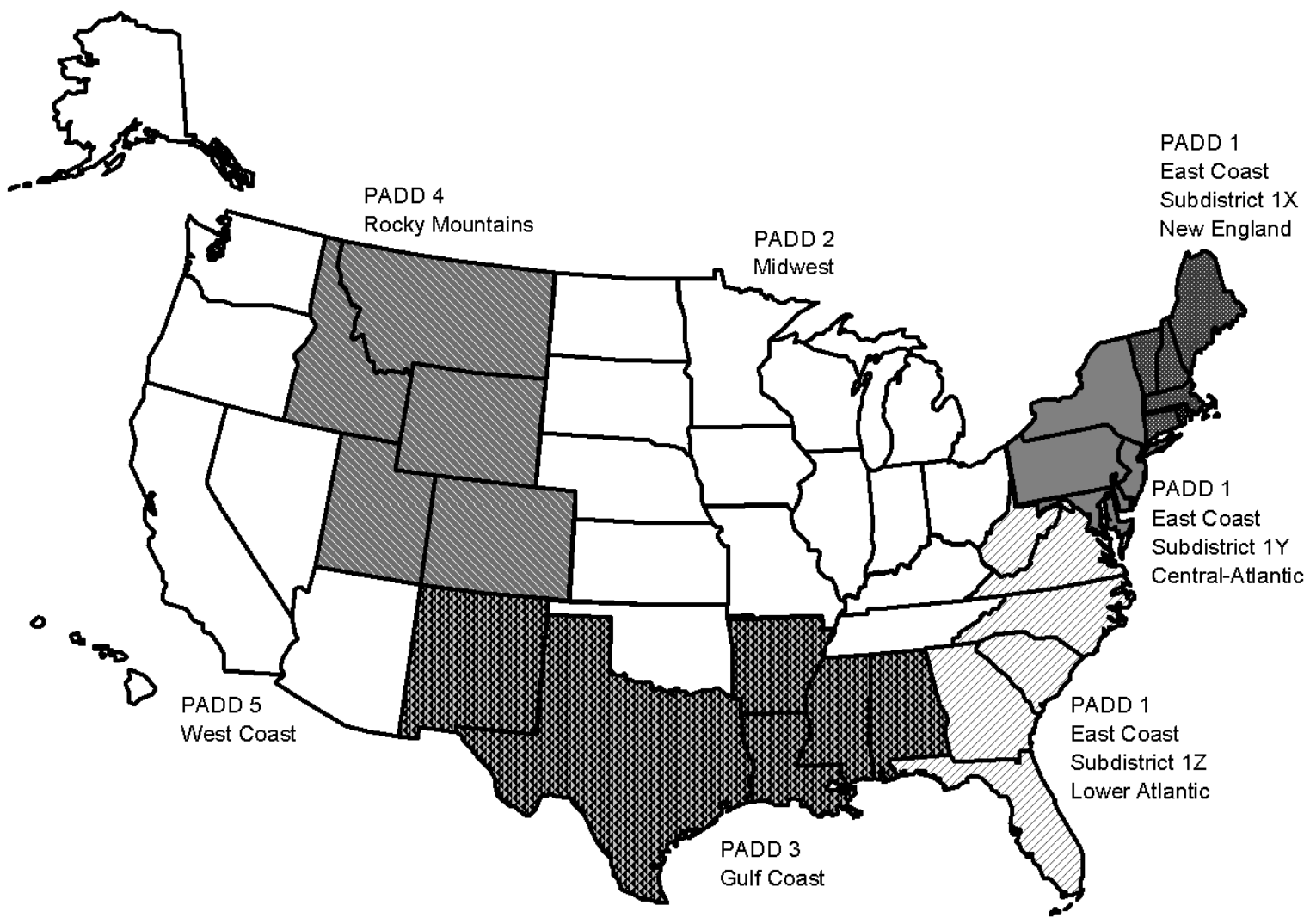

Source: See Table C.2. 


\section{GLOSSARY}

Acceleration power - Measured in kilowatts. Pulse power obtainable from a battery used to accelerate a vehicle. This is based on a constant current pulse for 30 seconds at no less than $2 / 3$ of the maximum open-circuit-voltage, at $80 \%$ depth-of-discharge relative to the battery's rated capacity and at $20^{\circ} \mathrm{C}$ ambient temperature.

Air Carrier - The commercial system of air transportation consisting of certificated air carriers, air taxis (including commuters), supplemental air carriers, commercial operators of large aircraft, and air travel clubs.

Certificated route air carrier: An air carrier holding a Certificate of Public Convenience and Necessity issued by the Department of Transportation to conduct scheduled interstate services. Nonscheduled or charter operations may also be conducted by these carriers. These carriers operate large aircraft (30 seats or more, or a maximum payload capacity of 7,500 pounds or more) in accordance with Federal Aviation Regulation part 121.

Domestic air operator: Commercial air transportation within and between the 50 States and the District of Columbia. Includes operations of certificated route air carriers, Pan American, local service, helicopter, intra-Alaska, intra-Hawaii, all-cargo carriers and other carriers. Also included are transborder operations conducted on the domestic route segments of U.S. air carriers. Domestic operators are classified based on their operating revenue as follows:

$$
\begin{aligned}
& \text { Majors - over } \$ 1 \text { billion } \\
& \text { Nationals - \$100-1,000 million } \\
& \text { Large Regionals - \$10-99.9 million } \\
& \text { Medium Regionals - \$0-9.99 million }
\end{aligned}
$$

International air operator: Commercial air transportation outside the territory of the United States, including operations between the U.S. and foreign countries and between the U.S. and its territories and possessions.

Supplemental air carrier: A class of air carriers which hold certificates authorizing them to perform passenger and cargo charter services supplementing the scheduled service of the certificated route air carriers. Supplemental air carriers are often referred to as nonscheduled air carriers or "nonskeds." 
Alcohol - The family name of a group of organic chemical compounds composed of carbon, hydrogen, and oxygen. The molecules in the series vary in chain length and are composed of a hydrocarbon plus a hydroxyl group. Alcohol includes methanol and ethanol.

Amtrak - See Rail.

Anthropogenic - Human made. Usually used in the context of emissions that are produced as the result of human activities.

Automobile size classifications - Size classifications of automobiles are established by the Environmental Protection Agency (EPA) as follows:

Minicompact - less than 85 cubic feet of passenger and luggage volume.

Subcompact - between 85 to 100 cubic feet of passenger and luggage volume.

Compact - between 100 to 110 cubic feet of passenger and luggage volume.

Midsize - between 110 to 120 cubic feet of passenger and luggage volume.

Large - more than 120 cubic feet of passenger and luggage volume.

Two seater - automobiles designed primarily to seat only two adults.

Station wagons are included with the size class for the sedan of the same name.

\section{Aviation - See General aviation.}

Aviation gasoline - All special grades of gasoline for use in aviation reciprocating engines, as given in the American Society for Testing and Materials (ASTM) Specification D 910. Includes all refinery products within the gasoline range that are to be marketed straight or in blends as aviation gasoline without further processing (any refinery operation except mechanical blending). Also included are finished components in the gasoline range which will be used for blending or compounding into aviation gasoline.

Barges - Shallow, nonself-propelled vessels used to carry bulk commodities on the rivers and the Great Lakes.

Battery efficiency - Measured in percentage. Net DC energy delivered on discharge, as a percentage of the total DC energy required to restore the initial state-of-charge. The efficiency value must include energy losses resulting from self-discharge, cell equalization, thermal loss compensation, and all battery-specific auxiliary equipment.

Btu - British thermal unit. The amount of energy required to raise the temperature of 1 pound of water 1 degree Fahrenheit at or near 39.2 degrees Fahrenheit. An average Btu content of fuel is the heat value per quantity of fuel as determined from tests of fuel samples.

Bunker - A storage tank. 
Bunkering fuels - Fuels stored in ship bunkers.

Bus -

Intercity bus: A standard size bus equipped with front doors only, high backed seats, luggage compartments separate from the passenger compartment and usually with restroom facilities, for high-speed long distance service.

Motor bus: Rubber-tired, self-propelled, manually-steered bus with fuel supply on board the vehicle. Motor bus types include intercity, school, and transit.

School and other nonrevenue bus: Bus services for which passengers are not directly charged for transportation, either on a per passenger or per vehicle basis.

Transit bus: A bus designed for frequent stop service with front and center doors, normally with a rear-mounted diesel engine, low-back seating, and without luggage storage compartments or restroom facilities.

Trolley coach: Rubber-tired electric transit vehicle, manually-steered, propelled by a motor drawing current, normally through overhead wires, from a central power source not on board the vehicle.

Calendar year - The period of time between January 1 and December 31 of any given year.

Captive imports - Products produced overseas specifically for domestic manufacturers.

Carbon dioxide $\left(\mathbf{C O}_{2}\right)$ - A colorless, odorless, non-poisonous gas that is a normal part of the ambient air. Carbon dioxide is a product of fossil fuel combustion.

Carbon monoxide (CO) - A colorless, odorless, highly toxic gas that is a by-product of incomplete fossil fuel combustion. Carbon monoxide, one of the major air pollutants, can be harmful in small amounts if breathed over a certain period of time.

Car-mile (railroad) - A single railroad car moved a distance of one mile.

Cargo ton-mile - See Ton-mile.

Certificated route air carriers - See Air carriers.

Class I freight railroad - See Rail.

Coal slurry - Finely crushed coal mixed with sufficient water to form a fluid. 
Combination trucks - Consist of a power unit (a truck tractor) and one or more trailing units (a semi-trailer or trailer). The most frequently used combination is popularly referred to as a "tractor-semitrailer" or "tractor trailer".

Commercial sector - An energy-consuming sector that consists of service-providing facilities of: businesses; Federal, State, and local governments; and other private and public organizations, such as religious, social or fraternal groups. Includes institutional living quarters.

Commuter railroad - See Rail.

Compact car - See Automobile size classifications.

Constant dollars - A time series of monetary figures is expressed in constant dollars when the effect of change over time in the purchasing power of the dollar has been removed. Usually the data are expressed in terms of dollars of a selected year or the average of a set of years.

Consumer Price Index (CPI) - An index issued by the U.S. Department of Labor, Bureau of Labor Statistics. The CPI is designed to measure changes in the prices of goods and services bought by wage earners and clerical workers in urban areas. It represents the cost of a typical consumption bundle at current prices as a ratio to its cost at a base year.

Continuous discharge capacity - Measured as percent of rated energy capacity. Energy delivered in a constant power discharge required by an electric vehicle for hill climbing and/or highspeed cruise, specified as the percent of its rated energy capacity delivered in a one hour constant-power discharge.

Corporate Average Fuel Economy (CAFE) standards - CAFE standards were originally established by Congress for new automobiles, and later for light trucks, in Title V of the Motor Vehicle Information and Cost Savings Act (15 U.S.C.1901, et seq.) with subsequent amendments. Under CAFE, automobile manufacturers are required by law to produce vehicle fleets with a composite sales-weighted fuel economy which cannot be lower than the CAFE standards in a given year, or for every vehicle which does not meet the standard, a fine of $\$ 5.00$ is paid for every one-tenth of a mpg below the standard.

Crude oil - A mixture of hydrocarbons that exists in the liquid phase in natural underground reservoirs and remains liquid at atmospheric pressure after passing through surface separating facilities. Crude oil production is measured at the wellhead and includes lease condensate. 
Crude oil imports - The volume of crude oil imported into the 50 States and the District of Columbia, including imports from U.S. territories, but excluding imports of crude oil into the Hawaiian Foreign Trade Zone.

Curb weight - The weight of a vehicle including all standard equipment, spare tire and wheel, all fluids and lubricants to capacity, full tank of fuel, and the weight of major optional accessories normally found on the vehicle.

Current dollars - Represents dollars current at the time designated or at the time of the transaction. In most contexts, the same meaning would be conveyed by the use of the term "dollars." See also constant dollars.

Demand Response - A transit mode that includes passenger cars, vans, and small buses operating in response to calls from passengers to the transit operator who dispatches the vehicles. The vehicles do not operate over a fixed route on a fixed schedule. Can also be known as paratransit or dial-a-ride.

Diesel fuel - See distillate fuel oil.

Disposable personal income - See Income.

Distillate fuel oil - The lighter fuel oils distilled off during the refining process. Included are products known as ASTM grades numbers 1 and 2 heating oils, diesel fuels, and number 4 fuel oil. The major uses of distillate fuel oils include heating, fuel for on-and off-highway diesel engines, and railroad diesel fuel.

Domestic air operator - See Air carrier.

E85 - 85\% ethanol and 15\% gasoline.

E95 - 95\% ethanol and 5\% gasoline.

Domestic water transportation - See Internal water transportation.

Electric utilities sector - Consists of privately and publicly owned establishments which generate electricity primarily for resale.

Emission standards - Standards for the levels of pollutants emitted from automobiles and trucks. Congress established the first standards in the Clean Air Act of 1963. Currently, standards are set for four vehicle classes - automobiles, light trucks, heavy-duty gasoline trucks, and heavy-duty diesel trucks. 
Energy capacity - Measured in kilowatt hours. The energy delivered by the battery, when tested at $\mathrm{C} / 3$ discharge rate, up to termination of discharge specified by the battery manufacturer. The required acceleration power must be delivered by the battery at any point up to $80 \%$ of the battery's energy capacity rating.

Energy efficiency - In reference to transportation, the inverse of energy intensiveness: the ratio of outputs from a process to the energy inputs; for example, miles traveled per gallon of fuel (mpg).

Energy intensity - In reference to transportation, the ratio of energy inputs to a process to the useful outputs from that process; for example, gallons of fuel per passenger-mile or Btu per tonmile.

Ethanol $\left(\mathbf{C}_{2} \mathbf{H}_{5} \mathbf{O H}\right)$ - Otherwise known as ethyl alcohol, alcohol, or grain-spirit. A clear, colorless, flammable oxygenated hydrocarbon with a boiling point of 78.5 degrees Celsius in the anhydrous state. In transportation, ethanol is used as a vehicle fuel by itself (E100-100\% ethanol by volume), blended with gasoline (E85 - 85\% ethanol by volume), or as a gasoline octane enhancer and oxygenate ( $10 \%$ by volume).

\section{Fixed operating cost - See Operating cost.}

\section{Fleet vehicles -}

Private fleet vehicles: Ideally, a vehicle could be classified as a member of a fleet if it is:

a) operated in mass by a corporation or institution,

b) operated under unified control, or

c) used for non-personal activities.

However, the definition of a fleet is not consistent throughout the fleet industry. Some companies make a distinction between cars that were bought in bulk rather than singularly, or whether they are operated in bulk, as well as the minimum number of vehicles that constitute a fleet (i.e. 4 or 10).

Government fleet vehicles: Includes vehicles owned by all Federal, state, county, city, and metro units of government, including toll road operations.

Foreign freight - Movements between the United States and foreign countries and between Puerto Rico, the Virgin Islands, and foreign countries. Trade between U.S. territories and possessions (e.g. Guam, Wake, American Samoa) and foreign countries is excluded. Traffic to or from the Panama Canal Zone is included. 
Gas Guzzler Tax - Originates from the 1978 Energy Tax Act (Public Law 95-618). A new car purchaser is required to pay the tax if the car purchased has a combined city/highway fuel economy rating that is below the standard for that year. For model years 1986 and later, the standard is $22.5 \mathrm{mpg}$.

Gasohol - A mixture of $10 \%$ anhydrous ethanol and $90 \%$ gasoline by volume; $7.5 \%$ anhydrous ethanol and $92.5 \%$ gasoline by volume; or $5.5 \%$ anhydrous ethanol and $94.5 \%$ gasoline by volume. There are other fuels that contain methanol and gasoline, but these fuels are not referred to as gasohol.

Gasoline - See Motor gasoline.

General aviation - That portion of civil aviation which encompasses all facets of aviation except air carriers. It includes any air taxis, commuter air carriers, and air travel clubs which do not hold Certificates of Public Convenience and Necessity.

Gross National Product - A measure of monetary value of the goods and services becoming available to the nation from economic activity. Total value at market prices of all goods and services produced by the nation's economy. Calculated quarterly by the Department of Commerce, the Gross National Product is the broadest available measure of the level of economic activity.

Gross vehicle weight (gvw) - The weight of the empty truck plus the maximum anticipated load weight.

Gross vehicle weight rating (gvwr) - The gross vehicle weight which is assigned to each new truck by the manufacturer. This rating may be different for trucks of the same model because of certain features, such as heavy-duty suspension. Passenger cars do not have gross vehicle weight ratings.

\section{Heavy-heavy truck - See Truck size classifications.}

Household - Consists of all persons who occupy a housing unit, including the related family members and all unrelated persons, if any, who share the housing unit.

Housing unit - A house, apartment, a group of rooms, or a single room occupied or intended for occupancy as separate living quarters. Separate living quarters are those in which the occupants do not live and eat with any other persons in the structure and which have either (1) direct access from the outside of the building or through a common hallway intended to be used by the occupants of another unit or by the general public, or (2) complete kitchen facilities for the exclusive use of the occupants. The occupants may be a single family, one 
person living alone, two or more families living together, or any other group of related or unrelated persons who share living arrangements.

Hydrocarbon (HC) - A compound that contains only hydrogen and carbon. The simplest and lightest forms of hydrocarbon are gaseous. With greater molecular weights they are liquid, while the heaviest are solids.

\section{Income -}

Disposable personal income: Personal income less personal tax and non-tax payments.

National income: The aggregate earnings of labor and property which arise in the current production of goods and services by the nation's economy.

Personal income: The current income received by persons from all sources, net of contributions for social insurance.

Industrial sector - Construction, manufacturing, agricultural and mining establishments.

Inertia weight - The curb weight of a vehicle plus 300 pounds.

Intercity bus - See Bus.

Internal water transportation - Includes all local (intraport) traffic and traffic between ports or landings wherein the entire movement takes place on inland waterways. Also termed internal are movements involving carriage on both inland waterways and the water of the Great Lakes, and inland movements that cross short stretches of open water that link inland systems.

International air operator - See Air carrier.

International freight - See Foreign freight.

Jet fuel - Includes both naphtha-type and kerosene-type fuels meeting standards for use in aircraft turbine engines. Although most jet fuel is used in aircraft, some is used for other purposes such as generating electricity in gas turbines.

Kerosene-type jet fuel: A quality kerosene product with an average gravity of 40.7 degrees API and $10 \%$ to $90 \%$ distillation temperatures of 217 to 261 degrees centigrade. Used primarily as fuel for commercial turbojet and turboprop aircraft engines. It is a relatively low freezing point distillate of the kerosene type. 
Naphtha-type jet fuel: A fuel in the heavy naphtha boiling range with an average gravity of 52.8 degrees API and $10 \%$ to $90 \%$ distillation temperatures of 117 to 233 degrees centigrade used for turbojet and turboprop aircraft engines, primarily by the military. Excludes ramjet and petroleum.

Kerosene - A petroleum distillate in the 300 to 500 degrees Fahrenheit boiling range and generally having a flash point higher than 100 degrees Fahrenheit by the American Society of Testing and Material (ASTM) Method D56, a gravity range from 40 to 46 degrees API, and a burning point in the range of 150 to 175 degrees Fahrenheit. It is a clean-burning product suitable for use as an illuminant when burned in wick lamps. Includes grades of kerosene called range oil having properties similar to Number 1 fuel oil, but with a gravity of about 43 degrees API and an end point of 625 degrees Fahrenheit. Used in space heaters, cooking stoves, and water heaters.

Kerosene-type jet fuel - See Jet fuel.

Large car - See Automobile size classifications.

Lease Condensate - A liquid recovered from natural gas at the well or at small gas/oil separators in the field. Consists primarily of pentanes and heavier hydrocarbons (also called field condensate).

Light duty vehicles - Automobiles and light trucks combined.

Light truck - Unless otherwise noted, light trucks are defined in this publication as two-axle, fourtire trucks. The U.S. Bureau of Census classifies all trucks with a gross vehicle weight less than 10,000 pounds as light trucks (See Truck size classifications).

\section{Light-heavy truck - See Truck size classifications.}

Liquified petroleum gas (lpg) - Consists of propane and butane and is usually derived from natural gas. In locations where there is no natural gas and the gasoline consumption is low, naphtha is converted to lpg by catalytic reforming.

Load factor - Total passenger miles divided by total vehicle miles.

Low emission vehicle - Any vehicle certified to the low emission standards which are set by the Federal government and/or the state of California.

M85 - 85\% methanol and 15\% gasoline. 
M100 - 100\% methanol.

Medium truck - See Truck size classifications.

Methanol $\left(\mathrm{CH}_{3} \mathrm{OH}\right)$ - A colorless highly toxic liquid with essentially no odor and very little taste. It is the simplest alcohol and boils at 64.7 degrees Celsius. In transportation, methanol is used as a vehicle fuel by itself (M100), or blended with gasoline (M85).

Midsize car - See Automobile size classifications.

Minicompact car - See Automobile size classifications.

Model year - In this publication, model year is referring to the "sales" model year, the period from October 1 to the next September 31.

Motor bus - See Bus.

Motor Gasoline - A mixture of volatile hydrocarbons suitable for operation of an internal combustion engine whose major components are hydrocarbons with boiling points ranging from 78 to 217 degrees centigrade and whose source is distillation of petroleum and cracking, polymerization, and other chemical reactions by which the naturally occurring petroleum hydrocarbons are converted into those that have superior fuel properties.

MTBE - Methyl Tertiary Butyl Ether - a colorless, flammable, liquid oxygenated hydrocarbon containing 18.15 percent oxygen.

Naphtha-type jet fuel - See Jet fuel.

National income - See Income.

Nationwide Personal Transportation Survey (NPTS) - A nationwide survey of households that provides information on the characteristics and personal travel patterns of the U.S. population. Surveys were conducted in 1969, 1977, 1983, 1990, and 1995 by the U.S. Bureau of Census for the U.S. Department of Transportation.

Natural gas - A mixture of hydrocarbon compounds and small quantities of various nonhydrocarbons existing in the gaseous phase or in solution with crude oil in natural underground reservoirs at reservoir conditions.

Natural gas, dry: Natural gas which remains after: 1) the liquefiable hydrocarbon portion has been removed from the gas stream; and 2) any volumes of nonhydrocarbon gases have been removed where they occur in sufficient quantity to render the gas unmarketable. Dry 
natural gas is also known as consumer-grade natural gas. The parameters for measurement are cubic feet at 60 degrees Fahrenheit and 14.73 pounds per square inch absolute.

Natural gas, wet: The volume of natural gas remaining after removal of lease condensate in lease and/or field separation facilities, if any, and after exclusion of nonhydrocarbon gases where they occur in sufficient quantity to render the gas unmarketable. Natural gas liquids may be recovered from volumes of natural gas, wet after lease separation, at natural gas processing plants.

Natural gas plant liquids - Natural gas liquids recovered from natural gas in processing plants and from natural gas field facilities and fractionators. Products obtained include ethane, propane, normal butane, isobutane, pentanes plus, and other products from natural gas processing plants.

Nitrogen oxides $\left(\mathbf{N O}_{\mathbf{x}}\right.$ ) - A product of combustion of fossil fuels whose production increases with the temperature of the process. It can become an air pollutant if concentrations are excessive.

Oil Stocks - Oil stocks include crude oil (including strategic reserves), unfinished oils, natural gas plant liquids, and refined petroleum products.

\section{Operating cost -}

Fixed operating cost: In reference to passenger car operating cost, refers to those expenditures that are independent of the amount of use of the car, such as insurance costs, fees for license and registration, depreciation and finance charges.

Variable operating cost: In reference to passenger car operating cost, expenditures which are dependent on the amount of use of the car, such as the cost of gas and oil, tires, and other maintenance.

Organization for Economic Cooperation and Development (OECD) - Consists of Australia, Austria, Belgium, Canada, Czech Republic, Denmark, Finland, France, Germany, Greece, Hungary, Iceland, Ireland, Italy, Japan, Luxembourg, Mexico, Netherlands, New Zealand, Norway, Poland, Portugal, South Korea, Spain, Sweden, Switzerland, Turkey, United Kingdom, and United States. Total OECD includes the United States Territories (Guam, Puerto Rico, and the U.S. Virgin Islands). Total OECD excludes data for Czech Republic, Hungary, Mexico, Poland, and South Korea which are not yet available.

OECD Europe: Consists of Austria, Belgium, Czech Republic, Denmark, Finland, France, Germany, Greece, Hungary, Iceland, Ireland, Italy, Luxembourg, Netherlands, Norway, 
Poland, Portugal, Spain, Sweden, Switzerland, Turkey, and United Kingdom. OECD Europe excludes data for Czech Republic, Hungary, and Poland which are not yet available.

OECD Pacific: Consists of Australia, Japan, and New Zealand.

Organization for Petroleum Exporting Countries (OPEC) - Includes Saudi Arabia, Iran, Venezuela, Libya, Indonesia, United Arab Emirates, Algeria, Nigeria, Ecuador, Gabon, Iraq, Kuwait, and Qatar. Data for Saudi Arabia and Kuwait include their shares from the Partitioned Zone (formerly the Neutral Zone).

Arab OPEC - Consists of Algeria, Iraq, Kuwait, Libya, Qatar, Saudi Arabia and the United Arab Emirates.

Other single-unit truck - See Single-unit truck.

Oxygenate - A substance which, when added to gasoline, increases the amount of oxygen in that gasoline blend. Includes fuel ethanol, methanol, and methyl tertiary butyl ether (MTBE).

Particulates - Carbon particles formed by partial oxidation and reduction of the hydrocarbon fuel. Also included are trace quantities of metal oxides and nitrides, originating from engine wear, component degradation, and inorganic fuel additives. In the transportation sector, particulates are emitted mainly from diesel engines.

Passenger-miles traveled (PMT) - One person traveling the distance of one mile. Total passengermiles traveled, thus, give the total mileage traveled by all persons.

Passenger rail - See Rail, "Amtrak" and "Transit Railroad".

Persian Gulf countries: Consists of Bahrain, Iran, Iraq, Kuwait, Qatar, Saudi Arabia, and the United Emirates.

Personal Consumption Expenditures (PCE) - As used in the national accounts, the market value of purchases of goods and services by individuals and nonprofit institutions and the value of food, clothing, housing, and financial services received by them as income in kind. It includes the rental value of owner-occupied houses but excludes purchases of dwellings, which are classified as capital goods (investment).

Personal income - See Income. 
Petroleum - A generic term applied to oil and oil products in all forms, such as crude oil, lease condensate, unfinished oil, refined petroleum products, natural gas plant liquids, and nonhydrocarbon compounds blended into finished petroleum products.

Petroleum consumption: A calculated demand for petroleum products obtained by summing domestic production, imports of crude petroleum and natural gas liquids, imports of petroleum products, and the primary stocks at the beginning of the period and then subtracting the exports and the primary stocks at the end of the period.

Petroleum exports: Shipments of petroleum products from the 50 States and the District of Columbia to foreign countries, Puerto Rico, the Virgin Islands, and other U.S. possessions and territories.

Petroleum imports: All imports of crude petroleum, natural gas liquids, and petroleum products from foreign countries and receipts from Guam, Puerto Rico, the Virgin Islands, and the Hawaiian Trade Zone. The commodities included are crude oil, unfinished oils, plant condensate, and refined petroleum products.

Petroleum inventories: The amounts of crude oil, unfinished oil, petroleum products, and natural gas liquids held at refineries, at natural gas processing plants, in pipelines, at bulk terminals operated by refining and pipeline companies, and at independent bulk terminals. Crude oil held in storage on leases is also included; these stocks are know as primary stocks. Secondary stocks - those held by jobbers dealers, service station operators, and consumers -are excluded. Prior to 1975, stock held at independent bulk terminals were classified as secondary stocks.

Petroleum products supplied: For each petroleum product, the amount supplied is calculated by summing production, crude oil burned directly, imports, and net withdrawals from primary stocks and subtracting exports.

Processing Gain - The amount by which the total volume of refinery output is greater than the volume of input for given period of time. The processing gain arises when crude oil and other hydrocarbons are processed into products that are, on average, less dense than the input.

Processing Loss - The amount by which the total volume of refinery output is less than the volume of input for given period of time. The processing loss arises when crude oil and other hydrocarbons are processed into products that are, on average, more dense than the input. 
Proved Reserves of Crude Oil - The estimated quantities of all liquids defined as crude oil, which geological and engineering data demonstrate with reasonable certainty to be recoverable in future years from known reservoirs under existing economic and operating conditions.

Quad - Quadrillion, 10². In this publication, a Quad refers to Quadrillion Btu.

\section{Rail -}

Amtrak (American Railroad Tracks): Operated by the National Railroad Passenger Corporation of Washington, DC. This rail system was created by President Nixon in 1970, and was given the responsibility for the operation of intercity, as distinct from suburban, passenger trains between points designated by the Secretary of Transportation.

Class I freight railroad: Defined by the Interstate Commerce Commission each year based on annual operating revenue. A railroad is dropped from the Class I list if it fails to meet the annual earnings threshold for three consecutive years.

Commuter railroad: Those portions of mainline railroad (not electric railway) transportation operations which encompass urban passenger train service for local travel between a central city and adjacent suburbs. Commuter railroad service - using both locomotive-hauled and self-propelled railroad passenger cars - is characterized by multi-trip tickets, specific station-to-station fares, and usually only one or two stations in the central business district. Also known as suburban railroad.

Transit railroad: Includes "heavy" and "light" transit rail. Heavy transit rail is characterized by exclusive rights-of-way, multi-car trains, high speed rapid acceleration, sophisticated signaling, and high platform loading. Also known as subway, elevated railway, or metropolitan railway (metro). Light transit rail may be on exclusive or shared rights-of-way, high or low platform loading, multi-car trains or single cars, automated or manually operated. In generic usage, light rail includes streetcars, trolley cars, and tramways.

Residential sector - An energy consuming sector that consists of living quarters for private households. Excludes institutional living quarters.

Residential Transportation Energy Consumption Survey (RTECS) - This survey was designed by the Energy Information Administration of the Department of Energy to provide information on how energy is used by households for personal vehicles. It has been conducted five times since 1979, the most recent being 1991. 
Residual fuel oil - The heavier oils that remain after the distillate fuel oils and lighter hydrocarbons are boiled off in refinery operations. Included are products know as ASTM grade numbers 5 and 6 oil, heavy diesel oil, Navy Special Fuel Oil, Bunker C oil, and acid sludge and pitch used as refinery fuels. Residual fuel oil is used for the production of electric power, for heating, and for various industrial purposes.

Rural - Usually refers to areas with population less than 5,000.

Sales period - October 1 of the previous year to September 30 of the given year. Approximately the same as a model year.

Sales-weighted miles per gallon (mpg) - Calculation of a composite vehicle fuel economy based on the distribution of vehicle sales.

Scrappage rate - As applied to motor vehicles, it is usually expressed as the percentage of vehicles of a certain type in a given age class that are retired from use (lacking registration) in a given year.

School and other nonrevenue bus - See Bus.

Single-unit truck - Includes two-axle, four-tire trucks and other single-unit trucks.

Two-axle, four-tire truck: A motor vehicle consisting primarily of a single motorized device with two axles and four tires.

Other single-unit truck: A motor vehicle consisting primarily of a single motorized device with more than two axles or more than four tires.

Special fuels - Consist primarily of diesel fuel with small amount of liquified petroleum gas, as defined by the Federal Highway Administration.

Specific acceleration power - Measured in watts per kilogram. Acceleration power divided by the battery system weight. Weight must include the total battery system.

Specific energy - Measured in watt hours per kilogram. The rated energy capacity of the battery divided by the total battery system weight.

Subcompact car - See Automobile size classifications.

Supplemental air carrier - See Air carrier. 
Test weight - The weight setting at which a vehicle is tested on a dynomometer by the U.S. Environmental Protection Agency (EPA). This weight is determined by the EPA using the inertia weight of the vehicle.

Ton-mile - The movement of one ton of freight the distance of one mile. Ton-miles are computed by multiplying the weight in tons of each shipment transported by the distance hauled.

\section{Transmission types -}

A3 - Automatic three speed

A4 - Automatic four speed

A5 - Automatic five speed

L4 - Automatic lockup four speed

M5 - Manual five speed

Transit bus - See Bus.

Transit railroad - See Rail.

Transportation sector - Consists of both private and public passenger and freight transportation, as well as government transportation, including military operations.

Truck Inventory and Use Survey (TIUS) - Survey designed to collect data on the characteristics and operational use of the nation's truck population. It is conducted every five years by the U.S. Bureau of the Census. Surveys were conducted in 1963, 1967, 1972, 1977, 1982, 1987, and 1992. For the 1997 survey, it was renamed the Vehicle Inventory and Use Survey in anticipation of including additional vehicle types. However, no additional vehicle types were added to the 1997 survey.

Trolley coach - See Bus.

Truck size classifications - U.S. Bureau of the Census has categorized trucks by gross vehicle weight (gvw) as follows:

Light - Less than 10,000 pounds gvw (Also see Light Truck.)

Medium - 10,001 to 20,000 pounds gvw

Light-heavy - 20,001 to 26,000 pounds gvw

Heavy-heavy - 26,001 pounds gvw or more.

Two-axle, four-tire truck - See Single-unit truck.

Two seater car - See Automobile size classifications. 
Ultra-low emission vehicle - Any vehicle certified to the ultra-low emission standards which are set by the Federal government and/or the state of California.

Urban - Usually refers to areas with population of 5,000 or greater.

Vanpool - A transit mode made up of vans and sometimes small buses operating as a ridesharing arrangement to provide transportation to a group of individuals traveling directly between their homes and a regular destination within the same geographical area. Most vanpools are privately-operated, are not available to the public, and are not considered public transportation. Vanpool data in this report are for vanpools that are owned, purchased or leased by a public entity and are publicly available.

Variable operating cost - See Operating cost.

Vehicle Inventory and Use Survey - See Truck Inventory and Use Survey.

Vehicle-miles traveled (vmt) - One vehicle traveling the distance of one mile. Total vehicle miles, thus, is the total mileage traveled by all vehicles.

Zero-emission vehicle - Any vehicle certified to the zero emission standards which are set by the Federal government and/or the state of California. These standards apply to the vehicle emissions only. 


\section{TITLE INDEX}


Acquisitions

Federal Fleet Vehicle Acquisitions by Fuel Type, FY 1998-2001 . . . . . . . . . . . . . . . . . . . 7-7 Act

Energy Policy Act Purchase Requirements of Light Alternative Fuel Vehicles $\ldots \ldots \ldots \ldots \ldots \ldots \ldots \ldots .7-8$

Advanced

Sales and Specifications of Available Advanced Technology Vehicles $\ldots \ldots \ldots \ldots \ldots \ldots \ldots \ldots .6$ Age

Automobiles in Operation and Vehicle Travel by Age, 1970 and $2001 \ldots \ldots \ldots \ldots \ldots \ldots \ldots \ldots$. . . . . . . . . . . . .

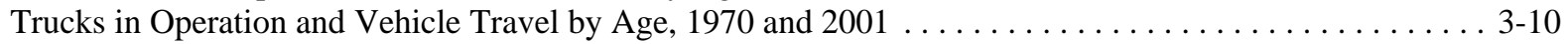

Average Age of Automobiles and Trucks in Use, 1970-2001 . . . . . . . . . . . . . . . . . . . 3-11

Average Age and Registrations of Automobiles and Trucks, $1970-2001$. . . . . . . . . . . . . . . . 3-12

Average Age of Vehicles by Household Vehicle Ownership, 2001 NHTS $\ldots \ldots \ldots \ldots \ldots \ldots \ldots \ldots \ldots . .12$

Average Annual Miles per Household Vehicle by Vehicle Age $\ldots \ldots \ldots \ldots \ldots \ldots \ldots \ldots \ldots \ldots . \ldots .13$ Agency

Federal Government Vehicles by Agency, Fiscal Year $2001 \ldots \ldots \ldots \ldots \ldots \ldots \ldots \ldots \ldots \ldots \ldots \ldots$ Air

Summary Statistics for U.S. Domestic and International Certificated Route Air Carriers (Combined

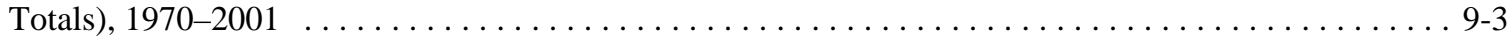

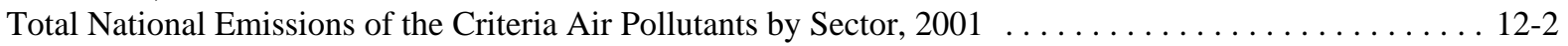
Alternative

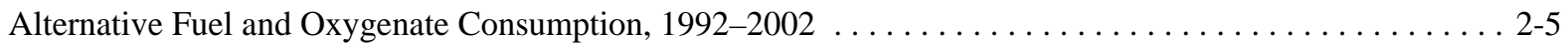

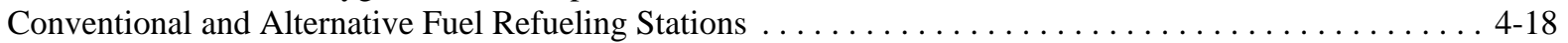

Estimates of Alternative Fuel Vehicles in Use, $1992-2002 \ldots \ldots \ldots \ldots \ldots \ldots \ldots \ldots \ldots \ldots \ldots \ldots \ldots$

Estimates of Alternative Fuel Vehicles by Ownership, 1996 and 2002 . . . . . . . . . . . . . . . . 6-4

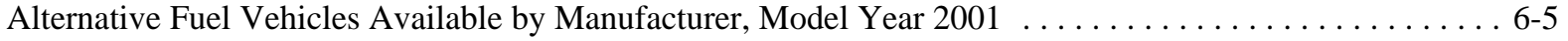

Number of Alternative Refuel Sites by State and Fuel Type, $2002 \ldots \ldots \ldots \ldots \ldots \ldots \ldots \ldots \ldots \ldots .6 \ldots$

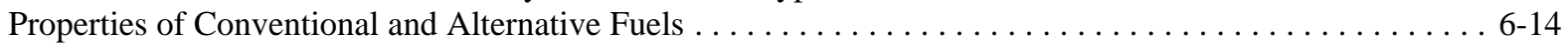

Energy Policy Act Purchase Requirements of Light Alternative Fuel Vehicles . . . . . . . . . . . . . 7-8 Amtrak

Summary Statistics for the National Railroad Passenger Corporation (Amtrak), $1971 \ldots \ldots \ldots \ldots \ldots . .99$ Annual

Average Annual Vehicle-Miles of Travel for Fleet Vehicles, $2000 \ldots \ldots \ldots \ldots \ldots \ldots \ldots \ldots \ldots \ldots .74$

Average Annual Expenditures of Households by Income, $2001 \ldots \ldots \ldots \ldots \ldots \ldots \ldots \ldots \ldots \ldots .4$

Average Annual Vehicle-Miles, Vehicle Trips and Trip Length per Household 1969, 1977, 1983, 1990, 1995 NPTS and 2001 NHTS . . . . . . . . . . . . . . . . . . . . . . . . . . . . . . 8 8 .

Average Annual Person-Miles Traveled, Person Trips and Trip Length per Household by Selected Trip

Purposes, 1983, 1990, 1995 NPTS and 2001 NHTS . . . . . . . . . . . . . . . . . . 8 8 8

Average Annual Miles per Vehicle by Household Vehicle Ownership, 2001 NHTS . . . . . . . . . . 8 8-12

Average Annual Miles per Household Vehicle by Vehicle Age ........................ 8-13

Self-Reported vs. Odometer Average Annual Miles, 1995 NPTS $\ldots \ldots \ldots \ldots \ldots \ldots \ldots \ldots \ldots \ldots . . .14$ Automobile

Automobile Registrations for Selected Countries, 1950-2001 . . . . . . . . . . . . . . . . . . . 3-2

Automobile Scrappage and Survival Rates 1970, 1980 and 1990 Model Years . . . . . . . . . . . . . . 3-13

New Retail Automobile Sales in the United States, 1970-2002 . . . . . . . . . . . . . . . . . . 4-5

Average Material Consumption for a Domestic Automobile, 1978, 1985, and 2001 . . . . . . . . . . 4-16

Automobile Corporate Average Fuel Economy (CAFE) Standards versus Sales-Weighted

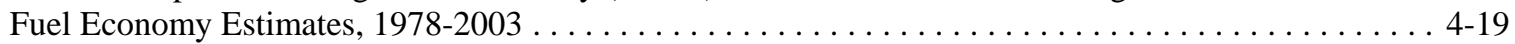

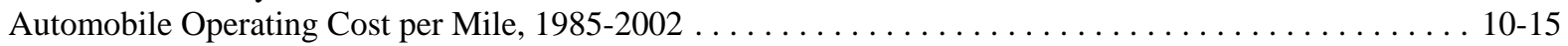

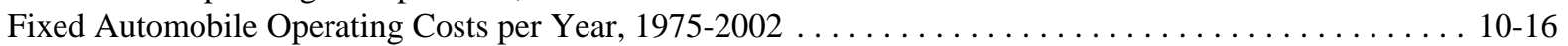

Automobiles

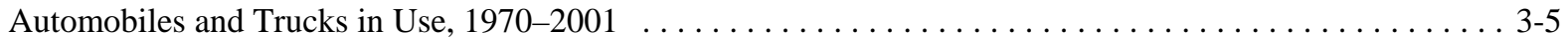

Automobiles in Operation and Vehicle Travel by Age, 1970 and $2001 \ldots \ldots \ldots \ldots \ldots \ldots \ldots \ldots$. . . . . . . . . . . . .

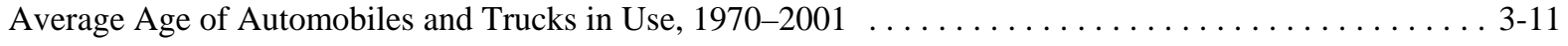


Automobiles (continued)

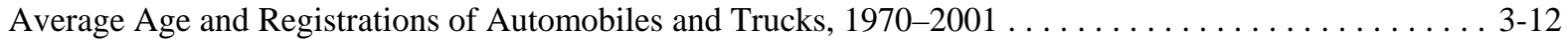

Import Automobiles, Selected Sales Periods 1976-2002 . . . . . . . . . . . . . . . . . . . . 4-7

Sales-Weighted Engine Size of New Domestic and Import Automobiles by Size Class, Sales Periods

$1976-2002 \ldots \ldots \ldots \ldots \ldots \ldots \ldots \ldots \ldots \ldots \ldots \ldots \ldots \ldots \ldots \ldots \ldots \ldots \ldots \ldots \ldots \ldots \ldots \ldots$

Sales-Weighted Curb Weight of New Domestic and Import Automobiles by Size Class, Sales Periods

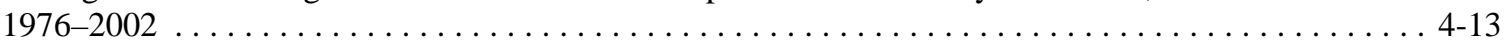

Sales-Weighted Interior Space of New Domestic and Import Automobiles by Size Class, Sales Periods

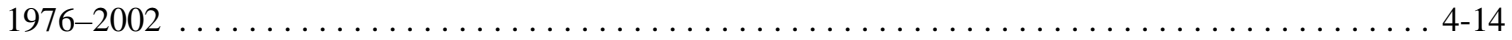

Sales-Weighted Wheelbase of New Automobiles and Light Trucks, Sales Periods 1976-2002 . . . . . . . . 4-15

Period Sales, Market Shares, and Sales-Weighted Fuel Economies of New Domestic and Available

Alternative Fuel Vehicles Available by Manufacturer, Model Year $2001 \ldots \ldots \ldots \ldots \ldots \ldots$

Sales and Specifications of Available Advanced Technology Vehicles . . . . . . . . . . . . . . 6-8 Average

Average Age of Automobiles and Trucks in Use, 1970-2001 . . . . . . . . . . . . . . . . . 3-11

Average Age and Registrations of Automobiles and Trucks, $1970-2001 \ldots \ldots \ldots \ldots$. . . . . . . . . . 3

Average Material Consumption for a Domestic Automobile, 1978, 1985, and $2001 \ldots \ldots$. . . . . . . 4-16

Automobile Corporate Average Fuel Economy (CAFE) Standards versus Sales-Weighted

Fuel Economy Estimates, $1978-2003 \ldots \ldots \ldots \ldots \ldots \ldots \ldots \ldots \ldots \ldots \ldots \ldots \ldots \ldots$

Light Truck Corporate Average Fuel Economy (CAFE) Standards versus Sales-Weighted

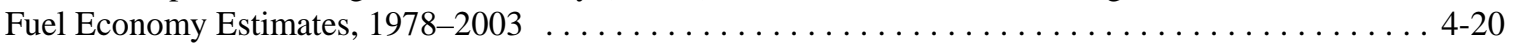

Corporate Average Fuel Economy (CAFE) Fines Collected, 1983-2001 . . . . . . . . . . . . . . . 4-21

Average Length of Time Business Fleet Vehicles are in Service, $2001 \ldots \ldots \ldots \ldots \ldots$

Average Annual Vehicle-Miles of Travel for Fleet Vehicles, $2000 \ldots \ldots \ldots \ldots \ldots \ldots \ldots$

Average Miles per Domestic Federal Vehicle by Vehicle Type, $2001 \ldots \ldots \ldots \ldots \ldots \ldots$

Average Annual Expenditures of Households by Income, $2001 \ldots \ldots \ldots \ldots$. . . . . . . . . . . . . . 8-4

Average Annual Vehicle-Miles, Vehicle Trips and Trip Length per Household 1969, 1977,

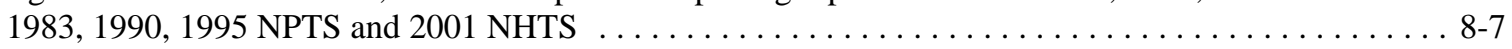

Average Annual Person-Miles Traveled, Person Trips and Trip Length per Household by Selected Trip

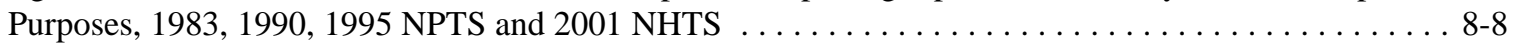

Average Number of Vehicles and Vehicle Travel per Household, 1990 NPTS and

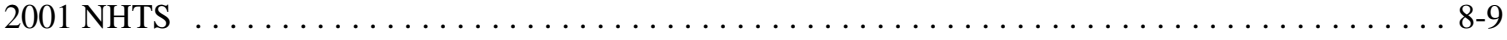

Average Vehicle Occupancy by Vehicle Type, 1995 NPTS and 2001 NHTS . . . . . . . . . . . . . 8-10

Average Vehicle Occupancy by Trip Purpose, 1977 NPTS and 2001 NHTS . . . . . . . . . . . . . . . 8-11

Average Annual Miles per Vehicle by Household Vehicle Ownership, 2001 NHTS . . . . . . . . . . . . 8-12

Average Age of Vehicles by Household Vehicle Ownership, 2001 NHTS . . . . . . . . . . . . . 8-12

Average Annual Miles per Household Vehicle by Vehicle Age . . . . . . . . . . . . . . . . . . . 8-13

Self-Reported vs. Odometer Average Annual Miles, 1995 NPTS . . . . . . . . . . . . . . . . . 8 8

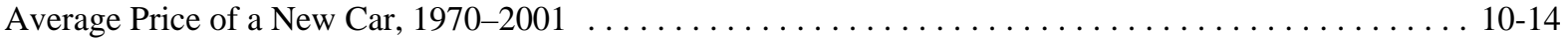
Aviation

Summary Statistics for General Aviation, $1970-2001 \ldots \ldots \ldots \ldots \ldots \ldots$

Refiner Sales Prices for Aviation Gasoline and Jet Fuel, 1978-2002 . . . . . . . . . . . . . . . . . . . . 10-9 Axle

Summary Statistics for Two-Axle, Four-Tire Trucks, $1970-2001 \ldots \ldots \ldots \ldots$ Barrel

Refinery Yield of Petroleum Products from a Barrel of Crude Oil, 1978-2002 . . . . . . . . . . . . . 1-15

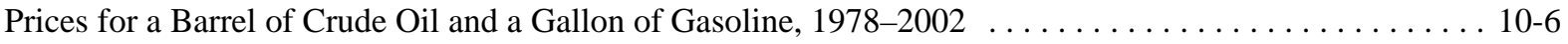
Bicycle

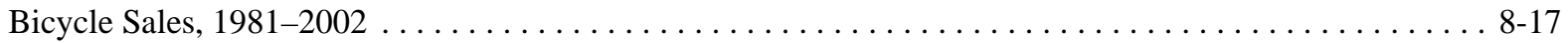

Bike

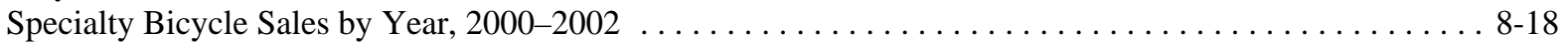
Boating

Walk and Bike Trips by Trip Purpose, 2001 NHTS . . . . . . . . . . . . . . . . . . . . . . 8-19

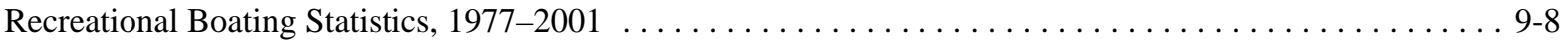


Breakdown

Breakdown of Domestic Marine Cargo by Commodity Class, 2001 . . . . . . . . . . . . . . . . . . . 9-7 Bus

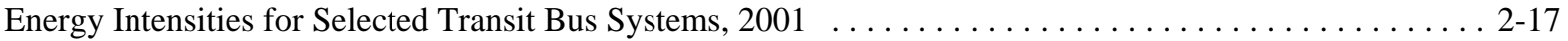

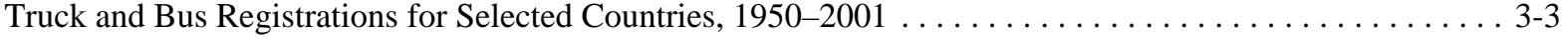
Buses

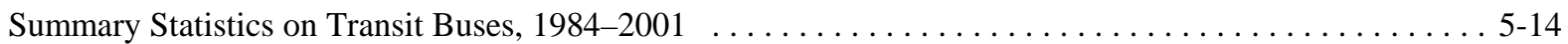

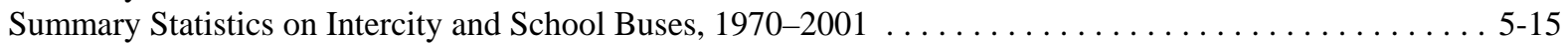

Business

CAFE

Average Length of Time Business Fleet Vehicles are in Service, 2001 ..................... 7-4

Automobile Corporate Average Fuel Economy (CAFE) Standards versus Sales-Weighted Fuel

Economy Estimates, 1978-2003 . . . . . . . . . . . . . . . . . . . . . . . . . . . . 4-19

Light Truck Corporate Average Fuel Economy (CAFE) Standards versus Sales-Weighted Fuel

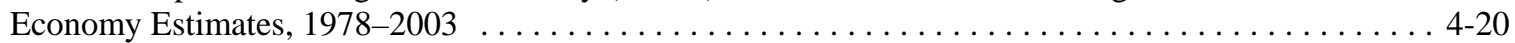

Corporate Average Fuel Economy (CAFE) Fines Collected, $1983-2001 \ldots \ldots \ldots \ldots \ldots \ldots \ldots \ldots \ldots . .4 .21$

Calendar

Vehicle Stock and New Sales in the United States, 2001 Calendar Year ..................... 3-7

California

California Passenger Cars and Light Trucks Emission Certification Standards for Model

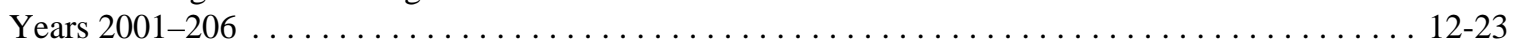

Car

California Vehicle Emission Reduction for Passenger Cars and Light Trucks . . . . . . . . . . . . 12-24

Carbon

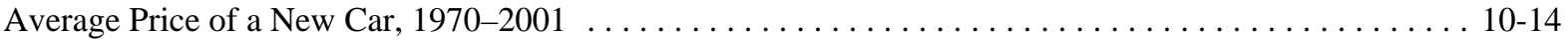

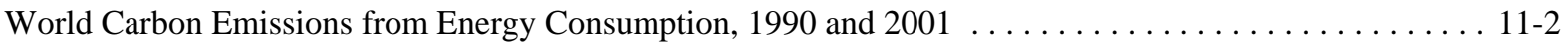

Numerical Estimates of Global Warming Potentials Compared with Carbon Dioxide . . . . . . . . . . 11-3

U.S. Carbon Dioxide Emissions from Fossil Energy Consumption by End-Use Sector, 1990-2001 . . . . . . 11-5

U.S. Carbon Dioxide Emissions from Energy Use in Transportation Sector, 1990-2001 . . . . . . . . 11-6

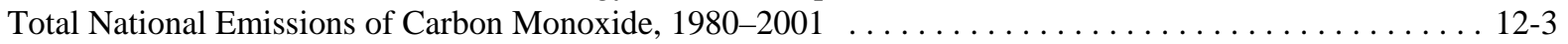

Cargo

Emissions of Carbon Monoxide from Highway Vehicles, $1980-2001 \ldots \ldots \ldots \ldots \ldots \ldots \ldots \ldots \ldots \ldots \ldots \ldots$

Carloads

Breakdown of Domestic Marine Cargo by Commodity Class, $2001 \ldots \ldots \ldots \ldots \ldots \ldots \ldots \ldots$

Railroad Revenue Carloads by Commodity Group, 1974 and 2001 . . . . . . . . . . . . . . . . . . . . 9 9-11

Carriers

Summary Statistics for U.S. Domestic and International Certificated Route Air Carriers (Combined

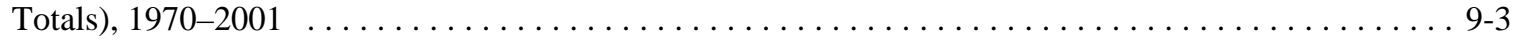

Cars

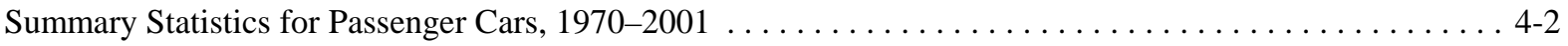

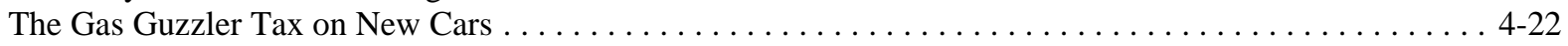

Tier 2 Emission Standards for Cars and Light Trucks Effective for 2004-2009 Model Years . . . . . . . . . 12-14

California Passenger Cars and Light Trucks Emission Certification Standards for Model Years

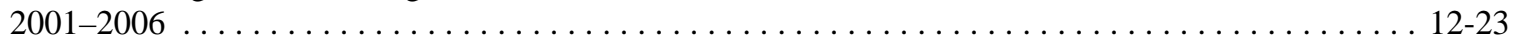

California Vehicle Emission Reduction for Passenger Cars and Light Trucks . . . . . . . . . . . . . 12-24

Category

U.S. and World Hydrogen Consumption by End-Use Category, 1999 . . . . . . . . . . . . . . . . 6-12

Federal Exhaust Emission Certification Standards for Gasoline- and Diesel-Powered Light Trucks

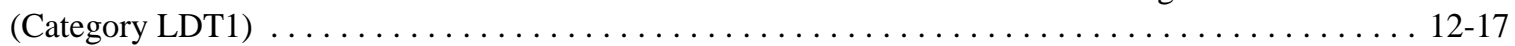

Federal Exhaust Emission Certification Standards for Gasoline- and Diesel-Powered Light Trucks

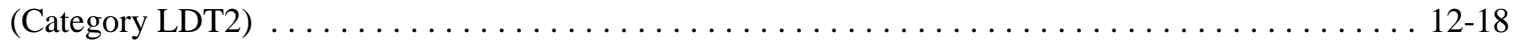

Category (continued)

Federal Exhaust Emission Certification Standards for Gasoline- and Diesel-Powered Light Trucks 
(Category LDT3) . . . . . . . . . . . . . . . . . . . . . . . . . . . . . . . . . . . . . . . . . 12-19

Federal Exhaust Emission Certification Standards for Gasoline- and Diesel-Powered Light Trucks

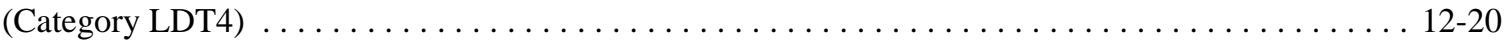

Cell

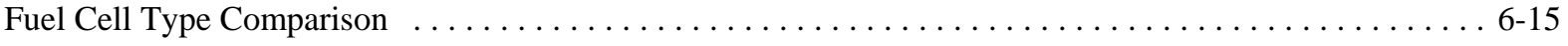

Census

Household Vehicle Ownership, 1960-2000 Census . . . . . . . . . . . . . . . . . . . . . 8-5

Means of Transportation to Work, 1980, 1990 and 2000 Census . . . . . . . . . . . . . . . . 8-15

Workers by Commute Time, 1990 and 2000 Census $\ldots \ldots \ldots \ldots \ldots \ldots \ldots$

Certificated

Summary Statistics for U.S. Domestic and International Certificated Route Air Carriers (Combined

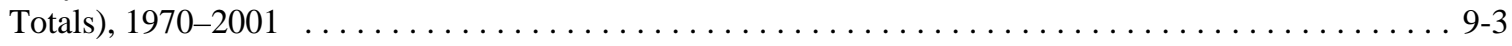

Certification

Federal Exhaust Emission Certification Standards for Gasoline- and Diesel-Powered Light Vehicles . . . 12-16

Federal Exhaust Emission Certification Standards for Gasoline- and Diesel-Powered Light Trucks

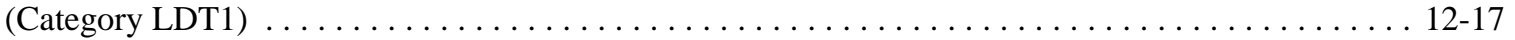

Federal Exhaust Emission Certification Standards for Gasoline- and Diesel-Powered Light Trucks

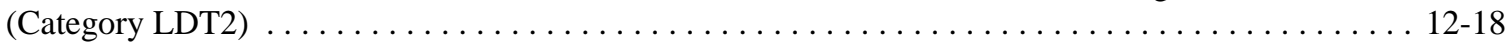

Federal Exhaust Emission Certification Standards for Gasoline- and Diesel-Powered Light Trucks (Category LDT3) . . . . . . . . . . . . . . . . . . . . . . . . . . . . . . . . . . . . . . . 12-19

Federal Exhaust Emission Certification Standards for Gasoline- and Diesel-Powered Light Trucks

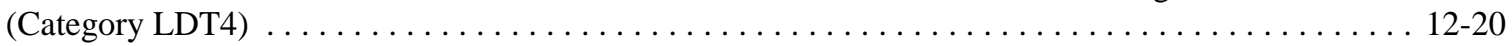

Federal Exhaust Emission Certification Standards for Gasoline- and Diesel-Powered Light Heavy Trucks . . . . . . . . . . . . . . . . . . . . . . . . . . . . 12-21

Federal Exhaust Emission Certification Standards for Gasoline- and Diesel-Powered Heavy Heavy Trucks . . . . . . . . . . . . . . . . . . . . . . . . . . . . 12-22

California Passenger Cars and Light Trucks Emission Certification Standards for Model Years

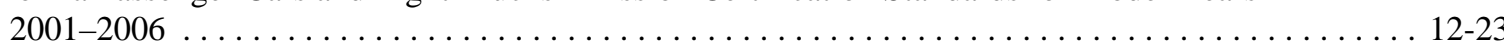

Cities

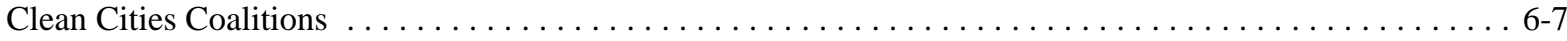

City Class

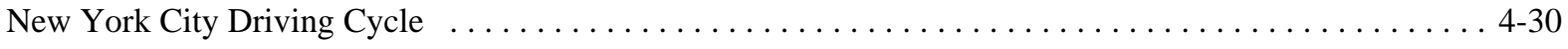

Summary Statistics on Class 1 , Class $2 \mathrm{a}$, and Class $2 \mathrm{~b}$ Light Trucks . . . . . . . . . . . . . . . . . . . .

Sales Estimates of Class 1, Class 2a, and Class 2b Light Trucks, 1989-1999 . . . . . . . . . . . . . . . . 4 4-4

Light Vehicle Market Shares by Size Class, Sales Periods 1976-2002 . . . . . . . . . . . . . . . . . . 4-9

Sales-Weighted Engine Size of New Domestic and Import Automobiles by Size Class, Sales

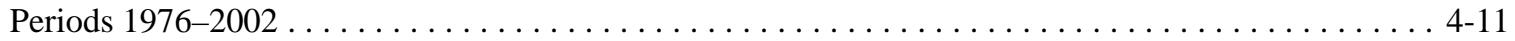

Sales-Weighted Engine Size of New Domestic and Import Light Trucks by Size Class, Sales

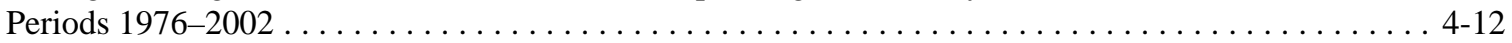

Sales-Weighted Curb Weight of New Domestic and Import Automobiles by Size Class, Sales

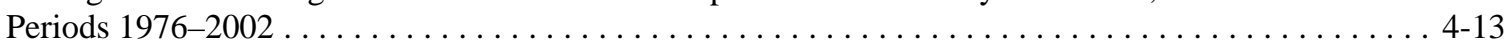

Sales-Weighted Interior Space of New Domestic and Import Automobiles by Size Class, Sales

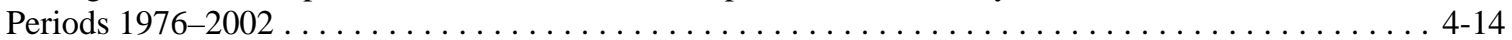

Truck Statistics by Gross Vehicle Weight Class, $1997 \ldots \ldots \ldots \ldots \ldots \ldots \ldots \ldots \ldots$

Truck Harmonic Mean Fuel Economy by Size Class, 1992 and $1997 \ldots \ldots \ldots \ldots$

Breakdown of Domestic Marine Cargo by Commodity Class, $2001 \ldots \ldots \ldots$. . . . . . . . . . . . . 9-7

Class I Railroad Freight Systems in the United States Ranked by Revenue Ton Miles, 2001 . . . . . . . . 9-9

Summary Statistics for Class I Freight Railroads, $1970-2001$. . . . . . . . . . . . . . . . . . . . . . 9-10 Clean

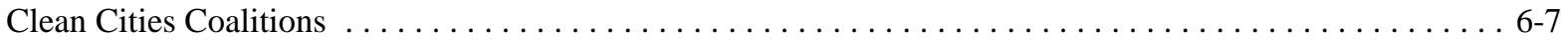

Coalitions

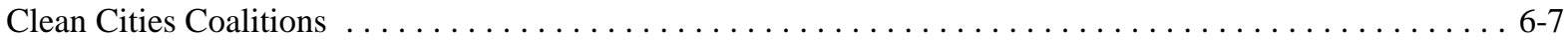

Collected 
Corporate Average Fuel Economy (CAFE) Fines Collected, 1983-2001 . . . . . . . . . . . . . . . . 4 4-21

Commerce

Tonnage Statistics for Domestic and International Waterborne Commerce, 1970-2001 . . . . . . . . . . 9-5

Summary Statistics for Domestic Waterborne Commerce, 1970-2001 . . . . . . . . . . . . . . . . . . 9-6

Commodity

Growth of Freight in the United States: Comparison of the 1997 and 1993 Commodity Flow Surveys . . . . 5-12

Growth of Freight Miles in the United States: Comparison of the 1997 and 1993 Commodity

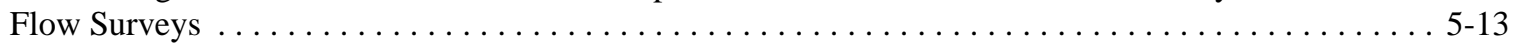

Breakdown of Domestic Marine Cargo by Commodity Class, $2001 \ldots \ldots \ldots \ldots \ldots \ldots \ldots \ldots$. . . . . . . . . . .

Commute

Railroad Revenue Carloads by Commodity Group, 1974 and 2001 . . . . . . . . . . . . . . . . . . . . . 9-11

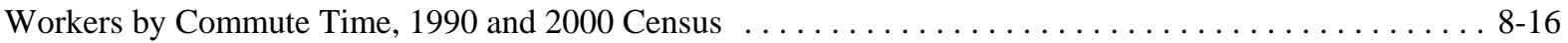

Commuter

Summary Statistics for Commuter Rail Operations, $1984-2001 \ldots \ldots \ldots \ldots \ldots \ldots \ldots \ldots \ldots \ldots \ldots$. . . . . . . . . . . . . .

Compounds

Total National Emissions of Volatile Organic Compounds, $1980-2001 \ldots \ldots \ldots \ldots \ldots \ldots \ldots \ldots \ldots$. . . . . . . . . .

Constant

Emissions of Volatile Organic Compounds from Highway Vehicles, $1980-2001$. . . . . . . . . . . 12-8

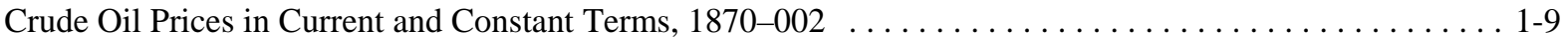

Consumed

Fuel Consumed by Federal Government Fleets, FY 1998-2001 . . . . . . . . . . . . . . . . . . . . . . . . 7-7

Consumer

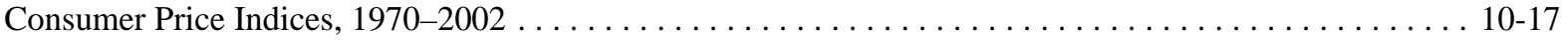

Consumption

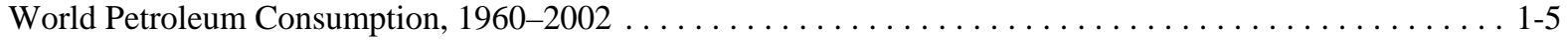

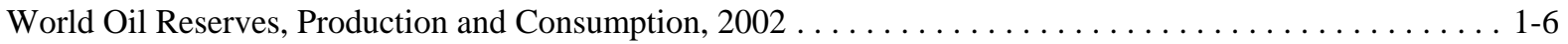

World Natural Gas Reserves, Production, and Consumption, $2000 \ldots \ldots \ldots \ldots \ldots \ldots \ldots \ldots \ldots \ldots \ldots \ldots \ldots$

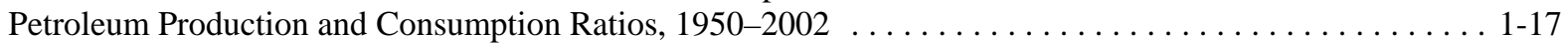

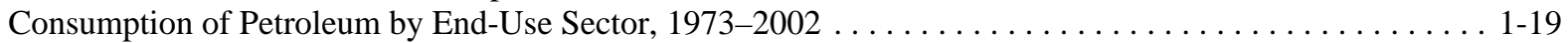

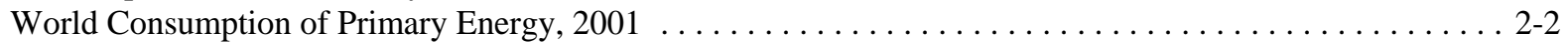

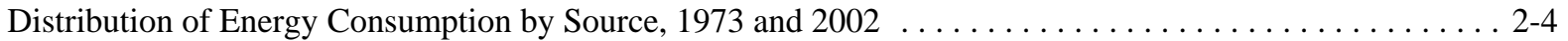

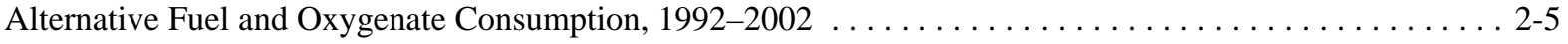

Domestic Consumption of Transportation Energy by Mode and Fuel Type, $2001 \ldots \ldots \ldots \ldots \ldots \ldots \ldots$. . . . . . .

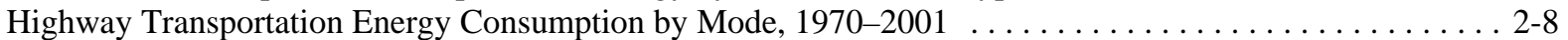

Nonhighway Transportation Energy Consumption by Mode, $1970-2001 \ldots \ldots \ldots \ldots$. . . . . . . . . . . . 2-9

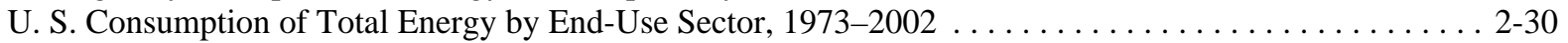

Average Material Consumption for a Domestic Automobile, 1978, 1985, and $2001 \ldots \ldots \ldots \ldots \ldots$. . . . 16

U.S. and World Hydrogen Consumption by End-Use Category, 1999 . . . . . . . . . . . . . . . . . 6-12

U.S. Carbon Dioxide Emissions from Fossil Energy Consumption by End-Use Sector, 1990-2001 . . . . . 11-5

Conventional

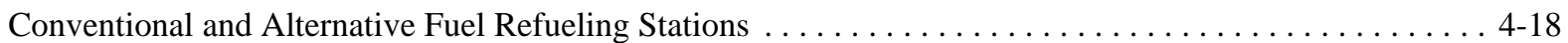

Properties of Conventional and Alternative Fuels . . . . . . . . . . . . . . . . . . . . . . . . 6 6-14

Corporate

Automobile Corporate Average Fuel Economy (CAFE) Standards versus Sales-Weighted Fuel

Economy Estimates, 1978-2003 . . . . . . . . . . . . . . . . . . . . . . . . . . . . . . . 4-19

Light Truck Corporate Average Fuel Economy (CAFE) Standards versus Sales-Weighted Fuel

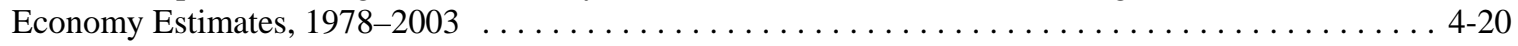

Corporate Average Fuel Economy (CAFE) Fines Collected, $1983-2001 \ldots \ldots \ldots \ldots \ldots \ldots \ldots \ldots \ldots .4 .21$

Corporation

Summary Statistics for the National Railroad Passenger Corporation (Amtrak), $1971 \ldots \ldots \ldots \ldots \ldots . .99-13$ Cost

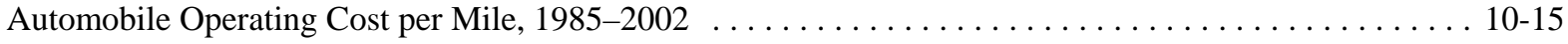

Costs

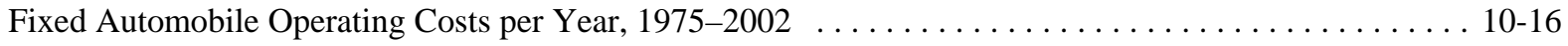


Countries

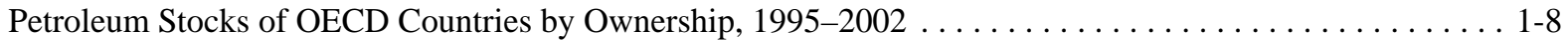

Automobile Registrations for Selected Countries, $1950-2001 \ldots \ldots \ldots \ldots \ldots \ldots$

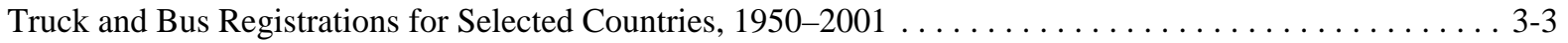

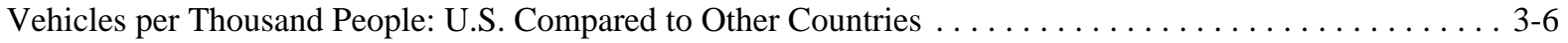

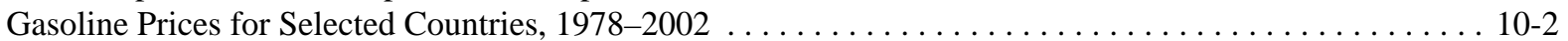

Diesel Fuel Prices for Selected Countries, 1978-2002 . . . . . . . . . . . . . . . . . . . . . . . . . . 10-4 Crash

Crashes by Crash Severity, Crash Type, and Vehicle Type, $2001 \ldots \ldots \ldots \ldots$ Crashes

Crashes by Crash Severity, Crash Type, and Vehicle Type, $2001 \ldots \ldots \ldots \ldots \ldots \ldots$

Percent Rollover Occurrence in Fatal Crashes by Vehicle Type, $2001 \ldots \ldots \ldots \ldots \ldots$. . . . . . . . . . . 4 37 Criteria

Total National Emissions of the Criteria Air Pollutants by Sector, $2001 \ldots \ldots \ldots$. . . . . . . . . . 12-2

Crude

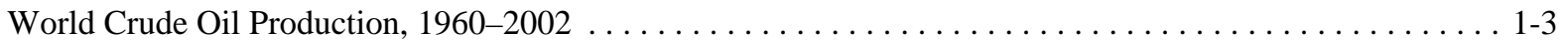

Crude Oil Prices in Current and Constant Terms, $1870-2002 \ldots \ldots \ldots \ldots \ldots \ldots$

U.S. Refinery Input of Crude Oil and Petroleum Products, $1987-2002 \ldots \ldots \ldots \ldots \ldots$

Refinery Yield of Petroleum Products from a Barrel of Crude Oil, 1978-2002 . . . . . . . . . . . . . 1-15

Prices for a Barrel of Crude Oil and a Gallon of Gasoline, 1978-2002 . . . . . . . . . . . . . . . . . . 10-6 Curb

Sales-Weighted Curb Weight of New Domestic and Import Automobiles by Size Class, Sales

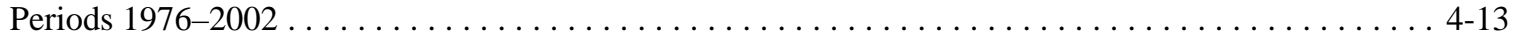

Current Cycles

Crude Oil Prices in Current and Constant Terms, 1870-2002 . . . . . . . . . . . . . . . . . . . . 1-9

Projected Fuel Economies from U.S., European, and Japanese Driving Cycles . . . . . . . . . . . . . 4-32

Comparison of U.S., European, and Japanese Driving Cycles $\ldots \ldots \ldots \ldots \ldots \ldots \ldots$

Dealerships

New Light Vehicle Dealerships and Sales, $1970-2000 \ldots \ldots \ldots \ldots \ldots \ldots \ldots$

Defending

Summary of 1996 Military Expenditures for Defending Oil Supplies from the Middle East . . . . . . . . . 1-12

Demographic

Demographic Statistics, 1969, 1977, 1983, 1990, 1995 NPTS and 2001 NHTS . . . . . . . . . . . . 8-6

Destination

Long-Distance Trips by Destination, $1995 \ldots \ldots \ldots \ldots \ldots \ldots \ldots \ldots$

Diesel

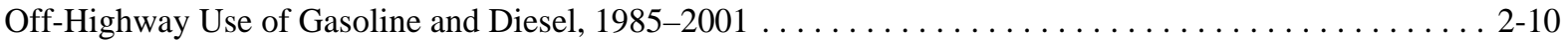

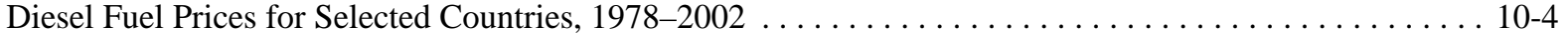

Refiner Sales Prices for Propane and No. 2 Diesel, 1978-2002 . . . . . . . . . . . . . . . . . . . 10-8

Federal Exhaust Emission Certification Standards for Gasoline- and Diesel-Powered Light Vehicles . . . 12-16

Federal Exhaust Emission Certification Standards for Gasoline- and Diesel-Powered Light Trucks

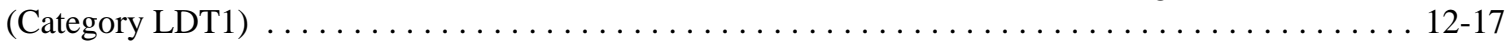

Federal Exhaust Emission Certification Standards for Gasoline- and Diesel-Powered Light Trucks

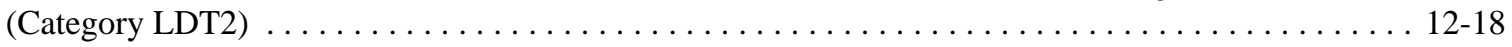

Federal Exhaust Emission Certification Standards for Gasoline- and Diesel-Powered Light Trucks

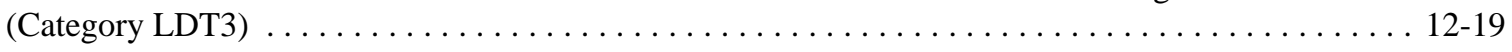

Federal Exhaust Emission Certification Standards for Gasoline- and Diesel-Powered Light Trucks

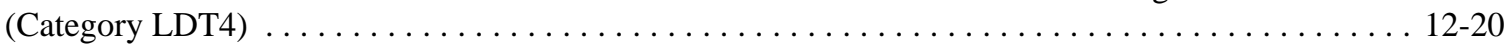

Diesel (continued)

Federal Exhaust Emission Certification Standards for Gasoline- and Diesel-Powered Light

Heavy Trucks . . . . . . . . . . . . . . . . . . . . . . . . . . . . . 12-21

Federal Exhaust Emission Certification Standards for Gasoline- and Diesel-Powered Heavy 
Dioxide

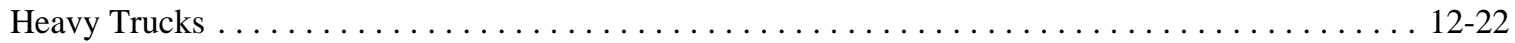

Numerical Estimates of Global Warming Potentials Compared with Carbon Dioxide . . . . . . . . . . . 11-3

U.S. Carbon Dioxide Emissions from Fossil Energy Consumption by End-Use Sector, 1990-2001 . . . . . 11-5

U.S. Carbon Dioxide Emissions from Energy Use in Transportation Sector, 1990-2001 . . . . . . . . 11-6 Distance

Long-Distance Trips by Destination, $1995 \ldots \ldots \ldots \ldots \ldots \ldots \ldots \ldots \ldots \ldots$

Long-Distance Trips by Mode and Purpose, $1995 \ldots \ldots \ldots \ldots \ldots \ldots \ldots \ldots \ldots$

Shares of Long-Distance Person Trips by Mode and Household Income, $1995 \ldots \ldots \ldots$. . . . . . . . . 8 22

Distribution

Distribution of Energy Consumption by Source, 1973 and $2002 \ldots \ldots \ldots \ldots \ldots$

Domestic

Domestic Consumption of Transportation Energy by Mode and Fuel Type, $2001 \ldots \ldots \ldots$

Period Sales, Market Shares, and Sales-Weighted Fuel Economies of New Domestic and Import

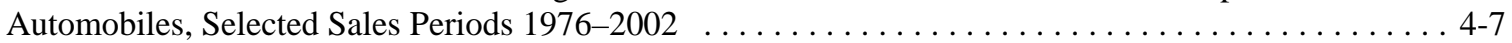

Period Sales, Market Shares, and Sales-Weighted Fuel Economies of New Domestic and Import Light Trucks, Selected Sales Periods $1976-2002 \ldots \ldots \ldots \ldots \ldots \ldots \ldots \ldots \ldots \ldots \ldots \ldots \ldots \ldots \ldots$

Sales-Weighted Engine Size of New Domestic and Import Automobiles by Size Class, Sales

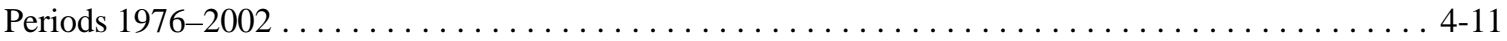

Sales-Weighted Engine Size of New Domestic and Import Light Trucks by Size Class, Sales

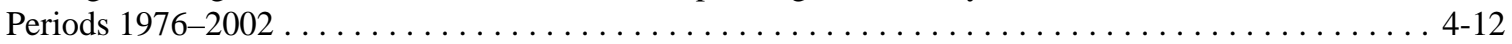

Sales-Weighted Curb Weight of New Domestic and Import Automobiles by Size Class, Sales

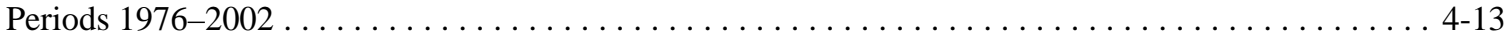

Sales-Weighted Interior Space of New Domestic and Import Automobiles by Size Class, Sales

Periods $1976-2002 \ldots \ldots \ldots \ldots \ldots \ldots \ldots \ldots \ldots \ldots \ldots \ldots \ldots \ldots \ldots \ldots \ldots \ldots \ldots$

Average Material Consumption for a Domestic Automobile, 1978, 1985, and $2001 \ldots \ldots \ldots$

Average Miles per Domestic Federal Vehicle by Vehicle Type, $2001 \ldots \ldots \ldots$. . . . . . . . . . . . . . . 7-5

Summary Statistics for U.S. Domestic and International Certificated Route Air Carriers (Combined

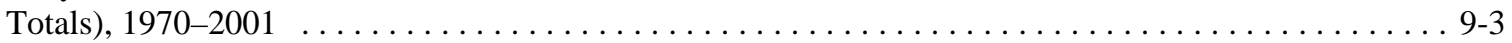

Tonnage Statistics for Domestic and International Waterborne Commerce, 1970-2001 . . . . . . . . . 9-5

Summary Statistics for Domestic Waterborne Commerce, 1970-2001 . . . . . . . . . . . . . . . . 9-6

Driving

Breakdown of Domestic Marine Cargo by Commodity Class, $2001 \ldots \ldots \ldots \ldots \ldots$

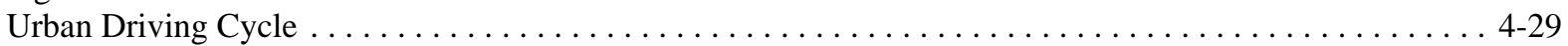

Highway Driving Cycle $\ldots \ldots \ldots \ldots \ldots \ldots \ldots \ldots \ldots \ldots \ldots \ldots \ldots \ldots \ldots \ldots \ldots \ldots$

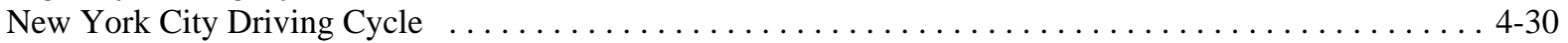

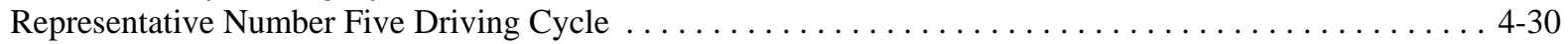

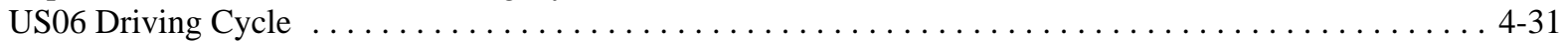

Projected Fuel Economies from U.S., European, and Japanese Driving Cycles . . . . . . . . . . . . . . 4-32

Comparison of U.S., European, and Japanese Driving Cycles $\ldots \ldots \ldots \ldots \ldots \ldots \ldots$ East

Summary of 1996 Military Expenditures for Defending Oil Supplies from the Middle East . . . . . . . . . 1-12 Economic

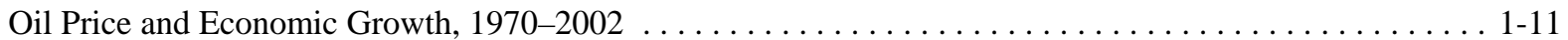

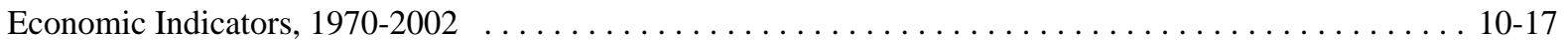
Economies

Period Sales, Market Shares, and Sales-Weighted Fuel Economies of New Domestic and Import

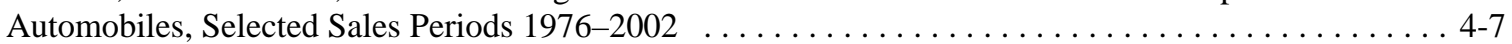

Period Sales, Market Shares, and Sales-Weighted Fuel Economies of New Domestic and Import

Light Trucks, Selected Sales Periods $1976-2002 \ldots \ldots \ldots \ldots \ldots \ldots \ldots \ldots \ldots$

Projected Fuel Economies from U.S., European, and Japanese Driving Cycles . . . . . . . . . . . . . . . 4-32 Economy

Automobile Corporate Average Fuel Economy (CAFE) Standards versus Sales-Weighted Fuel

Economy Estimates, 1978-2003 4-19 
Light Truck Corporate Average Fuel Economy (CAFE) Standards versus Sales-Weighted Fuel

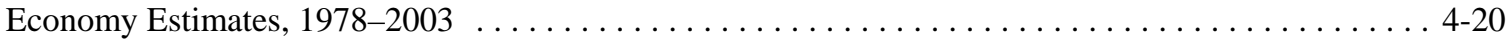

Corporate Average Fuel Economy (CAFE) Fines Collected, 1983-2001 . . . . . . . . . . . . . . . . . 4-21

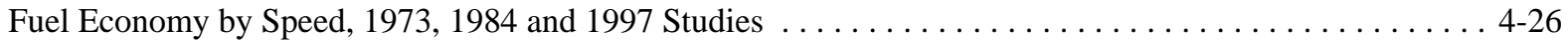

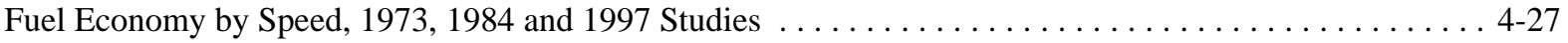

Steady Speed Fuel Economy for Vehicles Tested in the 1997 Study . . . . . . . . . . . . . . . . . 4-28

Truck Harmonic Mean Fuel Economy by Size Class, 1992 and 1997 . . . . . . . . . . . . . . . . . . . . . 5-6 Effective

Tier 2 Emission Standards for Cars and Light Trucks Effective for 2004-2009 Model Years . . . . . . . . 12-14 Emission

Tier 2 Emission Standards for Cars and Light Trucks Effective for 2004-2009 Model Years . . . . . . . . 12-14

Light Vehicle Exhaust Emission Standards in Effect in 2009 when U.S. Tier 2 Standards are Final . . . . 12-15

Federal Exhaust Emission Certification Standards for Gasoline- and Diesel-Powered Light Vehicles . . . 12-16

Federal Exhaust Emission Certification Standards for Gasoline- and Diesel-Powered Light Trucks

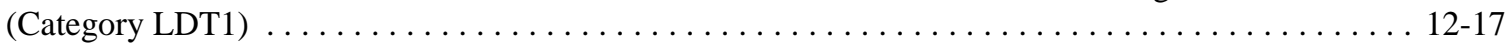

Federal Exhaust Emission Certification Standards for Gasoline- and Diesel-Powered Light Trucks

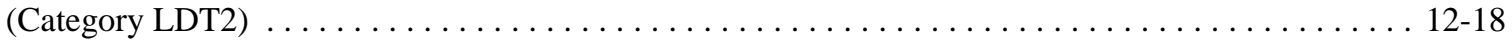

Federal Exhaust Emission Certification Standards for Gasoline- and Diesel-Powered Light Trucks (Category LDT3) . . . . . . . . . . . . . . . . . . . . . . . . . . . . . . . . . . 12-19

Federal Exhaust Emission Certification Standards for Gasoline- and Diesel-Powered Light Trucks

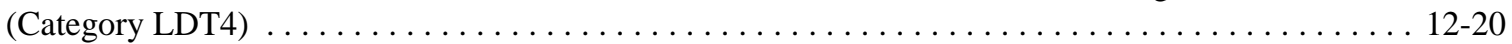

Federal Exhaust Emission Certification Standards for Gasoline- and Diesel-Powered Light Heavy Trucks . . . . . . . . . . . . . . . . . . . . . . . . . . . . 12-21

Federal Exhaust Emission Certification Standards for Gasoline- and Diesel-Powered Heavy Heavy Trucks . . . . . . . . . . . . . . . . . . . . . . . . . . . . . . . . . 12-22

California Passenger Cars and Light Trucks Emission Certification Standards for Model Years

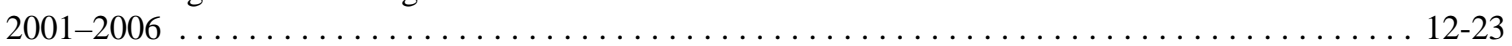

California Vehicle Emission Reduction for Passenger Cars and Light Trucks . . . . . . . . . . . . . . 12-24 Emissions

World Carbon Emissions from Energy Consumption, 1990 and $2001 \ldots \ldots \ldots \ldots$

Estimated U.S. Emissions of Greenhouse Gases, 1990-2001 . . . . . . . . . . . . . . . . . . . . 11-4

U.S. Carbon Dioxide Emissions from Fossil Energy Consumption by End-Use Sector, 1990-2001 . . . . . 11-5

U.S. Carbon Dioxide Emissions from Energy Use in Transportation Sector, 1990-2001 . . . . . . . . . 11-6

Total National Emissions of the Criteria Air Pollutants by Sector, $2001 \ldots \ldots \ldots \ldots \ldots$

Total National Emissions of Carbon Monoxide, $1980-2001 \ldots \ldots \ldots \ldots \ldots$

Emissions of Carbon Monoxide from Highway Vehicles, $1980-2001 \ldots \ldots \ldots \ldots$

Total National Emissions of Nitrogen Oxides, $1980-2001 \ldots \ldots \ldots \ldots \ldots \ldots$

Emissions of Nitrogen Oxides from Highway Vehicles, 1980-2001 . . . . . . . . . . . . . . . . 12-6

Total National Emissions of Volatile Organic Compounds, 1980-2001 . . . . . . . . . . . . . . . . . 12-7

Emissions of Volatile Organic Compounds from Highway Vehicles, 1980-2001 . . . . . . . . . . . . . 12-8

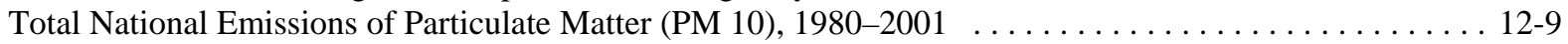

Emissions of Particulate Matter (PM 10) from Highway Vehicles, 1980-2001 . . . . . . . . . . . . . . . 12-10

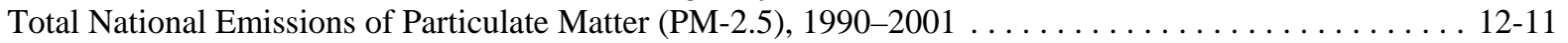

Emissions of Particulate Matter (PM-2.5) from Highway Vehicles, 1990-2001 . . . . . . . . . . . . . 12-12 Employment

Transportation-related Employment, 1993 and $2002 \ldots \ldots \ldots \ldots \ldots \ldots \ldots \ldots$ Energy

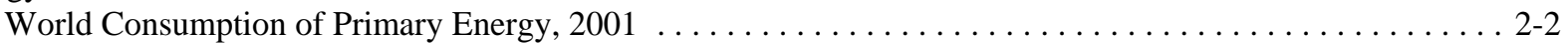

U. S. Consumption of Total Energy by End-Use Sector, $1973-2002 \ldots \ldots \ldots \ldots \ldots \ldots$

Distribution of Energy Consumption by Source, 1973 and $2002 \ldots \ldots \ldots \ldots \ldots$

Domestic Consumption of Transportation Energy by Mode and Fuel Type, $2001 \ldots \ldots \ldots$

Transportation Energy Use by Mode, $2000-2001 \ldots \ldots \ldots \ldots \ldots \ldots \ldots \ldots \ldots$

Highway Transportation Energy Consumption by Mode, $1970-2001 \ldots \ldots \ldots$. . . . . . . . . . . . . 2-8

Nonhighway Transportation Energy Consumption by Mode, $1970-2001 \ldots \ldots$. . . . . . . . . . . . . . 2-9 


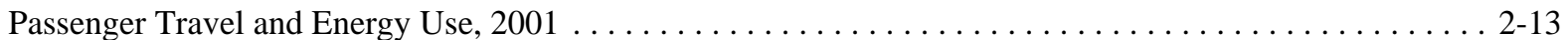

Energy Intensities of Highway Passenger Modes, 1970-2001 . . . . . . . . . . . . . . . . . . . . . . 2-14

Energy Intensities of Nonhighway Passenger Modes, 1970-2001 . . . . . . . . . . . . . . . . . . . . 2-15

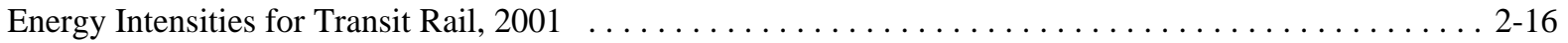

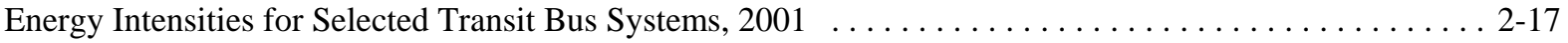

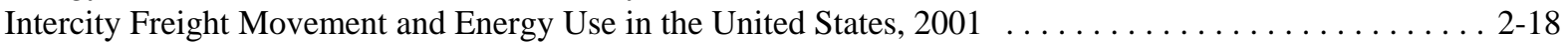

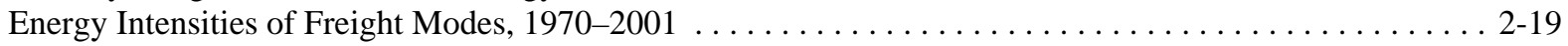

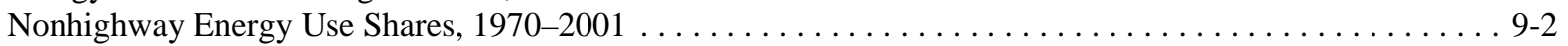

Energy Policy Act Purchase Requirements of Light Alternative Fuel Vehicles . . . . . . . . . . . 7-8

U.S. Carbon Dioxide Emissions from Fossil Energy Consumption by End-Use Sector, 1990-2001 … . . 11-5 Engine

U.S. Carbon Dioxide Emissions from Energy Use in Transportation Sector, 1990-2001 . . . . . . . . . 11-6

Sales-Weighted Engine Size of New Domestic and Import Automobiles by Size Class, Sales Periods

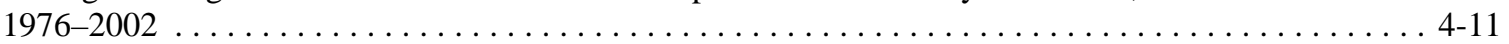

Sales-Weighted Engine Size of New Domestic and Import Light Trucks by Size Class, Sales Periods

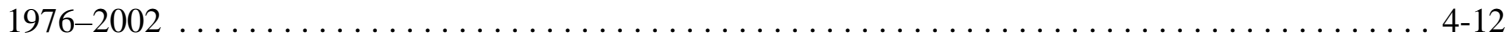

Estimated

Estimated U.S. Emissions of Greenhouse Gases, 1990-2001 . . . . . . . . . . . . . . . . . . 11-4 Estimates

Sales Estimates of Class 1, Class 2a, and Class 2b Light Trucks, 1989-1999 . . . . . . . . . . . . . . 4-4

Automobile Corporate Average Fuel Economy (CAFE) Standards versus Sales-Weighted Fuel

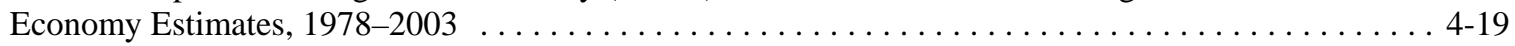

Light Truck Corporate Average Fuel Economy (CAFE) Standards versus Sales-Weighted Fuel

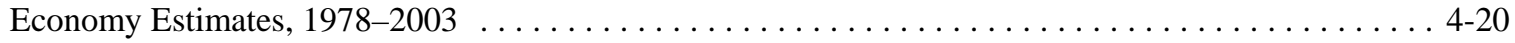

Estimates of Alternative Fuel Vehicles in Use, $1992-2002 \ldots \ldots \ldots \ldots \ldots \ldots \ldots \ldots \ldots \ldots \ldots .6 \ldots$

Estimates of Alternative Fuel Vehicles by Ownership, 1996 and 2002 . . . . . . . . . . . . . . . 6-4

Numerical Estimates of Global Warming Potentials Compared with Carbon Dioxide $\ldots \ldots \ldots \ldots \ldots \ldots$ 11-3 Ethanol

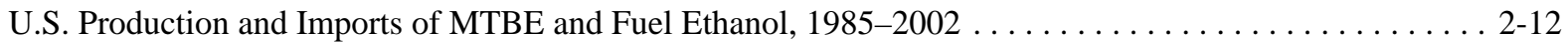

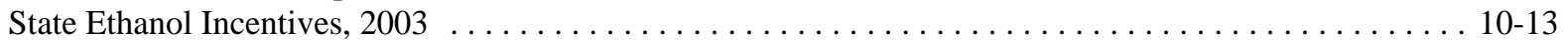

European

Projected Fuel Economies from U.S., European, and Japanese Driving Cycles . . . . . . . . . . . . 4-32 Excise

Comparison of U.S., European, and Japanese Driving Cycles $\ldots \ldots \ldots \ldots \ldots \ldots \ldots \ldots \ldots \ldots .4 . \ldots \ldots$

Federal Excise Taxes on Motor Fuels . . . . . . . . . . . . . . . . . . . . . . . . . . . . . . . . . . . . 10-12

Exemptions

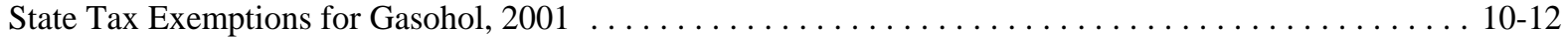

Exhaust

Light Vehicle Exhaust Emission Standards in Effect in 2009 when U.S. Tier 2 Standards are Final . . . . . 12-15

Federal Exhaust Emission Certification Standards for Gasoline- and Diesel-Powered Light Vehicles . . . 12-16

Federal Exhaust Emission Certification Standards for Gasoline- and Diesel-Powered Light Trucks

(Category LDT1) . . . . . . . . . . . . . . . . . . . . . . . . . . . . . . . . . . . 12-17

Exhaust (continued)

Federal Exhaust Emission Certification Standards for Gasoline- and Diesel-Powered Light Trucks

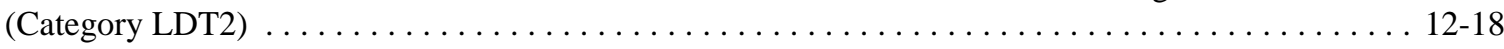

Federal Exhaust Emission Certification Standards for Gasoline- and Diesel-Powered Light Trucks (Category LDT3) . . . . . . . . . . . . . . . . . . . . . . . . . . . . . . . . . 12-19

Federal Exhaust Emission Certification Standards for Gasoline- and Diesel-Powered Light Trucks

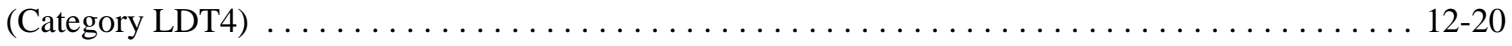

Federal Exhaust Emission Certification Standards for Gasoline- and Diesel-Powered Light

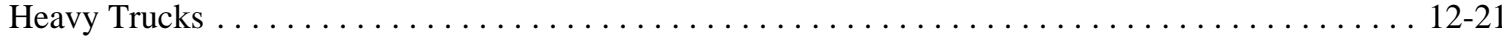

Federal Exhaust Emission Certification Standards for Gasoline- and Diesel-Powered Heavy 


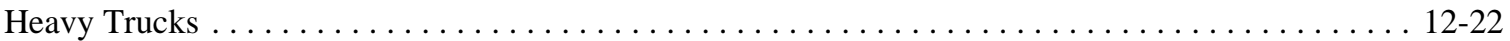

Expenditures

Summary of 1996 Military Expenditures for Defending Oil Supplies from the Middle East . . . . . . . . . 1-12 Exports

Average Annual Expenditures of Households by Income, $2001 \ldots \ldots \ldots \ldots \ldots \ldots \ldots \ldots \ldots \ldots . .4$

United States Petroleum Production, Imports and Exports, 1950-2002 . . . . . . . . . . . . . . 1-16

Facility

Percentage of Trucks by Fleet Size and Primary Fueling Facility, $1997 \ldots \ldots \ldots \ldots \ldots \ldots \ldots \ldots \ldots \ldots . . \ldots \ldots$

Percentage of Trucks by Major Use and Primary Fueling Facility, $1997 \ldots \ldots \ldots \ldots \ldots \ldots \ldots \ldots \ldots$. . . . . . . . . .

Fatal

Percent Rollover Occurrence in Fatal Crashes by Vehicle Type, $2001 \ldots \ldots \ldots \ldots \ldots \ldots \ldots \ldots \ldots \ldots .4-37$

Fatalities

Occupant Fatalities by Vehicle Type and Nonoccupant Fatalities, $1975-2000$. . . . . . . . . . . . 4 4-34

February

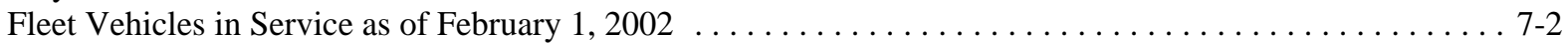

Federal

Average Miles per Domestic Federal Vehicle by Vehicle Type, 2001 . . . . . . . . . . . . . . . . . . 7-5

Federal Government Vehicles by Agency, Fiscal Year $2001 \ldots \ldots \ldots \ldots \ldots \ldots \ldots \ldots \ldots \ldots \ldots \ldots .6 \ldots$

Federal Fleet Vehicle Acquisitions by Fuel Type, FY 1998-2001 . . . . . . . . . . . . . . . . . . . 7-7

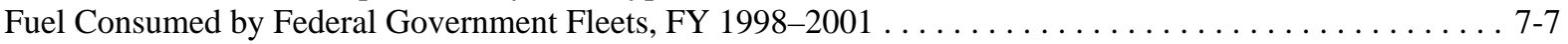

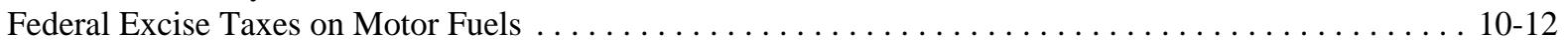

Federal Exhaust Emission Certification Standards for Gasoline- and Diesel-Powered Light Vehicles .. 12-16

Federal Exhaust Emission Certification Standards for Gasoline- and Diesel-Powered Light Trucks

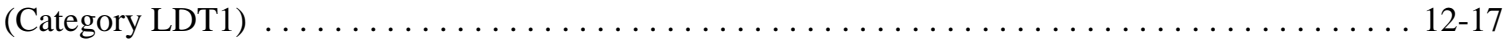

Federal Exhaust Emission Certification Standards for Gasoline- and Diesel-Powered Light Trucks

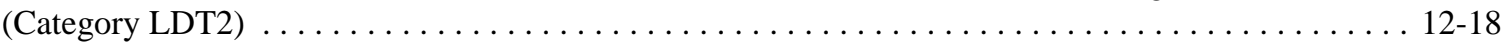

Federal Exhaust Emission Certification Standards for Gasoline- and Diesel-Powered Light Trucks

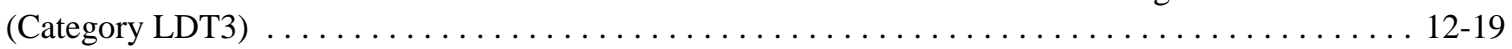

Federal Exhaust Emission Certification Standards for Gasoline- and Diesel-Powered Light Trucks

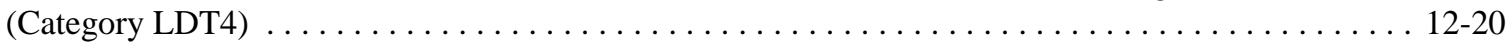

Federal Exhaust Emission Certification Standards for Gasoline- and Diesel-Powered Light

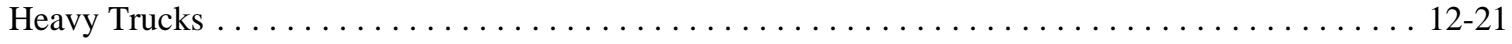

Federal Exhaust Emission Certification Standards for Gasoline- and Diesel-Powered Heavy

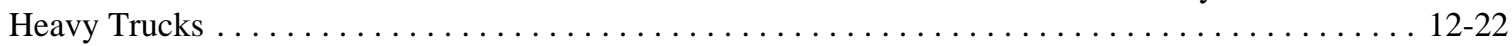

Final

Light Vehicle Exhaust Emission Standards in Effect in 2009 when U.S. Tier 2 Standards are Final . . . . 12-15 Fines

Fiscal

Corporate Average Fuel Economy (CAFE) Fines Collected, 1983-2001 . . . . . . . . . . . . . . . . 4 4-21 Fixed

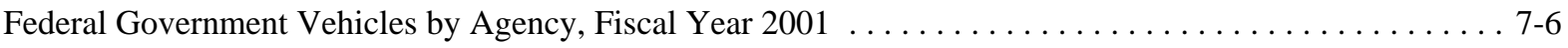
Fleet

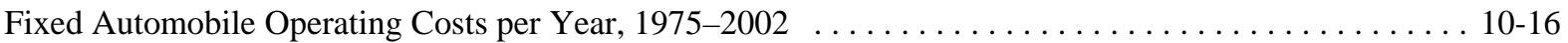

Percentage of Trucks by Fleet Size and Primary Fueling Facility, $1997 \ldots \ldots \ldots \ldots \ldots \ldots \ldots \ldots \ldots \ldots$. . . . . . . . . .

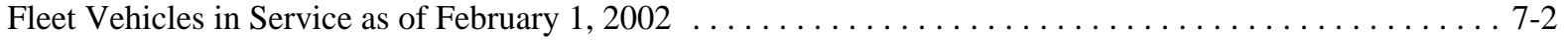

New Light Fleet Vehicle Purchases by Vehicle Type, $2000 \ldots \ldots \ldots \ldots \ldots \ldots \ldots \ldots \ldots \ldots \ldots \ldots .7 . \ldots \ldots$

Average Length of Time Business Fleet Vehicles are in Service, 2001 . . . . . . . . . . . . . . . . . 7-4

Average Annual Vehicle-Miles of Travel for Fleet Vehicles, $2000 \ldots \ldots \ldots \ldots \ldots \ldots \ldots \ldots \ldots \ldots \ldots \ldots$. ${ }^{4}$

Federal Fleet Vehicle Acquisitions by Fuel Type, FY 1998-2001 . . . . . . . . . . . . . . . . . . 7-7 Fleets

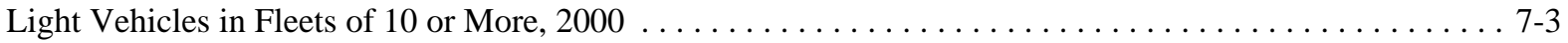
Flow

Fuel Consumed by Federal Government Fleets, FY 1998-2001 . . . . . . . . . . . . . . . . . . . . 7-7

Growth of Freight in the United States: Comparison of the 1997 and 1993 Commodity Flow Surveys . . . . 5-12 
Growth of Freight Miles in the United States: Comparison of the 1997 and 1993 Commodity

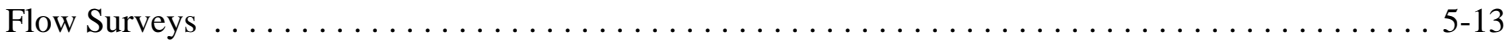

Fossil

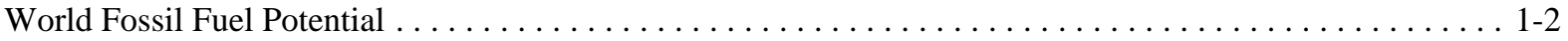

U.S. Carbon Dioxide Emissions from Fossil Energy Consumption by End-Use Sector, 1990-2001 . . . . 11-5 Freight

Intercity Freight Movement and Energy Use in the United States, $2001 \ldots \ldots \ldots$

Energy Intensities of Freight Modes, 1970-2001 . . . . . . . . . . . . . . . . . . . . . . . . . 2-19

Growth of Freight in the United States: Comparison of the 1997 and 1993 Commodity Flow Surveys . . . . 5-12

Growth of Freight Miles in the United States: Comparison of the 1997 and 1993 Commodity

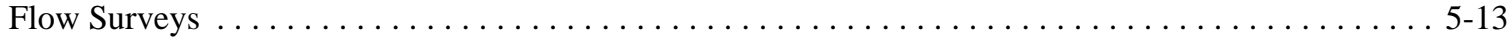

Class I Railroad Freight Systems in the United States Ranked by Revenue Ton Miles, 2001 . . . . . . . . 9-9

Summary Statistics for Class I Freight Railroads, 1970-2001 . . . . . . . . . . . . . . . . . . . . . . 9-10 Fuel

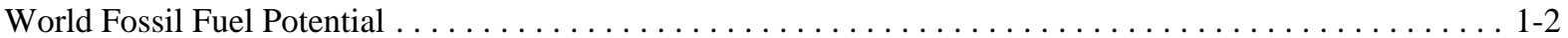

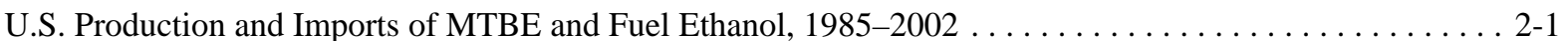

Alternative Fuel and Oxygenate Consumption, $1992-2002 \ldots \ldots \ldots \ldots \ldots \ldots \ldots \ldots$

Domestic Consumption of Transportation Energy by Mode and Fuel Type, 2001 . . . . . . . . . . . . . . 2-6

Period Sales, Market Shares, and Sales-Weighted Fuel Economies of New Domestic and Import

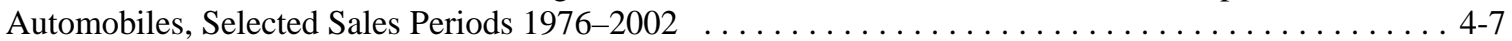

Period Sales, Market Shares, and Sales-Weighted Fuel Economies of New Domestic and Import Light

Trucks, Selected Sales Periods $1976-2002 \ldots \ldots \ldots \ldots \ldots \ldots \ldots \ldots \ldots$

Conventional and Alternative Fuel Refueling Stations . . . . . . . . . . . . . . . . . . 48

Automobile Corporate Average Fuel Economy (CAFE) Standards versus Sales-Weighted Fuel

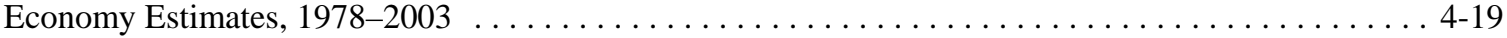

Light Truck Corporate Average Fuel Economy (CAFE) Standards versus Sales-Weighted Fuel

Economy Estimates, $1978-2003 \ldots \ldots \ldots \ldots \ldots \ldots \ldots \ldots \ldots \ldots \ldots \ldots \ldots \ldots \ldots$

Corporate Average Fuel Economy (CAFE) Fines Collected, 1983-2001 . . . . . . . . . . . . . . . . . . 4-21

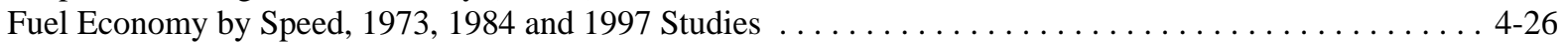

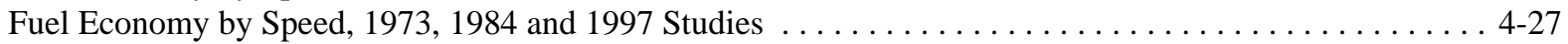

Steady Speed Fuel Economy for Vehicles Tested in the 1997 Study . . . . . . . . . . . . . . . . . . 4-28

Projected Fuel Economies from U.S., European, and Japanese Driving Cycles . . . . . . . . . . . . . . . 4-32

Truck Harmonic Mean Fuel Economy by Size Class, 1992 and $1997 \ldots \ldots \ldots \ldots$

Estimates of Alternative Fuel Vehicles in Use, $1992-2002 \ldots \ldots \ldots \ldots \ldots \ldots \ldots \ldots \ldots \ldots$

Estimates of Alternative Fuel Vehicles by Ownership, 1996 and 2002 . . . . . . . . . . . . . . . . . 6-4

Alternative Fuel Vehicles Available by Manufacturer, Model Year $2001 \ldots \ldots \ldots$

Number of Alternative Refuel Sites by State and Fuel Type, $2002 \ldots \ldots \ldots \ldots \ldots \ldots$

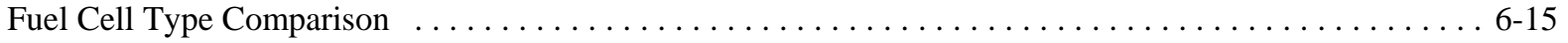

Federal Fleet Vehicle Acquisitions by Fuel Type, FY 1998-2001 . . . . . . . . . . . . . . . . . . . 7-7

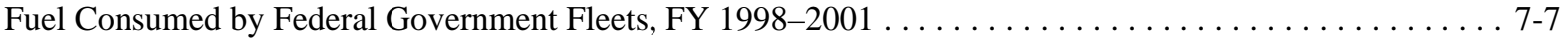

Energy Policy Act Purchase Requirements of Light Alternative Fuel Vehicles . . . . . . . . . . . . 7-8

Fuel (continued)

Diesel Fuel Prices for Selected Countries, $1978-2002 \ldots \ldots \ldots \ldots \ldots \ldots \ldots$

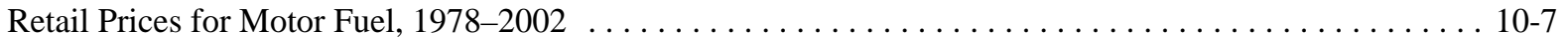

Refiner Sales Prices for Aviation Gasoline and Jet Fuel, 1978-2002 . . . . . . . . . . . . . . . . . 10-9

Fueling

Percentage of Trucks by Fleet Size and Primary Fueling Facility, $1997 \ldots \ldots \ldots \ldots$

Percentage of Trucks by Major Use and Primary Fueling Facility, 1997 . . . . . . . . . . . . . . . . 5-10 Fuels

Highway Usage of Gasoline and Special Fuels, 1973-2001 . . . . . . . . . . . . . . . . . . . . . . 2-11

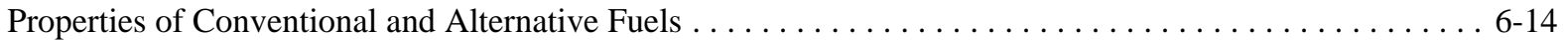

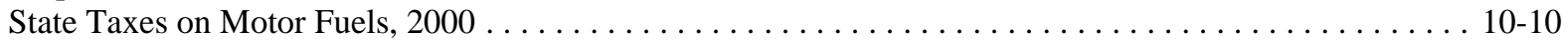

Federal Excise Taxes on Motor Fuels . . . . . . . . . . . . . . . . . . . . . . . . . . . . . 10-12 
FY Gallon

Federal Fleet Vehicle Acquisitions by Fuel Type, FY 1998-2001 . . . . . . . . . . . . . . . . . . . 7-7

Fuel Consumed by Federal Government Fleets, FY 1998-2001 . . . . . . . . . . . . . . . . . . . . 7-7 Gas

Prices for a Barrel of Crude Oil and a Gallon of Gasoline, $1978-2002 \ldots \ldots \ldots \ldots \ldots$

World Natural Gas Reserves, Production, and Consumption, $2000 \ldots \ldots \ldots \ldots \ldots$

The Gas Guzzler Tax on New Cars . . . . . . . . . . . . . . . . . . . . . . . . . . . . . . . 4-22

Tax Receipts from the Sale of Gas Guzzlers, $1980-2001 \ldots \ldots \ldots \ldots \ldots \ldots \ldots$ Gases

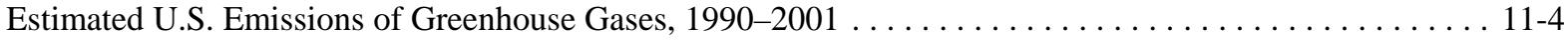

Gasohol

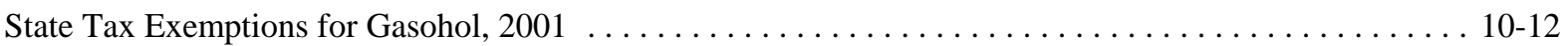

Gasoline

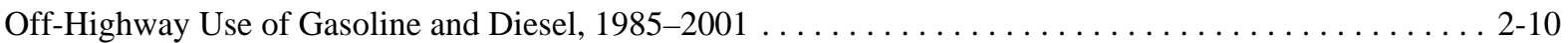

Highway Usage of Gasoline and Special Fuels, $1973-2001 \ldots \ldots \ldots \ldots \ldots \ldots \ldots \ldots \ldots$

Gasoline Prices for Selected Countries, $1978-2002 \ldots \ldots \ldots \ldots \ldots \ldots \ldots \ldots \ldots \ldots$

Prices for a Barrel of Crude Oil and a Gallon of Gasoline, 1978-2002 . . . . . . . . . . . . . . . 10-6

Refiner Sales Prices for Aviation Gasoline and Jet Fuel, 1978-2002 . . . . . . . . . . . . . . . . . 10-9

Federal Exhaust Emission Certification Standards for Gasoline- and Diesel-Powered Light Vehicles . . . 12-16

Federal Exhaust Emission Certification Standards for Gasoline- and Diesel-Powered Light Trucks

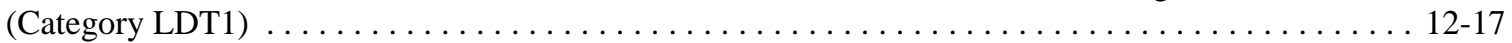

Federal Exhaust Emission Certification Standards for Gasoline- and Diesel-Powered Light Trucks

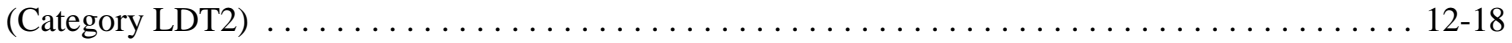

Federal Exhaust Emission Certification Standards for Gasoline- and Diesel-Powered Light Trucks

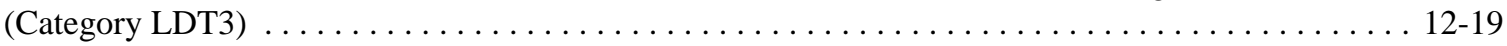

Federal Exhaust Emission Certification Standards for Gasoline- and Diesel-Powered Light Trucks

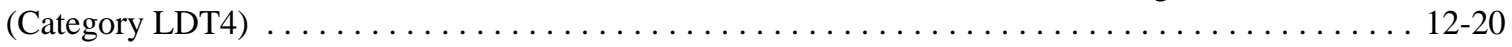

Federal Exhaust Emission Certification Standards for Gasoline- and Diesel-Powered Light Heavy Trucks . . . . . . . . . . . . . . . . . . . . . . . . . . . . 12-21

Federal Exhaust Emission Certification Standards for Gasoline- and Diesel-Powered Heavy

Global Heavy Trucks . . . . . . . . . . . . . . . . . . . . . . . . . . . . 12-22

Numerical Estimates of Global Warming Potentials Compared with Carbon Dioxide . . . . . . . . . . 11-3 Government

Federal Government Vehicles by Agency, Fiscal Year $2001 \ldots \ldots \ldots \ldots \ldots \ldots$

Fuel Consumed by Federal Government Fleets, FY 1998-2001 . . . . . . . . . . . . . . . . . . . . 7-7

Greenhouse Gross

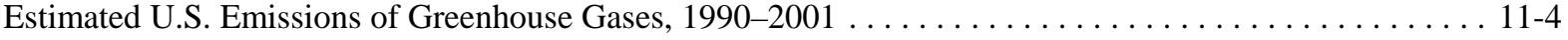

Refinery Gross Output by World Region, $2002 \ldots \ldots \ldots \ldots \ldots \ldots \ldots \ldots \ldots$

New Retail Truck Sales by Gross Vehicle Weight, 1970-2002 . . . . . . . . . . . . . . . . . . . . . . . . 5-4

Truck Statistics by Gross Vehicle Weight Class, $1997 \ldots \ldots \ldots \ldots \ldots \ldots \ldots$ Growth

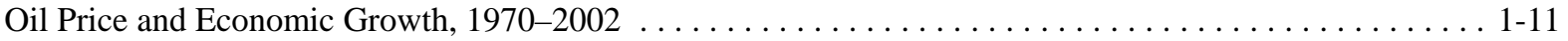

Growth of Freight in the United States: Comparison of the 1997 and 1993 Commodity Flow Surveys . . . . 5-12

Growth of Freight Miles in the United States: Comparison of the 1997 and 1993 Commodity Guzzler(s)

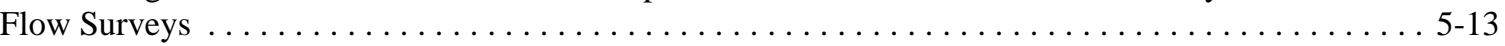

The Gas Guzzler Tax on New Cars . . . . . . . . . . . . . . . . . . . . . . . . . 4 -22

Tax Receipts from the Sale of Gas Guzzlers, $1980-2001 \ldots \ldots \ldots \ldots \ldots \ldots \ldots \ldots \ldots$ GVW

New Retail Sales of Trucks 10,000 pounds GVW and Less in the United States, 1970-2002 . . . . . . . . 4-6 Harmonic 
Truck Harmonic Mean Fuel Economy by Size Class, 1992 and 1997 . . . . . . . . . . . . . . . . . 5-6 Heavy

Heavy Truck Scrappage and Survival Rates . ............................... 3-17

Federal Exhaust Emission Certification Standards for Gasoline- and Diesel-Powered Light

Heavy Trucks ........................................... 12-21

Federal Exhaust Emission Certification Standards for Gasoline- and Diesel-Powered Heavy

Heavy Trucks . . . . . . . . . . . . . . . . . . . . . . . . . . . . . . . . . . . . 12-22

Highway

Highway Transportation Energy Consumption by Mode, $1970-2001 \ldots \ldots \ldots \ldots \ldots \ldots \ldots \ldots \ldots \ldots$

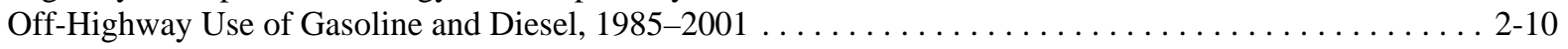

Highway Usage of Gasoline and Special Fuels, 1973-2001 . . . . . . . . . . . . . . . . . . . . . . 2-11

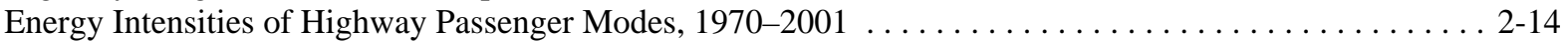

Shares of Highway Vehicle-Miles Traveled by Vehicle Type, $1970-2001 \ldots \ldots \ldots \ldots \ldots \ldots$. . . . . . . . . 3-8

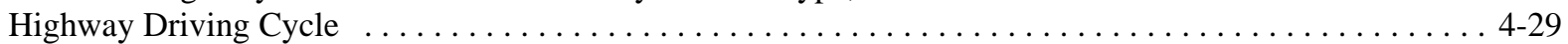

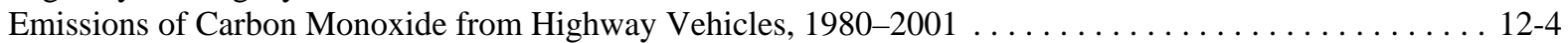

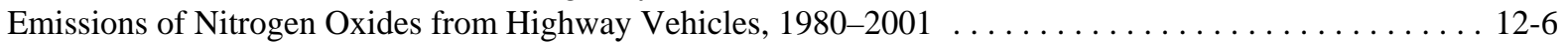

Emissions of Volatile Organic Compounds from Highway Vehicles, $1980-2001$. . . . . . . . . . . . . 12-8

Emissions of Particulate Matter (PM 10) from Highway Vehicles, 1980-2001 . . . . . . . . . . . . . . 12-10

Emissions of Particulate Matter (PM-2.5) from Highway Vehicles, 1990-2001 . . . . . . . . . . . . . 12-12

Household

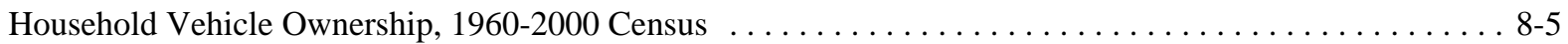

Average Annual Vehicle-Miles, Vehicle Trips and Trip Length per Household 1969, 1977, 1983, 1990,

1995 NPTS and 2001 NHTS ....................................... 8 . . .

Average Annual Person-Miles Traveled, Person Trips and Trip Length per Household by Selected Trip

Purposes, 1983, 1990, 1995 NPTS and 2001 NHTS ............................ 8-8

Average Number of Vehicles and Vehicle Travel per Household, 1990 NPTS and 2001 NHTS . . . . . . . 8 8-9

Average Annual Miles per Vehicle by Household Vehicle Ownership, 2001 NHTS . . . . . . . . . . . . 8-12

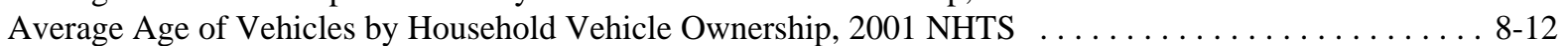

Average Annual Miles per Household Vehicle by Vehicle Age . . . . . . . . . . . . . . . . 8 8-13

Shares of Long-Distance Person Trips by Mode and Household Income, 1995 . . . . . . . . . . . . . . 8 8-22

Households

Average Annual Expenditures of Households by Income, $2001 \ldots \ldots \ldots \ldots \ldots \ldots \ldots \ldots \ldots \ldots .4$

Hydrogen

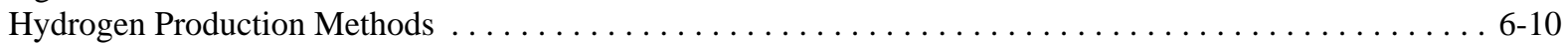

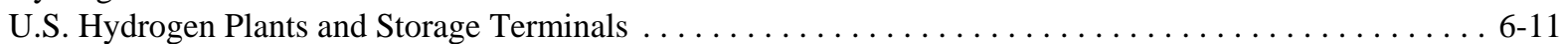

U.S. and World Hydrogen Consumption by End-Use Category, $1999 \ldots \ldots \ldots \ldots \ldots \ldots \ldots \ldots \ldots \ldots \ldots$. . . . . . . . . . . . . . Import

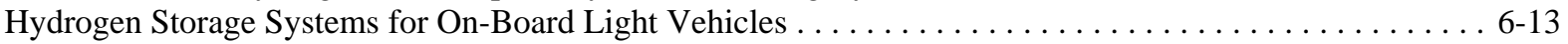

Period Sales, Market Shares, and Sales-Weighted Fuel Economies of New Domestic and Import Automobiles, Selected Sales Periods $1976-2002$. . . . . . . . . . . . . . . . . . . . . . . . 4 4

Period Sales, Market Shares, and Sales-Weighted Fuel Economies of New Domestic and Import

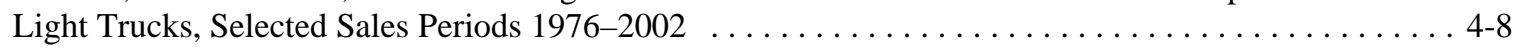

Sales-Weighted Engine Size of New Domestic and Import Automobiles by Size Class, Sales Periods

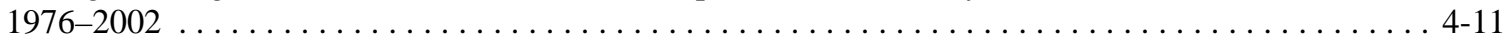

Sales-Weighted Engine Size of New Domestic and Import Light Trucks by Size Class, Sales Periods

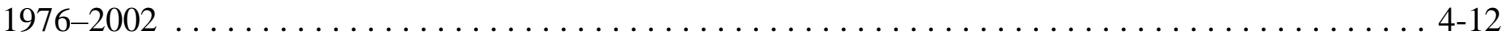

Sales-Weighted Curb Weight of New Domestic and Import Automobiles by Size Class, Sales Periods

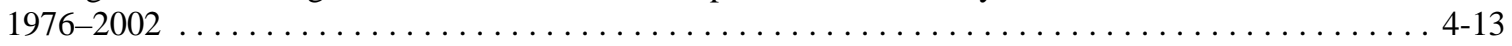

Sales-Weighted Interior Space of New Domestic and Import Automobiles by Size Class, Sales Periods

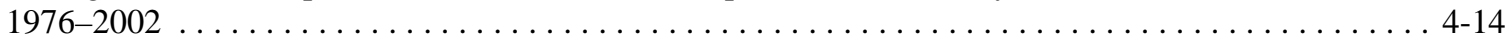

Imports

U.S. Petroleum Imports by World Region of Origin, 1960-2002 . . . . . . . . . . . . . . . . . 1-10

United States Petroleum Production, Imports and Exports, 1950-2002 . . . . . . . . . . . . . . . . 1-16

U.S. Production and Imports of MTBE and Fuel Ethanol, 1985-2002 . . . . . . . . . . . . . . . . 2-12 
Incentives

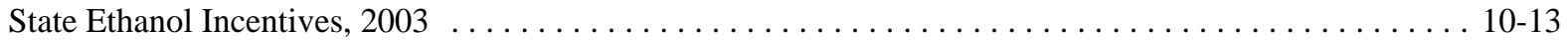

Income

Average Annual Expenditures of Households by Income, $2001 \ldots \ldots \ldots \ldots \ldots \ldots \ldots$

Shares of Long-Distance Person Trips by Mode and Household Income, $1995 \ldots \ldots \ldots$. . . . . . . . . . 8-22 Indicators

Economic Indicators $1970-2002 \ldots \ldots \ldots \ldots \ldots \ldots \ldots \ldots \ldots \ldots \ldots$

Indices

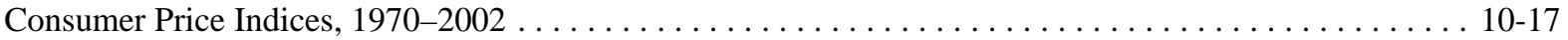
Input

U.S. Refinery Input of Crude Oil and Petroleum Products, $1987-2002 \ldots \ldots \ldots \ldots \ldots$ Intensities

Energy Intensities of Highway Passenger Modes, 1970-2001 . . . . . . . . . . . . . . . . . . . . . 2-14

Energy Intensities of Nonhighway Passenger Modes, 1970-2001 . . . . . . . . . . . . . . . . . 2-15

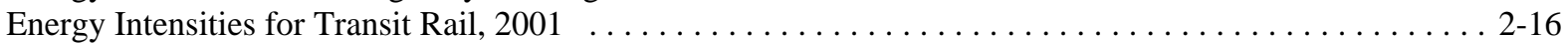

Energy Intensities for Selected Transit Bus Systems, $2001 \ldots \ldots \ldots \ldots \ldots \ldots \ldots$

Energy Intensities of Freight Modes, $1970-2001 \ldots \ldots \ldots \ldots \ldots \ldots \ldots \ldots$

Intercity

Intercity Freight Movement and Energy Use in the United States, $2001 \ldots \ldots \ldots \ldots$

Summary Statistics on Intercity and School Buses, 1970-2001 . . . . . . . . . . . . . . . . . . . . 5-15 Interior

Sales-Weighted Interior Space of New Domestic and Import Automobiles by Size Class, Sales Periods

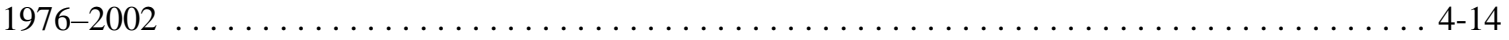

Intermodal

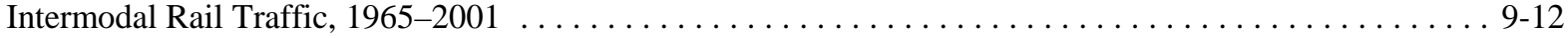

International

Summary Statistics for U.S. Domestic and International Certificated Route Air Carriers (Combined

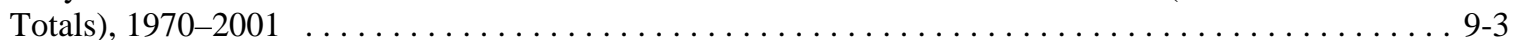

Tonnage Statistics for Domestic and International Waterborne Commerce, 1970-2001 . . . . . . . . . 9-5 Japanese

Projected Fuel Economies from U.S., European, and Japanese Driving Cycles . . . . . . . . . . . . . 4-32

Jet

Comparison of U.S., European, and Japanese Driving Cycles $\ldots \ldots \ldots \ldots \ldots \ldots \ldots \ldots \ldots$

Refiner Sales Prices for Aviation Gasoline and Jet Fuel, 1978-2002 . . . . . . . . . . . . . . . . . . 10-9 LDT1

Federal Exhaust Emission Certification Standards for Gasoline- and Diesel-Powered Light Trucks

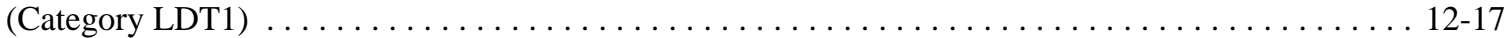

LDT2

Federal Exhaust Emission Certification Standards for Gasoline- and Diesel-Powered Light Trucks

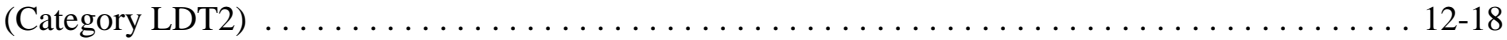

LDT3

Federal Exhaust Emission Certification Standards for Gasoline- and Diesel-Powered Light Trucks

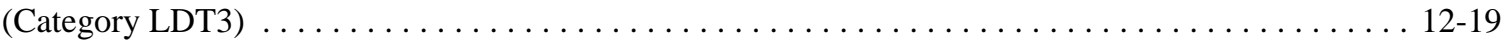

\section{LDT4}

Federal Exhaust Emission Certification Standards for Gasoline- and Diesel-Powered Light Trucks

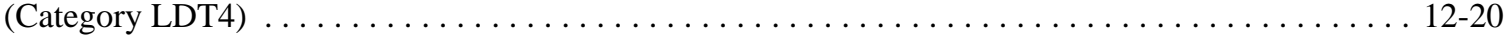

Length

Average Length of Time Business Fleet Vehicles are in Service, 2001 . . . . . . . . . . . . . . . . . 7-4

Average Annual Vehicle-Miles, Vehicle Trips and Trip Length per Household 1969, 1977, 1983, 1990,

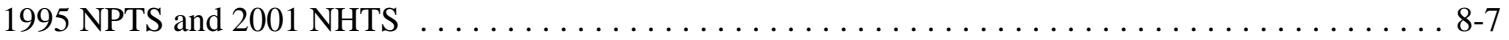

Average Annual Person-Miles Traveled, Person Trips and Trip Length per Household by Selected Trip

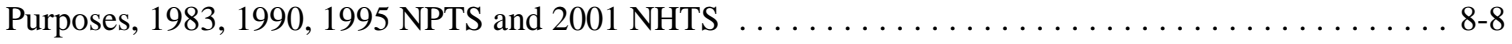

Light

Light Truck Scrappage and Survival Rates 
Summary Statistics on Class 1 , Class $2 \mathrm{a}$, and Class $2 \mathrm{~b}$ Light Trucks $\ldots \ldots \ldots \ldots \ldots \ldots \ldots$

Sales Estimates of Class 1, Class 2a, and Class 2b Light Trucks, 1989-1999 . . . . . . . . . . . . . . . . . 4-4

Period Sales, Market Shares, and Sales-Weighted Fuel Economies of New Domestic and Import Light

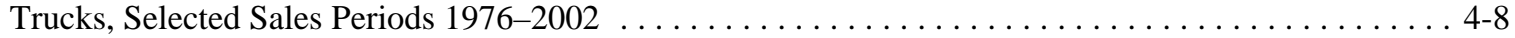

Light Vehicle Market Shares by Size Class, Sales Periods 1976-2002 . . . . . . . . . . . . . . . . . . . 4 4-9

Sales-Weighted Engine Size of New Domestic and Import Light Trucks by Size Class, Sales Periods

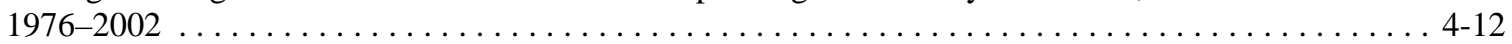

Sales-Weighted Wheelbase of New Automobiles and Light Trucks, Sales Periods 1976-2002 . . . . . . . 4 4-15

New Light Vehicle Dealerships and Sales, $1970-2000 \ldots \ldots \ldots \ldots \ldots \ldots \ldots \ldots$

Light Truck Corporate Average Fuel Economy (CAFE) Standards versus Sales-Weighted Fuel

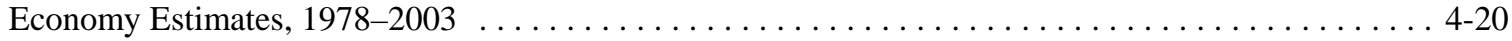

Light Vehicle Occupant Safety Data, $1975-2001 \ldots \ldots \ldots \ldots \ldots \ldots \ldots \ldots \ldots$

Summary Statistics on Light Transit Vehicles, $1994-2001 \ldots \ldots \ldots \ldots \ldots \ldots$

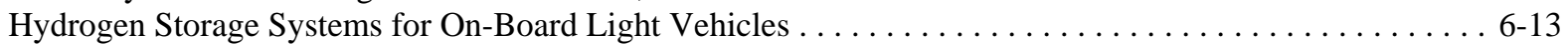

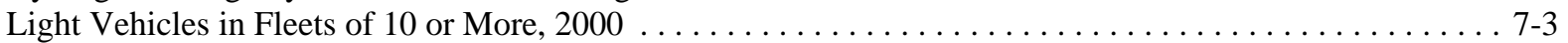

New Light Fleet Vehicle Purchases by Vehicle Type, $2000 \ldots \ldots \ldots \ldots \ldots \ldots \ldots \ldots \ldots$

Energy Policy Act Purchase Requirements of Light Alternative Fuel Vehicles . . . . . . . . . . . . . . 7-8

Tier 2 Emission Standards for Cars and Light Trucks Effective for 2004-2009 Model Years . . . . . . . . 12-14

Light Vehicle Exhaust Emission Standards in Effect in 2009 when U.S. Tier 2 Standards are Final . . . . . 12-15

Federal Exhaust Emission Certification Standards for Gasoline- and Diesel-Powered Light Vehicles . . . 12-16

Federal Exhaust Emission Certification Standards for Gasoline- and Diesel-Powered Light Trucks

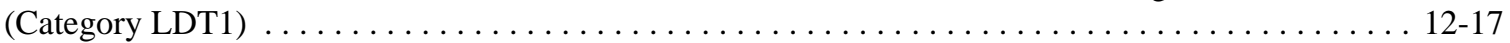

Federal Exhaust Emission Certification Standards for Gasoline- and Diesel-Powered Light Trucks

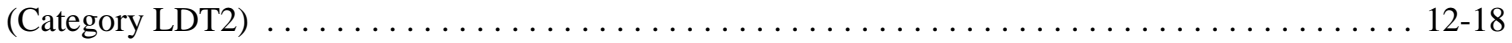

Federal Exhaust Emission Certification Standards for Gasoline- and Diesel-Powered Light Trucks (Category LDT3) . . . . . . . . . . . . . . . . . . . . . . . . . . . . . . . . . . . 12-19

Federal Exhaust Emission Certification Standards for Gasoline- and Diesel-Powered Light Trucks

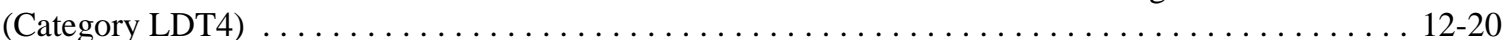

Federal Exhaust Emission Certification Standards for Gasoline- and Diesel-Powered Light

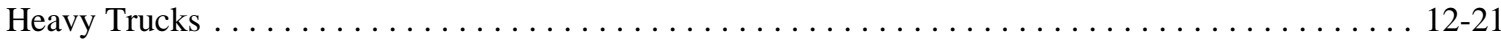

California Passenger Cars and Light Trucks Emission Certification Standards for Model Years

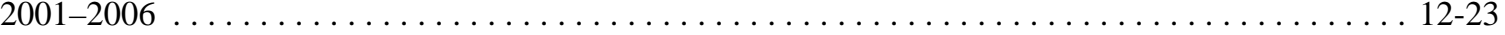

California Vehicle Emission Reduction for Passenger Cars and Light Trucks . . . . . . . . . . . . . . 12-24 Manufacturer

Alternative Fuel Vehicles Available by Manufacturer, Model Year $2001 \ldots \ldots$. . . . . . . . . . . . . . 6 6-5 Marine

Breakdown of Domestic Marine Cargo by Commodity Class, $2001 \ldots \ldots \ldots$. . . . . . . . . . . 9-7 Market

Period Sales, Market Shares, and Sales-Weighted Fuel Economies of New Domestic and Import

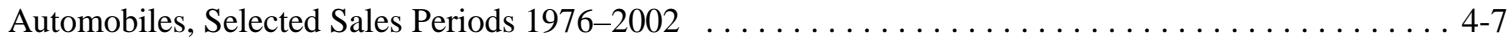

Period Sales, Market Shares, and Sales-Weighted Fuel Economies of New Domestic and Import Light

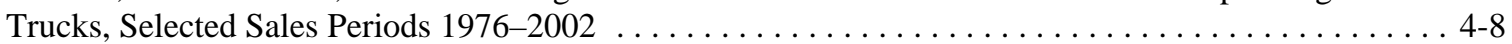

Light Vehicle Market Shares by Size Class, Sales Periods 1976-2002 . . . . . . . . . . . . . . . . . 4-9 Material Matter

Average Material Consumption for a Domestic Automobile, 1978, 1985, and $2001 \ldots \ldots \ldots$

Total National Emissions of Particulate Matter (PM 10), 1980-2001 . . . . . . . . . . . . . . . . . 12-9

Emissions of Particulate Matter (PM 10) from Highway Vehicles, 1980-2001 . . . . . . . . . . . . . . . 12-10

Total National Emissions of Particulate Matter (PM-2.5), 1990-2001 . . . . . . . . . . . . . . . . . 12-11

Emissions of Particulate Matter (PM-2.5) from Highway Vehicles, 1990-2001 . . . . . . . . . . . . 12-12 Mean

Truck Harmonic Mean Fuel Economy by Size Class, 1992 and 1997 . . . . . . . . . . . . . . . 5-6 Middle

Summary of 1996 Military Expenditures for Defending Oil Supplies from the Middle East . . . . . . . . 1-12 
Mile Miles

Automobile Operating Cost per Mile, $1985-2002 \ldots \ldots \ldots \ldots \ldots \ldots \ldots \ldots \ldots$

Ton-Miles of Petroleum and Petroleum Products in the U.S. by Mode, 1975-2001 . . . . . . . . . . . 1-20

Shares of Highway Vehicle-Miles Traveled by Vehicle Type, 1970-2001 . . . . . . . . . . . . . . . 3-8

Growth of Freight Miles in the United States: Comparison of the 1997 and 1993 Commodity

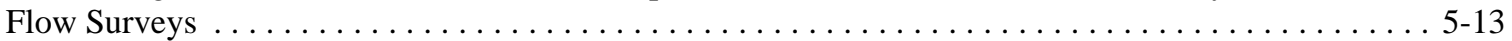

Average Annual Vehicle-Miles of Travel for Fleet Vehicles, $2000 \ldots \ldots \ldots \ldots \ldots \ldots$

Average Miles per Domestic Federal Vehicle by Vehicle Type, 2001 . . . . . . . . . . . . . . . . . 7-5

Average Annual Vehicle-Miles, Vehicle Trips and Trip Length per Household 1969, 1977, 1983, 1990,

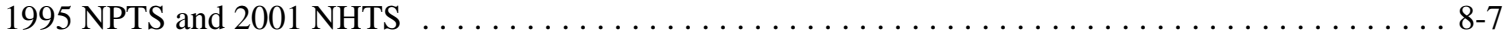

Average Annual Person-Miles Traveled, Person Trips and Trip Length per Household by Selected Trip

Purposes, 1983, 1990, 1995 NPTS and 2001 NHTS . . . . . . . . . . . . . . . . . . . 8-8

Average Annual Miles per Vehicle by Household Vehicle Ownership, 2001 NHTS . . . . . . . . . . . . . 8 8-12

Average Annual Miles per Household Vehicle by Vehicle Age . . . . . . . . . . . . . . . . . . . 8-13

Self-Reported vs. Odometer Average Annual Miles, 1995 NPTS . . . . . . . . . . . . . . . . . 8-14 Military

Class I Railroad Freight Systems in the United States Ranked by Revenue Ton Miles, 2001 . . . . . . . . 9-9 Mode

Summary of 1996 Military Expenditures for Defending Oil Supplies from the Middle East . . . . . . . . . 1-12

Ton-Miles of Petroleum and Petroleum Products in the U.S. by Mode, 1975-2001 . . . . . . . . . . . . 1-20

Domestic Consumption of Transportation Energy by Mode and Fuel Type, $2001 \ldots \ldots$. . . . . . . . . . 2-6

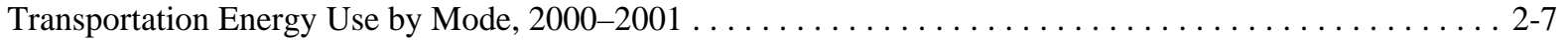

Highway Transportation Energy Consumption by Mode, $1970-2001 \ldots \ldots \ldots \ldots$

Nonhighway Transportation Energy Consumption by Mode, $1970-2001 \ldots \ldots \ldots$. . . . . . . . . . . . . 2-9

Long-Distance Trips by Mode and Purpose, $1995 \ldots \ldots \ldots \ldots \ldots \ldots \ldots \ldots \ldots$

Shares of Long-Distance Person Trips by Mode and Household Income, $1995 \ldots \ldots \ldots \ldots$. . . . . . . . . . 8 22 Model

Automobile Scrappage and Survival Rates 1970, 1980 and 1990 Model Years . . . . . . . . . . . . 3-13

Alternative Fuel Vehicles Available by Manufacturer, Model Year $2001 \ldots \ldots \ldots$. . . . . . . . . . . 6 6-5

Tier 2 Emission Standards for Cars and Light Trucks Effective for 2004-2009 Model Years . . . . . . . . 12-14 Modes

Energy Intensities of Highway Passenger Modes, $1970-2001 \ldots \ldots \ldots \ldots \ldots \ldots$. . . . . . . . . . . . . 2-14

Energy Intensities of Nonhighway Passenger Modes, 1970-2001 . . . . . . . . . . . . . . . . . . 2-15

Energy Intensities of Freight Modes, $1970-2001 \ldots \ldots \ldots \ldots \ldots$

Monoxide

Total National Emissions of Carbon Monoxide, $1980-2001 \ldots \ldots \ldots \ldots \ldots$ Motor

Emissions of Carbon Monoxide from Highway Vehicles, $1980-2001 \ldots \ldots \ldots$. . . . . . . . . . . . 12-4

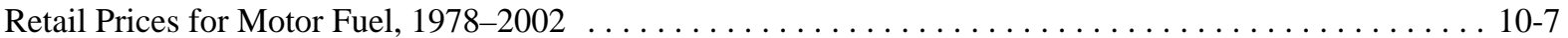

State Taxes on Motor Fuels, $2000 \ldots \ldots \ldots \ldots \ldots \ldots \ldots \ldots \ldots \ldots \ldots \ldots$

Federal Excise Taxes on Motor Fuels $\ldots \ldots \ldots \ldots \ldots \ldots \ldots \ldots \ldots \ldots \ldots \ldots$

Movement

Intercity Freight Movement and Energy Use in the United States, $2001 \ldots \ldots \ldots$ MTBE

U.S. Production and Imports of MTBE and Fuel Ethanol, 1985-2002 . . . . . . . . . . . . . . . 2-12 National

Summary Statistics for the National Railroad Passenger Corporation (Amtrak), $1971 \ldots \ldots \ldots$. . . . . . . 9 13

Total National Emissions of the Criteria Air Pollutants by Sector, $2001 \ldots \ldots \ldots$. . . . . . . . . . . 12-2

Total National Emissions of Carbon Monoxide, $1980-2001 \ldots \ldots \ldots \ldots \ldots \ldots$

Total National Emissions of Nitrogen Oxides, $1980-2001 \ldots \ldots \ldots \ldots \ldots \ldots \ldots$

Total National Emissions of Volatile Organic Compounds, 1980-2001 . . . . . . . . . . . . . . . . . 12-7

Total National Emissions of Particulate Matter (PM 10), 1980-2001 . . . . . . . . . . . . . . . . 12-9

Total National Emissions of Particulate Matter (PM-2.5), 1990-2001 . . . . . . . . . . . . . . . . . . . 12-11 
Natural

World Natural Gas Reserves, Production, and Consumption, $2000 \ldots \ldots \ldots \ldots \ldots$ NHTS

Demographic Statistics, 1969, 1977, 1983, 1990, 1995 NPTS and 2001 NHTS . . . . . . . . . . . . . 8-6

Average Annual Vehicle-Miles, Vehicle Trips and Trip Length per Household 1969, 1977, 1983, 1990,

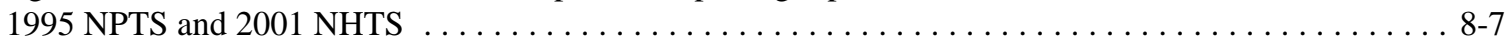

Average Annual Person-Miles Traveled, Person Trips and Trip Length per Household by Selected Trip

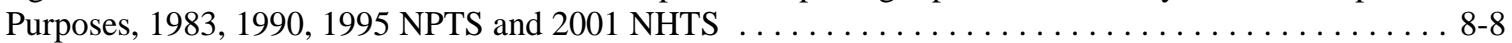

Average Number of Vehicles and Vehicle Travel per Household, 1990 NPTS and 2001 NHTS . . . . . . . 8-9

Average Vehicle Occupancy by Vehicle Type, 1995 NPTS and 2001 NHTS . . . . . . . . . . . . . 8-10

Average Vehicle Occupancy by Trip Purpose, 1977 NPTS and 2001 NHTS . . . . . . . . . . . . . . . 8-11

Average Annual Miles per Vehicle by Household Vehicle Ownership, 2001 NHTS . . . . . . . . . . . . 8 8-12

Average Age of Vehicles by Household Vehicle Ownership, 2001 NHTS . . . . . . . . . . . . . . . 8-12

Walk and Bike Trips by Trip Purpose, 2001 NHTS . . . . . . . . . . . . . . . . . . . . . . 8-19

Nitrogen

Total National Emissions of Nitrogen Oxides, 1980-2001 . . . . . . . . . . . . . . . . . . . . . . 12-5

No

Emissions of Nitrogen Oxides from Highway Vehicles, $1980-2001 \ldots \ldots \ldots \ldots$

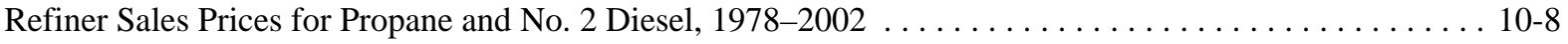

Nonhighway

Nonhighway Transportation Energy Consumption by Mode, 1970-2001 . . . . . . . . . . . . . . . . . . 2-9

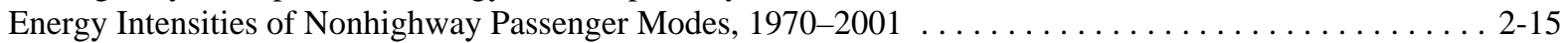

Nonhighway Energy Use Shares, $1970-2001 \ldots \ldots \ldots \ldots \ldots \ldots \ldots \ldots$

Nonoccupant

Occupant Fatalities by Vehicle Type and Nonoccupant Fatalities, 1975-2000 . . . . . . . . . . . . . 4-34 NPTS

Demographic Statistics, 1969, 1977, 1983, 1990, 1995 NPTS and 2001 NHTS . . . . . . . . . . . . . 8-6

Average Annual Vehicle-Miles, Vehicle Trips and Trip Length per Household 1969, 1977, 1983, 1990,

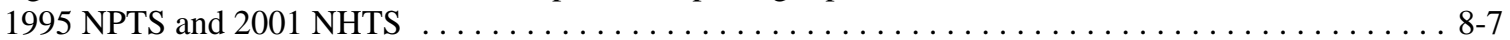

Average Annual Person-Miles Traveled, Person Trips and Trip Length per Household by Selected Trip

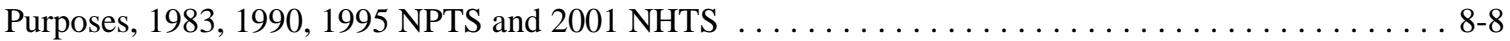

Average Number of Vehicles and Vehicle Travel per Household, 1990 NPTS and 2001 NHTS . . . . . . . 8-9

Average Vehicle Occupancy by Vehicle Type, 1995 NPTS and 2001 NHTS . . . . . . . . . . . . . . 8-10

Average Vehicle Occupancy by Trip Purpose, 1977 NPTS and 2001 NHTS . . . . . . . . . . . . . . . 8 8-11

Self-Reported vs. Odometer Average Annual Miles, 1995 NPTS . . . . . . . . . . . . . . . . . . . . 8-14

Numerical

Numerical Estimates of Global Warming Potentials Compared with Carbon Dioxide . . . . . . . . . . . 11-3

Occupancy

Average Vehicle Occupancy by Vehicle Type, 1995 NPTS and 2001 NHTS . . . . . . . . . . . . . . 8-10

Average Vehicle Occupancy by Trip Purpose, 1977 NPTS and 2001 NHTS . . . . . . . . . . . . . . . 8-11

Occupant

Occupant Fatalities by Vehicle Type and Nonoccupant Fatalities, 1975-2000 . . . . . . . . . . . . . . . . 4-34

Light Vehicle Occupant Safety Data, $1975-2001 \ldots \ldots \ldots \ldots \ldots \ldots \ldots$

Odometer OECD

Self-Reported vs. Odometer Average Annual Miles, 1995 NPTS . . . . . . . . . . . . . . . . . . 8 8-14

Petroleum Stocks of OECD Countries by Ownership, $1995-2002 \ldots \ldots \ldots \ldots \ldots$ 
Oil

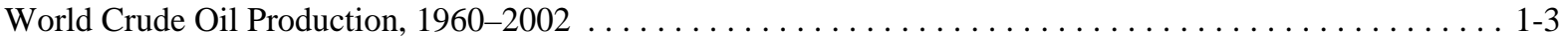

World Oil Reserves, Production and Consumption, $2002 \ldots \ldots \ldots \ldots \ldots \ldots \ldots$

Crude Oil Prices in Current and Constant Terms, $1870-2002 \ldots \ldots \ldots \ldots \ldots \ldots \ldots$

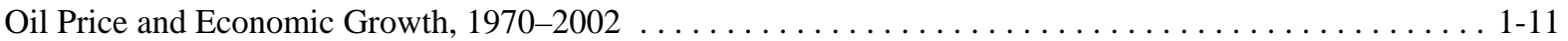

Summary of 1996 Military Expenditures for Defending Oil Supplies from the Middle East . . . . . . . . 1-12

U.S. Refinery Input of Crude Oil and Petroleum Products, 1987-2002 . . . . . . . . . . . . . . . . . 1-14

Refinery Yield of Petroleum Products from a Barrel of Crude Oil, 1978-2002 . . . . . . . . . . . . . . 1-15

Prices for a Barrel of Crude Oil and a Gallon of Gasoline, 1978-2002 . . . . . . . . . . . . . . . 10-6

Operating

Automobile Operating Cost per Mile, $1985-2002 \ldots \ldots \ldots \ldots \ldots \ldots \ldots \ldots \ldots \ldots$

Fixed Automobile Operating Costs per Year, $1975-2002 \ldots \ldots \ldots \ldots \ldots \ldots \ldots$

Operation

Automobiles in Operation and Vehicle Travel by Age, 1970 and 2001 . . . . . . . . . . . . . . . . . 3-9

Trucks in Operation and Vehicle Travel by Age, 1970 and $2001 \ldots \ldots \ldots \ldots$. . . . . . . . . . . . . 3-10

Operations

Summary Statistics for Commuter Rail Operations, 1984-2001 . . . . . . . . . . . . . . . . . . 9-14

Summary Statistics for Rail Transit Operations, $1970-2001 \ldots \ldots \ldots \ldots \ldots \ldots$

Organic

Total National Emissions of Volatile Organic Compounds, 1980-2001 . . . . . . . . . . . . . . . . 12-7

Emissions of Volatile Organic Compounds from Highway Vehicles, 1980-2001 . . . . . . . . . . . . . 12-8 Origin

Output

U.S. Petroleum Imports by World Region of Origin, $1960-2002 \ldots \ldots \ldots \ldots \ldots$

Refinery Gross Output by World Region, $2002 \ldots \ldots \ldots \ldots \ldots \ldots \ldots \ldots \ldots$

Ownership

Petroleum Stocks of OECD Countries by Ownership, 1995-2002 . . . . . . . . . . . . . . . 1-8

Estimates of Alternative Fuel Vehicles by Ownership, 1996 and $2002 \ldots \ldots \ldots \ldots \ldots$

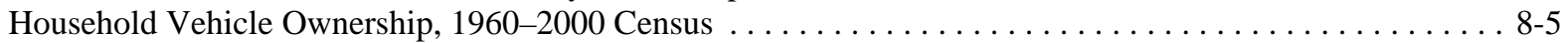

Average Annual Miles per Vehicle by Household Vehicle Ownership, 2001 NHTS . . . . . . . . . . . . 8-12

Oxides

Average Age of Vehicles by Household Vehicle Ownership, 2001 NHTS . . . . . . . . . . . . . . . 8-12

Total National Emissions of Nitrogen Oxides, $1980-2001 \ldots \ldots \ldots \ldots \ldots \ldots$

Emissions of Nitrogen Oxides from Highway Vehicles, $1980-2001 \ldots \ldots$. . . . . . . . . . . . . . 12-6

Oxygenate

Alternative Fuel and Oxygenate Consumption, $1992-2002 \ldots \ldots \ldots \ldots \ldots$

Particulate

Total National Emissions of Particulate Matter (PM 10), 1980-2001 . . . . . . . . . . . . . . . 12-9

Emissions of Particulate Matter (PM 10) from Highway Vehicles, 1980-2001 . . . . . . . . . . . . . . 12-10

Total National Emissions of Particulate Matter (PM-2.5), 1990-2001 . . . . . . . . . . . . . . . 12-11

Emissions of Particulate Matter (PM-2.5) from Highway Vehicles, 1990-2001 . . . . . . . . . . . . 12-12

Passenger

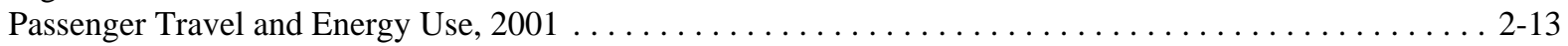

Energy Intensities of Highway Passenger Modes, $1970-2001 \ldots \ldots \ldots \ldots \ldots$. . . . . . . . . . . . . 2-14

Energy Intensities of Nonhighway Passenger Modes, $1970-2001 \ldots \ldots \ldots \ldots \ldots \ldots$

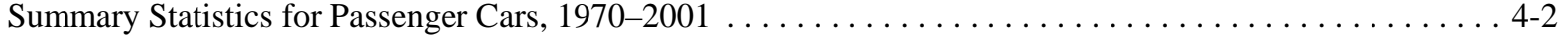

Summary Statistics for the National Railroad Passenger Corporation (Amtrak), $1971 \ldots \ldots$. . . . . . . . 9-13

California Passenger Cars and Light Trucks Emission Certification Standards for Model Years

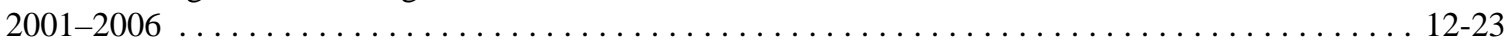

People

California Vehicle Emission Reduction for Passenger Cars and Light Trucks . . . . . . . . . . . . 12-24

Vehicles per Thousand People: U.S. Compared to Other Countries . . . . . . . . . . . . . . . . 3-6 
Percentage

Percentage of Trucks by Size Ranked by Major Use, $1997 \ldots \ldots \ldots \ldots \ldots \ldots \ldots$

Percentage of Trucks by Fleet Size and Primary Fueling Facility, $1997 \ldots \ldots \ldots \ldots$. . . . . . . . . . . . 5-9

Periods

Percentage of Trucks by Major Use and Primary Fueling Facility, $1997 \ldots \ldots \ldots \ldots$

Period Sales, Market Shares, and Sales-Weighted Fuel Economies of New Domestic and Import

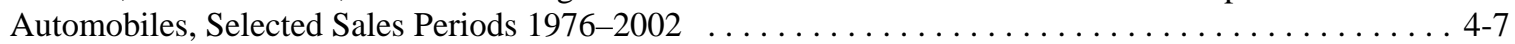

Period Sales, Market Shares, and Sales-Weighted Fuel Economies of New Domestic and Import Light Trucks, Selected Sales Periods $1976-2002 \ldots \ldots \ldots \ldots \ldots \ldots \ldots \ldots \ldots \ldots$

Light Vehicle Market Shares by Size Class, Sales Periods 1976-2002 . . . . . . . . . . . . . . . . . 4-9

Sales-Weighted Engine Size of New Domestic and Import Automobiles by Size Class, Sales Periods

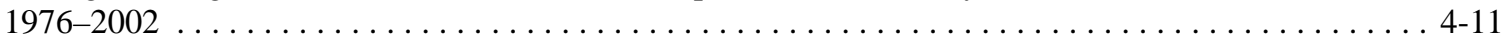

Sales-Weighted Engine Size of New Domestic and Import Light Trucks by Size Class, Sales Periods

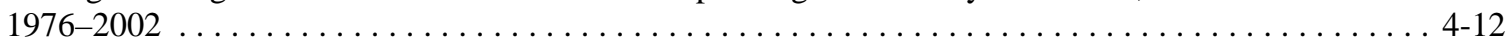

Sales-Weighted Curb Weight of New Domestic and Import Automobiles by Size Class, Sales Periods

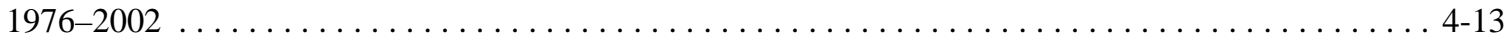

Sales-Weighted Interior Space of New Domestic and Import Automobiles by Size Class, Sales Periods

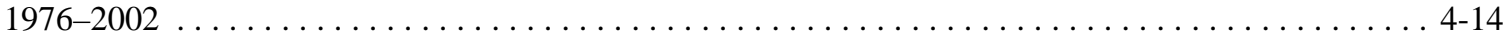

Sales-Weighted Wheelbase of New Automobiles and Light Trucks, Sales Periods 1976-2002 . . . . . . . . 4-15 Person

Average Annual Person-Miles Traveled, Person Trips and Trip Length per Household by Selected

Trip Purposes, 1983, 1990, 1995 NPTS and 2001 NHTS $\ldots \ldots \ldots \ldots \ldots \ldots$

Shares of Long-Distance Person Trips by Mode and Household Income, $1995 \ldots \ldots$. . . . . . . . . . . 8-22 Petroleum

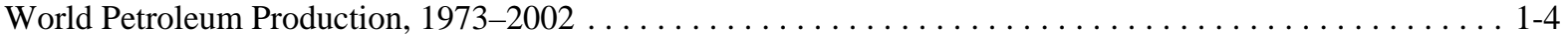

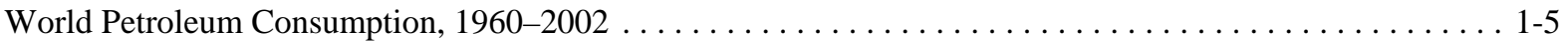

Petroleum (continued)

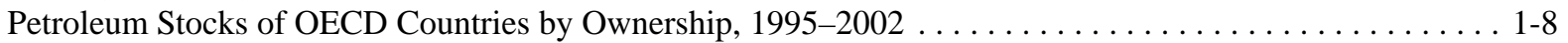

U.S. Petroleum Imports by World Region of Origin, $1960-2002 \ldots \ldots \ldots \ldots \ldots \ldots$

U.S. Refinery Input of Crude Oil and Petroleum Products, $1987-2002 \ldots \ldots \ldots \ldots \ldots$

Refinery Yield of Petroleum Products from a Barrel of Crude Oil, 1978-2002 . . . . . . . . . . . . . . 1-15

United States Petroleum Production, Imports and Exports, $1950-2002 \ldots \ldots \ldots \ldots \ldots$

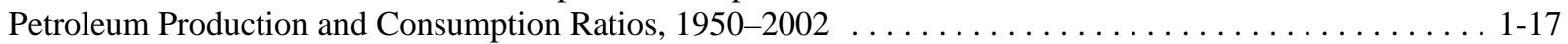

Consumption of Petroleum by End-Use Sector, 1973-2002 . . . . . . . . . . . . . . . . . . . . . . 1-19

PM

Ton-Miles of Petroleum and Petroleum Products in the U.S. by Mode, 1975-2001 . . . . . . . . . . . . 1-20

Total National Emissions of Particulate Matter (PM 10), 1980-2001 . . . . . . . . . . . . . . . 12-9

Emissions of Particulate Matter (PM 10) from Highway Vehicles, 1980-2001 . . . . . . . . . . . . . . 12-10

Total National Emissions of Particulate Matter (PM-2.5), 1990-2001 . . . . . . . . . . . . . . . 12-11

Emissions of Particulate Matter (PM-2.5) from Highway Vehicles, 1990-2001 . . . . . . . . . . . . 12-12 Policy

Energy Policy Act Purchase Requirements of Light Alternative Fuel Vehicles $\ldots \ldots \ldots \ldots \ldots$ Pollutants

Total National Emissions of the Criteria Air Pollutants by Sector, $2001 \ldots \ldots \ldots \ldots$ Population

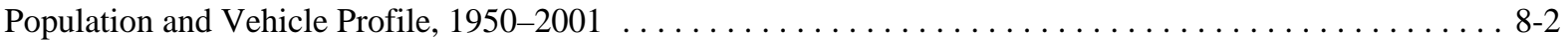

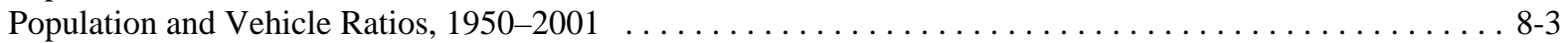

Potential

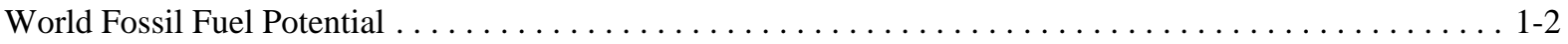

Potentials

Numerical Estimates of Global Warming Potentials Compared with Carbon Dioxide . . . . . . . . . . . 11-3 Pounds

New Retail Sales of Trucks 10,000 Pounds GVW and Less in the United States, 1970-2002 . . . . . . . . 4-6 
Powered

Federal Exhaust Emission Certification Standards for Gasoline- and Diesel-Powered Light Vehicles . . . 12-16

Federal Exhaust Emission Certification Standards for Gasoline- and Diesel-Powered Light Trucks

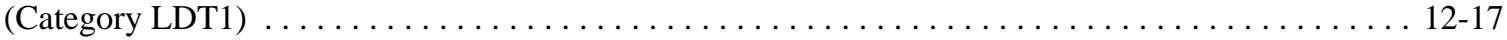

Federal Exhaust Emission Certification Standards for Gasoline- and Diesel-Powered Light Trucks

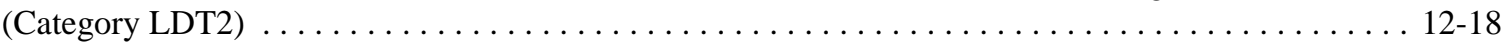

Federal Exhaust Emission Certification Standards for Gasoline- and Diesel-Powered Light Trucks

(Category LDT3) . . . . . . . . . . . . . . . . . . . . . . . . . . . . . . . . . . 12-19

Federal Exhaust Emission Certification Standards for Gasoline- and Diesel-Powered Light Trucks

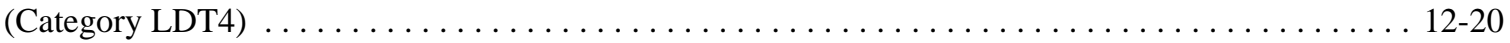

Federal Exhaust Emission Certification Standards for Gasoline- and Diesel-Powered Light

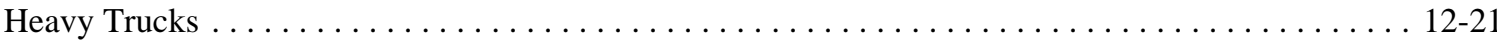

Federal Exhaust Emission Certification Standards for Gasoline- and Diesel-Powered Heavy

Heavy Trucks . . . . . . . . . . . . . . . . . . . . . . . . . . . . . . . . . . 12-22

Price

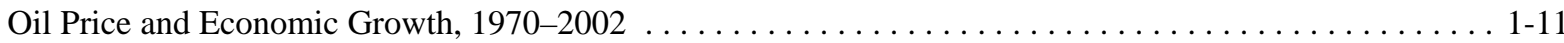

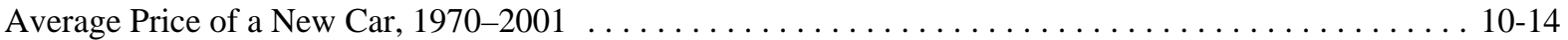

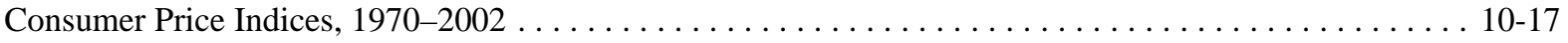

Prices

Crude Oil Prices in Current and Constant Terms, 1870-2002 . . . . . . . . . . . . . . . . . . . . 1-9

Gasoline Prices for Selected Countries, $1978-2002 \ldots \ldots \ldots \ldots \ldots \ldots \ldots \ldots$

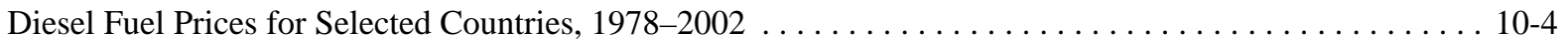

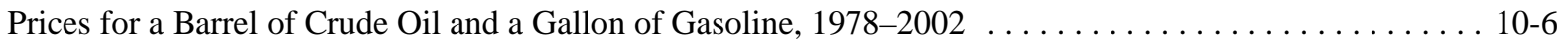

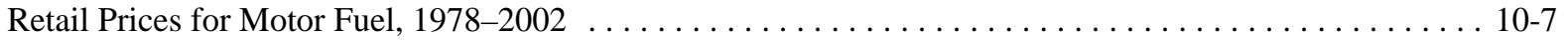

Refiner Sales Prices for Propane and No. 2 Diesel, 1978-2002 . . . . . . . . . . . . . . . . . . . 10-8

Primary

Refiner Sales Prices for Aviation Gasoline and Jet Fuel, 1978-2002 . . . . . . . . . . . . . . . . . . 10-9

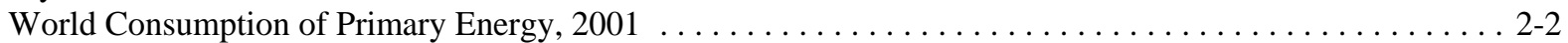

Percentage of Trucks by Fleet Size and Primary Fueling Facility, $1997 \ldots \ldots \ldots \ldots$

Percentage of Trucks by Major Use and Primary Fueling Facility, $1997 \ldots \ldots \ldots \ldots$

Production

Hydrogen Production Methods $\ldots \ldots \ldots \ldots \ldots \ldots \ldots \ldots \ldots \ldots \ldots \ldots \ldots \ldots \ldots \ldots \ldots \ldots \ldots \ldots$

U.S. Hydrogen Production Plants and Storage Terminals $\ldots \ldots \ldots \ldots \ldots \ldots \ldots \ldots \ldots$ Products

U.S. Refinery Input of Crude Oil and Petroleum Products, $1987-2002 \ldots \ldots \ldots \ldots \ldots$. . . . . . . . . . . . 1-14

Refinery Yield of Petroleum Products from a Barrel of Crude Oil, 1978-2002 . . . . . . . . . . . . . 1-15

Ton-Miles of Petroleum and Petroleum Products in the U.S. by Mode, 1975-2001 . . . . . . . . . . . . 1-20

Profile

Population and Vehicle Profile, $1950-2001 \ldots \ldots \ldots \ldots \ldots \ldots \ldots \ldots \ldots \ldots$

Projected

Projected Fuel Economies from U.S., European, and Japanese Driving Cycles . . . . . . . . . . . . 4-32

Propane

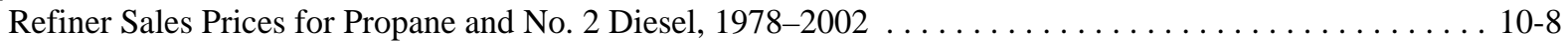

Purchase

Energy Policy Act Purchase Requirements of Light Alternative Fuel Vehicles . . . . . . . . . . . . . 7-8

Purchases

New Light Fleet Vehicle Purchases by Vehicle Type, $2000 \ldots \ldots \ldots \ldots \ldots \ldots \ldots \ldots$

Purpose

Average Vehicle Occupancy by Trip Purpose, 1977 NPTS and 2001 NHTS . . . . . . . . . . . . . 8 8-11

Walk and Bike Trips by Trip Purpose, 2001 NHTS $\ldots \ldots \ldots \ldots \ldots \ldots \ldots \ldots$

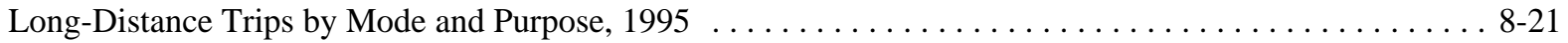

Purposes

Average Annual Person-Miles Traveled, Person Trips and Trip Length per Household by Selected

Trip Purposes, 1983, 1990, 1995 NPTS and 2001 NHTS 
Rail

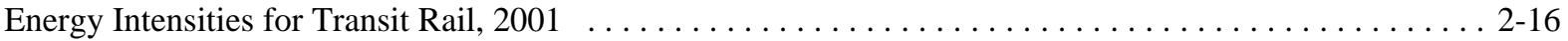

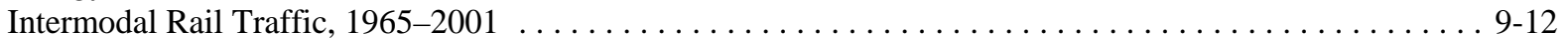

Summary Statistics for Commuter Rail Operations, $1984-2001 \ldots \ldots \ldots \ldots \ldots$. . . . . . . . . . . . . 9 . . . . . . . . . . . . . .

Summary Statistics for Rail Transit Operations, $1970-2001 \ldots \ldots \ldots \ldots \ldots \ldots \ldots$ Railroad

Class I Railroad Freight Systems in the United States Ranked by Revenue Ton Miles, 2001 . . . . . . . . . 9-9

Railroad Revenue Carloads by Commodity Group, 1974 and 2001 . . . . . . . . . . . . . . . . . . . . 9 9-11

Summary Statistics for the National Railroad Passenger Corporation (Amtrak), $1971 \ldots \ldots$. . . . . . . . 9-13

Railroads

Summary Statistics for Class I Freight Railroads, $1970-2001 \ldots \ldots \ldots \ldots \ldots \ldots \ldots$

Ranked

Percentage of Trucks by Size Ranked by Major Use, $1997 \ldots \ldots \ldots \ldots \ldots \ldots \ldots$

Class I Railroad Freight Systems in the United States Ranked by Revenue Ton Miles, 2001 . . . . . . . . . 9-9 Rates

Automobile Scrappage and Survival Rates 1970, 1980 and 1990 Model Years . . . . . . . . . . . 3-13

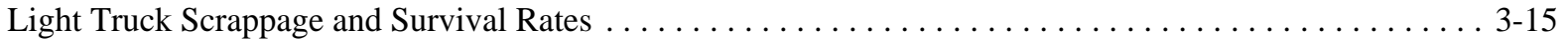

Ratios

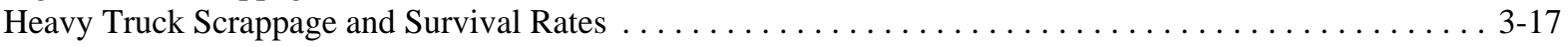

Petroleum Production and Consumption Ratios, $1950-2002 \ldots \ldots \ldots \ldots \ldots \ldots \ldots$

Population and Vehicle Ratios, $1950-2001 \ldots \ldots \ldots \ldots \ldots \ldots \ldots \ldots \ldots$

Receipts

Tax Receipts from the Sale of Gas Guzzlers, $1980-2001 \ldots \ldots \ldots \ldots \ldots \ldots \ldots$

Recreational

Recreational Boating Statistics, $1977-2001 \ldots \ldots \ldots \ldots \ldots \ldots \ldots \ldots \ldots$

Reduction

California Vehicle Emission Reduction for Passenger Cars and Light Trucks . . . . . . . . . . . . . . 12-24

Refiner

Refiner Sales Prices for Propane and No. 2 Diesel, 1978-2002 . . . . . . . . . . . . . . . . . . . 10-8

Refiner Sales Prices for Aviation Gasoline and Jet Fuel, 1978-2002 . . . . . . . . . . . . . . . . 10-9

Refinery

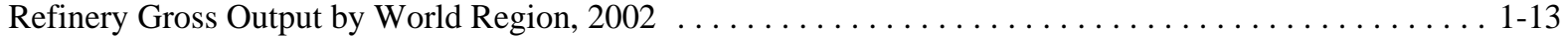

U.S. Refinery Input of Crude Oil and Petroleum Products, $1987-2002 \ldots \ldots \ldots \ldots \ldots \ldots$

Refinery Yield of Petroleum Products from a Barrel of Crude Oil, 1978-2002 . . . . . . . . . . . . . . 1-15 Refuel

Number of Alternative Refuel Sites by State and Fuel Type, $2002 \ldots \ldots \ldots \ldots$

Refueling

Region

Conventional and Alternative Fuel Refueling Stations $\ldots \ldots \ldots \ldots \ldots \ldots \ldots \ldots$

U.S. Petroleum Imports by World Region of Origin, $1960-2002 \ldots \ldots \ldots \ldots \ldots \ldots \ldots$

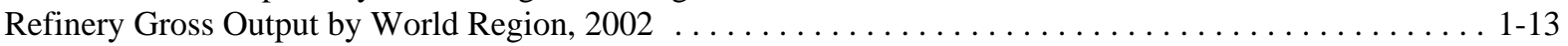

Registrations

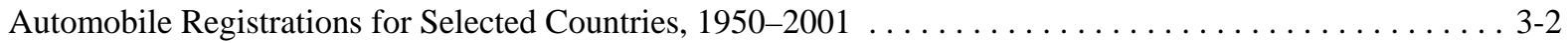

Truck and Bus Registrations for Selected Countries, 1950-2001 . . . . . . . . . . . . . . . . . . . 3-3

Average Age and Registrations of Automobiles and Trucks, 1970-2001 . . . . . . . . . . . . . . 3-12

Reported

Self-Reported vs. Odometer Average Annual Miles, 1995 NPTS . . . . . . . . . . . . . . . . . 8-14

Representative

Representative Number Five Driving Cycle $\ldots \ldots \ldots \ldots \ldots \ldots \ldots \ldots \ldots \ldots \ldots$

Reserves

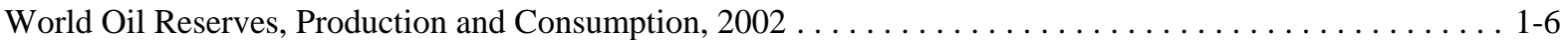

Retail

World Natural Gas Reserves, Production, and Consumption, $2000 \ldots \ldots \ldots \ldots \ldots$

New Retail Automobile Sales in the United States, $1970-2002$. . . . . . . . . . . . . . . . . . . . . . . . 4-5 
New Retail Sales of Trucks 10,000 pounds GVW and Less in the United States, 1970-2002 . . . . . . . . 4-6

New Retail Truck Sales by Gross Vehicle Weight, $1970-2002 \ldots \ldots \ldots \ldots \ldots \ldots$

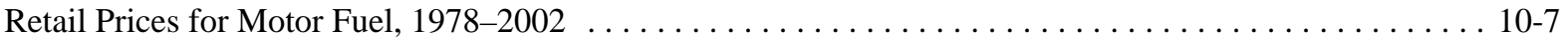

Revenue

Class I Railroad Freight Systems in the United States Ranked by Revenue Ton Miles, 2001 . . . . . . . . 9-9

Railroad Revenue Carloads by Commodity Group, 1974 and 2001 . . . . . . . . . . . . . . . . . . 9-11

Rollover

Percent Rollover Occurrence in Fatal Crashes by Vehicle Type, $2001 \ldots$. . . . . . . . . . . . . . . . . . 4-37

Route

Summary Statistics for U.S. Domestic and International Certificated Route Air Carriers (Combined

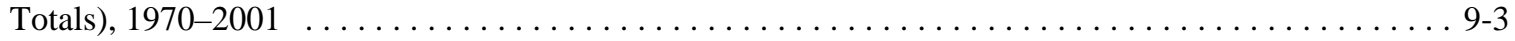

Safety

Light Vehicle Occupant Safety Data, $1975-2001 \ldots \ldots \ldots \ldots \ldots \ldots \ldots \ldots$

Sale

Sales

Tax Receipts from the Sale of Gas Guzzlers, $1980-2001 \ldots \ldots \ldots \ldots \ldots \ldots \ldots$

Vehicle Stock and New Sales in the United States, 2001 Calendar Year . . . . . . . . . . . . . . . 3-7

Sales Estimates of Class 1, Class 2a, and Class 2b Light Trucks, 1989-1999 . . . . . . . . . . . . . . . 4-4

New Retail Automobile Sales in the United States, 1970-2002 . . . . . . . . . . . . . . . . . 4-5

New Retail Sales of Trucks 10,000 pounds GVW and Less in the United States, 1970-2002 . . . . . . . . . 4-6

Period Sales, Market Shares, and Sales-Weighted Fuel Economies of New Domestic and Import

Automobiles, Selected Sales Periods $1976-2002 \ldots \ldots \ldots \ldots \ldots \ldots \ldots \ldots \ldots \ldots \ldots$

Period Sales, Market Shares, and Sales-Weighted Fuel Economies of New Domestic and Import

Light Trucks, Selected Sales Periods $1976-2002 \ldots \ldots \ldots \ldots \ldots \ldots \ldots \ldots \ldots \ldots \ldots \ldots \ldots \ldots$

Sales (continued)

Light Vehicle Market Shares by Size Class, Sales Periods 1976-2002 . . . . . . . . . . . . . . . . . . 4-9

Sales-Weighted Engine Size of New Domestic and Import Automobiles by Size Class, Sales Periods

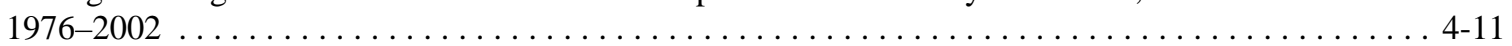

Sales-Weighted Engine Size of New Domestic and Import Light Trucks by Size Class, Sales Periods

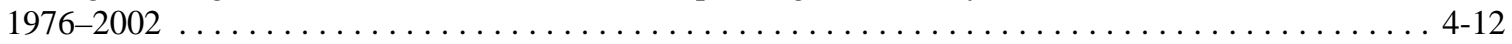

Sales-Weighted Curb Weight of New Domestic and Import Automobiles by Size Class, Sales Periods

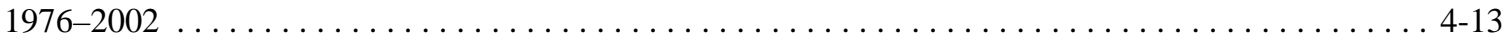

Sales-Weighted Interior Space of New Domestic and Import Automobiles by Size Class, Sales Periods

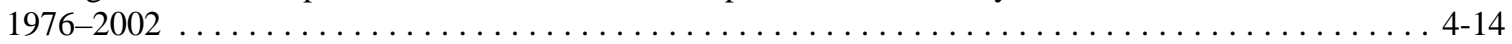

Sales-Weighted Wheelbase of New Automobiles and Light Trucks, Sales Periods 1976-2002 . . . . . . . . 4-15

New Light Vehicle Dealerships and Sales, $1970-2000 \ldots \ldots \ldots \ldots \ldots \ldots \ldots$. . . . . . . . . . . . 4

Automobile Corporate Average Fuel Economy (CAFE) Standards versus Sales-Weighted Fuel

Economy Estimates, $1978-2003 \ldots \ldots \ldots \ldots \ldots \ldots \ldots \ldots \ldots \ldots \ldots \ldots \ldots \ldots \ldots$

Light Truck Corporate Average Fuel Economy (CAFE) Standards versus Sales-Weighted Fuel

Economy Estimates, $1978-2003 \ldots \ldots \ldots \ldots \ldots \ldots \ldots \ldots \ldots \ldots \ldots \ldots \ldots \ldots \ldots$

New Retail Truck Sales by Gross Vehicle Weight, $1970-2002 \ldots \ldots \ldots \ldots \ldots \ldots$. . . . . . . . . . . . . 5-4

Sales and Specifications of Available Advanced Technology Vehicles $\ldots \ldots \ldots \ldots \ldots \ldots$

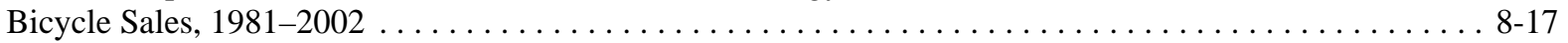

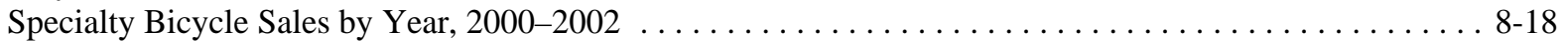

Refiner Sales Prices for Propane and No. 2 Diesel, 1978-2002 . . . . . . . . . . . . . . . . . . 10-8

Refiner Sales Prices for Aviation Gasoline and Jet Fuel, 1978-2002 . . . . . . . . . . . . . . . . 10-9 School

Summary Statistics on Intercity and School Buses, $1970-2001 \ldots \ldots \ldots \ldots \ldots$

Scrappage

Automobile Scrappage and Survival Rates 1970, 1980 and 1990 Model Years . . . . . . . . . . . . 3-13

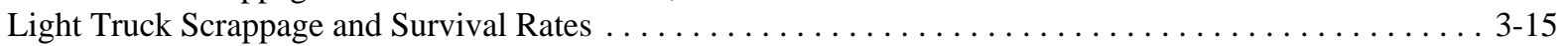

Sector

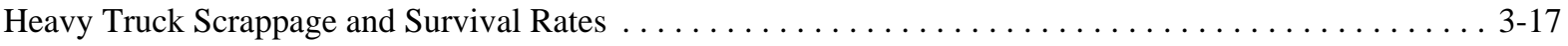

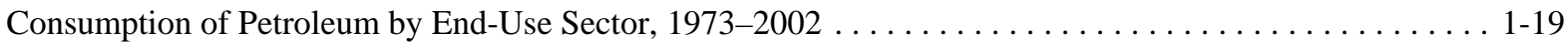


U. S. Consumption of Total Energy by End-Use Sector, 1973-2002 . . . . . . . . . . . . . . . . . . . . 2 2-3

U.S. Carbon Dioxide Emissions from Fossil Energy Consumption by End-Use Sector, 1990-2001 … . . 11-5

U.S. Carbon Dioxide Emissions from Energy Use in Transportation Sector, 1990-2001 . . . . . . . . . 11-6 Selected

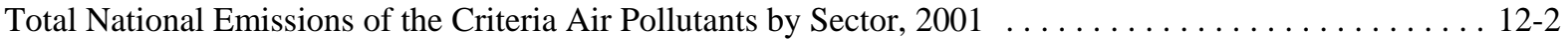

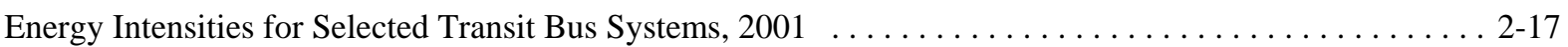

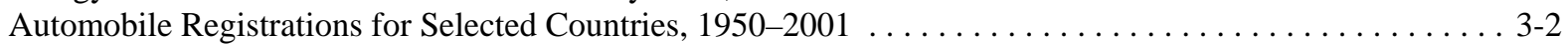

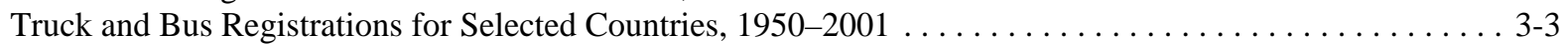

Period Sales, Market Shares, and Sales-Weighted Fuel Economies of New Domestic and Import Automobiles, Selected Sales Periods 1976-2002 . . . . . . . . . . . . . . . . . . . . . . . . . 4-7

Period Sales, Market Shares, and Sales-Weighted Fuel Economies of New Domestic and Import Light Trucks, Selected Sales Periods 1976-2002 . . . . . . . . . . . . . . . . . . . . . . 4-8

Average Annual Person-Miles Traveled, Person Trips and Trip Length per Household by Selected

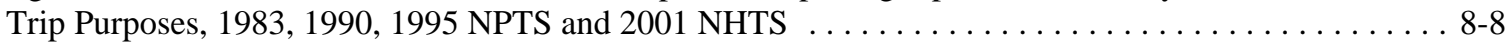

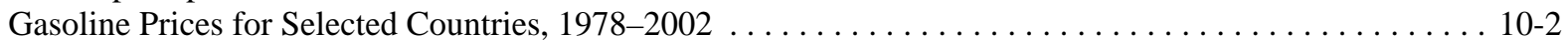

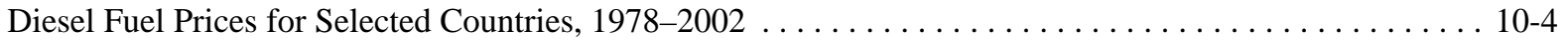
Self

Self-Reported vs. Odometer Average Annual Miles, 1995 NPTS $\ldots \ldots \ldots \ldots \ldots \ldots \ldots \ldots \ldots \ldots . . .14$ Service

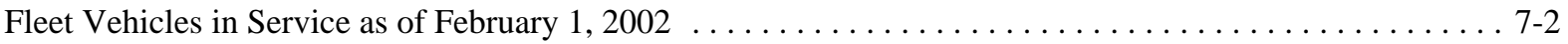

Average Length of Time Business Fleet Vehicles are in Service, $2001 \ldots \ldots \ldots \ldots \ldots \ldots \ldots \ldots \ldots$. . . . . . Severity Shares

Crashes by Crash Severity, Crash Type, and Vehicle Type, $2001 \ldots \ldots \ldots \ldots \ldots \ldots \ldots \ldots \ldots \ldots .4 .36$

Shares of Highway Vehicle-Miles Traveled by Vehicle Type, 1970-2001 . . . . . . . . . . . . . . . 3-8

Period Sales, Market Shares, and Sales-Weighted Fuel Economies of New Domestic and Import Automobiles, Selected Sales Periods $1976-2002$. . . . . . . . . . . . . . . . . . . . . 4-7

Period Sales, Market Shares, and Sales-Weighted Fuel Economies of New Domestic and Import

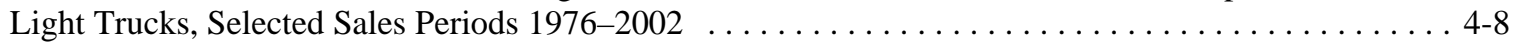

Light Vehicle Market Shares by Size Class, Sales Periods 1976-2002 . . . . . . . . . . . . . . . . 4-9

Shares of Long-Distance Person Trips by Mode and Household Income, 1995 . . . . . . . . . . . . . 8-22

Nonhighway Energy Use Shares, $1970-2001 \ldots \ldots \ldots \ldots \ldots \ldots \ldots \ldots \ldots \ldots \ldots \ldots . . \ldots \ldots \ldots \ldots .2$ Single

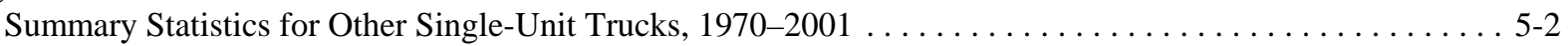

Sites

Number of Alternative Refuel Sites by State and Fuel Type, $2002 \ldots \ldots \ldots \ldots \ldots \ldots \ldots \ldots \ldots \ldots$ Source

Distribution of Energy Consumption by Source, 1973 and 2002 ........................ 2-4 Space

Sales-Weighted Interior Space of New Domestic and Import Automobiles by Size Class, Sales Periods

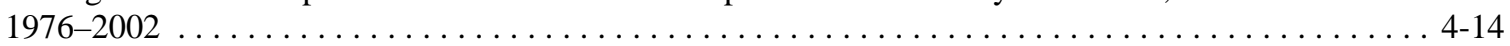

Specialty

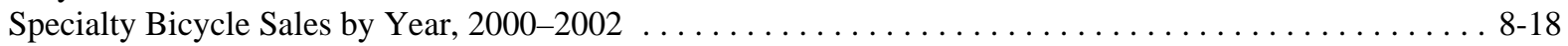

Specifications

Vehicle Specifications for Vehicles Tested in the 1997 Study . . . . . . . . . . . . . . . . . . 4-25

Sales and Specifications of Available Advanced Technology Vehicles . . . . . . . . . . . . . . . . 6-8 Speed

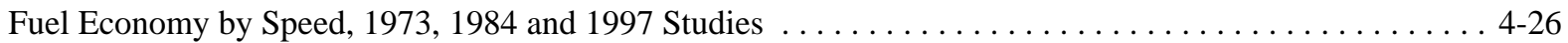

Fuel Economy by Speed, 1973, 1984 and 1997 Studies . . . . . . . . . . . . . . . . . . . . . 4-27

Steady Speed Fuel Economy for Vehicles Tested in the 1997 Study $\ldots \ldots \ldots \ldots \ldots \ldots \ldots \ldots \ldots \ldots .4-28$ 
Standards

Automobile Corporate Average Fuel Economy (CAFE) Standards versus Sales-Weighted Fuel

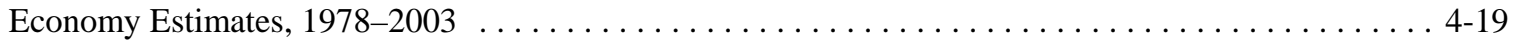

Light Truck Corporate Average Fuel Economy (CAFE) Standards versus Sales-Weighted Fuel

Economy Estimates, 1978-2003 . . . . . . . . . . . . . . . . . . . . . . . . . 4-20

Tier 2 Emission Standards for Cars and Light Trucks Effective for 2004-2009 Model Years . . . . . . . . 12-14

Light Vehicle Exhaust Emission Standards in Effect in 2009 when U.S. Tier 2 Standards are Final . . . . 12-15

Federal Exhaust Emission Certification Standards for Gasoline- and Diesel-Powered Light Vehicles . . . 12-16

Federal Exhaust Emission Certification Standards for Gasoline- and Diesel-Powered Light Trucks

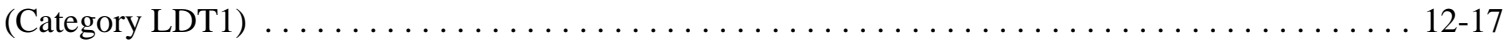

Federal Exhaust Emission Certification Standards for Gasoline- and Diesel-Powered Light Trucks

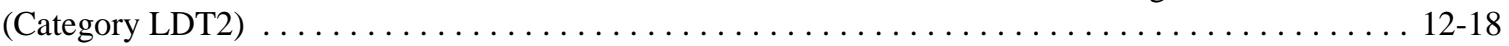

Federal Exhaust Emission Certification Standards for Gasoline- and Diesel-Powered Light Trucks

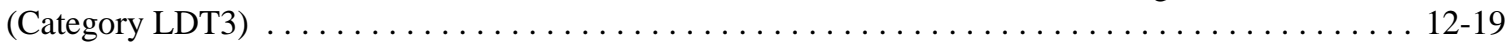

Federal Exhaust Emission Certification Standards for Gasoline- and Diesel-Powered Light Trucks

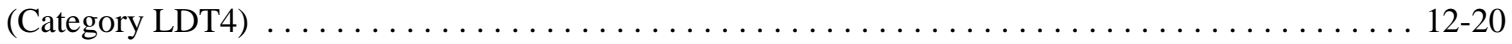

Federal Exhaust Emission Certification Standards for Gasoline- and Diesel-Powered Light

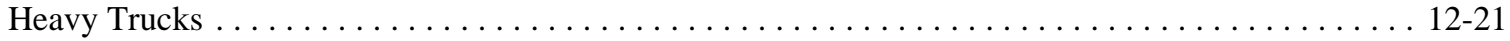

Federal Exhaust Emission Certification Standards for Gasoline- and Diesel-Powered Heavy Heavy Trucks . . . . . . . . . . . . . . . . . 22

California Passenger Cars and Light Trucks Emission Certification Standards for Model Years

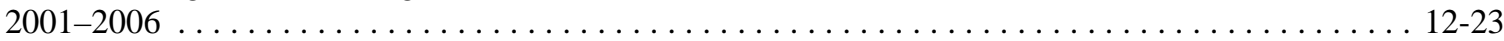

Stations

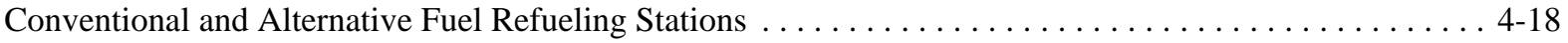
Statistics

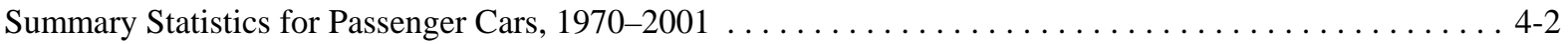

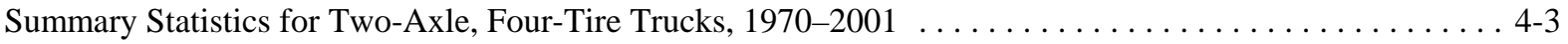

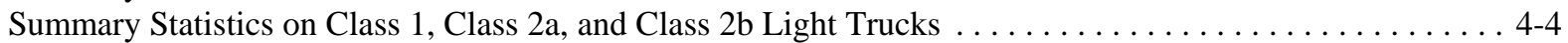

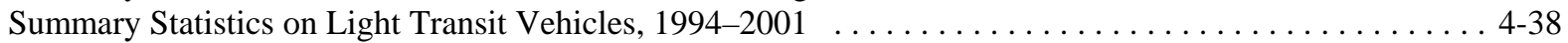

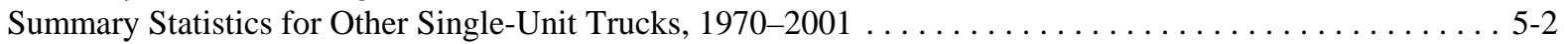

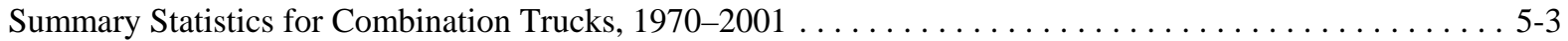

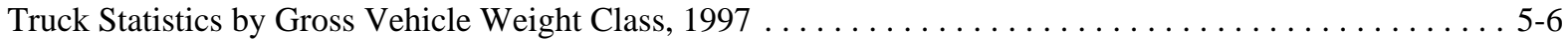

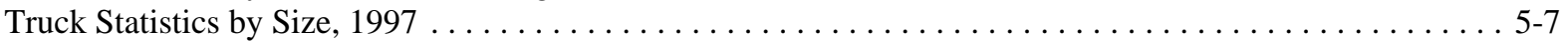

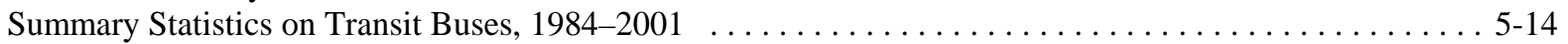

Summary Statistics on Intercity and School Buses, 1970-2001 . . . . . . . . . . . . . . . . . . . 5 5-15

Demographic Statistics, 1969, 1977, 1983, 1990, 1995 NPTS and 2001 NHTS . . . . . . . . . . . . 8-6

Summary Statistics for U.S. Domestic and International Certificated Route Air Carriers (Combined

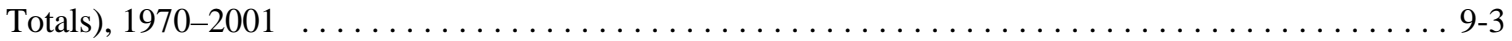

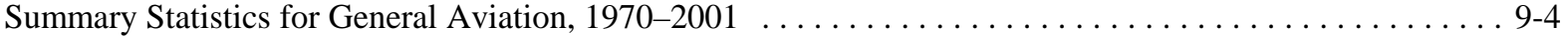

Tonnage Statistics for Domestic and International Waterborne Commerce, 1970-2001 . . . . . . . . . . 9 9-5

Summary Statistics for Domestic Waterborne Commerce, $1970-2001 \ldots \ldots \ldots \ldots \ldots \ldots \ldots$. . . . . . . . . .

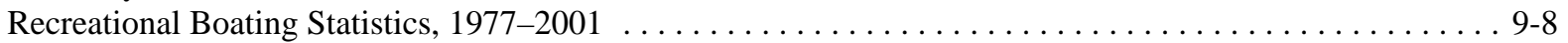

Summary Statistics for Class I Freight Railroads, $1970-2001 \ldots \ldots \ldots \ldots \ldots \ldots \ldots \ldots \ldots \ldots \ldots . \ldots \ldots$

Summary Statistics for the National Railroad Passenger Corporation (Amtrak), $1971 \ldots \ldots \ldots \ldots \ldots \ldots$. . . 13

Summary Statistics for Commuter Rail Operations, 1984-2001 . . . . . . . . . . . . . . . . . . . . 9-14 Steady

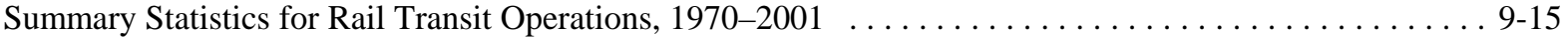
Stock

Steady Speed Fuel Economy for Vehicles Tested in the 1997 Study $\ldots \ldots \ldots \ldots \ldots \ldots \ldots \ldots \ldots .4 .28$ Stocks

Vehicle Stock and New Sales in the United States, 2001 Calendar Year . . . . . . . . . . . . . . . . 3-7 Storage

Petroleum Stocks of OECD Countries by Ownership, 1995-2002 ...................... 1-8

U.S. Hydrogen Production Plants and Storage Terminals $\ldots \ldots \ldots \ldots \ldots \ldots \ldots \ldots \ldots \ldots \ldots \ldots \ldots \ldots \ldots \ldots \ldots$ 
Hydrogen Storage Systems for On-Board Light Vehicles $\ldots \ldots \ldots \ldots \ldots \ldots \ldots \ldots \ldots \ldots \ldots \ldots \ldots .6 \ldots \ldots \ldots$ Summary

Summary of 1996 Military Expenditures for Defending Oil Supplies from the Middle East . . . . . . . . 1-12

Summary Statistics for Passenger Cars, $1970-2001 \ldots \ldots \ldots \ldots \ldots \ldots \ldots \ldots \ldots \ldots \ldots \ldots \ldots .2$

Summary Statistics for Two-Axle, Four-Tire Trucks, 1970-2001 . . . . . . . . . . . . . . . . . . . . 4-3

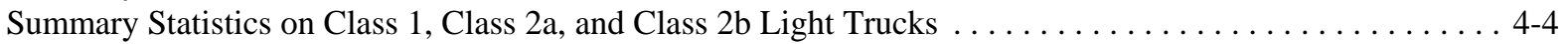

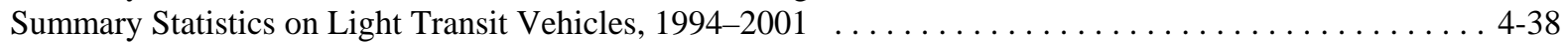

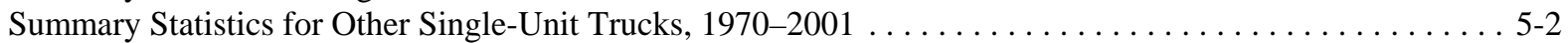

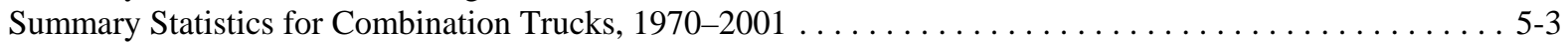

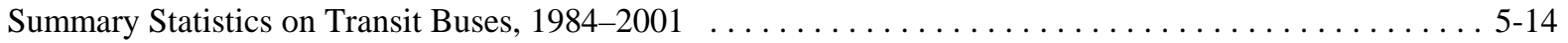

Summary Statistics on Intercity and School Buses, 1970-2001 . . . . . . . . . . . . . . . . . . . 5-15

Summary Statistics for U.S. Domestic and International Certificated Route Air Carriers (Combined

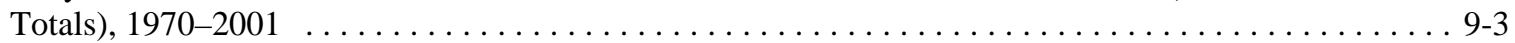

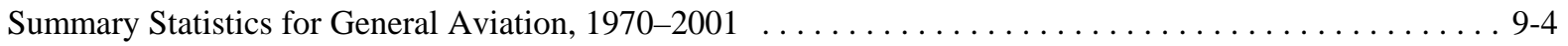

Summary Statistics for Domestic Waterborne Commerce, $1970-2001 \ldots \ldots \ldots \ldots \ldots \ldots \ldots$. . . . . . . .

Summary Statistics for Class I Freight Railroads, 1970-2001 . . . . . . . . . . . . . . . . . . . . . 9-10

Summary Statistics for the National Railroad Passenger Corporation (Amtrak), 1971 . . . . . . . . . . . 9 9-13

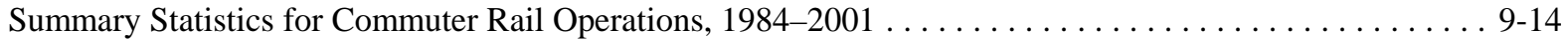

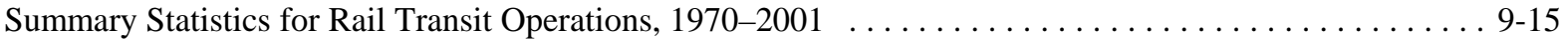

Supplies

Summary of 1996 Military Expenditures for Defending Oil Supplies from the Middle East . . . . . . . . 1-12

Surveys

Growth of Freight in the United States: Comparison of the 1997 and 1993 Commodity Flow Surveys . . . . 5-12

Growth of Freight Miles in the United States: Comparison of the 1997 and 1993 Commodity

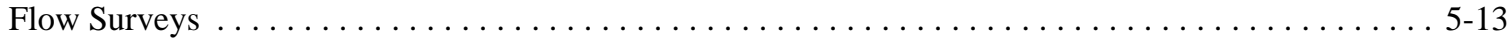

Survival

Automobile Scrappage and Survival Rates 1970, 1980 and 1990 Model Years . . . . . . . . . . . . . 3-13

Light Truck Scrappage and Survival Rates .................................. 3-15

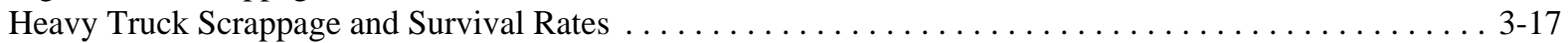

Systems

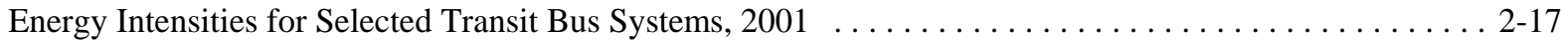

Class I Railroad Freight Systems in the United States Ranked by Revenue Ton Miles, 2001 . . . . . . . . 9-9 Tax

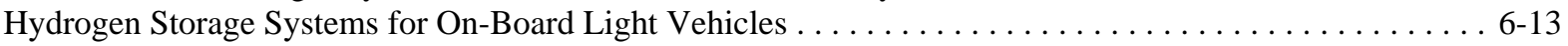

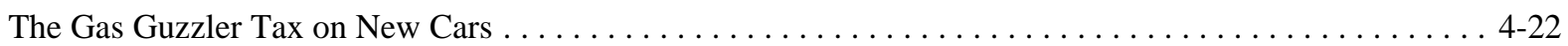

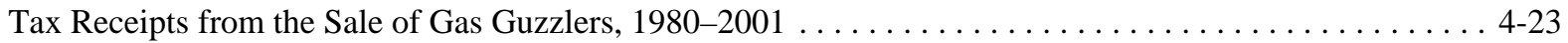

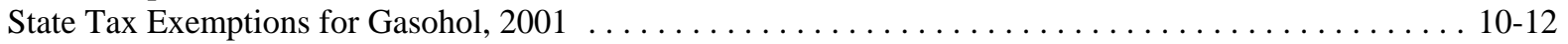

Taxes

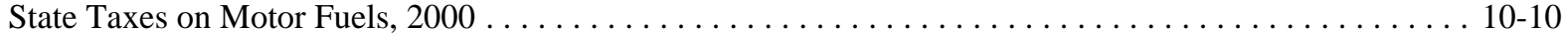

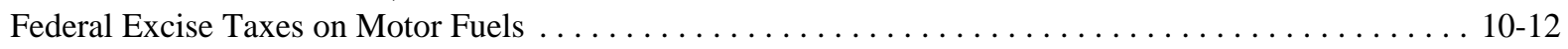

Technology

Sales and Specifications of Available Advanced Technology Vehicles . . . . . . . . . . . . . . . 6 6-8

Terminals

Terms

U.S. Hydrogen Production Plants and Storage Terminals $\ldots \ldots \ldots \ldots \ldots \ldots \ldots \ldots \ldots \ldots \ldots \ldots \ldots .6 \ldots \ldots \ldots \ldots$

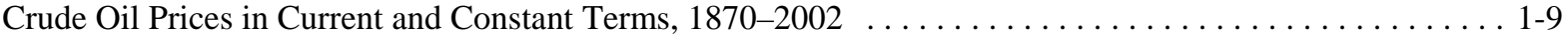

Tested

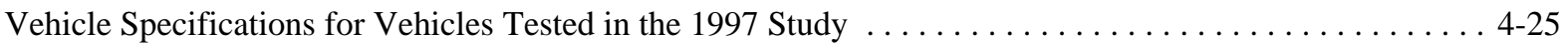

Steady Speed Fuel Economy for Vehicles Tested in the 1997 Study . . . . . . . . . . . . . . . . . 4-28

Thousand

Vehicles per Thousand People: U.S. Compared to Other Countries ........................ 3-6 Tier

Tier 2 Emission Standards for Cars and Light Trucks Effective for 2004-2009 Model Years . . . . . . . . 12-14

Light Vehicle Exhaust Emission Standards in Effect in 2009 when U.S. Tier 2 Standards are Final . . . . . 12-15 
Time

Average Length of Time Business Fleet Vehicles are in Service, $2001 \ldots \ldots \ldots \ldots \ldots$ Tire

Workers by Commute Time, 1990 and 2000 Census $\ldots \ldots \ldots \ldots \ldots \ldots \ldots$ Ton

Summary Statistics for Two-Axle, Four-Tire Trucks, $1970-2001 \ldots \ldots \ldots \ldots \ldots \ldots$

Ton-Miles of Petroleum and Petroleum Products in the U.S. by Mode, 1975-2001 . . . . . . . . . . . . . 1-20

Class I Railroad Freight Systems in the United States Ranked by Revenue Ton Miles, 2001 . . . . . . . . 9-9

Tonnage Totals

Tonnage Statistics for Domestic and International Waterborne Commerce, 1970-2001 . . . . . . . . 9-5

Summary Statistics for U.S. Domestic and International Certificated Route Air Carriers (Combined

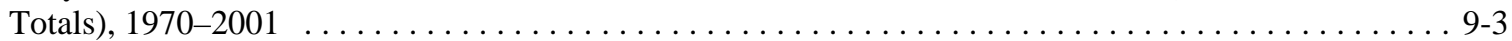

Traffic

Intermodal Rail Traffic, $1965-2001 \ldots \ldots \ldots \ldots \ldots \ldots \ldots \ldots \ldots \ldots \ldots$

Transit

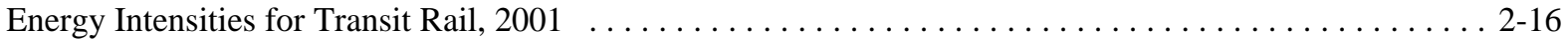

Energy Intensities for Selected Transit Bus Systems, $2001 \ldots \ldots \ldots \ldots \ldots \ldots \ldots \ldots \ldots$

Summary Statistics on Light Transit Vehicles, $1994-2001 \ldots \ldots \ldots \ldots \ldots \ldots$

Transit (continued)

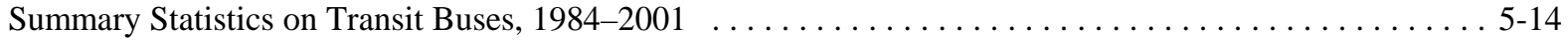

Summary Statistics for Rail Transit Operations, $1970-2001 \ldots \ldots \ldots \ldots \ldots \ldots$

Transportation

Domestic Consumption of Transportation Energy by Mode and Fuel Type, $2001 \ldots \ldots \ldots$

Transportation Energy Use by Mode, $2000-2001 \ldots \ldots \ldots \ldots \ldots \ldots \ldots \ldots$

Highway Transportation Energy Consumption by Mode, $1970-2001 \ldots \ldots \ldots$. . . . . . . . . . . . . 2-8

Nonhighway Transportation Energy Consumption by Mode, $1970-2001 \ldots \ldots \ldots$. . . . . . . . . . . 2-9

Means of Transportation to Work, 1980, 1990 and 2000 Census $\ldots \ldots \ldots \ldots \ldots$

Transportation-related Employment, 1993 and $2002 \ldots \ldots \ldots \ldots \ldots \ldots \ldots \ldots \ldots \ldots$

U.S. Carbon Dioxide Emissions from Energy Use in Transportation Sector, 1990-2001 . . . . . . . . . . 11-6 Travel

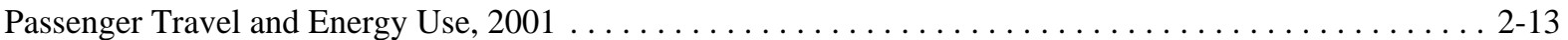

Automobiles in Operation and Vehicle Travel by Age, 1970 and 2001 . . . . . . . . . . . . . . . . . . 3-9

Trucks in Operation and Vehicle Travel by Age, 1970 and $2001 \ldots \ldots \ldots \ldots \ldots$

Average Annual Vehicle-Miles of Travel for Fleet Vehicles, $2000 \ldots \ldots \ldots \ldots \ldots \ldots$

Traveled

Average Number of Vehicles and Vehicle Travel per Household, 1990 NPTS and 2001 NHTS . . . . . . . 8-9

Shares of Highway Vehicle-Miles Traveled by Vehicle Type, 1970-2001 . . . . . . . . . . . . . . . 3-8

Average Annual Person-Miles Traveled, Person Trips and Trip Length per Household by Selected

Trip Purposes, 1983, 1990, 1995 NPTS and 2001 NHTS . . . . . . . . . . . . . . . . . . 8-8

Trip

Average Annual Vehicle-Miles, Vehicle Trips and Trip Length per Household 1969, 1977, 1983, 1990,

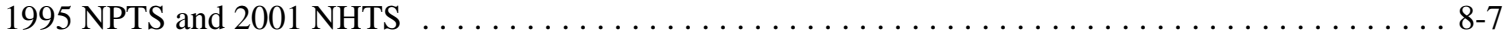

Average Annual Person-Miles Traveled, Person Trips and Trip Length per Household by Selected

Trip Purposes, 1983, 1990, 1995 NPTS and 2001 NHTS . . . . . . . . . . . . . . . . 8-8

Average Vehicle Occupancy by Trip Purpose, 1977 NPTS and 2001 NHTS . . . . . . . . . . . . . 8-11

Walk and Bike Trips by Trip Purpose, 2001 NHTS . . . . . . . . . . . . . . . . . . . . . . . 8-19 Trips

Average Annual Vehicle-Miles, Vehicle Trips and Trip Length per Household 1969, 1977, 1983, 1990,

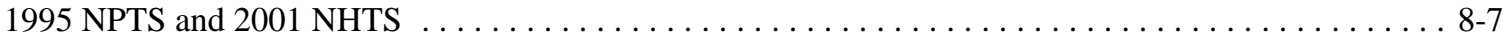

Average Annual Person-Miles Traveled, Person Trips and Trip Length per Household by Selected

Trip Purposes, 1983, 1990, 1995 NPTS and 2001 NHTS 


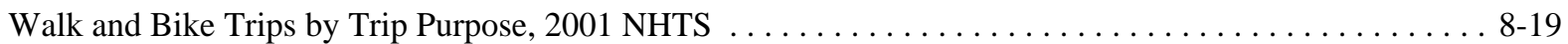

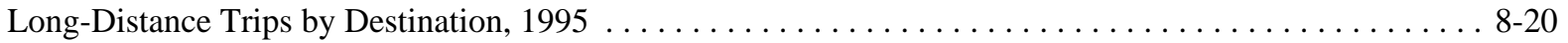

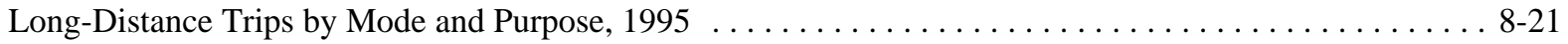

Truck

Shares of Long-Distance Person Trips by Mode and Household Income, $1995 \ldots \ldots \ldots \ldots \ldots \ldots \ldots . . .28$

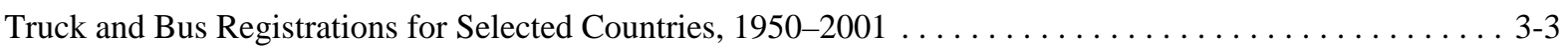

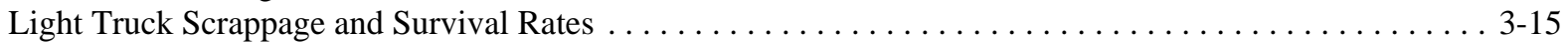

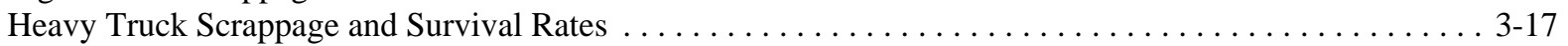

Light Truck Corporate Average Fuel Economy (CAFE) Standards versus Sales-Weighted Fuel

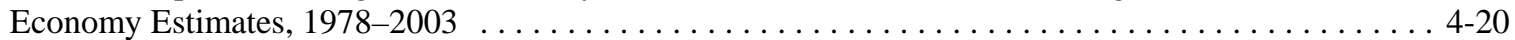

New Retail Truck Sales by Gross Vehicle Weight, $1970-2002 \ldots \ldots \ldots \ldots \ldots \ldots \ldots \ldots \ldots \ldots \ldots . . \ldots \ldots$

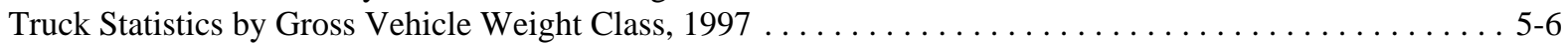

Truck Harmonic Mean Fuel Economy by Size Class, 1992 and $1997 \ldots \ldots \ldots \ldots \ldots \ldots \ldots \ldots \ldots \ldots$

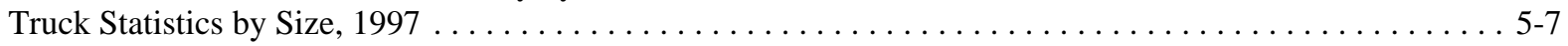

Trucks

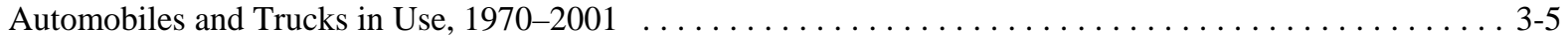

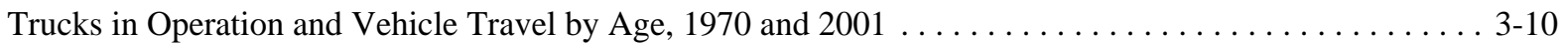

Average Age of Automobiles and Trucks in Use, $1970-2001 \ldots \ldots \ldots \ldots \ldots \ldots \ldots \ldots \ldots \ldots \ldots . .11$

Average Age and Registrations of Automobiles and Trucks, $1970-2001 \ldots \ldots \ldots \ldots \ldots \ldots \ldots \ldots \ldots . .12$

Summary Statistics for Two-Axle, Four-Tire Trucks, $1970-2001 \ldots \ldots \ldots \ldots \ldots \ldots \ldots \ldots \ldots \ldots$

Trucks (continued)

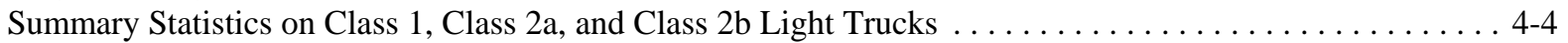

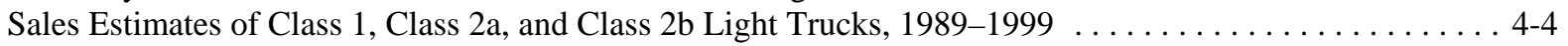

New Retail Sales of Trucks 10,000 pounds GVW and Less in the United States, 1970-2002 . . . . . . . 4 4-6

Sales-Weighted Engine Size of New Domestic and Import Light Trucks by Size Class, Sales Periods

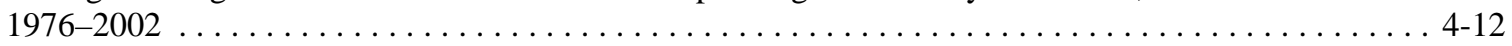

Sales-Weighted Wheelbase of New Automobiles and Light Trucks, Sales Periods 1976-2002 . . . . . . . 4-15

Period Sales, Market Shares, and Sales-Weighted Fuel Economies of New Domestic and Import

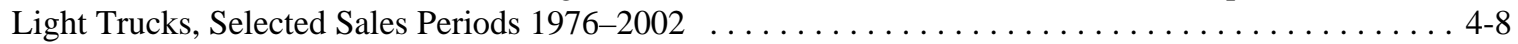

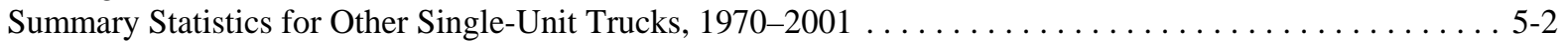

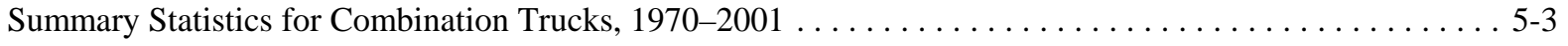

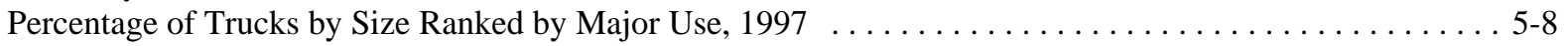

Percentage of Trucks by Fleet Size and Primary Fueling Facility, $1997 \ldots \ldots \ldots \ldots \ldots \ldots \ldots \ldots \ldots \ldots$. . . . . . . . .

Percentage of Trucks by Major Use and Primary Fueling Facility, 1997 . . . . . . . . . . . . . . . . . . 5-10

Tier 2 Emission Standards for Cars and Light Trucks Effective for 2004-2009 Model Years . . . . . . . . 12-14

Federal Exhaust Emission Certification Standards for Gasoline- and Diesel-Powered Light Trucks

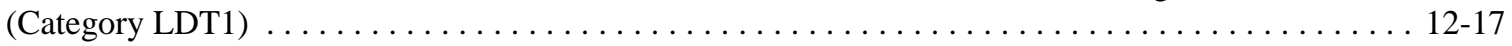

Federal Exhaust Emission Certification Standards for Gasoline- and Diesel-Powered Light Trucks

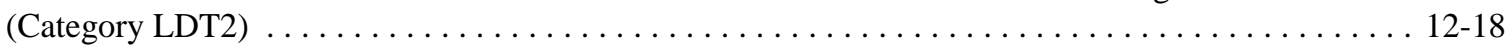

Federal Exhaust Emission Certification Standards for Gasoline- and Diesel-Powered Light Trucks (Category LDT3) ............................................. 12-19

Federal Exhaust Emission Certification Standards for Gasoline- and Diesel-Powered Light Trucks

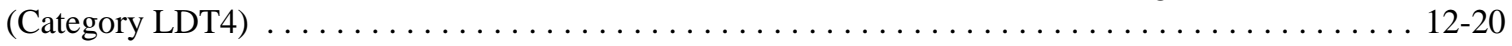

Federal Exhaust Emission Certification Standards for Gasoline- and Diesel-Powered Light

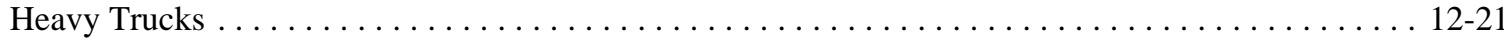

Federal Exhaust Emission Certification Standards for Gasoline- and Diesel-Powered Heavy

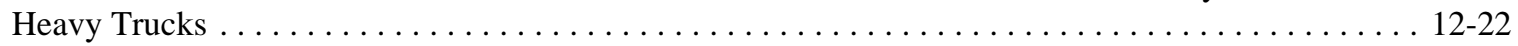

California Passenger Cars and Light Trucks Emission Certification Standards for Model Years

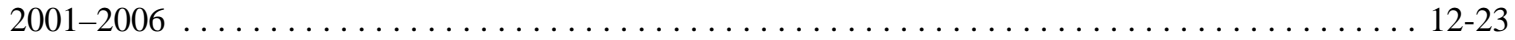
Unit

California Vehicle Emission Reduction for Passenger Cars and Light Trucks $\ldots \ldots \ldots \ldots \ldots \ldots \ldots . . \ldots \ldots$ 12-24 United

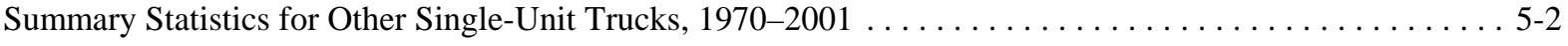


United States Petroleum Production, Imports and Exports, $1950-2002 \ldots \ldots \ldots \ldots$

Intercity Freight Movement and Energy Use in the United States, $2001 \ldots \ldots \ldots \ldots$

Vehicle Stock and New Sales in the United States, 2001 Calendar Year . . . . . . . . . . . . . . . 3-7

New Retail Automobile Sales in the United States, 1970-2002 . . . . . . . . . . . . . . . . . . 4-5

New Retail Sales of Trucks 10,000 pounds GVW and Less in the United States, 1970-2002 . . . . . . . . . 4-6

Growth of Freight in the United States: Comparison of the 1997 and 1993 Commodity Flow Surveys . . . . 5-12

Growth of Freight Miles in the United States: Comparison of the 1997 and 1993 Commodity

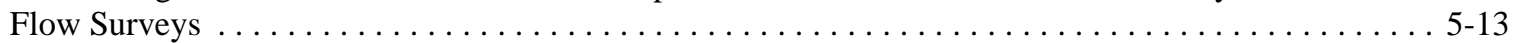
Urban

Class I Railroad Freight Systems in the United States Ranked by Revenue Ton Miles, 2001 . . . . . . . . 9-9 US06

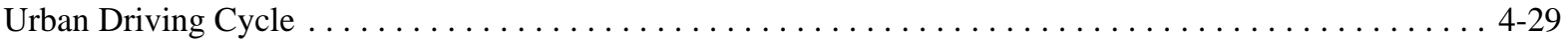

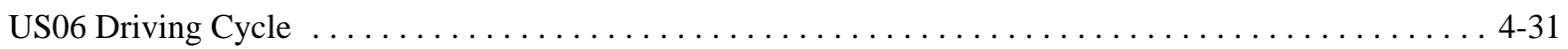

Vehicle

Vehicle Stock and New Sales in the United States, 2001 Calendar Year . . . . . . . . . . . . . . . . 3-7

Shares of Highway Vehicle-Miles Traveled by Vehicle Type, 1970-2001 . . . . . . . . . . . . . . . 3-8

Automobiles in Operation and Vehicle Travel by Age, 1970 and $2001 \ldots \ldots \ldots \ldots$

Trucks in Operation and Vehicle Travel by Age, 1970 and $2001 \ldots \ldots \ldots \ldots \ldots$

Light Vehicle Market Shares by Size Class, Sales Periods 1976-2002 . . . . . . . . . . . . . . . . . . 4-9

New Light Vehicle Dealerships and Sales, $1970-2000$. . . . . . . . . . . . . . . . . . . . . 4-17

Vehicle (continued)

Vehicle Specifications for Vehicles Tested in the 1997 Study . . . . . . . . . . . . . . . . . . 4-25

Occupant Fatalities by Vehicle Type and Nonoccupant Fatalities, 1975-2000 . . . . . . . . . . . . 4-34

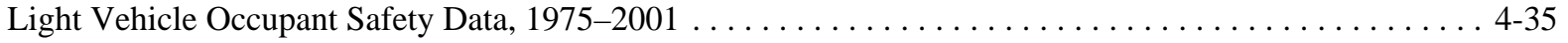

Crashes by Crash Severity, Crash Type, and Vehicle Type, $2001 \ldots \ldots \ldots \ldots \ldots$

Percent Rollover Occurrence in Fatal Crashes by Vehicle Type, $2001 \ldots \ldots \ldots$. . . . . . . . . . . . . . . 4-37

New Retail Truck Sales by Gross Vehicle Weight, 1970-2002 . . . . . . . . . . . . . . . . . . . . . 5-4

Truck Statistics by Gross Vehicle Weight Class, $1997 \ldots \ldots \ldots \ldots \ldots \ldots \ldots \ldots$

New Light Fleet Vehicle Purchases by Vehicle Type, $2000 \ldots \ldots \ldots \ldots \ldots \ldots \ldots \ldots$

Average Annual Vehicle-Miles of Travel for Fleet Vehicles, $2000 \ldots \ldots \ldots \ldots \ldots \ldots$

Average Miles per Domestic Federal Vehicle by Vehicle Type, $2001 \ldots \ldots \ldots \ldots \ldots$

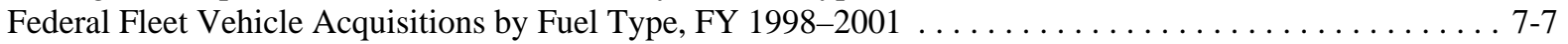

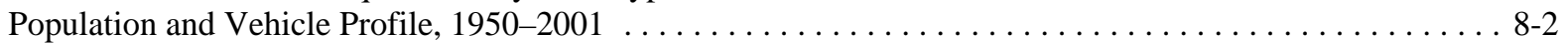

Population and Vehicle Ratios, $1950-2001 \ldots \ldots \ldots \ldots \ldots \ldots \ldots \ldots \ldots$

Household Vehicle Ownership, 1960-2000 Census . . . . . . . . . . . . . . . . . . . . . . 8-5

Average Annual Vehicle-Miles, Vehicle Trips and Trip Length per Household 1969, 1977, 1983, 1990,

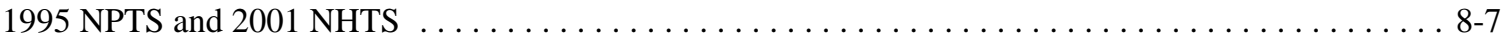

Average Number of Vehicles and Vehicle Travel per Household, 1990 NPTS and 2001 NHTS . . . . . . . 8-9

Average Vehicle Occupancy by Vehicle Type, 1995 NPTS and 2001 NHTS . . . . . . . . . . . . . . 8-10

Average Vehicle Occupancy by Trip Purpose, 1977 NPTS and 2001 NHTS . . . . . . . . . . . . . 8-11

Average Annual Miles per Vehicle by Household Vehicle Ownership, 2001 NHTS . . . . . . . . . . . . . 8 8-12

Average Age of Vehicles by Household Vehicle Ownership, 2001 NHTS . . . . . . . . . . . . . 8-12

Average Annual Miles per Household Vehicle by Vehicle Age . . . . . . . . . . . . . . . . . . . 8-13

Light Vehicle Exhaust Emission Standards in Effect in 2009 when U.S. Tier 2 Standards are Final . . . . 12-15

California Vehicle Emission Reduction for Passenger Cars and Light Trucks . . . . . . . . . . . . . . 12-24 Vehicles

Vehicles per Thousand People: U.S. Compared to Other Countries . . . . . . . . . . . . . . . 3-6

Vehicle Specifications for Vehicles Tested in the 1997 Study . . . . . . . . . . . . . . . . . . . . . . . 4-25

Steady Speed Fuel Economy for Vehicles Tested in the 1997 Study . . . . . . . . . . . . . . . . . 4-28

Summary Statistics on Light Transit Vehicles, $1994-2001 \ldots \ldots \ldots \ldots \ldots \ldots \ldots$

Estimates of Alternative Fuel Vehicles in Use, $1992-2002 \ldots \ldots \ldots \ldots \ldots \ldots \ldots$

Estimates of Alternative Fuel Vehicles by Ownership, 1996 and $2002 \ldots \ldots \ldots \ldots$

Alternative Fuel Vehicles Available by Manufacturer, Model Year $2001 \ldots \ldots$. . . . . . . . . . . . . . 6-5 
Sales and Specifications of Available Advanced Technology Vehicles . . . . . . . . . . . . . . 6 6-8

Hydrogen Storage Systems for On-Board Light Vehicles . . . . . . . . . . . . . . . . . . . 6 6-13

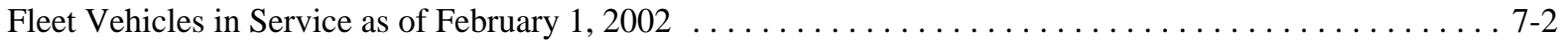

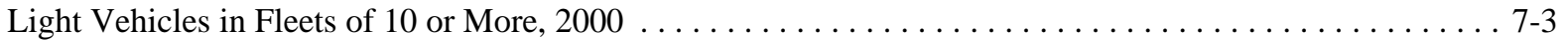

Average Length of Time Business Fleet Vehicles are in Service, 2001 . . . . . . . . . . . . . . . . 7-4

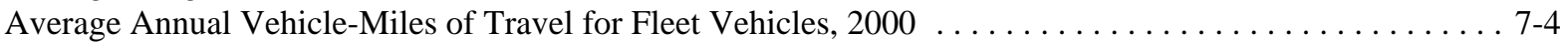

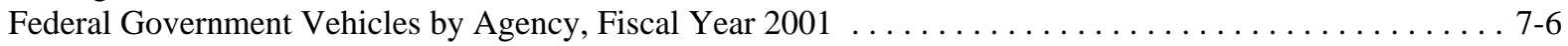

Energy Policy Act Purchase Requirements of Light Alternative Fuel Vehicles . . . . . . . . . . . . . 7-8

Average Number of Vehicles and Vehicle Travel per Household, 1990 NPTS and 2001 NHTS . . . . . . . 8-9

Average Age of Vehicles by Household Vehicle Ownership, 2001NHTS . . . . . . . . . . . . . . . . . 8 8-12

Emissions of Carbon Monoxide from Highway Vehicles, 1980-2001 . . . . . . . . . . . . . . . . . . 12-4

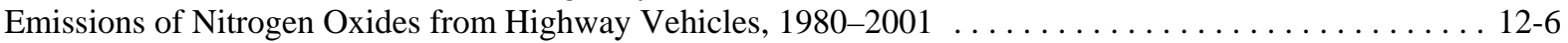

Emissions of Volatile Organic Compounds from Highway Vehicles, $1980-2001$. . . . . . . . . . . . . 12-8

Emissions of Particulate Matter (PM 10) from Highway Vehicles, $1980-2001 \ldots \ldots \ldots \ldots \ldots \ldots \ldots \ldots$. . . . . . . . . .

Emissions of Particulate Matter (PM-2.5) from Highway Vehicles, 1990-2001 . . . . . . . . . . . . 12-12

Federal Exhaust Emission Certification Standards for Gasoline- and Diesel-Powered Light Vehicles . . . 12-16 Volatile

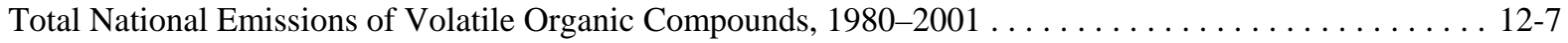
Walk

Emissions of Volatile Organic Compounds from Highway Vehicles, $1980-2001$. . . . . . . . . . . . 12-8

Walk and Bike Trips by Trip Purpose, 2001 NHTS $\ldots \ldots \ldots \ldots \ldots \ldots \ldots \ldots \ldots \ldots \ldots \ldots . \ldots \ldots$. . . . . . . . . . . . . Warming

Numerical Estimates of Global Warming Potentials Compared with Carbon Dioxide $\ldots \ldots \ldots \ldots \ldots \ldots . . \ldots 11-3$ Waterborne

Tonnage Statistics for Domestic and International Waterborne Commerce, 1970-2001 . . . . . . . . . . 9-5

Summary Statistics for Domestic Waterborne Commerce, $1970-2001 \ldots \ldots \ldots \ldots \ldots \ldots \ldots .6 .6 \ldots$ Weight

Sales-Weighted Curb Weight of New Domestic and Import Automobiles by Size Class, Sales Periods

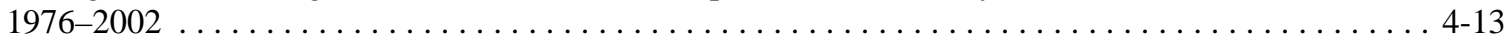

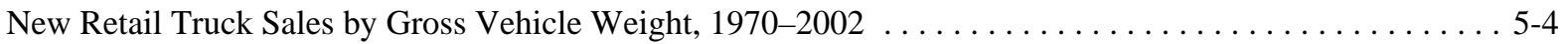

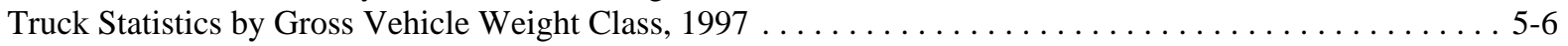
Weighted

Period Sales, Market Shares, and Sales-Weighted Fuel Economies of New Domestic and Import Automobiles, Selected Sales Periods $1976-2002$. . . . . . . . . . . . . . . . . . . . . 4-7

Period Sales, Market Shares, and Sales-Weighted Fuel Economies of New Domestic and Import Light Trucks, Selected Sales Periods $1976-2002$. . . . . . . . . . . . . . . . . . . . . 4-8

Sales-Weighted Engine Size of New Domestic and Import Automobiles by Size Class, Sales Periods

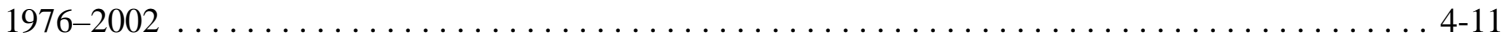

Sales-Weighted Engine Size of New Domestic and Import Light Trucks by Size Class, Sales Periods

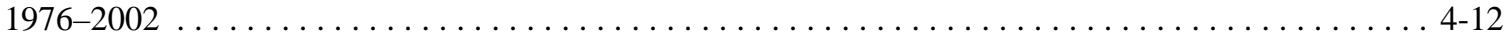

Sales-Weighted Curb Weight of New Domestic and Import Automobiles by Size Class, Sales Periods

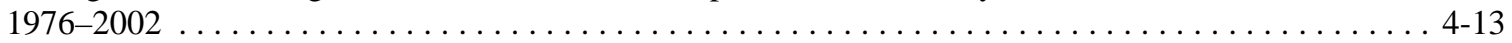

Sales-Weighted Interior Space of New Domestic and Import Automobiles by Size Class, Sales Periods

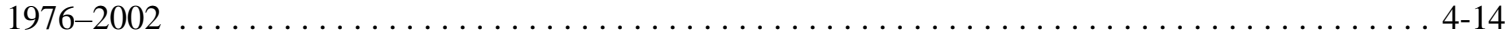

Sales-Weighted Wheelbase of New Automobiles and Light Trucks, Sales Periods 1976-2002 . . . . . . . 4 4-15

Automobile Corporate Average Fuel Economy (CAFE) Standards versus Sales-Weighted Fuel Economy Estimates, 1978-2003 . . . . . . . . . . . . . . . . . . . . . . . . . . . . . . 4-19

Light Truck Corporate Average Fuel Economy (CAFE) Standards versus Sales-Weighted Fuel

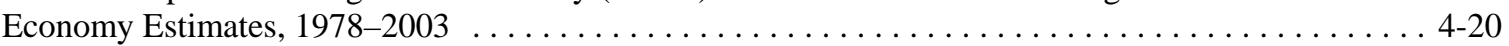
Wheelbase

Sales-Weighted Wheelbase of New Automobiles and Light Trucks, Sales Periods 1976-2002 . . . . . . . . 4-15 Work

Means of Transportation to Work, 1980, 1990 and 2000 Census 
Workers

Workers by Commute Time, 1990 and 2000 Census $\ldots \ldots \ldots \ldots \ldots \ldots \ldots$ World

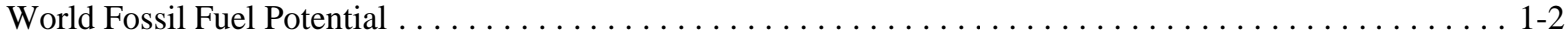

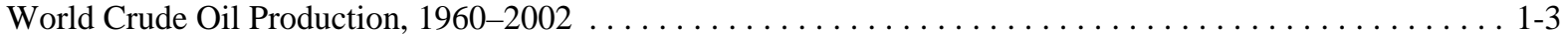

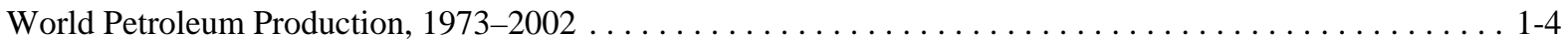

World Petroleum Consumption, $1960-2002 \ldots \ldots \ldots \ldots \ldots \ldots \ldots \ldots \ldots \ldots \ldots$

World Oil Reserves, Production and Consumption, $2002 \ldots \ldots \ldots \ldots \ldots \ldots \ldots$

World Natural Gas Reserves, Production, and Consumption, $2000 \ldots \ldots \ldots \ldots \ldots$

U.S. Petroleum Imports by World Region of Origin, $1960-2002 \ldots \ldots \ldots \ldots \ldots \ldots$

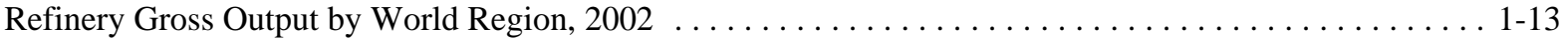

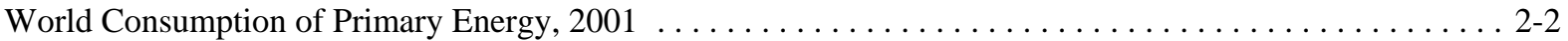

U.S. and World Hydrogen Consumption by End-Use Category, 1999 . . . . . . . . . . . . . . . . . . . 6-12

World Carbon Emissions from Energy Consumption, 1990 and $2001 \ldots \ldots \ldots \ldots$ Years

Automobile Scrappage and Survival Rates 1970, 1980 and 1990 Model Years . . . . . . . . . . . . 3-13

Tier 2 Emission Standards for Cars and Light Trucks Effective for 2004-2009 Model Years . . . . . . . . 12-14 Yield

Refinery Yield of Petroleum Products from a Barrel of Crude Oil, 1978-2002 